\title{
RESEARCH IN PROGRESS
}

BETWEEN 1 JULY 1991 and 30 JUNE 1992

\section{U.S. ARMY RESEARCH OFFICE}

(REPORT EXEMPT FROM REPORTS CONTROL

UNDER AR 335-15, PARAGRAPH 5-2b(9))

P.O. Box 12211

Research Triangle Park, N.C. 27709-2211

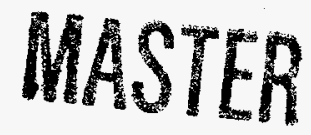

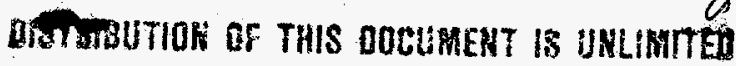




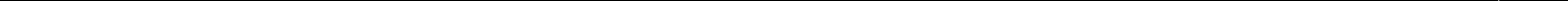




\section{DISCLAIMER}

This report was prepared as an account of work sponsored by an agency of the United States Government. Neither the United States Government nor any agency thereof, nor any of their employees, make any warranty, express or implied, or assumes any legal liability or responsibility for the accuracy, completeness, or usefulness of any information, apparatus, product, or process disclosed, or represents that its use would not infringe privately owned rights. Reference herein to any specific commercial product, process, or service by trade name, trademark, manufacturer, or otherwise does not necessarily constitute or imply its endorsement, recommendation, or favoring by the United States Government or any agency thereof. The views and opinions of authors expressed herein do not necessarily state or reflect those of the United States Government or any agency thereof. 


\section{DISCLAIMER}

Portions of this document may be illegible in electronic image products. Images are produced from the best available original document. 


\section{Foreword}

The U.S. Army Research Office, under the U.S. Army Materiel Command (AMC), is responsible for coordinating and supporting research in the physical and engineering sciences, in materials science, geosciences, biology, and mathematics.

This report describes research directly supported by the Army Research Office as well as research supported through this office by the Advanced Research Projects Agency, and several AMC and other Army commands. A separate section is devoted to the research program at the U.S. Army Research, Development and Standardization Group - United Kingdom.

The present volume includes the research program in physics, chemistry, biological sciences, mathematics, engineering sciences, metallurgy and materials science, geosciences, electronics, and the European Research Program. It covers the 12-month period from 1 July 1991 through 30 June 1992.

Additional copies of this report may be requested by writing to this office, attention AMXRO-RT-IP.

GERALD J. IAFRATE

Director 


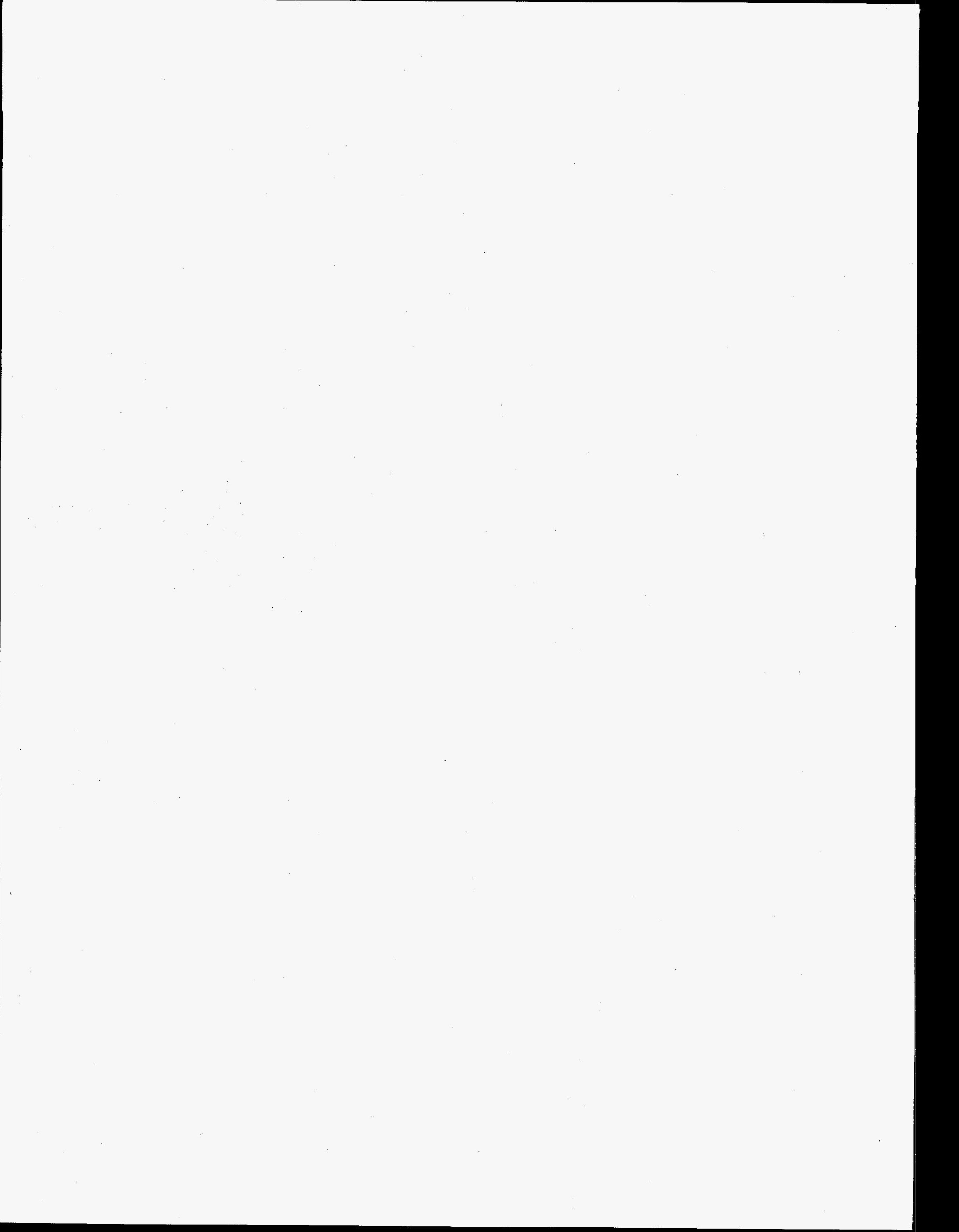




\section{Table of Contents}

Foreword

List of Abbreviations . ................. vii

\section{Physics}

A. General Physics ................. 1

B. Atomic and Molecular Physics ......... 1

C. Fluid and Plasma Physics ........... 10

D. Solid State Physics ................ 10

E. Acoustics, Optics and Cross

Disciplinary

\section{Chemistry}

A. Inorganic, Analytical, and Electrochemistry ................. 33

B. Organic Synthesis and Mechanisms ..... 39

C. Structural, Surface Chemistry and Spectroscopy ................... 43

D. Kinetics and Thermodynamics ........47 47

E. Polymer Chemistry ............... 53

\section{Biological Sciences}

A. Combat Ration Research ...........6 61

B. Biodegradation $\ldots \ldots \ldots \ldots \ldots \ldots \ldots 66,62$

C. Biotechnology .................6 64

D. Defense Against Chemical and Biological

Weapons ..................... 70

E. Sensory Factors in Performance

Enhancement ................. 74

F. Optimization of Physical Principles in

Biological Systems . .

\section{Mathematics}

A. Applied Analysis and Physical

Mathematics ................... 79

B. Numerical Methods and Scientific

Computing ..................... 85

C. Statistics and Probability ........... 94

D. Systems, Control, Modeling and

Artificial Intelligence ............. 103

E. Logistics and Operation Methods ..... 106

F. Special Projects ................ 110

\section{Engineering Sciences}

A. Solid Mechanics $\ldots \ldots \ldots \ldots \ldots \ldots . . .121$

B. Fluid Dynamics $\ldots \ldots \ldots \ldots \ldots \ldots \ldots \ldots \ldots$

C. Combustion ................... 134

D. Structures and Dynamics ........... 142

E. Multidisciplinary Mechanics ......... 148

\section{Metallurgy and Materials Science}

A. Degradation, Protection, and

Reaction ....................... 151

B. Mechanical Behavior .............. 159

C. Processing ..................... 163

D. Physical Behavior ............... 172

E. High Strain Rate Behavior of

Materials ........................ 179

F. Miscellaneous .................. 181

\section{Geosciences}

A. Geomorphology/Hydrology .......... 185

B. Snow, Ice and Frozen Ground ......... 187

C. Other (Terrestrial) .................. 189

D. Propagation ..................... 189

E. Atmospheric Remote Sensing ......... 193

F. Small-Scale Atmospheric

Processes ........................ 194

G. Aerosol Research ................ 196

\section{Electronics}

A. Physical Electronics ............... 199

B. Electron Devices ................ 203

C. Antennas and EM Detection ......... 212

D. Circuits, Networks and Related Systems .....................218

E. Signal Processing, Communications and Related Systems .................. 218

F. External Program .................226

G. Joint Services Electronics Program . . . . . 229

H. Miscellaneous ................. 232

\section{European Research Program}

A. Mathematics and Physics ...........233

B. Chemistry and Biological Sciences .....235

C. Aeronautics and Mechanics ..........238

D. Materials Science .................. 238

E. Environmental Sciences ............242

F. Electronics and Computer

Sciences ........................244

\section{Indexes}

Proposal Numbers ...................247

Scientific Liaison and Scientific Cognizance

Representatives ..................251

Contractors and Grantees ..............2257

Principal Investigators . . . . . . . . . . . 263

Subject Index ...................271 


\section{Abbreviations}

ACAA

AERO DIR

AFAL

AFESC

AFOSR

AIRMICS

AMBRDL

AMCCOM

AMRICD

AMSAA

AP TEC DIR

ARDEC

ARI

ARL

ARL/BED

ARL/BELVOIR2

ARL/EPSD

ARL/HRED

ARL/MD

ARL/VPD

ARL/VSD

ARL/WTD

ARO

ARPA

ATCOM

ATHAMA

BRADEC

BWL

CCAD

CECOM

CERL

CRREL

DPG

EOARD

ERDEC

ETL

MICOM

NRDEC

NRL

NVESD

OASA (RDA)

ONR

RIA

$\mathrm{SC}$

SDC

SDIO/IST

SL

SMO
U.S. Army Concepts Analysis Agency

Aeroflightdynamics Directorate, ATCOM

Air Force Astronautics Laboratory (AFSC)

Air Force Engineering and Service Center

Air Force Office of Scientific Research

Army Institute for Research in Management Information and Computer Sciences

U.S. Army Medical Bioengineering Research and Development Laboratory

U.S. Army Armament, Munitions and Chemical Command

U.S. Army Medical Research Institute of Chemical Defense

U.S. Army Materiel Systems Analysis Activity

Aviation Applied Technology Directorate, ATCOM

U.S. Army Armament Research, Development and Engineering Center

U.S. Army Research Institute for the Behavioral and Social Sciences

U.S. Army Research Laboratory

U.S. Army Research Laboratory/Battlefield Environments Directorate

U.S. Army Research Laboratory/Nonlinear Optical Devices Branch

U.S. Army Research Laboratory/Electronics \& Power Sources Directorate

U.S. Army Research Laboratory/Human Research and Engineering Directorate

U.S. Army Research Laboratory/Materials Directorate

U.S. Army Research Laboratory/Vehicle Propulsion Directorate

U.S. Army Research Laboratory/Vehicle Structures Directorate

U.S. Army Research Laboratory/Weapons Technology Directorate

U.S. Army Research Office

Advanced Research Projects Agency

U.S. Army Aviation and Troop Command

U.S. Army Toxic and Hazardous Materials Agency

U.S. Army Belvoir Research, Development and Engineering Center

Benet Weapons Laboratories, CCAC, ARDEC

Corpus Christi Army Depot

U.S. Army Communications-Electronics Command

Construction Engineering Research Laboratory

U.S. Army Cold Regions Research and Engineering Laboratory

U.S. Army Dugway Proving Ground

European Office of Aerospace R\&D

Edgewood Research, Development and Engineering Center

Army Engineer Topographic Laboratories

U.S. Army Missile Command

U.S. Army Natick Research, Development and Engineering Center

Naval Research Laboratory

Night Vision and Electronic Sensor Directorate

HODA, Office of the Assistant Secretary of the Army

Office of Naval Research

Rock Island Arsenal, ARRCOM

Scientific Cognizance

Strategic Defense Command

Strategic Defense Initiative Organization, Innovative Science and Technology

Scientific Liaison

U.S. Army Survivability Management Office 
SWC

TACOM

TECOM

TRADOC

USAIS

VAL

WES

WRAIR

WSMR
U.S. Army Signals Warfare Center CECOM

U.S. Army Tank-Automotive Command

U.S. Army Test and Evaluation Command

Training and Doctrine Command

U.S. Army Infantry School

U.S. Army Vulnerability Assessment Laboratory

Waterways Experiment Station

Walter Reed Army Institute of Research

White Sands Missile Range 


\section{PHYSICS}

\section{A. General Physics}

\section{ADVANCED NONLINEAR OPTICAL WAVEGUIDES, SWITCHES, LASERS AND MODULATORS FOR INTEGRATED INTERCONNECTS AND SYSTEMS}

G. Stegeman

University of Central Florida

SL: DARPA, ARL/EPSD

SC: ARI

GaAs/AlGaAs heterostructures of high quality, as evidenced from low-temperature photoluminescence spectra, have been grown by atmospheric pressure MOCVD. Impurity induced disordering of GaAs/AlGaAs MQW heterostructures has been obtained by $\mathrm{Zn}$ diffusion through $\mathrm{SiO}_{x}$ barriers. It was also demonstrated that the depth to which disordering would occur depends on the thickness of the $\mathrm{SiO}_{x}$ layer. Graded diffusion depths of $\mathrm{Zn}$ have been obtained with a graded $\mathrm{SiO}_{x}$ barrier. The barrier thickness is being tailored to vary the thickness of the disordered MOW layer from maximum at the laser section to a minimum at the modulator section of the MQW-SQW integrated structure. SQW laser structures fabricated using $\mathrm{Zn}$ diffused cladding layers show that the activation of $\mathrm{Zn}$ is lower than that required. A waveguide taper connecting a MQW and a SOW waveguide has been fabricated. A more efficient $M Q W$ modulator using $F$ implanted strained GaAsP/AlGaAs MQWs is being investigated. The potential of using this technique (impurity induced disordering) for integration has been demonstrated. It was observed, for the first time, that unlike AlGaAs/GaAs quantum wells fluorine impurity induced disordering in strained GaAsP/AlGaAs can be used to increase or decrease the effective band gap by varying the implantation energy of fluorine. $F$ implantation can be used to disorder the top MOW layer without affecting the SQW laser.
Reports:

1. Tailoring of Electron and Hole Energies in Strained GaAsP/AIGaAs Quantum Wells Using Fluorine Impurity Induced Layer Disordering, by Utpal Das et al., Appl Phys Let 60,210(1992). AD A249695

\section{B. Atomic and Molecular Physics}

\section{HIGH POWER SWITCHING AND OTHER HIGH POWER DEVICES}

Martin A. Gundersen

University of Southern California

Super-emissive cathode back-lighted thyratron (BLT) switch R\&D is transitioning to applications. Results include high current $(\approx 80 \mathrm{kA}$, single aperture), $100 \mathrm{kV}$ operation (3-gap in lab device, 2 gap in hermetically sealed switch), and other areas. There is strong interest for many different applications. Spin-offs of the switch to applications such as accelerators, plasma opening switches, lasers, high power pulse modulators, pollution control, are being explored. Interest has been expressed by most major pulsed power companies, national laboratories, and through invited talks at universities and meetings. Spin-off of BLT is high brightness $\left(10^{10} \mathrm{~A} / \mathrm{m}^{2} \mathrm{rad}^{2}\right)$ e-beam with very favorable properties (rugged, not poisoned, instant start, low power, low cost, long life) in comparison with presently available sources (dispenser, $\mathrm{LaB}_{6}$, graphite, velvet, $\mathrm{Cs}_{3} \mathrm{Sb}$, Os33b). Results show two phases of operation, leading to improved understanding of physics and applications. First phase is a hollow cathode phase, which produces the very interesting electron beam. Second phase is a super-emissive phase, which also produces a (different) electron beam, and is responsible for the high conduction current.

\section{Reports:}

No. 1-25 in previous editions.

26. Avalanche Injection Model for the Lock-On Effect in Photoconductive Power Switches, by H. Zhao et al., MS, J Appl Phys. 
27. A Comparative Study of Si and GaAs-Based Devices for Repetitive, High Energy, Pulsed Switching Applications, by P. Hadizad et al., MS, J Appl Phys.

28. Hollow Cathode and Super-Emissive Cathode Properties of Pseudospark and Back-Lighted Thyratrons, by M.A. Gundersen et al., MS.

29. Long Pulse Electron Beam Generation From The BackLighted Thyratron, by R-L. Liou et al., MS, Appl Phys Let.

30. Observation of Pulsed Microwave and Millimeter Wavelength Radiation During Hollow Cathode Discharge Operation of the Back Lighted Thyratron, by R. Liou et al., MS, Appl Phys Let.

31. A High-Power Electron Beam Source Based on the Superemissive Cathode, by T-Y.Hsu and M.A. Gundersen, MS, Appl Phys Let.

32. A Comparative Study of Si- and GaAs-Based Devices for Repetitive, High-Energy, Pulsed Switching Applications, by P. Hadizad et al., J Appl Phys 71,3586(1992).

\section{THE INTERACTIONS OF RADIATION WITH MATTER}

Steven T. Manson

Georgia State University

A paper has been prepared in which interactions between target and projectile electrons leading to ionization of one of the collision partners and simultaneous excitation of the other are investigated for fast clothed particle - clothed particle collisions. For $\mathrm{H}$ atom impact, the first Born approximation is used to demonstrate that the low energy electron emission is dominated by electron-electron rather than by electron-nucleus interaction processes. For a broad class of structured particle collision systems, the electron-electron interaction is shown to play an important, non-neglibible, role. Doubly differential cross sections for energetic $\mathrm{H}$ - He collisions illustrate this point. Comparison between sophisticated R-Matrix and simple central-field calculations for photoionization of $\mathrm{Al}^{+6}$ over a broad energy range is made. Except for the autoionization resonance, which is not included in the central-field model, agreement is excellent. Reasons for, and implications of, this agreement are discussed in another paper.

Reporis:

No. $1-5$ in previous editions.

6. Absolute Cross Sections for the Photoionization of the 6s6p P Excited State of Barium, by L.-W. He et al., Phys Rev Let 67,2131(1991). AD A249631

7. X-90, 15th International Conference on X-Ray and InnerShell Processes, by Manfred O. Krause et al., Comments Atom Mol Phys 26,247(1991). AD A249 350

8. Photoionization of the Outer Shells of Radon and Radium: Relativistic Random Phase Approximation for High-Z Atoms, by Pranawa C. Deshmukh et al., MS, Phys Rev.
9. Photoionization of Inner Shells of Excited Atoms: Dominance of Two-Electron Transitions, by Zineb Felfli and Steven T. Manson, MS, Phys Rev Let.

\section{ELECTRON-BEAM CONTROLLED SEMICONDUCTOR SWITCHES}

Karl H. Schoenbach

V.K. Lakdawala

Old Dominion University

\section{SL: ARL/EPSD}

An improved doping configuration for the cathode region of electron-beam controlled $\mathrm{GaAs}$ switches was developed which consists of an $n^{+}-p^{+}$structure. The $1000 \AA$ thick $n$-type layer is deposited on top of the cathodoluminescent $p$-type layer. This $n$-layer should prevent electron-beam generated electrons from reaching the surface and recombining nonradiatively, an effect which limits the internal quantum efficiency in the cathode region of electron-beam activated switches. GaAs samples with the desired properties were obtained and will be tested with a low-energy electron-beam. The construction of a low-energy, high-current density electron-beam gun is being continued. The components of the nanosecond e-beam gun, the pulse power circuit, the diagnostic circuit and the $\mathrm{N}_{2}$ - laser, which is used to generate photoelectrons from a dispenser cathode, have been tested. The assembly of the various components is underway. The feasibility of the electron-beam controlled closing and opening GaAs-switch concept with $\mathrm{MHz}$ repetition rate capability has been demonstrated.

\section{EXCIMER EMISSION FROM ALKALI DIATOMIC AND ALKALINE-EARTH-NOBLE-GAS MOLECULES}

John P. Huennekens

Lehigh University

\section{SL: NVESD}

A paper has been published which reports the observation of several six-wave mixing processes which result in broadly tunable coherent emission in the wavelength range $1.20-1.45 \mu \mathrm{m}$. These emissions are produced in potassium vapor which is simultaneously pumped by two pulsed dye lasers. One set of processes is produced when the frequency of the first laser is fixed to the potassium $4 \mathrm{~S} \rightarrow 6 \mathrm{~S}$ two-photon transition frequency, while the frequency of the sec- 
ond laser is tuned. The second set of processes is observed when both lasers are tuned, but with their sum frequency fixed to the $4 S \downarrow 6 S$ two-photon transition. Peak output energies of $\sim 10 \mathrm{~nJ} /$ pulse have been observed. The heteronuclear diatomic molecule $\mathrm{NaK}$ was excited using a single-mode ring dye laser over the wavelength range of $700-780 \mathrm{~nm}$. The resulting bound-free emission on the $1^{3} \Pi \rightarrow 1^{3} \Sigma^{+}$ band consists of broad oscillatory continua in the near-infrared. These spectra were inverted to determine parameters describing the $1^{3} \Sigma^{+}$repulsive wall above the dissociation limit. The inversion of the spectra was completed by comparison of experimental spectra with spectra calculated by full quantum mechanical techniques using a potential of the form $A e^{-B R}+C$ for the $1^{3} \Sigma^{+}$state.

Reports:

No. $1-4$ in previous editions.

5. Broadly Tunable Near-Infrared Six-Wave Mixing Processes in Potassium Vapor, by Z.J. Jabbour et al., Appl Phys B52,281(1991). AD A245 033

\section{DIAGNOSTICS OF DIAMOND FILM DEPOSITION PLASMAS}

Kenneth R. Stalder

Jay B. Jeffries

SRI Internationai

SL: ARO

Work has concentrated on mass spectroscopic measurements of arcjet produced plasmas and on laserinduced fluorescence measurements of $\mathrm{OH}$ radicals. Much of the effort expended on the arcjet was in design and construction of the components necessary for these measurements. Actual experiments using the quadrupole mass spectrometer system began in August 1991. The mass spectroscopic sampling was accomplished by impinging the arcjet plasma onto a tantalum surface at normal incidence. A number of experiments, with subsequent Raman analysis and microscopic inspection, confirmed that diamond films grew on the tantalum near the stagnation point (i.e., the point on the surface where the radial and axial velocity of the flow is zero). A portion of the gas in the stagnated flow diffused to the surface and was sampled through orifices and differential pumping sections such that a molecular beam of the arcjetproduced gases entered a quadrupole mass spectrometer. A tuning fork-type beam chopper and lock-in amplifier were used to detect the modulated molecular beam so that background effects could be eliminated. Mass scans of the beam were surveyed and qualitative agreement with model predictions of the appearance of various chemical species were confirmed. Other efforts measured the absolute concentration of the $\mathrm{OH}$ radical observed by laser-induced fluorescence (LIF) in the hot filament diamond CVD reactor. The calibration of LIF measurements to provide absolute number density of molecules is a complex task which is seldom done properly. One must know the absolute number density of $\mathrm{OH}$ to assess the importance of $\mathrm{OH}$ etching of the growing diamond/graphite surface. To do this absolute calibration three different quantities must be considered. First, the response and transmission of the optical detection system to a known number of photons per solid angle per optical bandwidth must be measured. Second, the collisional quenching of the excited state as a function of temperature and pressure must be determined. Third, the fraction of ground state molecules excited by the laser from the spatially resolved temperature measurements for the Boltzmann fraction and spectroscopic data on $\mathrm{OH}$ line strengths determined some ten years ago in the researchers' laboratories.

Reports:

No. 1-3 in previous editions.

4. Temperature Measurements by Laser-Induced Fluorescence of the Hydroxyl Radical, by David R. Crosley and Jay B. Jeffries, MS.

\section{FEMTOSECOND LASER STUDIES OF EXCITED STATE DYNAMICS}

Peter P. Sorokin

IBM Research Center

A practical scheme was demonstrated for both (1) generating femtosecond $193-\mathrm{nm}$ seed pulses at the $\mu \mathrm{J}$ pulse energy level and (2) amplifying these pulses to the $\mathrm{mJ}$ level in an ArF excimer gain module. In somewhat greater detail, third-order, difference-frequency $\left(2 \omega_{1}-w_{2}\right)$ mixing in xenon gas was utilized to generate the 193-nm seed pulses. All previous efforts to generate femtosecond UV seed pulses for subsequent amplification in excimer gain modules involved second-order, sum-frequency $\left(w_{1}+w_{2}\right)$ mixing in crystals such as KTP and BBO. The UV cut-off for efficient third-harmonic generation in BBO is 197.3 $\mathrm{nm}$. Thus, for the generation of femtosecond pulses further in the UV, one must rely upon the use of third-order mixing in gases. New schemes were also derived by which the UV and VUV pulses generated 
by third-order mixing in gases can be strongly amplified. Femtosecond-time-scale experiments, in which absorption spectra of fragments produced by photodissociation of gas-phase molecules are recorded in real time, are discussed. The observed spectra can generally be classified into two distinct categories: (1) impact spectra and (2) statistical spectra. It is shown that both types of spectra can be used to provide significant information about the difference potentials in the systems being studied.

Reports:

No. $1-4$ in previous editions.

5. Femtosecond Broadband Absorption Spectroscopy of Photodissociating Molecules, by J.H. Glownia et al., MS.

6. Amplification of 193-nm Femtosecond Seed Pulses Generated by Third-Order, Non-Resonant, Difference-Frequency Mixing in Xenon, by J.H. Glownia et al., Opt Let 17,337(1992). AD A249 468

\section{OPTICAL FREQUENCY DIVISION USING AN OPTICAL PARAMETRIC OSCILLATOR}

Ngai Chuen Wong

Massachusetts Institute of Technology

\section{SL: NVESD}

SC: ARL/EPSD

A paper has been prepared which presents experimental results of frequency tuning and stabilization of a type-II phase-matched potassium titanyl phosphate (KTP) doubly resonant optical parametric oscillator. Four tuning elements were employed to control the stability and tuning of the parametric oscillator. Discrete frequency tuning of a nearly degenerate optical parametric oscillator over a range of $\sim 3 \mathrm{THz}$ was obtained by crystal angle tuning and cavity length scanning. Continuous frequency tuning has been achieved over a $0.5-\mathrm{GHz}$ range through the use of temperature and electro-optic tuning of the KTP crystal. Using these frequency control techniques, researchers have phase locked the signalidler beat frequency to an external microwave frequency source, thus demonstrating tunable optical frequency division. The signal-idler beat-note linewidth was measured to be $25 \mathrm{mHz}$. Characteristics of two different cavity designs, their operations, tuning behavior, and stability issues are examined.

Reports:

No. 1 in previous edition.

2. Tunable Optical Frequency Division Using a Phase Locked Optical Parametric Oscillator, by D. Lee and N.C. Wong, Opt Let 17,13(1992). AD A249 519
3. Gravity-Wave Detection via an Optical Parametric Oscillator, by N.C. Wong, Phys Rev A45,3176(1992). AD A249520

4. Proposal for a $10 \mathrm{THz}$, Precision Optical Frequency Comb Generator, by N.C. Wong, MS, Electron Let.

5. Optical Frequency Counting From the UV to the Near-IR by N.C. Wong, MS, Opt Let.

\section{A HIGH FLUX RADICAL BEAM FOR DIAMOND GROWTH}

J.E. Lawler

L.W. Anderson

University of Wisconsin - Madison

\section{SL: ARO}

The goal of this investigation has been to develop a detailed understanding of the microscopic physics and chemistry of the chemical vapor deposition (CVD) of diamond thin films. A major scientific milestone has been achieved by developing a highly sensitive spectroscopic experiment for determining the absolute gas phase densities of the methyl radical, $\mathrm{CH}_{3}$, and other species during CVD of diamond. There is substantial evidence that $\mathrm{CH}_{3}$ is a key gas phase precursor of diamond. The method for monitoring $\mathrm{CH}_{3}$ densities during $\mathrm{CVD}$ of diamond uses the deep UV absorption feature of $\mathrm{CH}_{3}$ at $216 \mathrm{~nm}$. The sensitivity and simplicity of the experiment gives it a huge advantage over competing techniques for detecting $\mathrm{CH}_{3}$ and has permitted rapid progress. The initial report of the new diagnostic experiment has been published in Phys Lett A. Researchers have since used the experiment to measure effective activation energies for production of $\mathrm{CH}_{3}$ from both $\mathrm{CH}_{4}$ and $\left(\mathrm{CH}_{3}\right)_{3} \mathrm{COOC}\left(\mathrm{CH}_{3}\right)_{3}$. The technique can also be used in an intense glow discharge. It appears that the primary effect of the glow discharge is to dissociate $\mathrm{H}_{2}$ to make atomic hydrogen which subsequently drives gas phase chemistry which is similar to that in hot filament CVD systerns.

\section{UNCERTAINTY LIMITED ATOMIC POSITION MEASUREMENT USING OPTICAL FIELDS}

John E. Thomas

Duke University

The first demonstration of Raman induced resonance imaging of moving atoms was successfully completed. Employing a magnetic field gradient to yield a spatial variation of the Raman resonance frequency of $10^{9} \mathrm{~Hz} / \mathrm{cm}$, the experiments achieved a 
spatial resolution of 1.7 microns. This is at or near the theoretical limit for the atomic beam collimation employed in the experiments. To demonstrate the method, the image of the shadow cast in the atomic beam by a 25 micron gold coated tungsten wire was recorded. This work is viewed as an important new tool in the study of atomic optics, a newly emerging field. At present, a new experiment to demonstrate an atomic interferometer by spatial rephasing is being constructed. In this experiment, an atomic beam crosses two spatially separated optical regions which act as atomic diffraction gratings. These regions each consist of resonant laser fields which pass through a birefringent wedge. This generates a $\sigma+$ polarized field for which the intensity varies spatially with a period of twenty microns. Downstream from the atomic diffraction gratings, an atomic interference pattern will rephase in a Raman induced resonance imaging region where the pattern will be measured. Currently, the design of the region where the birefringent wedges are mounted is being finished. The initial vacuum system is complete and the wedges have been obtained and appear to conform to specifications. A primary goal of the research is the demonstration of suboptical wavelength atomic localization and position measurement using spatially varying light-shifts in the Raman induced resonance imaging scheme. Researchers have completed a study of optical pumping with the new atomic beam system and have attained $800-1000$ reduction in the final state population. This will be adequate for the first 0.1 micron resolution experiments.

\section{INFRARED/SUBMILLIMETER-WAVE DOUBLE RESONANCE STUDIES OF MOLECULAR COLLISION KINETICS}

David D. Skatrud

Duke University

A paper on the IR/millimeter-wave double resonance studies of rotational and vibrational relaxation in $\mathrm{CH}_{3} \mathrm{CI}$ was presented at the South Eastern Section of the American Physical Society. This paper was an expansion of the work presented at the Molecular Spectroscopy Symposium in June. That work showed initial indications that the $\Delta K=3 n$ rate might not be thermal in both $K$ and $J$, as it was for the previous work on $\mathrm{CH}_{3} \mathrm{~F}$. Since that time the data set has been greatly expanded: $K$ manifold in both $J=4-5$ and $J=6-7$ were obtained in addition to better signal/noise data on the $J=8-9$ transitions. Further- more, data were also obtained with a different pump transition. This additional data is crucial to examining the nonthermal $\Delta K=3 n$ rate since deviations from thermal are expected to be largest for levels far from the pumped transition. The data is currently being analyzed with models that employ master equation computer fits. Much of the reporting period was used to increase the signal/noise ratio of the data by improving the pump. In the previous period, the sample cell was modified to provide more uniform overlap of the pump and probe beams. The new cell also has a larger cross-sectional area, allowing larger pump intensities without saturation of the pumped transition. To utilize this capability the Q-switch was switched from the home built $\mathrm{CO}_{2}$ laser to a higher power commercial laser. Additional polarization discrimination had to be added to the laser because the larger gain of the commercial laser made it oscillate CW with the Q-switch in the "off" polarization. It is now operating with a factor of $\sim 5$ times more peak Q-switched power, which is a significant factor in obtaining the additional data described above.

\section{ELECTROMAGNETIC PROPAGATION AT ULTRAHIGH INTENSITIES}

\section{Charles Rhodes}

University of Illinois at Chicago

\section{SC: ARL/BED}

Recent theoretical and experimental studies have led to a fundamental breakthrough in the development of high-brightness $\mathrm{x}$-ray sources. These results affect researchers ability to controllably apply very high power densities in materials, the basic issue for the creation of bright and efficient sources of radiation in the $\mathrm{x}$-ray range. The main significance of this work is the establishment of the scaling law concerning the energy requirements for $x$-ray amplification. The critical governing issue, which determines the scaling relationship between the required excitation energy and the amplifier gain of $x$-ray lasers, is the spatial control the deposited energy. Recent experiments, which are supported by carefully developed theoretical analysis, have demonstrated the basic physics of a new form of self-channeled propagation exactly of the type necessary for the implementation of $x$-ray lasers. The confirming agreement between the theoretical predictions and the experimental finding can only be viewed as remarkable. These results repre- 
sent a profound change in the field of $x$-ray laser research because they alter drastically the fundamentall scaling relationships among the relevant physical variables.

Reports:

1. Theory of Stable Channeled Propagation of Intense Radiation in Plasmas Arising From Relativistic and Charge-Displacement Mechanisms, by A.B. Borisov et al., MS.

2. Excitation of Molecules and Solids with Intense Subpicosecond Ultraviolet Radiation, by T.S. Luk et al., MS.

3. Isotopic Studies of Atomic Site-Selectivity in Molecular Multiphoton Ionization of $\mathrm{N}_{2} \mathrm{O}$, by T.S. Luk et al., MS.

4. Observation of Relativistic/Charge-Displacement SelfChanneling of Intense Subpicosecond Ultraviolet (248 nm) Radiation in Plasmas, by A.B. Borisov et al, MS.

5. Method of Concentration of Power in Materials for X-Ray Amplification, by $\mathrm{K}$. Boyer et al., MS.

6. Studies of Plasmas Excited by Intense Subpicosecond Radiation for X-Ray Generation, by A. Zigler et al., MS.

7. Relativistic and Charge-Displacement Self-Channeling of Intense Short-Duration Laser Pulses in Plasmas, by A.B. Borisov et al., MS.

\section{SEMICONDUCTOR FILM CERENKOV LASERS}

John E. Walsh

Dartmouth College

$$
\text { SC: ARL }
$$

A mildly relativistic electron beam (3-3.6 MeV) has been used to generate Smith-Purcell (S-P) radiation over a spectral range extending from lower-millimeter to far-infrared wavelengths. The electron-beam was generated in a van der Graaf accelerator and was focussed and transported through an interaction region which contained a metal grating. Gratings with periods of 450,760 and $1000 \mu \mathrm{m}$ were used to produce the radiation. The emitted power was reflected from a plane mirror whose angle could be varied onto an off-axis, paraboloidal mirror and on into a diagnostic region. Radiation was detected with an $\mathrm{InSb}$ cryogenically-cooled detector and the spectrum was analyzed with a variety of filters and a grating spectrometer. Coincidence of the measured wavelength and the prediction from the S-P formula was verified in detail. Thus, the radiation consisted mainly of spontaneous S-P emission. However, the results indicate that a widely-tunable, FIR, S-P-free-electron laser is a practical possibility.

Reports:

1. Radio-Frequency-Injector-Driven, Cerenkov, Free-Electron Laser, by John E. Walsh and Emily E. Fisch, MS, Nucl Inst and Meth.
2. The Sapphire Cerenkov Laser, by Emily E. Fisch, PhD Thesis, 1991, 133 pp.

3. Operation of the Sapphire Cerenkov Laser, by E.E. Fisch and J.E. Walsh, Appl Phys Let 60,1298(1992). AD A249 110

4. Multimode Operation of the Grating-Coupled Oscillator at Submillimeter/FIR Wavelengths, by E.J. Praice and J.E. Walsh, MS.

5. First Observation of Smith-Purcell Radiation From Relativistic Electrons, by G. Doucas et al., MS, Phys Rev Let.

6. A New Geometric Approach to Wavelength Tuning of the Cerenkov Free-Electron Laser, by Emily E. Fisch and John E. Walsh, MS, Opt Let.

\section{SEMICONDUCTOR DEPOSITION AND ETCHING INTERACTIONS OF LASER-GENERATED TRANSLATIONALLY HOT ATOMS AND RADICALS}

Stephen R. Leone

University of Colorado

\section{SC: ARL/EPSD, ARL/MD}

Plasma etching of silicon is a complex process involving charged species, neutral molecules and radicals, and surface reactions. However, it is difficult to determine which reactions are central to the materials removal process and which are subsidiary. Recently, well-defined beams of translationally fast neutral chlorine molecules have been developed using a laser vaporization technique, and these beams have been used to study the etching process. This method allows the chemistry of the etching to be explored with selective energies in much more precise detail. The translationally energetic beam source is produced by laser vaporization of films. Chlorine molecules are deposited onto a cold transparent substrate, and the $355 \mathrm{~nm}$ output of an intense Nd:YAG pulsed laser is used to vaporize the film. The resulting superheated film evolves chlorine molecules with kinetic energies of 1-10 eV. These energies of the reactive molecules are sufficient to break the chemical bonds of atoms at the surface. The chlorine molecules impinge on a substrate of silicon $\mathrm{Si}(100)$ in an ultrahigh vacuum chamber and the pulsed production of silicon chloride products is detected with a mass spectrometer. Using thermal chlorine emanating from an effusive beam, no detectable etching is observed, but at the increased kinetic energies of 1-6 eV, sustained etching is detected on room temperature silicon. In addition, when the kinetic energy is increased, the rate of etching increases dramatically. A factor of 30 enhancement of the etch rate on room temperature silicon has already been observed. 
28569 VACUUM MICROELECTRONIC DEVICES AND THEIR

APPLICATIONS USING COMPOUND SEMICONDUCTOR TECHNOLOGY

Umesh K. Mishra

University of California, Santa Barbara

$$
\begin{aligned}
& \text { SL: ARL/EPSD } \\
& \text { SC: ARL }
\end{aligned}
$$

An innovative hot electron source will be developed for use in vacuum microelectronic devices. Hot electron emission in a novel planar doped barrier will be investigated in $\mathrm{GaAs}, \mathrm{AlGaAs}, \mathrm{Si}$, and wide bandgap semiconductors. The initial work will focus on the design, fabrication and testing of the vacuum electronic emitter. The innovative design relies in a drift region of dimension smaller than the mean free path of the electrons. In conjunction with the experimental work, a detailed theoretical study will be performed on the nature and magnitude of the injecting barrier under the constraints of this short drift region. After establishing a preferred vacuum emitter design, efforts will be directed towards the development of the requisite technologies for gate structures and incorporation in transistors and klystrons.

\section{NEW PYROOPTIC TECHNIQUES FOR IMAGING OF LONG WAVELENGTH IR-RADIATION}

L. Eric Cross

Amar S. Bhalla

The Pennsylvania State University

SL: NVESD

SC: ARL/EPSD

From a simple consideration of the thermal conversion efficiency which can be realized in the non-contact pyrooptic system it has been shown that for $f / 1$ optics a scene temperature change of $0.1^{\circ} \mathrm{C}$ will realize a change at the detector of $10^{-3{ }^{\circ}} \mathrm{C}$ for a detector thickness of $0.1 \mu \mathrm{m}$, chopping frequency of $25 \mathrm{~Hz}$. For a simple pixel element $50 \mu \times 50 \mu \times 0.1 \mu$ the RMS thermal noise limit in SbSI is calculated to be $3.5 \mathrm{x}$ $10^{-5} \mathrm{C}$, well below the expected signal level. Using a very simple ellipsometric setup working into a photomultiplier detector it was shown that for SbSI single crystals and thin films, temperature oscillations of $10^{-3^{\circ}} \mathrm{C}$ can be detected above noise level. Clearly many additional steps are needed to realize first a point detector, then an IR imaging system.

\section{RESEARCH STUDIES ON ELECTROMAGNETICALLY INDUCED TRANSPARENCY}

Stephen E. Harris

Stanford University

\section{SC: CECOM}

A key result was the realization that electromagnetically induced transparency in a dense media is not a Beer's law superposition of the single-atom response. It was shown that, when an arbitrarily shaped pulse is applied to an ensemble of population-trapped atoms, the atoms will generate a matching pulse shape on the complementary transition and, after a characteristic distance, will render themselves transparent. This result bears on all aspects of nonlinear optics, lasers without inversion, and transmission using this type of transparency phenomena. To propagate through an otherwise opaque media one could, using nonlinear optical techniques, prepare matched pulses at the two transition frequencies. In the same sense as in phase conjugation, the amplitude and phase distortion of these pulses, as well as distortion produced by the media, will tend to be removed by this type of phenomena. Researchers have developed an oscillator which produced $20 \mathrm{fs}$ pulses with peak powers of about $1 / 2 \mathrm{MW}$. The oscillator is a regeneratively-initiated, self-mode-locked Ti:sapphire laser. The key to the design was the minimization of cavity cubic phase errors and systematic increase of the phase-locked spectral content of the laser pulse. Present effort is aimed at developing mirror optics for the amplification of these pulses.

\section{Reports:}

1. Pulses of Atomic Time Scale Inherent to High-Order Harmonic Generation, by S.E. Harris et al., MS, Phys Rev Let.

2. Ultrafast Laser Generation of Hard X-Rays, by J.D. Kmetec, MS, IEEE J Quant Electron.

3. Dispersive Properties of Electromagnetically Induced Transparency, by S.E. Harris et al., MS, Phys Rev Rapid Commun.

4. Vacuum-Rabi Splitting Induced Transparency, by J.E. Field, MS, Phys Rev Let.

5. Quantum Nondemolition Measurement Using Electromagnetically Induced Transparency, by A. Imamoglu, MS, Phys Rev Let.

6. Studies of a 96.9-nm Laser in Neutral Cesium, by C.P.J. Barty et al., MS 
29015 EXPERIMENTAL STUDIES OF FUNDAMENTAL ONE- AND TWO-PHOTON LASING PROCESSES

Thomas W. Mossberg University of Oregon

Results were published on the first observation of continuous-wave two-photon laser operation. Lasing based on two-photon gain has been discussed theoretically for nearly 30 years. Realization of the process experimentally will allow researchers to study many of the properties predicted. The switching and statistical properties of two-photon lasers may be of technological importance. An effort has been initiated to observe two-photon lasing in a gain medium consisting of driven atoms confined in a magnetic optical trap. The high optical thicknesses previously demonstrated elsewhere in trap environments will allow one to observe two-photon lasing over much wider ranges of parameters than possible in the original atomic-beam based apparatus. Another project has been initiated to observe inversionless lasing in a driven three-level atomic system.

\section{ULTRAFAST BROADBAND PHOTODETECTORS FOR $\mathrm{HIGH}_{-} \mathrm{T}_{c}$ SUPERCONDUCTIVE OPTOELECTRONICS}

Philippe M. Fauchet

University of Rochester

\section{SC: ARO}

Researchers have built a laser-writing station, which includes an Ar-ion cw laser, focusing microscope, and computer controlled stage with a small gas chamber to control the ambient atmosphere during the process. A number of test structures (microbridges of various lengths and widths) have been fabricated by laser-writing in initially oxygen-rich (superconducting) Y-Ba-CuO (YBCO) films and measured. Since the goal is to demonstrate a high-speed, broadband photodetector, one must first identify the mechanisms that will allow a fast optoelectronic response. Researchers have performed a series of experiments on YBCO films using femtosecond optical pulses generated by a CPM dye laser. Additional femtosecond measurements will be performed on samples with varying degrees of oxygen, including semiconducting samples, and the range of wavelengths over which both excitation and probing are performed will be extended. This will allow one to identify the nature of the optical response of YBCO films. In addition, optoelectronic measurements will be performed on YBCO bridges. This will allow one to measure and optimize the electrical response of the photodetector structures following optical excitation with an ultrashort optical pulse.

\section{MILLIMETER AND SUBMILLIMETER STUDIES OF NONAMBIENT ENVIRONMENTS}

Frank C. Delucia

Ohio State University

The research objective is to develop new applications for nonambient and nonequilibrium molecular systems in the millimeter-wave and submillimeter-wave spectral region.

\section{LASER PROGRAMMED CONDUCTING POLYMERS: A NEW TECHNOLOGY IN THE PRODUCTION OF INTEGRATED ELECTRONIC CIRCUITS}

\section{R. Sauerbrey}

Rice University

\section{SC: ARL/MD}

A slight modification of a previously developed technique used to produce sub-100 $\mathrm{nm}$ linewidth nanostructures in polyimide was combined with the technique to permanently change the electrical conductivity of polymers by laser irradiation to produce an array of electrically conducting lines with $0.5 \mu \mathrm{m}$ linewidth. The conductivity along the wires was similar to that of macroscopic regions of laser induced electrical conductivity in polyimide, whereas the conductivity perpendicular to the wires was smalled by a factor greater than $10^{7}$. This experiment establishes the ability to use excimer lasers to modify the electrical conductivity of polyimide with high spatial resolution. In collaboration with Oak Ridge National Laboratories through the DOE sponsored share program, characterization of the laser induced electrically conducting polymers has continued. Transmission electron microscopy has revealed the formation of small $(\sim 10 \mathrm{~nm})$ carbon rich clusters in the electrically conducting material. Parallel electron 
energy loss spectroscopy (PEELS) has confirmed that nitrogen and oxygen are removed by the laser processing, consistent with pyrolysis studies. Electron diffraction has shown an increase in the short range order of the conducting material, and PEELS has also indicated an increase in $s p^{2}$ bonding, indicating a graphitization process is involved. These findings are consistent with the concept of a three dimensional metal-insulator percolative phase transition at the onset of laser induced electrical conductivity. The temperature dependence of the laser induced electrical conductivity has been measured, both in macroscopic regions and in the submicron wires. The results of both measurements are consistent with a phonon assisted variable range hopping conduction mechanism.

\section{THE INTERACTION OF ULTRAVIOLET LASER RADIATION WITH METAL AND SEMICONDUCTOR SURFACES}

R.M. Osgood, Jr.

Columbia University

\section{SC: ARL, NVESD}

Work has focussed on improving the sensitivity and the flexibility of the two-photon photoemission system. In order to accomplish this, researchers have worked on observing extremely short-lived imageresonance features on copper surfaces. These states have not been observed before due to their very short lifetimes and, hence, extremely weak photoemission signals; thus their observation provides a serious experimental measurement challenge to the sensitivity of the apparatus. In fact, after much work it was possible to measure the resonance system on $\mathrm{Cu}(110)$. In addition, researchers have found in the past that a major experimental issue with which to contend in two-photon studies is the presence of space-charge broadening due to residual one-photon emission from the Fermi tail. They therefore examined the efficacy of bichromatic two-photon photoemission as a technique for reducing the magnitude of the space-charge cloud. In this technique, a weak uv pulse is used in conjunction with a strong visible pulse. This procedure allows one to achieve the same pumping rates of the image state as with the use of two intense uv pulses but without achieving the space charge limitations. Experimentally, the technique worked extremely well, making a valuable approach for future experiments.

\section{PULSED POWER PLASMA DEVICES BASED ON HOLLOW AND SUPER-EMISSIVE CATHODES}

Martin Gundersen

University of Southern California

Electron beam lithography using a robust, high current density $\left(>10 \mathrm{~A} / \mathrm{cm}^{2}\right)$, high brightness $\left(>10^{10}\right.$ $\left.\mathrm{A} / \mathrm{m}^{2} \mathrm{rad}^{2}\right)$, broad area $(>1 \mathrm{~cm}$ diameter) electron beam source is reported in a paper. The electron beam is produced during the hollow cathode discharge phase of a back-lighted thyratron. The generated beam propagates in soft vacuum ( $<200 \mathrm{mTorr})$ and is collimated using cylindrical dielectric waveguides, resulting in uniform intensity over an area exceeding $\sim 1 \mathrm{~cm}^{2}$, along with a small divergence angle ( $<1.5$ degrees). Masked lithography for replication of fine line structures has been achieved, demonstrating the potential for a high throughput $(-30-40$ wafers/hour $)$, high resolution $(<0.25 \mu \mathrm{m})$ lithography system that is competitive with much more sophisticated $\mathrm{x}$-ray and short wavelength optical systems.

\section{NEW DEVELOPMENTS IN ATOM INTERFEROMETRY}

David E. Pritchard

Massachusetts Institute of Technology

SL: MICOM

The research objective is to further develop a recently demonstrated optically stabilized atomic interferometer and to apply the interferometer to several fundamental and applied scientific problems.

\section{TWO-PHOTON COOPERATIVE CASCADE SUPERFLUORESCENCE}

Sven R. Hartmann

Columbia University

Prior data has yielded little insight into superfluorescence. With the intent of calibrating the sample density, researchers decided to look for fluorescence from $6 \mathrm{P}_{1 / 2}$ to $6 \mathrm{~S}_{1 / 2}$ after single photon excitation on that same transition and fluorescence 
from $6 \mathrm{D}_{3 / 2}$ to $6 \mathrm{P}_{1 / 2}$ after two-photon excitation on the $6 \mathrm{~S}_{1 / 2}$ to $6 \mathrm{D}_{3 / 2}$ transition. All of the experiments suffered from fluctuations in the excitation efficiency which cannot be compensated for by simply monitoring the total excitation pulse energy. From a simple experiment, these fluctuations may be due to the frequency of the excitation varying from shot to shot: the total excitation pulse energy and the energy transmitted through a spectrometer were monitored simultaneously; the spectrometer output was not well correlated with the total energy implying that the carrier frequency was not stable. To negate for this behavior, researchers will monitor both the total excitation energy and the energy resonant with the desired transition and select events which satisfy a certain relation between these two outputs. All of the experiments will be conducted with the additional excitation pulse monitoring. This should allow one to better determine the condition of the sample immediately after excitation.

\section{Fluid and Plasma Physics}

\section{PHOTOEXCITATION OF CARBON FULLERENES}

Hanspeter Helm

Donald C. Lorents

SRI International

A supersonic beam source was constructed for embedding $\mathrm{C}_{60}$ molecules in a pulsed stream of helium gas. The pulsed helium source is kept at room temperature and separated by a quartz capillary from the oven that is used to vaporize $\mathrm{C}_{60}$ which is present in powdered form. The oven can be heated up to $800 \mathrm{~K}$ and from it gaseous $\mathrm{C}_{60}$ is injected into the helium stream to be rotationally and vibrationally cooled. The first experiments will be on pure $C_{60}$ to establish a spectroscopic base for understanding its discrete energy level structure. Two color resonant ionization coupled with mass analysis of the ionization product will be used to achieve spectroscopic characterization. Researchers have just succeeded in doping fullerenes with praseodymium atoms. Attempts will be made to determine the modification of the energy level structure of the praseodynium atom by the carbon cage.

\section{Solid State Physics}

\section{ELECTRONIC TRANSPORT IN HETEROJUNCTION SUPERLATTICES}

D.C. Tsui

M. Shayegan

Princeton University

The goal of this project is to fabricate and study nearly-free, quasi-three-dimensional, dilute electron systems. It has been proposed theoretically that such systems, under appropriate conditions, will exhibit new phenomena resulting from the electron-electron interactions. Researchers have grown wide, quadratically graded $\mathrm{AlGaAs}$ quantum wells in which the electron density is nearly uniform over a distance as wide as $\sim 1000 \AA$. They have calculated the subband structure of the electron system, and have found the results in remarkable agreement with experimental measurements. The structures have already provided evidence for new magnetooptical and transport effects in tilted magnet fields. More recently, it was possible to produce very high quality electron systems in parabolic wells. In these structures, researchers were able to study, for the first time, the dependence of the energy gap for the fractional quantum Hall states on the electron layer thickness. A dramatic decrease was observed in the measured energy gaps as the electron-layer thickness increases, which can be interpreted as the collapse of the correlated fractional states. By superimposing a periodic modulation on the parabolic potential, researchers have been able to realize a novel superlattice structure which contains a low-disorder degenerate electron system. This structure has the highest mobility reported so far for a degenerate electron system in a superlattice.

\section{Reports:}

No. 1-3 in previous editions.

4. Missing Integral Quantum Hall Effect in a Wide Single Quantum Well, by Y.W. Suen et al., Phys Rev B44,5947(1991). AD A249 218

5. Fabrication of Quasi-Three-Dimensional Electron Systems and Superlattices in Wide Parabolic Wells, by M. Santos et al., J Cryst Growth 111,366(1991). AD A249 403

\section{RADIATIONLESS TRANSITIONS AND EXCITED-STATE ABSORPTION IN TUNABLE-LASER MATERIALS}


Ralph H. Bartram

University of Connecticut

SC: ARL

$\mathrm{Pb}^{+}(1)$ centers in alkaline-earth fluorides consisting of a substitutional $\mathrm{Pb}^{+}$ion next to a $\mathrm{F}^{-}$vacancy have shown IR emissions when excited in their $\mathrm{A}_{1}$ absorption bands. In contrast to the analogous $\mathrm{Pb}^{+}$(1) centers in $\mathrm{KMgF}_{3}$ they could not be brought to lase. It is shown that this failure occurs because of a loss mechanism in which excited-state absorption leads to an upconversion emission peaking at $2.37 \mathrm{eV}$ for $\mathrm{CaF}_{2}$, $2.361 \mathrm{eV}$ for $\mathrm{SrF}_{2}$ and $2.370 \mathrm{eV}$ for $\mathrm{BaF}_{2}$. Its intensity depends quadratically on the pump power and it can also be excited in the $\mathrm{A}_{3}$ band in the UV. A theoretical calculation shows that the upconversion emission originates from the relaxed excited state of a F-center model rather than from a $6 \mathrm{~s}^{2} 7 \mathrm{~s}$ excited $\mathrm{Pb}^{+}(1)$ state and explains its observed temperature dependence. The upconversion is observed in the alkaline-earth fluorides because of an accidental energy resonance $2 \mathrm{~A}_{2}=\mathrm{A}_{3}$ that does not occur in $\mathrm{KMgF}_{3}$.

Reports:

No. 1 in previous edition.

2. Computational Modeling of Lattice Statics and Dynamics in Crystals of the Elpasolite Structure, by Robert Stephen Sinkovits, PhD Thesis, 1991, 262 pp.

3. Molecular Cluster Modeling of Chromium Complexes in Elpasolite Ctystals, by Amanda May Woods, PhD Thesis, 1991, $166 \mathrm{pp}$.

4. Computer Modeling of Lattice Dynamics in Halide Elpasolites, by R.S. Sinkovits and R.H. Bartram, J Phys Chem Sol 52,1137(1991). AD A244 684

5. Optical Studies at High Pressure on Chromium-Doped Ordered Perovskite Crystals, by Andrew Gabriel Rinzler, $\mathrm{PhD}$ Thesis.

6. Embedded Cluster Modeling of Excited Chromium Impurities in Halide Elpasolites, by R.H. Bartram et al., Radiation Effects and Defects in Solids 119,627(1991). AD A244 735

7. Two-Photon Excitation Spectroscopy of $\mathrm{Cr}^{3+}: \mathrm{K}_{2} \mathrm{NaScF}_{6}$, by G. Robert Wein, PhD Thesis, 1992, 174 pp.

\section{A NOVEL APPROACH FOR NEW RADIATION SOURCES BASED ON SOLID STATE PLASMA INSTABILITIES}

Pradip Bakshi

K. Kempa

Boston College

An investigation of the effects of introducing a grating coupler on the surface of the layered system has led to a paper. This is a first principles calculation involving proper matching of the appropriate fields at all boundaries. The main results are: $(a)$ for existing modes the dispersion relation is slightly modified due to the presence of the grating, (b) a new, grating generated mode was discovered, and $(c)$ the efficiency of emission of the electromagnetic radiation was obtained for all modes. Researchers have continued the investigation of the effects of introducing a magnetic field perpendicular to the $2 \mathrm{D}$ carrier layers. A scatteringless environment is achieved in the quantum Hall domain, and a cold-beam (two-stream) type of instability develops. An investigation was made of the possibility of employing quantum dot or quantum wire systems as couplers and resonators. A particularly interesting development is in recognizing the possibility of generating a modulated $2 \mathrm{D}$ electron gas by biasing a quantum wire grid placed above a uniform $2 \mathrm{D}$ electron gas. By varying the bias voltage the system can be varied from weak modulation to very strong modulation, which breaks up the 2D electron gas into parallel wires. Just prior to such a break-up the system can still carry a transverse current, which can give rise to current driven instabilities of a modulated $2 \mathrm{D}$ electron gas.

Reports:

No. 1-7 in previous editions.

8. Current Driven Plasma Instabilities in Layered Solid State Systems, by J. Cen, PhD Thesis, 1991, 166 pp.

9. Spontaneous Polarization in Quantum-Dot Systems, by $\mathrm{K}$. Kempa et al., Phys Rev B43,9343(1991). AD A244 738

10. Spontaneous Polarization of Electrons in Quantum Dashes, by P. Bakshi et al., J Appl Phys 70,5150(1991). AD A249700

11. Growth Rates of Current-Excited Plasma Waves in Semiconductor Layered Systems, by H. Xie et al., MS, J Appl Phys.

\section{SEARCH FOR FAR INFRARED RADIATION FROM OPTICALLY PUMPED DEFECT MODES}

\section{A.J. Sievers}

Cornell University

\section{SC: ARL}

The behavior of the far infrared radiation produced by the passage of a mm-long bunch of electrons through a vertical $0.44 \mathrm{~T}$ magnetic field is found to be consistent with the properties expected for coherent synchrotron radiation. The radiation intensity is proportional to the square of the number of electrons in the bunch. Averaged over the entire spectral bandwidth, the radiation is 82 percent polarized in the orbit bending plane. A novel metal mesh and hole 
filter spectrometer is used to determine that most of the radiation appears at wavelengths longer than 1 $\mathrm{mm}$ but the observation of shorter wavelength radiation suggests either a non-Gaussian bunch shape or a Gaussian with a submillimeter bunch length. A perturbed shell model calculation for the impurity system $\mathrm{KI}: \mathrm{Ag}^{+}$predicts three nearly degenerate localized gap modes of $\mathrm{A}_{1 \mathrm{~g}}, \mathrm{E}_{\mathrm{g}}$ and $\mathrm{T}_{1 \mathrm{u}}$ symmetry with unusual amplitude patterns: the displacements of the defect's fourth nearest neighbors are more than an order of magnitude larger than those of the impurity of its nearest neighbors. Far infrared isotope effect experiments directly confirm the existence of these localized pocket modes. Coherent transition radiation, generated by the passage of $\mathrm{mm}$ long bunches of electrons through a thin metal foil, has been observed in the far infrared. The intensity was compared to the smaller amount of Cherenkov radiation produced when $\mathrm{SF}_{6}$ gas was introduced into the electron path. Wakefield radiation was ruled out by varying the geometry of the metal chamber near the electron beam. The intensity, polarization, spectral, and angular distribution of the transition radiation was measured and its coherent nature was identified. Persistent IR spectral holes generated in the electronic ground state inside the inhomogeneously broadened vibrational hole burning spectra were described: (1) the hole and antihole in the same absorption line, (2) the antihole removed in frequency from the inhomogeneously broadened line, (3) multiple antiholes and (4) the burning at one vibrational mode frequency while monitoring the persistent changes produced in the other internal (IR) or external (FIR) modes with a high resolution FT interferometer. All molecule:chalcogenide glass combinations show persistent IR spectral holes and spontaneous hole filling at low temperatures. Persistence appears to be produced by molecular reorientation and/or local host restructuring.

\section{Reports:}

No. 1-2 in previous editions.

3. Far-Infrared Transmission of Superconducting $\mathrm{K}_{\mathbf{x}} \mathrm{C}_{6} \mathrm{O}$ Films, by S.A. FitzGerald et al., Phys Rev B45,10 165(1992).

4. Prediction and Observation of Pocket Vibrational Modes in Crystals, by K.W. Sandusky et a1., Phys Rev Let 67,871(1991). AD A244 552

5. Persistent Infrared Spectral Hole Burning of the Fundamental Stretching Mode of $\mathrm{SH}^{-}$in Alkali Halides, by C.E. Mungan and A.J. Sievers, J Opt Soc Am B9,746(1992).

6. Persistent Infrared Spectral Hole Burning of $\mathrm{Tb}^{3+}$ in the Glasslike Mixed Crystal Ba $1-x-y L_{x} \mathrm{~Tb}_{y} \mathrm{~F}_{2+x+y}$, by S.P. Love et al., J Opt Soc Am B9,794(1992).

\section{OPTICAL AND MAGNETIC RESONANCE INVESTIGATIONS IN SINGLE CRYSTAL HOSTS: CANDIDATES FOR TUNABLE SOLID-STATE LASERS}

David J. Singel

Harvard University

\section{SL: NVESD}

Detailed results were achieved for EPR (electron paramagnetic resonance) experiments through which the presence of a tetra-oxo $\mathrm{Cr}^{4+}$ center in $\mathrm{Cr}$-doped forsterite was demonstrated. Specifically, a spectrum was identified which was attributable to a ground state triplet spin species accommodated at the crystallographic $4 \mathrm{c}$ site; the intensity of the spectrum was found to be directly related to the $\mathrm{Cr}$ content of the crystal. While both octahedral $\mathrm{Mg}^{+2}$ centers and the tetrahedral $\mathrm{Si}^{4+}$ centers lie at $4 \mathrm{c}$ sites in forsterite, the presence of a very similar spectrum in $\mathrm{Cr}$-doped BMAG $\left(\mathrm{Ba}_{2} \mathrm{MgGe}_{2} \mathrm{O}_{7}\right)$, suggests similar coordination in both materials. Thus, inasmuch as the relevant spectrum in BMAG unambiguously derives from substitutional incorporation at the tetra-oxo Ge sites, the forsterite spectrum is, by analogy, assigned as a tetrahedral species. Recently experiments were completed that prove the common identity of the tetrahedral $\mathrm{Cr}^{4+}$ paramagnetic center characterized by EPR and the optically active laser center in Cr-doped forsterite. The approach was motivated by the work of the Yen group, who directly probed, in the NIR emission of Cr-doped forsterite, the ground-state sublevel structure, resolved through FLN (fluorescence line-narrowing). The $2 \mathrm{~cm}^{-1}$ splitting observed in their experiments accurately reflects the sublevel structure expected in zero external magnetic field for the triplet species characterized by $E P R$ (with $D \pm E \approx 2 \mathrm{~cm}^{-1}$ ). Work has continued on examining chromium doped garnet systems for EPR spectra attributable to tetra-oxo $\mathrm{Cr}^{4+}$. The Gd-garnets are not magnetically dilute and exhibit a ferritelike spectral absorption spanning 1T, and are thus unsuitable for the study of defects by EPR. Of more interest is the YAG system, particularly because it is the only other NIR laser system, besides forsterite, to be putatively $\mathrm{Cr}^{4+}$ based.

\section{Reports:}

1. Comparison of Chromium-Doped Forsterite and Akermanite Laser Host Crystals, by M.H. Garrett et al., MS.

2. EPR of $\mathrm{Cr}^{4+}$ in Laser Host Crystals, by David J. Singel et al., MS. 


\section{INVESTIGATION OF QUANTUM EFFECTS IN HETEROSTRUCTURES}

Leroy Chang

Emilio Mendez

IBM Research Center

A detailed study was made of the magneto-transport of $p$-type InMnAs, the new diluted magnetic semiconductor made of III- $\mathrm{V}$ compounds. The anomalous Hall effect dominates the Hall resistivity throughout the entire temperature range of $0.4 \mathrm{~K}$ to room temperature, and it plays a key role in the formation of partial ferromagnetic order below 7.5 $\mathrm{K}$. This magnetic order is the result of a cooperative phenomenon accompanied by carrier localization, in contrast to the conventional RKKY mechanism. The anomalous Hall effect originates from the interaction between the holes and the $3 \mathrm{~d}$ spins of the $\mathrm{Mn}$ ions. Both a remnant magnetization, unsaturated spins and a large negative magneto-resistance were observed at low temperatures. All these results are explained satisfactorily by the formation of large bound magnetic polarons. Pronounced many-body exciton effects have been observed through photoluminescence in modulation-doped n-type GaAsGaAlAs single quantum wells. The structure is designed to achieve a near-resonance coincidence between a transition involving two-dimensional electrons at the Fermi level in the first conduction subband and an exciton transition from the second conduction subband. Under these conditions, strong and distinct Fermi-edge singularities are observed. A magnetic field induces further enhancement of the effect and leads to strong oscillations in the luminescence. The large amplitude variations reflect the stability of the exciton in terms of the position of the Fermi level relative to the Landau levels. Recent time-resolved spectra are consistent with these results. By optically pumping a laser heterostructure whose active region consists of coupled quantum wells, researchers have succeeded in tuning the stimulated emission by external electric fields. This was made possible by the relatively large energy shifts of the quantum states with the field in such coupled structures.

Reports:

No. $1-13$ in previous editions.

14. Stark Ladders in Semiconductor Superlattices, by E.E. Mendez, Proc of 20th Intl Conf of the Physics of Semiconductors, 1990, p1206.

15. Optical and Magnetic Properties of Diluted Magnetic Semiconductor Heterostructures, by L.L. Chang et al., Condensed Systems of Low Dimensionality, 1991, p165. AD A249 056
16. Fractional States in Few-Electron Systems, by W. Hansen et al., Appl Phys Let 56,168(1990). AD A249 346

17. New Diluted Magnetic Semiconductors Based on III-V Compounds, by S. von Molnar et al., $J$ Magnetism and Magnetic Mater 93,356(1991). AD A249 150

18. Magnetic-Field-Induced Type-IA Type-II Transition in a Semimagnetic CdTe/Cd0.93Mno.07Te Superlattice, by E. Deleporte et al., Phys Rev B42,5891(1990). AD A249 149

19. Ultrafast Spin Dynamics in Diluted Magnetic Semiconductor Superlattices, by D.D. Awschalom et al., Surface Sci 229,145(1990). AD A249 342

20. Observation of Miniband Formation in the $\mathrm{CdTe} / \mathrm{Cd}_{1-\mathbf{x}} \mathrm{Mn}_{\mathbf{x}} \mathrm{Te}$ Quantum Well System, by Alex Harwit et al., Appl Phys Let 57,1769(1990). AD A249 057

21. Tunneling Between Two-Dimensional Electron Gases, by E.E. Mendez and L.L. Chang, Surface Sci 229,173(1990). AD A249 114

22. $p$-Type Diluted Magnetic III-V Semiconductors, by $\mathbf{H}$. Munekata et al., J Cryst Growth 111,1011(1991). AD A249 058

23. The Electronic Properties of the Miniband and the Effect of External Electric Fields in Superlattices, by C.Y. Fong et al., Superlattices and Microstructures 7,147(1990). AD A249 059

24. Interplay Between Landau and Stark Quantizations in GaAs/Ga0.65Al0.35As Superlattices, by A. Aalexandrou et al., Phys Rev B44,1934(1991).

25. New III-V Diluted Magnetic Semiconductors, by H. Ohno et al., J Appl Phys 69,6103(1991).

26. Femtosecond Spectroscopy of Carrier-Spin Relaxation in GaAs-Al $\mathrm{Gaa}_{\mathbf{x}} \mathrm{xAs}$ Quantum Wells, by M. Kohl et al., Phys Rev B44,5923(1991).

27. Resonant Magnetotunneling in Type II Heterostructures, by E.E. Mendez et al., Resonant Tunneling in Semiconductors, 1991, p51.

28. Interference and Dephasing by Electron-Electron Interaction on Length Scales Shorter than the Elastic Mean Free Path, by A. Yacoby et al., Phys Rev Let 66,1938(1991).

29. Growth and Novel Properties of Magnetic Heterostructures by Molecular Beam Epitaxy, by J.M. Hong et al., J Cyst Growth 111,1016(1991).

30. Resonant Interband Tunneling via Landau Levels in Polytype Heterostructures, by E.E. Mendez et al., Phys Rev B43,5196(1991).

31. A Perspective of Resonant Tunneling, by L.L. Chang, Resonant Tunneling in Semiconductors, 1991, p1.

32. Stark Quantization in Superlattices, by Raphael Tsu and L. Esaki, Phys Rev B43,5204(1991).

33. The Evolution of Semiconductor Quantum Structures in Reduced Dimensionality - Do-It-Yourself Quantum Mechanics, by L. Esaki, Electron Properties Multilayers \& LowDimensional Semiconduct Struct, 1990, p1.

27780 EXPERIMENTAL AND THEORETICAL S STUDIES OF IONIC MOLECULAR SOLIDS

John R. Hardy

Frank G. Ullman

University of Nebraska 


\section{SC: ARL/WTD, ARL/EPSD}

Major advances have been made in theoretical studies now coordinated with the experimental studies of Drs. Cornelison and Gauss at the Ballistics Research Laboratory (ARL/WTD). These studies have centered on the behavior of $\mathrm{KNO}_{3}, \mathrm{KNO}_{2}$, and $\mathrm{NaNO}_{2}$. In particular, theoretical work on the last material is described more fully in a paper just submitted to $P$ hys Rev Let (report 13, below). In general terms, researchers have developed a semi-quantitative understanding of the microwave losses found by the ARL/WTD group for their materials. In particular, researchers have a clear account of the "flare up" in these seen to occur just below the order-disorder transformations in $\mathrm{KNO}_{3}$ and $\mathrm{NaNO}_{2}$ and of the strong, but only weakly temperature dependent, losses observed in $\mathrm{KNO}_{2}$. Currently an account of the experimental aspects of this work is being prepared by the ARL/WTD group as a ARL/WTD Internal Report. Moreover, this work will be greatly aided by the "in cavity" measurement capability now on-line at ARL/WTD. Other work has resulted in developing a comprehensive understanding of the phase diagrams of incommensurate $\mathrm{A}_{2} \mathrm{BX}_{4}$ structures such as $\mathrm{K}_{2} \mathrm{ZnCl}$. In the experimental area, work has continued on the birefringence studies on $\mathrm{CsZnI}_{4}$, and previous work, which formed the basis of Dr. D. Liu's Dissertation, has either been published or submitted for publication. Finally, it should be observed that much of the future program will involve close collaboration with the ARL/WTD workers and may involve extended visits in the future.

Reports:

No. 1-7 in previous editions.

8. Simulations of Phase Transitions in $\mathrm{Rb}_{2} \mathrm{ZnCl}_{4}$, by H.M. Lu and J.R. Hardy, MS, Phys Rev.

9. Raman Scattering and Lattice-Dynamical Calculations of Alkali-Metal Sulfates, by D. Liu et al, Phys Rev B44,7387(1991). AD A244 857

10. First-Principles Study of Phase Transitions in $\mathrm{KNO}_{3}$, by H.M. Lu and J.R. Hardy, Phys Rev B44,7215(1991). AD A244 945

11. A New Optical Study of $\operatorname{Cs}_{2} \mathrm{ZnI}_{4}$, by D.P. Billesbach and F.G. Ullman, MS.

12. Raman Scattering and Lattice-Dynamical Calculations of Crystalline $\mathrm{KNO}_{3}$, by D. Liu et al., Phys Rev B45,2142(1992). AD A249 281

13. A Priori Prediction of the Order-Disorder Phase Transition in $\mathrm{NaNO}_{2}$, by H.M. Lu et al., MS, Phys Rev Let.

14. Lattice and Molecular Dynamics Studies of $\mathrm{CsLiSO}_{4}$, by V. Katkanant et al., MS, Phys Rev.

15. Ferroelectric Transitions, Molecular Dynamics and Intrinsic Surface Problems, by J.R. Hardy and H.M. Lu, MS, Ferroelectrics.
16. Anomalous Dynamics and High $T_{\mathrm{c}}$, by J.W. Flocken et al., High-Temperature Superconductivity, p331.

\section{FAST OPTO-ELECTRONIC QUANTUM WELL AMPLITUDE MODULATOR DEVICE DEVELOPMENT}

Gary L. Duerksen

Lawrence C. West

AT \& T Technology Systems

\section{SL: DARPA}

An asymmetric semiconductor electron wave FabryPerot interference filter with two above-barrier quasi-bound states has been designed. The upper state was designed to have an electronic lifetime which is greater than three times that of the lower state (which was in turn designed to be less than $\mathbf{1 0 0}$ $f s$ ). Such lifetime characteristics, which are nearly impossible for below-barrier states, satisfy the criteria required for achieving population inversion. Both bound-to-quasi-bound and quasi-bound-toquasi-bound transitions were observed using roomtemperature infrared absorption measurements. These experiments confirmed that the dipole matrix element between the above-barrier states is quite large (at least $2.4 \mathrm{~nm}$ ) and that the lifetime of the upper state is significantly larger than that of the lower state. This structure could represent the basis for a new type of room-temperature infrared semiconductor laser.

Reports:

1. An Integrated-Optical Amplitude Modulator Based on Electrical Modulation of Intersubband Transitions, by G.L. Duerksen et al., MS.

\section{BLUE SEMICONDUCTOR LASERS BASED ON WIDE-BAND GAP II-VI MATERIALS}

\section{J.F. Schetzinaa}

North Carolina State University

SC: ARL

Substantial progress has been made in the synthesis of $\mathrm{ZnS}$ by MBE using elemental sources for both $\mathrm{Zn}$ and $S$. This required modifications to the MBE chamber, which have been completed, and also the design and fabrication of a new MBE furnace specifically for use with elemental $S$. S has a very high vapor pressure and, as a consequence, the MBE source had 
to be designed for stable use at $70-100^{\circ} \mathrm{C}$. Two $\mathrm{S}$ sources have now been fabricated for use in two separate MBE systems. The first system is being used to grown $\mathrm{ZnS}$ on $\mathrm{GaP}$ substrates. The quality of this epitaxy is now beginning to approach that of $\mathrm{ZnSe}$ on GaAs, as judged by double crystal x-ray diffraction measurements. Chlorine, in the form of $\mathrm{ZnCl}_{2}$, has been successfully used to dope $\mathrm{ZnS}$ films n-type to a level in excess of $10^{17}$ electrons $/ \mathrm{cm}^{3}$, as measured by Hall effect studies. Quantum well structures of $\mathrm{ZnS} / \mathrm{ZnCdS}$ have also been prepared for the first time. These new structures exhibit intense photoluminescence in the UV spectral region at about 3.6 $\mathrm{eV}$. Additional work in this area is continuing. The second $S$ source is being used for the growth of $\mathrm{ZnSSe} / \mathrm{ZnSe} / \mathrm{ZnCdSe}$ quantum well structures for blue/green light emission applications.

\section{THEORETICAL AND NUMERICAL PREDICTION OF STOPPING PROPERTIES OF COUNTERPART THIN FILMS AND SOLIDS}

\section{John R. Sabin}

University of Florida

\section{SC: ARL/WTD}

Work has concentrated on investigation of the direct calculation of generalized oscillator strengths and their integration to give stopping cross sections in the first Born approximation directly. Using $\mathrm{He}$ as a test case, it has become clear that extensive basis sets including high angular momentum functions must be used if one wishes to satisfy the Bethe sum rule at high momentum transfer. One of the problems with the study of stopping at low projectile velocity and with heavy projectiles is the determination of the charge state of the projectile. Work is beginning on this problem. Studies are also being made on electronic and structural properties of a simple but realistic ionically bonded monolayer, $\mathrm{LiF}$. The objective is to compare stopping properties and behavior in metallic, covalent, and ionically bonded systems in a procedurally unambiguous way. Work is also in progress on a so-far unsolved problem of "local normalization" of the momentum density in the $\mathrm{Na}$ monolayer. Resolution of the difficulty is critical to completion of the $\mathrm{Na}$ monolayer stopping study as well as to undertaking the $\mathrm{Li}$ mono-through-five layer study. The apparent problem is a peculiar first Brillouin Zone position dependence of the momentum density normalization that seems to be related to the order of limits in the approach to thermodynamic size
(Limit of number cells $\rightarrow \infty$ ). Very substantial progress has been made on diagnosing and correcting a bug in the solid FLAPW code WIEN. Heretofore this bug has prevented doing zincblende symmetries. It now seems to be traced to the $\mathrm{Y}_{3} 2$ spherical harmonic contribution to the potential.

Reports:

No. $1-2$ in previous editions.

3. Prediction of the Low-Pressure Crystalline Phases of Lithium, by J.A. Nobel and S.B. Trickey, MS, Phys Rev.

4. Structure, Energetics, and Molecular- to Atomic-Ordering Transitions in Hydrogen Thin Films, by J.Z. Wu et al., Phys Rev B45,8610(1992).

5. Quantum Size Effects in Equilibrium Lithium Ultrathin Layers, by J.C. Boettger and S.B. Trickey, Phys Rev B45,1363(1992).

6. Interplanar Binding and Lattice Relaxation in a Graphite Dilayer, by S.B. Trickey et al., Phys Rev B45,4460(1992). AD A249 803

7. Low-Pressure Crystalline Phases of Lithium, by J.A. Nobel et al., Phys Rev B45,5012(1992). AD A249 619

8. Near-Equilibrium Ordering of the Crystalline Phases of Atomic Hydrogen, by J.A. Nobel et al., Intl J Quant Chem 42,1037(1992).

9. Comment on "Total-Energy Calculations of Solid H,Li, Na,K,Rb, and Cs", by J.C. Boettger et al., Phys Rev B45,7503(1992).

10. Status of the Calculation of the Energy Loss of Swift Ions in Molecules, by John R. Sbain and Jens Oddershede, $\mathrm{NuCl}$ Inst and Meth in Phys Res B64,678(1992).

\section{THEORY OF TRANSPORT FOR INTERACTING MANY-BODY SYSTEMS}

Walter Poetz

University of Illinois at Chicago

A coherent-state functional integral approach has been developed to describe coupled fermion-boson systems in and out of thermal equilibrium. This method is rather general; here researchers are primarily concerned with charge transport theory in normal solids. They consider a typical situation. For times smaller than zero, the system is in thermal equilibrium which is established by coupling to a bath with which energy and particles may be exchanged. An external perturbation, in form of an external electric field, drives the system out of equilibrium. Depending on the nature of the system and the time-dependence of the electric field, the system evolves into a new equilibrium or steady state. The whole evolution, including the initial state, may be treated within the present method at various levels of sophistication. It is demonstrated that, similar to coupled electron systems, coupled electron-phonon sys- 
tems can be treated approximately within mean-field approximations which neither require weak interparticle interactions nor weak perturbations. The latter assumptions and/or oversimplified electronphonon coupling mechanisms have most frequently been used in previous theories on charge transport. As compared to Feynman path integrals, the present approach maintains a fully quantum-mechanical description of the system, even within lowest order of approximation. In particular, quantum confinement is fully accounted for. Nevertheless, and in contrast to most other approaches, the present approach has the potential for numerical application to realistic systems, such as semiconductor heterostructures and mesoscopic electronic devices. Semiconductor alloys are used in virtually all mesoscopic electronic devices, yet a (quantitative) theoretical understanding of many basic properties, such as the nature of their electronic structure, is still largely missing. Here, a general semi-empirical approach has been developed to provide the electronic density of states of tetrahedral semiconductors, including heterostructures, alloys, and amorphous materials.

\section{Reports:}

1. On the Current Response in Semiconductor Heterostructures Within the Independent-Electron Picture, by W. Potz, MS, Phys Rev.

2. Coherent-State Functional Integral Approach to HighField Transport in Coupled Electron-Phonon Systems, by W. Potz and J. Zhang, MS.

\section{ADDENDUM TO A MICROSCOPIC THEORY OF QUANTUM OPTICS AND NEW PHOTON-LOCKED BISTABLE STATES}

Mikael Ciftan

L.C. Biedenharn

Duke University

Work has continued in the area of phase transitions in magnetism: In ferromagnetism, it has been shown that the dynamical aspects of phase transitions need to be looked at microscopically because by doing so it revealed that the celebrated classic equilibrium theorem of Mermin and Wagner miss metastable equilibrium states; specifically the Mermin - Wagner Theorem states that at no temperature above absolute zero can one have ferromagnetism stemming from the Heisenberg Hamiltonian of a lattice of 3 dimensional spins if the lattice is the two dimensional plane. What they missed was that above absolute zero there is a large temperature range within which the system of spins show the Kosterlitz - Thouless type ordering of the spins in clusters where the correlation of spins grows, if not exponentially but certainly as a power law; furthermore the system undergoes a clear-cut phase transition above this Kosterlitz Thouless region, giving a very nice curve for the magnetization plotted versus temperature. In addition several other criteria for phase transitions are satisfied, such as the behavior of specific heat, susceptibility and the scaling of these functions with respect to the size of the lattice being considered.

\section{Reports:}

1. Ferromagnetism in Two Dimensions $I$, by R.G. Brown and M. Ciftan, MS

2. Ferromagnetism in Two Dimensions II, by R.G. Brown and M. Ciftan, MS

3. Quantum Statistical Microdynamics and Critical Phenomena, by R.G. Brown and M. Ciftan, MS.

4. N-Atom Optical Bloch Equations: A Microscopic Theory of Quantum Optics, by Robert G. Brown and Mikael Ciftan, Phys Rev A40,3080(1989). AD A244 630

5. Multipolar Expansions for Multiple Scattering Theory, by R.G. Brown and M. Ciftan, MS.

6. The 2D/3D Classical Heisenberg Ferromagnet, by R.G. Brown and M. Ciftan, MS.

\section{OPTICS WITH SEMICONDUCTORS: ULTRAFAST PHYSICS FOR DEVICES}

Philippe Fauchet

University of Rochester

The gain dynamics of thin GaAs films was measured near the band edge with femtosecond time resolution following photoexcitation of a large carrier density with $\sim 100$-fs pulses at $2 \mathrm{eV}$. The subpicosecond onset of gain was attributed to very efficient cooling of carriers near the $\Gamma$ point of the Brillouin zone, which implies nearly instantaneous equlibration and preferential intervalley scattering of high-energy electrons. Evidence for a heated hole distribution on a subpicosecond time scale is presented, which points to the importance of electron-hole interactions. Pump-probe techniques were used to perform a series of measurements on intrinsic GaAs samples at room temperature with a temporal resolution of 75-100 fs. Changes of both absorption coefficient and refractive index are measured over a wide spectral region $(550-950 \mathrm{~nm})$ for various carrier densities $\left(\sim 10^{17}-10^{19} \mathrm{~cm}^{-3}\right)$ injected at $2 \mathrm{eV}$. These measurements provide insight on the fundamental properties of nonequilibrium carriers, including electron-electron scattering, electron-hole scattering, electronphonon intervalley scattering, band-gap 
renormalization, plasma screening of Coulomb interactions, and free-carrier absorption. Various carrier scattering mechanisms are responsible for the recovery of the spectral hole burning observed in pump and probe femtosecond optical measurements. An experimental study was performed on fsec hole burning in several semiconductors (GaAs, AlGaAs, InP), either in the absence of a cold plasma or in the presence of electrons and/or holes produced by doping or by another fsec laser pulse. Two of the most striking results are that the initial scattering rate of hot electrons can decrease when the plasma density increases and the peak of the spectral hole can be downshifted by up to $100 \mathrm{meV}$ with respect to the peak of the pump pulse spectrum. These and other results are analyzed in terms of the various carriercarrier and carrier-phonon scattering mechanisms.

\section{Reports:}

1. Femtosecond Gain Dynamics in Thin GaAs Films, by P.M. Fauchet et al,, MS.

2. Femtosecond Nonlinearities and Hot-Carrier Dynamics in GaAs, by T. Gong and P.M. Gauchet, MS.

3. Femtosecond Gain Dynamics Due to Initial Thermalization of Hot Carriers Injected at $2 \mathrm{eV}$ in GaAs, by T. Gong et al., Phys Rev B44,6542(1991). AD A249 693

4. Femtosecond Refractive and Absorptive Nonlinearities Due to Real Carriers in GaAs, by T. Gong and P.M. Fauchet, OSA Proceedings on Picosecond Electronics and Optoelectronics, Vol 9, 1991, p253. AD A249 694

\section{CHARACTERIZATION OF SOLID STATE LASER AND NONLINEAR OPTICAL MATERIALS}

Richard C. Powell

Oklahoma State University

Research on frequency conversion materials focused on the characterization of an optical parametric oscillator (OPO) based on KTA. The properties of this OPO were studied using a tunable alexandrite laser as a pump source. This resulted in the first OPO made from this material, which has better nonlinear optical properties than KTP. Threshold energies and slope efficiencies for a doubly resonant oscillator configuration were measured for pump wavelengths between 700 and $800 \mathrm{~nm}$, and the characteristics of pump-wavelength-tuning were demonstrated. In addition, the frequency mixing of the near infrared output with the pump beam input was found to give tunable coherent radiation in the green spectral region. Investigations of photorefractive materials focused on $\mathrm{Bi}_{2} \mathrm{TeO}_{5}$ (BTO) crystals. The c.w. four-wave mixing studies of this new material show a strong photorefractive signal with a multicomponent decay. The signal was measured as a function of wavelength, intensity, polarization direction, and crossing angle of the laser write beams and the sample orientation. Both absorption and phase gratings were observed as the signal evolves in time from a defect population grating, to trapped charge gratings involving two different types of traps, to fixed gratings produced by oxygen ion displacements. The photorefractive effect in BTO is found to be of the photoconductive type and it produces a larger diffraction efficiency than other sillenite materials. BTO has excellent properties for an optical information storage material. Research on solid state laser materials continued to focus on Tm,Ho:YAG. The results of the transient laser-induced population gratings in the $\mathrm{Tm}^{3+}$ ions are being analyzed in terms of a percolation theory model for transient grating experiments that have been developed here.

\section{Reports:}

1. Pump Wavelength Tuning of a Near Infrared Optical Parametric, by Mahendra G. Jani et al., TR, Sep 91,10 p. AD A244 304

2. Lasing Properties of Chromium-Aluminum-Doped Forsterite Pumped with an Alexandrite Laser, by Edward G. Behrens et al., IEEE J Quant Electron 27,2042(1991). AD A244 487

3. Nonradiative Energy-Transfer Processes in $\mathrm{Cd}_{1-\mathrm{x}} \mathrm{Mn}_{\mathrm{x}} \mathrm{F}_{2}$ Crystals, by Andrzej Suchocki et al., J Appl Phys 71,28(1992). AD A250 253

4. Characteristics of the Photorefractive Effect in $\mathrm{Bi}_{2} \mathrm{TeO} 5$, by I. Foldvari et al., SPIE Proc 1626,9(1992).

5. Pump Wavelength Tuning of Optical Parametric Oscillations and Frequency Mixing in $\mathrm{KTiOAsO}_{4}$, by Mahendra G. Jani et al., Appl Phys Let 60,2327(1992).

6. Investigation of the Photorefractive Effect in $\mathrm{Bi}_{2} \mathrm{TeO}$ s, by I. Foldvari et al., J Appl Phys 71,5465(1992).

7. Pump Wavelength Tuning of a Near-Infrared Optical Parametric Oscillator, by Mahendra G. Jani et al., Appl Opt 31,1998(1992).

\section{ANALYTICAL INVESTIGATIONS OF THE ACCELERATION SENSITIVITY OF ACOUSTIC BULK AND SURFACE WAVE RESONATORS}

Harry F. Tiersten

Rensselaer Polytechnic Institute

\section{SL: ARL/EPSD}

Calculation of the resultant of the normal and inplane acceleration sensitivities for the optimal sup- 
port orientation and aspect ratio of the contoured SC-cut quartz resonator, based on earlier analyses, are continuing in order to study the influence of the dimensioning and number of the clips, in accordance with the observations made in the most recent work. In addition, calculations of the resultant acceleration sensitivity of the trapped energy SC-cut quartz flat plate resonator are being performed for the optimal support configuration in order to see what differences in the acceleration sensitivity with fabrication error arise on account of the change in mode shape. In this work the computer programs for the biasing states are the same as for the contoured resonator. An analysis of the $\mathrm{SC}$-cut quartz trapped-energy resonator with an electroded flat cylindrical inner region surrounded by a contoured outer region is being performed. The analysis employs the mean planar isotropic form of the equations for the transversely varying thickness modes. The first perturbation in eigenfrequency resulting from the anisotropy vanishes for the harmonics and all anharmonics of every family of modes except for those anharmonics that have one angular nodal plane. The analytical results will be used in the calculation of the resonant frequencies and motional capacitances of all harmonic and anharmonic trapped energy modes.

\section{TOWARDS EXPERIMENTAL VERIFICATION AND DEVICE APPLICATIONS OF CURRENT DRIVEN PLASMA INSTABILITIES}

Pradip Bakshi

K. Kempa

Boston College

SC: ARL/WTD, ARL

Development of a formalism for grating coupler on layered systems. Earlier studies have dealt with establishing the feasibility of current driven plasma instabilities in layered solid state systems. The energy of the dc current is transferred to growing plasma waves under appropriate conditions. In order to make this system radiate electromagnetic radiation will emerge at the frequency of the corresponding plasma wave in the layered system. A grating placed on the surface of such a layered system can quench the momentum at discrete wave numbers, related to the grating period, and if plasma instability can occur at any of these wave numbers, the layered system with the grating will become a radiation source. A first principle formalism has been developed for the combined "grating plus single layer" current driven sys- tem. This is a generalization of previous work by W.L. Schaich on response of stable (non current driven) systems. Work on current driven systems is being carried out in collaboration with Schaich. The details will be presented in a series of papers under preparation. The main technique is to study the electromagnetic response of the full system to an externally incident electromagnetic wave, and in particular to obtain the reflection and transmission coefficients in the presence of the drift current. The normal modes of the system are given by the pole in the reflection coefficient. These are the self-generating modes of the system in the absence of the external incident wave. In the presence of a strong enough current, the mode frequency becomes complex and the plasma waves grow in time. These are the plasma instabilities due to the drift current. Studies so far show that there are two main effects due to the grating: (1) The dispersion relation is altered by the presence of the grating and (2) The second effect is that radiation is emitted from the system at the modified frequency of the growing plasma waves inside the system. This arrangement can be used either as a radiation source or as an amplifier. The ideal arrangement would be to trap the emitted radiation in a cavity surrounding the system, so that it can reenter the system through the grating, get amplified and come out at an increased strength. Repetition of this process can lead to high levels of emitted radiation.

\section{MAGNETIC, ELECTRONIC AND THERMAL PROPERTIES OF MAGNETIC MULTILAYERS}

R.E. Camley

D.L. Mills

University of Colorado at Colorado Springs

SC: ARL

Very recently there were the first experimental results on the properties of antiferromagnetic superlattices $\left(\mathrm{CoF}_{2} / \mathrm{FeF}_{2}\right)$. These results showed that for some samples two magnetic phase transitions occur, while for other samples only one phase transition occurs. These new antiferromagnetic superlattices may be important because, in contrast to most of the magnetic multilayers presently being studied, they are made out of insulators rather than metallics. From a fundamental point of view this is attractive because these systems can be properly modeled with localized spins interacting via exchange interactions. 
There are known insulating systems which are good representatives for Heisenberg systems as well as Ising and $X Y$ models. In addition, insulators are much more promising for signal processing applications because of lower losses and narrower linewidths. Also typical excitation frequencies for antiferromagnets lie in the infrared rather than in the microwave, which makes these materials of interest for high-frequency applications. Work to date has shown how the experimental results could be understood in terms of interface exchange coupling and film thickness. The key idea is that the coupling at the interfaces allows the material with the higher transition temperature to stabilize the material with the lower transition temperature. Since the stabilization only extends a few atomic layers away from the interface, this produces a significant effect for thin films, but only a small change for thicker films. A reasonable set of parameters resulted in agreement with experimental results. From these parameters, it was concluded that the magnetic films have fairly large exchange coupling at the interface, thus characterizing the new superlattices to a significant degree.

\section{HOT ELECTRON PHYSICS OF ALTERNATING - CURRENT THIN-FILM ELECTROLUMINESCENT DEVICES}

John F. Wager

Stephen M. Goodnick

Oregon State University

\section{SL: ARL/EPSD}

Ensemble Monte Carlo simulations of high-field transport in $\mathrm{ZnS}$ are performed which include polar optic phonon, acoustic phonon, and intervalley scattering and which treats the conduction band as three valleys. Nonparabolicity is found to be important for stabilizing the electron distribution which otherwise exhibits electron run-away above $100 \mathrm{kV} / \mathrm{cm}$. A calculated steady-state velocity-field curve is in agreement with previously published results of Brennan [J. Appl. Phys. 64, 4024 (1988)] at high fields but deviates from Brennan's result at low fields and is in better agreement with experimentally deduced low-field mobilities than that reported by Brennan. At 1 $\mathrm{MV} / \mathrm{cm}$ in steady-state, one finds a significant fraction of the transported electrons gain sufficient energy to excite luminescent impurities; this fact was obscured by Brennan's presentation of his data because he normalized the electron kinetic energy profile by the conduction band density of states. Nonstationary effects such as velocity overshoot do not appear to play an important role in establishing the high-field transport in $\mathrm{ZnS}$. An investigation is being made of the electrical response of $\mathrm{ZnS}: \mathrm{Mn}$ ACTFEL devices studied as a function of the voltage amplitude, pulse width, and rise/fall time of the applied voltage. Experimental turn-on voltage and polarization charge trends can be accurately simulated using an improved SPICE model for the ACTFEL in which a parallel resistor-capacitor network is included in the Zener diode branch of the equivalent circuit. The dynamic phosphor field characteristics can also be accurately reproduced using this improved SPICE model. Additionally, it is found that the dynamic phosphor field characteristics may be quantitatively explained without having to invoke the presence of space charge within the phosphor layer. Another project currently underway is estimation of the interface state density by measurement of the dynamic charge [i.e., $\mathrm{Q}(\mathrm{t})$ ] characteristics. The interface state density is inferred by computer simulation of the $Q(t)$ decay curve subsequent to a voltage pulse. The $Q(t)$ transient is attributed to the re-emission of electrons from interface trap states by thermal emission, thermally-assisted tunneling, and pure tunneling.

Reports:

1. Electrical Characterization and Modeling of ACTFEL Devices, by J.F. Wager et al., MS.

2. Distribution of Trapped Electrons at Interface States in ACTFEL Devices, by S. Kobayashi et al., MS.

3. Monte-Carlo Simulation of High-Field Electron Transport in Alternating-Current Thin-Film Electroluminescent Devices, by $\mathbf{K}$. Bhattacharyya et al., MS.

\section{SPUTTERED FERROELECTRIC THIN FILMS OF KTN FOR ELECTRO-OPTIC DEVICES}

\section{Sanat Sashital}

TRW, Inc.

Suitable hardware modification was made to an existing RF-PM sputtering machine. It included design, fabrication and installation of a suitable substrate heater assembly (Mo filament; heated up to $850^{\circ} \mathrm{C}$ with adequate temperature sensing and controls within $\pm 5^{\circ} \mathrm{C}$ ) for up to 4 inch Si wafers and other substrates. A ceramic KTN sputtering target was fabricated. A trial composition of $\mathrm{KTa} .5 \mathrm{Nb} .5 \mathrm{O}_{3}$ was 
chosen and starting materials synthesis and ceramic processing procedures were provided to the vendor. Hot isostatic pressing of sintered KTN ceramic powders yielded best results. X-ray analysis at TRW together with vendor's composition analysis confirmed the phase and anticipated stoichiometry. Thus, a stoichiometric $\mathrm{KTa} .5 \mathrm{Nb} .5 \mathrm{O}_{3} \mathrm{RF}-\mathrm{PM}$ target of good mechanical integrity was obtained with the above procedures.

\section{MODELING OF CHAOTIC FLUCTUATING CHANNELS FOR SIGNAL DETECTION}

Cory Myers

Henry Abarbanel

Lockheed Sanders, Inc.

SL: ARDEC

SC: ARL

Chaos poses a significant challenge for the time series analyst, since structure in strange attractors tends to be very intricate and nonuniform. Although frequently referred to as unpredictable deterministic behavior, chaotic systems are in fact forecastle over limited time scales. Techniques for construcient predictive models for chaotic dynamics are discussed, including a variety of functional interpolation schemes and several examples of connectionist approaches to the problem. Error estimates based on polynomial interpolation are provided. The underlying deterministic nature of chaotic signals motivates a nonlinear smoothing procedure for the reduction of noise.

\section{Reports:}

1. Effects of Convolution on Chaotic Signals, by Steven $H$. Isabelle et al., MS.

2. Mode1 Reconstruction of Chaotic Dynamics: First Preliminary Radar Results, by Simon Haykin and Henry Leung, MS.

3. Modeling of Chaotic Time Series for Prediction, Interpolation, and Smoothing, by John J. Sidorowich, MS.

4. Signal Processing in the Context of Chaotic Signals, by Alan V. Openheim et al., MS.

5. Synchronized Chaotic Signals and Systems, by Lou Pecora and Tom Carroll, MS

6. Codebook Prediction: A Nonlinear Signal Modeling Paradigm, by Andrew C. Singer et al., MS.

7. Chaotic Signals and Physical Systems, by Henry Abarbanel, MS.

8. Signal Separation for Nonlinear Dynamical Systems, by Cory Myers et al., MS.

9. Modeling Chaotic Systems with Hidden Markov Models, by Cory Myers et al., MS.

\section{ELECTRONIC STRUCTURE OF FINITE SYSTEMS}

P. Jena

Virginia Commonwealth University

\section{SL: BWL, ARL/MD}

SC: ARDEC

Theoretical investigations will be made on the dissociative chemisorption of hydrogen on cluster surfaces, pairing of hydrogen in transition and rare earth metals, the effects of symmetry and topology on magnetism in clusters. Ground state geometries, total energy, and electronic structure of hydrogen interacting with neutral and charged metal clusters, hydrogen-hydrogen interaction, and proton spin-lattice relaxation times in niobium, and magnetic moments of cobalt in clusters will be calculated using state-ofthe-art theoretical techniques, with an eye towards the development of new atomically engineered materials.

\section{MICROWAVE AND MILLIMETER WAVE MAGNETIC SOLITONS AND CHAOS IN MAGNETIC THIN FILMS AND THIN SUPERLATTICES}

Carl E. Patton

Colorado State University

There have been four major accomplishments in the soliton work. First, researchers completed a detailed study of the effect of surface spin pinning on the resonance spectra for YIG films. These pinning effects define the nature of the low wavenumber dispersion curves which control soliton properties. This work has resolved several long standing problems concerning the interpretation of resonance spectra for thin films. Second, a state-of-the-art system was built for the experimental study of microwave magnetic solitons in YIG films and applied to the study of forward volume wave solitons and surface wave solitons. These results confirm and expand upon the work of previous workers in the former Soviet Union and in Italy on microwave magnetic solitons and establish the CSU program as a center of unique expertise in this area. Third, researchers have observed two new types of microwave magnetic solitons, backward wave solitons and so-called dark solitons. The backward wave soliton offers new possibilities for signal processing applications. The dark soliton is an entirely new effect consisting essentially of a hole in a nonlinear cw magnetic signal. Fourth, 
considerable progress was made in modeling the properties of microwave magnetic solitons numerically. It is possible to match the experimentally observed profiles for the above soliton categories very well, based on numerical solutions to the nonlinear Schrödinger equation.

\section{LASER AND OPTICAL PHYSICS}

Melvin Lax

City University of New York

\section{SL: ERDEC}

SC: ARL

Statistical decision theory, such as the Neyman-Pearson procedure, will be used to investigate the scattering properties of multi-parameter small system of scatterers to arrive at distinguishing features of the scattering medium. For distributed feedback semiconductor lasers, the rate of information transmission is limited principally by "partition noise" which introduces bit errors at the receiving end down an optical fiber; both analytical and combined analyticnumerical methods will be used to determine the best set of design parameters to reduce the noise. A Green's function approach that permits space dependence will be used to model electron transport in real3D devices including tunneling through quantum barriers, transport through quantum wells with coupling to photons to account for nonequilibrium heating using a phonon packet representation, ultrafast relaxation of photoexcited electrons, and nonlinear high frequency conductivity in strong $\mathrm{AC}$ electric fields.

\section{HIGH SPEED INTEGRATED LIGHT MODULATORS}

David Bloom

Stanford University

SC: ARL

The work was focussed on two objectives: producing arrays of devices and increasing the yield. At the beginning of the effort a determination was made to redesign the structure for use with visible light in the first diffraction order. The design was tested with fixed gratings and color coordinates were measured. One dimensional arrays of devices were fabricated. Researchers measured 20.5 ns switching times and pronounced device hysteresis. The first step in rede- signing the devices for color/visible operation was the design, fabrication, and projection of a fixed grating structure that modelled a two dimensional low resolution ( $200 \times 200$ color pixels) display with 12 bits of color. An image was selected from a graphics library, pixellated, rendered in silicon, and then projected and the CIE color coordinates were measured. Arrays (4x64) of operational devices were designed, fabricated, and tested. The yield was unsatisfactorily low, so alternative release techniques were explored. A number of chemical and physical processes were tried with a focus on passivating the silicon surfaces with non-polar molecules. These experiments were successful in increasing yield. Also, dielectric coatings of the metallic surfaces also improved yield, but additional work in this area is still needed.

\section{FAR-INFRARED (THz) LASERS USING MULTIPLE QUANTUM WELLS}

\section{Qing $\mathbf{H u}$}

Clifton G. Fonstad

Massachusetts Institute of Technology

\section{SC: ARL/EPSD, ARL}

The researchers have ordered a cryostat with a 12tesla superconducting magnet. According to a new theory developed by Professor S. Geng at UCLA, the nonradiative relaxation rate in a quantum well (mainly dominated by Auger process) can be substantially decreased by applying a magnetic field, which will confine the electron motion in the transverse direction and reduce the dimensionality of the system. Consequently, the lasing efficiency can be improved and the lasing threshold reduced. Furthermore, the emission of optical photons, which is the main reason prohibiting the lasing frequency to be above $9 \mathrm{THz}$, is also suppressed. Thus, the frequency range of the proposed quantum-well lasers can be extended to mid-infrared frequencies. This superconducting magnet will enable the researchers to achieve this goal. The researchers have fabricated many multiple quantum-well structures with different well widths, barrier widths, and doping profiles. They have performed extensive tunneling spectroscopy and magnetotunneling spectroscopy on these devices. They have also obtained some key information for building the lasers. By measuring the resonant tunneling current at different temperatures, the researchers have measured the activation energy which is the difference between the Fermi energy at zero temperature and the resonant subband energy. 
The researchers have also pioneered a novel technique of magnetotunneling spectroscopy. In this technique, the researchers monitor a resonant current peak due to a LO phonon-assisted tunneling. The amplitude of this resonant current oscillates with a magnetic field applied in the longitudinal direction (along the current), similar to the de Haas-van Alphen oscillation. The period of oscillation (in $1 / B$ ) is a function of the difference between two subbands between which the phonon-assisted tunneling occurs. The researchers were to present this novel technique and the measurement results at the 1993 APS March meeting. Currently, the researchers are preparing a manuscript summarizing these tunneling and magnetotunneling results.

\section{NONLINEAR OPTICAL STUDIES OF EXCITONS IN SEMICONDUCTOR HETEROSTRUCTURES}

Duncan G. Steel

University of Michigan

This program focuses on the study of exciton dynamics, primarily in GaAs heterostructures. The experimental methods are based on the use of nonlinear laser spectroscopy methods which were developed earlier in this program on simpler systems. Recently, however, researchers have also begun to examine the basic origin of the nonlinear response in these systems, since measurements show behavior which does not seem to be explained by the existing theoretical descriptions. Research has focused on understanding the dynamical contribution to the nonlinear optical response which has not yet been considered in these systems as well as carrying out experiments in the area of nonlinear magneto-optics.

\section{SEQUENTIAL RESONANT TUNNELING AND ELECTRIC FIELD DOMAINS IN SEMICONDUCTOR SUPERLATTICES}

Roberto D. Merlin University of Michigan

SC: ARL/EPSD, ARL

In a series of experiments, studies were made of the time response of a quantum-well structure to a step- like optical perturbation. The system is such that it exhibits two electric-field domains under cw excitation. The data represent photoluminescence (PL) spectra obtained at various time delays with respect to time at which the laser is turned on. At a given $t$, there are two peaks in the spectra corresponding to the two electric-field domains. The intensity of the peaks varies in a quasiperiodic fashion. The corresponding frequencies were found to be extremely sensitive to the applied bias voltage. At this time, researchers are exploring theoretical approaches to understand this behavior. Tentatively, the results are ascribed to the highly nonlinear motion of the domain boundary. Other work consists of developing a cryogenic near-field scanning optical microscope system. The microscope centers around a fiber optic tip giving a lateral resolution of $\approx 400 \AA$. The tip can be scanned using a coarse differential spring and by controlling the bias voltage across a piezoelectric rod to which the fiber is attached. With this system, attempts will be made to probe the field or, alternatively, the PL-profile of a quantum-well structure a few wells - ideally one well - at a time.

\section{COMPOSITE NANOSTRUCTURED MATERIAL FABRICATION BY ELECTROCHEMICAL SCANNING PROBE MICROSCOPY}

Kenneth Douglas

University of Colorado

Progress has been made in characterizing the parameters needed to achieve selective electrodeposition onto a nanostructured surface and in techniques and procedures needed to create a nanoheterostructure, a class of composite solid-state/solid and bimolecular/solid state materials with nanometer-scale structure. A newly created device call a minicell, a minielectrochemical cell, proved to be very successful in terms of dealing with the small substrate size (usually less than $20 \mathrm{~mm}^{2}$ ) on which the nanostructures are formed. A properly designed electrochemical cell for these experiments should have the working-reference and working-counter electrode distances the same. It should also use a counter electrode with a large surface area exposed to the electrolyte, and one which is located over the whole working electrode, so that deposition occurs smoothly and uniformly across the entire substrate surface. 


\section{E. Acoustics, Optics and Cross Disciplinary}

\section{EXPERIMENTAL STUDIES OF FUNDAMENTAL PROBLEMS IN QUANTUM OPTICS}

Thomas W. Mossberg

Michael G. Raymer

University of Oregon

A number of years ago under ARO support the PI formulated a scheme for the storage of optical temporal waveforms in inhomogeneously broadened materials. The storage of waveforms and their subsequent regeneration can be used as the basis of an ultra-high speed and density memory and also, it turns out, as an optical waveform convolver crosscorrelator. Under continued ARO support, the scheme proposed and a number of variations of it were experimentally demonstrated to be feasible. Now the PI has discovered a radically new approach to the storage of temporal waveforms in frequency selective materials. This new approach, termed swept-carrier time-domain memory, provides a means to store extremely long data pulses, to fully utilize the spectral storage capabilities of materials over wide ranges of data input/output rates, and to avoid a number of fundamental problems associated with the original scheme when applied to real storage materials. This development should move the exciting capabilities of time-domain frequency-selective memories substantially closer to practical utilization. Two Phys. Rev. Letters report studies of the temporal and spatial coherence properties of stimulated Raman scattering. One letter discusses measurements of the spatial fluctuations caused by quantum noise and manifested as shot-to-shot changes in the direction of the generated beam. The second discusses a new technique for measuring very small amounts of vibrational coherence in a high-density gas of molecules. By spatially interfering two Stokes pulses, produced in sequence in the same sample of molecules, it is possible to detect on the order of 20 coherently vibrating molecules, in the presence of about $10^{16}$ incoherently vibrating molecules.

26695 FERROELECTRIC LIQUID

CRYSTAL OPTICAL

INTERCONNECT SWITCHING NETWORKS
Joseph W. Goodman

Stanford University

Researchers continued theoretical work aimed at understanding the design and performance of a waveguide $1 \times 2$ switch based on ferroelectric liquid crystals. The switch would use birefringent walkoff to allow either of two output positions for a light beam to be selected electrically, thereby creating the building block for a more complex switching network. A waveguide-based switch is attractive because of the lone interaction length that can be achieved. In birefringent waveguides, the ordinary and extraordinary modes are coupled. There is a set ratio between the field strength of ordinary and extraordinary modes depending on the propagation constant. This in turn determines the energy distribution in the ordinary and extraordinary beams, and therefore the energy distributions in the two states of the switch. However, the relation between the field ratio and the energy ratio is not a simple squared one. Through the Poynting vector, the energy ratio is found to be related to both the square of the field ratio and the propagation constant. The following issues have been investigated: (1) the number of propagating modes; (2) the ratio between the field strengths of the ordinary and extraordinary beams; and (3) the ratio between the energy distributions of the ordinary and extraordinary beams.

Reports:

No. $1-4$ in previous editions.

5. Ferroelectric Liquid Crystal Optical Switching Networks, by Robert N. McRuer, PhD Thesis, 1991, 153 pp.

6. Oblique-Incidence Liquid-Crystal-Tunable Etalon, by Larry R. McAdams et al., Opt Let 16,864(1991). AD A244 602

\section{STRUCTURE AND SWITCHING DYNAMICS IN FERROELECTRIC CRYSTAL AND LIQUID CRYSTAL THIN FILMS}

Noel A. Clark

James Scott

University of Colorado

Researchers have completed a theoretical analysis of the equations for flow of smectic $\mathrm{C}$ liquid crystals. This is a rigorous development which offers a proper foundation for understanding smectic $\mathrm{C}$ flow properties. The model treats both backflow (the generation of flow by molecular reorientation), transverse flow (the generation of molecular hopping between smectic layers by application of shear flow). Studies 
were made of the switching behavior of ferroelectric liquid crystals having more ordered smectic phases (smectics I and F), and in the compound 8SI, a careful study was made of the switching states. The results show a sequence of eight states appearing as the field is scanned from the large negative to large positive limit. The results confirm an understanding of the layer structure in these systems and address several incorrect interpretations in the literature. In addition, researchers have quantitatively characterized the d.c. leakage current in PZT thin-film ferroelectric memories. It was shown that the I (V) response is ohmic up to about $3 \mathrm{~V}$, above which it is perfectly quadratic. This implies that at the $5 \mathrm{~V}$ CMOS operating voltage these devices are spacecharge limited current carriers and that they are fully depleted. They have also developed a novel optical flow rate/pressure sensor based upon thermal focussing and surface convection in thin $(0.043 \mathrm{~cm}) \mathrm{PMN}$ ceramic films.

Reports:

No. $1-15$ in previous editions.

16. Quantitative Measurement of Space-Charge Effects in Lead Zirconate-Titanate Memories, by J.F. Scott et al., $J$ Appl Phys 70,382(1991). AD A244 650

17. Temporal Dependence of Thermal Self-Focusing in Ferroelectric $\mathrm{Ba}_{2} \mathrm{NaNb}_{5} \mathrm{O}_{15}$ and $\mathrm{Ce}^{3+}: \mathrm{Sr}_{\mathrm{B}} \mathrm{Ba}_{1-\mathrm{xN}} \mathrm{Nb}_{2} \mathrm{O}_{6}$, by Ting Chen et al., Ferroelectrics 120,115(1991). AD A244 737

18. Use of the Thermal Lens Effect at $T_{\mathrm{c}}=855 \mathrm{~K}$ in $\mathrm{Ba}_{2} \mathrm{NaNb}_{5} \mathrm{O}_{15}$ to Measure Order Parameter Evolution, by J.F. Scott et al., Ferroelectrics 117,21(1991). AD A244 736

19. Low Temperature Properties of Incommensurate Barium Sodium Niobate, by William F. Oliver III and James F. Scott, Ferroelectrics 117,63(1991). AD A244 991

20. Evaluation of Critical Exponents From Raman Intensities, by J.F. Scott and Ting Chen, Phase Transitions 32,235(1991).

21. An Approach to the Design of Ferroelectric Liquid Crystals with Large Second Order Electronic Nonlinear Optical Susceptibility, by David M. Walba et al., MS.

22. Director-Polarization Reorientation via Solitary Waves in Ferroelectric Liquid Crystals, by I. Abdulhalim et al., MS, Appl Phys Let.

23. Optical Bistability in Ceramic Ferroelectrics Due to Thermal Focussing, by T. Chen et al., MS, Appl Phys Let.

24. Total Internal Reflection Studies of a Ferroelectric Liquid Crystal-Anisotropic Solid Interface, by Zhiming Zhuang and Noel A. Clark, SPIE Proc 1455,105(1991). AD A249655

25. Use of the Boulder Model to Predict Coupling Between Dipoles in the Core and Chiral Tail of Fluorinated Ferroelectric Liquid Crystals, by M.D. Wand et al., MS, Mol Cyst and Liq Cryst. AD A249 472

26. Thermal Focusing in Ferroelectrics Near $T_{c}$ : Effect of Conjugate Electric Fields, by Shou-Jong Sheih et al., Ferroelectrics 123,1(1991). AD A248 749

27. Raman Spectroscopy of Incommensurate $\mathrm{Ba}_{2} \mathrm{NaNb}_{5} \mathrm{O}_{15}$, by A. Shawabkeh and J.F. Scott, Phys Rev B43,999(1991). AD A249 305
28. Ferroelectric Memories: A Comparison with Other HighSpeed Digital Devices, by J.F. Scott et al., Ferroelectrics 116,147(1991). AD A249 003

\section{NONEQUILIBRIUM SCREENING AND EXCITON DYNAMICS PROBED BY FEMTOSECOND LASER PULSES}

Nasser Peyghambarian

Stephan Koch

University of Arizona

Resonant coherent lattice vibrations were observed in the vicinity of the indirect absorption edge in a Bil 3 layered crystal in a femtosecond pump-probe experiment. Coherent optical photons that are impulsively excited by femtosecond pump pulses modulate the phase of probe pulses, causing oscillation of the probe spectrum in time. This oscillation, which has a period given by the period of the oscillation, continues for more than 100 cycles. Optical dephasing, i.e., the decay of the polarization field in a semiconductor, is a direct consequence of electron and hole scattering. Under high excitation conditions or in a semiconductor amplifier/laser, carrier-carrier scattering is often the dominant relaxation mechanism, which also leads to energy-level broadening and dynamical screening of the Coulomb interaction potential. The theoretical analysis of carrier-carrier scattering based on the quantum Boltzmanan equation. Even though this equation is well known in the many-body literature, its solution for high excitation conditions and nonequilibrium carrier distributions is a substantial challenge. To study nonequilibrium carrier relaxation and optical dephasing, researchers performed direct numerical integrations of the timedependent Boltzmann equation where the dynamical screening of the Coulomb interaction potential was consistently treated at the same level as the scattering probabilities. The solution of the quantum Boltzmann equation yields the dynamic changes of the electron and hole distribution functions. These changes can be expressed in terms of scattering rates, which determine the average time an electron or hole remains in a quantum state. In generalization of the well-known relation between optical dephasing and excited state lifetime in atomic systems, the sum of the lifetimes of the semiconductor electrons and holes in a state determine the dephasing of that state. 
Reports:

No. $1-5$ in previous editions.

6. Comparison of Optical Nonlinearities of Type II and Type I Quantum Wells, by K. Meissner et al., Appl Phys Let 59,259(1991). AD A249 187

\section{RESEARCH IN}

\section{PHOTOREFRACTIVE CRYSTALS}

Amnon Yariv

California Institute of Technology

SC: ARL

Work has concentrated on photorefractive beam coupling in paraelectric $\mathrm{K}_{1-y} \mathrm{Liy} \mathrm{Ta}_{1-x} \mathrm{Nb}_{x} \mathrm{O}_{3}$ (KLTN) crystals. Researchers have investigated the frequency reflectivity of fixed holograms for use as narrow-band interference filters. Also, the properties of dynamic holography during phase modulation of the interfering beams were studied. Two beam coupling in photorefractive crystals is described by wellknown coupled mode equations. Researchers have applied the theory to several new problems. First, they solve the coupled mode equations which gives the index modulation in the crystals. Then the resultant index grating is considered to be fixed, and reflection as a function of frequency is calculated. A similar theory was used to describe the effect of two-beam coupling in a material with a fixed grating. This theory was applied to describe the performance of an all-optical microphone/vibration sensor.

\section{Reports:}

1. Frequency Response and Tunability of Grating-Assisted Directional Couplers, by Giora Griffel and Amnon Yariv, IEEE J Quant Electron 27,1115(1991). AD A244 982

2. Fixing of a Photorefractive Grating in $\mathrm{KTa}_{1-\mathrm{x}} \mathrm{Nb}_{\mathbf{x}} \mathrm{O}_{3}$ by Cooling Through the Ferroelectric Phase Transition, by Victor Leyva et al., Opt Let 16,554(1991). AD A244 980

3. Determination of the Physical Parameters Controlling the Photorefractive Effect in $\mathrm{KTa}_{1-\mathrm{x}} \mathrm{Nb}_{\mathbf{x}} \mathrm{O}_{3}: \mathrm{Cu}, \mathrm{V}$, by Fictor Leyva et al., J Opt Soc Am B8,701(1991). AD A244 981

4. Optical Interferometry Between Image-Bearing Beams and Their Redirected Phase Conjugates, by Mordechai Segev and Amnón Yariv, Opt Let 17,145(1992). AD A249 310

5. Interferometric Electrooptical Signal Processors with Partially Coherent Illumination, by Joseph Roen et al., MS, $J$ Opt Soc Am.

6. A Fundamental Limitation on the Performance of Photorefractive Waveguide Devices, by Mordechai Segev and Amnon Yariv, MS, Opt Let.

\section{SELECTIVE REACTIVITY, ULTRAFAST ENERGY TRANSFER, AND THE DEVELOPMENT OF CHEMICALLY PUMPED VISIBLE LASERS}

James L. Gole

Georgia Institute of Technology

Research has been concerned with the development of chemically pumped visible chemical laser systems. Efforts have involved three major thrusts. Work has continued on development of energy transfer based visible chemical laser amplifier and oscillator systems based on fast energy transfer from metastable metalloid oxides. These energy transfers pump and create population inversions on select metal atom transitions. Researchers have now completed definitive gain measurements and have demonstrated significant proof of continuous laser action based on the $\mathrm{Na}$ $4 d^{2} D-3 p 2 P$ transition (569nm) pumped by SiO metastables. They are well into the testing of an up-scaled supersonic expansion system to study the chemically pumped $\mathrm{Na} 2$ amplifiers produced from the $\mathrm{Na}_{3}-x(\mathrm{Cl}, \mathrm{Br}, \mathrm{I})$ reaction systems in order to convert these amplifiers into continuous visible chemical laser oscillators and have completed modifications of their Phase I Na3 system to study the $\mathrm{Na}_{3}-\mathrm{F}_{2}$ reactions. Finally, attempts are being made to extrapolate the Na3-halogen atom concept to the reactions of the alkaline earth trimers ( $\left.\mathrm{Mg}_{3}-\mathrm{Ba}_{3}\right)$ in the hopes of producing excimer based amplifiers.

\section{PICOSECOND DEMODULATION AND COHERENT ELECTRON BEAMS}

\section{H. Craig Casey}

Bob D. Guenther

Duke University

Researchers have continued with the solid state device design for this project (silicon on sapphire microcircuits for generation and detection of millimeter and submillimeter microwaves). The graduate student sponsored by this project has spent considerable time studying microwave design techniques and has performed a preliminary study in the design of filters using coplanar transmission lines. The fabrication of the microcircuits has followed from this work. Most recently researchers have become extremely interested in Ti:sapphire lasers as a means of driving the solid state devices. Ti:sapphire lasers are cur- 
rently reported producing $150 \mathrm{fS}$ pulses at $150 \mathrm{MHz}$ repetition rates, operating between 700 and 800 nanometers with average power of 0.2-2.0 watts. These characteristics far exceed those of a CPM laser and are well matched to this work using SOS devices for microwave generation and detection.

\section{OPTICAL SIGNAL PROCESSING}

Anthony VanderLugt

North Carolina State University

\section{SC: ARL}

A surprising number of traditional topics, such as the Rayleigh resolution criterion, spatial filtering, bandlimited signals, the sampling theorem, phase contrast microscopy, and white light holography can be explained using simple interference theory. These basic results are then easily extended to the $\mathrm{N}$-source case to introduce diffraction theory, and facilitates the teaching of the elements of modern optics to junior and senior level students. A paper has been prepared which develops the general scanning relationships of an acousto-optic system using both a purely geometrical optics and a physical optics approach; each approach provides useful insights into the scanning relationships. The diffraction approach reveals that there are four basic scanning configurations: a long or short chirp scanner, either aperture or repetition rate limited. The throughput rate for a scanner is always maximized if we use the short chirp scanning, repetition rate limited mode of operation. The maximum rate may be achieved with other configurations but at the expense of a decrease in some of the other performance parameters. Examples are given of how these design relationships are used. Digital radio transmission systems use complex modulation schemes that require powerful signal processing techniques to correct channel distortions and to minimize bit error rates. In a paper, acousto-optic processors are used, in conjunction with adaptive digital equalizers, to reduce the bit error rate of the digital radio receiver. The acousto-optic processor implements an inverse channel filter that is rapidly adaptable to time varying distortions. A specific architecture is identified and a laboratory system is tested to verify the ability of the processor to track and correct time varying channels. Computer simulations are used to show that the hybrid acousto-optic and digital equalizer allows a four-fold increase in the modulation capacity of the radio, relative to all digital equalization, while improving the bit error rate performance. In another paper, combined analog and digital processors are investigated for minimizing the mean square error of the radio receiver. The analog filters are implemented using acousto-optic processing since rapidly adaptable, inverse channel filters can be produced for either minimum or non-minimum phase channels. A specific architecture is identified and a laboratory system is tested to verify the ability of the processor to track and correct time varying channels. Computer simulations are used to show that the hybrid acousto-optic and digital equalizer allows a 16-fold increase in the modulation capacity of the radio, relative to all digital equalization, while maintaining similar equipment signatures.

\section{Reports:}

No. 1-12 in previous editions.

13. Equalization of Microwave Digital Radio Channels with a Hybrid Acousto-Optic and Digital Processor, by Chris S. Anderson, MS.

14. Acousto-Optic Channel Equalization for Microwave Digital Radios, by Chris A. Anderson, MS, Appl Opt.

15. Hybrid Acousto-Optic and Digital Equalization of Microwave Digital Radios, by Christopher S. Anderson, PhD Thesis, 1991, $253 \mathrm{pp}$.

16. Short Pulse Detection by Acousto-Optic Processing, by A. VanderLugt, MS, SPIE Proc.

17. Time Delay Detection of Short Pulses by Fresnel and Fourier Transformations, by A. VanderLugt et al., MS, Appl Opt.

\section{SCANNING NEAR FIELD OPTICAL MICROSCOPY USING A VIBRATING KNIFE EDGE OR STYLUS}

\section{Adrian Korpel}

University of Iowa

The experiment was completed in which attempts were made to verify predictions about two-dimensional image formation. Two methods were used: (1) the detection of the difference frequency between the two orthogonal knife edges, vibrating at $1000 \mathrm{~Hz}$ and $1100 \mathrm{~Hz}$, in a phase synchronous fashion, and (2) scanning with one vibrating knife edge only and deriving the derivative in the non-scan direction by computer processing. No attempt was made to achieve submicron resolution at this stage. The difference frequency experiment was quite successful, both quantitatively (signal levels) and qualitatively. The simulation experiment was designed to resemble as closely as possible the actual operation of the device. A square opaque rectangle was made to vibrate in two directions simultaneously on the computer screen, and quasi-periodically obscured a 
bright pixel that served as a spatial impulse. The resulting record of obscurations (zeros) and transmissions (ones) was stored in a file and analyzed for the presence of the difference frequency component. As predicted, the two-dimensional impulse response function appeared to be the product of two identical functions: $h(x, y)=g(x) g(y)$. A horizontal and a diagonal cross section through $h(x, y)$ showed close agreement with theoretical values.

\section{SYNCHROTRON X-RAY DIFFRACTION ANALYSIS OF PHOTOREFFRACTIVE CRYSTALS AND THEIR GRATINGS}

Mark Cronin-Golomb

Tufts University

\section{SL: DARPA}

A trip was made to the NSLS during which researchers successfully recorded the first X-ray typographs of photorefractive gratings. These gratings were written by a CW diode pumped frequency doubled YAG laser. Nuclear emulsion plates were used to record images of gratings with $40 \mu \mathrm{m}$ and $7 \mu \mathrm{m}$ period, and CCD camera images of the longer period gratings were recorded so that the emulsion plate densities could be calibrated. Gratings imaged in surface typographs showed contrast reversal when passing through the needle like features that had previously been tentatively identified as 180 degree domain structures. The contrast reversal confirms this identification: the electrooptic coefficients have opposite signs in 180 degree domains, so the crystal stresses are opposite in different domains. True calibration of the images is still in progress.

\section{FUSION OF MULTIPLE SENSING MODALITIES FOR MACHINE VISION}

\author{
J.K. Aggarwal \\ University of Texas at Austin
}

\section{SL: NVESD, WSMR}

Work has resulted in (1) an interpretation system for high-level reasoning for the prototype knowledgebased system to interpret ground-based, kilometerrange laser radar images; (2) a review of various approaches to estimate motion parameters from a sequence of two range images; (3) a new algorithm to detect and classify straight lines in images of complex scenes using the principles of perceptual organization to group features according to categories of relations; (4) a new approach to detection of large man-made objects in outdoor non-urban scenes using a single monochrome image using a computational framework based upon the principles of perceptual organization of perceptual grouping; (5) a new technique for estimating the position and pose of an autonomous mobile robot navigating in an outdoor, urban environment consisting of polyhedral building, in which the free space of the robot is partitioned into a set of distinct, non-overlapping regions called "edge visibility regions"; and (6) an algorithm to compute the structure of stationary imaged objects and the motion of a moving camera from a sequence of video images, which exploits the principle of the invariance of rigid configurations during motion.

\section{DEVELOPMENT OF AN ANALYTICAL PHOTON SCANNING TUNNELING MICROSCOPE}

Michael A. Paesler

North Carolina State University

\section{SC: ARL/EPSD, ARL/MD}

The optics program has been expanded to incorporate other similar optical instruments that fall under the general category of near-field scanning optical microscopy (NSOM). This expansion strengthened the microscopic capabilities and broadened the range of materials that one can investigate. These new configurations serve as complements to the existing photon scanning tunneling microscopy (PSTM) program. Experimental progress includes advances in tip fabrication (which is useful for any scanning optical microscopy configuration); the demonstration of NSOM images; and the development of a novel technique that enhances image contrast. The latter, which involves rapid modulation of the microscope sęnsing tip in a direction perpendicular to the surface plane of the sample, also yields information about tip structure and the local electric field distribution. A micro-pipette puller (typically used for micro-biology applications) has been adapted for use in drawing single-mode fibers down to tip radii of approximately $70-100 \mathrm{~nm}$. Incorporated in the puller is a $\mathrm{CO}_{2}$ laser which is focused onto one 
side of the fiber and retro-reflected onto the other side to provide a source of relatively uniform heat. The morphology of the pulled tip can be controlled by adjusting pulling parameters which include the pull strength, laser intensity, heating area, and timing mechanisms. Tips are coated with an Al film approximately 200-800 ̊̊ thick. Tips are currently coated at each of four directions orthogonal to the tip axis. Images show evidence that tip coating is quite nonuniform and asymmetric. Consequently an instrument is currently being built that will rotate the tips while evaporation is taking place. This will provide more uniform, symmetric coatings. A conventional NSOM image gives the transmitted signal as a function of location in the $x-y$ plane of the sample. In what is called the illumination NSOM mode, light is incident on one side of the sample through the drawn tip and is collected in transmission on the opposite side of the sample with conventional optical lenses. Superresolution is provided by the illuminating tip. A demonstrably different image is obtained when the sample tip is rapidly dithered in a direction normal to the surface of the sample while imaging. Typical amplitudes of oscillation are $10-100 \mathrm{~nm}$ and the frequency is generally near $18 \mathrm{kHz}$ (i.e., much higher than the scan rate). The modulated signal is passed through a lock-in amplifier and the amplitude of the modulated component of the signal is sent to the $z$-channel of the control and imaging electronics.

\section{HIGH-POWER CW DIODE-LASER-ARRAY-PUMPED SOLID STATE LASERS AND EFFICIENT NONLINEAR OPTICAL FREQUENCY CONVERSION}

Robert L. Byer

Stanford University

\section{SL: DARPA}

Work is continuing on the construction of a semiconductor-diode-laser-pumped Nd:YAG laser to operate with frequency-and amplitude-stable single-mode 100-watt continuous-wave output at $1064 \mathrm{~nm}$. A number of related studies are simultaneously under way. These topics include resonator design, stable laser operation, injection locking techniques, laser and nonlinear optical materials characterization, and techniques of nonlinear optical frequency conversion such as external resonant cav- ity harmonic generation optical parametric oscillation. The $100-\mathrm{W}$ laser project involves a number of problems in engineering design, electrical and mechanical assembly. The initial lot of ten 10-W diodelaser arrays has been received. Other hardware to support these diode lasers is being developed and built.

\section{Reports:}

1. Measurement of Nonlinear Optical Coefficients by PhaseMatched Harmonic Generation, by Robert C. Eckardt and Robert L. Byer, SPIE Proc 1561,119(1991). AD A249 469

\section{RESEARCH IN OPTICAL SCIENCES: JOINT SERVICES OPTICS PROGRAM}

Robert R. Shannon

Michael A. Cusanovich

University of Arizona

\section{SL: AFOSR}

SC: TACOM

Research efforts have been concerned with the following: modeling of infrared staring sensors using charge transfer devices, wide field-of-view micro-optics, optical elements for X-UV wavelength, fundamental physics of MBE heterostructures, MBE growth of novel semiconductor heterostructures, optical nonlinearities in low-dimensional semiconductor structures, carrier relaxation studies in semiconductor lasers, amplifiers, and in novel $\mathrm{GaAs}$ quantum-well structures, spectral hole burning and instabilities in semiconductor lasers, light interactions with metal clusters on semiconductors, propagation of short optical pulses in passive and active nonlinear all-optical switches, and atom optics.

Reports:

1. MBE Growth of Novel Semiconductor-Material Structures, by Galina Khitrova, TR, 1991, 20 pp.

2. Fundamental Physics of MBE Heterostructures, by Hyatt M. Gibbs, TR, 1991, 28 pp.

3. Induced Focusing of Optical Pulses in a Self-Defocusing Semiconductor Medium, by G. Khitrova et al., MS.

4. Interaction of a Single Metal Cluster with Light, by Dror Sarid and Sam Howells, TR, 1991, 12 pp.

5. Optical Nonlinearities and High-Contrast Modulation in Strained-Layer InGaAs/GaAs Multiple Quantum Wells, by K. Okada et al., MS.

6. High Contrast, Submilliwatt Power InGaAs/GaAs Strained-Layer Multiple-Quantum-Well Asymmetric Reflection Modulator, by R. Jin et al., MS, Appl Phys Let. 
7. Evidence for CW Spectral Hole Burning in a Vertical-Cavity Surface-Emitting Laser, by H.M. Gibbs et al., MS.

8. High Contrast, Submilliwatt Power InGaAs/GaAs Strained-Layer Multiple-Quantum-Well Asymmetric Reflection Modulator, by R. Jin et al., MS.

9. Spatial Solitons in a Self-Focusing Semiconductor Gain Medium, by G. Khitrova et al., MS.

10. Annual Report - Research in Optical Sciences, by Robert R. Shannon, TR, Feb 92, 53 pp.

\section{ITERATIVE ENCODING METHODS FOR COMPUTER GENERATED HOLOGRAMS}

Michael R. Feldman

University of North Carolina at Charlotte

$$
\begin{array}{ll}
\text { SL: ARL } \\
\text { SC: TACOM }
\end{array}
$$

Work has led to the development of the Recursive Mean Squared Error (RMSE) algorithm for designing CGH's. Also efforts were concentrated on fabrication of holograms designed with this method, to test simulation results. Three holograms were identified for fabrication: a 9 by 9 spot array, binary phase hologram with a simulated diffraction efficiency of 78.1 percent, a 10 by 10 spot array hologram with a simulated diffraction efficiency of 83.5 percent, and a 32 spot array hologram with a simulated diffraction efficiency of 75.7 percent. The holograms will be fabricated by reactive ion etching in a 3 etching step process. These 3 steps will be used to generate 8 phase levels on two of the three holograms. Three sets of masking data are therefore needed for each hologram. The mask data for each of these holograms was generated and the masks were fabricated by electron beam lithography. The results of experimental testing of an eight phase level holographic lens along with previous simulation results were compiled in a paper entitled, "Iterative Discrete On-axis Encoding for Radially Symmetric Computer Generated Holograms." This paper was submitted to Applied Optics for publication. In addition an article appeared in Laser Focus World describing some of the results of this project. Although the RSIDO method can provide extremely high diffraction efficiency for holographic lenses, it is limited to radially symmetric holograms. In order to avoid this limitation, efforts are being made to develop a modified version of this method to extend its applicability to non-radially symmetric systems. With the modified RSIDO method, the hologram is divided into a series of regions. Within each region, a radially symmetric model is assumed. This allows for non-radial solu- tions to be generated for fringes that cross over several regions. Several holograms have been designed for this method for an optical interconnect project sponsored by ARPA.

\section{Reports:}

1. Recursive Mean Squared Error Algorithm for Iterative Discrete On-Axis Encoded Holograms, by Jared D. Stack and Michael R. Feldman, MS, Appl Opt.

\section{AN ADAPTIVE LIQUID CRYSTAL TELEVISION BASED JOINT TRANSFORM CORRELATOR AS APPLIED TO REAL TIME PATTERN RECOGNITION}

Francis T.S. Yu

The Pennsylvania State University

\section{SL: MICOM, NVESD}

The research objective is to study an adaptive liquid crystal television based joint transform correlator for real time pattern recognition. The following topic will be explored: (a) evaluation of the phase modulation properties of a liquid crystal TV; $(b)$ investigate the use of an iterative phase correction scheme for a phase only filter; and (c) evaluate the use of phase only circular filters for rotation invariant pattern recognition.

\section{PHOTOREFRACTIVE NONLINEAR OPTICS}

Mark Cronin-Golomb

Tufts University

SL: DARPA

SC: MICOM, NVESD

A paper has been prepared which presents information on large-amplitude oscillations in the phase conjugate intensity which have been observed when one of the pumping beams of an externally pumped photorefractive phase conjugate mirror deviates slightly from the Bragg angle. The Fourier transform of the experimental time series data exhibits the broad frequency spread characteristics of a chaotic signal. A phase space plot is constructed, and is found to exhibit some of the features associated with strange attractors. The fractal dimension of the attractor is determined to be approximately 5.7, indicating chaotic behavior. The number of dynamical dimensions for this attractor is found from Taken's theorem to be approximately 13 , considerably greater than what is predicted by the standard scalar four-wave mixing 
equations, and an explanation for such high dimensionality is presented in terms of a vector field description of photorefractive four-wave mixing. Additionally, evidence is presented to support the conclusion that the chaotic behavior results from the off-Bragg detuning of one of the pumping beams. In another paper researchers show that the double phase conjugate mirror may be made achromatic by using material dispersion to compensate the dispersion of the photorefractive grating. It is shown that the bandwidth of the entire visible spectrum may be accommodated in barium titanate crystal. The achromatic conditions found in the experiment are in good agreement with theory. Broadband to single frequency image conversion is demonstrated.

\section{Reports:}

1. Whole Beam Method for Photorefractive Nonlinear Optics, by Mark Cronin-Golomb, Opt Commun 89,276(1992).

2. Photorefractive Two-Beam Coupling with Reduced Spatiotemporal Coherence, by Hongzhi Kong et al., Opt Let 16,1183(1991). AD A244 940

3. Temporal and Spatial Coherence Effects in Photorefractive Nonlinear Optics, by Hongzhi Kong, PhD Thesis, 1992, 164 pp.

4. Phase-Conjugate Interferometric Analysis of Thin Films, by Elaine R. Parshall and Mark Cronin-Golomb, Appl Opt 30,5090(1991). AD A249529

5. Experimental Study of Achromatic Volume Holography with Dispersive Compensation in Barium Titanate, by Hongzhi Kong et al., Opt Let 17,297(1992). AD A249 223

6. Femtosecond Temporal Encoding in Barium Titanate, by L.H. Acioli et al., Opt Let 16,1984(1991). AD A249 224

7. Achromatic Double Phase Conjugate Mirror, by Hongzhi Kong et al., MS, Opt Commun.

\section{ACOUSTIC PROCESSING IN PHOTOREFRACTIVE SYSTEMS}

Dana Z. Anderson

University of Colorado

\section{SC: ARO, ARL/EPSD, ARL, MICOM}

This period of research focused primarily on developing the full, one-dimensional time-delay system. This system was demonstrated in real time using a liquid crystal spatial light modulator (LCM) driven by a computer. An acoustic signal, either speech or music from a $C D$ player, is digitized and time-window Fourier analyzed by the computer in real time. The Fourier power spectrum is placed as a one-dimensional grey scale image onto the LCM, which imposes the Fourier spectrum onto a laser beam. This beam is then the signal input to the photorefractive time-delay. Researchers have shown theoretically that the time-delay device is a true time-delay, meaning that the signal reproduced by the device at some time is a replica of the signal at an earlier time, except for an overall amplitude factor. In other words, the frequency of the signal is reproduced. The hologram can also be used to generate sound as well as to record and replay sound. One could, in fact, use a hologram as a general waveform synthesizer.

\section{DEVELOPMENT OF NOVEL SUBWAVELENGTH LIGHT SOURCES AND LASERS}

Aaron Lewis

Hebrew University

Researchers have been actively extending the development of a subwavelength stand alone light source in the direction of making it the central component of an ultrahigh density, near-field optical memory system. The principal directions have been to improve the methods of producing the electroluminescent subwavelength light sources that have been developed and to integrate this unique device into an ultrahigh density optical memory system that will allow for the rapid access times that are required for practical systems.

\section{GROWTH, CHARACTERIZATION AND APPLICATIONS OF KTN FAMILY NONLINEAR OPTICAL CRYSTALS}

Amnon Yariv

California Institute of Technology

Work has continued on growth and characterization of photorefractive $\mathrm{KTa}_{1-x} \mathrm{Nb}_{x} \mathrm{O}_{3}(\mathrm{KTN})$ and $\mathrm{K}_{1-y} \mathrm{Li}_{y}$ Tal- $x \mathrm{Nb}_{x} \mathrm{O}_{3}$ (KLTN) crystals. Recent efforts have concentrated on the photorefractive effect observed in these crystals without an applied field. Researchers have determined that the zero external field photorefractive (Zefpr) effect seen in paraelectric KTN and KLTN crystals is due to a Jahn-Teller strain modulation which leads to an index grating via the photoelastic effect. This effect has not previously been documented in photorefractive materials. Beam coupling resulting from Zefpr gratings has been shown to have unique properties stemming from the local response of the medium. One application of the Zefpr effect which was predicted and demonstrated is its use as a sensitive vibration detector/microphone. Here, a grating is written in a Zefpr material with two coherent laser beams. One of the beams is reflected off of an acoustic membrane which 
vibrates in response to sound waves. Because of the nature of the Zefpr effect, the phase modulation of the laser beam by the motion of the membrane is translated linearly into an intensity variation of the two interfering beams. This phenomenon has been experimentally established in the demonstration of an all-optical microphone.

\section{OPTICAL PROCESSING, HOLOGRAPHY, INTERFEROMETRY WITH FEMTOSECOND PULSES}

Emmett N. Leith

University of Michigan

\section{SC: ARL/BELVOIR2, ARL/WTD}

A paper has been prepared in which different holographic methods for imaging through biological tissue are evaluated and compared. The role of the source autocorrelation function is analyzed. A graphical plot for performance evaluation is introduced. Experimental results for the various methods are given and possibilities for further development are indicated. Another paper describes a lensless imaging system, in which the image is formed by the mutual intensity function instead of by a conventional intensity distribution. Both theoretical and experimental results are given. In a third paper a theoretical model is established for finding the resolution of imaging through random media with the first-arriving-light method. With this model, analytical mathematical forms of the point spread function are derived for transillumination and confocal scanning imaging modes combined with the first-arriving-light method. Finally, experiments were carried out with the holographic gating technique to demonstrate the validity of the theory. The experimental results show that the first arriving-light method improves the image resolution by a factor up to 20 over the conventional transillumination mode. 


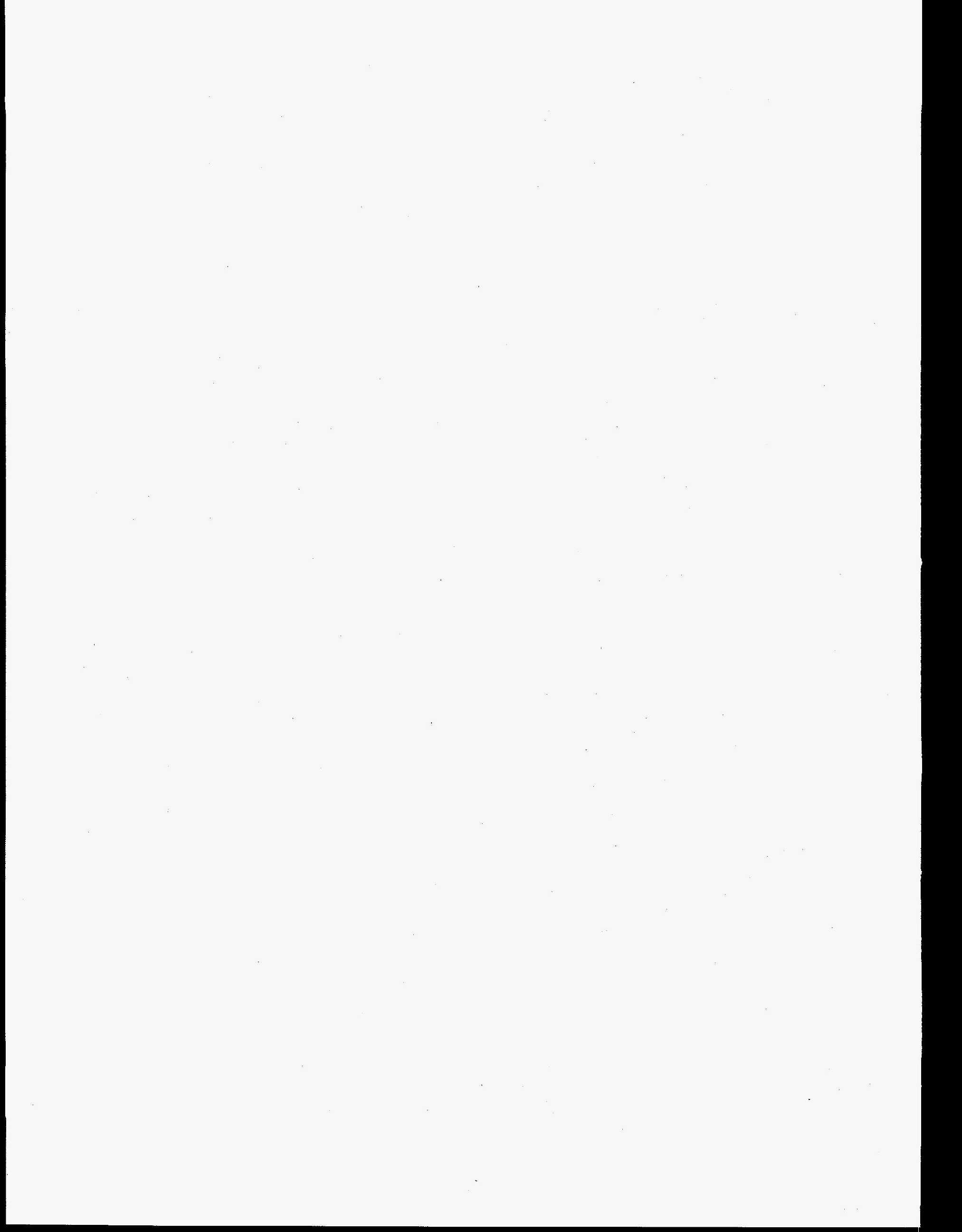




\section{CHEMISTRY}

\section{A. Inorganic, Analytical, and Electrochemistry}

\section{DIELECTRICS FOR THE SUPERCAPACITOR}

Sergio Petrucci

Polytechnic Institute of New York, Farmingdale

SL: ARL/EPSD, ARL/MD

SC: AROE, ARL/EPSD

Microwave data are being taken of the complex permittivity $\varepsilon^{\prime}=\varepsilon^{\prime}-\mathrm{J} \varepsilon^{\prime \prime}$, infrared and visible refractive indices and infrared attenuation coefficients for dimethylcarbonate, chloroform and their mixtures at $25^{\circ} \mathrm{C}$. For pure dimethylcarbonate, the real part of the complex permittivity $\varepsilon^{\prime} v$. frequency shows the presence of two relaxation domains: the microwave frequency range, already studied and interpreted as the rotational relaxation of the methoxy groups $-\mathrm{O}-\mathrm{CH}_{3}$ around the carbonate $\rangle \mathrm{C}=\mathrm{O}$ moiety, and the infrared frequency range with a newly discovered relaxation domain. Pure $\mathrm{CHCl}_{3}$ has revealed in the profile of $n^{2}$ IR (the squared infrared refractive index) as a function of frequency, a new dielectric phenomenon previously hinted by literature data in the far-IR region. This phenomenon has been explained qualitatively by current theories in terms of collision induced dipoles. $\mathrm{CHCl}_{3}$ and dimethylcarbonate have been used in mixtures because of their potential interactions through $\mathrm{H}$-bonding as opposed to inert $\mathrm{CCl}_{4}$ mixed with dimethylcarbonate. Mixtures of dimethylcarbonate and $\mathrm{CHCl}_{3}$ show a microwave dielectric spectrum, deeply altered from that expected at molar fraction $X_{D M C}=0.50$, if the two components did not interact with each other. A mixture of dimethylcarbonate and $\mathrm{CCl}_{4}$ of similar composition, namely $\mathrm{X}_{\mathrm{DMC}}=0.50$ does not reveal the same dielectric phenomenon as for the $\mathrm{DMC}-\mathrm{CHCl}_{3}$ mixtures, thus confirming that the source of the phenomenon at microwave frequencies is attributable to the interaction of $\mathrm{DMC}$ with $\mathrm{CHCl}_{3}$.
Reports:

No. 1 in previous edition.

2. Static, Microwave, Infrared, and Visible Permittivity Related to Chemical Structure; N-Methylacetamide, NDimethyl Acetamide and Their Mixtures in $\mathrm{CCl}_{4}$ at $32^{\circ} \mathrm{C}$, by Paul Firman et al., TR, Jun 91, 30 p. AD A246 364

3. Microwave and Infrared Dielectric Relaxation of Alkylcarbonates, Chloroform and Their Mixtures at $25^{\circ} \mathrm{C}$, by R. Chandra et al., TR, Jun 92, 45 p.

\section{SINGLE SOURCE PRECURSORS FOR THE OMCVD OF III/V COMPOUND SEMICONDUCTORS}

Richard A. Jones

John G. Ekerdt

University of Texas at Austin

SL: ARO

SC: AROE, ARL/EPSD, ARL/MD

Low pressure growth studies on single source precursors have focussed on $\left[\mathrm{Me}_{2} \mathrm{Ga}\left(\mu-t-\mathrm{Bu}_{2} \mathrm{As}\right)\right]_{2}$, $\left[\mathrm{Et}_{2} \mathrm{Ga}\left(\mu-t-\mathrm{Bu}_{2} \mathrm{As}\right)\right]_{2}$, and $\left[\mathrm{Me}_{2} \mathrm{Ga}\left(\mu-i-\mathrm{Pr}_{2} \mathrm{As}\right)\right]_{3}$. The results of the work have been accepted for publication. Details on the growth of epitaxial (100) GaAs films using the single source precursor $\left[\mathrm{Me}_{2} \mathrm{Ga}\left(\mu-t-\mathrm{Bu}_{2} \mathrm{As}\right)\right]_{2}$ have also been accepted for publication. Several other classes of compounds have been evaluated for film growth. These include a base free diphosphadigalletane [Ar'PGa-t-Bu$]_{2}$ $\mathrm{Ar}^{\prime}=\left(2,4,6-t-\mathrm{Bu}_{3}-\mathrm{C}_{6} \mathrm{H}_{2}\right)$; aluminum based compounds which feature $\mathrm{PH}_{2}$ or $\mathrm{AsH}_{2}$ units [t$\left.\mathrm{Bu}_{2} \mathrm{Al}\left(\mathrm{EH}_{2}\right)\right]_{3}(\mathrm{E}=\mathrm{P}, \mathrm{As}) ; \mu-t-\mathrm{BuPh}$ and $\mu-t-\mathrm{BuAsH}$ bridged dimers $\left[t-\mathrm{Bu}_{2} \mathrm{Ga}(\mu-t-\mathrm{BuPH})\right]_{2}$ and $[t-$ $\left.\mathrm{Bu}_{2} \mathrm{Ga}(\mu-t-\mathrm{BuAsH})\right]_{2}$ as well as cubanes of the type $\left[\phi_{3} \mathrm{SiEM}-t-\mathrm{Bu}\right]_{4}(\mathrm{M}=\mathrm{Al}, \mathrm{Ga})$. In addition, examples of a novel class of monomeric precursor which features $\mathrm{M}-\mathrm{H}$ bonds have been isolated and characterized.

\section{Reports:}

1. An Aluminum - Phosphorus Cubane. A New Aluminum Phosphide Precursor, by Alan H. Cowley et al, MS. 
2. III/V Precursors with P-H or As-H Bonds. A Low Temperature Route to Gallium Arsenide and Gallium Phosphide, by Alan H. Cowley et al., Organometal 10,652(1991). AD A244 770

3. Decomposition Pathways of the Novel GaAs Precursors $\left[\mathrm{Me}_{2} \mathrm{Ga}(\mathrm{m}-i-\mathrm{Pr} 2 \mathrm{As})\right]_{3}$ and $[\mathrm{MeGa}(\mathrm{m}-\mathrm{t}-\mathrm{Bu} 2 \mathrm{As})] 2$, by James E. Miller et al., MS, Chem Mater.

4. Reaction of $\left(t-\mathrm{BuGaCl}_{2}\right) 2$ with $\mathrm{Ar}^{\prime} \mathrm{PHLi}\left(\mathrm{Ar}^{\prime}=2,4,6-t\right.$ $\left.\mathrm{Bu}_{3} \mathrm{C}_{6} \mathrm{H}_{2}\right)$ : Preparation of the Chloride-Bridged Dimer $\left(t-\mathrm{BuGa}(\mathrm{Cl}) \mathrm{P}(\mathrm{H}) \mathrm{Ar}^{\prime}\right)$, , by Alan H. Cowley et al., Heteroatom Chem 2,11(1991). AD A244 772

5. X-ray Crystal Structure of the Dimethylgallium Azide Polymer and Its Use as a Gallium Nitride Precursor, by David A. Atwood et al., J Organometal Chem 394,C6(1990). AD A234 145

6. Synthesis and Structure of a Diphosphadigalletane: A Novel Base-Stabilized Ga2P2 Ring System, by Alan H. Cowley et al., Angew Chem 29,1150(1990). AD A233 974

7. Growth and Characterization of Gallium Arsenide Using Single-Source Precursors: OMCVD and Bulk Pyrolysis Studies, by James E. Miller et al., Chem Mater 2,589(1990). AD A234 144

8. Primary Amido and Amine Adduct Complexes of Gallium: Synthesis and Structures of $\left[t-\mathrm{Bu}_{2} \mathrm{Ga}(\mathrm{m}-\mathrm{NHPh})\right]_{2}$ and $t$ $\mathrm{Bu}_{3} \mathrm{GaNH}_{2} \mathrm{Ph}$, by David A. Atwood et al., Polyhedron 10,1897(1991).

9. A Novel Gallium-Phosphorus Cage Compound, by Alan $\mathbf{H}$. Cowley et al., Angew Chem 30,1141(1991).

10. Synthesis and Structures of Two Bulky Gallium Chlorides, by David A. Atwood et al., J Coord Chem 25,233(1992)

11. Synthesis and Structure of the First-Base-Free Diphosphadigalletane, by D.A. Atwood et al., J Am Chem Soc 113,7050(1991). AD A249 375

12. Tetrameric Gallium and Aluminum Chalcogenides, [tBuME]4 $(\mathrm{M}=\mathrm{Al}, \mathrm{Ga} ; \mathrm{E}=\mathrm{S}, \mathrm{Se}, \mathrm{Te})$. A New Class of Cubane, by Alan H. Cowley et al., Angew Chem 30,1143(1991). AD A249 217

13. Isopropylphosphido and Arsenido Derivatives of Gallium and Indium. Isolation of Gallium-Phosphorus and IndiumPhosphorus Dimers and Trimers, by Alan H. Cowley et al., Organometal 10,1635(1991). AD A244 771

14. Synthesis and X-Ray Structure of $\mathrm{Me}_{2} \mathrm{InI}\left(\mathrm{NH}_{2}-t-\mathrm{Bu}\right)$. The First Structurally Characterized Amine Adduct of a Dialkyl Indium lodide, by David A. Atwood et al., MS.

15. Synthesis and Structural Characterization of $o$ Tol3GaOEt2, by David A. Atwood et al., MS.

16. Primary Amide and Amine Complexes of Gallium and Indium: X-Ray Crystal Structures of $\left[\mathrm{Me}_{2} \mathrm{Ga}(\mathrm{m}-\mathrm{NH}(t-\right.$ $\mathrm{Bu}))]_{2}, \mathrm{Me}_{3} \mathrm{GaNH}_{2}(t-\mathrm{Bu})$ and $\mathrm{Me}_{3} \mathrm{InNH} 2(t-\mathrm{Bu})$, by David A. Atwood et al., MS, J Organometal Chem.

17. Synthesis and Structures of $\left[\mathrm{NMe}_{2}(\mathrm{~m}-\mathrm{NMe} 2) \mathrm{GaCl}\right]_{2}$ and $[\mathrm{TMP}(\mathrm{m}-\mathrm{OEt}) \mathrm{GaCl}]_{2} \quad(\mathrm{TMP}=2,6$ Tetramethylpipyridine), by David A. Atwood et al., MS.

18. Pyrolysis Studies of the Single-Source GaAs Precursors $\left[\mathrm{Me}_{2} \mathrm{Ga}(\mathrm{m}-\mathrm{As}-\mathrm{i}-\mathrm{Pr})\right] 3$, [Me2 $\left.\mathrm{Ga}(\mathrm{m}-\mathrm{AsMe} 2)\right] 3,[\mathrm{Me} 2 \mathrm{Ga}(\mathrm{m}-$ As-t-Bu2 $)] 2$, and $[\mathrm{Et} 2 \mathrm{Ga}(\mathrm{m}-\mathrm{As}-\mathrm{t}-\mathrm{Bu} 2)] 2$, by James E. Miller et al., Chem Mater 4,447(1992).

19. Growth of Epitaxial (100) GaAs Films Using the Single Source Precursor $\left[\mathrm{Me}_{2} \mathrm{Ga}(\mathrm{m}-\mathrm{t}-\mathrm{Bu} 2 \mathrm{As})\right]_{2}$, by James E. Miller and John G. Ekerdt, Materials 4,7(1992).
20. Single Source Precursors for III-V OMCVD Growth and Pyrolysis Studies, by Richard A. Jones et al., Mat Res Soc Symp Proc 204,73(1991). AD A249 234

\section{STRUCTURAL AND DYNAMICAL ASPECTS OF ELECTRODEPOSITION}

Hector D. Abruna

Cornell University

\section{SL: NVESD \\ SC: AROE, ARL/EPSD}

Studies have continued on the underpotential deposition (UPD) of copper and of silver onto well defined Pt(111) single crystal electrode surfaces. In the case of copper UPD, the voltammetric response is extremely sensitive to the surface preparation and/or pretreatment. In fact, the voltammetric response appears to be even more sensitive than the traditional voltammetry in sulfuric acid alone where the socalled "butterfly" response is taken as indicative of a clean and well-ordered surface. Electrodes that yield the characteristic "butterfly" response in sulfuric acid can give rather different $\mathrm{Cu}$ UPD voltammetry. Copper UPD in sulfuric acid might be a better test of long range order of the surface. UPD of silver on Pt(111) appears to be very dependent on the nature of the surface and, again, electrodes that give the characteristic "butterfly" response can give different voltammetric responses during Ag UPD. In situ structural investigations of the underpotential deposition of copper on an iodine covered platinum surface have been carried out using $x$-ray standing waves generated by specular (total external) reflection and Bragg diffraction. Surface coverage isotherms derived from both electrochemical and x-ray measurements were also compared. The growth mode of the copper ad-layer appears to be strongly influenced by the electrode's surface morphology.

Reports:

1. Underpotential Deposition of Silver on $\operatorname{Pt}(111)$ : Part I. Concentration Dependence, by J.F. Rodriguez et al., MS.

2. In situ Structural Studies of the Underpotential Deposition of Copper onto an Iodine Covered Platinum Surface using X-Ray Standing Waves, by G.M. Bommarito et al., MS, SPIE Proc. 


\section{REACTOR STUDIES OF METAL IONS ASSOCIATED WITH CHROMATOGRAPHIC SILICA}

Stanley H. Langer

University of Wisconsin - Madison

\section{SC: CRDEC, ARL/MD, NRDEC}

Stable, reproducible, ferric ion-modified silica stationary phases have been prepared in aqueous media by a batch process. The catalytic activity of this modified stationary phase for hydroquinone oxidation to benzoquinone was considerably greater than that of earlier ferric ion-modified silicas from nonaqueous media. Capacity factors of selected solutes were used to compare the retention properties of the aqueous ferric ion-modified silica with those of the unmodified silica. The capacity factors for solutes containing hydroxyl groups tended to increase for the ferric ion-modified silica relative to those for the unmodified silica column, whereas the capacity factors for carbonyl-containing compounds (ester, ketones, and aldehydes) became smaller or were unaffected.

Reports:

No. $1-4$ in previous editions.

5. Liquid Chromatographic Reactor Studies of Ferric Ion Association with Silica, by Chawn-Ying Jeng, PhD Thesis, $1991,310 \mathrm{p}$.

\section{SYNTHESIS OF TUNGSTEN NITRENE COMPLEXES PRECURSORS FOR TUNGSTEN NITRIDE}

Lisa McElwee-White

Stanford University

SC: AROE

The reaction of $c i s$-azobenzene with a series of $p$-substituted phenyl carbons $(\mathrm{CO})_{5} \mathrm{~W}=\mathrm{C}(\mathrm{OMe}) p-\mathrm{X}$ $\mathrm{C}_{6} \mathrm{H}_{4}\left(\mathrm{X}=\mathrm{H}, \mathrm{OMe}_{2} \mathrm{CF}_{3}\right)$ was carried out in both non-coordinating and coordinating solvents. The stability and reactivity of the initially formed zwitterionic species (CO) 5 WHPhNPhC(OMe) $\left(p-\mathrm{XC}_{6} \mathrm{H}_{4}\right)$ depended on the substituent $X$. In non-coordinating solvents, the unsubstituted zwitterionic species (CO) $5 \mathrm{WHPhNPhC} \mathrm{OMe}) \mathrm{C}_{6} \mathrm{H}_{6}$ was converted into an isomeric zwitterionic intermediate and a 2,4diazametallacycle. Both isomeric zwitterions and the 2,4-diazametallacycle ultimately decomposed to yield the metathesis product $\mathrm{PhN}=\mathrm{C}(\mathrm{OMe}) \mathrm{Ph}$. The mechanism of 2,4-diazametallacycle formation was shown to involve the intermediacy of a coordinated diaziridine in which the metal subsequently inserts into the $\mathrm{N}-\mathrm{N}$ bond. When the unsubstituted zwitterion was decomposed in $\mathrm{CH}_{3} \mathrm{CN}$, the coordinated diaziridine was displaced by solvent, inhibiting formation of the 2,4-diazametallacycle.

Reports:

1. Metathesis and Diaziridination Reactions of $(\mathrm{CO}) 5 \mathrm{~W}=\mathrm{C}(\mathrm{OMe}) p-\mathrm{X}-\mathrm{C}_{6} \mathrm{H}_{4}$ with $c i s-A z o b e n z e n e . ~ E l e c-$ tronic and Solvent Effects, by Claudia Tata Maxey et al., $J$ Am Chem Soc 114,5153(1992).

2. Stabilization of Zero-Valent Hydrazido Complexes by Phosphine Ligands. Crystal Structure of fac$(\mathrm{CO})_{3}$ (DPPE) W $=\mathrm{NNMe}_{2}$, a Nitrene Analogue to Fischer Carbenes, by Bruce A. Arndtsen et al., MS, J Am Chem Soc.

28402 ENCAPSULATED ALKALINE-EARTH ORGANO-METALLICS AS CONTROLLED SOURCES OF CALCIUM, STRONTIUM, AND BARIUM IONS

Timothy P. Hanusa

Vanderbilt University

\section{SC: AROE, ARL/MD}

The major development that occurred centered on the continuing synthesis of new "encapsulated" metallocenes containing highly substituted cyclopentadienyl or cyclooctatetrenyl rings and an evaluation of the structural features that contribute to their air/thermal stability and volatility. Some of the earlier rationalizations for the properties of encapsulated systems now appear to require some refinement. Key features of the findings include the following: the origin of the high levels of air stability (up to $30 \mathrm{~min}$ ) displayed by metallocenes containing the new cyclopentadienyl ligand $\mathrm{C}_{5} \mathrm{H}_{3}\left(\text { cyclo }-\mathrm{C}_{6} \mathrm{H}_{5}\right)_{3}$ is under investigation, and the tetraphenylcyclopentadienyl ligand seems ideally suited for generating encapsulated metallocenes, but in the past reactions using it often produced only low yields of desired products. Multidentate ethers such as DME have been used as solvents to prepare alkaline-earth octaphenylmetallocenes in much higher purity than before. Preliminary studies of their reactivity suggests that they are behaving as would be expected for encapsulated species. 


\section{DECONTAMINATION AND REDOX CATALYSIS}

Craig L. Hill

Emory University

\section{SL: NRDEC}

SC: AROE, CRDEC, ARL/MD

The direct thermal oxidation of thioethers such as mustard (HD) by some polyoxometalates can form the basis of new catalytic cycles for decontamination of $\mathrm{HD}$ and related compounds by $\mathrm{O}_{2}$ catalyzed by the polyoxometalates. Mechanistic work has begun. A polyoxotungstate with catalytic activity has been functionalized with aryl phosphonyl groups. This chemistry provides an entry into covalent immobilization of catalytically active polyoxometalates on surfaces and fibers. From extensive NMR and other studies, one now understands several features of the hydrolytic chemistry of the organic "handles" attached to these redox active clusters. A comprehensive paper on these new classes of polyoxometalates will be published. Further mechanistic work on the facile thermal dehalogenation of a range of aliphatic and aromatic halocarbons by the twoelectron reduced form of decatungstate, $\mathrm{W}_{10} \mathrm{O}_{32} 6^{-}$, has been accomplished. Much data are consistent with rate determining dissociative electron transfer or halogen atom abstraction as the C-halogen cleavage step.

Reports:

1. Comparative Study of Polyoxometalates and Semiconductor Metal Oxides as Catalysts. Photochemical Oxidative Degradation of Thioethers, by R. Carlisle Chambers and Craig L. Hill, Inorg Chem 30,2776(1991). AD A244 240

\section{THE REDOX CHEMISTRY OF SOME PEROXO AND SUPEROXO METAL ION COMPLEXES}

\author{
Richard C. Thompson \\ University of Missouri at Columbia \\ SL: NRDEC \\ SC: AROE, CRDEC
}

Measurements have been made of the Mo-95 NMR spectra of peroxomolybdenum(VI) complexes formulated as $\mathrm{Mo}\left(\mathrm{O}_{2}\right)_{4}{ }^{2-}, \quad \mathrm{MoO}\left(\mathrm{O}_{2}\right) 3^{2-}$, $\mathrm{MoO}(\mathrm{H})\left(\mathrm{O}_{2}\right)_{2}-\left(\mathrm{H}_{2} \mathrm{O}\right)^{-}, \mathrm{MoO}\left(\mathrm{O}_{2}\right)_{2}\left(\mathrm{H}_{2} \mathrm{O}\right)_{2}$, and $\mathrm{MoO}\left(\mathrm{O}_{2}\right)-\left\{\mathrm{C}_{5} \mathrm{H}_{3} \mathrm{~N}\left(\mathrm{CO}_{2}\right)_{2}\right\}\left(\mathrm{H}_{2}\right)$. Discrete peaks were found for each from -100 to $-500 \mathrm{ppm}$ relative to $\mathrm{MoO}_{4}{ }^{2-}$ as a standard. Assignment of the peaks was greatly simplified by previous equilibrium stud- ies, and the NMR results support that work at least semiquantitatively. The first five complexes comprise all the peroxo species of Mo(VI) (in the absence of heteroligands) over the $\mathrm{pH}$ region $0-12$. The approximate line width of each peak gives some qualitative information about the relative symmetry about the central molybdenum atom. Kinetic studies have been completed on the interconversions of di-, tri-, and tetraperoxo $\mathrm{Mo}(\mathrm{VI})$ complexes in basic solution. The most significant observation is that the interconversion of the di- and triperoxo complexes is extremely rapid, nearly too rapid for stopped-flow monitoring, whereas the conversion of the tri- to the tetraperoxo complex is much slower. Progress has been made on studies of peroxo complexes containing heteroligands. The quite stable oxodiperoxomolybdenum(VI) complex can be converted into an oxomonoperoxo species with dipicolinic acid in strongly acidic solution. Progress has also been made on a study of the product distribution when (en) ${ }_{2} \mathrm{Co}\{-\mathrm{S}-\mathrm{R}-\mathrm{R}-\mathrm{S}-\}^{+}$is oxidized by $\mathrm{H}_{2} \mathrm{O}_{2}$ or $\mathrm{MoO}(\mathrm{O})\left(\mathrm{O}_{2}\right)^{-}$. The issue is whether two oxygens are first transferred to one sulfur of the dithiol complex and none to the other, or, as one would predict, one to each sulfur atom.

\section{INORGANIC HALOGEN OXIDIZERS}

\section{Karl O. Christe}

Rockwell International Corporation

\section{SL: BRADEC, MICOM \\ SC: AROE, CRDEC, MICOM}

$\mathrm{NF}_{4}{ }^{+}$salts are the key ingredient in solid propellant $\mathrm{NF}_{3}-\mathrm{F}_{2}$ gas generators which are important for applications such as HF-DF lasers. Although a great number of $\mathrm{NF}_{4}{ }^{+}$salts have been prepared and characterized under ARO sponsorship, the theoretically highest performing candidates $\left(\mathrm{NF}_{4}{ }^{+}\right)_{3} \mathrm{AlF}_{6}{ }^{3-},\left(\mathrm{NF}_{4}{ }^{+}\right)_{2} \mathrm{BeF}_{4}{ }^{2-}$ and $\mathrm{NF}_{4}{ }^{+} \mathrm{F}$ have not been accessible so far because of the high basicity of these anions and the lack of a nonacidic solvent system. Researchers have now found the $\mathrm{NF}_{4}{ }^{+}$salts can be handled at low temperatures in $\mathrm{CH}_{3} \mathrm{CN}$ solution. This discovery opens up a new potential route towards new $\mathrm{NF}_{4}{ }^{+}$salts containing more basic anions using metathesis. In order to obtain the solubility data required for such metathetical reactions, solubilities of numerous $\mathrm{NF}_{4}{ }^{+}$and alkali metal salts in $\mathrm{CH}_{3} \mathrm{CN}$ were measured. Work on this interesting problem continues. The possibility of synthesizing novel catenated nitrogen fluorides was explored. These compounds would provide new improved ox- 
idizers which would be earth-storable, but have the increased performance of a space-storable liquid. Recent synthesis of anhydrous $\mathrm{N}\left(\mathrm{CH}_{3}\right)_{4}{ }^{+} \mathrm{F}$ and its use as a highly soluble source of naked fluoride ion has resulted in the synthesis and characterization of numerous novel ions possessing unusually high coordination numbers and oxidation states. New radical anions $\mathrm{SF}_{4}^{-}$and $\mathrm{SF}_{4} \mathrm{O}^{-}$were prepared by low-temperature $\gamma$-irradiation of $\mathrm{SF}_{5}{ }^{-}$and $\mathrm{SF}_{5} \mathrm{O}^{-}$salts. They were characterized by EPR spectroscopy and LDF calculations. Fullerene ("buckyball") chemistry is presently the hottest and fastest moving field of chemistry. Of particular interest are ions derived from $\mathrm{C}_{60}$ since $\mathrm{C}_{60}$ anions have been shown to exhibit super-conductivity at high temperatures. Whereas $\mathrm{C}_{60}$ anions can be prepared with relative ease, the corresponding $\mathrm{C}_{60}$ cations have so far not been reported. Several oxidation reactions of $\mathrm{C}_{60}$ were carried out. Using $\mathrm{O}_{2}{ }^{+} \mathrm{AsF}_{6}{ }^{-}$as the oxidant, materials have been prepared which appear to be the desired $\mathrm{C}_{60}{ }^{+} \mathrm{AsF}_{6}{ }^{-}$salts. Characterization of these materials is difficult, but preliminary EPR spectra and magnetic measurements look promising.

\section{Reports:}

1. New Synthesis, Crystal Structure and Vibrational Spectra of Tetramethylammonium Azide and Reactions of the Fluoride Anion with $\mathrm{HN}_{3}$ and of the Azide Anion with $\mathrm{HF}$, by Karl O. Christe et al, J Am Chem Soc 114,3411(1992).

2. The Pentabromine ${ }^{1+}$ Cation, Brs ${ }^{+}$. Local Density Functional Calculations and Vibrational Spectra, by K.O. Christe et al., MS.

3. Nitrosyl Hypofluorite: Local Density Functional Study of a Problem Case for Theoretical Methods, by David A. Dixon and Karl $O$. Christe, MS.

4. A Quantitative Scale for the Oxidizing Strength of Oxidative Fluorinators, by Karl O. Christe and David A. Dixon, $J$ Am Chem Soc 114,2978(1992).

5. Osmium Tetrafluoride Dioxide, $\mathrm{OsF}_{4} \mathrm{O}_{2}$ : A New Os. mium $^{+\mathrm{VIII}}$ Oxide Fluoride, by Karl $\mathrm{O}$. Christe and Roland Bougon, MS, J Chem Soc Chem Commun.

6. Nitrogen Pentafluoride: Covalent NFs versus Ionic $\mathrm{NF}_{4}+\mathrm{F}$ and on the Stability of the Latter, by Karl $\mathrm{O}$. Christe and William W. Wilson, MS, J Am Chem Soc.

\section{FUNDAMENTAL PROCESSES OCCURRING AT ELECTRODES}

Fred C. Anson

California Institute of Technology

\section{SC: AROE, CRDEC}

Work on the electrochemical behavior of soluble polyelectrolytes associated with or bonded to electroactive probes has been extended to polyacrylates. Attempts are underway to prepare a cobalt(III) com- plex coordinated to the carboxylate groups of the polyanion to variable extents. The preparative chemistry has proved challenging but is being pursued. A new line of experimentation has been initiated in which water soluble "starburst" dendrimers, a recently characterized new class of mixed polyelectrolytes, are reacted with redox probes and examined electrochemically. The preliminary results appear quite interesting and differ substantially from the other classes of polyelectrolytes examined in previous studies.

Reports:

1. Association of Electroactive Counterions with Polyelectrolytes. 4. Coordinative Binding of Ru(edta) to Poly(4vinylpyridine), by Rongzhong Jiang and Fred C. Anson, $J$ Phys Chem 96,452(1992). AD A249 311

\section{CHEMISTRY OF BORANE ANIONS: FORMATION OF PRECURSORS AND THEIR CONVERSION TO BORON, ALUMINUM NITRIDES AND METAL BORIDES}

Sheldon G. Shore

Ohio State University

\section{SC: BRADEC, CRDEC, MICOM, ARL/MD}

Higher boron hydride anions derived from pentaborane will be the focus for synthesis of a wide variety of heteroboranes ranging from main group to lanthanide metal derivatives. Also studies of boride, diboride, methide, and ethane will be carried out. Materials studies will be carried out in the area of the lanthanide borides and boron nitrides. Pentaborane, from government stockpiles, will be converted into the various boron anions which will be further converted by boron techniques to heteroatomboranes. Conversion of lanthanide borohydride to lanthanide borides will be investigated, and further studies will take place into the conversion of boranes to boron nitride.

\section{FULLERENE SUPERCONDUCTORS}

\section{Richard B. Kaner}

University of California, Los Angeles

\section{SL: DARPA}

The objective of the research is to explore the superconducting state properties of a number of materials based on the buckminsterfullerene molecule. A number of high quality powders and films of known 
fullerene superconductors will be prepared and their properties studied to permit the rational exploration of novel compounds with high transition temperatures.

\section{METALLACARBORANE-BASED PRECURSORS FOR ELECTROACTIVE MATERIALS}

Russell N. Grimes

University of Virginia

The research will center on the design and synthesis of metallacarborane sandwich complexes and their derivatives, and their conversion to new solid and liquid-state materials, with the ultimate goal of tailoring such products to have desired electronic or metallic properties. The research to be performed will include the synthesis, structural and physical characterization, and controlled modification of organometallic carborane-metal-delocalized oligomers and polymers. Detailed investigation of their molecular and electronic structures will be carried out. Modern spectroscopic and electrochemical tools and $\mathrm{x}$-ray crystallography will be employed.

Reports:

1. Organotransition-Metal Metallacarboranes. 28. Controlled Substitution at Cage Carbon Atoms in $\mathrm{CoC}_{2} \mathrm{~B}_{4}$ Clusters. Linkage of bis(Cobaltacarborane) Sandwich Complexes via Carbon-Bound Bridging Groups, by Mark A. Benvenuto and Russell N. Grimes, MS, Inorg Chem.

2. Organotransition-Metal Metallacarboranes. 29. Synthesis of Selectively C- and B-Substituted Double- and TripleDecker Sandwiches. (Eta $\left.{ }^{5}-\mathrm{C}_{5} \mathrm{Mes}\right) \mathrm{Co}(\mathrm{III})\left(\mathrm{Eta}^{5}\right.$ $\left.\mathrm{R}_{2} \mathrm{C}_{2} \mathrm{~B}_{3} \mathrm{R}_{3}^{\prime} \mathrm{H}_{2}\right)$ Cobalticinium Analogues, by Mark A. Benvenuto et al., MS.

3. Boron-Carbon Ring Ligands in Organometallic Synthesis, by Russell N. Grimes, Chem Rev 92,251(1992).

\section{DETECTION AND IDENTIFICATION OF AIRBORNE CONTAMINANTS}

\author{
Alan R. Katritzky \\ University of Florida \\ SL: CRDEC \\ SC: ARL/MD, NRDEC
}

The research objective is to prepare starburst dendrimer polymers which are functionalized on their outer surface for use as sensor coatings on Surface Acoustic Wave (SAW) devices. Surface modifications will be covalently bonded onto the ester-terminated dendrimers in a maximum of three well established chemical reactions. A variety of chemical groups that have previously shown promising activity in microsensors will be used. Specifically, the groups to be attached include 4-dimethylaminopyridine surfactants, $\mathrm{N}$-oxides of the foregoing type, ionic type surfactants, o-iodoso- and o-iodoxy-benzoic acid residues, $\mathrm{N}$-chlorocyanurates, oximes, a variety of ligand groups to be converted to metal chelates, diverse heterocycles, and phosphonic acids together with their esters and salts.

\section{DEVELOPMENT AND DEMONSTRATION OF ADVANCED TECHNOLOGIES FOR DIRECT ELECTROCHEMICAL OXIDATION OF HYDROCARBONS (METHANOL, METHANE, PROPANE)}

Anthony B. LaConti

John A. Kosek

Giner, Inc.

\section{SL: DARPA}

It has been demonstrated that $\mathrm{Pt}-\mathrm{Ru}$ is superior to $\mathrm{Pt}$ for methanol oxidation in both aqueous carbonate fuel cell (ACFC) and proton exchange membrane fuel cell (PEMFC) half-cell testing. An evaluation was made of the effect of electrode structure and operating conditions, including temperature, methanol concentration and concentration of the supporting electrolyte, on methanol oxidation in PEM half-cell testing. This should assist in the design of electrode/membrane structure for submission to JPL for advanced testing. Testing of an ACFC full cell operating on methanol fuel was initiated.

\section{STUDIES OF $\mathrm{C}_{60}, \mathrm{C}_{70}$ AND \\ OTHER FULLERENES: PREPARATION, SYNTHESIS, ELECTROCHEMISTRY, LUBRICANT PROPERTIES AND NEW COMPOSITES}

John L. Margrave

Richard E. Smalley

Rice University

\section{SL: ARPA}

The research objective is to explore the chemistry and electrochemistry of the buckminster fullerene molecule with a view towards applications in tribology, energy technology, and composite materials. 
The group will explore pure carbon composites made from $\mathrm{C}_{60}, \mathrm{C}_{70}$ and $\mathrm{C}_{80}$.

\section{B. Organic Synthesis and Mechanisms}

\section{ORGANIC SYNTHESIS AND MECHANISMS}

A. Paul Schaap

Wayne State University

The goals of the research include the successful synthesis of phosphate-substited dioxetanes with potential use with phosphatase-linked immunoassay and DNA probes, as well as dioxetanes with tethered fluorescers which emit in other spectral regions including the infrared. Chemiluminescent efficiency will be determined, and the effects of structural variations determined. Synthesis efforts will focus on the hydroxybenzothiazole which has shown encouraging preliminary results. Turnover rates and chemiluminescence efficiencies will be determined for enzymatic triggering. The effects of structure, such as length of tether, on the efficiency of energy transfer will be determined. The work has high potential relevance to the detection of chemical and biological agents (NBC) which can be applied to either direct detection via a simple device, or the general systems for the development of a chemical protective system.

\section{NUCLEOPHILIC DECONTAMINATION AGENTS}

\section{J. Milton Harris \\ Samuel P. McManus \\ SL: CRDEC, MICOM \\ SC: CRDEC, DPG}

Some time ago researchers here published a work with mustard tosylate showing that unusually powerful nucleophiles were required to provide SN2 displacement on mustards in aprotic solvents. Yet there was a report in the literature by Bordwell claiming that mustard reacts by an SN2 mechanism with iodide ion in acetone. Studies have now been performed with deuterium-labeled 2-(thiophenyl)ethyl chloride in acetone which clearly show that the product iodide is scrambled and that direct displacement by iodide on neutral substrate is not occurring. Interestingly, however, control studies with mustard iodide and mustard bromide show that these materials do not form stable products; possibly the sulfonium ion is the most stable species in these solutions. Researchers have learned a great deal in the past about mustard reactions in liquid solvents. Now they are beginning pioneering studies of these reactions in solid polymer films. These new reaction media are of interest since they could be used to adsorb mustard and then to destroy it. Thus a study has been undertaken of mustard reactions in polymer films, both to demonstrate feasibility and to begin characterization of polymer films as reaction media. The initial efforts were with 2-(thiophenyl)ethyl 2-naphthalenesulfonate. This ester was chosen because it exhibits a significant UV change upon hydrolysis. The four films that were chosen for these initial studies were poly(methyl methacrylate), poly(vinylpyrolidone), poly(vinyl acetate) and poly(vinyl pyridine). To conduct the experiment, thin films on quartz slides were prepared containing the mustard model. Previous work has shown that these films rapidly adsorb moisture, so water is present as a reactant in the films. Reaction occurred as monitored by UV spectroscopy. Interestingly the rates observed were nicely correlated by the Kamlet-Taft solvatochromic equation that researchers had previously derived for reactions in liquid solvents. Also humidity has a dramatic effect on the polymer as a reaction medium.

\section{CATALYSTS FOR THE OXIDATION OF THIOETHERS AND AMINES IN MICROEMULSIONS, EMULSIONS AND FILMS}

Russell S. Drago

University of Florida

\section{SL: CRDEC \\ SC: AROE, CRDEC, DPG, ARL/MD, NRDEC}

The research has seen several developments in the application of $\mathrm{H}_{2} \mathrm{O}_{2}$ activation to the decontamination of simulant compounds for mustard and VX. Activation of $\mathrm{H}_{2} \mathrm{O}_{2}$ by transition metal catalysts in the oxidation of the mustard simulant, $n$-butyl sulfide, was investigated in the solvent $\mathrm{N}$ methylpyrrolidinone (NMP) under ambient conditions. Complete oxidation of the sulfide $(1.6 \times$ $10^{-1} \mathrm{M}$ ) occurred using the catalyst, $\mathrm{V}(\mathrm{O})(\mathrm{pic})_{2}$ (pic $=$ pyridine -2 -carboxylate $)\left(1.6 \times 10^{-2} \mathrm{M}\right)$, in 2 hours using a $1: 3$ substrate to $\mathrm{H}_{2} \mathrm{O}_{2}$ (30 percent aqueous solution) molar ratio. The employment of $\mathrm{Mo}(\mathrm{O})_{2}$ (acac) 2 as the catalyst in an analogous system resulted in 99 percent of the substrate being oxidized to sulfoxide and sulfone in 5 minutes. Another set of 
experiments involving the use of a 1:1 substrate to $\mathrm{H}_{2} \mathrm{O}_{2}$ system $\left(1.6 \times 10^{-1} \mathrm{M}\right.$ in each) with these catalysts in NMP showed a very efficient use of $\mathrm{H}_{2} \mathrm{O}_{2}$ in the oxidation of the substrate with longer reaction times. Use of a solvent system consisting of NMP and $\mathrm{H}_{2} \mathrm{O}(50 / 50, \mathrm{v} / \mathrm{v})$ led to decrease in the oxidation of the substrate. It was also noted that the addition of maleic anhydride $(1.6 \mathrm{M})$ to the solvent NMP enabled $\mathrm{H}_{2} \mathrm{O}_{2}\left(4.9 \times 10^{-1} \mathrm{M}\right)$ to oxidize $n$-butyl sulfide $\left(1.6 \times 10^{-1} \mathrm{M}\right)$ completely to sulfone in 1 hour without the use of metal catalysts. Decreasing the amount of $\mathrm{H}_{2} \mathrm{O}_{2}$ from $4.9 \times 10^{-1} \mathrm{M}$ to $1.6 \times 10^{-1} \mathrm{M}$ led to selective oxidation to sulfoxide (100 percent) in 14 hours, with 100 percent efficient use of $\mathrm{H}_{2} \mathrm{O}_{2}$. Experiments were designed to demonstrate that maleic anhydride was catalytic in this reaction. An evaluation of the maleic anhydride/ $/ \mathrm{H}_{2} \mathrm{O}_{2}$ oxidative system using a less reactive mustard simulant, diphenyl sulfide, was performed. A complete oxidation of the substrate to sulfoxide (14 percent) and sulfone ( 86 percent) was observed in 1 hour. The use of a metal catalyst gave similar results. Oxidative systems involving $\mathrm{H}_{2} \mathrm{O}_{2}$ activation were extended to the $\mathrm{VX}$ simulant compound, Monitor (O, S-dimethyl phosphoramidothioate). Utilization of the maleic anhydride $/ \mathrm{H}_{2} \mathrm{O}_{2}$ system resulted in the complete oxidation of the VX simulant. The oxidation of another VX simulant compound, DIMP (diisopropylmethane phosphonate), is being investigated.

Reports:

No. $1-2$ in previous editions.

3. Hydroxylation of Methane by a Sterically Hindered Ruthenium Complex, by Alan S. Goldstein and Russell S. Drago, J Chem Soc Chem Commun p 21, Issue 1 (1991). AD A245 137

\section{PHOTODEGRADATION AND PHOTOPHYSICS OF LASER DYES}

Guilford Jones, II

Boston University

SL: CECOM, MICOM

SC: ARL/WTD, NRDEC

The influence of the solvent medium and polymer and other additives on the photophysical and photochemical properties of laser dyes has been investigated. The coumarin and rhodamine families of dyes have been studied in the initial phases of the work. These hydrophobic molecules or ions are sequestered in microdomains created by the hypercoiling of selected polyelectrolytes when the polymers are folded into globular conformations under certain conditions in water. Poly (methacrylic acid) (PMAA) behaves in this fashion for aqueous dilute solutions at $\mathrm{pH}$ 3.0. Experiments conducted to date include measurement of fluorescence quantum yields, lifetimes, and anisotropy (polarization). These parameters for the dyes under study are highly dependent on solvent polarity and viscosity. The results reveal sharp changes in photophysical properties on binding the coumarins and rhodamines in the PMAA hydrophobic pockets. Quantum yields and lifetimes of fluorescence are raised signifying a relatively dry interior for the polymer globule; polarization measurements further reveal a high local viscosity. The most recent effort involves polymer hypercoil binding of the merocyanine dye, Styrel 7 . This dye shows some of the most dramatic results to date regarding systematic alteration of the Stokes shift of emission and the improvement in quantum efficiency for fluorescence in the $680 \mathrm{~nm}$ region. These studies show promise for the solubilization of laser dyes in water and the protection of these dyes against adventitious quenching and undesirable photodegradation under flash excitation.

\section{THE DEVELOPMENT OF STABLE, HIGH EFFICIENCY LASER DYES}

George R. Bird

Rutgers, The State University of New Jersey

\section{SL: CECOM, MICOM \\ SC: CRDEC, NRDEC}

Experimental and computational determinations have been made of the excited state characteristics for several perylene derivatives. Some of these materials are candidates for use as laser dyes capable of wide-band tuning and efficient lasing action. Experimental triplet-triplet absorptions and oxygen triplet quenching rates were observed under the conditions found in a dye laser cavity: moderately concentrated solutions at room temperature (with or without equilibrium oxygen (air) concentrations) receiving intense irradiation from the driving pulses of a rare gas halide ultraviolet laser. Soluble derivatives of sevenringed or larger aromatic systems were produced by introducing relatively rigid out-of-plane substituents to prevent the strongly attractive intermolecular "graphitic" packing at $3.4 \AA$ separation. An unexpected chiral distortion in the normally planar backbone of the perylene system solubilized some ring-chlorinated derivatives and grossly altered the shape of the absorption bands. 
Reports:

No. 1 in previous edition.

2. Lasing Action in a Family of Perylene Derivatives: Singlet Absorption and Emission Spectra: Triplet Absorption and Oxygen Quenching Constants: Molecular Mechanics and Semiempirical INDO/S Molecular Orbital Calculations, by Mahin Sadrai et al., MS, J Phys Chem.

28013 ARTIFICIAL PHOSPHOESTERASE: METAL ACTIVATION BY STRAIN

Jik Chin

McGill University

SL: AMRICD, NRDEC

SC: AROE, CRDEC

Work has been concerned with hydrolyzing unactivated phosphate diesters like dimethyl phosphate. Prior to this study only activated phosphate diesters (e.g., bis( $p$-nitrophenyl)phosphate) have been hydrolyzed with a variety of artificial enzymes. However, the structural requirement of a catalyst for hydrolyzing activated substrates is not necessarily the same as that for hydrolyzing unactivated substrates. In order to hydrolyze the phosphate diesters backbone of DNA, it is important to develop catalysts that will hydrolyze unactivated phosphate diesters like dimethyl phosphate. A major barrier to studying the hydrolysis of dimethyl phosphate at neutral $\mathrm{pH}$ is the stability of the diester (P-O bond cleavage). The estimated water rate for dimethyl phosphate hydrolysis $\left(1.9 \times 10^{-19} \mathrm{~s}^{-1}\right.$ at $25^{\circ} \mathrm{C}$ is $10^{8}$ times slower than the water rate for bis( $p$-nitrophenyl)phosphate hydrolysis $\left(3.8 \times 10^{-11} \mathrm{~s}^{-1}\right.$ at $\left.25^{\circ} \mathrm{C}\right)$. In order to be able to detect the hydrolysis of dimethyl phosphate at neural $\mathrm{pH}$, a highly efficient metal complex that gives an enormous rate-acceleration for the hydrolysis reaction is required. Efforts in this area resulted in the first hydrolysis of an unactivated phosphate diester at neutral $\mathrm{pH}$. It is important to test catalysts for hydrolyzing phosphate esters with poor leaving groups like DNA or VX since the catalysts that are efficient for hydrolyzing esters with good leaving groups may not be active for hydrolyzing esters with poor leaving,groups.

\section{Reports:}

1. Developing Artificial Hydrolytic Metalloenzymes by a Unified Mechanistic Approach, by Jik Chin, Accnts Chem Res 24,145(1991). AD A244 603

\section{SUPER HYDRIDES}

Herbert C. Brown

Purdue University

SC: AROE

The importance of chiral compounds containing fluorine atoms in organic chemistry needs no restatement. Their utility, especially in medicinal chemistry, has been summarized in several reviews. As part of an ongoing program in chiral reductions, researchers are interested in reducing prochiral fluorinated aromatic and aliphatic ketones using the versatile reagent, $B$-chlorodiisopinocampheylborane (Aldrich: DIP-Chloride ${ }^{\mathrm{TM}}$ ). Asymmetric reduction of prochiral ketones is one of the important methodologies to prepare optically pure alcohols. Several reagents have been introduced in the last decade which are extremely efficient for the reduction of many classes of ketones. One of the successful reducing agents introduced here is DIP-Chloride. This reagent is extremely efficient in reducing prochiral aralkyl ketones and hindered aliphatic ketones in very high ee. Several other mono-, di- and trifluoromethyl ketones are being studied in an effort to distinguish between the steric and electronic influence of the fluorine atom on chiral reductions. In conclusion, DIP-Chloride reduces fluorinated ketones to the corresponding alcohols in very high ee. These alcohols are of extreme importance in organic chemistry. Investigations are being made of monofluoro- difluoro- and trifluoromethyl ketones of different classes (aliphatic, alicyclic, aromatic, heterocyclic, acetylenic, olefinic, keto esters) to understand the effect of fluorine atom on chiral reduction.

Reports:

1. This number not used.

2. Asymmetric Reduction with Chiral Organoboranes Based on a-Pinene, by Herbert C. Brown and P.V. Ramachandran, MS.

3. b-Chlorodiiso-2-Ethylapopinocampheylborane-An Extremely Efficient Chiral Reducing Agent for the Reduction of Prochiral Ketones of Intermediate Steric Requirements, by Herbert C. Brown et al., MS, Tetrahedron Let 32,6691(1991). 
28656 THE USE OF COLLOIDAL ASSEMBLIES IN OXIDATIVE AND HYDROLYTIC DECONTAMINATION

Clifford A. Bunton

University of California, Santa Barbara

\section{SL: CRDEC}

SC: CRDEC, DPG

Cationic micelles increase first order rate constants of reactions of $\mathrm{OH}^{-}$with $p$-nitrophenyl diphenyl phosphate ( $p$ NPDPP), 2,4-dinitro-chloronaphthalene (DNCN) and DDT. The micellar rate effects with moderately concentrated $\mathrm{OH}^{-}(<0.5 \mathrm{M})$ are larger than predicted by pseudophase ion-exchange treatments with constant fractional micellar ionization, $\alpha$. A treatment based on solution of the PoissonBoltzmann equation, which includes both nonspecific, Coulombic, and specific ion-micelle interactions, fits the data reasonably well with modest increase of the aggregation number and a "rough", rather than a "smooth", micellar surface. Estimated second order rate constants of reactions of dilute $\mathrm{OH}^{-}$with $p$ NPDPP and DNCN at the micellar surface based on a "smooth" micelle agree with the new values for reactions with $0.5 \mathrm{M} \mathrm{OH}^{-}$.

Reports:

1. Ion Binding and Reactivity at Charged Aqueous Interfaces, by Clifford A. Bunton et al.,Accnts Chem Res 24,357(1991). AD A249 668

\section{VESICLE STABILIZATION AND DESTABILIZATION FOR APPLICATION IN CHEMICAL REAGENT STORAGE AND RELEASE}

David A. Jaeger

University of Wyoming

\section{SC: CRDEC, ARL/MD, NRDEC}

In an ongoing study of the ability of surfactant aggregates to control the regioselectivity of Diels-Alder reactions of surfactant 1,3-diene with dienophiles. It has been demonstrated that interfacial and related orientational effects associated with surfactant aggregation can impart regioselectivity control in thermal cycloaddition reactions.

\section{PHOSPHOROLYTIC AND MEMBRANE MIMETIC CHEMISTRY}

R.A. Moss

Rutgers, The State University of New Jersey

SL: CRDEC, NRDEC
SC: ARLMD

Cyclopropanated dihydrosterculoyl (3-F, 3-NF) and unsaturated oleoyl (cis-2-F, cis-2-NF) ammonium ion lipids were prepared. Liposomes were created by sonication of 1:10 functional/nonfunctional lipid

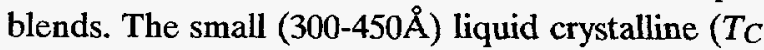
$<15^{\circ} \mathrm{C}$ ) liposomes were surface differentiated by exovesicular glutathione in an endovesicular/exovesicular $\mathrm{pH}$ gradient of $3.9 / 8$. At $25^{\circ} \mathrm{C}$, the cyclopropyl liposomes of 3 reequilibrated by transbilayer lipid migration much more slowly $\left(t_{1 / 2}=16\right.$ $\mathrm{min})$ than the oleoyl liposomes of $2\left(t_{1 / 2}<1 \mathrm{~min}\right)$. The origins of this difference were traced to variations in the lipids' acyl chain conformational states. Fourier transform IR studies revealed that the acyl chains of 3 contained fewer highly disruptive double gauche conformational sites than the chains of 2 . This can be associated with better chain packing, increased chain order, and the observed enhanced resistance to lipid "flip-flop" in liposomes of 3.

\section{A NEW TECHNIQUE FOR GENERATION OF 3-COORDINATE PHOSPHORYL SPECIES; APPLICATIONS IN SURFACE MODIFICATIONS}

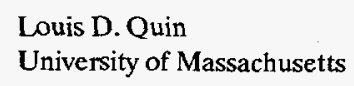

\section{SC: CRDEC, NRDEC}

The objective of the research is to investigate new techniques for generation of 3-coordinate phosphoryl species, and phosphorylation of solid surfaces with these metaphosphate species. Attempts will be made to extend metaphosphate and other low-coordination chemistry to reaction with hydroxyl groups on various surfaces including alumina, silica, zeolites, and cellulosic materials. New precursors will be investigated with the goal of developing practical, large scale surface phosphorylation techniques. 


\section{Structural, Surface Chemistry and Spectroscopy}

\section{APPLICATIONS OF LOW-COORDINATION PHOSPHORUS CHEMISTRY IN THE CHEMICAL MODIFICATION OF SURFACES}

Louis D. Quin

University of Massachusetts

\section{SC: AROE, CRDEC, ARL/MD}

The thermal fragmentation of $\mathrm{O}$-alkyl $\mathrm{N}$-substituted phosphoramidates of general structure has been developed into a practical method for the generation of alkyl metaphosphates. Several derivatives have been prepared by the general method, and all were found to decompose smoothly on heating in inert solvents. When an alcohol was present in the medium, it intercepted the metaphosphate efficiently with formation of a dialkyl phosphate. Kinetics studies have confirmed that a least for one compound the reaction is first-order in the phosphoramidate, and the identity and concentration of the alcohol plays no role, which is consistent with an elimination-addition mechanism. The fragmentation of the bicyclic system continues to be of interest and has been used to prepare two novel forms of phosphorylated silica gel. The photolysis of the 2,3-oxaphosphabicyclo[2.2.2] octene system has also been of interest as a means of generating metaphosphates at room temperature. Photolysis at low temperature seemed to offer the advantage of generating the species under conditions where it might be stable and observable spectroscopically, a feat never before accomplished with esters but used by by the P.I. to observe metaphosphoramides (as THF solvates). He synthesized two precursors for this purpose with large $\mathrm{O}$ substituents to assist in stabilizing the product. At room temperature, both compounds fragmented smoothly by the expected pathway with the elimination of the metaphosphate. However, both compounds gave major amounts of an entirely different product at low temperature $\left(-75^{\circ}\right)$ than observed at room temperature. These products were characterized by ${ }^{31} \mathrm{P} n m r$ signals at about $\delta 50$. The adamantyl ester was isolated as a crystalline solid; its analysis showed it to be an isomer of the starting material.
Reports:

No. $1-4$ in previous editions.

5. Chemistry of the 2,3-Oxaphosphabicyclo[2.2.2] Octene Ring System: Extrusion of Metaphosphates, by Louis D. Quin et al., Heteroatom Chem 2,99(1991). AD A239 749

6. Diels-Alder Adducts of 4-Chloro-1,6-Dihydrophosphinine Derivatives: A New Precursor of 2-Phosphapropene, by Louis D. Quin et al., Heteroatom Chem 2,283(1991). AD A239 747

7. Reaction of Epoxides with Metaphosphoric Acid Derivatives, by Ryszard Bodalski and Louis D. Quin, J Org Chem 56,2666(1991). AD A239 746

8. Products Derived from Oxidations of 3,4-Dimethyl-1Phenylphosphole-1-Oxide: A 3-Phenyl-5,6-Dimethyl-2,3Oxaphosphabicyclo[2.2.2]Octene-3-Oxide Derivative as a Precursor of Phenyl Metaphosphonic Anhydride, by Louis D. Quin and Xiao-Ping Wu, Heteroatom Chem 2,359(1991). AD A239 748

9. Mechanistic Studies on Metaphosphate Generated From Fragmentation of 2,3-Oxaphosphabicyclo[2.2.2] Octene Derivatives, by Stefan Jankowski and Louis D. Quin, $J A m$ Chem Soc 113,7011(1991). AD A249 207

10. Photoinduced Phosphorylation by [a-(Hydroxyimino)Benzyl]Phosphonates Through Fragmentation to Monomeric Metaphosphates, by Eli Breuer and Mahmoud Mahajna, $J$ Org Chem 56,4791(1991). AD A249 208

\section{PHOSPHORUS-, NITROGEN-, SULFUR-, AND CHLORINE- CONTAINING MOLECULES ON SURFACES}

John M. White

University of Texas at Austin

\section{SC: AROE, CRDEC}

Construction has been completed and modifications made to an ultrahigh vacuum Fourier Transform Mass Spectrometry (FTMS) system specially designated for investigating the chemistry of sulfur-, nitrogen-and chlorine-containing molecules on the metal surfaces. This system also has a conventional quadrupole mass spectrometer and a single pass Auger electron spectrometer. Researchers have achieved an excellent mass resolution and a reasonable sensitivity for FTMS. They have successfully combined FTMS with laser-induced thermal desorption (LITD) technique to detect and identify surface species. The above system is being used to study the chemistry of DMMP, DFP and 2-chloroethyl ethyl sulfide on Pt(111). In the case of DMMP and DFP on Pt(111), both molecules adsorb molecularly at 85 $\mathrm{K}$ and the decomposition, which occurs above $300 \mathrm{~K}$, is a minor channel. At most, $0.03 \mathrm{ml}$ of adsorbed DMMP decomposes to produce gaseous carbon monoxide, hydrogen and water and surface $P$, and $0.01 \mathrm{ml}$ of adsorbed DFP decomposes to produce 
gaseous carbon monoxide and hydrogen and surface carbon and phosphorus. The surface intermediates leading to these products are undetectable by FTMS/LITD because the concentration is too low. For 2-chloroethyl ethyl sulfide, its adsorption and thermal decomposition behavior was examined on clean and oxygen-covered $\operatorname{Pt}(111)$ using FTMS/LITD, temperature programmed desorption and Auger electron spectroscopy. 2-Chloroethyl ethyl sulfide is strongly yet molecularly adsorbed on $\mathrm{Pt}(111)$ at $85 \mathrm{~K}$. For a monolayer coverage, molecular desorption which occurs between 250 and $380 \mathrm{~K}$ is a minor channel. Extensive decomposition and reaction occur between 300 and $400 \mathrm{~K}$, producing gaseous hydrogen, hydrogen chloride, ethylene and diethyl sulfide, and surface sulfur and ethylidyne. Ethylidyne dehydrogenates at higher temperatures, leaving carbon and sulfur, thereby poisoning the substrate. On atomic oxygen-covered $\mathrm{Pt}(111)$, additional reaction products, water and carbon dioxide, are found. Compared to oxygen-free $\mathrm{Pt}(111)$, the extent of decomposition does not change significantly. At the end, the surface is poisoned by nearly the same amount of sulfur as, but less amount of carbon than, in the absence of oxygen.

\section{MODIFICATION OF SEMICONDUCTOR SURFACE PROPERTIES WITH CHEMICALLY BOUND MOLECULAR FILMS}

David L. Allara

S. Ashok

The Pennsylvania State University

\section{SC: AROE, CRDEC, ARL/EPSD}

In early 1991, it was discovered that alkane thiol molecules $\left[\mathrm{CH}_{3}\left(\mathrm{CH}_{2}\right)_{n} \mathrm{SH}\right]$ can be chemically bound to the GaAs (100) surface to form a highly organized monolayer film. A paper detailing the discovery for actadecane thiol $(n=17)$ was submitted to the Journal of the American Chemical Society. It has been shown that the monolayers on (100) GaAs can be prepared from the general class of molecules $\mathrm{X}\left(\mathrm{CH}_{2}\right)_{n} \mathrm{SH}$, where $\mathrm{X}=\mathrm{CH}_{3}, \mathrm{CO}_{2} \mathrm{H}, \mathrm{CO}_{2} \mathrm{CH}_{3}$ and $\mathrm{CH}_{2} \mathrm{OH}$ for $n=17$ and $\mathrm{X}=\mathrm{CH}_{3}$ for $n=10-22$. For $n \geq 17$ the films appear to consist of conformationally ordered alkyl chains, as observed by the infrared vibrational spectra, but for $n \leq 17$ the films begin to show evidence of conformational disorder with the disorder increasing with decreasing $n$. The wetting properties of the $\mathrm{X}=\mathrm{CH}_{3}$ films also show a decrease in hydrophobicity with decreasing $n$. This phenome- non appears to be due to the loss of intermediate chain-chain interaction energy with decreasing $n$ and a consequent increasing tendency towards thermal disordering. The above studies have been complemented with a detailed study of the modification of GaAs surface/interface electrical properties through Schottky barrier contact formed on the organic filmmodified GaAs surface. In continuation of the current-voltage (I-V) and capacitance-voltage (C-V) measurements reported earlier, studies were made of the influence of the metal as well as the number of $\mathrm{C}$ atoms in the alkane thiol self-assembled monolayer. The study of GaAs surface passivation by $\mathrm{P}_{2} \mathrm{~S}_{5}$ was extended and with variation of the metal there is preliminary evidence that the phosphorus pentasulfide passivation tends to unpin the surface Fermi level, thereby making the Schottky barrier height a more sensitive function of the metal work function.

\section{NANO-SCALE METAL OXIDE PARTICLES AS MATERIALS FOR AIR PURIFICATION}

Kenneth J. Klabunde

Kansas State University

\section{SL: CRDEC}

SC: AROE, BRADEC, CRDEC

Nano-scale particles of $\mathrm{FeO}$ were prepared by controlled pyrolysis of iron oxalate dihydrate $\left(\mathrm{FeC}_{2} \mathrm{O}_{4} \cdot 2 \mathrm{H} 2 \mathrm{O}\right)$. The use of these $\mathrm{FeO}$ particles as deoxygenation reagents was studied with $\mathrm{CO}_{2}$. At low pressure of $\mathrm{CO}_{2}(<0.6 \mathrm{~atm})$ a reaction proceeds at $600{ }^{\circ} \mathrm{C}$ to yield $\mathrm{Fe}_{3} \mathrm{O}_{4}$ and $\mathrm{CO}$ with about 50 percent conversion of $\mathrm{CO}_{2}$. At pressures near $1 \mathrm{~atm}$ the reaction proceeds somewhat differently and graphite is a product. The activity of the FeO particles varies with prior heat treatment temperature. About $600{ }^{\circ} \mathrm{C}$ appears to be optimum both for the heat treatment (evacuation/activation) step and the reaction temperature. The activation step can be carried out in vacuo or under flowing nitrogen. As an alternative to incineration of chlorinated aromatics, an investigation is being made of $\mathrm{MgO}$ and $\mathrm{CaO}$ as destructive adsorbents. On an in-situ reactor-GCMS system, researchers have examined the decomposition of polychlorinated benzene (PCB's simulant): chlorobenzene (CB), 1,3- and 1,4dichlorobenzene (DB) and 1,3,5-trichlorobenzene (TB) on $\mathrm{MgO}$ and $\mathrm{CaO}$. For comparison, the decomposition process in the reactor without such oxides (thermal decomposition) has also been carried out. Several samples of nano-scale $\mathrm{MgO}$ powders were 
prepared and heat treated at $500^{\circ} \mathrm{C}$. Using the $\mathrm{Al}(\mathrm{ET})_{3}$ titration procedure, the number of surface$\mathrm{OH}$ groups $/ \mathrm{nm}^{2}$ were determined and these ranged from $1.7-2.1 \mathrm{OH} / \mathrm{nm}^{2}$. The samples were placed in a quartz microbalance apparatus and evacuated. They were then treated with $\mathrm{SO}_{2}$ or $\mathrm{CO}_{2}$ at 10 torr pressure at $25^{\circ} \mathrm{C}$. The weight of the $\mathrm{CO}_{2}$ or $\mathrm{SO}_{2}$ adsorbed was determined.

\section{Reports:}

No. $1-6$ in previous editions.

7. Nano-Scale Metal Oxide Particles as Chemical Reagents. Intrinsic Effects of Size on Hydroxyl Content and Reactivity, and Acid/Base Properties of Well Defined, Magnesium Oxide Clusters, by Hidenobu Itoh et al., MS, J Am Chem Soc.

8. Heterophasic Isotope Exchange in Nanoscale Metal Oxide Particles. Lattice Oxygen and Surface-OH Groups with Water Vapor ( $\mathrm{D}_{2} \mathrm{O}$ and $\mathrm{H}_{2}{ }^{18} \mathrm{O}$ ), by Yong- $\mathrm{Xi} \mathrm{Li}$ and Kenneth J. Klabunde, Chem Mater 4,611(1992).

9. The Reaction of Carbon Dioxide with Fine Particles of Iron (II) Oxide, by Hidenobu Itoh and Kenneth J. KJabunde, MS, Chem Mater.

10. Adsorption and Decomposition of Organophosphorus Compounds on Nano-Scale Metal Oxide Particles. In-Situ GC-MS Studies of Pulsed Microreactions Over Magnesium Oxide, by Yong-Xi Li et al., Chem Mater 4,323(1992).

11. Fourier Transform Infrared Photoacoustic Spectroscopy Study of the Adsorption of Organophosphorus Compounds on Heat-Treated Magnesium Oxide, by Yong-Xi Li et al, Langmuir 7,1394(1991). AD A249 302

28319 TRANSITIONS IN LIQUID CRYSTALS INITIATED WITH CIRCULARLY POLARIZED LIGHT

Gary B. Schuster

University of Illinois

SL: ARL/MD

SC: AROE, CRDEC, NRDEC

Attempts are being made to use irradiation with unpolarized and circularly polarized light to modify the pitch of twisted nematic liquid crystal phases. As part of this investigation, the photochemistry of a series of chiral 1,1'-binaphthyl derivatives in isotropic solution and in liquid crystals was explored. The mechanism for photoracemization of these compounds is revealed and an efficient, light-induced conversion of a cholesteric to a nematic liquid crystal was studied. Specifically, the photochemistry and photophysics of a series of optically active 1,1'binaphthyl derivatives was examined by time resolved laser spectroscopy. It was found that photoracemization occurs in the triplet state by atropisomerism. The efficiency of photoracemiza- tion is controlled primarily by the efficiency of intersystem crossing and the magnitude of the activation barrier of atropisomerism. The latter value is perturbable by the specific nature of the binaphthyl derivative. Attempts to partially photoresolve these binaphthyl derivatives in fluid solution and in liquid crystals were unsuccessful. However, their photoracemization induces a cholesteric to compensated nematic transition in doped liquid crystalline $\mathrm{K}-\mathbf{1 5}$.

Reports:

1. Photoracemization of Optically Active $1,1^{\prime}$-Binaphthyl Derivatives: Light Initiated Conversion of Cholesteric to Compensated Nematic Liquid Crystals, by Mingbao Zhang and Gary B. Schuster, MS, J Phys Chem.

\section{CHARACTERIZATION OF EXCITED STATE OXYGEN-ORGANIC MOLECULE COMPLEXES}

Peter R. Ogilby

University of New Mexico

\section{SC: AROE, CRDEC, ARL/MD, NRDEC}

The argon ion laser required for several of the proposed studies has been received. An electromagnet has been ordered for the proposed magnetic field studies. The modifications are being made to existing time-resolved spectrometers that are required to perform experiments as a function of magnetic field strength.

\section{COLLISIONAL AND DISSOCIATIVE PROCESSES INVOLVING OPEN-SHELL MOLECULAR FREE RADICALS}

Paul J. Dagdigian

Millard H. Alexander

The Johns Hopkins University

SL: ARDEC

SC: AROE, CRDEC

The dynamics of the $\mathrm{NH}+\mathrm{NO} \rightarrow \mathrm{OH}+\mathrm{N}_{2}$ reaction has been investigated by measurement of the nascent $\mathrm{OH}$ product internal state distribution in a crossed beam experiment. As in previous studies involving the NH radical, this species was prepared by $193 \mathrm{~nm}$ 2-photon photolysis of ammonia. As $\mathbf{N H}_{2}$ is also found in the photolysis of ammonia, workers distinguished between these possible reactants by monitoring the signals due to the reagents and the $\mathrm{OH}$ 
product v. the photolysis laser pulse energy. Despite the very large exothermicity of this reaction $(408 \pm 2 \mathrm{~kJ} / \mathrm{mol})$, only a moderate internal excitation of the $\mathrm{OH}$ product was found. Workers also studied another reaction involving the imidogen radical, namely $\mathrm{O}+\mathrm{NH} \rightarrow \mathrm{H}+\mathrm{NO}$, and have derived the internal state distribution of the NO product. This experiment was carried out in a cell in which a flow of oxygen atoms, produced in a microwave discharge, was mixed with ammonia. The reaction was initiated by the $193 \mathrm{~nm}$ photolysis of ammonia to yield the NH reagent. Additionally, workers carried out full closecoupled calculations of state-to-state cross sections for rotationally inelastic collisions of $\mathrm{NH}$ in its $\mathrm{A}^{3} \Pi$ electronic state with helium, based on the recently calculated $a b$ initio potential energy surfaces of Jonas and Staemmler [Z.Phys. D 14, 143 (1989)]. In a major theoretical advance, a new method has been found for the study of the mechanism of inelastic atomic and molecular collisions. This involves the determination of the current density associated with, separately, the incoming and outgoing scattering wave functions in either an asymptotic or locally adiabatic basis. This yields a picture of how the incoming flux, initially associated with a given internal state, redistributes itself as a function of the interparticle separation both as the particles approach, and subsequently, as the particles recede. A simple extension of the researchers' linear-reference-potential, log-derivative propagation technique can be used to compute the redistribution of the initial flux. Analysis in a fully adiabatic basis, which corresponds to the local eigenvectors of the collision system, provides the most meaningful physical insight. This method provides mechanistic insight which is complementary to that given by application of time-dependent wavepacket methods. A first application was to the study of collision-induced transitions among excited electronic states of atomic $\mathrm{Ca}$.

Reports:

1. Quantum Scattering Studies of Inelastic Collisions of NH(A3P) with Helium: Fine-Structure and 1-Doublet Propensities, by Millard H. Alexander et al., $J$ Chem Phys 95,5036(1991). AD A244 944

2. The Dynamics of the $\mathrm{NH}\left(\mathrm{X3S}^{-}\right)+\mathrm{NO}\left(\mathrm{X}^{2} \mathrm{P}\right)$ Reaction: Internal State Distribution of the $\mathrm{OH}\left(\mathrm{X}^{2} \mathrm{P}\right)$ Product, by Dipti Patel-Misra and Paul J. Dagdigian, J Phys Chem 96,3232(1992).

3. Quantum Study of the Redistribution of Flux During Inelastic Collisions, by Millard H. Alexander, J Chem Phys 95,8931(1991). AD A249 186

4. Dynamics of the $\mathrm{O}^{3} \mathrm{P}+\mathrm{NH}_{2}$ Reaction: the $\mathrm{HNO}+\mathbf{H}$ Product Channel, by Dipti Patel-Misra and Paul J. Dagdigian, Chem Phys Let 185,387(1991). AD A244 581
5. Quantum Flux Studies of the Mechanism of $\mathrm{Ca}\left(4 \mathrm{~s} 5 \mathrm{p}{ }^{1} \mathrm{P} \rightarrow\right.$ $\mathrm{Ca}\left(4 \mathrm{~s} 5 \mathrm{p}^{3} \mathrm{P}\right)$ Collisions, by Millard $\mathrm{H}$. Alexander, MS, $J$ Chem Phys.

6. Determination of the Internal State Distribution of $\mathrm{NO}\left(\mathrm{X}^{2} \mathrm{P}\right)$ Produced in the $\mathrm{O}^{3} \mathrm{P}+\mathrm{NH}\left(\mathrm{X}^{3} \mathrm{~S}\right)$ Reaction, by Yu-Lin Huang and Paul J. Dagdigian, $J$ Chem Phys 97,180(1992).

7. Potential-Energy Surface Control of the NH Product State Distribution in the Decomposition Reaction $\mathrm{HN}_{3}\left(\mathrm{X}^{1} \mathrm{~A}^{\prime}\right) \rightarrow$ $\mathrm{NH}\left(\mathrm{a}^{1} \mathrm{D}+\mathrm{N}_{2}\left(\mathrm{X}^{1} \mathrm{Sg}^{+}\right)\right.$, by Millard H. Alexander etal., Faraday Discuss Chem Soc 91,319(1991). AD A249 206

8. Predissociation of the NH/ND $\left(c^{1} \mathrm{P}, \mathrm{V}^{\prime}, \mathrm{J}^{\prime}\right)$ States, by $B$. Bohn et al., J Chem Phys 96,5059(1992).

\section{PHOTO AND THERMAL EXCITATION OF SURFACE CHEMISTRY ON MODEL CATALYST SURFACES}

John T. Yates

University of Pittsburgh

\section{SL: ARL/MD}

Pure phosphorus is being studied under oxidizing conditions on a Mo (110) surface. The techniques being employed are temperature programmed desorption (TPD), Auger electron spectroscopy (AES), and low energy electron diffraction (LEED). A comprehensive study of the oxidation of Mo(110) is underway using high resolution electron energy loss spectroscopy (HREELS) and other ultrahigh vacuum techniques. The decomposition and oxidation of phosphine has been studied on an $\mathrm{MoO}_{3} / \mathrm{Al}_{2} \mathrm{O}_{3}$ supported catalyst using transmission IR spectroscopy and mass spectroscopy in the temperature range $300-800 \mathrm{~K}$. Phosphine decomposes on $\mathrm{MoO}_{3}$ at $573 \mathrm{~K}$ and is oxidized to a surface species containing the $\mathrm{H}-\mathrm{P}=\mathrm{O}$ moiety, exhibiting a characteristic $\mathrm{H}-\mathrm{P}$ mode at $2490 \mathrm{~cm}^{-1}$ and a $\mathrm{P}=\mathrm{O}$ mode at $\sim 1100 \mathrm{~cm}^{-1}$. Further oxidation at $673 \mathrm{~K}$ under $\mathrm{O}_{2}(\mathrm{~g})$ produces a surface species $(\mathrm{HO})_{x}-\mathrm{P}=\mathrm{O}(\mathrm{a})$ which has probably migrated to the support. The (HO) $)_{x}-\mathrm{P}=\mathrm{O}$ species is characterized by an $\mathrm{O}-\mathrm{H}$ stretching mode at $3672 \mathrm{~cm}^{-1}$; it is desorbed from the surface at temperatures near $773 \mathrm{~K}$. The two sequential oxidation steps have been performed for several cycles using additional $\mathrm{PH}_{3}$ adsorbate. These observations suggest that the $\mathrm{MoO}_{3} / \mathrm{Al}_{2} \mathrm{O}_{3}$ catalyst may be effective for the continuous catalytic oxidation of organophosphorus compounds.

Reports:

1. Catalytic Oxidation of Phosphorus on $\mathrm{MoO}_{3}$ as Studied by Infrared Spectroscopy, by Dilip K. Paul et al., MS, $J$ Phys Chem. 
2. Surface Chemistry: New Developments Aid Technologies, by John T. Yates, Jr., MS.

3. The Adsorption and Dissociation of Carbon Monoxide on Clean and Oxygen-Modified Mo(110) Surfaces, by M.L. Colajanni et al., J Am Chem Soc 114,3735(1992).

4. Oxygen on Mo(110): Low-Temperature Adsorption and High-Temperature Oxidation, by M.L. Colajanni et al., MS, Surface Sci.

\section{MOLECULAR LEVEL ASSEMBLIES OF POLYPYRIDYL COMPLEXES AT METAL OXIDE AND GLASS INTERFACES}

Thomas J. Meyer

University of North Carolina at Chapel Hill

The research objective is to develop a systematic basis for transferring the known excited state and electron transfer chemistry of polypyridyl complexes of ruthenium, osmium and rhenium in solution to glass or metal oxide surface. The complexes will be fixed to the surface by ester link formation and the properties of the photochemistry and photophysics of the resulting combined surface structures will be compared to solution chemistry using electrochemical, spectroscopic and photochemical techniques.

\section{SPECTROSCOPIC STUDY OF REACTION INTERMEDIATES AND MECHANISMS IN NITRAMINE DECOMPOSITION AND COMBUSTION}

\section{Marilyn E. Jacox}

National Institute of Standards and Technology

\section{SL: ARDEC}

The research objective is to measure spectra of likely intermediate of nitramine combustion thus enabling spectroscopy of solid propellant combustion. $\mathrm{H}$ atoms and isotopically labelled $\mathrm{H}_{2} \mathrm{O}$ will be reacted with model compound (e.g., nitromethane, monomethylnitramine) in low temperature rare gas matrices. This will generate stabilized radicals whose vibrational and electronic spectroscopy can be determined.

\section{Kinetics and Thermodynamics}

\section{MECHANISMS OF DECOMPOSITION AND OXIDATION OF BORANE COMPOUNDS}

Simon H. Bauer

Cornell University

\section{SC: ARDEC, AROE, ARL/WTD, MICOM}

The following major items in the Nd/YAG laser had to be replaced: the Q-switch, the high voltage relay, and the polarizer. Studies of the reaction: $\mathrm{B}_{10} \mathrm{H}_{14}+$ $O\left({ }^{3} \mathrm{O}\right)$ is the third member of the borane series which has been studied; $\mathrm{B}_{2} \mathrm{H}_{6}$ and $\mathrm{B}_{5} \mathrm{H}_{9}$ have been completed. Due to its low vapor pressure at room temperature the flow-tube reactor had to be modified for operation at variable temperatures $30-100{ }^{\circ} \mathrm{C}$. A "flow through" vaporizer was constructed. All lines leading to the reactor, as well as the reactor tube, were wrapped with heating tapes and lagged to obtain uniform temperatures throughout the operating system. Five thermocouples were inserted in strategic locations, and currents through the tapes were adjusted to establish temperatures to $\pm 2{ }^{\circ} \mathrm{C}$. Before the present difficulties with $\mathrm{Nd} / \mathrm{YAG}$ laser, two sets of runs with $\mathrm{B}_{10} \mathrm{H}_{14}$ were recorded. No kinetic analyses have yet been undertaken.

\section{CHEMICAL DYNAMICS STUDIES OF UNIMOLECULAR REACTIONS IN ENERGETIC MATERIALS}

Donald L. Thompson

Oklahoma State University

\section{SL: ARDEC, ARL/WTD \\ SC: AMCCOM, AROE, BRADEC, MICOM}

Molecular dynamics simulations were used to study the flexibility of hexahydro-1,3,5-trinitro-1,3,5-triazine (RDX). A potential-energy surface was constructed using spectroscopic and theoretical data. The activation barrier for the boat-to-twist-boat pseudorotation was calculated to be $0.8 \mathrm{kcal} \mathrm{mol}^{-1}$. The entropy change for the chair-to-boat inversion was calculated to be $6 \pm 3 \mathrm{cal} \mathrm{mol}^{-1} \mathrm{~K}^{-1}$. Using the time averaged potential-energy difference between the chair and boat conformations of $1.5 \pm 0.4 \mathrm{kcal}$ $\mathrm{mol}^{-1}$ and neglecting PV effects, the free energy change for the chair-to-boat inversion is $0 \pm 1 \mathrm{kcal}$ $\mathrm{mol}^{-1}$. Constrained classical trajectory calculations were carried out to investigate intramolecular vibra- 
tional energy redistribution (IVR), cis-trans isomerization, and rotation-vibration interactions in HONO. Relaxation of the $\mathrm{OH}$ bond stretching mode, initially excited to the second overtone state, was monitored by using a local-mode approximation. In order to determine the roles of the various modes in the IVR and cis-trans isomerization, the dihedral, HON bending, or ONO bending angle was dynamically constrained by incorporating Lagrangian multipliers into Hamilton's equations of motion. The results show that the in-plane HON bending and out-of-plane torsional motions play important roles in the IVR. A strong interaction between the $\mathrm{OH}$ stretching and HON bending modes is a major factor in causing cis-HONO to isomerize at significantly greater rates than trans-HONO. Molecular rotation significantly enhances IVR; however, it has little effect when the torsional coordinate is frozen at its equilibrium value. The collisional excitation and relaxation of para-difluorobenzene ( $p$-DFB) by light atoms have been studied by a wave-packet scattering method. In this model the light atom is represented by a two-dimensional wave packet and the vibrations of $p$-DFB molecule are treated classically. Researchers found that the v30 mode, which is the mode with the lowest vibrational frequency and one which involves an out-of plane motion of the fluorine atoms, is most easily excited or relaxed in the collisions. This is in agreement with the experimental and other theoretical results. This study shows that this combined trajectory/wave packet approach can be used to treat intermolecular energy transfer in collisions of atoms with polyatomic molecules.

Reports:

No. $1-12$ in previous editions.

13. Classical Dynamics Study of HONO Using Constrained Trajectories, by Yue Qin and Donald L. Thompson, J Chem Phys 96,1992(1992). AD A249 823

14. Mode Selectivity in the Classical Power Spectra of Highly Vibrationally Excited Molecules, by Thomas D. Sewell et al., MS, J Chem Phys.

15. Comparisons of Statistical and Nonstatistical Behavior for Bond Fission Reactions in 1,2-Difluoroethane, Disilane, and the 2-Chloroethyl Radical, by Thomas D. Sewell et al., J Chem Phys 95,8089(1991). AD A249 736

16. Collision-Induced Energy Transfer in $p$-Difluorobenzene by Light Atoms: A Wave-Packet Scattering Calculation, by Huadong Gai and Donald L. Thompson, Chem Phys Let 191,441(1992)

\section{KINETICS AND DYNAMICS OF REACTING SYSTEMS}

Sidney Redner

Boston University

\section{SC: AROE, CRDEC, ARL/EPSD, ARL/MD, NRDEC}

An investigation is being made of catalytic processes in which the rate-limiting step is the dissociation of a molecule into its atomic constituents. This feature may underlie some of the intriguing behavior found in the process, $\mathrm{NO}+\mathrm{CO}_{2} \rightarrow 1 / 2 \mathrm{~N}_{2}+\mathrm{CO}_{2}$. In work on the coarsening of population distributions in a model of self-replicating and competing species, the basic parameter that controls the growth rate of domains was found. New analytical tools were developed to study simple catalytic reactions. One method is the construction of both subcritical and supercritical perturbation expansions, with which one can probe the properties of non-equilibrium critical points. Both of these methods have been applied to a variety of simple model systems. Finally, new geometric features of the spatial organization in two species annihilation have been investigated.

\section{ULTRAFAST RESPONSE OF ENERGETIC SOLIDS TO LIGHT, HEAT AND SHOCK PULSES}

Dana D. Dlott

University of Illinois

\section{SC: ARL/WTD}

Ultrafast temperature jump experiments $(\Delta T=$ $140{ }^{\circ} \mathrm{C} \quad d c T / d t=10^{12} \mathrm{deg} / \mathrm{s}$ ) were performed on polymers doped with small concentrations of dyes which function as point sources of phonons (molecular heaters) and thermometers. Internal conversion, multiphonon up pumping and vibrational cooling of dyes in the polymer, occurring on the 0.1 to $100 \mathrm{ps}$ time scale, were investigated. Applications of tunable infrared picosecond pulse free-electron lasers were considered in a paper. The difficulties of combining an FEL with an intricate experimental apparatus, together with the performance specifications which an FEL must achieve in order to perform sophisticated applications, were discussed. Some novel applications in which FELs should excel due to their unique properties were described. These include measurements of vibrational dynamics in condensed phases, hyper Raman scattering, and a novel class of experiments, nanoheating. In nanoheating experiments, a specific nanometer-sized object is abruptly heated to high temperatures while the surroundings 
remain mostly unaffected. Potential applications of nanoheating include viral vaccine production and cell fusion.

Reports:

No. $1-4$ in previous editions.

5. Molecular Dynamics Simulation of Nanoscale Thermal Conduction and Vibrational Cooling in a Crystalline Naphthalene Cluster, by Hackjin Kim and D.D. Dlott, $J$ Chem Phys 94,8203(1991). AD A245 113

6. Multiphonon Up-Pumping and Molecular Hot Spots in Superheated Polymers Studied by Ultrafast Optical Calorimetry, by Xiaoning Wen et al., Chem Phys Let 192,315(1992).

7. Applications of Infrared Free-Electron Lasers: Basic Research on the Dynamics of Molecular Systems, by Dana D. Dlott and Michael D. Fayer, IEEE J Quant Electron 27,2697(1991). AD A249 404

8. Applications of Ultrafast Temperature Jump Spectroscopy to Condensed Phase Molecular Dynamics, by Sheah Chen et al., MS.

9. Direct Measurement of Polymer Temperature During Laser Ablation Using a Molecular Thermometer, by I-Yin Sandy Lee et al., MS, J Appl Phys.

10. Dynamics of Laser Ablation Transfer Imaging Investigated by Ultrafast Microscopy, by I-Yin Sandy Lee et al., J Imaging Sci Tech 36,180(1992).

\section{THE REMOVAL OF HETEROATOMS FROM ORGANIC COMPOUNDS BY SUPERCRITICAL WATER}

Thomas Houser

Western Michigan University

\section{SC: AROE, ATHAMA, CRDEC}

Interest in the reactions of supercritical water (SW) with organic compounds containing $\mathrm{N}, \mathrm{S}$, and $\mathrm{O}$ atoms was initiated by the potential use of $\mathrm{SW}$ as a solvent in the conversion of coal, since at these conditions SW not only acts as a solvent but also as a reactant. Up to this time the studies have been limited to organic compounds containing only $\mathrm{N}$ or $\mathrm{O}$. The kinetics of the benzaldehyde-SW reaction have been determined in excess water and appear to be 2nd-order in benzaldehyde with inhibition by product(s). The results of the reactions of benzylamine, bibenzyl, and benzaldehyde with SW have led to the conclusion that if a CN group is attached to a benzene ring, those with multiple bonds hydrolyze followed rapidly by decarboxylation to form benzene, ammonia and carbon dioxide. However, if the $\mathrm{CN}$ is a single bond it may rupture to form benzyl and amino radicals or it may loose hydrogen to become a multiple bond and react as previously described. Once the benzyl radical forms it will pick up a hydrogen to form toluene without oxidation of the side chain. The compounds which are of current interest are those that model certain hazardous materials which may in the future require large scale destruction or conversion to innocuous products. These are: (a) 1-chlorohexane, (b) ethylbenzilate, (c) trihexyl amine, (d) quinuclidine, (e) phenylpiperidine, and (f) phenylpyridine.

Reports:

No. 1 in previous edition.

2. Reactions of Supercritical Water with Benzaldehyde, Benzylidenebenzylamine, Benzyl Alcohol and Benzoic Acid, by C.C. Tsao et al., MS, J Supercritical Fluids.

3. The Removal of Heteroatoms from Organic Compounds by Supercritical Water, by Thomas J. Houser et al., MS.

\section{THIN FILM LASER PYROLYSIS OF NITRAMINE PROPELLANTS}

Charles A. Wight

University of Utah

\section{SL: ARDEC, ARL/WTD \\ SC: AFAL, AROE}

Efforts have been focused on the transient $\mathrm{CO}_{2}$ laser pyrolysis of thin film samples of RDX. The main intent of these experiments is to investigate the initial thermal decomposition mechanism of nitramine propellants. The laser fluence threshold for causing detectable chemical or structural changes in the film is quite sharp, and above-threshold there is a strong tendency to lose material from the surface of the window. A "sandwich" experimental technique has been devised whereby a thin film is deposited onto an IR-transparent substrate ( $\mathrm{NaCl}, \mathrm{KBr})$ and subsequently covered with a second transparent window. This assembly is attached to a copper retainer inside a vacuum dewar vessel and cooled to liquid nitrogen temperature. Laser photolysis of the film then causes transient heating and vaporization of the film, but the early-time reaction products are rapidly trapped on the surfaces of the confining windows at $77 \mathrm{~K}$. Using this apparatus, no detectable changes were observed in the RDX samples upon irradiation with $\mathbf{P}(20)$ (10.6 micron) line of the $\mathrm{CO}_{2}$ laser below a threshold of $1.5 \mathrm{~J} / \mathrm{cm}^{2} /$ pulse.

\section{Reports:}

1. Thermal Decomposition of Solid RDX Begins with N-N Bond Scission, by Charles A. Wight and Tod R. Botcher, MS, J Am Chem Soc. 


\section{INSENSITIVE ENERGETIC MATERIALS: THE IMPORTANCE OF PHYSICAL PROPERTIES AND MOLECULAR STRUCTURE}

Richard Behrens

Sandia National Laboratories

\section{SL: ARDEC}

SC: AROE, CRDEC

The work has concentrated on analyzing the results of thermal decomposition experiments on RDX in the solid and liquid phases. In these experiments the reaction pathways of the products formed in the thermal decomposition of RDX have been traced by identifying the decomposition products and measuring the temporal behaviors of their gas formation rates. In addition, the inter- $v$. intra-molecular origin of the products formed in the thermal decomposition of RDX has been traced by isotopic cross-over experiments using mixtures of differently labeled analogues of RDX. The isotopic analogues of RDX used in the experiments include: ${ }^{2} \mathrm{H},{ }^{13} \mathrm{C},{ }^{15} \mathrm{~N}$, and ${ }^{18} \mathrm{O}$. The fraction of isotopic scrambling and the extent of the deuterium kinetic isotope effect (DKIE) were determined for the different thermal decomposition products. Analysis of the results from these experiments with RDX have shed considerable light on the mechanisms that control the identities and rates of formation of the various products formed during its thermal decomposition. Results are encouraging in that a new understanding now provides a basis for further exploration of the processes that control the thermal decomposition of these nitramines. One of the first steps in this regard is to explore the thermal decomposition of the mononitroso analogue of RDX that is formed as an intermediate in one of the primary decomposition pathways.

Reports:

No. 1 in previous edition.

2. Thermal Decomposition of Energetic Materials II: Deuterium Isotope Effects and Isotopic Scrambling in Condensed-Phase Decomposition of Octahydro-1,3,5,7Tetranitro-1,3,5,7-Tetrazocine, by Richard Behrens, Jr., and S. Bulusu, J Phys Chem 95,5838(1991). AD A244 773

3. Thermal Decomposition of Energetic Materials III: Temporal Behaviors of the Rates of Formation of the Gaseous Pyrolysis Products From Condensed-Phase Decomposition of 1,3,5-Trinitro-hexahydro-s-triazine, by Richard Behrens, Jr., and S. Bulusu, MS, J Phys Chem.

4. Thermal Decomposition of Energetic Materials IV: Deuterium Isotope Effects and Isotopic Scrambling (H/D, $\left.{ }^{13} \mathrm{C} /{ }^{18} \mathrm{O},{ }^{14} \mathrm{~N} /{ }^{15} \mathrm{~N}\right)$ in Condensed-Phase Decomposition of 1,3,5,-Trinitro-hexahydro-s-triazine (RDX), by Richard Behrens, Jr., and S. Bulusu, MS, J Phys Chem.

\section{CONDENSED AND GAS PHASE DIAGNOSTICS OF NITRAMINE PROPELLANT COMBUSTION}

Charles E. Kolb

Aerodyne Research, Inc.

\section{SL: ARL/WTD \\ SC: AROE, CRDEC}

The research goal is to develop the technique of fast-response spectral infrared fiber probing of burning propellant strands, and demonstrate it in mechanism studies of condensed phase processes in nitramine propellant combustion. The strand burner has been assembled. This camera system will not only be used in observing the eventual experiments in the strand burner, but will be required in the next round of rapid fiber heating studies. A fast resistance heater has been built and observations will begin with it when the camera arrives which will allow the P.I. to correlate the melting wave position with the infrared transmission. These tests of fiber transmission are just one of several studies of components of the full experiment. Several other component studies will proceed in parallel including characterizing the infrared transmission of the solid propellants, and learning how to cut strands, insert fibers, and reseal the strands.

\section{DESTRUCTION OF HAZARDOUS CHEMICALS BY OXIDATION IN SLPERCRITICAL WATER: A THEORETICAL AND COMPUTATIONAL TREATMENT}

Jefferson W. Tester

Massachusetts Institute of Technology

SL: AFESC

SC: AROE, ATHAMA, CRDEC, MICOM, NRDEC

Oxidation in a supercritical water environment is an efficient method for treating wastes without formation of harmful by-products. Complete oxidation of organics is often limited by conversion of carbon monoxide to carbon dioxide. The kinetics of carbon monoxide oxidation have been studied previously, but efforts to establish kinetic parameters for the direct oxidation pathway $\left(\mathrm{CO}+1 / 2 \mathrm{O}_{2} \rightarrow \mathrm{CO}_{2}\right)$ were complicated by the reaction of carbon monoxide with water via the water-gas shift reaction pathway ( $\mathrm{CO}$ $+\mathrm{H}_{2} \mathrm{O} \rightarrow \mathrm{CO}_{2}+\mathrm{H}_{2}$ ) during preheating of the reactor feeds. The kinetics of carbon monoxide oxidation have been re-examined in an updated experimental apparatus with improved 
temperature-measurement and hydrogen-detection capabilities, and the studied range of $\mathrm{O}_{2} / \mathrm{CO}$ feed ratios have been extended to the substoichiometric regime. Using the results of heat-transfer experiments, the temperature profiles within the feed preheater were established. The experimentally determined temperature profiles compared favorably with profiles predicted by conventional heattransfer correlations. These temperature profiles were used in determining new kinetic parameters for the water-gas shift pathway and for the direct oxidation of carbon monoxide in supercritical water. The two kinetic pathways can apparently be treated as separable under the conditions of this study. Regressed kinetic rate forms were similar to those obtained in the earlier study. The new oxidation data exhibit a fractional-order dependence on oxygen concentration, consistent with gas-phase studies of carbon monoxide oxidation.

\section{Reports:}

1. Carbon Monoxide Oxidation in Supercritical Water: The Effects of Heat Transfer and the Water-Gas Shift Reaction on Observed Kinetics, by H. Richard Holgate et al., MS.

2. Supercritical Water Oxidation Technology: A Review of Process Development and Fundamental Research, by J.W. Tester et al., MS.

3. Solubilities of Sodium Chloride and Sodium Sulfate in Suband Supercritical Water Vapor, by Fred J. Armellini and Jefferson W. Tester, MS.

\section{A MOLECULAR DYNAMICS SIMULATOR FOR OPTIMAL CONTROL OF MOLECULAR MOTION}

Herschel Rabitz

Princeton University

\section{SC: AROE}

The purpose of this research is to develop a realistic laboratory molecular dynamics simulator, controlled by the same optimal control techniques and software being employed in a parallel study on molecular systems. Careful quantitative measurements were made on a collinear molecular dynamics simulator, using mechanical springs as in the earlier work. In this case, an emphasis was placed on anharmonicity in the simulated bonds, and it was concluded that it would be more flexible to change to magnetic "springs". Such "springs" are made by judiciously locating small ceramic high intensity permanent magnets on the frictionless carts representing the atoms in the simulator. Theoretical calculations using magnetostatics indicated that realistic interatomic potentials could be readily simulated in this fashion, and laboratory demonstrations of this point have been made. Considerable effort has gone into the measurement of the corresponding inter-cart (atomic) forces, and unlike the static techniques employed for mechanical springs, in the present case, it was most useful to make dynamical measurements and extract the underlying potential from the measurements.

\section{EXCITED ELECTRONIC STATES OF ENERGETIC MATERIALS - STRUCTURE, DYNAMICS, REACTIVITY}

Elliot R. Bernstein

Colorado State University

\section{SL: ARDEC \\ SC: AROE, ARL/WTD, MICOM}

Studies of Rydberg states of molecules and clusters have been very successful. The main thrusts for the initial part of the studies of highly excited electronic states of energetic materials are to observe Rydberg spectra of new model systems and of clusters. Both aspects of this work have been highly successful. Cluster spectra of 1,4-dioxane have been obtained with $\mathrm{Ar}, \mathrm{Kr}, \mathrm{CH}_{4}, \mathrm{CD}_{4}, \mathrm{CF}_{4}, \mathrm{C}_{2} \mathrm{H}_{2}, \mathrm{C}_{3} \mathrm{H}_{8}, \mathrm{C}_{6} \mathrm{H}_{12}$, $\mathrm{C}_{6} \mathrm{D}_{12}, \mathrm{SiH}_{4}$, and $\mathrm{Si}\left(\mathrm{CH}_{3}\right)_{4}$. The structure and van der Waal vibrational modes of these clusters have been calculated. In addition to these cluster studies with dioxane, new Rydberg spectra have been obtained for diethyl ether, diazobicyclo[2.2.2]octane (DABCO), tetrahy-dro-furan (THF) and methyl vinyl ether, diisopropyl ether. These spectra are very rich in structure and sharp, potentially revealing a great deal of information about the Rydberg states of many molecules. Spectra are obtained by 2 -color multi-photon processes so fragmentation is controlled. It is now possible to access both $\mathrm{O}$ and $\mathrm{N}$ Rydberg transitions so the potential for observing many different compounds (e.g., energetic materials) now becomes a reality.

\section{STATE RESOLVED DIFFERENTIAL CROSS SECTIONS FOR REACTIONS IMPORTANT TO THE DECOMPOSITION OF ENERGETIC MATERIALS}


Paul L. Houston

Cornell University

SL: ARDEC, ARL/WTD

SC: CRDEC

State-selected differential cross sections have been obtained by directly imaging the products of collisions in crossed molecular beams. The new technique allows final state resolution and simultaneous detection of all scattering angles. The method has been used to study inelastic collisions between $\mathrm{Ar}$ and $N O\left({ }^{2} \prod_{1 / 2}, v=O, J=0.5\right)$ at a collision energy of $0.21 \mathrm{eV}$. Rotational rainbows in the product angular distribution are directly observed to change in position as a function of the final rotational state; the peak of the angular distribution moves toward the backward hemisphere and the angular distribution broadens with an increase in final rotational quantum number. The method relies on multiphoton ionization of the product, but is otherwise generally applicable to reactive as well as inelastic collisions.

Reports:

1. Differential Cross Sections for State-Selected Products by Direct Imaging: Ar + NO, by A.G. Suits et al., MS, JChem Phys.

\section{OPTIMALLY CONTROLLED MOLECULAR DYNAMICS}

Herschel Rabitz

Princeton University

\section{SL: ARDEC}

This research is concerned with the observation that controlled pulsed lasers are being created in the laboratory with very high duty cycles (i.e., the ability to create one pulse, and then another very different shape, etc., under computer control with high rapidity). This capability may in principle be combined with advanced learning algorithms to close the loop in the laboratory and teach lasers how to control molecular motion. This work has been concerned with simulating this process on the computer, to test its efficacy. Recently, particular attention has been paid to issues of robustness, in the sense that the learning process has been simulated under conditions of excessive laboratory noise, and only modest laboratory resolution of the product being steered into the desired $\mathrm{v}$. an undesired state. It has been found that the quality of the results is learning algorithm-dependent, and this observation can be taken advantage of, in particular, by employing algorithms that untiringly continue to seek better solutions. It is possible to show that the presence of laboratory noise, or poor detector resolution, merely slows down the learning process, but does not detract from the ultimate quality of the results. This behavior is very akin to the well-known experience that a "slow learner" may eventually achieve the same goal, but it may simply take longer than usual. In the present case, this translates to the need for more pulsed experiments to get the desired results. Sample simulations have been carried out for controlling rotational, vibrational, and electronic degrees of freedom.

\section{ENERGY AND STATE RESOLVED STUDIES OF THE PATHWAYS OF MOLECULAR DECOMPOSITION}

\section{F. Fleming Crim}

University of Wisconsin - Madison

$$
\begin{array}{ll}
\text { SL: ARDEC } \\
\text { SC: ARL/WTD, CRDEC }
\end{array}
$$

The objective of the research is to produce, characterize, and dissociate energetic molecules containing weak N-O and N-N bonds. Vibrational overtone excitation will be used to prepare highly vibrationally excited molecules, probe them using laser induced fluorescence, and photodissociation and ionization to study simple organic nitrites, nitrates, nitramines and nitro-compounds.

\section{THERMAL DECOMPOSITION PATHWAYS IN NITRAMINE PROPELLANTS}

Francis I. Lovas

Richard D. Suenram

National Institute of Standards and Technology

$$
\text { SC: ARL/WTD }
$$

The objective of the research is to study the chemical species that appear as reaction intermediates when nitramines are decomposed. Nitramines will be decomposed using laser vaporization and pyrolysis. Species formed will be identified using microwave spectroscopy. 


\section{IN-SITU NEUTRALIZATION OF ACIDS GENERATED BY SUPERCRITICAL WATER OXIDATION}

Michael Modell

Modell Development Corporation

The research objective is to study in-situ neutralization of acids generated by chemical reactions in supercritical water. This project tests the feasibility of mixing a slurry of base with the organic wastes before they enter the reactor. Metal carbonate slurries will be added to a feed solution of organic chlorides (reacting to $\mathrm{HCl}$ ) before it enters the reactor.

\section{COST-EFFECTIVE RED WATER DISPOSAL BY ELECTRON BEAM RADIOLYSIS}

\author{
Daniel Goodman \\ Jonah Jacob \\ Science Research Laboratory, Inc.
}

The research objective is to determine the efficiency of destruction of undesirable chemicals in red water by electron beam irradiation, and to determine the by-products resulting from the process. Proof-ofprinciple experiments will be performed to determine the technical and economic feasibility of using electron beam irradiation to decompose the undesirable chemicals present in red water resulting from TNT manufacture.

\section{FREE PISTON PRESSURE RECOVERY FUEL CELL POWER PLANT}

David P. Bloomfield

Analytic Power Corporation

The research objective is to develop a free piston pressure recovery system for small fuel cell systems so that small fuel cells can be pressurized (and deliver the higher performance associated with increased concentration of the reactants) at an acceptable energy cost. Recently available free piston linear motor compressors will be modified to recover the exhaust energy from pressurized PEM fuel cells.

\section{E. Polymer Chemistry}

\section{DETECTION AND/OR DOSIMETRY IN POLYMERIC MATERIALS}

Harry L. Frisch

Walter Gibson

State University of New York at Albany

SL: DARPA

SC: CRDEC, ARL/MD, NRDEC

High-resolution ${ }^{19} \mathrm{~F}$ solid-state NMR spectroscopy was employed in order to study the sorption properties of hexafluorobenzene (HFB) and 3,5bis(trifluoromethyl)aniline (TFMA) in polystyrene (PS) and butyl rubber (BR). The NMR spectra indicate that the penetrants undergo dual-mode sorption in the glassy polymer (PS), but are highly mobile in the rubbery polymer (BR). In addition, the NMR method was applied to the experimental determination of bulk diffusion coefficients for the HFB/PS, TFMA/PS, and HFB/BR systems through desorption studies. The technique could be utilized successfully for either thin film or hollow cylindrical samples. The diffusion results for the TFMA/PS case agreed very well with those previously obtained by resonance nuclear reaction analysis. Interestingly, DSC traces for the samples, taken before exposure to the penetrants and after desorption was complete, displayed essentially identical $T_{g}$ 's, thus indicating little or no physical changes inside the polymers.

Reports:

No. $1-15$ in previous editions.

16. The Case II Diffusion Study of Fluorinated Hydrocarbon in Poly(Styrene) by Resonance Nuclear Reaction Analysis, by Kenji Umezawa and Walter M. Gibson, MS.

17. Diffusion Through a Membrane From a Sessile DropletLimiting Case of the Large Approximation for Short Times, by H.L. Firsch, TR, Aug 91, 9 pp. AD A244 853

18. Method for Determining the Distribution of Josephson Coupling Energies in High- $T_{c}$ Superconductors, by G. Forgacs et al., Physica C177,67(1991). AD A249 080

19. Percolation and the Superconductive Transition of $\mathrm{YBa}_{2} \mathrm{Cu}_{3} \mathrm{O}_{7}$ Films, by G. Forgacs et al., MS, Phys Rev Let.

20. Thermal Conduction in Simple Models of Foams, by W.K. den Otter et al., MS, J Appl Phys.

21. Partially Directed Percolation on Compact Clusters. Exact Solution in Two Dimensions, by G. Forgacs, MS, J Phys.

22. Drop Evaporation Through a Thin Membrane, by Joseph B. Keller and Harry L. Frisch, MS.

23. High-Resolution Solid-State ${ }^{19}$ F NMR Spectroscopy of the Sorption and Diffusion of Fluorine-Containing Aromatic Molecules in Polymeric Media, by Paul J. Toscano et al., MS. 


\section{CHAIN PROPAGATION/STEP PROPAGATION POLYMERIZATION CHEMISTRY}

Ken B. Wagener

University of Florida

\section{SC: ARDEC, AROE, BRADEC, CRDEC, ARL/MD, NRDEC}

Dimethyldichlorosilane and methylcyanopropylsilane in a 37/1 ratio were hydrolytically polymerized as before, and after conversion of the cyano to the carboxyl group, grafting of $\delta$ Valerolactone was carried out. The product was characterized by IR, NMR, and GPC. The formation of the graft was confirmed by the increase in $\mathrm{Mn}$ from 6500 for the siloxane to 15000 for the graft. Grafting of $\varepsilon$ caprolactan to the same siloxane copolymer was achieved in a two-step process: $250^{\circ} \mathrm{C}$, sealed tube, and $180^{\circ} \mathrm{C}, 1 \mathrm{~atm}$. The product was washed several times with ether and dichloromethane until no more siloxane was extracted, as indicated by ${ }^{1} \mathrm{H}$ NMR evaluation of the $\mathrm{CH}_{3}-\mathrm{Si}$ of the siloxane versus the $\mathrm{CH}_{2}-\mathrm{N}$ signals of the lactone; the residue was dissolved in $m$-cresol, recrystallized 3 times by addition of acetone and dried under vacuum. Films were cast from a solution in hexafluoroisopropanol. Characterization was carried out by IR and MNR.

\section{COLLOIDAL CATALYSTS IN WATER}

Warren T. Ford

Oklahoma State University

\section{SC: CRDEC, ARL/MD}

Efforts were made to understand how the surface structures of colloids affect their activity as catalysts and to design catalysts in aqueous colloids that have general activity for decontamination. Researchers have bound catalysts into the gel phase and onto the surface of copolymer latexes for autoxidation and hydrolysis reactions. These colloidal ion exchange resins could be precipitated to obtain an easily packaged and transported catalyst, and redispersed quickly into water for use in the field. The colloids might also be incorporated into latex coatings having catalytic activity for decontamination.

Reports:

No. $1-9$ in previous editions.

10. Oxidations of Alkenes Catalyzed by a Mn(III) Porphyrin and Cationic Polymer Latexes, by Weiming Zhu and Warren T. Ford, J Polym Sci Polym Chem 30,1305(1992).
11. Peroxomolybdate Ions Bound to Latexes as Epoxidation Catalysts, by Sanjay Srinivasan and Warren T. Ford, Polymeric Mater Sci and Eng 64,355(1991). AD A239 588

12. Surface vs. Internal Reactivity of Solid Polymers, by Warren T. Ford, Chemically Modified Surfaces, 1992, p155.

13. Oxidation of Lignin Model Compounds in Water with Dioxygen and Hydrogen Peroxide Catalyzed by Metallophthalocyanines, by Weiming Zhu and Warren T. Ford, MS, J Org Chem.

14. Polymer Colloids as Catalyst Supports, by Warren T. Ford et al., Am Chem Soc Symp Series: Preparation Properties \& Appi of Polym Latexes, 1992, p25.

15: Autoxidation of 2-Mercaptoethanol Catalyzed by $\mathrm{CO}-$ balt(II) Phthalocyaninetetrasulfonate on Colloidal Particles, by Sunkara Hari Babu and Warren T. Ford, MS, J Polym Sci Polym Chem.

\section{MOLECULAR LEVEL CHARACTERIZATION BY NMR OF THE TRANSPORT AND STRUCTURAL ENVIRONMENT OF GASES AND CONDENSED PENETRANTS}

Paul T. Inglefield

Alan A. Jones

Clark University

SL: ARL/MD

SC: AROE, CRDEC

The probes and accessories for the pulse field gradient experiments to measure diffusion coefficients were ordered and recently delivered. Work is now underway to set up the equipment for the proposed experiments. The first system for study will be butyl rubber and toluene. Work was continued on the measurement of carbon dioxide in elastomers and glasses. The general conclusions from that work supported the proposition that sorbed gas behavior in rubbers is characterized by a single mode whereas in glasses the so called "dual mode" picture is appropriate. Extensive measurements were made on $\mathrm{CO}_{2}$ in polystyrene and polyisobutylene. The measurements, primarily of NMR relaxation, have been partially interpreted in terms of the state of the sorbed gas. The data can be explained in terms of a two site exchange model analogous to the "dual-mode" picture. There are $(a)$ dissolved sites involving liquidlike motion of the $\mathrm{CO}_{2}$ and (b) langmuir (or hole) sites involving solid-like motion of the $\mathrm{CO}_{2}$. Final detailed interpretation of this data including the connection to macroscopic permeability behavior is underway. 


\section{FUNDAMENTAL STUDIES OF POLYPHOSPHAZENE AND POLYSILOXANE POLYELECTROLYTES FOR ELECTROCHEMICAL DEVICES}

Duward F. Shriver

Mark A. Ratner

Northwestern University

SL: ARL/EPSD

SC: AROE, ARL/EPSD, ARL/MD, NRDEC

Research included both experimental studies of new polymer electrolytes and theoretical investigations of ion motion. The new systems include: (a) new siloxy linked polyethers with good low-temperature performance. (b) A New polycarbonate polymer, poly(diethylene glycol carbonate), and its salt complexes were synthesized and characterized. The ionic conductivity was found to be low for this material. Apparently the advantages of its high dielectric constant are offset by low segmental motion. (c) Spectroscopic studies of the very promising phosphazene polyelectrolytes containing $\mathrm{Mg}^{2+}$-cryptand complexes. Ion pairing is the issue being addressed here. (d) Molecular dynamics studies were performed on ion motion in a Stockmayer fluid. These simulations provide considerable insight into the role of ion pairing and ion screening on the role of ion transport.

\section{Reports:}

No. $1-4$ in previous editions.

5. Molecular Dynamics Simulations of Highly Concentrated Salt Solutions, by M.A. Ratner et al., MS, Sol St Ionics.

6. Polymer Solid Electrolytes: Charge Transport Mechanisms, by M.A. Ratner, Mater Forum 15,1(1991).

\section{SYNTHESIS AND \\ POLYMERIZATION OF \\ AZAETHYLENES AND \\ AZA-1,3-DIENES, NEW \\ REACTIVE MONOMERS}

Henry K. Hall, Jr.

University of Arizona

3-Aza-3-cyano-acrylonitrile, (N,C-dicyanometh-animine $\mathrm{NC}-\mathrm{CH}=\mathrm{N}-\mathrm{CN}$ ) was too reactive to work with even in dilute solution. Although its existence could be demonstrated by trapping with cyclopentadiene, trapping experiments with various other dienes failed. These included 2,3-dimethylbutadiene, furan, and 1,3-diphenylisobenzofuran. Attempts to synthesize bis imines of o-phthalaldehyde as monomers for imine cyclopolymerization failed. III-de- fined disproportionations, the $\mathrm{N}$ analog of Cannizzaro reactions, predominated. This effort was also terminated. N-Arylsulfonyl derivatives of 1azabutadienes proved to be satisfactory new comonomers. Readily available in crystalline form by the direct reaction of methacrolein with benzene- or other sulfonamides in presence of $\mathrm{TiCl}_{4}-\mathrm{Et}_{3} \mathrm{~N}$, they copolymerized readily and spontaneously with electron-rich styrenes. Use of acrolein in place of methacrolein did not give the analogous azabutadienes with no methyl group. The direct synthesis using $\mathrm{TiCl}_{4}-\mathrm{Et}_{3} \mathrm{~N}$ with aldehydes and amides has now been shown to succeed with cyanamide. Cinnamaldehyde under these conditions gave the corresponding $\mathrm{N}$-cyanoimine as yellow crystalline solid whose polymerization is now being studied. As to electron-rich imines, formaldehyde oxime, $\mathrm{CH}_{2}=\mathrm{NOH}$, was found to be stable above its ceiling temperature of $50-60^{\circ}$, but cyclotrimerized at room temperature. Its o-benzyl ether was even stable in its monomeric form, and acetaldehyde oxime was only seen as monomer. None of these underwent cationic homo- or free radical polymerizations.

Reports:

No. 1-3 in previous editions.

4. Synthesis and Polymerization of Imines and Azadienes: New Polymerizable Monomers, by H.K. Hall, Jr., Makromol Chem and Macromol Symp 54/55,73(1992).

\section{COPOLYMERS AND BLENDS WITH ENHANCED POLYMER FILM DIELECTRIC PROPERTIES}

John R. Reynolds

University of Texas at Arlington

\section{SL: ARL/WTD, ARL/EPSD \\ SC: ARL/MD}

Structural and thermal analyses of the homopolymers poly ( $\alpha$-fluoroacrylonitrile)(PFAN) and poly ( $\alpha$-ethylfluoroacrylate)(PEFA), along with the copolymers poly ( $\alpha$-fluoroacrylonitrile-co- $\alpha$ ethylfluoroacrylate) $[\mathrm{P}(\mathrm{FAN}-\mathrm{co}-\mathrm{EFA})]$ and $\operatorname{poly}(\alpha-$ fluoroacrylonit rile-co-et hylvinylether) [P(FAN-co-EVE)] have been accomplished using a combination of FTIR, ${ }^{1} \mathrm{H}$ NMR, ${ }^{13} \mathrm{C}$ NMR, ${ }^{19} \mathrm{~F}$ NMR, GPC, viscosity, DSC and TGA methods. PFAN has a molecular weight of $130,000 \mathrm{~g} \mathrm{~mol}^{-1}$ while $P(F A N-c o-E F A)$ has a molecular weight of $30,000 \mathrm{~g} \mathrm{~mol}^{-1}$. Both copolymers studied exhibit a relatively random and atactic structure. The highest dielectric permittivity has been measured for PFAN with a value of 8.9 at $50 \mathrm{~Hz}$. 
Reports:

1. Poly(1,3-Cyclohexadiene-alt- $\alpha$-Fluoroacrylonitrile): A Thermally Stable, Alternating Copolymer, by V. Panchalingam and John R. Reynolds, MS, J Polym Sci Polym Chem.

2. Poly( $\alpha$-Fluoroacrylonitrile) and Poly( $\alpha$-Fluoroacrylonitrile-co-Ethylvinylether) Copolymers: Potential 1:1 Alternating Copolymers with Applications as Dielectric Materials, by Mark W. Victor et al., MS, Am Chem Soc

\section{SOLUBILIZATION OF HYDROPHOBIC SUBSTANCES INTO BLOCK COPOLYMER MICELLES IN AQUEOUS MEDIA AND THEIR RELEASE}

Petr Munk

Stephen E. Webber

University of Texas at Austin

\section{SC: AROE, CRDEC, ARL/MD}

Earlier work has emphasized the takeup and release of hydrophobic probe molecules from block polymer micelles and the effect of the micelle formation on the photophysics of a fluorescent probe (naphthalene) covalently bound to the polystyrene end of a diblock polymer. Recent work is attempting to elucidate the properties of the core and corona of these micelle units. Diblock polymers containing an average of one 2-vinylnaphthalene unit per chain have been prepared in which the naphthalene resides either at the polystyrene end of the diblock, or at the interface between the polystyrene and poly(butylmethacrylate) blocks. A triblock polymer of the type PtBMA-PS-N-N-PS-PtBMA was also studied, in which P.tBMA stands for a $t$-butyl methacrylate block, PS indicates polystyrene and N-N represents two 2-vinylnaphthalenes separated by the initiating distyryl anion. Because the distribution of naphthalenes per chain is random, some chains contain two or more naphthalenes so a weak excimer fluorescence is observed. The time-dependence of excimer formation clearly demonstrates the loss of segmental mobility as the micelle is formed by the addition of a non-solvent for the core (polystyrene). Time-dependent depolarization of fluorescence permits an assessment of the rotational mobility of the fluorophore. These studies demonstrate that rotational mobility also ceases as micelles form. Subtle differences in the behavior of the residual anisotropy as more non-solvent is added implies that there are small differences in the rotational restrictions depending on the location of the probe. However in broad outline it may be stated that all parts of the polystyrene segments are essentially immobilized as micelles form, consistent with the simple idea of a fully collapsed polystyrene core.

Reports:

No. 1 in previous edition.

.2. Time-Resolved Fluorescence Studies of the Chain Dynamics of Naphthalene-Labeled Polystyrene-BlockPoly(Methacrylic Acid) Micelles in Aqueous Media, by K. Prochazka et al., MS, Macromol.

3. Fluorimetric and QELS Study of the Solubilization of NonPolar Low-Molar Mass Compounds into Water-Soluble Block-Copolymer Micelles, by D. Kiserow et al., MS, Macromol.

4. Styrene-t-Butyl Methacrylate and Styrene-Methacrylic Acid Block Copolymers: Synthesis and Characterizations, by C. Ramireddy et al., MS, Macromol.

\section{MACROMOLECULAR CHARACTERIZATION AND SOLUTION BEHAVIOR OF POLYMER ADDITIVES}

\section{Benjamin Chu}

State University of New York at Stony Brook

\section{SL: ARL/MD \\ SC: AROE, BRADEC, CRDEC}

The phase behavior of a ternary system consisting of zinc-sulfonated polystyrene, poly(ethyl acrylate- 4 vinyl pyridine) and tetrahydrofuran (THF), which was expected to form interpolymer aggregates (or complexes), was investigated in comparison with that of the non-charged "parent" polymer mixture of polystyrene, poly(ethyl acrylate) and THF. The thermodynamic stability of the homogeneous phase of the ternary system could be decreased by adding the extra interpolymer interactions in a low-dielectricconstant solvent. This observation is in agreement with the theoretical prediction.

\section{Reports:}

1. Magnet Enhanced Optical Falling Needle/Sphere Rheometer, by Benjamin Chu and Jian Wang, Rev Sci Instr 63,2315(1992).

2. Phase Equilibria in the Ternary System Zinc Sulfonated Polystyrene/Poly(Ethyl Acrylate-4-Vinylpyridine)/Tetrahydrofuran, by Jian Wang et al., Macromol 25,2566(1992). 


\section{SYNTHESIS, STRUCTURE AND PROPERTIES OF SEGMENTED POLYMERS CONTAINING MESOGENS}

William J. MacKnight

University of Massachusetts

\section{SL: ARL/MD, NRDEC}

SC: AROE, CRDEC

Efforts have been concentrated on the use of molecular simulation techniques to develop predictive techniques for generating phase diagrams in segmented polyurethanes containing rigid or semi-rigid hard segments. When polymers are mixed, free energy of mixing is often interpreted in terms of a lattice model. In this expression the combinatorial entropic contribution to the free energy of mixing is derived by assuming that polymer chains are extremely flexible. Even though more complete models have taken into consideration segmental size, shape, and local concentration, the assumption of totally flexible chains is maintained. This assumption is not applicable for many polymers including model polyurethanes. The combinatorial entropic expression of generalized Flory-Huggins models cannot be applied to rigid or semi-rigid segments because excluded volume effect and the relative orientations for the rigid chains are not considered. Furthermore, most of these improved mean field models introduce a $\chi$ parameter with a van Laar term to describe the enthalpic contribution to the free energy of mixing. Additional modifications to the van Laar term have been considered. These developments nevertheless cannot be applied to a locally ordered phase. Phase separation behavior using generalized Flory-Huggins models depends only on the sign and magnitude of the $\chi$ parameter. In this study, a different set of thermodynamic expressions have been developed that can be used to describe the phase separated structure involving rigid chains and flexible chains such as polyurethanes. When expressions describing the formation of an anisotropic phase in liquid crystals are incorporated, a new set of equations can be developed to describe the phase separated structures of polyurethanes. Many of the molecular parameters included in the newly derived expressions have been obtained by using molecular simulation techniques.
28373 REACTIVE POLYMERS: MAIN-CHAIN COORDINATION POLYMERS FOR AIR SEPARATION

G. Ronald Husk

Rey T. Chern

North Carolina State University

SC: NRDEC

This project has explored the notion that the positive oxygen binding stability effects of combining Schiffbase/Co(II) chelates with polymers might be markedly improved by incorporating the oxygen-binding sites into the backbone of the polymer chain. Studies have indicated that the inclusion of bulky cardo groups, such as phenophthalein and hexafluoroisopropylidene, in the chain backbone coupled with the addition of cobalt after, rather than before, the polymerization has resulted in the formation of soluble cobalt-containing polymers. Moreover, preliminary qualitative results suggest that the cobalt may be activated by axial ligands to absorb oxygen. Current effort is directed towards: $(a)$ completing the polymer characterization (molecular weight, molecular weight distribution, cobalt concentration, and spectroscopic (UV and IR) studies of the nature and extent of cobalt binding to the Schiff base sites), (b) using the polymer characterization information to formulate strategies to improve yields and molecular weights of these polymers, and (c) performing quantitative oxygen transport and diffusion studies to assess the membrane performance of these materials.

\section{REACTIVE COMPATIBILIZATION OF POLYMER BLENDS}

Donald R. Paul

University of Texas at Austin

\section{SL: ARL/MD}

SC: ARDEC, AROE, BRADEC, CRDEC, NRDEC

Poly( $m$-xylene adipamide), MXD6, was melt mixed at $290^{\circ} \mathrm{C}$ in a Brabender with the aliphatic polyamides: nylon 6,6, nylon 6,9 , nylon 6,10 , nylon 6,12 , and nylon 12,12 . MXD6 was found to be physically immiscible with each nylon $x, y$; however, due to interchange reactions during melt mixing, the mixtures eventually became one phase. The time required for this reaction-induced phase homogenization was followed visually and by differential scanning calorimetry (loss of ability to crystallize and change in glass transition behavior) and was found to increase significantly as the ratio of methylene to amide units, 
$\mathrm{CH}_{2} / \mathrm{NHCO}$ ratio, in the aliphatic polyamide increased. Previous work showed that blends of a maleic anhydride functionalized triblock copolymer (with an ethylene/butane mid-block and styrene endblocks or SEBS-g-MA) with nylon 6,6 gives supertough materials with complex shaped rubber particles that are just below the upper critical particle sized for toughening. Similar blends of SEBS-g-MA with nylon 6 are not toughened because the rubber particles are evidently too small; i.e., below a proposed lower critical size for toughening. It was further proposed that this difference in morphology stems from the difunctional (nylon 6,6) versus monofunctional (nylon 6) character of these polyamides for reaction with anhydrides. This proposal is supported here by the observation that blends of SEBSg-MA with nylon 12,12 give large complex rubber particles like those in nylon 6,6 while nylon 11 and nylon 12 give smaller particles like nylon 6 .

\section{Reports:}

1. Effect of Polyamide Functionality on the Morphology and Toughness of Blends with a Functionalized Block Copolymer, by Y. Takeda et al., MS, Polymer.

2. Morphology of Nylon 6 Blends with Styrenic Polymers, by Y. Takeda and D.R. Paul, MS, J Polym Sci Polym Phys.

3. The Effect of Physical Interactions on Melt Phase Homogenization of Mixtures of Poly( $m$-xylene adipamide) with Aliphatic Polyamides Induced by Interchange Reactions, by Y. Takeda and D.R. Paul, MS, Polymer.

4. Phase Homogenization of Mixtures of Poly( $m$-xylene adipamide) and Nylon 6 by Interchange Reactions, by Y. Takeda and D.R. Paul, Polymer 32,2771(1991). AD A249 826

5. Rubber Toughening of Polyamides with Functionalized Block Copolymers: 1. Nylon-6, by A.J. Oshinski et al., Polymer 33,268(1992). AD A249590

6. Morphology of Toughened Aliphatic Polyamides, by B. Majumdar et al., MS.

7. Reactive Compatibilizers for Blends of Nylon 6 with Styrenic Polymers, by I. Park and D.R. Paul, MS

8. Toughened Nylons, by H. Keskkula and D.R. Paul, MS.

9. Reactive Coupling of Core-Shell Impact Modifiers to Polyamide Matrices Using Styrene-Maleic Anhydride Copolymers, by M. Lu et al., MS, Polymer.

\section{WELL-DEFINED}

POLYPHOSPHAZENES BY

ANIONIC POLYMERIZATION OF PHOSPHORANIMINES

Krzysztof Matyjaszewski

Carnegie-Mellon University

SL: ARL/MD, NRDEC

SC: ARDEC, AROE, BRADEC, CRDEC
Work has focused on two synthetic methods towards novel polyphosphazenes. The first method employs catalyzed condensation of phosphoranimines which proceeds with anionic intermediates. The second method is based on the thermal polymerization of phosphine azides which can either be isolated or prepared in situ by metathesis of the trimethylsilyl azides and the corresponding organophosphorus compounds with $\mathrm{P}-\mathrm{Cl}$ and $\mathrm{P}-\mathrm{OR}$ linkages. Catalyzed polymerization of phosphoranimines: Various $\mathrm{N}$ trimethylsilyl phosphoranimines have been prepared by the Saudinger reaction of phosphites with trimethylsilyl azide. Stability and reactivity of phosporanimines depends on the substituents at phosphorus. Tris(trifluoroethyl)phosphite provides at $100^{\circ} \mathrm{C}$ phosphoranimine in above 90 percent yield. The phosphoranimine is thermally stable till $200^{\circ} \mathrm{C}$ and can be polymerized in the presence of strong nucleophiles and compounds with strong affinity towards silicon such as ammonium halides but also uncharged species such as N,N-dimethylaminopyridine and imidazole. Triphenylphosphite reacts with the azide at $70^{\circ} \mathrm{C}$ but the phosphoranimine is thermally unstable and decomposes in situ to poly(diphenoxyphosphazene). Mixed phosphite (bis[trifluoroethyl]phenylphosphite) gives stable phosphoranimine which decomposes to a random copolymer. Reaction of phosphinites and phosphonites with trimethylsilyl azide gives a mixture of phosphoranimine and phosphine azide. The latter decomposes thermally to polyphosphazene. Phosphine azides can be also prepared by the reaction of the corresponding chlorophosphines with trimethylsilyl azide. Phosphine azides can be polymerized in situ and can also be isolated by removal of the volatile trimethylsilyl chloride or alkoxysilane.

\section{Reports:}

1. Synthesis of Poly(Phenyltrifluoroethoxyphosphazene) by Direct Reaction of Silyl Azide with Phosphonite, by Krzysztof Matyjaszewski et al., MS, J Polym Sci Polym Chem.

2. Preparation of Inorganic and Organometallic Polymers with Controlled Structures, by Krzysztof Matyjaszewski, MS, J Inorg and Organometallic Polym.

3. New Synthesis Routes Towards Polyphosphazenes, by Kuzysztof Matyjaszewski et al., MS, Makromol Chem.

4. Towards Well-Defined Polysilylenes and Polyphosphazenes, by Krzysztof Matyjaszewski, MS.

5. Catalysts and Initiators for Controlling the Structure of Polymers with Inorganic Backbone, by Krzysztof Matyjaszewski, MS. 


\section{SYNTHESIS AND EXAMINATION OF NEW POLYMERIC MATERIALS}

Fredric M. Menger

Emory University

\section{SL: CRDEC, NRDEC}

SC: AROE, CRDEC, ARL/MD

The Army Research Office has been instrumental in supporting the "systems research" which has been summarized now in a long Angewandte Chemie article. This article describes a decade of experiments into the structure of self-aggregating systems and into their catalytic properties. Micelles, microemulsions, vesicles, laminates, reverse micelles, films, etc., are included in the discussion. In the course of investigating cobalt complexes for possible activity toward nerve agents, an unusual compound was prepared in which sulfate acts as a bidentate ligend to $\mathrm{Co}$ (III), the first such compound of its type. The x-ray structure of the complex has now been published in Inorganic Chemistry. Unfortunately, the complex does not show high activity toward phosphorus compounds. Template synthesis of polymers has been used by Shea to incorporate easily accessible functional groups at the surface of highly crosslinked polymers. Previous efforts in this group have resulted in the design of surface functionalized copper loaded catalysts from commercial chlorobenzyl polystyrene and from functional monomers incorporated in water pools. A report has been prepared which describes the synthesis of diamine functionalized high surface area polystyrenes in the presence of toluene as an inert diluent. The number of accessible functional groups was determined by the complexation of the diamine groups with $\mathrm{Cu}^{2+}$ ions. The catalytic activity of these polymers have been evaluated by the copper catalyzed hydrolysis of $p$-nitrophenyl diphenyl phosphate.

\section{THE SYNTHESIS AND STRUCTURE OF POLYPHOSPHAZENES}

Harry R. Allcock

The Pennsylvania State University

\section{SL: ARDEC, NRDEC}

SC: AROE, BRADEC

The objective of the research is to design and find methods for the synthesis of new polymers that will be useful to the Army in a broad array of applications that range from high performance elastomers and protective coatings, to materials for use in communications technology. The principles being developed involve the incorporation of inorganic elements into polymers in ways that provide improved materials, properties compared to existing polymeric materials. Poly (heteroelemento-phosphazenes): Work is continuing on the synthesis and properties of poly(carbophosphazenes) and poly(thiophosphazenes) with respect to $(a)$ cyclic monomer synthesis, $(b)$ model reactions carried out at the level of small-molecule cyclic carbophosphazenes, (c) polymerization studies, $(d)$ polymer substitution reactions, and $(e)$ materials' properties of the polymers in the solid state.Poly (phosphazo (phosphazenes): These are new polymers with $\mathrm{NPR}_{3}$ side groups attached to the phosphorus atoms of a phosphazene chain. Research is proceeding on polymerization reactions, polymer substitution chemistry, and polymer physical properties. Several of these polymers have prospective uses as high performance elastomers. Organometallic Polyphosphazenes: Two topics are the focus of recent work. First, the polymerization mechanism of ferrocene-bearing phosphazenes has been studied in detail, with an emphasis on the role of ring-strain in the polymerization process. The electrochemistry of these polymers is being developed through a collaborative progam with Professor Royce Murray at the University of North Carolina. The gas permeability of these polymers is also being examined through another collaborative arrangement. Surface Chemistry: Researchers continue to examine the ways in which the surface characteristics of polyphosphazenes can be changed by simple surface reactions. Earlier work with fluoroalkoxy phosphazenes is being extended to aryloxyphosphazene materials. Structure-Property Relationships: Molecular mechanics/molecular graphics methods are being applied to a range of phosphazene cyclic and short-chain linear species. These studies are based on single crystal X-ray data produced in the program. The objective is to refine the understanding of the relationship of polymer molecular structure to materials' properties so that synthetic chemists can respond quickly when a need arises for new materials with specific combinations of properties.

Reports:

No. 1 in previous edition.

2. Synthesis of Poly(Bis-Phosphazo)Phosphazenes Bearing Aryloxy and Alkoxy Side Groups, by Harry R. Allcock and Dennis C. Ngo, Macromol 25,2802(1992).

3. This number not used.

4. Strained Inorganic Heterocyclic Compounds and Their Conversion to Macrocycles and High Polymers, by Harry R. Allcock, MS. 
5. Strain-Induced Ring-Opening Polymerization of Ferrocenylorganocyclotriphosphazenes: A New Synthetic Route of Poly(Organophosphazenes), by Harry R. Allcock et al., J Am Chem Soc 113,9596(1991). AD A249818

6. Polyphosphazenes Bearing Polymerizable Pyrrole, Thiophene and Furan Side Groups: Synthesis and Chemical Oxidation, by Harry R. Allcock and Jeffrey A. Dodge, MS, Chem Mater.

7. Cyclic and Short-Chain Linear Phosphazenes with Hindered Aryloxy Side Groups, by Harry R. Allcock et al., $J$ Chem Soc Dalton Trans p1687, (1992).

8. Poly(Thiophosphazenes): New Inorganic Polymers, by Harry R. Allcock et al., MS, Macromol.

9. This number not used.

\section{ADHEREND SURFACE EFFECTS ON EPOXY CURE BY NMR}

Paul T. Inglefield

Clark University

SL: ARL/MD

SC: ARDEC, AROE

A schedule has been organized for sample preparation in conjunction with the Materials Technology Laboratory of the U.S. Army. In this regard, a custom synthesis of 4,4' - Diamino ${ }^{15} \mathrm{~N}_{2}$ - diphenyl sulfone has been commissioned. This ${ }^{15} \mathrm{~N}$ enriched crosslinking agent will be used to generate the epoxies to be used for MAS NMR experiments to elucidate both structure and dynamics in the cure. Deuteron $\left({ }^{2} \mathrm{H}\right)$ solid state measurements have been made using both $1 D$ and $2 \mathrm{D}$ NMR in both linear and 4,4 methylene dianiline (MDA) crosslinked bisphenol-A epoxy resins with ${ }^{2} \mathrm{H}$ labelling on the bisphenol aromatic rings. Both 1D and 2D deuteron spectroscopy on these resins suggest that the dynamics are represented by a superposition of rigid and fast $180^{\circ}$ flips of the bisphenol-A aromatic rings. The population of fast flippers is temperature dependent and greatly enhanced in the linear epoxy. Quantitatively the ring dynamics seems to follow that of pure BPAPC with a $40^{\circ}$ temperature shift (to slower motion) for the crosslinked material. The chain deuterated material has not been measured yet but will yield information on the chain dynamics near $T_{g}$. Quantification of both below $T_{g}$ (ring motion) and at $T_{g}$ (chain motion) should be successful.

\section{SYNTHESIS AND CHARACTERIZATION OF POLY(ALKYL/ARYLPHOSPHAZENES) AND THEIR FUNCTIONALIZED DERIVATIVES}

Robert H. Neilson

Patty Wisian-Neilson

Texas Christian University

SL: NRDEC
SC:

A multifaceted investigation will be made of the synthesis, characterization, and chemical modification of poly(alkyl/arylphosphazenes) as well as their small-molecule Si-N-P precursors. The synthetic process, including the catalysis of the polymerization, the chemical modification of the preformed polymers, the improved synthesis of the precursors, and the characterization and structure-property relationships will be the focus of this program using methods pioneered by the principal investigators. 


\section{BIOLOGICAL SCIENCES}

\section{A. Combat Ration Research}

\section{FOOD RELATED STUDIES}

\author{
Myron Solberg \\ Jozef L. Kokini \\ Rutgers, The State University of New Jersey
}

\section{SL: NRDEC}

After redefinition and refocusing, the water phenomena project has as broad goals, to develop mechanistic understanding of the role of water as it affects flavor, texture, appearance and ultimate quality and to predict and control physical and chemical changes as a function of environment and time occurring during processing, storage and distribution of foods. To estimate the two parameters describing moisture history during isothermal drying of plates, slabs and spheres, five key parameters were necessary and helped optimize the drying process. Work on sugar solutions at low temperatures resulted in the observation that all carbohydrates studied (glucose through starch) have the same gravimetric $\mathrm{C}_{\mathrm{g}}$ (maximum freeze - concentration) of 80 percent. A newly developed gradient slab gel electrophoresis system allows detection of a fraction of unreacted native lysozyme. Quantification of the amount of monomeric and polymeric lysozyme will provide the foundation for kinetic studies. Comparison of computer simulated pressure, temperature and velocity fields, mixing characteristics, residence time distributions and shear rate variation inside tangential, self-wiping twin screw extruders and single screw extruders, showed an improved mixing and very similar pressure and temperature profiles in twin screw extruders. The results also agree qualitatively with the experimental observations. In slit rheometer studies, a prototype for non-reactive systems was designed to overcome limitations of the slit measuring section; also a control loop was designed. Both new components have been installed for system tests. In thermomoisture probe studies, a prototype was designed and the manufacturer was selected. Proto- types were ordered for trial. A powder test cell was designed.

Reports:

1. Lipid-Protein and Lipid-Carbohydrate Interactions During Extrusion, by Chi-Tang Jo and Mark T. Izzo, Food Extrusion Science and Technology, 415. AD A249564

2. Molecular Transformations of Starch and Protein During Twin-Screw Extrusion Processing; of Cornmeal, by Bruce P. Wasserman et al., Food Extrusion Science and Technology, 325. AD A249 766

3. Water Diffusivity in Extrusion Cooking of Starch Materials, by V.T. Karathanos and G.D. Saravacos, Food Extrusion Science and Technology, 177. AD A249 473

4. Application of an Energy Equivalent Concept to the Studies of the Kinetics of Starch Conversion During Extrusion, by S.S. Wang et al., Food Extiusion Science and Technology. AD A249 378

5. Numerical Simulation of Fluid Flow and Heat Transfer in a Twin-Screw Extruder, by T. Sastrohartono et al., Food Extrusion Science and Technology, 89. AD A249290

6. Modeling of Starch Gelatinization in a Single Screw Extruder, by S. Gopalak ishna and Y. Jaluria, MS.

7. Molecular Restructuring and Complexation During Extrusion of Cornmeal, by George Strauss et al., MS.

8. Effect of Extrusion Operation Variables on Functionality of Extrudates, by S.W. Halek and K.L.B. Chang, MS.

9. The Role of Rhrological Properties on Extrudate Expansion, by Jozef L. Kokini et al., Food Extrusion Science and Technology, 631. AD A249 765

\section{MECHANISM OF RESISTANCE IN MICROBIAL SPORES}

Philipp Gerhardt

Robert E. Marquis

Michigan State University

\section{SL: CRDEC}

Thermograms of the model exosporiumless dormant spores of Bacillus megaterium ATCC 33729, obtained by differential scanning calorimetry, showed three major irreversible endothermic transitions with peaks at 56,100 , and $114^{\circ} \mathrm{C}$, and a major irreversible exothermic transition with a peak at $119^{\circ} \mathrm{C}$. A reversible endothermic transition with a peak at $91^{\circ} \mathrm{C}$, identified as the melting of DNA in vegetative cells, was 
not revealed in the dormant spores or in germinated spores. Thermograms of heat-activated spores did not show the $56^{\circ} \mathrm{C}$ transition, and those of coat-divested spores did not show the $100^{\circ} \mathrm{C}$ transition. Thus, the endothermic $100^{\circ} \mathrm{C}$ transition was apparently associated with denaturation of the rate-limiting critical target in heat killing of the dormant spores. Following an onset at $73^{\circ} \mathrm{C}$, the ascending part of this transition corresponded to a first-order reaction and was correlated with spore death; i.e, 99.9 percent of the spores were killed when the peak at $100^{\circ} \mathrm{C}$ was reached. Most of the spore killing occurred before the release of most of the dipicolinic acid and other intraprotoplast materials.

\section{Reports:}

1. Heat Killing of Model Bacterial Spores Analyzed by Differential Scanning Calorimetry, by Brian H. Belliveau et al., MS, J Bacteriology.

\section{KINETICS AND MECHANISM OF CHEMICAL MARKER \\ FORMATION AND \\ WATER-ACTIVATED HEAT GENERATION}

Kenneth Kustin

Brandeis University

\section{SL: NRDEC}

The effort on chemical markers has been concerned with marker identity, comparison of bacterial lethality with chemical marker formation to validate the use of chemical markers in thermally processed food, and evaluation of the behavior of the chemical marker over a range of temperatures and temperature variations. Several interesting and informative, but relatively minor activities, connected with wateractivated heat generation include working on alternatives to the iron-promoted magnesium-water flameless ration heater (FRH), laboratory tests of alternative chemical heat generators, and laboratory testing of competitive brands of the magnesiumwater FRH. The major activity has been the development of a successful strategy to suppress gaseous $\mathrm{H}_{2}$ evolution that accompanies heat generation. Other important activities have been connected with chemical dynamics in a calorimeter; namely, selection of a system for kinetics calibration and development of a self-consistent technique of extracting rate constants and heats of reaction from chemical dynamics data in a calorimeter. A careful study by scanning electron microscopy of the surface properties of the magnesium-iron blend has also been carried out.

\section{THE DESIGN AND \\ CONSTRUCTION OF AN ION \\ TRAP BASED SYSTEM FOR \\ LASER PYROLYSIS/MASS \\ SPECTROMETRY OF SINGLE \\ ORGANIC AEROSOL PARTICLES}

Henk I.C. Meuzelaar

Neil S. Arnold

University of Utah

The effort has been directed toward modeling and design considerations for the experimental apparatus. A model has been constructed to evaluate aerosol injection parameters via a capillary restrictor to the reduced pressure environment. Preliminary analyses indicate that particles in the range of 1 to $10 \mu \mathrm{m}$ in diameter should be captured at pressures as low as 10 torr. The exact values will depend largely on charging efficiency for the particles and will be experimentally verified. If high charging efficiencies can be obtained, lower pressures may be possible along with a larger range of particle sizes trapped in a monitoring scenario. Present expectations are that using a given set of operating parameters, particles differing in radii by a factor of 3 may be monitored simultaneously. A system design is largely in place and construction has begun.

\section{B. Biodegradation}

\section{LIMITING FACTORS, ENHANCEMENT AND KINETICS OF BIODEGRADATION}

Martin Alexander
Cornell University

\section{SC: CRDEC, CRREL}

Research on the biodegradation of organic compounds present in nonaqueous phase liquids has continued. The mineralization of di(2-ethylhexyl) phthalate (DEHP) in soil was suppressed or prevented if it was present in hexadecane, heptamethylnonane, or dibutyl phthalate but not cyclohexane. Phenanthrene mineralization in a subsoil was reduced to varying extents if the hydrocarbon was in hexadecane, DEHP, cyclohexane, or heptamethylnonane, but the addition of $N$ and $P$ substantially increased the biodegradation. These NAPLs themselves differ markedly in susceptibility to microbial degradation. In contrast, the rate and extent of hexadecane mineralization in the subsoil 
were increased when alkane was present in heptamethylnonane, DEHP, or cyclohexane. Hexadecane mineralization in lake water was more rapid if in heptamethylnonane than in DEHP. Studies of pure cultures acting on compounds in NAPLs are being conducted with Pseudomonas acting on phenanthrene and arthrobacter degrading hexadecane dissolved in various solvents, apparently by difference mechanisms. Additional isolates are being sought which can use biphenyl and phenanthrene in several NAPLs.

Reports:

No. 1-9 in previous editions.

10. Microbial Mineralization of Biphenyl Sorbed to Sediments and Synthetic Beads, by Rochelle Araujo and Martin Alexander, MS, Env Toxicology and Chem.

11. Effect of a Nonionic Surfactant Added to the Soil Surface on the Biodegradation of Aromatic Hydrocarbons Within the Soil, by Boris N. Aronstein and Martin Alexander, MS, Appl and Environ Microbiol.

12. Effect of Diffusion and Sorption on the Kinetics of Biodegradation: Theoretical Considerations, by Kate M. Scow and John Hutson, Soil Sci Soc Am J 56,119(1992).

\section{MICROBIAL DEGRADATION OF POLY-B-ESTERS: A MECHANISTIC STUDY}

Richard A. Gross

Shan S. Wong

University of Lowell

\section{SL: NRDEC}

SC: CRREL, NRL

A poly ( $\beta$-hydroxyalkanoate), PHA, depolymerase exoenzyme has been isolated from Penicillium funiculosum (ATCC 9644). The enzyme was purified using a hydrophobic Norleucine Sepharose column to homogeneity as determined by SDS-PAGE. This PHA depolymerase has a pI, optimal $\mathrm{pH}$, and temperature of $5.8,6.0$, and $30^{\circ} \mathrm{C}$, respectively. The enzyme is a monomer with a molecular weight of 38,000 as determined by gel filtration. It was determined that the enzyme is completely inactivated by DAN and EPNP, which are aspartic acid protease inhibitors. The $K_{m}$ values for PHB and the corresponding copolymers with 7 percent, 16 percent, and 22 percent ethyl side chain copolymer compositions are 14, 7.6, 1.07 , and $2.7 \mathrm{mg} / \mathrm{mL}$, respectively. These results may be attributed to optimal hydrophobic-hydrophobic interactions or possibly crystalline morphology effects. Synthetic methodology has been developed which allows the preparation of poly $(\beta$ hydroxyalkanoates), of variable composition and with control of polymer stereochemistry. The synthetic scheme involves the alkylation of $\beta$-diketo ester dianions by reaction with alkyl bromides, asymmetric hydrogenation of the $\beta$-keto functional group using ruthenium 2,2'-bis (diphenylphosphino)-1, $1^{\prime}$ binaphthyl as catalyst, saponification to form the corresponding $\beta$-hydroxycarboxylic acid, and thermolysis with either triethyl orthoacetate or tetraethyl orthoacetate (depending on the $\beta$-propiolactone substituent) to form the lactone. The polymerization of $[R]-\beta$-pentyl- $\beta$-propiolactone with a diethylzinc/water catalyst has been carried out to produce a homopolymer with a heat of fusion measured by DSC of $10 \mathrm{cal} / \mathrm{g}$.

\section{EVOLUTION OF REGULATORY GENES GOVERNING BIODEGRADATION IN "ACINETOBACTER CALCOACETICUS"}

L. Nicholas Ornston

Yale University

Two manuscripts have been published on comparative aspects of genes associated with benzoate metabolism in Acinetobacter calcoaceticus and Pseudomonas putida. The benzoate genes share common ancestry but are clustered with other genes associated with different metabolic pathways. In the first manuscript, it was possible to demonstrate that the $A$. calcoaceticus ben $A B C$ genes could be traced to an ancient lineage of genes that gave rise to progeny as divergent as chloroplast ferredoxin and ferredoxin reductase. In the second manuscript evidence was presented supporting the view that the $P$. putida $x y l X Y Z$ genes diverged relatively recently from the $A$. calcoaceticus ben $A B C$ genes. Divergence of the $x y l Z$ gene appears to have been accompanied by acquisition of complex DNA slippage patterns suggesting that intragenic sequence exchange created mutations leading to the present primary structure of the $x y l Z$. The possible roles of the DNA slippage structures in transcriptional control or genetic repair is yet to be established. In some respects the $A$. calcoaceticus pob $A$ system affords great advantages for examining the role of secondary DNA structures in slippage and repair. The $A$. calcoaceticus pob $A$ gene has been sequenced and the deduced primary structure of the enzyme bears 62.4 percent amino acid sequence identity to the pob $A$ gene product from Pseudomonas fluorescens. The crystal structure of the latter enzyme has been solved, and it is possible to fit the 
sequence of the $A$. calcoaceticus enzyme into this structure. This alignment reveals strict conservation of sites associated with substrate binding, and relatively extensive divergence in other regions. Intriguingly, conservation is observed at positions corresponding to protein-protein interfaces in the dimeric protein, although this region makes no obvious contribution to substrate binding.

\section{Reports:}

1. Nucleotide Sequences of the Acinetobacter calcoaceticus benABC Genes for Benzoate 1,2-Dioxygenase Reveal Evolutionary Relationships Among Multicomponent Oxygenases, by Ellen L.Neidle et al., J Bacteriology 173,5385(1991). AD A249 658

2. Potential DNA Slippage Structures Acquired during Evolutionary Divergence of Acinetobacter calcoaceticus Chromosomal ben $A B C$ and Pseudomonas putida TOL pWWO Plasmid $x y L X Y Z$, Genes Encoding Benzoate Dioxygenases, by Shigeaki Harayama et al., J Bacteriology 173,7540(1991). AD A249 295

3. Genetic Analysis of Supraoperonic Clustering by Use of Natural Transformation in Acinetobacter calcoaceticus, by Beate Averhoff et al., MS, $J$ Bacteriology.

\section{BIOCHEMICALLY VULNERABLE SITES FOR ANTIFUNGAL INTERCESSION IN THE CONTROL OF FUNGAL GROWTH}

Shirley R. Tove

Leo Parks

North Carolina State University

Sterol auxotrophic strains of Saccharomyces cerevisiae were grown and allowed to conjugate on media supplemented with various sterols. The mating efficiency of the auxotrophs is perturbed by the replacement of the yeast sterol, ergosterol, with other sterols. After four hours of mating, cells grown on ergosterol exhibited a 30 -fold higher productive mating percentage than those cells grown on stigmasterol. Aberrant budding by the conjugants was enhanced following incubation on the non-ergosterol sterols. Using light and electron microscopy, researchers demonstrated that there is a reduced ability for stigmasterol-grown cells to undergo cytoplasmic fusion during conjugation. Many of the mated pairs remained adherent but prezygotic even after twelve hours of incubation. The addition of ergosterol to cells previously grown on stigmasterol rescued the organisms, allowing for zygote formation and normal budding.
Reports:

1. Effect of Sterol Alterations on the Efficiency of Conjugation in Sterol Auxotrophs of the Yeast, Saccharomyces cerevisiae, by Michele E. Tomeo et al., MS.

\section{Biotechnology}

\section{PLASMID STABILIZATION TO INSURE GENE EXPRESSION}

Arnold L. Demain

Massachusetts Institute of Technology

A fragment of $C$. thermocellum chromosomal DNA, originally isolated because of its ability to encode a polypeptide which crossreacts with antibodies raised against the $S_{L}$ subunit of the $C$. thermocellum cellulase, was further found to encode another distinct polypeptide. This new polypeptide, termed EXGA, does not however crossreact with anti-S $S_{L}$ antibodies. Sub-cloning experiments showed that this polypeptide is encoded by a different portion of the same $C$. thermocellum extracellular protein with a molecular weight of $62 \mathrm{kDa}$, i.e. $\mathrm{J}_{5}$ of the sub-cellulosome preparation. Anti- $\mathrm{J}_{5}$ antibodies, when used against crude cellulase after SDS-PAGE, bind very specifically to the $62 \mathrm{kDA}$ band. The sub-cellulosome preparation is a much simpler enzyme complex than that of the cellulosome. It is obtained by cation-exchange chromatography and lectin-affinity chromatography of $C$. thermocellum extracellular broth. The sub-cellulosome preparation consists of 6 main proteins with molecular weights ranging from $210 \mathrm{kDa}\left(\mathrm{J}_{1}=\mathrm{S}_{\mathrm{L}}\right)$ to $58 \mathrm{kDa}\left(\mathrm{J}_{6}\right)$. The discrepancy in size, $49 \mathrm{kDa}$ for the protein encoded by exgA and $62 \mathrm{kDa}$ for native $\mathrm{J}_{5}$, as well as the fact that it was not possible to align the $\mathrm{N}$-terminal amino acid sequence of $\mathrm{J}_{5}$ with any of the reading frames on $\mathrm{pRV} 4.3$, indicates that researchers are dealing with two different proteins. It was possible to show that of the six proteins of the sub-cellulosome, only $S_{L}$ has a detectable degree of glycosylation and thus ruled out the possibility that the difference in size can be attributed to glycosylation of EXGA.

Reports:

No. $1-7$ in previous editions.

8. Purification and Characterization of a New Endoglucanase from Clostridium thermocellum, by M.P.M. Romaniec et al., MS, Biochem J.

9. Cloning and Expression of a Clostridium thermocellum DNA Fragment that Encodes a Protein Related to Cellulosome Component $\mathrm{S}_{\mathrm{L}}$, by T. Kobayashi et al., Appl Bioch Biotech 31,119(1991). 
10. Cloning and Expression in Escherichia coli of Clostridium thermocellum DNA Encoding Sub-Cellulosomal Proteins, by T. Kobayashi et al., MS.

\section{SYNTHETIC HELIZYME ENZYMES}

John M. Stewart

University of Colorado at Denver

\section{SC: NRDEC}

Efforts have resulted in a system for HPLC that allows one to purify chymohelizyme-1 (CHZ-1). Although the material is still not homogeneous, the best fractions now show catalytic activity at least twice thơse reported earlier. There is very significant catalysis of hydrolysis of benzyloxycarbonyltyrosine- $p$ nitrophenyl ester, and slower, but real, hydrolysis of benzoyltyrosine ethyl ester. Although the first synthesis of CHZ-1 went remarkably well, all subsequent attempts at resynthesis met with great difficulties; until it was realized that the commercial synthesis resins available clearly were not suitable for synthesis of the complex CHZ molecule. The first synthesis had used the last of a batch of remarkably good resin. A source has been found for synthesis resin having the desired properties. Computer design work has continued for improved versions of $\mathrm{CHZ}_{-1}$, in consultation with the others in the helizyme group. An improved design for $\mathrm{CHZ}-1$ was completed and a significantly different design for a chymotrypsin-like helizyme should show much improved properties.

\section{Reports:}

No. 1 in previous edition.

2. Studies on "Chymohelizyme-1", a Designed Synthetic Enzyme, by John M. Stewart et al., MS.

3. Solid Phase Synthesis of a Peptide Designed to Have Enzymic Activity, by John M. Stewart et al., MS.

4. Design and Synthesis of "Chymohelizyme-1", a Peptide Having Chymotrypsin-Like Catalytic Activity, by John M. Stewart et al., Peptides, 1991, p575. AD A246 737

\section{TOXIN MEDIATED TRANSFER AND EXPRESSION OF GENES IN NERVE CELLS}

Gregory P. Mueller

Uniformed Services of the Health Sciences

\section{SC: CRDEC}

While there are several methods for introducing foreign genes into mammalian cells, none of these approaches selectively targets gene expression into the central nervous system (CNS). This research investigates the feasibility of using receptor-mediated gene transfer as a mechanism for introducing the expression of foreign genes in the mammalian brain. Gene carrier systems are constructed using neuronal ligands that are rapidly internalized by receptor-mediated endocytosis. The ligands are complexed with high expression reporter genes and evaluated as vectors for transfer into nerve cells in vitro and in vivo. Findings showed that foreign genes can be expressed in the CNS of adult rats. Experiments demonstrated liposome-mediated transfer and expression of a reporter gene (RSV- $\beta$-GAL) in the choroid plexus and circumventricular organs of rats. Further, low level expression was also observed in the parenchyma. This appears to be the first evidence that plasmid DNA can be expressed within the mammalian CNS. Researchers continue to systematically determine optimal conditions for the (a) construction of targeted DNA carrier complexes and (b), uptake and expression of these systems by cells grown in culture. Further, the research now involves the use of tetanus toxin Fragment-C as a targeting ligand. Dr. Paul Fishman has developed the techniques for the bulk production of Fragment-C from crude tetanus toxin ( $J$. Neurosci. Meth., in press). In addition, purified Fragment-C has recently become available commercially through both conventional and recombinant techniques. He has tested these preparations in vivo for capacity for neuronal uptake and transport. Although all the preparations have neurobiological activity, the recombinant preparation is the most avidly internalized.

\section{BACTERIAL SPORE ULTRAVIOLET LIGHT RESISTANCE AND REGULATION OF THE ACTIVITY OF A SPORE PROTEASE}

Peter Setlow

Connecticut University Health Center

\section{SL: NRDEC}

Work has continued on the analysis of the effect of $\alpha / \beta$-type SASP on DNA structure and properties. Structural studies include initiating a collaborative effort with Drs. Alex Rich (MIT) and Scott Mohr (Boston University) to crystallize an $\alpha / \beta$-type SASP/oligonucleotide complex and determine the structure of such a complex by x-ray crystallography. Preliminary work has also begun with Dr. Mohr and R. John Lee (Harvard Medical School) to carry out an NMR structural determination of an $\alpha / \beta$-type SASP with and without an appropriate deoxyoligonucleotide. Work to date has indicated 
that the project is feasible in that researchers can make more than enough protein, and that small (1220) double stranded DNA ologomers are tightly bound. Micro-co-crystals of an SspC/oligonucleotide complex have been obtained. The preliminary NMR work has shown that the protein alone has very little structure. In a continuation of work on the effects of $\alpha / \beta$-type SASP binding on DNA photochemistry in vitro (and thus presumably UV resistance in vivo) researchers have shown that all wild type $\alpha / \beta$-type SASP block UV induced formation of cyclobutane type cytosine-cytosine dimers and cytosine-thymine dimers, as well as 6/4 photoproducts between adjacent cytosine, or cytosine and thymine residues. This adds further evidence of the key role these proteins play in spore UV resistance. Preliminary work has been initiated to analyze the effects of $\alpha / \beta$-type SASP on DNA depurination. To date this work has shown that $\alpha / \beta$-type SASP binding to DNA in vitro reduces the rate of depurination in DNA at $\mathrm{pH} 5.0$ and $70^{\circ}$ over 20 fold. This finding may have great relevance to spore heat resistance, since spores lacking most $\alpha / \beta$-type SASP have lost some heat resistance.

Reports:

No. $1-4$ in previous editions.

5. Changes in Forespore Chromosome Structure During Sporulation in Bacillus Species, by P. Setlow, Dev Biol 2,55(1991). AD A245 251

6. Effects of Mutant Small, Acid-Soluble Spore Proteins From Bacillus subtilis on DNA in Vivo and in Vitro, by Federico Tovar-Rojo and Peter Setlow, J Bacteriology 173,4827(1991). AD A245 085

7. Ultraviolet Irradiation of DNA Complexed with $\alpha / \beta$-Type Small, Acid-Soluble Proteins From Spores of Bacillus or Clostridium Species Makes Spore Photoproduct But Not Thymine Dimers, by Wayne L. Nicholson et al., Proc Natl Acad Sci 88,8288(1991). AD A244 492

8. Condensation of the Forespore Nucleoid Early in Sporulation of Bacillus Species, by Barbara Setlow et al., J Bacteriology 173,6270(1991). AD A245 124

9. Properties of Bacillus megaterium and Bacillus subtilis $\mathrm{Mu}$ tants Which Lack the Protease That Degrades Small, AcidSoluble Proteins During Spore Germination, by Jose-Luis Sanchez-Salas et al.,J Bacteriology 174,807(1992). AD A249 648

10. Interaction Between DNA and $\alpha / \beta$-Type Small, Acid-Soluble Spore Proteins: A New Class of DNA-Binding Protein, by Barbara Setlow et al., J Bacteriology 174,2312(1992). AD A249 692

11. Binding of DNA to $\alpha / \beta$-Type Small, Acid-Soluble Proteins From Spores of Bacillus or Clostridium Species Prevents Formation of Cytosine Dimers, Cytosine-Thymine Dimers, and Bipyrimidine Photoadducts after UV Irradiation, by Heather Fairhead and Peter Setlow, $J$ Bacteriology 174,2874(1992).

12. I Will Survive: Protecting and Repairing Spore DNA, by Peter Setlow, J Bacteriology 174,2737(1992).

\section{INVESTIGATION OF PRESSURE REGULATION IN ENZYMES}

Alan J. Russel

University of Pittsburgh

\section{SC: CRDEC}

During work on the purification of a pressure sensitive hydrogenase from Methanococcus jannaschii, the presence of an extremely stable protease was discovered. This protein is of such interest that efforts were focused on its isolation and characterization. This work has led to the discovery of a number of interesting discoveries related to protein purification from the archaebacter. In addition, it has been possible to purify and sequence the first 25 amino acids of the methyl-coenzyme $\mathrm{C}$ reductase from Methanococcus jannaschii. The partially purified sample was used for the studies to determine in which class of protease it may be. The samples were incubated for one hour at $90^{\circ} \mathrm{C}$ with the different inhibitors. The volume of the sample was $700 \mathrm{ml} .10 \mu 1$ of each of the inhibitors was used; $100 \mathrm{mM}$ PMSF in DMSO, .1 mg/ml pepstain in DMSO, $100 \mathrm{mM} 1,10$ phenanthroline in DMSO, $10 \mathrm{mM} \mathrm{E}-64$ in water. 100 $\mu l$ of $200 \mathrm{mM}$ EDTA in water was also used. An 11 percent drop in the rate was seen in the sample with 1,10 phenanthroline, and a 9 percent drop in the rate was seen with EDTA. The rest of the samples had the same rate as the sample without an inhibitor. This information may suggest a metal binding protein. In general, the proteins from this organism agglutinate, without precipitation, under a wide variety of conditions. This phenomenon can only be relaxed by increased temperature or the presence of mild detergents.

\section{BIOCATALYTIC PROCESSING OF POLYMERS IN SUPERCRITICAL FLUIDS}

Alan J. Russell

Eric J. Beckman

University of Pittsburgh

\section{SL: CRDEC, NRDEC}

SC: AROE, CRDEC

Biocatalytic transesterification of methylmethacrylate is possible in many different solvents. The reaction rate is readily controlled by variation in solvent physical properties. The reaction proceeds better in hydrophobic solvents, and activity can be restored in hydrophilic solvents by the addition of water. Supercritical carbon dioxide is not a good solvent for the 
reaction between 2-ethylhexanol and methylmethacrylate. It appears that the supercritical carbon dioxide may either alter the $\mathrm{pH}$ of the microaqueous environment associated with the protein, or reversibly form covalent complexes free amine groups on the surface of the enzyme. Although supercritical carbon dioxide is a poor solvent for acrylate transesterification, many other supercritical fluids (ethane, ethylene, sulfur hexafluoride, and fluoroform) are better than most conventional solvents. Using a set of standard equations, it has been possible to calculate the role of internal and external mass transfer in limiting the rate of enzyme-catalyzed reactions in anhydrous organic solvents and supercritical fluids. A manuscript has been prepared on the solubilization and activity of proteins in supercritical fluids. In summary, researchers have used microemulsion formation in pressurized gases to solubilize protein. The pressure of the system controls the formation of microemulsion, and drives the extraction process.

\section{Reports:}

1. Should the High Diffusivity of a Supercritical Fluid Increase the Rate of an Enzyme-Catalyzed Reaction?, by Alan J. Russell and Eric J. Beckman, MS, Enzyme Microb Technol.

2. Enzyme Activity in Supercritical Fluids, by Alan J. Russell and Ric J. Beckman, Appl Bioch Biotech 31,197(1991).

3. Effect of Hydration on the Morphology of Enzyme Powder, by Kristin Rozeiwski and Alan J. Russell, MS, Biotech and Bioeng.

4. Protein Extraction and Activity in Reverse Micelles of a Non-Ionic Detergent, by Guadalupe A. Ayala et al., MS, Biotech and Bioeng.

5. Biocatalytic Synthesis of Acrylates in Organic Solvents and Supercritical Fluids I. Optimization of Enzyme Environment, by Sanjay Kamat et al., MS, Biotech and Bioeng.

6. Role of Diffusion in Non-Aqueous Enzymology: 1. Theory, by Sanjay Kamat et al., MS, Enzyme Microb Technol.

\section{STRUCTURE-FUNCTION ASPECTS OF MEMBRANE ASSOCIATED PROKARYOTIC DNA REPLICATION}

William Firshein

Wesleyan University

\section{SC: NRDEC, WRAIR}

Continuing the studies with membrane associated prokaryotic DNA replication, substantial progress was made in cloning a gene which codes for a membrane associated protein that may act as a repressor of initiation of DNA replication in Bacillus subtilis. Using a lambda virus packing system, a $B$. subtilis genomic library was prepared and probed with an oligonucleotide coding for the first 13 amino acids of the purified protein. Seven positive clones were detected and attempts are being made to determine whether the protein is synthesized by these positive lambda clones in their Escherichia coli hosts. In another study, researchers have been using an origin plasmid (OriC) of $B$. subtilis constructed by Japanese colleagues to determine where it can be replicated in vitro using the membrane system developed in this laboratory. Two different approaches have been employed. One is to add the OriC plasmid exogenously to extracted membrane, while the second is to extract the membrane from cells transfected with $O$ riC plasmid. Thus far, DNA replication has been obtained under both conditions but the "exogenous" system exhibits more repair activity than the "endogenous" system. Attempts are being made to define conditions in which repair activity is suppressed in favor of initiation activity. Finally, in studies with the resistance plasmid RK2 cultured in E. coli, attempts are being made to determine whether replication of this plasmid occurs in association with the inner or outer membrane. It was previously shown that most of the plasmid encoded proteins including the initiation protein were present in the inner but not outer membrane. However, DNA synthesis can be detected with both membrane domains. Whether the synthesis is different in each domain still remains to be determined.

\section{Reports:}

1. The Temporal Expression of a Membrane-Associated Protein Putatively Involved in Repression of Initiation of DNA Replication in Bacillus-Subtilis, by Bethanie Eident-Wilkinson et al., MS, J Bacteriology.

\section{ELECTROPORATION OF BACTERIA AND YEAST: AN EXPERIMENTAL STUDY OF MOLECULAR TRANSPORT}

James C. Weaver

Arnold L. Demain

Massachusetts Institute of Technology

\section{SC: CRDEC, NRDEC}

Previous studies in the laboratory have shown that the electroporative uptake of molecules is heterogeneous, i.e., different cells take up different numbers of the desired molecule. In these studies, media with known concentrations of fluorescent molecules are used to suspend microorganisms of interest. Then, following one or more electric field pulses to cause electroporation, a waiting time for membrane recov- 
ery is used, and the cells then washed and analyzed by flow cytometry. By suitable calibration of the fluorescence, each cell is measured in terms of the number of fluorescent molecules taken up. A previous study investigated and developed a method for rapidly determinating the clonal growth of individual cells. In this method, individual microorganisms are incorporated into gel microdrops (GMDs). In work with microorganisms to date, GMDs about 20 to $40 \mu$ in diameter are formed from agarose. The GMDs are "diffusionally transparent", capable of gently confining cells upon replication, so that microcolonies are formed. The GMDs are also sufficiently small and robust that they can be handled much like cells, e.g., suspended, pipetted and centrifuged. GMDs can also be analyzed by flow cytometry, so that the distribution of microcolony size can be quickly determined, and the distribution of clonal growth within a subpopulation rapidly determined.

\section{SPIDER SILK STRUCTURE AND MECHANISMS OF FUNCTION}

Randolph V. Lewis

University of Wyoming

SL: NRDEC

SC: CRDEC

The sequencing has been completed of both strands of the second spider dragline silk protein (Spidroin 2). It was necessary to sequence both strands due to compression problems which created difficulties in accurate sequencing in certain regions. This protein has no relationship to any protein in the current protein or genetic databases. It consists of regions of poly-alanine as is present in the first protein (Spidroin 1) but the other region is a series of five amino acid repeats of Gly-Pro-Gly-Gln-Gln or GlyPro-Gly-Gly-Tyr. These regions are almost certainly a series of $\beta$-turns and the Ala regions are $\beta$-sheet. This varies greatly from what the structure of Spidroin 1 was originally thought to be but is consistent with some data from that protein as well. Interestingly despite the lack of similarity between Spidroins 1 and 2, there is a region in the C-terminal non-repeating region which is nearly identical in amino acid sequence in both. Thus, this region may play an important role in secretion, processing or assembly. Progress has been made on both cocoon silk and minor ampullate șilks. However, the clones are very unstable in plasmid vectors and thus sequencing has been difficult. Researchers have determined the water content of several spider silk fibers and are writing a manuscript about that. The bottom line is that the silk proteins are extremely dry. Progress in expression of the substantial quantities of the spider silk protein clones continues to be a problem. The inserts are unstable which leads to low levels of expression.

\section{CLONING OF CDNA FOR TWO GLUTAMATE RECEPTOR PROTEINS}

Elias K. Michaelis

Mary K. Michaelis

University of Kansas

Two proteins were isolated which appear to be part of the glutamate/NMDA receptor-ion channel complex, a glutamate binding protein of $\sim 70 \mathrm{kDa}$ and a CPP binding protein of $\sim 58 \mathrm{kDa}$ protein. Two smaller proteins were detected co-purifying with the glutamate and CPP binding proteins (molecular masses of 42 and $36 \mathrm{kDa}$ ). When all four proteins (the $70,58,42$, and $36 \mathrm{kDa}$ bands) are present in isolated fractions, one can demonstrate all the expected activities of the NMDA receptor-ion channel complex, i.e., ligand binding for NMDA, glutamate, glycine, PCP, Mk801, CPP, and the polyamines. In addition, even the most highly purified preparations of these proteins exhibit ion channel characteristics when they are reconstituted into planar lipid bilayers. Researchers have cloned and sequenced a full-length cDNA for the largest subunit of the NMDA receptor complex, and the glutamate-binding protein. The inferred sequence of the protein describes unique protein structure with four transmembrane domains. The protein expressed in $E$. Coli reacts with the antibodies raised against the brain glutamate-binding proteins, binds L-glutamate with the same affinity and specific activity as the brain protein, and has been purified by glutamate affinity chromatography. The cDNA for this protein has been used to screen additional cDNA libraries. The original clone for the glutamate binding protein was inserted into a baculovirus and is currently being stably expressed in the insect cells of Spodoptera frugipeda. Primary neuronal cultures and sophisticated fluorescence ratio imaging techniques were developed to monitor the activity of NMDA receptors. These methods are used to explore the correlation between the expression of the glutamate binding protein and the sensitivity of the cells to NMDA receptor agonists. 
28680 MECHANISM OF ACTION OF BACTERIOPHAGE T4 TRANSLATIONAL REPRESSOR REGA PROTEIN

Eleanor K. Spicer

Yale University

\section{SL: NRDEC}

The bacteriophage $T 4$ regA protein $\left(M_{r}=14,600\right)$ is a translational repressor of a group of $\mathrm{T} 4$ early mRNAs. To identify a domain of regA protein that is involved in nucleic acid binding, ultraviolet light was used to photochemically crosslink regA protein to $\left[{ }^{32} \mathrm{P}\right] \mathrm{p}(\mathrm{dT})_{16}$. The crosslinked complex was subsequently digested with trypsin and peptides were purified using anion-exchange HPLC. Two tryptic peptides crosslinked to $\left[{ }^{32} \mathrm{P}\right] \mathrm{p}(\mathrm{dT})_{16}$ were isolated. Gas-phase sequencing of the major cross-linked peptide yielded the following sequence: VISXKQKHEWK, which corresponds to residues 103-113 of regA protein. Phenylalanine 106 was identified as the site of crosslinking, thus placing this residue at the interface of the regA protein:p(dT) ${ }_{16}$ complex. The minor crosslinked peptide corresponded to residues 31-41 and the site of crosslinking in the peptide was tentatively assigned to Csy 36 . The RNA binding domain of regA protein was further examined by chemical cleavage of regA protein into six peptides using $\mathrm{CNBr}$. Peptide $\mathrm{CN6}$, which extends from residue 95 to 122 , retains both the ability to crosslink to $\left[{ }^{32} \mathrm{P}\right] \mathrm{p}(\mathrm{dT}){ }_{16}$ and the non-specific binding characteristics of the intact protein. However, peptide $\mathrm{CN} 6$ does not exhibit the binding specificity of the intact protein. Three of the other individual $\mathrm{CNBr}$ peptides have no measurable affinity for RNA, as assayed by photo-crosslinking or gel mobility shifts.

28699 ENZYME DESIGN FOR NONAQUEOUS MEDIA: OPTIMIZATION OF ENZYMATIC CATALYSIS FOR ORGANIC SOLVENT SYSTEMS

Jonathan S. Dordick

Douglas S. Clark

University of Iowa

SL: NRDEC

SC: CRDEC

The catalytic efficiency of enzymes have been improved in organic solvents through the use of protein engineering. Specifically, increasing the polarity of the active site of subtilisin BPN' increases the relative activity of the mutant enzyme compared to the native enzyme. In aqueous buffer, however, the mutations led to diminished catalytic function. One of the polar mutants (M222Q) has been studied by electron paramagnetic (EPR) spectroscopy. Evidence indicated the presence of an extra water molecule on the Q222 as compared to the wild-type enzyme and this greater degree of hydration may result in the observed increase in enzymatic activity in anhydrous organic solvents.

\section{Reports:}

1. Solvent Dielectric Effects on Protein Dynamics, by Rhett Affleck et al., MS, Proc Natl Acad Sci.

2. Engineering Subtilisin for the Use in Organic Solvents, by Zu-Feng Xu et al., MS, Ann NY Acad Sci.

\section{NOVEL APPROACHES TO THE CHARACTERIZATION OF SPECIFIC PROTEIN-PROTEIN INTERACTION IMPORTANCE IN GENE EXPRESSION}

Ronald L. Somerville

Purdue University

Work has continued on characterizing by genetic, biochemical and physiological means the TrbA protein of Escherichia coli. The Trb notation refers to a Trp repressor binding species. This protein (198 amino acids) was earlier identified and purified on the basis of its reactivity toward anti-idiotype antibodies that were elicited in rabbits in response to injections of Trp repressor. The TrbA protein, purified to homogeneity from extracts of an engineered overproduction strain of $E$. coli, was found to enhance the formation and/or stability of specific complexes between the Trp holorepressor and its cognate operator. A null mutation within the trbA gene was constructed via in vitro manipulation of cloned DNA, then introduced into the chromosome of a haploid strain of $E$. coli. The resulting trbA mutants were indistinguishable from wild-type strains with respect to long-term survival (two weeks) in rich medium, but had new nutritional requirements that are presently under investigation. Repression of transcription at the trp promoter during the entry of cells into stationary phase was evaluated in wild-type cells and trbA mutants. The trp promoter was shut down 2-4 fold more efficiently in trbA ${ }^{+}$cells than in their mutant counterparts. The current model for the TrbA protein plays an important role in the establishment and maintenance of stationary phase physiology by medi- 
ating the efficient "lockdown" of the trp promoter and possibly other promoters.

\section{INTERACTING SITES IN NOVEL POLYMERIC PROTEINS}

Steven T. Case

University of Mississippi Medical Center

\section{SL: NRDEC}

A challenge in this research is to synthesize genes encoding monomeric [C+SR] and multimeric $[\mathrm{C}+\mathrm{SR}] n$ repeats from spIa that can be expressed at high levels in bacterial cells. It can be reported that researchers have several cell lines which, upon appropriate induction, apparently express a recombinant spIa $[\mathrm{C}+\mathrm{SR}]$ monomer. While conditions of expression have not yet been optimized, nearly $1 \mathrm{mg}$ spIa $[\mathrm{C}+\mathrm{SR}]$ monomer $/ 10 \mathrm{ml}$ of bacterial culture has been obtained. The identity of the recombinant protein has been confirmed by directly sequencing the first 30 amino acid residues. Additional $\mathrm{C}$ and $\mathrm{SR}$ peptides have been synthesized and await purification. Progress in this section will accelerate when recombinant $[\mathrm{C}+\mathrm{SR}]$ proteins have been purified to homogeneity.

Reports:

1. Secretory Proteins of Chironomus Salivary Glands: Structural Motifs and Assembly Characteristics of a Novel Biopolymer, by Steven T. Case and Lars Wieslander, MS.

\section{COFACTOR-ASSISTED ANTIBODY CATALYSIS}

\section{Donald Hilvert}

The Scripps Research Institute

Arylseleninic acids catalyze the oxidation of olefins, sulfides and other molecules by hydrogen peroxide. To generate arylseleninic acid dependent antibody catalysts researchers prepared hapten 1 , which resembles the putative transition state for attack of an ethyl aryl sulfide on an aryl perseleninic acid. The thyroglobulin conjugate of 1 was used to elicit a strong immune response in mice. Twenty hybridomas that secrete anti-1 antibodies have been identified from a single fusion and are currently being propagated in mouse ascites. Screening for catalysts will commence once the monoclonals have been purified to homogeneity. Preliminary experiments indicate that analytical HPLC will be the method of choice for assaying the oxidation of sulfide to sulfoxide. Attempts to develop alternate methods that are both simple and reliable for monitoring the kinetics of this reaction, including spectroscopic differentiation of substrates and products and/or their complexes with $\mathrm{Fe}(\mathrm{II}), \mathrm{Mn}$ (II) and $\mathrm{Ni}(\mathrm{II})$ ions, have not yet been successful. The possibility of detecting sulfoxides through the formation of specific complexes with $\mathrm{Fe}$ (II)-pyrazine derivative is still under investigation.

\section{DEVELOPMENT OF A YEAST RNA VECTOR}

Michael J. Leibowitz

University of Medicine and Dentistry of New Jersey

\section{SL: ARL/EPSD \\ SC: CRDEC, NRDEC}

The research objective is to develop a vector system for the yeast Saccharomyces cerevisiae based on the cytoplasmic double-stranded RNA of killer virus of yeast. Transfection methods will be developed using both standard methods and some innovative methods of the principal investigator. The proposed vector will be tested and will eventually be improved to include many signal sequences.

Reports:

1. Gene Expression from Multicopy T7 Promoter Vectors Proceeds at Single Copy Rates in the Absence of T7RNA Polymerase, by Ronald L. Somerville et al., MS, Biochem and Biophys Res Commun.

\section{Defense Against Chemical and Biological Weapons}

\section{THE RAPID DETECTION OF BACTERIA AND OTHER MICROORGANISMS: APPLICATION OF UV RESONANCE RAMAN SPECTROSCOPY}

Wilfred $\mathbf{H}$. Nelson

University of Rhode Island

\section{SL: CRDEC, WRAIR}

SC: CRDEC

The sensitivity limits of the UV resonance Raman experiment for bacterial detection and identification have been investigated. For that purpose a speciallydesigned micro-Raman spectrometer capable of exciting single cells and small groups of cells selectively has been built. Spectra of single cells to date have been of uncertain quality even though nucleic acid Raman peaks can be detected reproducibly. This 
suggests that under certain conditions more than one cell needs to be excited if quality spectra are to be produced. However, considering the 3 percent throughput of the spectrograph used, conservatively, in practice it will be possible to increase the signal level by at least a factor of 10 without changing the optical system or the laser power. Consequently, it is clearly possible with present technology to detect single bacterial cells using UV resonance Raman spectroscopy. Furthermore, with additional instrument improvements it appears very likely that such spectra will be of high enough quality to allow the use of all resonance Raman information currently extracted from bulk samples. Hence, it may be possible to use such spectra for bacterial identification.

\section{Reports:}

No. $1-9$ in previous editions.

10. UV Resonance Raman Studies of Bacteria, by W.H. Nelson and R. Manoharan, MS, Appl Spectrosc.

11. UV Resonance Raman Spectra of Bacillus Spores, by E. Ghiamati et al., Appl Spectrosc 46,357(1992). AD A249 767

12. The Rapid Detection of Single Bacterial Cells by Deep UV Micro Raman Spectroscopy, by W.H. Nelson and J.F. Sperry, FR, Apr 92, 19pp. AD A249 811

\section{IDENTIFICATION OF CHEMICAL MARKERS FOR MICROORGANISMS BY PYROLYSIS GC-MS}

Stephen L. Morgan

Alvin Fox

Unjversity of South Carolina

\section{SL: CRDEC}

SC: CRDEC, NRDEC

This research seeks to identify the relevant biological fingerprints generated by pyrolysis that are capable of differentiating or characterizing important biological threats. The investigators will use a selected group of microorganisms, cell fractions, and model compounds, and will subject them to analytical pyrolysis followed by high resolution capillary gas chromatography-mass spectrometry to obtain information on relevant chemical markers for target agent identification.

\section{ROLE OF AXON-SCHWANN CELL INTERACTIONS IN NERVOUS SYSTEM IONIC HOMEOSTASIS}

Edward M. Lieberman

East Carolina University

\section{SC: CRDEC}

Researchers have continued the study of the role of glutamate in the signaling between axons and glia and have now shown that glutamate released from the axon during excitation activates a Schwann cell receptor of the quisqualate/kainate type. Receptor activation induces an increase in intracellular inositol phosphates leading to internal calcium release. The internal calcium causes the release of acetylcholine and activation of the previously reported Schwann cell membrane potential changes via a reduction in chloride conductance. Another major project is related to interests in metabolic properties of glia and in particular how glia and nerve interact in this regard. One project has dealt with the relationship between energy utilization by nerve and its associated glia. In keeping with the overall goal of the research program of this laboratory in the understanding of the role of the Schwann cell and glia in the nervous system in all animals, researchers searched for and began the development of an intact vertebrate nerveglial cell preparation which shows great promise as a model of the role of the Schwann (glial) cell at the synaptic junction.

Reports:

No. $1-3$ in previous editions.

4. Determination of the Membrane Potential of Cultured Mammalian Schwann Cells and Its Sensitivity to Potassium Using a Thiocarbocyanine Fluorescent Dye, by P.T. Hargittai et al., GLLA 4,611(1991). AD A249 476

5. Mechanisms of Glutamate Activation of Axon-ToSchwann Cell Signal in the Squid, by Edward M. Lieberman and Eric Sanzenbacher, MS, Neurosci.

6. Role of Glutamate in Axon-Schwann Cell Signal in the Squid, by Edward M. Lieberman, MS, Ann NY Acad Sci.

\section{EXPRESSION CLONING OF THE HIGH AFFINITY CHOLINE TRANSPORTER}

Cameron B. Gundersen

University of California, Los Angeles

\section{SC: CRDEC}

A paper has been submitted to NEURON that describes in detail findings concerning a small protein that appears to be essential for the expression in Xenopus oocytes of N-type, omega-conotoxin-sensitive calcium channels. The cDNA clone is designated $\mathrm{CCCS}_{1}$ for candidate calcium channel subunit1. Current interest is in a recent paper that was published 
in Science by Williams et al., in which they present evidence that a novel alpha1 sub-unit cDNA is sufficient for the expression of $\mathrm{N}$-type calcium channels in human embryonic kidney cells (HEK 293 cells). Since the data for $\mathrm{CCCS}_{1}$ in oocytes imply that $\mathrm{CCCS}_{1}$ is essential for the expression in cells of $\mathrm{N}$ type calcium channels from Torpedo, either the $\mathrm{N}$ type calcium channel that Williams and co-workers are studying is fundamentally different from the fish protein or perhaps the HEK293 cells express a $\mathrm{CCCS}_{1}$ homolog. Efforts are being made to resolve these questions.

\section{ELECTROSTATIC CONTROL OF ACETYLCHOLINESTERASE REACTIVITY}

Harvey A. Berman

State University of New York at Buffalo

\section{SL: NRDEC}

SC: NRDEC

Research addressed two salient features of AchE concerning interaction of the peripheral anionic site with the active center. The first feature notes that the kinetics of substrate hydrolysis are essentially independent of ionic composition of the surrounding medium. Such characteristics stand in contrast to the marked ionic strength dependence of ligand occupation of the peripheral anionic site and the active center. Second, the capacity of peripheral site ligands to alter substrate hydrolysis is critically dependent on the electrostatic nature of the substrate. That is, peripheral site ligands cause linear inhibition of hydrolysis of cationic substrates, nonlinear inhibition of uncharged substrates, and antagonize inhibition by uncharged methylphosphonates only incompletely. On this basis, it is concluded that the peripheral anionic site interacts with the active center principally through an electrostatic mechanism. The relationship between ionic strength and the near-constancy of substrate turnover have been documented. Research examined the influence of ionic composition of the bulk medium on AchE conformation. Enzyme conformation was assessed through utilization of (NBD)aminoalkyl methylphosphonofluoridates. The findings indicate that while substrate turnover is invariant with ionic composition of the medium, fluorescence intensity of the (NBD)aminoalkyl methylphosphonyl reporter ligands tethered within the active center undergo measurable changes with subtle changes in ionic composition of the medium. These findings nicely substantiate the view that one functional role for the peripheral anionic site is to maintain the high catalytic efficiency of substrate turnover, compatible with correspondingly efficient termination of neuromuscular transmission. An electrostatic interaction between discrete loci on the enzyme subunit plays one role in mediating such a function.

Reports:

No. $1-3$ in previous editions.

4. Function of the Peripheral Anionic Site ofAcetylcholinesterase, by Harvey Alan Berfman et al., Cholinesterases: Structure, Function, Mechanism, Genetics, and Cell Biology, 1991, p229. AD A249 082

5. Fluorescence Studies on the Interactions of Myelin Basic Protein in Electrolyte Solutions, by Mark W. Nowak and Harvey Alan Berman, Biochem 30,7642(1991). AD A249 083

6. Interaction of Tetrahydroaminoacridine with Acetylcholinesterase and Butyrylcholinesterase, by Harvey Alan Berman and Kathryn Leonard, Mol Pharm 41,412(1992). AD A249 081

\section{THE GENETIC AND BIOCHEMICAL MANIPULATION OF A BROAD-SPECTRUM ORGANOPHOSPHATE DEGRADING SYSTEM}

James R. Wild

Frank M. Raushel

Texas A \& M University

$$
\text { SL: NRDEC }
$$

SC: CRDEC

Extensive biochemical research into the physical characteristics of the phosphotriesterase expressed from the bacterial opd gene has been used to define its enzymatic mechanism and its capacity to hydrolyze a wide-spectrum of OP-thiolesters and triesters (P-O bond cleavage) as well as OP-based nerve agents (P-F bond breakage). Subsequent studies have demonstrated that the hydrolytic capacity of this enzyme for phosphotriesters is extremely efficient and approaches diffusion-limited catalysis. The ability to genetically manipulate the opd cistron has resulted in the initiation of site-directed mutageneisis oriented toward affecting intracellular membrane trafficking. The post-translational modification of the enzyme has been manipulated by the introduction of specific amino acid substitutions in the signal sequence in order to alter the stability and protein processing of the enzyme. It has been possible to expand the use of nylon-immobilized enzyme preparations to provide a practical method for detoxification of organophosphate pesticides. 
Reports:

No. 1-3 in previous editions.

4. Mechanism-Based Inactivation of a Bacterial Phosphotriesterase by an Alkynyl Phosphate Ester, by Jeffrey N. Blankenship et al., J Am Chem Soc 113,8560(1991). AD A249566

\section{ENZYME DECONTAMINATION OF O-P TOXINS}

Michael Syvanen

University of California, Davis

SL: CRDEC, NRDEC

SC: CRDEC

Researchers have described the cloning and partial sequencing of three new glutathione transferases (GST) from the housefly. Because these new clones were all missing sequences at their $5^{\prime}$ end, they had to go back and construct another phage $\lambda$-cDNA bank from housefly transcripts. This time they size fractionated the cDNA product to ensure that they would have full length GST copies. They have established $(a)$ that they now have the complete nucleotide sequence for MdGST2 and MdGST3 and (b) that MdGST3 is the most likely candidate for enzyme responsible for detoxifying the organophosphate triesters neurotoxins. The evidence supporting the conclusion in point $(b)$ is based on molecular genetic analysis of the housefly Cornell-R mutants. CornellHR is resistant to OP triesters because it overproduces a GST (the $\mathrm{OP}^{\mathrm{R}}$ GST). Cornell-R* is a sensitive revertant to $O P$ sensitivity that also produces less GST activity than does Cornell-HR. They have now shown that MdGST3 transcripts are overproduced in Cornell-HR relative to Cornell- $R^{*}$. No such correlation in transcript level with OP resistance is observed for the MdGST1, MdGST2, and MdGST4 genes.

Reports:

1. Changes in House Fly Glutathione $S$-Transferase Transcripts After Induction with Phenobarbital or Sodium Barbital, by Steven McCommas et al., MS.

2. DNA Twist is a Transcriptional Sensor for Environmental Changes, by Jian-Ying Wang and Michael Syvanen, MS, Mol Microbiol.

3. Effect of Recombination on the MtDNA Clock Within a Species, by Michael Syvanen, MS, J Mol Evol.

\section{SPECIFIC PATHOGEN DETECTION IN $2.5 \mathrm{M}$ GUSCN}

David Gillespie

Hahnemann University

\section{SL: CRDEC, WRAIR \\ SC: CRDEC}

The proposed sequence detection system requires a capture nucleic acid which is immobilized on a plastic surface. Originally, researchers proposed to use plastic with streptavidin covalently attached, to which a biotin-conjugated capture probe would be attached. However, they learned that the density of immobilized streptavidin was too low to be useful in the proposed system. A novel system was devised for covalently attaching the capture nucleic acid directly to a plastic surface. It was learned that diethylpyrocarbonate (DEPC) unexpectedly stimulated the binding of RNA to a modified butadienepolystyrene surface. However, poly(U) bound poorly to this surface and, in any event, the modification process was a proprietary one to Xenopore, Inc. Subsequently, a plastic surface with available imino groups was obtained that bound poly(U) well in the presence of DEPC. This plastic surface is available but is expensive. Poly $(U)$ bound to this surface resisted heating in chaotropic salt or strong detergent and resisted incubation in concentrated salt. Therefore, the binding was neither hydrophobic nor electrostatic and was probably covalent. The bound RNA was sensitive to RNase and consequently can be expected to be available for immobilization by molecular hybridization with poly(dA)-containing capture probes. On the order of $1 \mu \mathrm{g}$ of poly(U) per $\mathrm{cm}^{2}$ of plastic could be bound, which is a capacity that is suitable for the proposed research.

\section{IDENTIFICATION OF BIOMARKERS IN BACTERIA BY PYROLYSIS TANDEM MASS SPECTROMETRY}

Kent J. Voorhees

Colorado School of Mines

\section{SL: CRDEC}

The work on this project has concentrated on the thermal decomposition of oligopeptides. Eight peptides have been studied by Curie-point tandem mass spectrometry (Py-MS/MS). These are PHE-LEUMET; PHE-MET-LEU; MET-LEU-PHE; ALAPHE-LEU-MET; ALA-MET-LEU-PHE; ALA-PHE-LEU-MET-TYR; ALA-TYR-LEUMET-PHE; and ALA-TYR-LEU-MET-PHEPHE. Each of these compounds has been pyrolyzed at $510^{\circ} \mathrm{C}$ and the major mass spectral peaks identified 
by electron ionization tandem mass spectrometry. Based on previous results the data have been interpreted mechanistically. The following conclusions have been formulated: $(a)$ mass spectral molecular ions are observed with tripeptides, nothing for the higher peptides; (b) all compounds studied were observed to decompose by a similar mechanism involving cyclization to form a diketopiperazine (DKP) as was observed for the dipeptides; ( $c$ ) for even numbered oligopeptides, the possible DKPs are formed with no single amino acids identified; (d) for odd numbered oligopeptides, the possible DKPs are formed with the C-terminal amino acid being observed as a free amino acid not associated with any DKP; and (e) decomposition of the DKPs follow the same sequence as was observed for the dipeptides.

\section{COUPLING OF BRAN MUSCARINIC RECEPTORS TO SECOND MESSENGERS}

Esam E. El-Fakahany

University of Minnesota, Minneapolis

Researchers studied the selectivity of a series of muscarinic cholinergic agonists in activating phosphoinositide (PI) hydrolysis. Chinese hamster ovary cells transfected with the genes of muscarinic receptor subtypes $\mathrm{m} 1, \mathrm{~m} 3$ and $\mathrm{m} 5$ were used as a test system. Pilocarpine was more efficacious at $\mathrm{m} 1$ as compared to $\mathrm{m} 3$ and $\mathrm{m} 5$ receptors.

\section{E. Sensory Factors in Performance Enhancement}

\section{DIFFERENTIAL GENE EXPRESSION IN MAMMALIAN LIVER DURING HIBERNATION}

Sandra L. Martin

University of Colorado, Health Science Center

SC: NRDEC

Mammalian hibernators experience dramatic reductions in body temperature, metabolic rate, respiratory rate, and heart rate during hibernation. These changes are precisely controlled and reversible with only internally driven mechanisms, suggesting specific biochemical regulation. A model was presented that integrates observations of differential liver gene expression during preparation for, and maintenance of, the hibernating state, with the known phylogenetic interspersion of hibernating species in several major mammalian lineages. This model predicts a major role for the differential expression of existing mammalian genes in the biochemical regulation of hibernation.

\section{F. Optimization of Physical Principles in Biological Systems}

\section{PRIMARY EVENTS IN OLFACTORY RECEPTION}

Robert R.H. Anholt

Duke University Medical Center

\section{SC: CRDEC, NRDEC}

Studies have continued on olfactomedin, a recently discovered olfactory glycoprotein. Olfactomedin is produced by sustentacular cells and submucosal glands of the olfactory epithelium and is the major component of the lower mucus layer adjacent to the chemosensory membrane. Researchers have invested considerable effort in isolating cDNAs which encode olfactomedin from a cDNA library of frog olfactory tissue. In order to generate suitable oligonucleotide or antibody probes for the identification of such clones, they have confirmed and extended previous analysis of the $\mathrm{N}$-terminal sequence of the purified protein by conventional protein sequencing methods. The sequence of the first 21 amino acids is FVAQXATGILAGKDHDVXEDL. The fifth and eighteenth amino acids could not be identified. Since the first amino acid is phenylalanine rather than methionine, it is evident that olfactomedin like other secretory protein is synthesized with a leader sequence. Moreover, the N-terminal amino acid sequence of olfactomedin shows no homologies to any other known polypeptides, which indicates that olfactomedin's sequence may be unique.

Reports:

No. 1 in previous edition.

2. Molecular Aspects of Olfaction, by Robert R.H. Anholt, MS.

3. Odor Recognition and Olfactory Transduction: The New Frontier, by Robert R.H. Anholt, Chem Senses 16,421(1991). AD A249 111

4. Olfaction, Olfactomedin and the Dynamic Range of Smell, by Robert R.H. Anholt et al., MS.

5. Cyclic Nucleotide-Activated Channels, by Anna Menini and R.H. Anholt, MS. 


\section{FUNCTIONAL RECONSTITUTION OF OLFACTORY RECEPTOR FOR ANALYTICAL APPLICATION}

Vitali Vodyanoy

Auburn University

SL: AMRICD

SC: CRDEC

This research is concerned with the functional reconstitution of chemosensitive receptors from olfactory epithelium of the rat and also with study of olfaction associated ion transport molecular mechanisms. Studies are being made of the interactions of the odorants, (+) and (-)-carvone (5-isopropenyl-2methyl-2-cyclohexenone), with L-a-1,2-dipalmitoylsn-glycerol-3-phosphocholine (L-DPPC) monolayers. Data indicate that the molecular area occupied by the L-DPPC monolayer becomes larger when the monolayer spread on the subphase containing (-)-carvone, and, in contrast, it becomes smaller when the monolayer is exposed to $(+)$-carvone. Both spreading and condensing effects are dependent on temperature. L-DPPC monolayers absorb twice as much heat as those with $(+)$-carvone when compressed at $30^{\circ} \mathrm{C}$. Under the same conditions, monolayers with (-)-carvone undergo a larger entropy change than monolayers with $(+)$-carbone. Variations in molecular areas and thermodynamic properties may contribute to membrane recognition of optical isomers. Researchers have developed and have built a new set up for measurements of ultra small currents in membrane vesicles. It has been demonstrated that purified AMPA receptors can be functionally reconstituted in phospholipid bilayers, they can be specifically agonized by kinate and AMPA and can be inhibited by 6,7-dinitroquinoxaline-2,3-dione (DNQX). The activity mediated by AMPA can be significantly enhanced by the nootropic compound aniracetam.

Reports:

No. $1-4$ in previous editions.

5. Chiral Recognition of Odorants + - and --Carvone by Phospholipid Monolayers, by Suram Pathirana et al., J Am Chem Soc 114,1404(1992). AD A251 509

28775 LIGANDS FOR STRETCH-ACTIVATED ION CHANNELS
Frederick Sachs

State University of New York at Buffalo

The most important finding is that researchers believe they have found a spider venom that has a component of about $30 \mathrm{kD}$ capable of blocking stretch-activated ion channels at less than $\mathrm{nM}$ concentrations. They have submitted a patent disclosure on the toxin to the university. A fluorescence assay was developed for the toxin using the elevation of calcium that follows hypotonic stress in $\mathrm{GH} 3$ cells. The toxin blocks this elevation of calcium. It also blocks stretch activated ion channels in patch clamp experiments on heart cells and oocytes.

\section{SYNAPTIC PLASTICITY IN VISUAL CORTEX: FROM SYSTEM PROPERTIES TO MEMBRANES AND RECEPTORS}

Leon N. Cooper

Brown University

An important consideration in modeling synaptic plasticity in the visual cortex is how to represent the sensory input which is converging on straite cortex from the LGN. In the past, a simplified representation of visual input has made the analysis of the behavior of the model less cumbersome. The input to the network of BCM neurons consisted of a set of non-orthogonal vectors. This set of vectors represented the visual presentation of a contrast border to the network, and each vector represented different orientation. Vectors representing adjacent orientations overlapped more than vectors whose orientations were far apart. Current work extends this representation of visual input. A model of the early retinal signal processing is used to create the vectors. The visual input to the network is no longer constrained to the one dimensional abstract patterned input. The retinal model produces two dimensional patterns which present the activity of the ganglion cells distributed across the retina. In this model the cells are arranged in a hexagonal array which represents their spacing across the retina. The receptive fields of the ganglion cells are either symmetric oncenter, off-surround or off-center, on-surround. The weighting of the center and surround areas is balanced so that an increase in overall illumination of the receptive field does not change the activity of the cell. The new input vectors are simply generated by presenting the retinal model with a bar of light with random orientations. The activity of the ganglion cells is the new input to the cortical model. One 
benefit of using the new input is in the number of ways the visual stimulus can be manipulated. If the bar of light is swept across the visual field, instead of being statically flashed, the effects of motion can be examined. Also, the receptive fields of the neurons in the cortical model can be mapped using a discrete spot of light. The properties of the new model relate much more closely to the properties of visual system in animals which are used for the biological experiments the researchers are considering.

\section{DEVELOPMENT AND APPLICATION OF LOW TEMPERATURE AFM}

Jie Yang

University of Virginia

\section{SL: CRDEC, NRDEC}

The design of the low temperature atomic force microscope cryostat and the liquid nitrogen buffer dewar has been completed. These two most important parts of this project are now being fabricated. Various electronics components have been tested at liquid nitrogen temperature, including laser diode and driver, pre-amplifiers and photodetector, and various mechanical parts, including piezo scanner, mounting gears and metal components. To facilitate current experiments and gain sufficient experience on various aspects of atomic force microscope, an x-y motion stage was built for the AFM to improve the very limited scan range (from $12 \mu \mathrm{m}$ to $3 \mathrm{~mm}$ ). Red blood cells were studied by atomic force microscopy in air and extended using a home made fluid cell in HEPES (pH 7.3) with the softest cantilever $(k=0.06$ $\mathrm{N} / \mathrm{m}$, while the stiffest one has a $k$ of $0.58 \mathrm{~N} / \mathrm{m}$ ). It was found that, in a dried condition, the height of the red blood cell was only about half of the value in a buffer solution, although the general shape and lateral dimensions are correct. A 2 percent glutaraldehyde fixation is useful to facilitate cell adhesion to the glass substrate, but fails to improve cell rigidity at high resolution. The surface resolution of the whole cell has been found to be about $10 \mathrm{~nm}$, not sufficient to make positive identification with the abundant transmembrane band 3 protein. AFM was used to study ionic salt crystals: $\mathrm{NaCl}, \mathrm{NaNO}_{3}$, and $\mathrm{NaClO}_{3}$. Atomic resolution images of all above salts have been obtained, yet it has been found that the structures observed by AFM only correspond to one charge component of the salt. Polysterene beads of various sizes have been imaged by AFM in air. AFM images of these beads have shown that a concentration in the range of 0.1 percent in weight tends to facilitate the formation of two dimensional crystals of these beads on mica when dried in air. Beads of diameter larger than $100 \mathrm{~nm}$ readily form two dimensional crystals with a hexagonal packing. Cubic packing sometimes also appears at the edge of the crystal. Since these 2-d crystals can be relatively stable, it should be possible to couple other materials to the surface of the beads. Therefore, they can be used as an intermediate substrate for the study of proteins. Various schemes are being tested for this purpose.

Reports:

1. Fractional Power Law Concentration Dependence of the Freezing Temperature in Dipolar Systems, by Jie Yang, MS, Phys Rev.

2. Atomic Force Microscopy of DNA Molecules, by Jie Yang et al., FEBS 301,173(1992).

\section{MOLECULAR PROBES OF GATING AND OPEN CHANNEL CONFORMATIONAL TRANSITIONS OF MECHANOSENSITIVE ION CHANNELS}

Jeffry B. Lansman

University of California, San Francisco

An investigation was made of the action of aminoglycoside antibiotics as probes of the transitions that give rise to the formation of L-type and mechanosensitive $\mathrm{Ca}^{2+}$ channels. The goal of this work was to evaluate the role of aminoglycosides as possible structural probes of mechanosensitive channel function. To understand the general mechanisms by which this compound inhibits ion channels, studies were made of the interaction of various aminoglycosides that varied in net charge on L-type $\mathrm{Ca}^{2+}$ channels and mechanosensitive ion channels in intact muscle fibers isolated from mice. The use of intact fibers circumvents problems associated with the variability of channel activity in cultured cells and allows examination of both types of channels which are found in these cells. When aminoglycosides were added to a patch recording electrode, discrete transitions between the open and closed channel levels were observed in recordings of the activity of single L-type channels. This behavior resembled classic open channel block in its concentration dependence, but otherwise had features inconsistent with a model in which the drug enters and occludes the open channel. Analysis of the action of these compounds on mechanosensitive ion channels showed a novel dual inhibitory action in which the aminoglycosides produce a very rapid block of the open channel as well 
as fluctuations between the open and a subconductance level.

\section{CHROMOPHORE ATTACHMENT IN THE LIGHT-HARVESTING PROTEINS OF CYANOBACTERIA}

Lamont Anderson

University of Tulsa

The research objective is to define the structural determinants in one class of biliproteins that are required for proper chromophore attachment in vivo; examine structural differences at analogous chromophore sites in two different biliproteins, looking for factors that direct differential chromophore attachment. Research will utilize x-ray crystal structural information, biliprotein gene engineering and a cyanobacterial gene transfer system to examine the attachment of bilin chromophores to biliproteins during phycobilisome biosynthesis. 


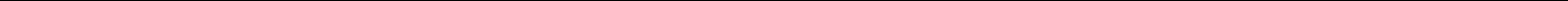




\section{MATHEMATICS}

\section{A. Applied Analysis and Physical Mathematics}

\section{NONLINEAR PROBLEMS AND NUMERICAL METHODS IN DIFFERENTIAL EQUATIONS AND APPLIED PHENOMENA}

H.B. Keller

California Institute of Technology

SC: ARL/WTD, ETL

A model for sharp fronts in glassy polymers was derived and analyzed. The major effect of a diffusing penetrant on the polymer entanglement network is taken to be the inducement of a differential viscoelastic stress. This couples diffusive and mechanical processes through a viscoelastic response where the strain depends upon the amount of penetrant present. Analytically, the major effect is to produce explicit delay terms via a relaxation parameter. This accounts for the fundamental difference between a polymer in its rubbery state and the polymer in its glassy state, namely the finite relaxation time in the glassy state due to slow response to changing conditions. Researchers adapted a recently proposed model for non-Fickian diffusion of penetrants into polymers and use it to study a drug-delivery problem. The model modifies Fick's diffusion equation by the addition of stress-induced flux. A stress evolution equation incorporating aspects of the Maxwell and Kelvin-Voight viscoelastic stress models completes the model. The relaxation time in the polymer is taken as a function of the penetrant concentration. The system is studied under the assumption that the diffusivity is large. Singular perturbation techniques are used to show that the concentration and stress evolve diffusively for small time, but exhibit steep fronts in a narrow region within the domain for larger time. These predictions are verified numerically for specified parameter values. Finally, the equations are studied in the steady state and are found to predict the evolution of shocks.

\section{STRESS SENSITIVITY OF DIELECTRIC RESONATORS}

Peter C.Y. Lee

Princeton University

\section{SL: ARL/EPSD}

A detailed report on the guided EM waves in infinite dielectric plates has been finished. First, the exact solutions, modes, dispersion relations from the three-dimensional Maxwell's equations are obtained for plates with $(a)$ perfect electric faces, $(b)$ perfect magnetic faces, and $(c)$ faces in contact with free space. These results give the important insight and understanding of the effect of boundary conditions on the propagation of waves. They are also needed as a basis for comparison in evaluating the accuracy of the approximate theories. Secondly, the 2-D governing equations are derived and presented, from which dispersion relations are obtained and compared with the "exact" ones. Finally, a uniqueness theorem of the solutions of the system of the 2-D equations is derived from which the initial and boundary conditons are obtained. Closed form solutions of the 2-D governing equations are obtained for the vibrations of circular disk dielectric resonators embedded in free space. Predicted results are compared with the experimental data by Cohn, Chow, and $Y e e$, and with the computational results by Chow and Yee. It may be seen that present predictions agree well overall with different sets of experimental data and for modes of various types and order.

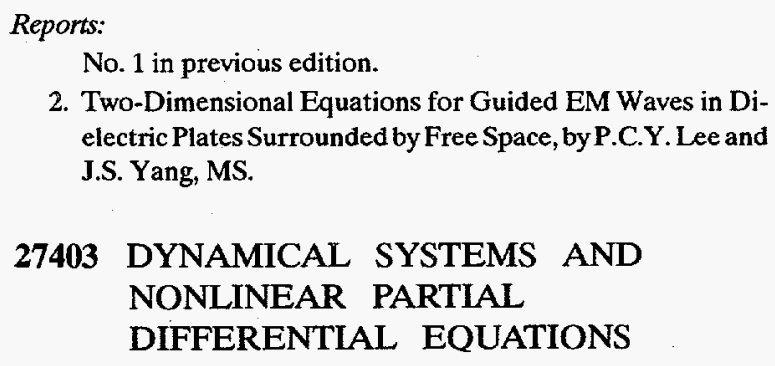

2. Two-Dimensional Equations for Guided EM Waves in Dielectric Plates Surrounded by Free Space, by P.C.Y. Lee and J.S. Yang, MS.

\section{DYNAMICAL SYSTEMS AND NONLINEAR PARTIAL DIFFERENTIAL EQUATIONS}




\section{C.M. Dafermos}

Brown University

SL: CRDEC

SC: MICOM

The formation of sawtooth-shaped patterns has been established in solutions of strictly hyperbolic, genuinely nonlinear systems of two conservation laws under periodic initial data. A study is being made of the spectrum of various time-periodic parabolic PDE's, in the spirit of Floquet theory. Work has continued on the weak formulation of propagating fronts and in particular on phase transitions and generalized motion by mean curvation, front propagation and phase field theory and uniqueness of rotationally symmetric surfaces moving by mean curvature. Other work has established the gain of regularity of dispersive waves, in a very general context.

Reports:

No. 1-3 in previous editions.

4. Gain of Regularity for Equations of KdV Type, by Walter Craig et al., MS.

5. Numerical Analysis. Convergence of MUSCL Type Method for Scalar Conservation Laws, by P.L. Lions and P.E. Souganiđis, MS.

6. Boundary Layer Phenomena for Differential-Delay Equations with State Dependent Time Lags, I, by John MalletParet and Roger D. Nussbaum, MS.

7. Front Propagation for Reaction-Diffusion Equations of Bistable Type, by G. Barles et al., MS.

\section{NUMERICAL METHODS FOR SINGULAR INTEGRAL EQUATIONS}

Ram P. Srivastav

State University of New York, Stony Brook

SL: CRDEC

SC: MICOM, ARL/MD

The analysis of the stress concentration in a layer, with a cylindrical cavity, which is bonded to a halfspace has been completed using Weber's integral transforms. It leads to a singular integral equation, which facilitates the analysis of the singular behavior. The researcher plans to present the results at the forthcoming SIAM Summer Meeting in the special session on Special Functions. Progress has been made towards determining the singular behavior numerically for Cauchy singular integral equations, when the coefficients are not constants. The constant coefficient case has been found to be amenable to the Stenger's formula for numerical integration, and Newton's method. The latter case was presented at the meeting of the Society for Engineering Science at University of Florida, Gainesville in November 1991.

\section{NONLINEAR MATHEMATICS APPLIED TO THE SCIENCE OF MATERIALS}

Morton E. Gurtin

William 0 . Williams

Carnegie-Mellon University

Work has concentrated on the study of material instabilities, such as phase transitions for fluids and alloys. A "blow-up" method was introduced that allows one to study lower semicontinuity properties of functionals on the space of Radon measures. Researchers have studied a simple model for the dynamics of fine structure. They characterized the creation and propagation of oscillations; the analysis relied on the theory of compensated compactness. A theory of deformable two-phase continua has been developed in which the two phases are solid, in which the interface supports stress, and in which the phase transition is either coherent or incoherent. Researchers have developed a dynamical theory of coarsening (Ostwald ripening). The theory is mechanical, involving diffusional mass transport and stress. They have also been developing a theory of highly nonequilibrium phase transitions. A systematic procedure for obtaining corrections to the standard measure of stress for classical continua that permits one to measure contact forces as if microfracture were not occurring. For elastic-plastic materials such as metals undergoing large deformations, where the microfracture occurs as microslip, this procedure yields a "corrected stress" that will be used in constitutive relations in plasticity and shows how the standard stress should depend upon natural measures of permanent deformation; for mixtures of continua such as bubbly liquids or mixtures of gases, where the microfracture represents dispersion at the microscopic level, the procedure yields a natural concept of partial stress, relates the partial stress to the standard measure of stress, and provides a consistence relation among the partial stresses for different constituents of the mixture.

\section{ANALYTICAL THEORY OF CONTINUED FRACTIONS AND TIME EVOLUTION IN MANY-BODY SYSTEMS}

\section{Howard Lee}

University of Georgia

The main progress has been in three areas: (a) Mechanisms of slow decay in the autocorrelation function. Using a microscopic model of magnetism (spin 
model), success was achieved in calculating the asymptotic behavior of the spin autocorrelation function exactly. From this work, one can extract the physical and mathematical mechanisms of slow decay. (b) Finite-temperature susceptibility. To understand the thermal behavior of a plasma gas at finite temperatures, an expression was derived for the temperature-dependent derived susceptibility. This expression shows how temperature dependence emerges from the zero-temperature susceptibility. (c) Correlation Inequalities. The susceptibilities are bounded functions. The origin of these bounds has been a subject of interest in mathematical physics as an application of convexity theory. Researchers have given the first analytic verification of the bounds on a model. The work also provides an alternative derivation of the bounds, when nonconvex functions are encountered.

\section{SOME PROBLEMS IN NONLINEAR ANALYSIS}

Paul H. Rabinowitz

University of Wisconsin - Madison

A paper has been prepared which describes some recent joint work with V. Coti Zelati on the existence of infinitely many "multibump" or homoclinic like solutions of a semilinear elliptic PDE on $\mathbf{R}^{n}$. There are 3 other pieces of work in progress. In one, researchers obtained the existence of generalized periodic orbits of prescribed energy for a class of singular Hamiltonian systems. These solutions are in fact collision orbits and pass through the singularity of the potential energy. The second piece of work in progress is a lengthy paper joint with F. Giannoni on the existence of homoclinic solutions of Hamiltonian systems on manifolds. The final piece of research in progress also involves some of the ideas of joint work with Coti Zelati but in a new setting where both homoclinic and heteroclinic solutions are involved. This is a fairly complicated situation but good progress is being made.

\section{Reports:}

No. 1 in previous edition.

2. Homoclinic Type Solutions for a Semilinear Elliptic PDE on $\mathbf{R}^{\mathbf{n}}$, by Vittorio Coti Zelati, MS.

3. On a Class of Nonlinear Schrödinger Equations, by Paul $\mathbf{H}$. Rabinowitz, MS.

27869

VISCOSITY SOLUTIONS OF FULLY NONLINEAR EQUATIONS
Michael G. Crandall

University of California, Santa Barbara

The research objective is to investigate problems involving fully nonlinear differential equations of first order in infinite dimensions and of second order of finite and infinite dimensions. The method of viscosity solutions incorporating variational inequalities and boundary controls will be used.

\section{STABILITY AND THERMAL INFLUENCES IN NONLINEAR CONTINUUM MECHANICS AND MATERIALS SCIENCE}

Morton E. Gurtin

Carnegie-Mellon University

\section{SC: ARL/MD}

Force and moment balance laws are derived for structured continua. The approach consists in the use of two hypotheses: the availability of a "microscope", mathematized by the introduction of a scale parameter $\varepsilon$; the existence of total and fine power expenses and the invariance of the corresponding power functionals in the limit $\varepsilon \rightarrow 0+$. When a phase is quenched into a metastable state, the late stages of the phase transformation process are usually characterized by a dissolution of second-phase domains with large interfacial curvature at the expense of domains with low interfacial curvature. This process, known as coarsening (Ostwald ripening), occurs in a wide variety of two-phase materials and has a significant impact on the high temperature stability of many technologically important materials. However, despite the wealth of experimental data showing that elastic stress has a profound influence on the morphological development of particles during ripening in solids, there has been little theoretical work on the effects of elastic stresses; in fact, there is no encompassing continuum mechanical framework that is dynamic in nature and of sufficient generality to study two-phase phenomena such as these, in which deformation and stress accompany diffusion. This framework is presented in a paper. For realistic interfacial energies the equations of anisotropic motion-by-curvature exhibit backward-parabolic behavior over portions of their domain, thereby inducing phenomena such as the formation of facets and wrinkles. A physically consistent regularized equation was derived that may be used to analyze such phenomena. 
28548 NONLINEAR WAVES IN MECHANICS AND GAS DYNAMICS

Tai-Ping Liu

Stanford University

A study is being made of the $\mathrm{H}$-theorem for hyperbolic conservation laws with relaxation. In a paper being written, researchers show that dissipative convex entropy functions exist only if the frozen characteristics interlace with the equilibrium characteristics. This relates the $\mathrm{H}$-theorem for the kinetic theory with the stability interion for the analogous nonlinear p.d.e. with relaxations. Researchers also perform the asymptotic expansion of the Chapman-Enskog type for the general system and show that the resulting parabolic system is well-posed if the entropy function is dissipative. For the $2 \times 2$ model proposed earlier by the author, an incompressible near-equilibrium limit can be derived and justified as the frozen characteristics tend to infinity and the relaxation time tends to zero. Currently, the researchers continue their study of time-asymptotic behavior viscous conservation laws. During these past months, a new approach in nonlinear analysis has been introduced which is more hyperbolic in nature and able to study the coupling of waves of different characteristic families more effectively. The approach has been applied successfully to MHD model with Freistuhler and to multi-phase model with Zumbrun.

Reports:

1. A Scalar Combustion Model, by Tai-Ping Liu and Tong Zhang, Arch Ratl Mech Anal 114,297(1991). AD A249 182

\section{THE STROH FORMALISM FOR ANISOTROPIC ELASTICITY WITH APPLICATIONS TO COMPOSITE MATERIALS}

T.C.T. Ting

University of Illinois, Chicago

Manuscripts have been submitted for publication. The first paper on anisotropic elastic semi-infinite strip deals with the stress decay in the strip. Aside from the physical importance of the problem, the authors consider various forms of boundary conditions which include the mixed boundary conditions as special cases. The Stroh formalism is generalized to accommodate general boundary conditions. A pleasant and surprising result is that the generalized version of the Stroh formalism is rather simple in appearance. Yet it applies to quite a wide range of boundary conditions. The paper on Green's functions offers an insightful look at the structure of Green's functions for anisotropic elastic media. It also provides the solution for the case when the material is degenerate, of which isotropic materials are a special case. Another paper is a review paper which is the content of an invited keynote speech at the Army Solid Mechanics Symposium.

Reports:

1. The Anisotropic Elastic Semi-Infinite Strip, by M.Z. Wang et al., MS, Quart Appl Math.

2. Synergism of Mechanics, Mathematics and Anisotropic Elastic Materials, by T.C.T. Ting, MS.

3. Anatomy of Green's Functions for Line Forces and Dislocations in Anisotropic Media and in Degenerate Materials, by T.C.T. Ting, MS, Physica Scripta.

4. The Motion of One-Component Surface Waves, by T.C.T. Ting, MS, J Mech Phys Sol.

5. Generalized Stroh Formalism for Anisotropic Elasticity for General Boundary Conditions, by T.C.T. Ting and M.Z. Wang, MS, Acta Mech Sinica.

\section{SYSTEMS OF HYPERBOLIC PARTIAL DIFFERENTIAL EQUATIONS}

Michael Shearer

North Carolina State University

Studies are being made of the formation of shear bands in elastoplastic materials. The mechanism of formation is that of change of type of the partial differential equations modeling the dynamic deformations. When the equations change type, stress localizes, and there is an associated unloading of the material adjacent to the shear band. Researchers formulated a model for this process, and proved an existence theorem for solutions of the equations, and provided the asymptotic form of the solution near the point and time of initiation of the shear band. This information is currently being incorporated into a computer code to simulate deformations that include shear bands. A paper has been prepared which describes the structure of solutions of Riemann problems for a simple model of phase transitions in elastic bars. The stress-strain law is approximated by the graph of a cubic function, for which one can perform explicit calculations. The structure of solutions includes nonuniqueness, a phenomenon that has some significance physically, but which can be removed using ad hoc ideas of nucleation due to Abeyarantne 
and Knowles. Similar calculations are used to analyze traveling wave solutions of the modified KdV-Burgers equation (in which the usual quadratic nonlinearity is replaced by a cubic). Numerical results confirm the unusual structure of solutions of Riemann problems, and their stability.

Reports:

1. Scale-Invariant Initial Value Problems in One Dimensional Dynamic Elastoplasticity, with Consequences for Multidimensional Nonassociative Plasticity, by David G. Schaeffer and Michael Shearer, MS, European J Appl Math.

2. Explicit Solutions of a Class of Riemann Problems of Mixed Type, by Yadong Yang, PhD Thesis, 1991, 128 pp.

\section{MATHEMATICAL ANALYSIS OF GASDYNAMIC WAVE INITIATION}

David R. Kassoy

University of Colorado

SC: ARL/WTD

Researchers have completed the formal verification of the Dold "ignition-kernel variable" grouping which gives a precise, accurate description of the final time solution profile for the reactive-diffusive self-ignition process. This description of the progress of self-ignition right up to the time when combustion ceases in the core of the hot spot predicts a process of reactive self-heating, very little hindered by diffusive losses. This leads to further asymptotic models appropriate for describing the final stages which determine the way in which self-ignition leads to the emergence of self-propagating flames. For physically important quasilinear heat equations with ignitionlike source terms, a class of perturbed models has been identified for which the blowing up self-similar behavior is similar. It was also discovered that this behavior can be much different than in the similinear case. Extension of the numerical study of detonation initiation and evolution in Sileem, Hayashi and Kassoy (1992) has shown that oscillatory relaxation to C.J.-type detonation wave propagation disappears when sufficient resolution is used in the MacCormack scheme, at least for the physico-chemical parameter used in the original study. New parameter ranges are being investigated with CRAY-based computations in order to develop "moving pictures" of the evolving detonation process.

\section{6}

\section{MATHEMATICAL ANALYSIS OF STRONG FLUID MECHANICAL EFFECTS IN REACTIVE AND NONREACTIVE FLOWS}

Andrew J. Majda

Princeton University

\section{SL: CRDEC \\ SC: ARO, ARL/WTD}

Research has been concerned with: (a) development of a new theory to explain transition to detonation in reactive granular materials (two papers, one recently published and one accepted); (b) development of first computational results which exhibit transition to fully developed turbulence in the wake of unstable detonation fronts (three papers, two accepted and one submitted); first asymptotic theories for nonlinear instability of anti-parallel vortex filaments and turbulence as well as theories for turbulent vortex filaments in a general background flow field (two papers, one accepted and one submitted); and development of higher order statistics in exactly solvable models for turbulent transport.

\section{THREE PROBLEMS IN FLUID DYNAMICS}

Daniel D. Joseph

Matthew Tirrell

University of Minnesota, St. Pau]

\section{SL: CRDEC}

Three problems are under study. The first is to determine the fluid dynamics of spin-up of two fluids in a rotating container. The spin-up can be convection or diffusion-dominated, depending on the viscosities of the liquids and other parameters of the two-fluid system. The applications of these studies are to the problem of stabilization of rotating liquid-filled artillery shells in free flight and to the determination of the rheological properties of blends of polymers used to fabricate plastic composites with improved material properties. The second problem is to study the position and motion of solid particles in a flowing viscous fluid by direct numerical simulation of the Navier-Stokes equations and the particles equations of motion. This is a kind of "molecular dynamics" based on correct equations which tell how a particle will fall in the hydrodynamic field created by its motion, like a falling bomb. The calculations give exact results for solid-liquid flow of up to four 
particles interacting with the flow and with each other. Two different working codes have been developed to do these problems. The third problem is to identify correct physical mechanisms to regularize ill-posed problems which arise in the study of twophase flows of bubbly mixtures and in spinodal regions of phase change mixtures of vapor bubbles in liquids. The idea is to apply a newly discovered method of regularizing ill-posed problems of fluidized suspensions of solids by taking into account the finite size of particles to the problem of change of phase in liquid-vapor flow.

\section{SOME PROBLEMS IN THE MECHANICS OF SOLIDS WITH PHASE MIXTURES}

Roger Fosdick

University of Minnesota, Minneapolis

\section{SL: ARL/WTD}

A paper has been prepared which involves non-convex minimization theory and the generation of microstructure under severe loads. Researchers have studied the regularity properties of a solution to an anti-plane shear minimization problem and determined the existence and general nature of those subregions of the body (bordered by free boundaries) which carry a highly discontinuous strain field.

\section{TRANSITIONS AND DEFECTS IN CRYSTALS}

Mitchell Luskin

Richard James

University of Minnesota, Minneapolis

\section{SL: ARL/WTD}

SC: ARL/MD

Work has continued on the microstructure and behavior of the highly magnetostrictive material $\mathrm{TbDyFe}_{2}$. One of the main conclusions of this work is that the growth twins in this material do not inhibit the magnetostriction, as was thought by most workers in this field. In this respect the work is having an impact on the thinking in this field, as several prominent researchers have changed their minds. It now appears that improvements in this material will be achieved by making the twins more regular, i.e., removing the waviness of the twin boundaries and converting grain boundaries into exact twin boundaries.
But the improvements will be limited with regard to the maximum achievable magnetostrictive strain. Researchers have done the first numerical computations for the dynamics of the development of twinned microstructure and the propagation of the austeniticmartensitic interface. The computations approximated a three-dimensional model for the dynamics of the indium-thallium alloy which used a stress tensor computed from the Ericksen-James energy density and which included viscous dissipation. One set of the computations were initialized by the macroscopic deformation for the mixture of two variants. The second set of computations were initialized by the deformation for the macroscopic deformation for the austenitic-martensitic interface. At a temperature below the transformation temperature, austenite was transformed into martensite near the interface and the interface moved into the austenitic region. At a temperature above the transformation temperature, martensite was transformed into austenite near the interface and the interface moved into the martensitic region. The stability of $x /|x|$ as a solution of the liquid crystal system is important to understand since it is both the simplest solution, the only universal solution, and yet it is unstable for a wide range of material constants.

\section{MATHEMATICAL PROBLEMS IN MICROMECHANICS AND COMPOSITE MATERIALS}

Robert V. Kohn

New York University Courant Institute of Mathematics

$$
\text { SC: BWL }
$$

A paper has been prepared in which the dynamic response of isotropic composites of two viscoelastic isotropic phases mixed in fixed proportions is considered in the frequency range where the acoustic wavelength is much larger than the inhomogeneities. The effective bulk-modulus bounds of Hashin-Shtrikman-Walpole are extended to viscoelasticity in this quasi-static regime, where the properties of the isotropic composite can be described by complex bulk and shear moduli. The effective bulk modulus is shown to be constrained to a lens-shaped region of the complex plane bounded by the outermost pair of four circular arcs (three circular arcs in the case of two-dimensional elasticity). This is proved using a new variational principle for viscoelasticity together with two established techniques for deriving bounds on effective moduli, namely the translation method and the Hashin-Shtrikman method. In this applica- 
tion the Hashin-Shtrikman method needs to be generalized to allow the reference tensor to have an associated quasiconvex energy. Microstructures are identified which have bulk-moduli that correspond to various points on each of the circular arcs. Thus these microstructures have extremal viscoelastic behavior when the associated arc forms one of the outermost pairs. The bounds and the extremal microstructures are similar to those obtained for the complex dielectric constant, but the methods used here are entirely different. Additional results appear in the reports listed below.

Reports:

1. Renormalization Theory for Eddy-Diffusivity in Turbulent Transport, by Marco Avellaneda and Andrew J. Majda, MS, Phys Rev Let.

2. Invariant Properties of the Stress in Plane Elasticity and Equivalent Classes of Composites, by Andrei Cherkaev et al., MS, Proc Roy Soc (London).

3. Cross-Property Relations for Transport in Porous Media: Rigorus Link Between Fluid Permeability, Conductivity and Relaxation Times, by S. Torquato and M. Avellaneda, MS.

4. Effective Medium Theories for Piezoelectric Composites, by Marco Avellaneda and Tamara Oison, MS.

5. Extremal Microstructures for Composite Materials, by Robert V. Kohn and Graeme W. Milton, MS.

6. Branching of Twins Near an Austenite/Twinned-Martensite Interface, by Robert V. Kohn and Stefan Muller, MS, Phil Mag.

7. Surface Energy and Microstructure, by Robert V. Kohn and Stefan Muller, MS.

8. Effective Equations and the Inverse Cascade Theory for Kolmogorov Flows, by Weinan E. and Chi-Wang Shu, MS, Phys Fluids.

9. Effective Dielectric and Elastic Constants of Piezoelectric Polycrystals, by Tamara Olson and Marco Avellaneda, MS, J Appl Phys.

\section{B. Numerical Methods and Scientific Computing}

\section{MULTIVARIATE SPLINE APPROXIMATION}

Carl de Boor

Amos Ron

University of Wisconsin - Madison

SL: ARL/WTD

One topic successfully pursued was the use of radial functions in the fitting to scattered data. The attraction is the simplicity of the formulation in any number of dimensions and for any pointset $P$. As it turned out, though, the approximation properties of this very simple and versatile multivariate interpolation scheme had been determined so far only when the points in $P$ form a regular grid, the very situation in which researchers already have plenty of good interpolation schemes, like tensor product spline interpolation. A quite simple method was found which converts most of the approximation results on regular grids to non-regular grids which satisfy some uniformity assumption. In addition, stimulated by recent as yet unpublished work by Hakopian and Sahakian, researchers gave a full analysis of the problem of interpolating by polynomials to data given on a given family of hyperplanes, and, more generally, on all $s$-dimensional intersections of such hyperplanes, thus extending earlier work which covered the special cases $s=0$. In the process, a better understanding was gained of the space of all polynomials in a box spline space, of interest in the study of approximations from such a space.

Reports:

No. 1-2 in previous editions.

3. Approximation From Shift-Invariant Subspaces of L2(IRd), by Carl de Boor et al., MS, Ann Math.

4. Fourier Analysis of the Approximation Power of Principal Shift-Invariant Spaces, by Carl de Boor and Amos Ron, MS, Constr Approx.

5. Box-Spline Tilings, by Carl de Boor and KJaus Hollig, Am Math Month 98,793(1991). AD A249 710

6. The Structure of Finitely Generated Shift-Invariant Spaces in $\mathrm{L}_{2}$ (IRd), by Carl de Boor et al., MS, J Functional Anal.

7. On the Construction of Multivariate (pre) Wavelets, by Carl de Boor et ál., MS.

8. On the Error in Multivariate Polynomial Interpolation, by C. de Boor, MS, J Appl Numer Anal.

9. On Ascertaining Inductively the Dimension of the Joint Kernel of Certain Commuting Linear Operators, by Carl de Boor et al., MS, Adv in Math.

\section{A NUMERICAL SOLVER FOR INITIAL AND BOUNDARY VALUE PROBLEMS IN DIFFERENTIAL-ALGEBRAIC SYSTEMS}

Stephen L. Campbell

Kenneth D. Clark

North Carolina State University

SL: TACOM

Differential algebraic equations (DAEs) are systems of differential and algebraic equations which have 
not been reworked into an explicit format. Many engineering problems are initially modeled as DAEs. This project is to develop numerical methods for working directly with these original implicit equations. Previously developed numerical methods for DAEs such as backward differentiation (BDF) and implicit Runge-Kutta (IRK) methods experience numerical difficulities with the higher index problems that arise in such areas as constrained mechanics, for example with models for vehicular systems. Even when these numerical methods can be used it is important, and often difficult, to determine sufficiently accurate initial conditions for all variables. In 1988 , one the principal investigators outlined a more general numerical approach for nonlinear DAEs. While computationally more intensive than BDF and IRK methods, this approach had several potential advantages. It can work when the other methods do not. It can also be used to compute consistent initial conditions. This general approach involved symbolic differentiations of the original equations followed by a singular Newton's method which was carried out numerically. One current thrust of the research is to develop the theoretical basis of the general method. This has proven to be highly nontrivial. One issue currently being resolved is that alterations of the numerical iteration in order to speed up the computation, such as less frequent Jacobian updating, can alter the equations characterizing the limit of the iteration. Thus each numerical modification must be accompanied with a corresponding theoretical analysis. It has been shown that nonlinear index three Hessenberg systems can be solved by this method. This result is important since many mechanics and control problems have this form. A second thrust of the research has been to develop numerical methods and guidelines for their use. A software package is being developed that will carry out the entire general procedure for both integrating DAEs and for finding initial conditions. The user will then need only to write down the equations and specified desired quantities. The software will then symbolically differentiate the equations several times, generate Jacobians of the corresponding derivative arrays, generate FORTRAN code for these arrays and Jacobians, and finally carry out the numerical calculations in FORTRAN. This approach has several advantages. However, it has the disadvantage that it does not reimpose the explicit and implicit constraints that are present in a DAE. Thus there can be drift off the constraint manifold. This is often undesirable, particularly in simulations of vehicular and other systems. Researchers are currently examining the use of the ideas from the general approach to design an integrator which will work for unstructured higher index DAEs and also conserve contraints. The modification being considered first is a new method similar in spirit to an Adams-Moulton.

Reports:

No. 1-7 in previous editions.

8. Progress on a General Numerical Method for Nonlinear Higher Index DAEs, by Stephen L. Campbell and Edward Moore, MS.

9. Explicit Formulae for Completions of Linear Time Varying Singular Systems of Differential Equations, by Stephen L. Campbell and Marek Rakowski, MS.

10. Utilization of Automatic Differentiation in Control Algorithms, by Stephen L. Campbell et al., MS.

11. Solvability of General Differential Algebraic Equations, by Stephen L. Campbell, MS.

\section{LINEAR ALGEBRAIC COMPUTATION ON DISTRIBUTED MEMORY PARALLEL MACHINES}

Stanley C. Eisenstat

Yale University

SC: TACOM

A current focus is sparse $L U$ factorization with partial pivoting. Most implementations compute the factors one row or column at a time, with each step involving both symbolic operations (to determine the nonzero structure) and numeric operations. With the development of fast floating-point hardware and vector processors, the symbolic operations have come to represent a non-trivial fraction of the overall factorization time. The technique of symmetric reduction exploits structural symmetry to decrease the amount of structural information required for the symbolic factorization of a sparse unsymmetric matrix (i.e., for obtaining the nonzero structures of the factor matrices), thus decreasing the run-time. Working with Joseph Liu of York University, this investigator has shown how to use symmetric reduction to improve the performance of a class of sparse partial pivoting codes that includes Sherman's NSPFAC and a code of Gilbert and Peierls. For some problems the improvement is more than a factor of two. Work is now in progress on a supernodal implementation, which should offer even better performance and will be the basis for a parallel implementation. 


\section{ALGORITHMIC ISSUES IN HIGH-PERFORMANCE COMPUTING}

Jeffrey Vitter

Brown University

$$
\text { SC: TACOM }
$$

Mark Nodine and Jeff Vitter (SPAA '91) have recently presented an optimal deterministic sorting algorithm. The algorithm is an interesting variant of merge sort. In each merge pass, a priority scheme guarantees that each record ends up sufficiently close to its correct position, so that another pass of column sort can complete the merging. Jeff Vitter and P. Krishnan (FOCS '91) have developed optimal algorithms for prefetching that are novel in that they use data compression techniques that are both theoretically optimal and good in practice. The motivation is the intuition that in order to compress data, you have to be able to predict future data well, and thus good data compressors should be able to predict well for purposes of prefetching. They show for powerful rates incurred by their prefetching algorithms are optimal for almost all sequences of page accesses. Paul Howard and Jeff Vitter have done a rigorous analysis of dynamic arithmetic coding and have expressed the compression achieved in an elegant way in terms of a natural notion of entropy. Work on lossless image compression has resulted in algorithms that are significantly better than those currently used in practice. Jyh-Han Lin and Jeff Vitter have developed the first known approximation algorithms with provable performance guarantees for the $s$-median problem, the generalized assignment problem, and the tree pruning problem. These important problems have numerous applications to data compression, vector quantization, memory-based learning, computer graphics, image processing, clustering, network location, scheduling, and communication. Franco Preparata and Jeff Vitter have developed practical new methods for hidden-line elmination of polyhedral terrains. The algorithm meets the optimal bound, but its especially fast and easy to implement. Franco Preparata and Roberto Tamassia have devised a new data structure for point location in a convex cell complex. Their technique combines space-sweep, dynamic planar point location, and persistent data structures. Y.J. Chiang, F.P Preparata, and R. Tamassia have developed a new approach for dynamically maintaining the trapezoidal decomposition of a planar map, and apply it to the development of a unified dynamic data structure that supports point-location, rayshooting, and shortestpath queries in the planar map. This is the first polylog-time dynamic data structure for shortestpath and ray-shooting queries in planar maps, and also the first dynamic data structure for point-location queries in general planar maps with optimal query time and polylog update time.

Reports:

1. Practical Implementations of Arithmetic Coding, by Paul G. Howard and J.S. Vitter, MS.

2. Greed Sort: Optimal Deterministic Sorting on Parallel Disks, by Mark H. Nodine and Jeffrey Scott Vitter, MS.

3. Analysis of Arithmetic Coding for Data Compression, by Paul G. Howard and Jeffrey Scott Vitter, MS, IEEE Trans on Info Theory.

4. Efficient Point Location in a Convex Spatial Cell Complex, by Franco P. Preparata and Roberto Tamassia, MS, SLAM J Comput.

5. Parallel Transitive Closure and Point Location in Planar Structures, by Roberto Tamassia and Jeffrey S. Vitter, MS, SLAM J Comput.

6. Area Requirement and Symmetry Display of Planar Upward Drawings, by Giuseppe Di Battista and Roberto Tamassia, MS.

7. Dynamic Algorithms in Computational Geometry (Preliminary Version), by Yi-Jen Chiang and Roberto Tamassia, MS, Proc IEEE.

\section{RESEARCH IN GRAPH ALGORITHMS AND COMBINATORIAL OPTIMIZATION}

Serge A. Plotkin

Stanford University

\section{SC: ACAA}

The multicommodity flow problem is a natural generalization of the maximum-flow problem where, instead of a single commodity, there are several commodities, defined by demand/supply vectors, that have to be shipped through the network such that the total amount of flow through each edge is below its capacity. Instead of trying to satisfy all the demands, one can try to satisfy the maximum percentage of each demand. This modification is called the concurrent flow problem. Many optimization problems can be stated as special cases of multicommodity flow or concurrent flow. Applications include VLSI layout, network routing, and efficient simulations of one interconnection network by another. Generalized results have been obtained on the multicommodity flow to apply to a large class of linear programs, the so called "size" and "covering" programs. For example, one can view job scheduling as 
packing jobs into machines, where the "size" of each job is its execution time, and the "capacity" is the maximum amount of execution time to be assigned to a machine. The only previously known algorithms for solving these problems are based on general linear programming techniques. These techniques greatly outperform the general methods in many applications. The algorithm can be viewed as a Lagrangean relaxation; an important aspect of the results is that one obtains a theoretical analysis of the running time of a Lagrangean relaxation-based algorithm. The new approach yields several orders of magnitude of improvement over the best previously known running times for the scheduling of unrelated parallel machines in both the preemptive and the non-preemptive models, for the job shop problem, for the cutting-stock problem, and for the minimum-cost multicommodity flow problem. An important open problem is whether or not there exists a strongly polynomial algorithm for linear programming, i.e., an algorithm with running time that depends only on the number of inequalities and variables, and not on the size of the numbers involved. In recent years there has been substantial progress in this direction, and currently there are several special cases of linear programs for which strongly polynomial algorithms are known. A technique has been developed that extends the class of linear programs solvable in strongly-polynomial algorithms. In particular, this technique leads to the first strongly-polynomial algorithm for the concurrent multicommodity flow.

\section{PARALLELISM DETECTION AND SCHEDULING STRATEGIES FOR RELIABLE AND EFFICIENT EXECUTION ON MULTICOMPUTERS}

Dharma P. Agrawal

North Carolina State University

$$
\begin{array}{ll}
\text { SL: } & \text { SDC } \\
\text { SC: } & \text { ARL/EPSD }
\end{array}
$$

A study of various languages was completed and SISAL (Stream and Iterations In a Single Assignment Language) was selected to investigate the issues in exploiting dataflow function level parallelism, on a private memory machine. The reasons for selecting SISAL are: its clean semantics, and expressibility for dataflow parallelism. A compiler front-end was obtained from Lawrence Livermore National Labora- tory. The problem of scheduling tasks on private memory machines is known to be NP-complete in the strong sense. A new heuristic algorithm was introduced for scheduling SISAL programs, to minimize schedule length, and to investigate the tradeoff between the schedule length and the parallelism degree. None of the current approaches investigate this vital tradeoff that determines efficient usage of the processors without unduly increasing program execution time. A compile time scheduling method works on IF-2, an intermediate form to which SISAL programs are converted by the SISAL compiler front-end. Researchers will initially carry out a dependence analysis, to bind the implicit dependencies across IF-2 graph boundaries, followed by cost assignment based on Intel Touchstone i860 timings. The first phase of the two-phase scheduler finds the threshold as the difference between the earliest and the latest completion times of each task respectively. A global threshold is then selected for the complete graph. For those tasks whose threshold is below the global threshold, the scheduler attempts to find a new virtual processor. For all other tasks, the best actual dependence edge is found. The processor is then inherited from the corresponding predecessor task. The scheduler varies the value of global threshold, and determines the best value for minimal schedule length. In the second phase of the scheduler, the processors are merged to generate schedule for variable available number of processors. Schedule results for several benchmark programs have been investigated to demonstrate the effectiveness of the approach.

\section{FINITE ELEMENT TECHNOLOGY FOR PENETRATION PROBLEMS}

Ted Belytschko

Northwestern University

\section{SL: ARLWTD}

Research has focused on two topics: (a) the improvement of the pinball algorithm by the implementation of an automatic multi-pinball method; and $(b)$ the development of an hourglass control procedure for the three dimensional hexahedral element with 1point quadrature. The multi-pinball algorithm has been implemented both in shell elements and in hexahedral elements. In the original pinball algorithm, a single sphere was embedded in any element adjacent to a free surface for the purposes of the 
contact-impact algorithm. Impenetrability checks were then made on these embedded spheres, and a penalty method was used to enforce impenetrability. However, for skewed elements and for shell elements, a single pinball which was totally embedded within the element was often not a sufficiently accurate representation of the surface. In the multi-pinball or splitting pinball version, a hierarchy of pinballs is used. The parent pinball in this hierarchy encloses all nodes of the element, but is only used to make a preliminary check for the possibility of interpenetration. If this preliminary check indicates such a possibility, a hierarchy of smaller pinballs which more closely approximate the actual surface of the shell is constructed and the impenetrability condition and penalty forcers are computed through these higher level pinballs. This provides improved convergence in certain types of contact-impact problems. In work on hexahedral elements, a very robust hourglass control based on variational principles has now been developed for three dimensional elements. This is an extension of the work originally done in two dimensions by Belytschko and Bindeman. Preliminary checks of this algorithm show that it is very accurate for elastic beam bending and converges either at the optimal rate of 2 or close to it in the $\mathrm{L} 2$ norm.

\section{MODIFIED EIGENVALUE PROBLEMS, WITH APPLICATION TO STRUCTURAL DYNAMIC RE-ANALYSIS ON PARALLEL COMPUTERS}

Gene H. Golub

K.H. Law

Stanford University

\section{SC: AERO DIR, ARL/WTD}

A row-oriented sparse matrix factorization scheme has been developed to perform Cholesky factorization of a sparse or variable banded stiffness matrix arising from structural finite element analysis. This factorization scheme can factor a symmetric positive definite matrix having either a sparse or a profile structure. A sparse Lanczos algorithm has been implemented for the generalized eigenvalue problems arising from finite element structural dynamic analysis. Three different methods have also been implemented for the matrix factor modification problem on the Intel hypercube computer. The first two methods perform partial matrix re-factorization. The first method re-generates the matrix entries from the element stiffness matrices in the affected columns and then factorizes only the affected columns. The second method performs a partial matrix factor product to recover the affected columns then adds the entries from the modified element stiffness matrices and factorizes the affected columns. The third method uses househoulder transformations to update the matrix factor. A study has been initiated on the modified eigenvalue problem when the structural finite elements are modified in the dynamic re-analysis problems.

\section{NUMERICAL ANALYSIS AND COMPUTATION OF NONLINEAR PARTIAL DIFFERENTIAL EQUATIONS FROM APPLIED MATHEMATICS}

Donald A. French

University of Cincinnati

SC: ARL/WTD, BWL

In collaboration with J.T. King of the University of Cincinnati, this investigator produced two papers that deal with finite element approximations of optimal control problems. The first on control of an elliptic problem through Dirchlet boundary conditions has been accepted for publication by the journal, Numerical Functional Analysis. The second which is on parabolic problems with rough $\left(L^{2}\right.$ or $\left.L^{\infty}\right)$ Dirichlet boundary data has been submitted to Mathematics of Computation. These parabolic problems arise in control where the theory indicates "bangbang" boundary controls are present. Research continues on other related problems and has led to some other technical results.

Reports:

No. 1 in previous edition.

2. On the Numerical Approximation of an Evolution Problem in Nonlinear Viscoelasticity, by Donald A. French and Lars B. Wahlbin, MS, Comp Meth in Appl Sci and Eng.

3. Long Time Behaviour of Arbitrary Order Continuous Time Galerkin Schemes for Some One-Dimensional Phase Transition Problems, by Donald A. French and Soren Jensen, MS, IMA $\mathbf{J}$ Numer Anal.

4. Analysis of a Finite Element Approximation for a Parabolic Equation with Rough Boundary Data, by Donald A. French, MS.

5. A Space-Time Finite Element Method for the Wave Equation, by Donald A. French, MS. 


\section{NEW METHODS FOR NONLINEAR OPTIMIZATION}

Robert B. Schnabel

University of Colorado

SC: ARL/WTD, ARO

Several recent computational studies have shown that the symmetric rank-one (SR1) update is a very competitive quasi-Newton update on optimization algorithms. A paper has been prepared which gives a new analysis of a trust region SR1 method for unconstrained optimization, and shows that the method has $n+1$ step $q$-superlinear and $2 n$ step $q$-quadratic rates of convergence. The analysis makes neither of the assumptions of uniform linear independence of the iterates nor positive definiteness of the Hessian approximations that have been made in other recent analyses of SR 1 methods. The trust region method that is analyzed is fairly standard, except that it includes the feature that the Hessian approximation is updated after all steps, including rejected steps. The authors also present computational results that show that this feature, safeguarded in a way that is consistent with the convergence analysis, does not harm the efficiency of the SR 1 trust region method.

\section{MULTI-DIMENSIONAL HIGH ORDER NON-OSCILLATORY NUMERICAL METHODS FOR DISCONTINUOUS PROBLEMS IN PARALLEL STRUCTURE}

Chi-Wang Shu

Brown University

SL: ARL/BED

SC: ARL/WTD, WES

Efforts have concentrated on high order, non-oscillatory type shock capturing methods. These include finite difference (Essentially Non-Oscillatory, or ENO, schemes), finite elements (discontinuous Galerkin) and spectral methods. In ENO finite difference method, work has continued on the development and application of the high order ENO schemes based on point values and numerical fluxes coupled with TVD (total-variation-diminishing) high order Runge-Kutta type time discretization. Comparing with a 4th order finite-volume type ENO method, the point-value ENO schemes can save a factor of 4 in two space dimensions and a factor of 9 in three space dimensions. There is a good potential to apply this type of method to direct numerical simulations of turbulence. Another application area is the device simulation for semi-conductors. Researchers have successfully simulated a 2D MESFET containing high gradient regions in the solution with a coarse grid. In spectral methods, concentration is on designing approximation theory in recovering exponential accuracy in the maximum norm for piecewise smooth functions from their Fourier coefficients. Other efforts established theoretical results completely removing the Gibbs phenomenon and the theory on discontinuous functions has been tested.

Reports:

1. An Improved Energy Transport Model for Submicron Device Simulation, by Datong Chen et al., MS, IEEE Electron Device Let.

2. Energy Models for One-Carrier Transport in Semiconductor Devices, by Joseph W. Jerome and Chi-Wang Shu, MS.

3. On the Gibbs Phenomenon I: Recovering Exponential Accuracy From the Fourier Partial Sum of a Non-Periodic Analytic Function, by David Gottlieb et al., MS, J Comp and Appl Math.

4. Effective Equations and the Inverse Cascade Theory for Kolmogorov Flows, by Chi-Wang Shu, MS, Phys Fluids.

\section{MASSIVELY PARALLEL ITERATIVE METHODS: MULTISCALE PRECONDITIONERS AND IMPLICIT METHODS}

Tony F.C. Chan

University of California, Los Angeles

\section{SL: ARL/BED, ARL/WTD \\ SC: WES}

A paper has been prepared in which a domain decomposition method based on a simple change of basis on the interfaces and vertices is presented. It is shown that this leads to an effective preconditioner compared to the ones previously considered, such as the preconditioner by Bramble, Pasciak, and Schatz (BPS) [Math. Comp., 47 (1986), pp. 103-134] and the hierachical basis domain decomposition (HBDD) preconditioner by Smith and Widlund [SLAM J. Sci. Statistic Comput., 11 (1990), pp. 1212-1226]. This domain-decomposed preconditioner is based on Bramble, Pasciak, and Xu's multilevel nodal basis preconditioner [Math. Comp., to appear]. It is shown that analytically this method and the HBDD method give the same order of condition number, namely, $O\left(\log ^{2}(H / h)\right)$ for problems with smooth coefficients. Numerically this method appears to be more effective with little additional cost and for the model 
Poisson problem, the condition numbers appear to be $O(1)$. The interface probing technique, which was developed and used by Chan and Resasco and Keyes and Gropp, is an algebraic technique for constructing interface preconditioners in domain decomposition algorithms. The basic technique is to approximate interface matrices by matrices having a specified sparsity pattern. The construction involves only matrix-vector products, and thus the interface matrix need not be known explicitly. A special feature is that the approximations adapt to the variations in the coefficients of the equations and the aspect ratios of the subdomains. This preconditioner can then be used in conjunction with many standard iterative methods, such as conjugate gradient methods. In another paper, some old results are summarized and new ones are presented, both algebraic and analytic, about the interface probing technique and its applications to interface operators. Comparisons are made with some optimal preconditioners.

\section{THE QUANTUM HYDRODYNAMIC MODEL FOR SEMICONDUCTOR DEVICES}

\author{
Carl L. Gardner \\ Duke University
}

SL: ARO

The hydrodynamic model treats the propagation of charge carriers (electrons or holes) in a semiconductor device as the flow of a compressible charged fluid. The classical hydrodynamic equations consist of a set of nonlinear conservation laws for particle number, momentum, and energy, plus Poisson's equation for the electric potential. The nonlinear conservation laws are just the Euler equations of gas dynamics for a gas of charged particles in an electric field, with the addition of a heat conduction term. The hydrodynamic model was extended to include quantum tunneling effects. A new set of quantum hydrodynamic (QHD) equations has been proposed as a set of nonlinear conservation laws based on the quantum potential of Boehm and a quantum kinetic energy term due to Wigner. The QHD model allows for efficient simulation of the behavior of quantum devices that depend on quantum tunneling. It is proposed to simulate the steady-state behavior of $1 \mathrm{D}$ quantum semiconductor devices, including resonant tunneling diodes and transistors and superlattice de- vices. The classic quantum semiconductor device is the tunnel diode. The idea of tunneling in this device was extended to double barrier resonant tunneling diodes and transistors. Recently these resonant tunneling devices have been grown "vertically," which makes their fabrication in large numbers on chips practical, and has opened up many possibilities for new quantum devices. Resonant tunneling diodes exhibit negative differential resistance (a portion of an I-V curve where the current drops as the applied voltage increases), which makes them useful in many circuit applications; e.g., in high-frequency oscillators. For logic applications in digital computers, resonant tunneling transistors will play an important role in the near future. A resonant tunneling double barrier can be directly used to make up a transistor by modulating the barrier height by means of a controlling voltage. Multiple peaks can be obtained in the output current of a resonant tunneling transistor. The multiple peaks can be used for multiple-state logic devices. The resonant tunneling transistor can be modeled by a series of $1 \mathrm{D}$ simulations of a double barrier diode by modulating the barrier heights of the diode.

\section{HIGH-LEVEL PARALLEL PROGRAMMING TOOLS FOR FINITE ELEMENT ANALYSIS}

Paul Wang

Kent State University

\section{SC: TACOM}

Research under this project consists of two related areas: (a) parallel code generation - a portable code generator written in $\mathrm{C}(\mathrm{Lex} / \mathrm{Yacc})$ to produce parallel code; $(b)$ automatic derivation of parallel finite element routines - a common lisp based package for the automatic mapping of finite element computations on parallel processors and for deriving parallel procedures. A portable code generator to produce CFT77 (Cray Fortran) code has been created and a paper has been accepted by ACM TOMS. Work on porting this GENCRAY software to produce Encore/Sequent codes is on-going. Work is also in progress on the CL-based finite element code generator name PIER. One breakthrough is the design of a set of input specifications using very high-level text-book like notations to direct PIER to generate user specified sequential/parallel codes. 


\section{NONLINEAR AND STOCHASTIC NUMERICAL METHODS AND THEIR APPLICATIONS}

\section{R. Caflisch}

University of California, Los Angeles

Work is in progress on developing implicit numerical methods for a series of transport problems. The goal is to find numerical methods which perform well in the fluid dynamic limit. In this limit the solution varies on a slow fluid-dynamic time scale that is much slower than the collisional time scale, and plans are being made to use a numerical method that only resolves the fluid-dynamic scale. After careful choice of the method to insure consistency with the fluid-dynamic limit, the main remaining difficulty turns out to be one of stability, requiring use of implicit methods. The Broadwell model of the nonlinear Boltzmann equation is the simplest of the transport problems that were considered. For it a fully implicit or linearly implicit finite difference method or a Strang splitting method with an implicit collision step has been used. These methods have been successful in simulating shock waves, even if the collisional time scale was not well resolved. Researchers have collaborated on various problems involving multiphase flow and numerical methods for multiphase flows, and have developed an analytic formulation and a numerical method for describing bubble oscillations through vortex sheet dynamics, with the goal of simulating fingering, jet formation and collapse for bubbles. Other work is in progress on problems relating to vorticity production in time dependent separated flows and developing numerical methods for calculating the acoustic signals associated with incompressible flows.

\section{A SCALABLE PARALLEL LIBRARY FOR NUMERICAL LINEAR ALGEBRA}

Jack Dongarra

University of Tennessee, Knoxville

A paper has been prepared which presents a new numerical method for computing the GSVD of two matrices $A$ and $B$. This method is a variation on Paige's method. It differs from previous algorithms in guaranteeing both backward stability and convergence. There are two innovations. The first is a new preprocessing step which reduces $A$ and $B$ to upper triangular forms satisfying certain rank conditions. The second is a new 2 by 2 triangular GSVD algo- rithm, which consitutes the inner loop of Paige's method. The paper provides proofs of stability and convergence of the method, and demonstrates examples on which all previous algorithms fail.

Reports:

1. Computing the Generalized Singular Value Decomposition, by Zhaojun Bai and James W. Demmel, MS, SLAM J Math Anal Appl.

\section{PARALLEL ADAPTIVE TECHNIQUES FOR PARTIAL DIFFERENTIAL EQUATIONS USING QUADTREE AND OCTREE STRUCTURES}

\section{Joseph E. Flaherty}

Mark S. Shephard

Rensselaer Polytechnic Institute

The main goal of this research in the unification of parallel computation and automated adaptive techniques to create a user environment for the solution of large-scale scientific and engineering problems. The emphasis of this research will be on 3D time-dependent problems described by PDEs, using adaptive mesh and order variation methods on distributed memory computers. The mesh generation techniques are based on finite quadtree and octree methods which can efficiently handle the geometric information contained in the mesh via data structure techniques. New data structures will be investigated for representing the trees which reduce the total storage without losing associativity information between elements of the mesh, and which maintain the ability to represent general non-manifold geometric models. The investigators will also extend $h-p$ refinement techniques to 3D problems, develop efficient methods for octree-based unstructured mesh problems on parallel computers, and extend parallel adaptive techniques for 2D elliptic and hyperbolic problems on shared memory computers to $3 \mathrm{D}$ problems on distributed memory architectures.

\section{A STRUCTURED PROGRAMMING APPROACH TO LARGE-SCALE BATTLEFIELD SIMULATIONS}

Patrick J. Burns

Colorado State University

$$
\text { SC: ACAA }
$$

The research objective is to investigate the development of vectorized parallel algorithms for combat simulation. The approach will consist of transferring 
a vectorized version of ATCAL (ATrition using CALibrated parameters) into the Army large-scale combat simulation program CEM VI, performing a large number of simulations with multiple data sets to test the new version of the program, investigating the development of a graphics display package for display of the simulation results and adapting the earlier approaches to this research to a stochastic version of ATCAL.

\section{NUMERICAL SOLUTION OF DISCRETE BOUNDARY VALUE PROBLEMS}

Howard C. Elman

University of Maryland

$$
\text { SC: ARL/WTD }
$$

An analysis has been completed of block relaxation for linear systems derived from the two-dimensional convection-diffusion equation where one step of block Gaussian elimination has been applied to redblack ordered two-cyclic discretizations. The analysis generalizes the results of previous studies to systems that are nonsymmetrizable, in particular, for centered finite difference discretizations where one cell Reynolds number is less than one in absolute value and the other is greater than one. The analysis confirms previously observed empirical results, that two-line ordered relaxation displays fast convergence. An analytic study of the norms of relaxation operators associated with the one-dimensional convection-diffusion equation bas been completed. In contrast with traditional analyses for iterative methods based on spectral radii, the results give a precise picture of the interaction of physical characteristics of the problem, such as direction of flow, and computational aspects of the solution algorithm. The results explain the latency associated with performing relaxation against the flow, and with using relaxation with red-black ordering. A dissertation has been completed on multilevel preconditioners. This work includes development and analysis of a new algebraic multilevel preconditioner for second order elliptic operators, and exhaustive sets of numerical experiments comparing multilevel iterative methods, additional comparisons with algebraic methods such as ICCG. In addition, the researcher has developed new parallel algorithms for multilevel preconditioners, including an implementation of the Yserentant hierarchical basis requiring $O(\log \log n)$ steps, and an efficient $O(\log n)$ parallel algorithm for the Bramble, Pasciak, Xu preconditioner. A thesis has been completed on the use of BLAS3 linear algebra kernels to improve the performance of parallel implementation of $h p$-finite element solvers. The results show that roughly 90 percent of the performance of an Alliant FX/8 computer can be obtained.

\section{Reports:}

1. Ordering Effects on Relaxation Methods Applied to the Discrete One-Dimensional Convection-Diffusion Equation, by Howard C. Elman and MichaeJ P. Chernesky, MS.

2. Multilevel Preconditioners: Analysis, Performance Enhancements and Parallel Algorithms, by Xian-Zhong Guo, PhD Thesis, 1992, 102 pp.

\section{ADVANCED COMPUTATIONS AND MODELING FOR NONLINEAR AND STOCHASTIC WAVES}

James Glimm

State University of New York at Stony Brook

\section{SL: ARL/WTD, BWL, WES}

The research objective is to investigate computational methods and mathematical theory for fluid and material problems with interfaces and nonlinear waves. Attempts will be made to (a) develop parallel algorithms for computing nonlinear waves and interfaces based on multidimensional front-tracking and Eulerian conservation principles, effective computation for materials representing complex physics, and analyzing shock-induced instabilities; $(b)$ investigate relations between important length scales internal to wave structure and the large scale features such as wave shape and speed; and (c) for chaotic and fractal waves, investigate simplifying features which will allow for the prediction of stable and repeatable statistical patterns.

\section{NUMERICAL METHODS FOR DIFFERENTIAL-ALGEBRAIC EQUATIONS IN REAL-TIME INTEGRATION OF MECHANICAL SYSTEMS}

Linda Petzold

University of Minnesota, Minneapolis

\section{SL: ARL/WTD, TACOM}

The research objective is to extend analytical and numerical methods for low index differential-algebraic equations to higher index systems arising in constrained mechanical systems. Attempts will be made to investigate stable formulations of Euler-La- 
grange systems and analyze the stability and accuracy of numerical techniques for these systems, and investigate numerical methods for stiff and discontinuous DAE problems, and delay-differential DAE systems with a view to develop real-time integration algorithms for large mechanical systems simulation.

\section{H-P-ADAPTIVE FINITE ELEMENT METHODS FOR TIME DEPENDENT PROBLEMS WITH APPLICATIONS TO STRESS WAVES IN SOLIDS}

J. Tinsley Oden

University of Texas, Austin

SL: BWL, WES

The research objective is to investigate numerical techniques for $\mathbf{h}-\mathrm{p}$ adaptive finite element methods for second order hyperbolic systems of PDEs. Focus is on problems which arise in linear and nonlinear elastodynamics, e.g., stress waves in solids. Attempts will be made to develop a true h-p adaptive data structure with mesh size and spectral order as free parameters to be used to control local errors. Researchers will investigate space-time h-p approximations and develop a posteriori error estimates for $h-p$ approximations for problems in elastodynamics. They will also investigate order balancing of spatial and temporal approximations.

\section{Statistics and Probability}

\section{EFFICIENT ALGORITHMS FOR EVALUATION OF PLANAR NETWORK RELIABILITY}

A. Satyanarayana

Stevens Institute of Technology

\section{SC: AMSAA}

The central problem in the proposed research was the all-terminal reliability analysis of planar networks. The approach was and continues to be the characterization of extensive and important classes of planar networks that admit polynomial time algorithms for the all-terminal reliability computation. Significant progress has been made in enlarging the class of planar networks for which there exists efficient all-terminal reliability algorithms. The importance of this work is enhanced by the recent proof of
Vertigan that the all-terminal network reliability problem for the full class of planar networks is NPhard; thus it is very unlikely that an efficient polynomial algorithm will be found for general planar networks. A critical outgrowth of the work is the discovery of general techniques for algorithm construction that has the potential for providing $O(n \log$ $n$ ) algorithms for a greatly enlarged class of planar and nonplanar networks.

Reports:

No. $1-2$ in previous editions.

3. A Characterization of Quasi 4-Connected Graphs, by Themistocles Politof and A. Satyanarayana, MS.

4. Some Applications of Additive Group Theory to Connectivity, by Yahya Ould Hamidoune and Ralph Tindell, MS.

\section{TIME SERIES ANALYSIS AND} MULTIVARIATE ANALYSIS

Theodore Anderson

Stanford University

\section{SC: AMSAA, ARL/WTD, ETL, WSMR}

A paper has been prepared in which estimation of the parameters of a first-order Gaussian moving average model is treated in detail. Iterative methods in both the time and "frequency" domains are based on the maximization of the exact likelihood. Several methods for evaluating the necessary quadratic forms and traces are presented. The procedures are compared with each other and with alternative procedures. In another paper, the asymptotic distribution of a finite set of autocorrelations is obtained for a time series from a linear stochastic process. The disturbances (or innovations) are martingale differences with bounded variances and bounded mixed fourth-order moments satisfying a uniform conditional square integrability condition. The conditions are weaker than those used previously for such asymptotic distributions. In a third paper, the spectral distribution function of a stationary stochastic process standardized by dividing by the variance of the process is a linear function of the autocorrelations. The integral of the sample standardized spectral density (periodogram) is a similar linear function of the autocorrelations. As the sample size increases, the difference of these two functions multiplied by the square root of the sample size converges weakly to a Gaussian stochastic process with a continuous time parameter. A monotonic transformation of this parameter yields a Brownian bridge plus an independent random term. The distributions of functionals 
of this process are the limiting distributions of goodness of fit criteria that are used for testing hypotheses about the process autocorrelations. An application is to tests of independence (flat spectrum). The characteristic function of the Cramér-von Miese statistic is obtained; inequalities for the KolmogorovSmirnov criterion are given. Confidence regions for unspecified process distributions are found.

\section{Reports:}

No. $1-5$ in previous editions.

6. Goodness of Fit Tests for Spectral Distributions, by T.W. Anderson, TR, Oct 91,38 pp. AD A244 414

\section{RANK TRANSFORMATION TEST FOR BIB DESIGNS}

W.J. Conover

Texas Tech University

\section{SC: ARL/WTD}

The first objective of the research was to extend the theory of rank transformations to the balanced incomplete block design, so that the $F$ statistic computed on the ranks of the data can be shown to have asymptotically the chi-square distribution divided by its degrees of freedom, as in the regular analysis of variance on the data. This objective was completed. The second objective was to compare the exact null distribution of the $F$ statistic computed on ranks with the $F$ distribution with $t-1$ and $N-t-b+1$ degrees of freedom. This objective was completed. The third objective was to study the power of the rank transformed $F$ statistic as compared with other tests. This objective was completed. The fourth objective was to generalize the asymptotic results of the rank transformed $F$ statistic to include scores other than ranks. This objective was completed by arriving at the conditions required by the scores in order for the Lindeberg condition for asymptotic normality to hold. If these conditions hold, then many other types of scores, other than ranks, can be used. At this point normal scores do not meet the sufficient conditions found. Some more effort will be made to see if the sufficient conditions can be relaxed to include this important case. (Similar problems have been found by other researchers when using the rank transformation in experimental designs, and a breakthrough with the use of normal scores in BIB designs should take care of the problems with the other designs also).

\section{MODELING PROBABILISTIC AND LOGICAL RELATIONS WITH BELIEF FUNCTIONS}

Arthur P. Dempster

Harvard University

\section{SL: ARL/WTD}

The topic of a recent $\mathrm{PhD}$ thesis was the modeling of 24 hour time series and Bayesian estimation of pulse locations and amplitudes of blood concentrations of the hormone cortisol, a phenomenon with a strong circadian pattern that relates to human performance. The research focus is on the development of algorithms that sample marginal posterior distributions using Gibbs sampling, a relatively new approach that is currently revolutionizing applied Bayesian inference, making possible analyses until recently thought computationally intractable. A technical report is being prepared which shows that forecasting schemes proposed by nonstatisticians working under the rubric of "neural network" computing can be more effective than blind use of autoregressive statistical models, but they remain to be tried head to head against recent sophisticated nonlinear statistical techniques such as the MARs methods of Jerome Friedman. Work is continuing on developing models and algorithms for belief function networks with applications to case studies of probabilistic risk assessment that require the integration of statistical data sources and expert (e.g., engineering judgment) sources.

Reports:

1. Normal Belief Functions and the Kalman Filter, by A.P. Dempster, MS, Can J Stat.

\section{INTRODUCTION TO MODERN ANALYSIS OF VARIANCE}

J.W. Tukey

Princeton University

SC: ACAA, ARL/WTD, WSMR

Work on multiple comparisons, following on that appearing in Fundamentals of Exploratory Analysis of Variance and two other papers, has made significant advances, particulary in $(a)$ techniques appropriate to two-way tables, and $(b)$ techniques combining two or more approaches. Previous work on robust analysis of variance was expanded in scope and more 
usefully expounded. The techniques concerned allow for exotic fibers (one factor not constant), exotic sheets (two factors not constant), as well as exotic cells (all factors constant), (and exotic replications within a cell). A variety of real data examples have shown that it is essential to avoid limiting oneself to exotic cells (and exotic replications). The technology that make exoticity at different levels feasible and useful, also connects well with the general upsweeping-downsweeping approach of Fundamentals of $E x$ ploratory Analysis of Variance.

Reports:

1. Exbrids: Simple Nearly Symmetrizing Reexpressions for Exponentially Distributed Quantities, by John W. Tukey, The Art of Statistical Science, 1992, p183.

2. Tuning a Major Part of a Clustering Algorithm, by Katherine M. Hansen and John W. Tukey, Intl Stat Rev 60,21(1992). AD A249620

3. Tightening the Clinical Trial, by John W. Tukey, MS.

4. The Major Challenges for Multiple-Response (and Multiple-Adjustment) Analysis, by John W. Tukey, MS.

\section{COMPETITIVE TRADEOFF MODELING: METHODOLOGY, COMPUTATION AND TESTING}

Stephen M. Robinson

University of Wisconisn - Madison

SC: AMSAA

The main research activity was numerical testing and comparison of the algorithm developed for performing scenario analysis on large problems. It appears that the decomposition algorithm is significantly faster (up to 1,500 times faster) than the best current technology for this problem.

Reports:

1. Scenario Analysis for Optimization Under Uncertainty, by Bock Jin Chu et al., MS.

\section{FUNCTIONAL STATISTICAL DATA ANALYSIS AND MODELING}

Emanuel Parzen

Texas A \& M University

SC: WRAIR

Research is concerned with developing a general theory, called Change Analysis, which in the narrow sense provides new solutions to problems of detecting changes in probability distributions and provides in the wide sense new frameworks for classic statisti- cal problems of testing for homogeneity (no change). Change Analysis "in the narrow sense" is concerned with the problem of detecting and estimating slow and abrupt changes in the probability distributions of successive observations $Y(t)$ of a variable or system. Its literature (emerging since the 1960's) is vast (and is being developed in diverse fields: system adaptive control, signal detection, quality control, literary authorship, disorder detection). The aim is to develop the final phase of a Change Analysis as a curve smoothing problem which provides applications of wavelet methods and maximum entropy density estimation. A major technical development in the research is an approach to Change problems by introducing analysis of Score Change Processes (whose idea is to study if a model to a whole data set fails to fit it by "random walking" the parameter estimating equations). It also emphasized CoChange Analysis, which studies the relation between random variables $Y$ and $X$. A main problem in Change Analysis is choice of data transformations to be analyzed for change; researchers introduce Score Change Processes. To relate different change processes for a single data sequence they use comparison density functions and a new probability theory for expressing dependence between two random variables $X$ and $Y$ (which can be discrete or continuous).

Reports:

No. 1.4 in previous editions.

5. Hirotugu Akaike, Statistical Scientist, by Emanuel Parzen, TR, Jun 92, 10 pp.

6. History of Statistics in Real Time: Hammers and Nails, by Emanuel Parzen, TR, May 92, 8 pp.

7. Change Analysis and Fisher-Score Change Processes, by Emanuel Parzen, TR, May 92, 18 pp.

8. From Comparison Density to Two Sample Analysis, by Emanuel Parzen, TR, May 92, 20 pp.

9. Change Analysis, by Emanuel Parzen, TR, Jun 92, 10 pp.

\section{OBJECT ORIENTED SEGMENTATION OF IMAGES}

Jayant Shah

Northeastern University

The emphasis of the research has been on testing and evaluating the various alternatives offered by the new mathematical framework. The basic framework is a nonlinear system of diffusion equations for two interacting processes: one for non-uniform smoothing of the image intensity and the other for detection of the boundary. The latter process also is a non-uniform smoothing process; it obtains the boundary as a prob- 
ability distribution which, in turn, is nothing but smoothed and scaled gradient of image intensity. It is felt that such a framework offers a more flexible approach than the usual formulation of a single global energy functional. One of the objectives was to compare alternative methods of coupling the two diffusion processes. Very different behaviors may be produced by changing the way in which the boundary process controls the smoothing of the image intensity. The usual practice is to smooth the image intensity away from the boundary, thus requiring that smoothing should increase as the probability for the presence of a boundary diminishes. What is surprising is that better results are obtained by reversing this. The two processes compete; as the probability of a boundary rises at a point, the image is smoothed more, trying to reduce the probability. Another research objective was to explore the ways for thresholding the boundary so as to enhance significant boundaries and ignore extraneous ones. What is essential is a method for establishing local thresholds. This may be viewed as the problem of finding the points where the probability for the presence of a boundary is relatively high in the neighborhood. The method yields a regularized version of the boundary, smoothing out ragged or fragmented boundaries. The smoothing radius is the only parameter used in controlling the whole process.

Reports:

1. Segmentation by Nonlinear Diffusion, by Jayant Shah, IEEE Proceedings on Computer Vision and Pattem Recognition, 1991, p202. AD A244 918

2. Segmentation by Nonlinear Diffusion, II, by Jayant Shah, MS.

\section{TOPICS IN STATISTICAL ESTIMATION AND CONTROL}

Herbert Robbins

Rutgers, The State University of New Jersey

Consider the design of a trial for selecting the better of two treatments $A, B$ for treating a specified number $N$ of individuals. During the trial phase allocate the treatments randomly to $n$ pairs of individuals, $A$ to one and $B$ to the other, after which allocate the apparently superior treatment to all of the remaining $N-2 n$ individuals. The problem of how to choose $n$, $1 \leq n \leq N / 2$, in such a way as to minimize the expected total number of individuals who will have received the inferior treatment was treated by Anscombe (J.Am. Stat. Assn. 58 (1963)) and others, who showed that there exists sequential methods of choosing $n$ that have great advantages over any nonsequential method when $N$ is large. Researchers have found some useful inequalities that allow one to evaluate the performance of a particular sequential method for any $N$, when the random variables involved are normal, with known or unknown variance, or binomial. The problem was also studied by computer simulations, which indicate that the sequential rules have much better performance than designs with fixed sample sizes. When an individual is assigned to one of two treatments $A, B$ on the basis of whether a pre-treatment observation $X$ is $\leq a$ or $>$ $a$, it is not obvious how to evaluate the effects of the two treatments on each of the two subpopulations $\{X \leq a\}$ and $\{X>a\}$, since treatment $B$ will not have been used on the first of these nor treatment $A$ on the second. Under certain hypotheses, it has been shown (Biometrika, June, 1991) that this can be done when the effects of the treatments are normal, binomial, or Poisson. This work was extended to the non-parametric case when two pre-treatment observations $X$ and $X^{\prime}$ are available for each individual.

Reports:

1. Estimation in the Mean Residual Life Regression Model, by G. Maguluri and C.-H. Zhang, MS, Biometrika.

2. Estimating a Monotone Density From Censored Observations, by Youping Huang and Cun-Hui Zhang, MS, Ann Stat.

\section{PROBLEMS IN RELIABILITY, STATISTICS AND PROBABILITY}

Jayaram Sethuraman

Florida State University

SC: AMSAA, NVESD

Researchers considered Markov chains to simulate graphs with a fixed degree sequence and binary matrices with fixed row and column sums. By means of a combinatorial construction, they bound the subdominant eigenvalues of the chains. Under certain additional conditions, they show that the bounds are polynomial functions of the degree sequences and the row and column sums, respectively.

\footnotetext{
Reports:

No. 1-7 in previous editions.

8. The Role of a Module in the Failure of a System, by Emad El-Neweihi and Jayaram Sethuraman, TR, Jul 91, 10 pp. AD A244 277
} 
9. Order Statistics and Optimal Allocation Problems, by Emad El-Neweihi and Jayaram Sethuraman, TR, Jul 91, 9 pp. AD A244 301

10. Identification of Nonlinear Time Series From First Order Cumulative Characteristics, by Ian W. McKeague and MeiJie Zhang, TR, Aug 91, 24 pp. AD A239 822

11. The Role of a Group of Modules in the Failure of Systems, by A.M. Abouammoh et al., TR, Aug 91, 11 pp. AD A244 278

12. Testing the Minimal Repair Assumption in an Imperfect Repair Model, by Brett Presnell et al., TR, Sep 91, 21 pp. AD A244 786

13. Goodness-Of-Fit Tests for Additive Hazards and Proportional Hazards Models, by Ian W. McKeague and Klaus J. Utikal, Scand J Statist 18,177(1991). AD A244 655

14. Markov Chain Simulations of Binary Matrices, by William B. Krebs, TR, Jan 92, 18 pp. AD A249 265

15. A Diffusion Defined on a Fractal State Space, by William B. Krebs, Stochastic Processes and Appl 37,199(1991). AD A249 824

\section{FAILURE DATA ANALYSIS BASED ON ENGINEERING AND GEOMETRIC PRINCIPLES}

\section{Richard E. Barlow}

University of California at Berkeley

One of the objectives has been the development of multivariate probability distributions for observable engineering quantitites such as tensile, shear and compressive stresses. Using such models, experimental data on these quantities can then be used to analyze the strength of materials. The multivariate probability distribution model or likelihood model is, for example, conditional on the average distortion energy capacity of exchangeable units. Work is in progress on developing probability models for analyzing data relevant for research in the physical sciences and in particular in materials science. Work begins with well known deterministic theory such as the von Mises criterion used to predict the yield strength of isotropic linear elastic materials (or Hooke's law in the simplest case). Then research is focused on a parameter of interest such as the distortion energy relative to the yielding of a material. Using the principle of indifference in a Bayesian context, conditional probability models are determined for relevant quantities which can be measured either experimentally or in the field. Efforts are still underway on completing mathematical work on a new probabilistic notion of aging in order to determine appropriate life distribution models. Consider an $N$-tuple of exchangeable non-negative random variables, which can be interpreted as lifetimes of $N$ similar units, and assume that the joint survival func- tion is, in particular, Schur-concave. This condition is relevant since, as it has been recently shown, it provides a probabilistic model for aging in the subjectivistic set-up.

\section{Reports:}

1. The Construction of New Bivariate Exponential Distributions From a Bayesian Perspective, by Yu Hawakawa, MS, J Am Stat Assn.

\section{VISUALIZATION METHODS FOR EXPLORATION OF HIGH DIMENSIONAL DATA}

\section{Edward J. Wegman}

George Mason University

The visualization of multivariate data is crucial to the model building process since multivariate models are often based on linearity, Guassianity and other restrictive assumptions. Failure to verfiy whether or not these assumptions hold may lead to nonsensical inferences. In a paper, researchers proposed a nonparametric technique for finding structure in multivariate data by means of geometric ridges on the surfaces or hypersurfaces of multivariate densities. A $k$-ridge is a maximal $k$-dimensional feature (in a sense to be defined) on the hypersurface of a density in a space of $d$ dimensions where $0 \leq k \leq d$. They present two algorithms (orthogonal slicing and ridge tracing) for estimating the $k$-ridge. The ridge estimation method can be applied to the gradient of an image which allows the technique to be used as an edge detection algorithm in image processing applications. The investigators present some visualization techniques as implemented on a workstation. These include the use of lighting and rendering, transparency, 3-D stereoscopic display using red-green stereo, dynamic rotation and dynamic thresholding of both density surfaces and density contours. This combination of techniques allows the visual separation of as many as eight layers of surfaces giving an ability to visualize exceedingly complex density structures.

\section{BOOTSTRAP AND RECURSIVE PARTITIONING METHODS}

\section{Wei-Yin Loh}

University of Wisconsin - Madison
SL: USAIS
SC: ARL/WTD, WRAIR 
Results were obtained in the following five areas: Box-Cox Transformations - Proof has been obtained that the asymptotic efficiency of the two-sample $t$-test following a Box-Cox transformation relative to the same test without transformation is always at least 1. The effect of Box-Cox transformations for the two-class discrimination problem was examined where expressions for the asymptotic misclassification errors are obtained for Fisher's linear discriminant method with and without transformation of the data. Conditions are derived under which the method after transformation has smaller total error. Bootstrap Tests of Fit - A method for testing lack of fit of a regression model is proposed. It is asymptotically consistent against all alternative models and works by comparing the regression estimate of error variance with a nonparametric estimate. The null distribution of the test statistic is approximated by bootstrapping and studied by means of Edgeworth expansions. Tree-structured survival modeling - An algorithm for fitting Cox proportional hazards models to recursively stratified portions of the data is developed. Piecewise-polynomial Regression Trees - A method of piecewise-polynomial tree-structured regression is proposed. It is shown that the flexibility of polynomials permits impressive gains in accuracy, making the current method competitive or even better than other methods. A method of averaging the polynomial pieces together to form a continuous and differentiable whole is also described. Large-sample consistency properties are proved in the paper. Analysis of Factorial Experiments - A method of analysis of data from unreplicated two-level factorial experiments that is invariant of labeling of factor levels is proposed. By means of simultaneous confidience bands and computer simulation, critical values for determining the number of significant effects are given for practical use.

Reports:

No. $1-2$ in previous editions.

3. Bounds on AREs of Tests Following Box-Cox Transformations, by Hanfeng Chen and Wei-Yin Loh, MS, Ann Stat.

4. Application of Box-CoxTransformations to Discrimination for the Two-Class Problem, by Peng Qu and Wei-Yin Loh, MS, Commun Stat.

5. Bootstrap Tests of Fit for Nonparametric Regression Models, by Wei-Yin Loh and Bin Yu, MS, J Multivariate Anal.

6. Piecewise-Polynomial Regression Trees, by Probal Chaudhuri et al., MS, Statistica Sinica.

7. Tree-Structured Proportional Hazards Regression Modeling, by Hong-Shik Ahn and Wei-Y in Loh, MS, Biometric J.
8. Bounds on the Size of the Likelihood Ratio Test of Independence in a Contingency Table, by Wei-Yin Loh and Xujie Yu, MS, J Multivariate Anal.

9. Survival Modeling Through Regression Trees, by Hongshik Ahn, PhD Thesis, 1992, 238 pp.

\section{TOPICS IN EXPLORATORY AND SPECULATIVE DATA ANALYSIS}

James R. Thompson

Rice University.

\section{SL: ARL/WTD, USAIS \\ SC: WRAIR}

A major portion of effort has been spent in implementations of the SIMEST paradigm for the estimation of parameters characterizing a real world stochastic system for which the micro-axioms describing the system can be readily written down, but for which any closed form of the likelihood function or moment generating function is extremely difficult to obtain. Such systems are quite common, and their complexity is generally bypassed through the (usually inappropriate) use of a linear or loglinear model. SIMEST assumes a knowledge of the numerical values characterizing a stochastic system, generates many simulated universes based on these parameter values, and then compares the simulated data to that actually observed. This comparison provides the criterion for changing one's guess as to the true values of the parameters. Clearly, such an approach is highly computer intensive. However, by the use of parallelization, it has been possible to implement SIMEST using a relatively inexpensive (under 50K) desktop transputer system. The building of general use software is one of the goals of the project. In the case of the optimization software, some success has been achieved. A paper has been prepared which considers the personal computer market, in which $P C$ clones regularly appear and disappear, according to a nonstandard birth and death mechanism. The axioms of the model are straightforward, but the resulting likelihood function defies tractability. The use of SIMEST enabled satisfactory estimation of the characterizing parameters using as the data base the actual market entrances and exits in the PC market since its inception. Another addresses the classical homogeneous birth and death process. As in the previous paper the computer intensive tasks have been speeded up by parallelization. In another paper, the SIMEST algorithm for obtaining estimates of the parameters characterizing a stochastic process is implemented using a piecewise quadratic approxima- 
tion to a goodness of fit statistic. The implementation is motivated in part by the rotatable experimental designs of Box and Hunter. Here, however, an "experiment" is simply a computer simulation, so the cost of the experiment is essentially trivial. Parallelized computation is used on a Levco transputer system utilizating design points in a fashion so as to maximize the utilization of all transputers.

\section{Reports:}

1. Simulation Based Estimation for Birth and Death Processes, by Katherine B. Ensor et al., MS.

2. A Parallelized, Simulation Based Algorithm for Parameter Estimation, by Martin Lawera and James R. Thompson, MS.

\section{STOCHASTIC CONTROL AND TOPICS IN APPLIED PROBABILITY}

loannis Karatzas

Columbia University

\section{SL: NVESD}

Efforts were concentrated chiefly on adaptive stochastic control problems of the Bayesian type, formulated as questions of control under partial observations. Interest focused on establishing the optimality of the control law suggested by the certainty-equivalence (or separation) principle. A different activity concerned the study of the martingale representation property for the filtration generated by two independent semi-martingales. The results extend all those in the current literature.

Reports:

1. The Resolvent of a Degenerate Diffusion on the Plane, with Application to Partially-Observed Stochastic Control, by Ioannis Karatizas and Daniel L. Ocone, MS, Ann Appl Prob.

2. A Martingale Representation Theorem for Two Independent Semimartingales, by Xing-Xiong Xue, MS.

3. The Finite-Horizon Version for a Partially-Observed Stochastic Control Problem of Benses \& Rishel, by Ioannis Karatzas and Daniel L. Ocone, MS.

\section{PERFORMANCE AND}

ROBUSTNESS OF SELF-TUNING

AND ADAPTIVE ALGORITHMS

FOR IDENTIFICATION, CONTROL

AND FILTERING

\section{P.R. Kumar}

Tamer Baser

University of Illinois

\section{SC: AMSAA, TACOM}

The adaptive IIR filtering and output error identification algorithms are well known adaptive systems proposed more than a decade ago. However, their stability and asymptotic performance have been open theoretical problems for more than a decade now. A paper has been prepared which resolves their stability and asymptotic optimality. New algorithms were developed for adaptive feedforward control and adaptive active noise cancelling. Another paper addressed another problem, again open ever since the modern era of adaptive systems, concerning the robustness of the projection mechanism for adaptive control laws. Researchers established that by just projecting the parameter estimates into a compact convex set, one can ensure stability of the adaptive system to small unmodeled dynamics and large bounded disturbances. This demonstrates that many of the rather complex robustness fixes suggested by several research groups over the past decade are unnecessary. Recently, a new simulator was developed for complex discrete-time dynamic systems. The goal here is to bridge the gap between mathematical specification of dynamical systems, as found in technical papers, and their simulation. A paper has been prepared which formulates a graph theoretical model of the issues faced by simulation users, develops mathematical algorithms to automate the simulation process, and proves their validity. Finally, a simulator called QUICKSIM was developed which incorporates these algorithms. The net result is a total automation of the simulation process to the point where a user only has to more or less just type in verbatim the description of a complex discretetime system, as described in a typical journal, to obtain a simulation output.

\section{Reports:}

1. Robust Continuous Time Adaptive Control by Parameter Projection, by Sanjeev M. Naik et al., Proc of 30th Conference on Decision and Control, 1991, p742. AD A244 794

2. Stochastic Parallel Model Adaptation: Theory and Applications to Active Noise Canceling, Feedforward Control, IIR Filtering and Identification, by Wei Ren and P.R. Kumar, Proc of 30th Conference on Decision and Control, 1991, p1668. AD A244 860

3. Robustness of Extended Least Squares Based Adaptive Control, by Sanjeev M. Naik and P.R. Kumar, Proc of 30th Conference on Decision and Control, 1991, p754. AD A244 178

4. Adaptive IIR Filtering: Robustness Analysis, by Sanjeev M. Naik and P.R. Kumar, MS. 
5. The Theoretical Foundation of ISIM: A Software Package for the Simulation of Discrete Time Systems, by R. Douglas Ellis et al., MS.

6. Stochastic Adaptive System Theory for Identification, Filtering, Prediction and Control, by Wei Ren, PhD Thesis, 1991, 67 pp.

7. Automating the Simulation of Complex Discrete-Time Control Systems: A Mathematical Framework, Algorithms and a Software Package, by R. Douglas Ellis et al., MS, IEEE Trans on Auto Control.

8. Relating Simultaneous Learning and Simultaneous Estimation for Classes of Sets and Classes of Probabilities, by Kevin Buescher and P.R. Kumar, Proc of 25th Ann Confon Information Sciences and Systems, 1991, p108.

9. Robust Indirect Adaptive Control of Time-Varying Plants with Unmodeled Dynamics and Disturbances, by Sanjeev M. Naik and P.R. Kumar, MS, SIAM J Control and Optmztn.

\section{OPTIMAL UNIVERSAL CODING AND DENSITY ESTIMATION}

Bin $Y \mathbf{u}$

University of Wisconsin - Madison

\section{SL: ARL/WTD}

A paper has been prepared which investigates the density estimation problem in the supreme norm for dependent data, with applications to the Gibbs sampler - a very popular computer simulation method. It shows that the lid optimal minimax rates in the supreme norm are also optimal for smooth classes of stationary sequences satisfying certain mixing conditions. Empirical process methods have been proven to be an important tool deriving desirable limiting distributions in probability and statistics. Another paper shows almost minimal conditions for the convergence of empirical processes and U-processes over V-C subgraph classes and for mixing sequences. To address the mathematical/statistical problems involved in the design aspect of physical mapping, i.e., putting little pieces of chromosome back in the right linear order, a paper has been prepared which proposes a new criterion based on the mutual information and investigates the properties of this new criterion in the case of the pairwise comparison. It has recently been the focus of many papers to establish a sensible mathematical framework to model the process of "learning". Another paper illustrates the application of the MDL principle to a typical stochastic learning problem, where the features range over a continuum. Moreover, it shows that when the object to be learned, e.g., the probability function of the weight, lies in a parametric class, the best rate at which one can estimate it (or in other words, the best rate at which one can "learn" about it) is the same as the complexity of the model, that is, the minimum description length (MDL) of the model. When the model class is much larger, say a smooth nonparametric class, the "learning" rate is much slower.

\section{Reports:}

No. $1-4$ in previous editions.

5. Model Selection and Prediction: Normal Regression, by T.P. Speed and Bin Yu, MS.

6. MDL Learning, by J. Rissanen and Bin Yu, MS.

7. Bootstrap Tests of Fit for Nonparametric Regression Models, by Wei-Yin Loh and Bin Yu, MS, $J$ Multivariate Anal.

\section{DIFFERENTIAL GEOMETRICAL METHODS IN TIME SERIES}

Nalini Ravishanker

University of Connecticut

\section{SC: ARL/WTD}

Research has been a successful continuation of efforts to apply differential geometrical methods to time series. It focussed principally on the development of relative curvature measures of nonlinearity for time series models. The purpose of the work in this area has been to characterize these measures for the class of nonseasonal autoregressive integrated moving average (ARIMA) models and multiplicative seasonal ARIMA models with normal errors. For these models, and considering conditional likelihoods, the presence of the moving average parameters introduces nonlinearity in the model function or nonquadraticity in the sum of squares or log-likelihood function. In the time series literature, the assumption of quadraticity of the log-likelihood is extremely important for estimation and inference; however, no direct and computationally feasible method exists in general to verify this assumption. The relative curvature measures would be valuable diagnostics that verify this assumption. Research efforts have characterized the framework for computing these relative curvatures for the ARIMA models and calculated the maximum and root mean square intrinsic and parameter-effects curvature for 16 data sets modeled in the literature by ARIMA models. In more than half the cases, either the intrinsic or the parameter-effects curvature is significantly large. Further, in time series, an interesting finding is that the intrinsic curvature is larger, in general, than the curvature due to the particular parametrization.

\section{Reports:}

1. Relative Curvature Measurements of Nonlinearity for Time Series Models, by Nalini Ravishanker, MS, Biometrika. 
2. Bayesian Analysis of ARMA Processes: Complete Sampling Based Inference Under Full Likelihoods, by John Marriott et al., MS, J Am Stat Assn.

3. Shrinkage Estimation for Business Planning and Forecasting of Regional IBM Revenue, by N. Ravishanker et al., MS, J Am Stat Assn.

\section{STABILITY OF DYNAMICAL SYSTEMS IN THE PRESENCE OF NOISE}

Mark A. Pinsky

Northwestern University

\section{SL: NVESD}

Suppose that $H$ is a two-dimensional CartanHadamard manifold with sectional curvatures satisfying a weak negative upper bound and no lower bound. Then the angular part of Brownian motion on $H$ tends to a limit as time tends to infinity. Moreover this limit angle has a distribution which is dense on the circle at infinity. Let $p \Omega(t, x, y)$ denote the heat kernel on an euclidean domain $\Omega \in \mathrm{R}^{\mathrm{n}}$ with the Neumann (adiabatic) boundary condition. Researchers prove the following monotonicity property of the Neumann heat kernel: if $\Omega$ is a smooth convex domain and $D$ is a cell (multidimensional rectangle) containing $\Omega$, then for every pair of points $x, y$ in $\Omega$ and every $t>0$ there is $p \Omega(t, x, y) \geq p D(t, x, y)$. The proof is probabilistic and involves studying the sample path behavior of reflecting Brownian motion, the diffusion process whose transition density function is the Neumann heat kernel. Probabilistic counterpart of heat conduction in an insulated region is reflecting Brownian motion. The goal of this work is to study the refined sample path property of reflecting Brownian motion. Among other things, researchers will obtain a necessary and sufficient condition on the region for the reflecting Brownian motion to be a semimartingale. It turns out this property holds for a large class of regions whose boundaries are not very smooth.

Reports:

1. Extremal Character of the Lyapunov Exponent of the Stochastic Harmonic Oscillator, by Mark A. Pinsky, MS, Ann Appl Prob.

2. Lyapunov Exponent of a Stochastic Wave Equation, by Mark A. Pinsky, MS.

3. Fourier Inversion for Multi-Dimensional Characteristic Functions, by Mark A. Pinsky, MS, J Theortcl Prob.

4. A Domain Monotonicity Property of the Neumann Heat Kernel, by Elton P. Hsu, MS.

5. Limiting Angle of Brownian Motion in Certain Two-Dimensional Cartan-Hadamard Manifolds, by Elton P. Hsu and Wilfrid S. Kendall, MS

\section{PROBABILITY AND STATISTICS APPLIED TO THE THEORY OF ALGORITHMS}

J. Michael Steele

University of Pennsylvania

\section{SC: TRADOC}

Current work is represented by the article "Asymptotics of Euclidean Minimal Spanning Trees on Random Samples". This article solves an interesting conjecture of R. Bland's that the sum of the squares of the edges of the minimal spanning tree of a random sample from the unit square converges to a constant as the sample size goes to infinity. Although it is reinforcing to have resolved Bland's conjecture, the main contribution of the article is to provide a compelling example of the "objective method" whereby one obtains limit results by constructing an "infinite analog of the object of interest". The justification that one has the right infinite analog then requires showing that one's candidate provides concrete insight into the large sample behavior of the original finite problem. In the problem addressed with Aldous, both parts of the program were brought to a satisfying conclusion. The article "Applications of Mathematica to Stochastic Calculus" written with R. Stine reports on an implementation of the basic formalism that arises from Itô's formula in the Mathematica platform. The tool that results greatly shortens many of the routine calculations of the stochastic calculus. The article is one that has created considerable interest and there have been numerous requests for the code that was developed.

\section{RESEARCH IN STOCHASTIC PROCESSES AND THEIR APPLICATION}

Gopinath Kallianpur

University of North Carolina at Chapel Hill

SL: NVIESD

SC: MICOM

A paper has been prepared which contains a study of stochastic differential equations (SDE's) on duals of nuclear spaces driven by Poisson random measures. The existence of a weak solution is obtained by the Galerkin method. For uniqueness, a class of $l^{2}$-valued processes which are called Good processes is introduced. An equivalence relation is established between SDE's driven by Poisson random measures and those by Good processes. The uniqueness is established by extending the Yamada-Watanabe ar- 
gument to the SDE's driven by Good processes. This is an extension to discontinuous infinite dimensional SDE's of work done by G. Kallianpur, I. Mitoma and R. Wolpert for nuclear space valued diffusions. Another paper contains a study of a system of interacting stochastic differential equations taking values in duals of nuclear spaces driven by Poisson random measures. Researchers also consider the McKeanVlasov equation associated with the system. They show that under suitable conditions the system has a unique solution and the sequence of its empirical distributions converges to the solution of the McKean-Vlasov equation when the size of the system tends to infinity. The results are applied to the voltage potentials of a large system of neurons and a law of numbers for the empirical measure is obtained. A third paper considers several stochastic models arising from environmental problems. First, is a study of the pollution in a domain where undesired chemicals are deposited at random times and locations according to Poisson streams. Incorporated with drift and dispersion, the chemical concentration can be modeled by a linear stochastic partial differential equation (SPDE) which is solved by applying a general result. Various properties, especially the limit behavior of the pollution process, are discussed. Secondly, researchers consider the pollution problem when a tolerance level is imposed. The chemical concentration can still be modeled by a SPDE but is no longer linear. Its properties are investigated in this paper. Finally, the linear filtering problem is considered based on the data of several observation stations.

\section{CONTROL CHARTS FOR VALIDATING SIMULATION MODELS WITH CORRELATED OUTPUT}

Stuart J. Deutsch

Northeastern University

\section{SL: AMSAA}

The research objective is to develop extensions of the Space-Time Autoregressive, Moving Average (STARMA) model and appropriate multicriteria objective functions for use in the validation of combat simulation models.

29678 PDE'S RANDOM PROCESSES AND FIELDS: ASYMPTOTIC PROBLEMS
Mark Freidlin

University of Maryland

SL: NVESD

The objective of the research is to develop a theory of random perturbations of evolutionary partial differential equations and an associated asymptotic theory similar to those existing for finite dimensional systems. Averaging principle, or central limit theorem and large deviation type arguments will be employed.

\section{STUDIES IN NONLINEAR VIBRATIONS, DYNAMICS AND APPLICATIONS}

Lawrence N. Virgin

Duke University

The research objective is to investigate problems in nonlinear dynamics, nonlinear vibration, and vibrational control with emphasis on approximate methods and engineering applications. Various methods will be used for the study of nonlinear dynamical systems, e.g., the harmonic balance method, multiple time scales, and the method of averaging. New perturbation methods and symbolic computation tools will be developed; in particular, to extend the generalized harmonic balance method for a class of second order ODE with potential for handling moderate to large values of the parameters in these equations, and investigate the range of validity of this solution approach with respect to parameters. Experimental models, such as a ball rolling on a potential surface, a vibrating cantilever, and an impacting system will be constructed and used to analyze several relevant engineering applications such as the vibration of helicopter rotor blades or robot arms, and to verify the analytical techniques. Numerical methods will also be used for verification purposes.

\section{Systems, Control, Modeling and Artificial Intelligence}

26674 P.D.E., DIFFERENTIAL GEOMETRIC AND ALGEBRAIC METHODS IN NONLINEAR FILTERING 
Stephen Yau

University of Illinois at Chicago

\section{SC: AMSAA}

Estimation algebra turns out to be a crucial concept in the investigation of finite-dimensional nonlinear filters. In an earlier paper by the authors, a necessary and sufficient algebraic condition was derived for an exact estimation algebra to be finite-dimensional. In a paper, the investigation of the properties of finitedimensional exact estimation algebras is continued, and some structure and partial classification theorems for such algebras are proved.

Reports:

No. 1-4 in previous editions.

5. Recent Results on Classification of Finite Dimensional Estimation Algebras: Dimension of State Space $\leq 2$, by Stephen S.-T. Yau and Wen-Lin Chiou, MS.

\section{THE DESIGN AND ANALYSIS OF ROBUST FEEDBACK SYSTEMS}

Pramod P. Khargonekar

University of Michigan

\section{SL: ARDEC}

SC: AMSAA

Excellent progress is being made on a number of problem areas in the research program on the design and analysis of robust and $H_{\infty}$ optimal controller design, robust stability and performance analysis of sampled-data systems, and computation of $L_{\infty}$ and $L_{2}$ induced norm of sampled-data systems. The results here have led to explicitly checkable conditions for establishing robust stability and performance of uncertain sampled-data systems with structured uncertainty. For controller synthesis, constructive solutions were found to the $\mathrm{H}_{2}$ and $\mathrm{H}_{\infty}$ optimal controllers for sampled-data systems. These constructions involve solving Riccati equations. New results were obtained on the problem of identification in $H_{\infty}$ in the presence of worst case noise in frequency response measurements. Fast and efficient algorithms were developed for this problem. These algorithms use the well known FFT and SVD algorithms. Software for the algorithm is available as a MATLAB file and has been distributed to some interested researchers. Some progress was made on mixed $\mathrm{H}_{2} / H_{\infty}$ control problems for discrete-time systems. These problems have been reduced to convex optimization problems. Work is underway on developing numerical algorithms for computing the controllers.
Reports:

No. 1-17 in previous editions.

18. Induced Norms for Sampled-Data Systems, by $N$. Sivashankar and Pramod P. Khargonekar, MS, Automatica.

19. The Least Squares Algorithm, Parametric System Identification, and Bounded Noise, by H. Akcay and P. Khargonekar, MS, Automatica.

20. A Time-Domain Approach to Model Validation, by $\mathbf{K}$. Poolla et al., MS, IEEE Trans on Auto Control.

\section{AN INTERPOLATION THEORETIC APPROACH TO THE ROBUST CONTROL OF DISTRIBUTED AND NONLINEAR SYSTEMS}

Allen Tannenbaum

University of Minnesota, Minneapolis

SL: ARDEC
SC: AMSAA

Work has concentrated on the robust control of both linear and nonlinear systems using interpolation theory. A study is being made of a new structured interpolation method which has arisen out of research into the robust design of systems with structured uncertainty, and in particular the $\mu$-synthesis procedure. A large portion of the research effort has been devoted to nonlinear systems. This has led to work out an iterative commutant lifting theorem which gives an explicit design procedure for nonlinear systems and captures the $H_{\infty}$-control problem in the nonlinear framework. Researchers have been using a game theoretic (saddle point) approach to nonlinear system design. Along these lines, ideas have already been applied to certain systems with hard nonlinearities, e.g., saturations. In particular, ideas were utilized for a saturated double integrator problem (in collaboration with colleagues at Honeywell), with results that have been much better than those using more classical techniques. Work has continued on the utilization of functional analytic methods in $H_{\infty}$ optimization theory, especially for distributed parameter systems. A theory was applied to an unstable delay system which arose in connection with a flight control problem of the $X-29$, as well as to a two block mixed sensitivity problem for a distributed beam model. $H_{\infty}$ techniques have now also been implemented for sampled-data systems using a lifting approach.

\section{Reports:}

No. $1-3$ in previous editions.

4. A Lifting Technique for Linear Periodic Systems with Applications to Sampled-Data Control, by Bassam Bamieh et al., MS, Systs and Control Let. 
5. On the Optimal Solutions in Spectral Commutant Lifting Theory, by Hari Bercovici et al., MS, J Functional Anal.

6. Generalized Interpolation Theory and Its Application to Robust Control Design, by Allen Tannenbaum, MS.

7. Frequency Domain Analysis and Robust Control Design for an Ideal Flexible Beam, by Kathryn Lenz et al., MS, Automatica.

8. On Combined $\mathrm{H}_{\infty}-\mathrm{H}_{2}$ Suboptimal Interpolants, by Ciprian Foias et al., MS, Linear Alg and its Appl.

9. A Constructive Solution to the Gain-Phase Margin Problem, by Juan C. Cockburn and Yariv Sidar, MS.

10. On Affine Plane Curve Evolution, by Guillermo Sapiro and Allen Tannenbaum, MS, J Math Anal and Appl.

28511 ROBUST HYBRID STATE-SPACE SELF-TUNING CONTROL USING DUAL-RATE SAMPLING

L.S. Shieh

C.K. Koc

University of Houston

\section{SL: ARDEC}

Research results are presented in the reports listed below.

Reports:

1. Sequential Design of Linear Quadratic State Regulators with Prescribed Eigenvalues and Specified Relative Stability, by Sekar Ganesan et al., Comp Math with Appl 21,1(1991). AD A244 225

2. A Canonical State-Space Representation for SISO Systems Using Multipoint Jordan CFE, by Chyi Hwang et al., $J$ Franklin Inst 328,207(1991). AD A244 224

3. Hybrid State-Space Self-Tuning Control Using Dual-Rate Sampling, by Leang S. Shich et al., IEE Proc D138,50(1991). AD A244 731

4. Improved FET-Based Numerical Inversion of Laplace Transforms via Fast Hartley Transform Algorithm, by Chyi Hwang et al., Comput Math Applic 22,13(1991). AD A244 748

5. Optimal Momentum Management Controller for the Space Station, by J.W. Sunkel and L.S. Shieh, J Guidance Control Dynamics 13,659(1990). AD A244 430

6. Optimal Uniform-Damping Ratio Controller for Sequential Design of Multivariable Systems, by Leang G. Shieh et al., Intl J Systs Sci 22,1371(1991). AD A249 279

7. Parallel Algorithms for Nevanlinna-Pick Interpolation: The Scalar Case, by Cetin K. Koc and Guanrong Chen, Int J Comp Math 40,99(1991). AD A249 567

8. Comments on "Residue Arithmetic VLSI Array Architecture for Manipulator Pseudo-Inverse Jacobian Computation", by Cetin K. Koc, IEEE Trans on Robotics and Automation 7,715(1991). AD A249 474

9. Digital Redesign of an Optimal Momentum Management Controller for the Space Station, by J.W. Sunkel et al., $J$ Guidance Control Dynamics 14,712(1991). AD A249 665

10. Linear Quadratic Regulator Approach to the Stabilization of Matched Uncertain Linear Systems, by Y.H. Wang et al., J Guidance Control Dynamics 14,1074(1991). AD A249 664
28740

\section{ROBUST HYBRID-TIME AND TIME-VARYING SYSTEM DESIGN}

Gilead Tadmor

Northeastern University

SL: ARDEC

SC: TACOM

A paper has been prepared which suggests a state space oriented extension of Nehari Theorem to a time-varying system-theoretic setting. In that setting the theorem addresses the issue of measuring the distance between a non-causal bounded I/O operator and the family of causal I/O operators in stable linear systems. The analysis is based on the recent time-domain LQ optimization approach to robust control. The discussion includes a geometrical analysis of stable and anti-stable invariant subspaces, a short study of certain types of co-prime factorizations of I/O operators in time varying systems and a parametrization of all suboptimal solutions. Another paper addresses the problem of reduced order LQG optimization in a finite horizon, linear time-varying (LTV) system setting. First-order necessary conditions for local optimality in the parameter space is provided in terms of four coupled matrix differential equations. This result provides a transparent generalization of the optimal projection equations of $\mathrm{Hy}$ land and Bernstein for the optimal, steady-state compensation of linear time-invariant (LTI) plants.

\section{RAPID PROTOTYPING OF NONLINEAR CONTROLLER DESIGNS}

Shankar Sastry

Andrew Packard

University of California, Berkeley

\section{SL: AERO DIR, ARDEC}

A large activity has been initiated in developing software for nonlinear control systems design. The software is being evaluated and tested on numerous practical control systems design problems. Activity has continued in adaptive control of nonlinear systems, both adaptive regulation and adaptive tracking and also sliding mode control of nonlinear systems. A project has been initiated on the use of saturation functions for stabilizing systems which either had finite escape time or for which the presence of actuator constraints actually made the linearizing control laws difficult to implement. 


\section{VERY HIGH-SPEED REMOTE FILE SYSTEM}

Timothy J. Salo

Minnesota Supercomputer Center, Inc.

SC: ARL/WTD

Research is being conducted in protocols which will integrate TCP/IP networks with ATM networks; technologies which will enable gigabit-per-second local area networks (LANs) and high-performance hosts to use gigabit-speed ATM wide-area networks; and protocols and other technologies required to develop a shared, remote file system capable of transferring data to multiple remote supercomputers over gigabit wide-area networks. Some of the research results of the VHSRFS project will be used in the MAGIC Gigabit Testbed, which was recently funded by DARPA. The testbed will include: a wide-area, asynchronous transfer mode (ATM) network connecting heterogeneous elements including supercomputer systems, gigabit LANs, graphics workstations, mass storage systems, multimedia facilities, and ATM switches; a prototype, DoD-oriented application that enables a user to drive through or fly over and see terrain; a scalable image server system that is distributed and of sufficient performance to support the visualization application; data flow monitoring facilities at all levels of the image server system, the application, and the network to permit the experimentation with, and evaluation of, the interactions between the network and the distributed data and processing; and multiple diverse supercomputer applications that require a wide-area gigabit internetwork. The prototype terrain visualization application is expected to be hosted on the Army's CM-5 massively parallel processor located at the Army High Performance Computing Research Center (AHPCRC). The U.S Army Future Battle Laboratory will be the initial user of this application. In addition to the Minnesota Supercomputer Center, Inc., participants of the MAGIC Gigabit Testbed include: EROS Data Center, Lawrence Berkeley Laboratory, Sprint, SRI International and University of Kansas.

\author{
29397 STUDY TO PREPARE A REPORT \\ ON THE MATHEMATICAL \\ SCIENCES APPLIED TO \\ MATERIALS SCIENCE
}

Scott T. Weidman

National Academy of Sciences

SC: ARL/MD, BWL

Of the nearly 225 people from whom 2-page writeups were requested in June 1992, essays (ranging in length from one to eight pages) were received from about 100 people. Committee members have melded these, along with their own contributions, into a 127page "first rough" draft report.

\section{E. Logistics and Operation Methods}

\section{AN INFORMATION THEORETIC APPROACH TO RULE BASED EXPERT SYSTEMS}

Rodney M. Goodman

California Institute of Technology

SL: ARI

SC: AIRMICS, SWC

A paper has been prepared which describes a technique for automatically learning rules from network management databases. Motivations for this is to alleviate the knowledge acquisition bottleneck inherent in developing expert systems for integrated network management. Researchers outline their ITRULE rule induction algorithm, show how useful rules can be extracted from trouble ticket and alarms databases, and show how these rules can be automatically loaded into a standard expert system shell, thus virtually instantly producing a prototype expert system. In another paper, a framework for texture analysis is proposed based on information theoretic criteria. A feature extraction phase transforms the image space into an array of $\mathrm{N}$-dimensional feature vectors, in the frequency and orientation domains. The proposed model reduces the $\mathrm{N}$-dimensional representation, through unsupervised and supervised learning stages. In the unsupervised phase NeuralNetwork Vector Quantization is used for the quantization of the feature-vector attributes and projection onto a lower dimension clustered map for initial segmentation. A supervised stage follows in which labeling of the textured map is achieved. An information-measure is proposed by which the most informative correlations between the attributes and the texture class-specification are found. Thus, minimal representation for the textures are learned in a train- 
ing mode following which the classification of new test-images are achieved. Simulation results are shown both for artificial and natural texture classification.

\section{ARTIFICIAL INTELLIGENCE CENTER OF EXCELLENCE}

Aravind K. Joshi

Norman I. Bađler

University of Pennsylvania

\section{SL: MICOM}

An AI Center has been established to integrate and coordinate various research efforts in $\mathrm{AI}$ in which the unversity has excellent strengths. The key thrusts are in the following areas: $(a)$ natural language processing: language and speech; $(b)$ machine perception and robotics: exploration and perceptual development; $(c)$ task oriented computer animation; (d) programming structures for databases and knowledge bases; and (e) parallel processing in Artificial Intelligence.

Reports:

No. $1-15$ in previous editions.

16. A Kinemati: Model of the Human Spine and Torso, by Gary Monheit and Norman I. Badler, MS, IEEE Comp Graphics and Appl.

17. Human Strength Database and Multidimensional Data Display, by Susanna Wei, PhD Thesis, 1990, 109 pp.

18. Issues in Facial Animation, by Catherine Pelachaud et al., MS.

19. Interactive Behaviors for Bipedal Articulated Figures, by Cary B. Phillips and Norman I. Badler, MS.

20. Interpreting Prepositions Physically, by Jugal K. Kalita and Norman I. Badler, MS.

\section{MOTION PLANNING AND SENSORY PROCESSING IN A DYNAMIC ENVIRONMENT}

Ren C. Luo

David W. Hislop

North Carolina State University

Work has focused on two major tasks, namely, motion planning and landmark recognition. The successful operation of a Mobile Robot in a dynamic environment requires the consideration of imprecise sensory data. Most high level path planning algorithms do not take sensory errors into account and therefore must be supplemented by on-line correc- tions. Self referencing from known landmarks can facilitate such corrections. In this work, researchers have developed a detailed simulation model of the sonar subsystem on the mobile platform vehicle which is the actual robot base used in the project. The model incorporates impreciseness by adding noise to all sonar readings. A scan cone is used to reflect the conic scanning range of the sonar sensors on the vehicle. The odometer readings are also rendered imprecise by the addition of a uniformly distributed measurement error. The T-vector path planning algorithm is used in conjunction with a motion planner and self reference based on the model. The layout of the laboratory is used to generate an initial path for the robot. However, the robot used known landmarks to confirm its actual location with the expected values. Any significant deviation which may be due to cumulative odometer or sonar sensor reading errors causes the re-referencing of the robot and the resetting of all previous errors. The motion planner also incorporates a heuristic object mapper. In the landmark recognition area work has focused on two subsystems: landmark detection and landmark recognition. Landmarks are visual signs located randomly along the robot's path. These signs provide useful global and local information to the robot motion planning system. For example, mile markers provide distance information and street signs provide information about local traffic conditions. The detection of these landmarks in dynamic environments has been a problem faced by many researchers in the mobile robot area.

\section{CONTINUATION OF THE POSTGRES PROJECT}

Michael Stonebraker

University of California, Berkeley

\section{SL: SWC, TRADOC}

SC: SWC

Efforts have been concerned with implementing the algorithms for view support and version management which were developed under the previous ARO grant and published in STON90. These algorithms appear to be robust, and performance appears to be very good. Work is now in progress on examining several other higher level notations for rules. Furthermore, current implementations for rules provide "immediate activation", i.e., the action for each rule is triggered at the time the event specified in the rule 
becomes true. At times, a user would like "deferred execution", i.e. he would like rule activation to be delayed until the commit time of an enclosing transaction. An investigation is being made on how to perform deferred execution without having to maintain complex bookkeeping about the effects of a transaction during execution. Work is in progress on integrating tertiary memory into POSTGRES. An interface was designed for the large objects that occupy tertiary memory which has novel properties. First, it allows large objects to behave just like files. Second, it allows large objects to be optionally compressed with a user-defined compression function. Lastly, it allows compression to occur on a user-defined portion of a large object, independently of other portions of the same object. The POSTGRES optimizer is being extended to deal with functions which are expensive to compute.

\section{NEURODYNAMICAL SYSTEMS FOR COGNITION AND TARGET IDENTIFICATION}

Nabil H. Farhat

University of Pennsylvania

SL: ARI

SC: MICOM

Nearly all neural networks for "pattern recognition" being dealt with today are associative classifier or identifier networks that are not cognitive. To be $\operatorname{cog}$ nitive a net must be able to distinguish, on its own, between familiar and unfamiliar or novel sensory signals present at its input and this can not be done by associative classifiers. In a paper it will be argued and shown that in order to be truly cognitive a network must be nonlinear and dynamical and able to manifest bifurcation. This means it should be able to carry out phase-space computations with more than one type of attractor and to switch between these depending on whether the sensory input is familiar or novel. Cognition implies therefore bifurcation and computing with diverse attractors. Reasons for adopting this view, which stemmed from known biophysical observations and from the PI's neuromorphic target identification work, will be discussed. An example of a cognitive network that computes with both stationary (limit point) and dynamic (periodic) attractors will be given to illustrate the PI's thesis. The elements of a neuromorphic radar target identification system which employs these con- cepts and is capable of distortion invariant recognition of three targets with perfect score will be presented. The work presented will elucidate the role of periodic attractors in feature binding and cognition and the significance of cognition in autonomous systems.

\section{AUTOMATICALLY COMBINING CHANGES TO SOFTWARE SYSTEMS}

Valdis Berzins

Naval Postgraduate School

\section{SL: CECOM}

This project is investigating methods for achieving computer-aided software maintenance, with a specific focus on formal models and algorithms for combining modifications to programs that can provide guarantees of correctness. In addition to the published work, efforts are underway to refine models for merging changes to prototypes of real-time systems and for integrating change merging capabilities with support for project planning, job assignments, and design history management. As part of the first effort, researchers are analyzing previous approaches for merging changes to real-time systems to determine the exact conditions under which they do and do not yield correct results, and have proposed some decision criteria that can extend the types of changes that can be merged correctly and consistently. These efforts will continue until the methods have been sufficiently refined to produce effective algorithms for solving this problem. As part of the second effort, a literature search revealed some initial models that can be used to analyze the interaction between designer tasks and management/scheduling activities. These activities are still incomplete, and will be continued until they produce algorithms and data models that can automate team coordination and data control processes with a small communications overhead. This part of the effort is a preliminary step in the design and implementation of an engineering database system that provides support for evolutionary software development by teams of engineers.

Reports:

1. Software Merge: Semantics of Combining Changes to Programs, by Valdis Berzins, MS, ACM Trans on Programming Languages and Systems. 
2. Using Transformations in Specification-Based Prototyping, by V. Berzins et al., MS, IEEE Trans on Software Eng.

3. Applications and Meaning of Inheritance in Software Specifications, by Valdis Berzins et al., MS.

4. Software Merge: Models and Methods for Combining Changes in Programs, by Valdis Berzins, Proc of European Conference on Software Engineering, 1991, p229.

\section{DESIGN AND IMPLEMENTATION \\ OF LOGICAL DATABASE LANGUAGES}

Jeffrey D. Ullman

Stanford University

Further improvements have been made in the performance of the NAIL system. A graduate student completed the implementation of a number of additional optimizations for the GLUE language. He developed a suite of benchmarks, including GLUE code, and has measurements of performance improvements for each of these. Another researcher has improved the Iglue back end, introducing such performance enhancements as dynamic join reordering and dynamic index creation. She too has measurements based on the Phipps test suite that demonstrate the utility of (some of) these enhancements. Most interesting is that dynamic join reordering, with the right rule to decide when to attempt reordering, saves a factor of two in running time on a common and realistic example: bill-of-materials (parts-explosion). Consideration is being given to questions of nonmonotonic reasoning, or negation-as-failure rules for handling logical rules with negated subgoals. The idea is that all the recent approaches - well-founded semantics and the varieties of stable-model semantics - can be characterized in terms of "arguments", leading to new semantics for negation that resolve a number of awkward features of the well-founded and stablemodel approaches.

\section{ACCELERATING THE TRANSFER OF TECHNOLOGY FOR IMPLEMENTING DOMAIN SPECIFIC SOFTWARE ARCHITECTURE}

\section{Vincent P. Heuring}

University of Colorado

Contacts have been established in the DOD software engineering and use community. Contacts were made with the Naval Research Lab and with the Naval Postgraduate School. With the cooperation of the NPS personnel, researchers are developing a processor for their prototype system description language, PSDL. They have developed a formal grammar for the language, and can successfully parse a PSDL specification. They intend to continue to work to demonstrate the usefulness of the Eli system to the NPS, and ultimately to transfer it to them, so they may use it in further development efforts.

\section{INTELLIGENT SUPPORT FOR HUMAN-COMPUTER INTERACTION AND DECISION-MAKING IN DISTRIBUTION PLANNING AND SCHEDULING SYSTEM}

Steven F. Roth

Carnegie-Mellon University

The development of mechanisms that promote and maintain diversity within genetic search has been a central concern in Michigan-style learning classifier system (LCS) research. What is required of the genetic algorithm (GA) in a LCS context is not convergence to a single global optimum, as in the standard, optimization framework, but instead the generation of individuals (i.e., rules) that collectively cover the overall problem space. With regard to the problem of generating a covering population one can identify two general functions that the search must provide: an ability to identify appropriate classification niches and an ability to generate high performance rules within identified niches. Selection and reproduction according to basic measures of individual rule fitness (e.g., strength) contributes directly to generation of higher performance individuals within identified niches, but only indirectly addresses the problem of uncovering new niches. To deal with this problem, one body of work has focused on extensions to the basic selection/reproduction/replacement cycle (e.g. partial matching techniques to broaden the applicability of current rules in the population and further bias search toward expansion of coverage, triggered operators to explicitly fill gaps in coverage when they are recognized, restricted mating policies to enhance relevance of generated offspring). Other more recent work has alternatively demonstrated the viability of population dependent fitness calculation (in conjunction with the traditional genetic search cycle) as a means of discovering and maintaining independent subpopulations (implicitly accomplishing effects similar to the use of explicit sharing functions without their accompanying restrictive assumptions). 
29692 COMPUTATION AND IMPLEMENTATION OF NON-MONOTONIC DEDUCTIVE DATABASES

\section{V.S. Subrahmanian}

University of Maryland

The research objective is to study the design and implementation of algorithms for efficient computing for non-monotonic reasoning and deductive databases. Researchers will utilize linear programming techniques and logic programming to develop a semantics-based approach to non-monotonic (commonsense) reasoning, hence building a formal connection between numeric and symbolic computations.

\section{MODELING CLOTHES AND ANTHROPOMETRY}

Normal I. Badler

University of Pennsylvania

The objective of the research is to improve biostereometric contour figure bodies for anthropometric scaling, and movement limitations imposed by biomechanics and clothing. New computerized data will be incorporated into existing models in order to provide a more useful facility for addressing basic issues related to anthropometrics.

\section{Reports:}

1. Computer Graphics Research Laboratory Quarterly Progress Report, by Norman I. Badler, TR, May 92, 34 pp.

\section{F. Special Projects}

\section{SIGNAL DETECTION AND ESTIMATION}

C.R. Rao

The Pennsylvania State University

Researchers have been able to resolve some key issues in multitarget tracking. The main problems addressed are design of observations, efficient estimation of target positions almost continuously in time and establishing correct association between targets and the estimates. A complete package was developed for angles only tracking, which can be implemented in real time without unduly heavy computations. The method can be extended to tracking targets in terms of positional coordinates, and for a variety of models for the observations. For angles only tracking, simulation results indicate that the proposed method performs well for any number of targets and even under low signal-to-noise ratio.

Reports:

No. $1-18$ in previous editions.

19. Multiple Target Angle Tracking Using Sensor Array Outputs, by C.R. Rao et al., MS, IEEE Trans on Aerospace and Electron Systs.

20. Moderate Deviations for Some Weakly Dependent Random Processes, by Tiefeng Jiang et al., MS, Stochastic Processes and Appl.

21. This number not used.

22. Multivariate Analysis Under $M$-Estimation Theory Using a Convex Discrepancy Function, by C. Radhakrishna Rao and Z.J. Liu, MS, Biometric $J$.

23. Multivariate Aspects of Shape Theory, by Colin R. Goodall and Kanti V. Mardia, MS, Ann Stat.

24. Statistical Shape Models in Image Analysis, by K.V. Mardia et al., MS, Biometrika.

25. Limiting Behavior of $M$-Estimators of Regression Coefficients in High Dimensional Linear Models I. Scale-Dependent Case, by Z.D. Bai and Y. Wu, MS, JMultivariate Anal.

26. Multitarget Angle Tracking an Algorithm for Data Association, by C. Radhakrishna Rao et al., MS, IEEE Trans on Signal Proc.

27. Size and Shape Analysis of Landmark Data, by I.L. Dryden and K.V. Mardia, MS, Biometrika.

28. Laws of Large Numbers and Moderate Deviations for Stochastic Processes with Stationary and Independent Increments, by Jiang Tiefeng et al.. MS, Stochastic Processes and Appl.

29. Some Results on Strong Limits Theorems for (LB)-SpaceValued Random Variables, by Xiangchen Wang et al., MS, Proc Am Math Soc

30. Approximation to the Distributions of $M$-Estimates in Linear Models by Randomly Weighted Bootstrap, by C. Radhakrishna Rao and L.C. Zhao, MS, Sankhya.

31. On the Non-Null Distribution of Some Estimators of Interclass Correlation in Familial Data, by Raja Velu and $M$. Bhaskara Rao, MS, Biometrics.

32. Asymptotic Distribution of Statistics Based on Quadratic Entropy and Bootstrapping, by Z.J. Liu and C. Radhakrishna Rao, MS, J Stat Planning Inference.

33. Weak Representation of Regression Estimates in Multivariate Linear Models Obtained by Minimizing a Convex Function of Residuals, by Z.D. Bai et al., MS, Statistica Sinica.

34. On the Consistency of $M$-Estimate in a Linear Model $\mathrm{Ob}$ tained Through an Estimating Equation, by L.C. Zhao and C. Radhakrishna Rao, MS, Stat \& Prob Let.

35. Measurement of Genetic Variation for Evolutionary Studies, by R. Chakraborty and C.R. Rao, MS.

36. Asymptotic Behavior of Maximum Likelihood Estimates of Superimposed Exponential Signals, by C. Radhakrishna Rao and L.C. Zhao, MS, IEEE Trans on Signal Proc.

37. Analysis of Nonlinear Time Series (and Chaos) by Bispectral Methods, by T. Subba Rao, MS, J Time Series Analysis.

38. A Note on Multitarget Angle Tracking, by C. Radhakrishna Rao et al., MS. 
39. On Size and Shape Analysis of Palmar Interdigital Areas, by P.D.L. Constable and K.V. Mardia, MS, J Appl Stat.

\section{PERFORMANCE ANALYSIS OF SUBSPACE BASED METHODS}

Bhaskar D. Rao

University of California, San Diego

SC: SWC

The main goal of this research is to obtain a thorough understanding of the performance of the high resolution subspace based methods like MUSIC (MUltiple SIgnal Classification) and ESPRIT (Estimation of Signal Parameters via Rotational Invariant Techniques) for the problem of resolving closely spaced sources from measurements obtained using a sensor array. Also of interest are the Numerical and Implementation (parallel architectures) issues relating to these methods. The results of recent work are as follows: (1) In the area of statistical analysis of these methods, an important result was obtained in the context of a Uniform Linear Sensor Array. It was shown that Optimally weighted MUSIC and Optimally weighted State Space Methods/ESPRIT have identical performance. Currently, efforts in this area are focussed on spatial subspace smoothing. In these methods, the spatial smoothing is done using the subspace basis vectors as opposed to smoothing of the data. Such methods are computationally simpler and appear to have potential. Another area of focus is the application of array processing methods to the spatial localization of neural sources using magnetoencephalogram. (2) Work on finding parallel implementations for the subspace algorithms has made significant progress. In particular, attention was paid to the problem of partitioning large problems onto smaller systolic arrays, and new efficient scheduling methods have been developed. The new scheduling methods are efficient in terms of making the best tradeoffs between speed and hardware complexity (local memory, FIFO size etc.). They are illustrated with the help of standard matrix operations which are central to array processing methods and other signal applications.

\section{Reports:}

No. $1-10$ in previous editions.

11. Efficient Scheduling Methods for Partitioned Systolic Algorithms, by Prashanth Kuchibhotla and Bhaskar D. Rao, MS.

12. Weighted Subspace Methods and Spatial Smoothing: Analysis and Comparison, by Bhaskar D. Rao and K.V.S. Hari, MS, IEEE Trans on Signal Proc.

\section{MULTILEVEL-MULTIRESOLUTION METHOD FOR IMAGE PROCESSING, A BAYESIAN FRAMEWORK FOR RECONSTRUCTING AND REPRESENTING SHAPES IN ROBOT VISION AND SPEECH RECOGNITION}

Basilis Gidas

Brown University

A statistical procedure has been designed for multiple object invariant recognition from data that may contain clutter, occlusions, noise, and other sensor or atmospheric degrading effects. The algorithm can identify a large number (thousands to tens of thousands) of objects; in SDI scenarios, it may classify targets - missile tyres, decoys, etc., - from launch to impact. It involves massive off-line computations, but the on-line computations are minimal. The procedure is appropriate for various applications such as: wafer character identification, warfare mine detection and classification, text-to-speech. A coherent statistical framework has been developed for estimating 3-D shapes (topographic maps), and surface composition (albedo, or dielectric properties) from a single noisy image. The method has been tested with video data acquired under uncontrolled illumination. Currently, the method is being used to construct topographic maps of Venus by analyzing the Synthetic Aperture Radar data of the Magellan spacecraft. The method is based on Random Surface models, and leads to constrained optimization problems. In speech recognition studies, a procedure radically different from current methods is being used. Nonparametric statistics and the recently discovered wavelets are being used to model the raw speech waveform. The method involves a nonlinear predication model. Low frequency components are modelled nonparametrically, while high frequency components are parametrized via a wavelet basis. This representation of the acoustic signal induced center stochastic processes which are parametrized by high-order Markov chains. The parameters of these chains represent intrinsic features of the signal. The intention is to combine these features with global linguistic constraints.

\section{Reports:}

1. A Nonlinear Multi-Grid EM Algorithm for Emission Tomography, by Basilis Gidas and H.M. Hudson, MS.

2. Asymptotics of Maximum Likelihood Estimators for the Curie-Weiss Model, by Francis Comets and Basilis Gidas, Ann Stat 19,557(1991). 
3. Image Analysis and Computer Vision, by Donald Geman and Basilis Gidas, MS.

4. Metropolis-Type Monte Carlo Simulation Algorithms and Simulated Annealing, by Basilis Gidas, MS.

5. A Variational Method for Estimating the Parameters of MRF from Complete or Incomplete Data, by Murilo P. Almeida and Basilis Gidas, MS.

6. Parameter Estimation for Gibbs Distributions from Partially Observed Data, by Francis Comets and Basilis Gidas, MS, Ann Appl Prob.

\section{SVD-BASED SIGNAL PROCESSING ALGORITHMS}

Franklin T. Luk

Cornell University

\section{SC: SWC}

Research interest has been broadened to include real time control. An important problem in control, namely, linear prediction, can be solved via the singular value decomposition (SVD) of a product of three matrices. A new algorithm was developed for this product SVD problem, and researchers proved that this method will always compute an accurate decomposition in that all relevant residual elements will be small. Efforts are being made to examine the feasibility of solving the linear prediction problem in real time by implementing the product SVD algorithm on a systolic machine.

\section{PARALLEL STRUCTURED OPTIMIZATION ALGORITHMS FOR INVERSE PROBLEMS}

John E. Dennis

Rice University

Professor Dennis and some colleagues at Rice and at Boeing Corporation have discovered some novel ways to pose important practical problems that lead to very promising algorithmic ideas suitable for parallel architectures. The class of problems being considered is the optimization of a nonlinear objective subject to nonlinear differential equation constraints, especially cases where there are coupled differential equations of different types. For example, the aircraft industry is interested in this problem in the case of optimal airfoil design where one constraint is a system of partial differential equations that determine the pressure pattern on a wing of a certain shape caused by the air flowing across it at a certain speed and angle of attack; another constraint is a system of differential equations whose solution corrects the shape of the airfoil given a pressure pattern. Traditional black-box methods require the designer to resolve the coupled system consisting of both systems of differential equations before the optimization method is allowed to increment the basic shape parameters of the airfoil to improve the design objective. The approach here is very different. In this particular instance, researchers uncouple the two constraints by allowing the nonlinear programming algorithm to control as optimization variables the pressure pattern input to the structural analysis code and the shape input to the cfd code. This inconsistency is resolved only in the limit as optimality is approached by adding to the nonlinear programming formulation of the problem constraints that the structures and cfd codes match input and outputs with each other.

Reports:

No. $1-13$ in previous editions.

14. On the Superlinear and Quadratic Convergence of PrimalDual Interior Point Linear Programming Algorithms, by Yin Zhang et al., MS, SLAM J Control and Optmztn.

15. The Predictor-Corrector Interior-Point Method as a Composite Newton Method, by R. Tapia et al., MS, SLAM J Control and Optmztn.

16. A Study of Indicators for Identifying Zero Variables in Interior-Point Methods, by A.S. El-Bakry et al., MS, SLAM J Control and Optmztn.

17. Superlinear and Quadratic Convergence of Primal-Dual Interior-Point Methods for Linear Programming Revisited, by Y. Zhang and R.A. Tapia, MS, J Optmztn Theory and Appl.

18. On the Convergence of Interior-Point Methods to the Center of the Solution Set in Linear Programming, by Yin Zhang and R.A. Tapia, MS, Math Operations Res.

19. An Interior-Point Method with Polynomial Complexity and Superlinear Convergence for Linear Complementarity Problems, by Jun Ji et al., MS, SLAM J Optmztn.

20. On the Convergence of the Iteration Sequence in PrimalDual Interior-Point Methods, by R.A. Tapia et al., MS, Math Prog.

21. A Superlinearly Convergent Polynomial Primal-Dual Interior-Point Algorithm for Linear Programming, by Yin Zhang and R.A. Tapia, MS, SLAM J Optmztn.

22. A Quadratically Convergent Iteration Algorithm for Linear Programming, by Y. Ye et al., MS, Math Prog.

23. A Superlinearly Convergent Iteration Algorithm for Linear Programming, by Y. Ye et al., MS, SLAM J Optmztn.

27524 MATHEMATICAL METHODS AND ALGORITHMS FOR REAL-TIME APPLICATIONS 
Charles K. Chui

Texas A \& M University

SC: SWC

Emphasis of research activities was placed on the development of wavelet theory. It has been possible to classify all wavelets, and to characterize these wavelets in terms of certain identities of Littlewood and Paley type. Dyadic wavelets are used for bandpass filtering in the frequency domain with binary separation of the scale levels (or frequency bands) for continuous-time filtering. This type of wavelets include "frames" and has been used very successfully by $S$. Mallat and his colleagues in image compression. $R$-wavelets (usually called wavelets) are used for discrete-time filtering. There are three types of $R$-wavelets, namely: (1) orthogonal wavelets such as those of Daubechies, Meyer, and BattleLemarie; (2) semi-orthogonal wavelets, such as the spline-wavelets of Chui and Wang, and (3) nonorthogonal wavelets such as the biorthogonal wavelets of Cohen, Daubechies, and Feauveau. Researchers have also given a general framework for the construction of all these three types of wavelets. In addition, work is in progress on developing wavelet packets for finer wavelet decomposition, using nonorthogonal or semiorthogonal wavelets. This generalization of the orthogonal wavelet packets of Coifman and Meyer is necessary in order to avoid distortion as a result of applying the Daubechies wavelets. As a bonus, spline functions can now be used to construct wavelet packets. The difficulties are the structure of the duals and more importantly, the problem of stability. One of the goals on the study of wavelets was to explore their potential applications, particularly for problems that require real-time processing. Researchers have been very successful in the work in acoustic signal analysis. For instance, one can now remove various types of noise from both voice and instrumental music online. Other applications are removal of echoes and detection of radar signals.

\section{Reports}

No. $1-7$ in previous editions.

8. $N$ x Oversampling Preserves Any Tight Affine-Frame for Odd $N$, by C.K. Chui and X. Shi, MS.

9. Realization of Neural Networks with one Hidden Layer, by C.K. Chui and X. Li, MS.

10. Wavelets and Signal Analysis, by C.K. Chui, MS.

11. Bessel Sequences and Affine Frames, by C.K. Chui and X. Shi, MS.

12. Non-Orthogonal Wavelet Packets, by C.K. Chui and Chun $\mathrm{Lj}, \mathrm{MS}$.

13. Characterizations of Fundamental Scaling Functions and Wavelets, by C.K. Chui and X. Shi, MS.
14. Wavelets - With Emphasis on Spline-Wavelets and Applications to Signal Analysis, by C.K. Chui, MS.

15. On Trigonometric Wavelets, by C.K. Chui and H.N. Mhaskar, MS, Constr Approx.

16. Wavelets on a Bounded Interval, by C.K. Chui and E. Quak, MS.

17. Cardinal Interpolation with Differences of Tempered Functions, by C.K. Chui et al., MS, Comput Math Applic.

18. Characterizations of Convexity in Terms of Bezier Coefficients I: Surfaces Over Triangles, by Z. Cheng and C.K. Chui, MS.

19. A General Framework of Compactly Supported Splines and Wavelets, by C.K. Chui and J.Z. Wang, MS, J Approx Theory.

20. Wavelets and Spline Interpolation, by Charles K. Chui, MS.

21. Approximation by Ridge Functions and Neural Networks with One Hidden Layer, by Charles K. Chui and Xin Li, MS, J Approx Theory.

22. Compactly Supported Box-Spline Wavelets, by C.K. Chui et al., MS, Approx Theory and Its Appl.

23. An Analysis of Cardinal Spline-Wavelets, by C.K. Chui and J.Z. Wang, MS, J Approx Theory.

24. On the Density of Radial Function Spaces and Stability of Radial Function Approximations, by M.D. Buhmann and C.K. Chui, MS, $J$ Approx Theory.

25. Wavelets and Multiscale Interpolation, by C.K. Chui and X. Shi, MS.

26. Computational and Algorithmic Aspects of CardinalSpline Wavelets, by C.K. Chui and J.Z. Wang, MS.

27. On Wavelet Analysis, by C.K. Chui, MS.

\section{HIGH RESOLUTION SIGNAL PROCESSING}

Donald W. Tufts

University of Rhode Island

Faster, more accurate resolution of reflected or selfgenerated energy from targets, decoys, and interferers is important for fast, effective battlefield reaction; faster, more accurate localization and tracking of the potential targets is equally important. The research is especially motivated by digital beamforming and formation of synthetic apertures in radar, electronic warfare, and communications. New results have been obtained which contribute to these goals. Better performance stems from (1) more realistic modeling of received signals, including propagation effects and the effects of potential occlusion and local multipath (2) direct modeling of the signal arrivals, rather than summarizing the received data by a few attributes. The methods must be adaptive because of the lack of detailed prior information and the difficulty in acquiring such information from a rapidly varying environment. They must be robust because of the possibilities of impulsive noise, missing data and outliers. And they must have high resolution because of 
multiple signals or signal-like interferences which are close in angle, frequency, range or other signal attributes and parameters. The result of the research will be the preliminary design of a situation assessment system in which the data are processed by a number of methods, each having its own advantages and disadvantages. The system must quickly perform the job of an expert exploratory data analyst.

Reports:

No. 1-5 in previous editions.

6. Resolving the Components of Transient Signals by a Multistage Procedure, by S. Umesh and D.W. Tufts, MS.

7. Estimation of the Signal Component of a Date Vector: Analysis of Old and New Matrix Based Methods, by Abhijit A. Shah and Donald W. Tufts, MS.

8. The Arithmetic Fourier Transform (AFT): Iterative Computation and Image Processing Applications, by Donald W. Tufts et al., MS.

9. Iterative Realization of the Arithmetic Fourier Transform, by Donald W. Tufts and Haiguang Chen, MS, IEEE Trans on Signal Proc.

10. A Suboptimum Linear Receiver Based on a Parametric Channel Model, by Melbourne Barton and Donald $\mathrm{W}$. Tufts, MS, IEEE Trans on Commun.

11. A Low Rank Weighted Matrix Approximation Method for Robust Estimation of Sinusoid Parameters, by Geoffrey S. Edelson et al., MS.

12. Estimation of a Signal Waveform From Noisy Data Using Low-Rank Approximation to a Data Matrix, by Donald W. Tufts and Abhijit A. Shah, MS, IEEE Trans on Signal Proc.

\section{SINGULAR VALUE DECOMPOSITIONS: GENERALIZATION, ALGORITHMS AND APPLICATIONS}

Gene H. Golub

Stanford University

SC: SWC

The progress of VLSI and wafer-scale technology has led to the development of high-performance digital signal processing systems. Reliability of such systems is obviously a critical issue. Algorithm-based fault tolerance has been proposed to meet this need, since the most common practice of triplicating hardware is often too expensive and too bulky for real time signal processing. The weighted checksum scheme, originally developed by Abraham and students provides a low-cost error protection for basic matrix operations. Their work was extended by Luk et al. to applications that include matrix equation solvers, triangular decompositions, and recursive least squares. A theoretical framework for error correction was developed for the cases of one error, two errors, and multiple errors. Researchers have shown how very general sequences of polynomials such as orthogonal polynomials, can be used to generate certain checksum values, thus lessening the effects of numerical roundoffs, and they also describe how the Lanczos algorithm can be used to implement the error correction step, thus reducing the amount of required work. The Lanczos algorithm was originally devised by Lanczos as a procedure for reducing an arbitrary matrix to a tridiagonal form having the same eigenvalue as the original matrix. The method has been found to be particularly useful for large sparse matrices and has a number of optimality properties with respect to convergence in the symmetric case. For non-symmetric matrices the algorithm has been less well understood, but in recent years there has been major progress in the development and understanding.

\section{ALGORITHMS AND ARCHITECTURE FOR HIGH SPEED SIGNAL PROCESSING}

Thomas Kailath

Stanford University

SC: SWC

This work covers new approaches to designing fault tolerant processor arrays for scheduling multiprocessor systems. Results in fault tolerant arrays include new polynomial time reconfiguration algorithms for certain redundancy architectures as well as their performance predictions. In the area of scheduling, new algorithms have been devised and time-complexity results for the scheduling problems in a multiprocessor system when communication delays are considered. An array of identical processing elements is a common VLSI and WSI architecture for parallel processing. Fault tolerance through reconfiguring a faulty array into a healthy one by exploiting spare PE's and routing hardware is an attractive approach. Researchers proposed a new reconfiguration model that uses three tracks along every routing channel and one row (or column) of spare PEs along each boundary of the array and presented an efficient algorithm for reconfiguration of this model and evaluated its performance. They also considered a multiple-track model that uses $m 1 / 2$ track wide channels and $m$ spare rows (or columns) along each boundary of the array and proposed a polynomial time algorithm for solving the 
combinatorial problem that underlies the reconfiguration procedure for arbitrary $m$. In scheduling, multiprocessor architectures were studied in the presence of communication delays among processors. Given a set of dependent tasks, the scheduling problem allocates the tasks to processors such that the pre-specified precedence constraints among the tasks are obeyed and certain criteria (such as computation time) are minimized. Several cases of the scheduling problem are known to be NP-complete. Nevertheless there are some polynomial time algorithms for special cases, but these do not take into consideration the delays due to message passing among processors. In this model, workers incorporated communication delays and, in particular, addressed the important problem of scheduling In-forests (Out-forests) in a multiprocessor system of $m$ identical processors.

Reports:

No. $1-7$ in previous editions.

8. Divide-and-Conquer Solutions of Least-Squares Problems for Matrices with Displacement Structure, by J. Chun and T. Kailath, SLAM J Matrix Anal Appl 12,128(1991). AD A238 943

9. A General Weight Matrix Formulation Using Optimal Control, by Oluseyi Farotimi et al., IEEE Trans on Neural Networks 2,378(1991), AD A239 586

10. Generalized Displacement Structure for Block-Toeplitz, Toeplitz-Block, and Toeplitz-Derived Matrices, by J. Chun and T. Kailath, Numerical Linear Algebra, Digital Signal Processing and Parallel Algorithms, 1991, p215. AD A238 377

11. Systolic Array Implementation of the Square-Root Chandrasekhar Filter, by J. Chun and T. Kailath, MS, Intl J Control.

12. Multiple Invariance ESPRIT, by A. Swindlehurst et al., MS IEEE Trans on Acoust, Speech, and Signal Proc.

13. Systolic Array for Solving Toeplitz Systems of Equations, by J. Chung et al., MS, IEEE Trans on Acoust, Speech, and Signal Proc.

14. Fast Triangular Factorization of Hermitian Toeplitz and Related Matrices with Arbitrary Rank Profile, by Debajyoti Pal and Thomas Kailath, MS, SLAM J Matrix Anal Appl.

15. On the Localization of Algorithms for VLSI Processor Arrays, by V.P. Roychowdhury et al., MS, IEEE Trans on Comp.

16. A Performance Analysis of Subspace-Based Methods in the Presence of Model Errors - Part I: The MUSIC Algorithm, by A. Swindlehurst and T. Kailath, MS, IEEE Trans on Acoust, Speech, and Signal Proc.

17. Algorithms for Azimuth/Elevation Direction Finding Using Regular Array Geometries, by $A$. Swindlehurst and T. Kailath, MS, IEEE Transon Aerospace and Electron Systs.

18. Consistent Estimation of Cyclic Autocorrelation, by Michael J. Genossar et al., MS, IEEE Trans on Signal Proc.
19. Information Theoretic Inequalities, by Amir Dembo and Joy A. Thomas, IEEE Trans on Info Theory 37,1501(1991). AD A249 551

20. Depth-Size Tradeoffs for Neural Computation, by KaiYeung Siu et al., IEEE Trans on Comp 40,1402(1991). AD A249 470

21. Computing with Almost Optimal Size Threshold Circuits, by Kai-Yeung Siu et al., MS.

22. Circuit Complexity for Neural Computation, by Kai-Yeung Siu et al., MS.

\section{DESIGN AND ANALYSIS OF SCALABLE PARALLEL ALGORITHMS}

\section{Vipin Kumar}

University of Minnesota, Minneapolis

Research is continuing on the development of parallel algorithms and data structures for a variety of numeric and nonnumeric problems. Researchers are particularly interested in the analysis of the parallel formulations so that the performance and scalability of these algorithms can be predicted on different kinds of large-scale parallel architectures. The intention is to find what important practical problems can be solved cost-effectively on large-scale parallel processors, and what architectural features are most critical in obtaining good performance. Research has determined best scalable parallel formulations of algorithms such as FFT, matrix multiplication, sorting, shortest path for a variety of practically feasible architectures such as mesh and hypercubes. Work has also determined that under certain situations, parallel formulations of some important search algorithms can provide superlinear speedup. Work has continued on development of new methods and metrics for understanding the scalability behavior of parallel systems. In particular, exploring scalable load balancing techniques for solving problems such as finite elements, particle dynamics, quantitative simulations, etc. The scalability of different sparse matrix algorithms for large scale parallel computers is being analyzed.
28428 A NEW APPROACH TO
DISTRIBUTED DECISION-MAKING
IN THE MILITARY COMMAND
CONTROL AND
COMMUNICATIONS $\left(C^{3}\right)$ SYSTEMS 
Sumit Ghosh

Brown University

\section{SL: TRADOC}

The key issues in synthesizing a new approach to distributed decision-making in the military $C^{3}$ systems are (a) the identification and modeling of the actual decision processes including the important parameters, $(b)$ the modeling of the communications systems, and (c) the management of the overall system in the event that congestion is created by excess information traffic and erroneous decisions. In the past year, the investigator has focused only on $(b)$ and (c). This research program observes that the third issue has a parallel in the area of communications, namely, broadband-ISDN (B-ISDN). While B-ISDN is still under investigation, it has been known for some time that internet and phone outages are caused by the presence of excess traffic and positive feedback that quickly leads to catastrophic failure of the entire network. Currently, distributed algorithms are being investigated for dynamic self-healing in the event of link failures. In this investigation, a large multimedia military network is decomposed into groups. A group consists of a set of nodes, based on geographical proximity and other factors. Failure and self-healing information within a group are propagated only to the elements of the group. This prevents the network from being flooded with information from a single link failure. In essence, it represents a compromise between a (a) totally distributed approach where a failure must be broadcast to every node and every node must maintain massive information database and $a(b)$ centralized approach wherein failure information is localized to a single node that is responsible for recovery. However, failure of inter-group links are broadcast to all groups and, thereafter, each group recomputes, in a distributed manner, new routes to be used for communication with other groups. This research is expected to yield data on the performance of dynamic and distributed self-healing algorithms for realistic, multimedia traffic data.

\section{SIGNAL PROCESSING FOR HIGH-RESOLUTION IMAGE FORMATION}

A. S. Karalamangala

University of lllinois

A paper has been prepared which addresses the problem of tomographic reconstruction from a few discrete projections. When the projection data are discrete and few in number, the image formed by the convolution back-projection algorithm may not be consistent with the observed projections and is known to exhibit artifacts. Hence, the problem formulated is one of finding an image that is closest to a nominal and is consistent with the projection data and other convex constraints such as positivity. The measure of closeness used is a Hilbert space norm, typically a weighted sum/integral of squares, with weights used to reflect expected deviation from the nominal in different regions. In the absence of constraints, this approach leads to a direct, non-iterative algorithm (based on a simple matrix-vector computation) for construction of the image. When additional convex constraints such as positivity and upper-bounds need to be enforced on the reconstructed image to improve resolution, a quadratically-convergent Newton algorithm is suggested. Another paper addresses the problem of reconstructing band-limited signals from a finite number of observations. The algorithms presented in the paper exploit the reduced dimension of the set of signals which are band-limited and essentially timelimited. A criterion is presented for choosing the dimension of the reduced signal space based on knowledge of the passband, time-concentration interval, energy concentration factor, and bounds on the tolerable reconstruction error. The reconstruction is constrained to lie in this lower-dimensional signal space, and parameters characterizing the reconstruction are obtained from the data by solving a linear system of equations.

\section{SIMULATION METHODOLOGY}

Donald L. Iglehart

Peter W. Glynn

Stanford University

Research emphasized work on the initial transient problem in simulation output analysis. In a steadystate simulation, the term "initial transient" refers to the initial segment of the simulation that is contaminated by bias introduced by starting in some state that is not typical of the long-run behavior of the system. The principal approach used to deal with this problem is to delete an initial segment of the simulation before collecting observations to estimate the steadystate performance of the system. While the simulation community has been challenged by this problem 
for many years, no technique yet proposed satisfactorily solves this problem. This research deals with regenerative processes and Markov processes with a finite, countable, or general state space. It is shown that the tail of the distribution of the cycle length plays an important role in being able to detect stationarity. In fact it is shown that without some restrictions on the class of simulation considered, no universally satisfactory means for detecting stationarity in a stochastic simulation exists. However, when some special structure exists, algorithms are given for simulating a process which is close to being stationary.

\section{CENTER FOR NONLINEAR ANALYSIS}

Morton E. Gurtin

David Kinderlehrer

Carnegie-Mellon University

The Center is an ideal environment for graduate work. The intellectual activity revolves around workshops, seminars, and study groups on problems of current interest. These activities furnish an excellent opportunity for graduate students to become acquainted with current work, and to discuss their ideas and results with scientists of greatly varied backgrounds. The visitor and workshop programs involve both mathematicians and physical scientists, and maintain close connections with Carnegie-Mellon faculty in allied areas. This year the Center is host to postdocs and a large number of visiting scientists; the result is an environment in which new ideas are exchanged on a daily basis. The Center is a joint venture with Hampton University, a Historically Black University. In collaboration with Hampton, the Center has been trying to recruit qualified students from Historically Black Colleges or Universities or Minority Institutions. The Center with its workshops and visitors fosters collaboration with engineers and physicists; such collaborations are vital to the interdisciplinary development of science, raising the scientific level of all involved parties. In particular, the Research Workshops, which are intense workshops involving a small number of scientists working in a clearly defined area, are specifically intended to induce collaborative research.

\section{Reports:}

1. Micro-Structure Models of Diffusion in Fissured Media, by R.E. Showalter and N.J. Walkington, J Math Anal and Appl 155,1(1991). AD A249 395
2. A Hyperbolic Theory for the Evolution of Plane Curves, by Morton E. Gurtin and Paolo Podio-Guidugli, SLAM J Math Anal 22,575(1991). AD A249 291

3. A Free Boundary Problem Related to Singular Stochastic Control, by S.E. Shreve and H.M. Soner, Proc Imperial College Workshop on Applied Stochastic Analysis, 1989, p265. AD A249 396

4. An Example of Frustration in a Ferromagnetic Material, by Richard D. James and David Kinderlehrer, Nematics, 1991, p201. AD A249 397

5. Recent Developments in Liquid Crystal Theory, by D. Kinderlehrer, Frontiers in Pure and Applied Mathematics, 1991, p151. AD A249 174

6. On the Thermodynamics of Viscoelastic Materials of Single-Integral Type, by Morton E. Gurtin and William J. Hrusa, Quart Appl Math 49,67(1991). AD A249 398

7. Axially Symmetric Acoustic Wave Propagation Through Flows with Vorticity, by Noel J. Walkington, SLAM ISci Stat Comput 12,1438(1991).

8. On Thermomechanical Laws for the Motion of a Phase Interface, by Morton E. Gurtin, J Appl Math Phys 42,370(1991). AD A249 292

9. Global Existence of Smooth Solutions to the Vlasov Poisson System in Three Dimensions, by Jack Schaeffer, Commun in Partial Diff Eq 16,1313(1991).

10. Characterizations of Young Measures Generated by Gradients, by David Kinderlehrer and Pablo Pedregal, Arch Ratl Mech Anal 115,329(1991). AD A249 666

11. The Variety of Configurations of Static Liquid Crystals, by Robert Hardt et al., Variational Methods, 1990, p115. AD A249630

12. A Free Boundary Problem Related to Singular Stochastic Control: The Parabolic Case, by H,M. Soner and S.E. Shreve, Commun in Partial Diff Eq 16,373(1991). AD A249 118

13. Diffusion of Fluid in a Fissured Medium with Microstructure, by R.E. Showalter and N.J. Walkington, SLAM JMath Anal 22,1702(1991). AD A249 293

14. A Hyperbolic Stefan Problem, by R.E. Showalter and N.J. Walkington, Rocky Min J Math 21,787(1991). AD A249 294

15. Caracterisation des Mesures de Young associees a un Gradient, by David Kinderlehrer and Pablo Pedregal, $C . R$ Acad Sci Paris 313,765(1991). AD A249 413

16. Jacobians and Hardy Spaces, by P.L. Lions, MS.

17. Uniqueness and Singularities of Cylindrically Symmetric Surfaces Moving by Mean Curvature, by H.M. Soner, MS.

18. Second Variation of Liquid Crystal Energy at $x / x$, by David Kinderlehrer and Biao Ou, MS.

19. Two-Phase Continuum Mechanics with Mass Transport and Stress, by Morton E. Gurtin, MS.

20. On the Thermodynamics of Periodic Phases, by Bernard D. Coleman et al., MS, Arch Ratl Mech Anal.

21. The Continuum Mechanies of Coherent Two-Phase Elastic Solids with Mass Transport, by Morton E. Gurtin and Peter W. Voorhees, MS.

22. Co-Volume Methods for Degenerate Parabolic Problems, by Lisa $A$. Baughman and Noel Walkington, MS.

23. A Regularized Equation for Anisotropic Motion-by-Curvature, by Antonio Di Carlo et al., MS, SLAM J Math Anal.

24. Evolving Phase Boundaries in Deformable Continua, by Morton E. Gurtin, MS. 
25. Non-Monotonic Transformation Kinetics and the Morphological Stability of Phase Boundaries in Thermoelastic Materials, by Eliot Fried, MS.

26. Multi-Dimensional Finite-Fuel Singular Stochastic Control, by David S. Bridge and Steven E. Shreve, MS.

27. Singular Perturbations in Manufacturing, by $\mathrm{H}$. Mete Soner, MS, SLAMJ Control and Optmztn.

28. Anisotropic Motion of a Phase Interface. Well-Posedness of the Initial Value Problem and Qualitative Properties of the Interface, by Sigurd B. Angenent and Morton E. Gurtin, MS.

29. Frustration and Microstructure: An Example in Magnetostriction, by Richard D. James and David Kinderlehrer, MS.

30. On Mathematical Tools for Studying Partial Differential Equations of Continuum Physics: H-Measures and Young Measures, by Luc Tartar, MS.

31. Computation of Microstructure Utilizing Young Measure Representations, by R.A. Nicolaides and Noel J. Walkington, MS

32. On the Slow Dynamics for the Cahn-Hilliard Equation in One Space Dimension, by Lia Bronsard and Danielle Hilhorst, MS, Proc Roy Soc (London).

33. Memory Effects in Homogenisation Linear Second Order Equation, by Nenad Antonic, MS.

34. Thermodynamics and the Supercritical Stefan Equations with Nucleations, by Morton E. Gurtin, MS, Quart Appl Math.

35. Remarks About Gradient Young Measures Generated by Sequences in Soblev Spaces, by David Kinderlehrer and Pablo Pedregal, MS

36. On the Formulation of Mechanical Balance Laws for Structured Continua, by Morton E. Gurtin and Paolo PodioGuidugli, Z Angew Math Und Phys 43,181(1992).

37. Generalized Interface Evolution with the Neumann Boundary Condition, by Yoshikazu Giga and Moto-Hiko Sato, Proc Japan Academy 67,263(1991).

38. H-Measures, a New Approach for Studying Homogenisation, Oscillations and Concentration Effects in Partial Differential Equations, by Luc Tartar, Proc Roy Soc (Edinburg) 115A,193(1990). AD A249 212

\section{CENTER OF EXCELLENCE IN MATHEMATICAL SCIENCES}

Anil Nerode

Cornell University

Mathematical Sciences Institute at Cornell University developed long-term administrative links with the State University of New York at Stony Brook and with the University of Puerto Rico to coordinate research activities of the newly established Army Research Office Center of Excellence: the Center of Excellence in the Mathematical Sciences for Symbolic Method in Algorithmic Mathematics, the Center of Excellence in the Mathematical Sciences for Stochastic Analysis, and the Center of Excellence in the Mathematical Sciences for Nonlinear Analysis. In addition, significant research activities were undertaken in cooperation with the Harvey Mudd College Mathematics Program. The three Centers hosted seven workshops and conferences. Of these, three met in Ithaca, New York, two met in Stony Brook, New York, one met in Washington, DC, and one met in Claremont, California. Institute sponsorship continued for significant series of well-attended workshops on hybrid systems control and on computational methods. US Army scientists have attended and presented papers in both workshop series. The Mathematical Sciences Institute is recognized as a world leader in these research areas and further conferences are being planned. In addition, the MSIsupported workshop on patch dynamics, held during this reporting period, was extremely successful and this research will be extended during the coming year. Outreach activities included more than thirty public seminars presented by MSI-affiliated faculty, post-doctoral associates, graduate students, and staff. A major outreach to industrial consumers of mathematics was the MSI-cosponsorship of the Modern Computational Method in Industrial Mathematics workshop held this past summer in Claremont, California. Articles about research activities at the three Centers have appeared in the MSI News, the SIAM News, the CIT News, the Notices of the American Mathematical Society, the Comell Chronicle, the Ithaca Journal, and Connecting with Cornell. Reports on recent research will appear in the newsletters of various SIAM activity groups. The Mathematical Sciences Institute continues to expand its capabilities for the distribution of research results and announcements via electronic media.

Reports:

1. Fixation Results for Threshold Voter Systems, by Richard Durrett and Jeffrey E. Steif, MS, Ann Prob.

2. Extension Spaces of Oriented Matroids, by Bernd Sturmfels and Gunter M. Ziegler, MS

3. Estimating the Critical Values of Stochastic Growth Models, by L. Buttel et al., MS, I Appl Prob.

4. A Note on Polynomial Reduction, by Alyson Reeves and Bernd Sturmfels, MS.

5. On the Asymptotic Distribution of Large Prime Factors, by Peter Donnelly and Geoffrey Grimmett, MS, $J$ London Math Soc.

6. Maximal, by Beernd Sturmfels and Andrei Zelevinsky, MS.

7. On the Rate of Convergence of the Nonlinear Galerkin Methods, by Christophe Devulder et al., MS.

8. A Context for Belief Revision: Normal Logic Programs, by W. Marek et al, MS.

9. Theory Tableaux, by Ian Gent, MS. 
10. The Evaluation of the Anisotropy of a Polycrystalline Aggregate, by Ying Zhang and James T. Jenkins, MS, J Mech Phys Sol.

11. Multigraded Resultants of Sylvester Type, by Bernd Sturmfels and Andrei Zelevinsky, MS.

12. Computing Circumscriptive Deductive Databases, by Anil Nerode et al., MS.

13. Computation and Implementation of Non-Monotonic Deductive Databases, by Colin Bell et al., MS.

14. The Lexicographic Order Isn't Necessarily the Worst, by Alyson A. Reeves, MS.

15. Competitive Coexistence in a Seasonally Fluctuating Environment, by Toshiyuki Namba, MS.

16. On Branching Numbers of Normal Manifolds, by Daniel Ralph, MS.

17. Particle Systems and Reaction-Diffusion Equations, by R. Durrett and C. Neuhauser, MS, Ann Prob.

18. Computing Circumscriptive Databases, Part I: Theory and Algorithms, by Anil Nerode et al., MS.

19. A Fast Algorithm for Path Integration, by Dov Bai, MS.

20. Product Formulas for Sparse Resultants, by Paul Pedersen and Bernd Sturmfels, MS.

21. Approximating Oracle Machines for Combinatorial Optimization, by Shmuel Onn, MS.

22. A Dynamical Simulation Facility for Hybrid Systems, by Allen Back et al., MS.

23. Multigrid Preconditioning for the Biharmonic Dirichlet Problem, by M.R. Hanisch, MS.

24. The Expressiveness of Locally Stratified Programs, by Howard A: Blair et al., MS.

25. Nontrivial Dynamics in a Driven String with Impact Nonlinearity, by Theo $P$. Valkering, MS.

26. A Note on the Primal-Dual Affine Scaling Algorithms, by Levent Tuncel, MS.

27. On the Convergence of Primal-Dual Interior Point Methods with Wide Neighborhoods, by Levent Tuncel, MS.

28. Primitive Polynomial Remainder Sequences in Elimination Theory, by Michael Kalkbrener, MS, Applcbl Algebra in Eng, Commun and Comp.

29. A Generalized Euclidean Algorithm for Computing Triangular Representations of Algebraic Varieties, by Michael Kalkbrener, MS, $J$ Symb Computation.

30. Initial Complexes of Prime Ideals, by Michael Kalkbrener and Bernd Sturmfels, MS, J Algebraic Geometry.

31. A Generalized Euclidean Algorithm for Geometry Theorem Proving, by Michael Kalkbrener, MS.

32. Asymptotic Behavior of Excitable Cellular Automata, by Richard Durrett and David Griffith, MS.

\section{LARGE DEVIATION LIMIT THEOREMS, WITH APPLICATIONS}

N.R. Chaganty

Old Dominion University

For a complex digital communication system the symbol error probability is an important parameter in the determination of system performance. Hence the problem of evaluating error probability is an important one in the area of digital communication. The error probability goes by different names: it is known as false-alarm rate for engineers, tail probability for statisticians and the probability of large deviations for probabilists. For complex systems, detailed analyis of the false-alarm rate purely by analytical methods is almost impossible and one has to resort to Monte-Carlo methods, which requires the simulation of the whole system a large number of times. This may not be possible if one takes the cost factor into consideration and hence in most cases is almost impossible. In this research studies have been made of the performance of the nonparametric method, known as Bootstrap, to estimate the falsealarm rate. The Bootstrap method is basically a resampling procedure which can be used to simulate the sampling distribution of a given statistic based on the observed data. It is well known that this method works well for estimating standard deviation of the statistic, but what is not known is the performance of this method in the area of large deviations, that is, estimation of the false-alarm rate. The recent research has shown that indeed the bootstrap method does give consistent estimates for the probability of large deviations for almost all samples of observed data, which is a very positive result. It has been also shown that if one uses bootstrap estimates averaged over different observed samples, one will be slightly over-estimating the actual probability.

\section{DISCRETE EVENT DYNAMIC SYSTEMS MODELING AND OPTIMIZATION WITH APPLICATIONS TO $\mathrm{C}^{3} I$ PROBLEMS}

Yu-Chi Ho

Harvard University

A number of papers were published and submitted for publication. In one, authors present the stochastic comparison (SC) algorithm which is a further development of the ordinal optimization method. The SC algorithm is aimed at solving optimization problems with noisy estimates of the objective function to be optimized. This problem often occurs in the design of the computer/communication systems and many logistic problems. The basic idea of a stochastic comparison algorithm is to adopt a test number sequence which monotonicly increases to infinity and smoothly leads the optimization from ordinal-based comparisons at the initial stages to cardinal-based compari- 
sons in the end game. Proof of the convergence properties of the SC algorithm is given based on an innovative analysis for finite state Markov chains and the classical theory about the time inhomogeneous Markov chains. Numerical examples show that the SC algorithm outperforms existing algorithms. A second paper presents results of using general rational approximants as a performance evaluation tool for obtaining a response surface in stochastic discrete event systems with remarkable accuracy. Four papers report findings on derivative estimation in some discrete event systems and their possible application in communication networks. A powerful new parallel simulation scheme and its implementation on a massively parallel system was also reported.

\section{JUMP-DIFFUSION PROCESSES FOR OBJECT TRACKING AND DIRECTION FINDING}

Michael I. Miller

Washington University

$$
\begin{aligned}
& \text { SL: ARL/ACISD } \\
& \text { SC: NVESD }
\end{aligned}
$$

The objective of the research is to develop mathematical and computational models to address problems in spectrum estimation and direction finding when the underlying tracking scenarios have an unknown number of objects, dynamically moving past sensor arrays, with object tracks of variable length and shape. The research employs a Bayesian approach based on Gibbs models, under a Gaussian assumption. The order of the model (number of tracks) is not apriori known. Instead, the state space is taken to be a countable product space of models, each of finite order. A random sampling procedure, based on diffusions with invariant measures being the posterior densities of the particular models of varying order, is used to construct a jump diffusion process which generates most likely tracks.

\section{SIMULATION NETWORK}

Peter W. Glynn

Donald L. Iglehart

Stanford University

This effort is devoted to the development of an electronic network to facilitate information exchange within the stochastic simulation community. In the autumn of 1991, the principal investigators acquired a work station together with three $\mathrm{x}$-terminals. In addition, the software upon which the electronic network NA-NET is based was obtained from Oak Ridge National Laboratory; NA-NET is a network that links researchers in the field of numerical analysis. A number of compatibility problems had to be overcome, but the NA-NET software has now been modified to run on the DEC workstation, and to perform the basic postmaster duties envisioned for SNET. SNET will eventually include software libraries for test problems, papers, and output analysis algorithmis, in addition to its newsletter and electronic mail forwarding features.

\section{NEW ALGORITHMS FOR THE REDUCTION OF IMAGE DEGRADATION DUE TO ATMOSPHERIC TURBULENCE}

\section{B. Roy Frieden}

University of Arizona

The research objective is to establish the effectiveness of two algorithms for reconstructing an optical image in the presence of atmospheric turbulence and random motion of the optical image sensor. Attempts will be made to modify and extend the Knox-Thompson approach to image reconstruction. 


\section{ENGINEERING SCIENCES}

\section{A. Solld Mechanics}

\section{THREE-DIMENSIONAL ELASTIC STRESS ANALYSIS IN MATERIALS WITH MANY INHOMOGENEITIES}

\author{
Mark Kachanov \\ Tufts University \\ SL: ARL/MD \\ SC: ARDEC, ARL/WTD
}

Work has continued on stress analysis in elastic solids with multiple interacting holes. The method of analysis of many cracks' problems developed earlier was modified and extended to account for the interaction of closely spaced circular holes. The results thus obtained agree well with the available existing solution for several symmetric configurations. Several physically important aspects of the problem were examined (large hole, small hole interaction; interaction of a hole with a random field of microholes, etc.). Work on effective elastic properties of cracked solids continued. The possibilities provided by the continuum description of crack fields and limitations of such description were further investigated. The differential and self-consistent schemes were also examined, as well as the possibility of bounding the effective moduli. Work on mechanics of interactions of a crack with holes was started. This work has applications in mechanics of porous brittle materials and is currently in progress. A combined theoreticalexperimental study of microcracking in ceramics has been started.

Reports:

No. $1-8$ in previous editions.

9. On Continuum Characterization of Crack Arrays and Correlations Between Fracturing and Change of Effective Elastic Moduli, by Mark Kachanov, MS.

10. Effective Elastic Properties of Cracked Solids: Critical Review of Some Basic Concepts, by Mark Kachanov, MS, Appl Mech Rev.

\section{STUDIES IN PENETRATION MECHANICS}

Romesh C. Batra

University of Missouri at Rolla

\section{SL: ARL/WTD \\ SC: ARDEC}

Researchers studied the steady state axisymmetric deformations of a thick target being penetrated by a rigid cylindrical penetrator with a hemispherical nose and used three different constitutive relations, namely, the Litonski-Batra flow rule, the BodnerPartom flow rule, and the Brown-Kim-Anand flow rule, to model the thermoelastic-viscoplastic response of the target. Each of these constitutive relations uses an internal variable to account for the microstructural changes in the body. The three flow rules are calibrated to give virtually identical effective stress-logarithmic strain curves during the overall adiabatic plane strain compression of a block of the target material deformed at an average strain rate of $3300 \mathrm{~s}^{-1}$. It is found that the three constitutive relations give nearly the same value of the resisting force acting on the penetrator, temperature rise of material particles in the vicinity of the target-penetrator interface, and other macroscopic measures of deformation, such as the effective stress and logarithmic strain rate.

Reports:

No. 1-11 in previous editions.

12. Analysis of Steady State Axisymmetric Penetration Problems by the Finite Element Method Using a Mixed Formulation, by R. Jayachandran, PhD Thesis, 1991, 117 pp.

13. Steady State Axisymmetric Deformations of a Thick Elastic-Thermoviscoplastic Target, by R. Jayachandran and R.C. Batra, Intl J Eng Sci 30,1009(1992).

14. Effect of Constitutive Models on Steady State Axisymmetric Deformations of Thermoelastic-Viscoplastic Targets, by R.C. Batra and R. Jayachandran, MS, Intl J Impact Eng.

15. Effect of Frictional Force on the Steady State Axisymmetric Deformations of a Viscoplastic Target, by Xingju Chen and R.C. Batra, MS, Acta Mech. 


\section{WAVE PROPAGATION AND DYNAMIC RESPONSE OF LAMINATED STRUCTURES}

R.K. Kapania

J.N. Reddy

Virginia Polytechnic Institute and State University

SL: AP TEC DIR

SC: AERO DIR, ARL/WTD, ARL/VSD

The research continued on the use of reduction methods in reducing the enormous computational times otherwise required in determining the nonlinear impact response of imperfect laminated plates. The response was obtained using a 48 degree-of-freedom finite element developed previously by Kapania and coworkers. Reduction methods using two different types of expansion modes as the basis vectors were employed: $(a)$ those based on eigenvectors for the system under consideration, and $(b)$ those based on Ritz vectors. These methods were used with and without basis updating and the resulting responses were compared with those obtained by solving the full set of equations, the so called direct solution. It was observed that basis updating is essential to obtain accurate results. When applied to the impact response problem, it was seen that a large number of basis vectors are needed to account for the high frequency characteristics of the impact response. Furthermore, the reduction achieved in the computer time was found to be marginal (only about 7 percent). The inefficiency of the reduction methods was traced to the fact that most of the CPU time is spent in calculating the internal nodal force vector. Research is continuing to develop reduction methods that can reduce this time. Efforts are also being made to study the impact response of laminated plates analytically using higher-order theories. To that end, a free vibration analysis of general cross-ply laminated plates has been carried out using various shear deformation theories.

Reports:

1. A Study of Nonlinear Dynamic Equations of Higher-Order Shear Deformation Plate Theories, by A. Nosier and J.N. Reddy, Intl J Non-Linear Mech 26,233(1991). AD A245114

2. Prediction of Interlaminar Stresses in Laminated Plates Using Global Orthogonal Interpolation Polynomials, by Chansup Byun and R.K. Kapania, MS, ALAA J.

3. Free Vibration and Nonlinear Transient Analysis of Imperfect Laminated Structures, by Chansup Byun, PhD Thesis, 1991, 195 pp.

4. Nonlinear Impact Response of Thin Imperfect Laminated Plates Using a Reduction Method, by Chansup Byun and Rakesh K. Kapania, Composites Eng 2,391(1992)
5. Reduction Methods Based on Eigenvectors and Ritz Vectors for Nonlinear Transient Analysis, by Rakesh K. Kapania and Chansup Byun, MS, Comp Mech.

6. Nonlinear Impact Response of Thin Imperfect Laminated Plates Using a Reduction Method, by Chansup Byun and R.K. Kapania, MS, Composites Eng.

7. Vibrations of Imperfect Laminated Panels Under Complex Preloads, by Rakesh K. Kapania and Chansup Byun, Intl J Non-Linear Mech 27,51(1992). AD A249 669

\section{DURABILITY PREDICTIONS FOR ADHESIVELY BONDED JOINTS IN HUMID CONDITIONS}

\section{Gregory J. Rodin}

Kenneth M. Liechti

University of Texas at Austin

\section{SL: BRADEC, ARL/MD}

SC: ARDEC

Three temperature/humidity cabinets have been fabricated. They are each simultaneously controlled by a personal computer which allows arbitrary and independent temperature/humidity conditions to be specified. Mass uptake measurements are now being made on a modified Bisphenol A Epoxy with an amido amine hardener. This material has been chosen after insurmountable problems were encountered in producing defect-free specimens made of the adhesive 3-H. The material was extremely difficult to process and, in tensile creep testing, underwent shear banding at multiple sites which introduced an unacceptable degree of scatter in the tests. Unfortunately, the diffusion characteristics of the material had been obtained over a wide range of temperatures and humidity levels and the tests will now have to be repeated for the material. However, the availability of three additional environmental characters will greatly accelerate the process. The testing of miniature shear specimens in a modified shear test fixture is continuing. The material is bonded between aluminum adherends which have been treated with a silane coupling agent. The latter delays the onset of debonding until significant cohesive damage has occurred in the adhesive. The onset of shear banding is much better controlled than before and more consistent shear deformation data is being produced. However some uncertainty is produced by slight misalignments of the specimen and real time measurements of the displacements in the specimen are being made using the geometrical moire' technique. It is expected that the measurements will allow suitable modifications of the specimen loading to be made. 


\section{THEORETICAL, EXPERIMENTAL AND COMPUTATIONAL STUDIES IN PLASTICITY}

Elias C. Aifantis

Michigan Technological University

$$
\begin{array}{ll}
\text { SL: } & \text { BWL, ARL/MD } \\
\text { SC: } & \text { ARDEC, ARL/WTD }
\end{array}
$$

Progress has been made towards establishing and setting up the relevant experimental procedures and equipment; developing constitutive relations for large plastic deformations; and numerically investigating anisotropy and shear banding. In particular, the following has been established: $(a)$ The method of photoelasticity has been utilized to examine inhomogeneous plastic flow including evaluation of yield surfaces and localized shear banding. The method has been employed investigating the shear banding phenomenon in hot rolled 1018 steel and cold rolled 1018 steel at high strains, yielding approximate measurements for the band width. A number of photoelastic coatings with different thicknesses, adhesives and elongations has been examined. Moreover, a biaxial apparatus for measuring the distortion of the yield surface has been designed and manufactured. This is being used for determining the evolution of the yield loci of engineering materials with cruciform plate specimens. (b) The theoretical work has involved the development of a framework for describing plastic deformation with emphasis on predicting anisotropy, noncoaxiality and their implication to the phenomena of axial effects in torsion and shear banding at finite strains. To this end, models based on dislocation slip have been developed. They consider materials rotations (plastic spin), anisotropy and texture (internal or back stress) and plastic inhomogeneity (higher order strain gradients). (c) The numerical work includes the evaluation of the aforementioned models in predicting shear banding and axial effects in torsion; and their implication to specific problems such as high ballistic penetration and the manufacturing processes of deep drawing and metal cutting. The computational effort has been focused on the implementation of the models in finite element programs such as DYNA3d. In addition, new software has been developed for evaluating the effect of higher order strain gradients.

\section{Reports:}

1. On the Stability of Finite Plastic Deformations, by H.M. Zbib and E.C. Aifantis, MS.

2. On Finite Deformation Plasticity with Directional Softening Part II: Two-Component Model, by M.F. Shi et al., MS.
3. On the Structure and Width of Shear Bands in Finite Elastoplastic Deformations, by H.M. Zbib and E.C. Aifantis, MS.

4. Measurement of Shear Band Characteristics in Low Carbon Steel Using Photoelasticity, by G.L. Panger Jr. et al., MS.

\section{CONTINUUM MECHANICAL MODELING OF SOLID UNDERGOING A PHASE TRANSFORMATION}

Rohan Abeyaratne

Massachusetts Institute of Technology

\section{SC: ARL/WTD, ARL/MD}

Efforts are being made to develop a complete thermomechanical continuum model that describes the behavior of solids undergoing a thermoelastic phase transformation. The model is based on constructing an appropriate Helmholtz free-energy potential from which the theory then stems. A model has been constructed which accounts for the presence of the austenitic phase and two variants of martensite; each of these three phases is characterized by a constant elastic modulus, a constant specific heat, and a constant coefficient of thermal expansion. The isothermal mechanical response of the material at different temperatures and the thermal response of the material at different stress-levels have been examined. The model appears to predict many of the features observed in experiments on shape memory materials. Efforts have continued on a study of the role of surface structure; i.e., the energy and stresses within the interface separating two phases. The specific problem being examined is the stability of a steadily propagating planar phase boundary. Experimental observations show two types of phase boundaries: planar and zig-zag (needle-like). Typically, the planar boundaries propagate slowly while the needles propagate very rapidly. Attempts are being made to determine whether this is due to an instability, where a slow planar boundary becomes unstable and develops a needle-like geometry if it propagates too fast. If this was the case, it would be analogous to the Taylor-Saffman instability of the interface between two fluids.

\section{Reports:}

1. On the Occurrence of the Cavitation Instability Relative to the Asymmetric Instability Under Symmetric Dead-Load Conditions, by Rohan Abeyaratne and Hang-Sheng Hou, $J$ Mech Appl Math 44,429(1991). AD A245 242

2. Void Collapse in an Elastic Solid, by Rohan Abeyaratne and Hang-Sheng Hou, J Elasticity 26,23(1991). AD A244 759 
3. Reflection and Transmission of Waves From an Interface with a Phase-Transforming Solid, by Rohan Abeyaratne and James K. Knowles, J Intell Mater Syst and Struct 3,224(1992).

4. A Study of Combined Asymmetric and Cavitated Bifurcations in Neo-Hookean Material Under Symmetric Dead Loading, by Hang-Sheng Hou, MS, J Appl Mech.

\section{2 \\ D. Krajcinovic \\ M.P. Mignolet \\ Arizona State University}

DEFECTS IN COMPOSITES

SL: BWL, ARL,VSD

The first of two tasks was focused on the determination of stress and strain fields during the polymerization. During curing process the temperature in the matrix is not homogeneous. Temperature gradients develop due to the chemical shrinkage associated with cross-linking and temperature shrinkage associated with cooling. At the same time the rupture strength of the as yet incompletely cross-linked matrix is well below the strength of the fully cured matrix. The initial phase of the work on this problem was centered on the determination of the mean field estimates of the stress and strain field in a resin subjected to a given curing regime. The stresses and strain are determined as a function of the position (across the thickness) and time. The proposed model couples the exothermic heat diffusion equation and a viscoelastic stress-strain relationship in form of a hereditary integral. Both equations account for the change in transport properties attributable to the increase in cross-linking (related to the degree of cure). In the second task, an attempt to explain the fiber clustering in thermosets, the low Reynolds number flow through an array of rigid cylinders (fibers) has been investigated. The present study emphasized the determination of the forces exerted by the viscous fluid (resin) on the cylinders (fibers) forming an arbitrary array. These results were subsequently used to study the stability of a slightly imperfect array of fibers.

Reports:

1. Cure Induced Inelastic Deformation in Thermosetting Polymers, by Kaushik Mallick and D. Krajcinovic, MS.

2. Micromechanics of Critical States, by D. Krajcinovic et al., MS.

3. Fiber Clustering During the Curing of Composite Materials, by Marc P. Mignolet, MS.

\section{EFFECT OF NOSE SHAPE AND MASS OF THE IMPACTOR ON IMPACT DAMAGE OF LAMINATED COMPOSITE SHELLS}

Fu-Kuo Chang

Stanford University

$$
\text { SC: BWL }
$$

As the impact resistance of laminated composites strongly depends upon the interfacial strengths of the composites, the experiments have been focused on evaluating impact damage in composites with toughened interfaces. T800/3900-2 graphite/epoxy composite was selected and studied. The surfaces of the T800/3900-2 unidirectional prepregs are coated with thermoplastic amorphous polyamide particles during the manufacturing. The thin polyamide coating is to serve as a toughening agent for interface bonding. The impact test results were very interesting and differed significantly from what has been found for T300/976 composites toughened by 3M F300 thermoset polymer interleaves. Although T800/3900-2 had an impact velocity threshold relatively the same as T300/976, the delamination size of the former was substantially reduced, and was less than one-fifth of what was found in the F300-toughened T300/976. This implies that the impact damage resistance of the polyamide toughened composite is much better than the one toughened by F300 interleaves. Based on limited study, it suggests that polyamide coating is much more effective on suppression impact damage growth than FM300 thermoset interleaves. Work has been undertaken to develop an analytical model for predicting impact resistance of composite plates and cylinders with various types of impactors. The model will be implemented into the 3DIMPACT computer code which was developed previously under the ARO contract. (This code has been requested by many major companies and laboratories in the United States.)

\section{CONSIDERATION OF MICROSTRUCTURAL CHANGES IN THE STUDY OF ADIABATIC SHEAR BANDS}

Romesh C. Batra

University of Missouri at Rolla

$$
\text { SL: ARI/WTD }
$$

SC: BWL

A study was made of the development of shear bands in a long hollow cylindrical body made of a homoge- 
neous isotropic viscoplastic material and containing two thin voids on a radial line that are located symmetrically about the center. The material particles on the inner surface of the cylinder are assigned the same radial velocity, and the temperature that increases to the steady value in a small time. Two different values of the steady temperature on the inner surface are considered, namely, $100^{\circ} \mathrm{C}$ and $900^{\circ} \mathrm{C}$. It is found that a shear band initiates from each void tip and propagates toward the nearest bounding surface. A shear band will initiate from the void tip close to the inner surface sooner when the temperature on the inside surface of the cylinder is $100^{\circ} \mathrm{C}$ than when it is $900^{\circ} \mathrm{C}$. However, the temperature distribution on a line close to a void tip perpendicular to the shear band indicates that the temperature at a point on the line is higher when the inner surface temperature is $900^{\circ} \mathrm{C}$. An adaptive mesh refinement technique has been developed that generates elements such that the integral of the second invariant of the deviatoric strain-rate tensor over an element is nearly the same for all elements in the mesh. This technique has been applied to discern shear bands, which are narrow regions of intense plastic deformation, in a thermally softening viscoplastic block deformed in plane strain compression at an average strain-rate of $5000 \mathrm{sec}^{-1}$. A material defect has been modeled by introducing a temperature perturbation at the center of the block.

Reports:

1. Shear Band Development in a Viscoplastic Cylinder, by X.-T. Zhang and R.C. Batra, Anisotropy and Localization of Plastic Deformation, 1991, p103. AD A244 190

2. A Steady State Axisymmetric Penetration Problem for Rigid/Perfectly Plastic Materials, by T. Gobinath and R.C. Batra, Intl J Eng Sci 29,1315(1991). AD A244 715

3. Effect of Different Temperature Boundary Conditions on the Development of a Shear Band in a Viscoplastic Cylinder, by X.-T. Zhang and R.C. Batra, High Temperature Constitutive Modeling-Theory \& Appl Vol 121, 1991, p335. AD A249 583

4. Analysis of Shear Bands in Simple Shearing Deformations of Nonpolar and Dipolar Viscoplastic Materials, by R.C. Batra, Appl Mech Rev 45,S123(1992). AD A249 394

5. An Adaptive Mesh Refinement Technique for Two-Dimensional Problems and Its Application to the Analysis of Shear Bands, by Kwang I. Ko, PhD Thesis, 1992, 69 pp.

6. An Adaptive Mesh Refinement Technique for the Analysis of Shear Bands in Plane Strain Compression of a Thermoviscoplastic Solid, by R.C. Batra and K.-I. Ko, MS, Comp Mech.

7. Consideration of Phase Transformations in the Study of Shear Bands in a Dynamically Loaded Steel Block, by Z.G. Zhu and R.C. Batra, MS, JEng Mater Tech.

\section{HIGH VELOCITY IMPACT OF COMPOSITE LAMINATES}

C.T. Sun

Purdue University

SL: BWL

SC: ARL/WTD, ARL/VSD

Research efforts were focussed on the following three areas: (a) Development of a penetration model for composite laminates - A quasi-static penetration model for a blunt-ended projectile using finite element and fracture mechanics has been developed. This model is capable of predicting the load-displacement curve during penetration and the damage caused by penetration, such as delamination. To validate the accuracy of this model, a series of punch tests were performed. The agreement between the experimental and analytical results was found to be excellent; (b) Development of simple ballistic impact model - The model developed in item (a) requires the use of 3-D finite elements to carry out the stress analysis. It is computationally intensive. It is desirable to simplify the analysis while retaining the basic features of the penetration mechanisms. To achieve this goal, a shock wave analysis was performed to determine the region of laminate that is disturbed by the shock. This disturbed region is then used to estimate the resistance force to the projectile as a function of time and incorporated into the equations of motion for the plug; ( $c$ ) Development of multilayered composite/honeycomb laminates for improved impact resistance - Conventional sandwich constructions consist of two face sheets and a honeycomb core. This type of sandwich structure provides excellent bending stiffness and light weight. However, they are susceptible to ballistic impact damage. A possible remedy is to use additional reinforcing sheets to form multilayered honeycomb/fiber composite laminates. This new laminate construction displayed much greater high velocity impact resistance properties than the conventional sandwich construction.

\section{Reports:}

1. Modeling Penetration Process of Composite Laminates Subjected to a Blunt-Ended Punch, by R. Lee and C.T. Sun, MS.

2. A Quasi-Static Penetration Model for Composite Laminates, by R. Lee and C.T. Sun, MS, J Composite Mater. 
29040 LARGE DEFORMATION ANALYSIS OF NONLINEAR HOMOGENEOUS AND HETEROGENEOUS MEDIA USING AN ADAPTIVE ARBITRARY LAGRANGIAN-EULERIAN FINITE ELEMENT METHOD

Somnath Ghosh

Ohio State University

\section{SC: ARL/WTD, BWL, ARL/MD}

A method for incorporating the effect of second phase inclusions within each element has been completed. This is based on introducing a transformation strain in the heterogeneity, resulting in an additional consistency condition to be satisfied with the governing equations. An assumed stress hybrid formulation has been invoked for treating multi-noded Voronoi cells as finite elements. A number of test problems have been solved to depict the success of this formulation. This method has been coupled with the transformation strain formulation to analyze the response of particle reinforced composites. The average transformation strain method, though effective in the matrix material, was unable to predict stresses in the inclusion with desired level of accuracy. Based on these results, alternate methods to improve the accuracy are presently being looked at. Instead of an averaged consistency condition for the second phase, a point-wise description is being pursued. Also a formulation involving couple-stresses is introduced for accurate representation of composite material behavior. The ALE finite element code for large deformation, that was coupled with the $r$-method of node relocation, is now consolidated mesh enrichment techniques namely the $h$-method. The programming has been completed and test cases are presently being run to investigate the effectiveness of this method. Various criteria involving error analysis and important evolutionary variables are being experimented with. A strain localization model that is being formulated will then be coupled with this code.

\section{Reports:}

1. An Arbitrary Lagrangian-Eulerian Finite Element Model for Heat Transfer Analysis of Solidification Processes, by Somnath Ghosh and Suresh Moorthy, MS, Numer Heat Trans.

2. A Material Based Finite Element Analysis of Heterogeneous Media Involving Dirichlet Tessellations, by Somnath Ghosh and Sankar N. Mukhopadhyay, MS, Comp Meth in Appl Mech and Eng.

\section{A CRITICAL STUDY OF CONSTITUTIVE RELATIONS. FOR FINITE STRAIN INELASTICITY}

David L. McDowell

Georgia Institute of Technology

SL: ARL/WTD

SC: ARL/MD

The objective of the research effort is to conduct a combined experimental-computational-analytical investigation, at a fundamental level, of deformationinduced anisotropy of metals deformed to large strains under a variety of stress states. The experimental portion of the program consists of subjecting cylindrical specimens to incremental finite amounts of true strain in compression. Successive stages of compression will be performed on specimens cut out from the previous stage and compression axis rotated $90^{\circ}$. The role of plastic spin will be isolated and validated by $x$-ray diffraction studies of texture. The role of short range transients will be established by axial-torsional experiments on thin-walled tubular specimens. A cascading series of analyses and constitutive modelling evaluations in concert with the experimental portion is planned to incorporate specific features of deformation into the development of meaningful constitutive relations. Several simplifying assumptions of large strain plasticity theory will be critically examined for relevance to experimentally observed phenomena.

\section{B. Fluid Dynamics}

\section{ROTOR CRAFT CENTER OF EXCELLENCE}

Daniel P. Schrage

Georgia Institute of Technology

\section{SL: AERO DIR, AP TEC DIR, ATCOM, ARL/VSD SC: OASA (RDA)}

Research is being conducted in the following areas: studies in three-dimensional viscous aerodynamics, aerodynamic interactions, blade tip aerodynamics, unsteady aerodynamics for rotor aeroelasticity vibration and trim of elastic rotor blades with dynamic stall, unsteady aerodynamic testing of model rotors, nonlinear beam theory, rotorcraft vibrations and stuctural dynamics, damage resistance in rotorcraft structures and modern and active control research for rotorcraft applications. 
Reports:

No. $1-39$ in previous editions.

40. Smart Structures in the Active Control of Blade Vortex Interaction, by S. Hanagud et al., MS.

41. Helicopter Nonlinear Flight Control System Development, by J.V.R. Prasad and D.P. Schrage, MS.

42. Unified Nonlinear Analysis for Nonhomogeneous Anisotropic Beams with Closed Cross Sections, by A.R. Atilgan and D.H. Hodges, $A L A A J$ 29,1990(1991). AD A244 863

43. Chaos in a Single Equilibrium Point System: Finite Deformations, by E.K. Hall II and S.V. Hanagud, Nonlinear Dynamics 2,157(1991).

44. Modal Analysis with PVDF Films and Piezoceramic Transducers, by S.V. Hanagud et al., Smart Structures and Materials AMD-Vol 123,19(1991).

45. Mode Shape Measurement Using a Scanning Laser Doppler Vibrometer, by P. Sriram et al., MS, Intl JAnal and Exptl Modal Anal.

46. Crashworthy Behavior of Graphite/Epoxy Composite Sine Wave Webs, by Weiyu Zhou et al., MS, ALAA J Aircraft.

47. Vibration Control of a Smart Structure Using Fixed order Dynamic Compensation, by J.V.R. Prasad and S.V. Hanagud, MS, J Intelligent Mater Systs Struct.

48. Chaotic Vibrations of Beams: Numerical Solution of Partial Differential Equations, by N.S. Abhyankar et al., MS, $J$ Appl Mech.

49. Optimal Vibration Control by the Use of Piezoceramic Sensors and Actuators, by S. Hanagud et al., MS, J Guidance.

50. Adaptive Sensors and Actuators in the Vibration Control of Rotating and Pointing Systems, by S. Hanagud et al., MS.

51. Robustness Issues in Model Adaptive Controllers, by $S$. Hanagud and G.L. NageshBabu, MS.

52. Use of Fractal Dimension in the Characterization of Chaotic Structural Dynamic Systems, by E. Hall II et al., Appl Mech Rev 44,S107(1991).

53. Modeling Issues in the Vibration Control with Piezoceramic Actuators, by Girish Kulkarni and S.V. Hanagud, Smart Structures and Materials AMD-Vol 123,7(1991).

54. Applications of Smart Structures in the Control of Chaotic Vibrations, by E.K. Hall II and S.V. Hanagud, Smart Structures and Materials AMD-Vol 123,107(1991).

55. Coupled Waves in a Bar of Mooney-Rivlin Material, by N.S. Abhyankar and S.V. Hanagud.

56. A Three-Dimensional Navier-Stokes/Full-Potential Coupled Analysis for Rotor Blades, by Fu-Lin Tsung and L.N. Sankar, MS.

57. Numerical Simulation of Flow Separation for Rotors and Fixed Wings, by Fu-Lin Tsung and L.N. Sankar, $A L A A-92$, ALAA92-0635, 1992.

58. Quantification of Canard and Wing Interactions Using Spatial Correlation Velocimetry, by P.A. Fawcett et al., $A L A A$ 92, ALAA92-2687, 1992.

\section{ROTORCRAFT CENTERS OF EXCELLENCE}

Alfred Gessow

University of Maryland

SL: AERO DIR, AP TEC DIR, ATCOM, ARL/VSD

SC: OSA (RDA)
Research efforts are concentrating on experimental and theoretical investigations of rotor/body/lifting surface, rotor wake studies in hover and forward flight, modeling of unsteady aerodynamics and dynamic stall, helicopter flight dynamics, fabrication and testing of a new bearingless rotor model, dynamic analysis for tailored composite rotor blades, aeroelastic optimization of an advanced geometry helicopter rotor, dynamics of helicopters with dissimilar blades, coupled rotor/airframe vibration analysis, fabrication and testing of Froude-Scaled intelligent rotor, structural integrity of discontinuities in composites, material discontinuities, crashworthiness of composite structures and multiaxial loads, manufacture of foam-core composite box beams, active control of flexible structures and finite element modeling of composite beams.

Reports:

No. $1-33$ in previous editions.

34. An Analytical Model for Composite Box-Beams Including Thermal Effects, by Anita L. Tracy and Inderjit Chopra, MS.

35. Effect of Nonlinear Elastomeric Dampers on Aeromechanical Stability of Helicopters, by Gunjit S. Bir and Inderjit Chopra, MS.

36. Helicopter Rotor Blade Aeroelasticity in Forward Flight with an Implicit Structural Formulation, by Roberto Celi, MS.

37. Hingeless Rotor Dynamics in Coordinated Turns, by Roberto Celi, MS.

38. Optimum Aeroelastic Design of Helicopter Rotors for Longitudinal Handling Qualities Improvement, by Roberto Celi, $J$ Aircraft 28,49(1991).

39. Steady Stall and Compressibility Effects on Hingeless Rotor Aeroelasticity in High-G Turns, by Roberto Celi, Vertica 14,509(1990).

40. Aeromechanical Stability of Hingeless Helicopter Rotors in Forward Flight, by Wai Yip Chan and Inderjit Chopra, MS.

41. Vibration Characteristics of Composite I-Beams with Elastic Couplings Under Rotation, by Ramesh Chandra and Inderjit Chopra, MS.

42. Perspectives in Aeromechanical Stability of Helicopter Rotors, by Inderjit Chopra, Veriica 14,457(1990).

43. The Effect of Side Loads on the Energy Absorption of Composite Structures, by David C. Fleming et al., MS.

44. Aeroelastic Analysis of Rotor Blades with Advanced Tip Shapes, by Ki Chung Kim and Inderjit Chopra, ALAA 90 , ALAA 90-1118, 1990.

45. Blade Response Calculations Using Three-Dimensional Aerodynamic Modeling, by Ki-Chung Kim et al., J $\mathbf{A m}$ Helicopter Soc 36,68(1991).

46. Delamination Prevention in Tapered Composite Structures Under Uniaxial Tensile Loads, by Antonio S. Llanos et al., MS.

47. Effect of Hingeless Rotor Aeroelasticity on Helicopter Longitudinal Flight Dynamics, by Roberto Celi, J Am Helicopter Soc 36,35(1991). 
48. Formulation and Evaluation of an Analytical Model for Composite Box-Beams, by Edward C. Smith and Inderjit Chopra, MS.

49. An Analytical Model for Composite Box-Beams Including Thermal Effects, by Anita L. Tracy and Inderjit Chopra, MS.

50. An Analysis of Pitch and Plunge Effects on Unsteady Airfoil Behavior, by Joseph C. Tyler and J. Gordon Leishman, MS.

51. Strength of Laminated Composites with Longitudinal Discontinuities, by Anthony J. Vizzini, MS.

52. Engine-Rotor Interactions: A Dynamic Analysis in Hover, by Carl Jan Ockier, MS Thesis, 1990, 224 pp.

53. Experimental and Theoretical Analysis of Composite IBeams with Elastic Couplings, by Ramesh Chandra and Inderjit Chopra, MS.

54. Determination of the Energy Absorption of Composite Structures Under Combined Loadings, by David C. Fleming and Anthony J. Vizzini, MS.

55. Dynamic Analysis of Advanced Tip Rotors Including Three Dimensional Aerodynamics, by Ki Chung Kim, PhD Thesis, $1990,250 \mathrm{pp}$.

\section{FREE-WAKE COMPUTATION OF HELICOPTER ROTOR FLOW FIELDS FOR GENERAL FLIGHT REGIMES}

John Steinhoff

Flow Analysis Incorporated

$$
\begin{array}{ll}
\text { SL: } & \text { AERO DIR } \\
\text { SC: } & \text { OASA (RDA) }
\end{array}
$$

Improvements have been made to the rotor code, HELIX-II, which make it about 50 percent faster for forward flight. Also, a study has been done validating the compressible time-dependent equations. The unsteady terms have been included using a time linearization procedure. Since the density is unknown at the new time level, it is linearized about a known level and Newton iterations are performed at each time step to achieve a desired amount of convergence before marching to the next time step. In addition, an unsteady wake convection procedure has been included. This is required in a small region behind the trailing edge where the wake is represented by a discontinuity before being completely replaced by the Lagrangian convection. To validate this new solution procedure, computations have been made using HELIX-II on oscillating airfoils. Solutions have been obtained at a wide range of Mach Numbers extending up to 0.85 and for both high and low reduced frequencies. These computed results have been compared with experimental data and also with other computations. Very good comparisons have been obtained for unsteady aerodynamic loads, and chordwise pressure comparisons are good even for cases with strong shocks. HELIX-II has been applied to perform forward flight computations on a two blade rotor in forward flight at an advance ratio of 0.1 . This untwisted rotor has an aspect ratio of 12. A 400,000 point grid was used for this study which has 225 points in the azimuthal direction. The wake effects are the primary factor in determining the rotor loads at this low-speed condition, and these effects are clearly seen in the solution.

\section{Reports:}

1. Computational Vorticity Confinement: Two Dimensional Incompressible Flow, by John Steinhoff et al., MS.

\section{BOUNDARY LAYERS INDUCED BY THREE-DIMENSIONAL VORTEX LOOPS}

A.T. Conlisk

Ohio State University

\section{SL: AERO DIR}

The primary research activity has been the generation of the results of the boundary layer problem. Results have been produced for three types of mean flow. In the first case, the vortex filament is embedded in a stagnant environment; the induced boundary-layer results indicate that the flow separates in the sense that a closed eddy forms early in the calculation. In the second case where the vortex is embedded in a symmetric mean flow, the boundary layer flow results show the development of a more complex secondary eddy which is detached from the wall and appears to have a horseshoe-type shape. The head of the horseshoe is slightly lifted upward from the cylinder wall while its legs end at the cylinder wall and increase in length with time. The results for the boundary layer flow still indicate the existence of a secondary eddy that develops and grows in time and eventually is expected to lead to the breakdown of the boundary layer flow.

Reports:

No. 1 in previous edition.

2. A Simplified Model for the Interaction of a Rotor Tip Vortex with an Airframe. Part I: Theory, by $\mathrm{H}$. Affes and A.T. Conlisk, MS, ALAA $J$.

3. An Experimental and Analytical Study of the Interaction of a Vortex With an Airframe, by $\mathrm{H}$. Affes et al., MS.

4. The Three-Dimensional Boundary Layer Flow Due to a Vortex Filament Outside a Circular Cylinder, by H. Affes and A.T. Conlisk, MS.

5. A Simplified Model for the Interaction of a Rotor Tip Vortex with an Airframe. Part 2: Comparison with Experiment, by $H$. Affes et al., MS. 


\section{AN OSCILLATING THREE-DIMENSIONAL WING EXPERIMENT}

Peter F. Lorber

United Technologies Research Center

SL: AERO DIR, ARL/VSD

SC: AERO DIR, AFOSR, ARL/VPD

This experiment explored the transient compressible aerodynamic response of a three-dimensional wing undergoing pitching motions to high angles of attack. Surface pressures were measured on a rectangular wing during constant pitch rate ramps and sinusoidal oscillations, using 112 pressure transducers arranged in 5 chordwise arrays. Boundary layer transition and separation were determined using 16 surface hot film gages. The experiment was conducted in a compressible air flow ( $0.2 \leq M \leq 0.6)$, at realistic Reynolds numbers (2-6 million), and for swept and unswept geometries $\left(\Lambda=0,15\right.$, and $\left.30^{\circ}\right)$. The experiment obtained basic information applicable to helicopter rotors, to maneuvering fixed wing aircraft, and to the problem of incipient torsional stall flutter.

Reports:

No. 1-2 in previous editions.

3. Dynamic Stall of a Sinusoidally Oscillating Three-Dimensional Swept Wing in Compressible Flow, by Peter F. Lorber, MS.

4. Unsteady Transition Measurements on a Pitching ThreeDimensional Wing, by Peter F. Lorber and Franklin $O$. Carta, MS.

5. Compressibility Effects on the Dynamic Stall of a Three-Dimensional Wing, by P.F. Lorber, ALAA92, ALAA 92-0191, 1992. AD A249 553

6. Dynamic Stall of Sinusoidally Oscillating Three-Dimensional Swept and Unswept Wings in Compressible Flow, by Peter F. Lorber, MS.

\section{THEORETICAL STUDY OF TURBULENT MIXING BETWEEN HYPERSONIC STREAMS}

David C. Wilcox

DCW Industries, Inc.

\section{SL: ARL/WTD}

A careful examination has been made of the manner in which the $k$ - $\omega$ model predicts transition from laminar to turbulent flow. As a corollary result, it was demonstrated why the $k-\varepsilon$ model is so much more difficult to use than the $k-\omega$ for transition prediction. Most notably, the $k-\omega$ model has a unique laminarflow solution while, by contrast, the $k-\varepsilon$ model is extremely sensitive to initial conditions. The low-
Reynolds-number modifications to the $k$ - $\omega$ model have been reworked. Three important ends have been accomplished. First, the law of the wall for fully turbulent boundary layers is far less sensitive to the closure coefficients in the revised viscous modifications. Second, the revised viscous modifications yield much closer agreement with DNS results for incompressible channel flow than had been achieved previously. Third, the $k-\omega$ model accurately simulates effects of freestream turbulence on flat-plate boundary transition locations and width of the transition region. With the older viscous modifications, the $k-\omega$ model predicted too small a transition width. Work has been completed on the writing of all subroutines required to include the $\mathrm{k}-\omega$ turbulence model in the three-dimensional, thin-layer Navier-Stokes program known as CFL3DE. The subroutines have been isolated in a single "portable" subroutine package that can, in principle, be added to any compressible three-dimensional Navier-Stokes program. The portable subroutine version of the program is still malfunctioning. A new version also malfunctions, but researchers have eliminated several coding errors and isolated the source of the malfunction to eight subroutines that the portable and nonportable subroutine versions share.

Reports:

No. $1-4$ in previous editions.

5. Dilatation-Dissipation Corrections for Advanced Turbulence Models, by David C. Wilcox, MS, $A L A A J$.

6. The Remarkable Ability of Turbulence Model Equations to Describe Transition, by David C. Wilcox, MS.

\section{COMPUTATIONAL FLUID}

DYNAMICS RESEARCH ON

DYNAMICALLY ADAPTIVE MESH

METHODS FOR TRANSONIC FLOWS

Richard G. Hindman

Iowa State University of Science and Technology

\section{SC: ARL/WTD}

Work was done during the first year on a solution adaptive mesh scheme for the 1-D Euler equations. During the second year, significant effort has been spent investigating various methods of coupling the mesh dynamics equations with the fluid dynamics equations. The 1-D shock tube was the primary basis for the testing. Efforts to date promise to bring what has been a great amount of effort on various phases of the overall task into focus and integrate all of the knowledge gained in dynamically adaptive mesh gen- 
eration into a final product. Information has been generated which guides the researcher regarding when adaptive meshes are useful and when they just produce excess baggage. The knowledge gained is being documented and will be published as the documentation is completed. The various methods of coupling the mesh dynamics and the flow dynamics are still being explored. This is a crucial issue. The strong coupling procedure is the most elegant, and the most rigorous. It produces the best results in the cases studied so far. However, it is also the most expensive. The expense may turn out to be prohibitive in multiple dimensions. The mesh adapting procedures explored in this research are ideally suited for used in problems with moving and deforming boundaries. The work has been carefully guided to produce numerical procedures which are extendable to 3-D.

Reports:

No. 1-2 in previous editions.

3. Computation Fluid Dynamics Research on Dynamically Adaptive Mesh Methods for Transonic Flows, by Richard G. Hindman, TR, Nov 91, 21 pp.

\section{FLUID DYNAMIC MECHANISMS AND INTERACTIONS WITHIN SEPARATED FLOWS}

J. Craig Dutton

A.L. Addy

University of Illinois

\section{SL: ARL/WTD}

An experimental effort is underway to investigate plume-induced, boundary layer separation (PIBLS) in a planar, two-stream, supersonic flow using an "angle-induced" separation geometry. This study is being conducted in a small-scale wind tunnel operated in the blowdown mode. Flow visualization experiments have shown that all of the PIBLS flow fields are unsteady, and that the length scale of the separation shock wave motion is on the order of several boundary layer thicknesses. The unsteadiness of the separation process poses a problem when making laser Doppler velocimeter (LDV) measurements because it is impossible to distinguish between vellocity fluctuations caused by turbulent eddies and velocity fluctuations caused by the gross translational movement of the separation region. Since this investigation seeks to obtain LDV measurements throughout an unsteady PIBLS flowfield, a means must be found to overcome the problems posed by the unsteady separation process. Therefore, a conditional sampling technique for acquiring and analyzing the LDV data is under development using two fast-response pressure transducers. An experimental research program is being conducted to study the flowfield downstream of a body of revolution embedded in a supersonic flow. A blunt-based cylindrical afterbody is supported by a constant diameter string which runs upstream through the wind tunnel stagnation chamber in order to eliminate any support interference effects. An annular C-D nozzle produces a uniform flow approaching the base corner with a nominal Mach number of 2.5. Currently, the power-off configuration (no central jet) is being investigated. A research program is being conducted to develop a new Particle Image Velocimeter (PIV) system for use as a non-intrusive laser diagnostic tool in high-speed separated flow studies. The PIV system is capable of extracting instantaneous two-dimensional velocity maps within a flow by photographing images of seed particles within a double-pulsed light sheet and then examining particle image separations to determine local velocities.

\section{Reports:}

No. 1-3 in previous editions.

4. An Experimental Investigation of the Supersonic Axisymmetric Base Flow Behind a Cylindrical Afterbody, by Jeff Lee Herrin, MS Thesis, 1991, 104 pp.

5. Design and Validation of an Interrogation System for Particle Image Velocimetry, by Ronald Alan Rosner, MSThesis, $1991,66 \mathrm{pp}$.

6. Design of a Particle Image Velocimeter for High Speed Flows, by Ronald A. Rosner, MS.

7. An Experimental Investigation of Axisymmetric Power-Off Base Flow Phenomena, by Heff L. Herrin, MS.

\section{UNSTEADY FLOW PHENOMENA IN DISCRETE PASSAGE DIFFUSERS FOR CENTRIFUGAL COMPRESSORS}

Edward M. Greitzer

Massachusetts Institute of Technology

\section{SC: ARI/VPD, TACOM}

The work on this project has been focussed almost wholly on the necessary fabrication, rig modification, instrumentation, and data acquisition for the upcoming series of experiments. The specific tasks accomplished are: Rig modification and refurbishing. Both the thirty-eight passage diffuser and the vaneless space ring have been machined to accept the eight high response pressure sensors. This required that the previous instrumentation be partially removed to allow room for milling and drilling. A new scheme for 
passing the electrical and pressure connectors through the (Inconel) diffuser was developed since five additional connections are now needed. The traverse probe port was enlarged to allow the 0.039 inch diameter probe to pass through. The forty-one (41) diffuser static pressure taps have also been checked and repairs made where necessary. Traverse probe calibration and other instrumentation. The traverse probe has been calibrated using a free jet. The calibration consisted of six sets of 19 measurements to statistically determine the probe center angle to within approximately 0.2 degrees, which is the actuator accuracy. The calibration data also confirmed the current methodology for obtaining the stagnation pressure and the flow angle, based on analyzing the test data using twenty different permutations of polynomial fits and partial data sets. The latter were symmetric sets of various sizes about the mean center angle. One conclusion from the calibration exercise was that the data range (probe rotation range) should be larger than forty degrees about the center angle for acceptable accuracy, with sixty to seventy degrees being preferred. A second conclusion is that a low polynomial order fit of the data, i.e., second or third order, is the most appropriate model for the probe angle setting. In particular, the second order fit gave more consistent results when the span of the rotation about the center angle was reduced.

\section{Reports:}

1. Experimental Investigation of Flow Distortion Effects on the Performance of Radial Discrete-Passage Diffusers, by Victor G. Filipenco, PhD Thesis.

\section{COMPRESSIBILITY EFFECTS ON AND CONTROL OF DYNAMIC STALL OF OSCILLATING AIRFOILS}

M.S. Chandrasekhara

M.F. Platzer

Naval Postgraduate School

\section{SL: AERO DIR}

SC: AERO DIR

Considerable effort was devoted to development of interferometry fringe analysis software. There are several hundred interferograms that are required to be analyzed and the need for automation is very strong. New fringe filtering methods are being coded to clean-up the fringes so that proper fringe tracing can be accomplished even in regions of high fringe density. An analysis was carried out to identify the source of fringe distortion seen in the experimentally obtained interferogram images. The distortion was manifested in the images as a dark spot around the airfoil leading edge region. The surprising result was that the distortion was actually due to refraction through the 1 inch thick glass window, which caused the light to disappear from the image plane. A correction scheme is now being devised for reducing this effect in the post processing stage.

Reports:

No. $1-3$ in previous editions.

4. A Study of Compressibility Effects on Dynamic Stall of Rapidly Pitching Airfoils, by Lawrence W. Carr and M.S. Chandrasekhara, Comp Phys Commun 65,62(1991). AD A244 557

5. Schlieren Studies of Compressibility Effects on Dynamic Stall of Airfoils in Transient Pitching Motion, by M.S. Chandrasekhara et al., ALAA-90, ALAA-90-3038, 1990, p346. AD A234 788

6. Laser Velocimetry Measurements of Oscillating Airfoil Dynamic Stall Flow Field, by M.S. Chandrasekhara and S. Ahmed, $A L A A-91, A L A A-91-1799$. AD A244 546

7. A Quantitative Study of Unsteady Compressible Flow on an Oscillating Airfoil, by L.W. Carr et al., $A L A A-91, A L A A-$ 91-1683, 1991. AD A244 572

8. Oscillating Airfoil Velocity Field During Large Amplitude Dynamic Stall, by M.S. Chandrasekhara and R.D. Van Dyken, MS.

9. A Real Time Interferometry System for Unsteady Flow Measurements, by M.S. Chandrasekhara et al., Proc of 14th ICLASF Symposium, ICLASF' 91 Record, 1991. AD A244936

10. Leading Edge Velocity Field of an Oscillating Airfoil in Compressible Dynamic Stall, by R.D. Van Dyken and M.S. Chandrasekhara, $A L A A-92, A L A A-92-0193,1992$. AD A248 814

11. Laser Velocimetry Measurements in the Post Stall Field of an Oscillating Airfoil, by S. Ahmed and M.S. Chandrasekhara, MS.

12. Reattachment Studies of an Oscillating Airfoil Dynamic Stall Flow Field, by S. Ahmed and M.S. Chandrasekhara, ALAA-91, ALAA-91-3225, 1991. AD A244 854

13. Interferometry and Computational Studies of an Oscillating Airfoil Compressible Dynamic Stall Flow Field, by M.S. Chandrasekhara et al., MS.

\section{FAR-FIELD ROTOR NOISE}

Valana L. Wells

Arizona State University

\section{SL: AERO DIR \\ SC: ARL/VSD}

Development of an Acoustic Solution Methodology - Much current work in rotor acoustics focuses on isolation and characterization of the source terms in the acoustic analogy equation. There is, however, some speculation based on published results and discussions that nonlinear propagation, in addition 
to what one may term "nonlinear sources," is an important consideration in obtaining a solution to the governing equation which more closely approximates the measured values of sound pressure. Though the principal investigator has espoused this position for some time, recent discussions with Professor David Crighton during his visit to Arizona State University, have served to strengthen this conviction. It is even more likely that, far away from the source, the nonlinear propagation effects will be significant. Rotor Blade Aerodynamic Analysis - The Navier-Stokes solver has been tested and run for many rotor blades in both hover and forward flight. Additionally, two cases of a rotor blade in forward flight with feathering and prescribed flapping have been completed through a complete revolution. The results show good agreement with both experiment and other computional data. The code is currently undergoing further testing for grid convergence. Though the code appears to be very efficient, some improvements suggested by Professor Antony Jameson of Princeton University are also under evaluation.

\section{INTERACTIONS OF SPANWISE AND CHORDWISE VORTICITY ASSOCIATED WITH THREE-DIMENSIONAL DYNAMIC STALL OVER AN OSCILLATING WING}

\section{B.R. Ramaprian}

Washington State University

SL: AERO DIR

SC: AERO DIR

The 3-component velocity measurements in the unsteady trailing vortex generated by the wing-tip were continued to completion. The measurements were made using laser Doppler velocimetry (LDV) at several longitudinal locations in the wing wake starting from about 0.15 chord to 3 chord lengths downstream of the trailing edge. The LDV data have been used to obtain phase-locked values of the three components of velocity, vorticity and all the turbulent stress components. These are the first data available on the origin and evolution of the unsteady trailing vortex behind an oscillating wing. Extensive LDV measurements were made to obtain the three velocity components in the three-dimensional flow field in the near wake of the NASA 0015 rectangular wing of $1 \mathrm{ft}$ chord and $2 \mathrm{ft}$ semispan. These measurements were made in a cross-stream plane located $5 \mathrm{~cm}(0.17$ chord) downstream of the trailing edge. The measurement domain was approximately rectangular and extended from tip to 0.7 chord length inboard in the spanwise direction, and covered the entire vortical region in the cross-stream direction.

Reports:

1. LDV Measurements in the Three Dimensional Near Wake of a Stationary and Oscillating Wing, by Y. Zheng and B.R. Ramaprian, MS.

\section{VORTICAL FLOWS OVER} AXISYMMETRIC SLENDER BODY CONFIGURATIONS WITH APPENDAGES

Mukand Acharya

H. Nagib

Illinois Institute of Technology

The following work was carried out: 1 . Fabrication and testing of a model positioning system that enables the positioning of wind-tunnel models at desired yaw, pitch and roll angles under computer control. 2. Fabrication and testing of an axisymmetric wind-tunnel missile model instrumented for pressure measurements. The model is modular in construction to permit interchanging of three different nose sections as well as interchanging of instrumented body sections. In addition, the body section can be rolled through $360^{\circ}$ while a portion of the nose section $(1 / \mathrm{d}$ $=1$ ) and the tail section are held stationary. 3 . Development of the required software for model positioning in the wind tunnel. In addition, work was ongoing in the following areas: 1. Smoke-wire flow visualization study of the flow over the model with a cone-ogive forebody, at different angles of attack. The flow visualization brings out the differences in flow development over the port and starboard sides of the model, and captures the changes in the flow field, especially the onset of asymmetry in the separated flow. 2. Development of the required software for automated acquisition of surface pressure data over the model at different roll angles. 3. Design of a movable, instrumented finned-body section. The fin and body section will be instrumented for pressure measurements and will be used in the phase of this research project designed to investigate the interaction of the tip vortex with downstream control surfaces.

28249 WING-BODY JUNCTURE FLOW 
Richard J. Bodonyi

Ohio State University

$$
\text { SL: ARL/VPD }
$$

SC: ARL/WTD

Research efforts on the wing-body juncture problem have continued to be directed towards obtaining solutions of the finite-difference form of the nonlinear governing equations for order-one values of the wing thickness parameter. Grid size studies are still under study to determine the accuracy of the computations. Studies are also being made of various grid transformation schemes so as to be able to have relatively fine grid spacings around the wing surface to enhance the accuracy of the solutions in the wing-body region. This is especially important since the computer programs currently being used require a fair amount of memory and cpu time to run to convergence. The finite-difference form of the corresponding energy equation has also been developed and numerical solutions found to determine the temperature effects in the flow field. Since this equation is linear, no difficulties have been encountered in determining numerical solutions for the temperature distribution in the flow field. Studies of the effects of wall temperature and heat transfer on the flow will be considered in detail only after it is determined that the solutions for the velocity components and pressure are sufficiently accurate. Solutions have been found for the wall pressure and the streamwise wall shear stress along the line of symmetry as well as the corresponding contour plots for a biconvex airfoil placed on an otherwise flat surface.

\section{UNSTEADINESS OF SHOCK-INDUCED TURBULENT BOUNDARY LAYER SEPARATION-AN INHERENT FEATURE OF TURBULENT FLOW OR SOLELY A WIND TUNNEL PHENOMENON}

David S. Dolling

Richard Gramann

University of Texas at Austin

\section{SC: ARL/WTD}

Experiments have been conducted to examine the correlation between wall pressure and pitot pressure fluctuation in the incoming undisturbed turbulent boundary layer and the separation shock motion in a Mach 5 compression ramp interaction. The results to date show: $(a)$ strong correlation between wall pressure fluctuations in incoming boundary layer and separation shock wave motions; $(b)$ weak correlation between pitot pressure fluctuations measured near undisturbed boundary layer edge (i.e., in intermittent regions) and separation shock wave motions; $(c)$ "stronger" correlation between pitot pressure fluctuations measured in fully turbulent region of undisturbed boundary layer and separation shock wave motions - to be investigated further using pitot probes; $(d)$ correlation of the flapping of the shear layer near reattachment with motion of separation shock wave shows the reattachment motion lagging that at separation. No evidence of feedback from reattachment to separation.

\section{INCIPIENT SEPARATION IN SUBSONIC PITCHING AIRFOILS}

Doyle Knight

Rutgers, The State University of New Jersey

SL: AERO DIR

SC: AERO DIR, ARL NSD

The principal research accomplishment is the development of an explicit, unsteady, two-dimensional compressible Navier-Stokes code using unstructured triangular grids. The temporal discretization is a modified multi-stage Runge-Kutta method. Substantial progress has been achieved in the development of an implicit, unsteady, two-dimensional compressible Navier-Stokes code using triangular grids. The implicit code utilizes a substantial fraction of the explicit code for computation of the residual. The Jacobian matrices were derived analytically, and coding of the implicit algorithm for the Euler equations was nearly complete by December 1991. The implicit algorithm will permit substantial improvement in code efficiency by eliminating the Courant number restriction on the allowable time-step. This is particularly important for the study of incipient separation, where the requirements on grid resolution near the point of initial bursting of the boundary layer are particularly severe. Additional effort has focused on the modification of an existing unsteady two-dimensional compressible Navier-Stokes code using structured grids. The code employs a conventional boundary-fitted coordinate transformation and utilizes the implicit Beam-Warming algorithm ( $A L A A J, 1978$, p. 393). The code has been modified to incorporate $\mathrm{C}$-grid geometries and characteristicbased boundary conditions on the outer boundary. Further testing of the modified code is in progress. 
Reports:

1. A Fully Implicit Navier-Stokes Algorithm Using an Unstructured Grid and Flux Difference Splitting, by Doyle D. Knight, MS.

29049 VORTICITY DYNAMICS AND CONTROL OF DYNAMIC STALL

Patrick H. Reisenthel

Nielsen Engineering and Research Incorporated

\section{SL: AERO DIR}

The research objective is to develop an accurate efficient technique for the prediction of dynamic stall on pitching airfoils. Duhamel's convolution integral, together with a very accurate steady computational fluid dynamic analysis, will be innovatively employed to predict unsteady separation and dynamic stall behavior.

\section{NUMERICAL INVESTIGATION OF TRANSITIONAL AND TURBULENT AXISYMMETRIC WAKES AT SUPERSONIC SPEEDS}

Hermann Fasel

University of Arizona

\section{SC: ARL/WTD}

The research objective is to investigate transitional and turbulent axisymmetric wakes behind cylindrical bodies aligned with the flow at supersonic speeds. A supersonic Navier-Stokes code will be used to perform direct numerical simulations, and large eddy simulations, of the flow of interest. In addition, these numerical simulations will be complemented by a theoretical effort based on stability theory analysis and proper orthogonal decomposition techniques.

\section{Combustion}

\section{AUTOIGNITION AND} COMBUSTION IN DIESEL ENGINES UNDER COLD STARTING CONDITIONS

N.A. Henein

Wayne State University

SL: TACOM

SC: BRADEC, CRDEC, TACOM
The autoignition and combustion in diesel engines under cold starting conditions has been investigated both theoretically and experimentally. The theoretical work consisted of a detailed analysis of the autoignition reactions by using the chain reactions developed in the Shell Model. It has been found that the coefficients need to be calibrated in order to simulate the experimental data obtained on the LABECO-TACOM engine at different ambient temperatures. This work is still in progress to determine if the change in the calibration coefficients could be correlated to the changes in the inlet air temperature or any other related operating parameter. The experimental work is conducted on the LABECO-TACOM single cylinder direct injection water cooled diesel engine. The engine is located in the cold room where the ambient air temperature can be controlled, by a microprocessor, from normal room temperature to $-40^{\circ} \mathrm{C}$. Two types of tests have been conducted by using diesel fuel. The first set of tests was under steady state conditions in order to determine the effect of ambient temperature on the ignition delay. The next set of tests was conducted to simulate the cold starting conditions. The analysis of the data indicated that misfiring was not caused by the failure of the autoignition process but was caused by a lack of enough evaporated fuel to carry on the combustion process. This observation indicates that the fuel volatility plays a major role in the success of the combustion process. This phenomenon has been observed on another engine which is started by using a battery and an electric starter. A mathematical model is being developed for the fuel spray evaporation, autoignition and combustion at low ambient temperatures.

\section{PREDETONATIVE COMPRESSION OF PROPELLANTS}

\section{Neale Messina}

Princeton Combustion Research Laboratory, Inc.

SL: ARDEC

SC: ARL/NTD, MICOM

A new test method for the determination of propellant mechanical properties under dynamic uniaxial compressive loading has been developed, utilizing the PCRL ballistic compressor system. Direct measurement of the propellant strain and transmitted force (engineering stress) permits determination of elastic properties and plastic deformation behavior, including fracture toughness and failure modulus, over the operating temperature range of $-40^{\circ} \mathrm{C}$ to 
$65^{\circ} \mathrm{C}$. This dynamic pressure generating system imparts a ballistic-like loading to a propellant sample installed in a specially designed mechanical properties test chamber attached to the high pressure endcap of the ballistic compressor. A paper discusses the operation of the test apparatus, the instrumentation, and data acquisition and evaluation. The test method provides data on mechanical properties of propellant grains in accordance with a proposed NATO draft STANAG on uniaxial compressive gun propellant test methods. Dynamic mechanical properties data are presented for $\mathrm{M}-9, \mathrm{M}-30$, and HELP1 gun propellant grains at hot and cold conditioning temperature extremes. The test method is currently being extended to include dynamic biaxial compressive loading.

\section{STUDY OF STREAMWISE VORTICITY STIRRED COMBUSTION}

John McVey

A. Vranos

United Technologies Research Center

\section{SL: ARL/VPD}

The objective of this effort is to determine if the concept of combustion enhancement through the introduction of large-scale, intense, streaming vortices can be used to extend the capability and performance of small gas turbine combustion systems. The effort utilizes the recently developed technique of generating vortex arrays by the use of convoluted surfaces; it has been demonstrated that under certain conditions these vortex arrays produce intense large-scale mixing which is effective in promoting flame spreading in highly exothermic flows. The processes responsible for the rapid mixing are primarily inviscid and are related to the geometrical features of the convoluted surface and the chamber enclosure; because the processes are inviscid, the scale and the intensity of the mixing process can be tailored to the needs of the application. Water flow-visualization tests, in which the mixing characteristics of various primary and secondary mixer-lobe combinations were examined, resulted in the definition of four enhanced-mixing configurations that were characterized by strong interactions between the primary and secondary flows.

\section{DROP WEIGHT IMPACT INITIATION OF ENERGETIC MATERIALS}

A.M. Mellor

Vanderbilt University

$$
\begin{array}{ll}
\text { SL: } & \text { ARDEC } \\
\text { SL: } & \text { ARL/WTD, MICOM }
\end{array}
$$

At the Army Missile Command, drop-weight experiments are being conducted on both neoprene rubber and an HTPB/AP propellant. Using data from these tests, the drop-weight acceleration is correlated with the stress in both the drop-weight and the anvil measured via two PVDF gauges. The data indicate that the drop-weight stress from the time of impact up to the time of maximum load varies linearly with acceleration. This appears to be independent of both sample material and impact velocity. The anvil stress also appears to vary linearly with acceleration, but the correlation may depend on sample material and impact velocity. Tests are currently under way to clarify this and to determine the effect of sample geometry. The correlation of stress to acceleration will allow removal of the accelerometer in the drop-weight test. Modeling of sample deformation has continued. Recent modeling efforts show the effect of friction, sample constitutive relation, and sample geometry on three possible initiation criteria: (a) critical temperature for AP decomposition, (b) critical thickness for crushing AP particles, and (c) critical thickness for sample shear banding. Results indicate that critical temperature is a more likely initiation criterion than critical thickness for crushing AP. The effect of localizing the heating due to friction at the sample/machine interface was also studied, and the degree of localization was found to greatly influence sensitivity via the temperature rise. Results indicate that increasing sample flow stress decreases critical energy based on temperature, but increases critical energy based on the shear banding criterion. This indicates a need for additional modeling. Efforts in analysis of the drop weight impact test using DYNA2D focused on several areas. First, comparisons were made between several cases with different mesh sizes and default scaling factors. The more refined meshes resulted in significantly smaller oscillations in pressure-time plots. The optimal relationship between the stiffness and timestep scaling factors was also verified.

Reports:

No. $1-4$ in previous editions.

5. Dynamic Analysis of Drop Weight Impact Rocket Propellant Ignition, by K.P. Duffy et al., ALAA-91, ALAA-91-2193, 1991. AD A239 899

6. High Rate Deformation of Composite Solid Propellant Disks, by K.P. Duffy et al., MS. 
7. Relating Sample Deformation to Observations in the Drop Weight Impact Test, by P. Baker and A. Mellor, MS.

8. Mechanical Properties Test Methods as Related to Hazards Sensitivity of Energetic Materials, by K.P. Duffy and A.M. Mellor, MS.

\section{MECHANISM OF INTERMITTENT ATOMIZATION}

S.P. Lin

Clarkson University

\section{SC: ARL/WTD, TACOM}

The effect of compressibility of a liquid jet emanating from a nozzle into a compressible ambient gas was analyzed. An increase in the compressibility of the liquid jet is shown to promote generation of smaller droplets, although at slower rate. The slower rate is due to the loss of the available energy to the stored acoustic energy in the jet. The smaller droplets are probably due to the local enhancement of the frequency of pressure fluctuation upon the acoustic frequency. It was shown that in some parameter ranges, a small change in compressibility may result in a qualitative change of the mechanism of atomization, i.e., from convective instability to absolute instability of the jet.

Reports:

No. 1-2 in previous editions.

3. A Branching Liquid Jet, by S.P. Lin and D.R. Woods, Phys Fluids A3,241(1991). AD A239 895

4. Effects of Compressibility on the Atomization of Liquid Jets, by Z.W. Zhou and S.P. Lin, MS.

\section{MULTIPLE DROP-CONTAINING TURBULENT EDDIES EFFECTS ON EVAPORATION, IGNITION AND COMBUSTION OF CLUSTERS OF DROPS}

J. Bellan

Jet Propulsion Laboratory, California Institute of Technology

SC: ARL/VPD, TACOM

Considerable effort was devoted to preparing a manuscript for the 24th International Symposium on Combustion. In particular, the results obtained with the model for steady injection of identical clusters were again analyzed with special emphasis on the temporal variation of the fuel mass fraction in the gas phase outside of the cluster. Since the vortical motion results in the cluster forming a shell inside the vortex, the core of the vortex is devoid of drops. Thus there is a gas phase devoid of drops internally and exter- nally to the vortex, and fuel vapor is transported from the cluster in both directions. The fuel vapor transported outside the cluster in the increasing radial coordinate will meet oxygen and the high temperature surrounding ambient air and thus will initiate ignition. The fuel vapor which accumulates in the inner vortex core, being at a relatively lower temperature, will not burn until the drops have totally evaporated and the flame started to propagate from the periphery of the cluster to its interior. Results show that most of the fuel vapor accumulates in the vortex core, that the core of vortices having denser clusters are richer, and that for a fixed initial air/fuel mass ratio, entrainment has a minimal effect upon the value of the inner fuel vapor mass fraction. Progress has also been made in the development of the computer code for the unsteady injection and evaporation of clusters of drops embedded in vortices, each of these clusters and vortices having various characteristics.

\section{Reports:}

No. 1 in previous edition.

2. Evaporation of Steadily Injected, Identical Clusters of Drops Embedded in Jet Vortices, by J. Bellan and $\mathrm{K}$. Harstad, MS.

\section{HIGH PRESSURE PREIGNITION CHEMISTRY OF HYDROCARBONS AND HYDROCARBON MIXTURES}

Nicholas P. Cemansky

David L. Miller

Drexel University

\section{SL: ARL/VPD}

SC: BRADEC, TACOM

A detailed chemical kinetic mechanism for propane oxidation has been used to successfully model experimental data through the range of temperatures $(650$ to $800 \mathrm{~K}$ ) corresponding to the negative temperature coefficient (NTC) region encountered by engine end-gas just prior to knocking. An important feature of the mechanism is that it models alkylperoxy radical reactions using only uncoupled, parallel reaction paths. The results show that the overall mechanism is most sensitive to changes in the rate of the reactions which produce diperoxy radicals. Low temperature branching is dominated by decomposition of diperoxy radicals instead of alkyhydroperoxide branching. The calculated concentration versus temperature profiles for all species qualitatively reproduced the NTC behavior indicated by the 
experimental species data. The fuel consumption and the major product species, propene and carbon monoxide, were accurately predicted over the entire temperature range. While the calculations accurately reproduced the qualitative behavior of the profiles for the minor species, the concentration levels of these species were generally overpredicted. An attempt was made to model the experimental data using another propane oxidation mechanism which employs a coupled, multichannel unimolecular scheme to model alkylperoxy radical reactions and was found to be unable to reproduce the NTC behavior. However, when a direct path for the reaction $\mathbf{R}$ $+\mathrm{O}_{2} \rightarrow$ olefin $+\mathrm{HO}_{2}$ was added to the mechanism, NTC behavior emerged. Because an uncoupled, parallel alkylperoxy reaction scheme succeeded while a coupled, multichannel unimolecular alkylperoxy scheme failed to reproduce NTC behavior, a direct $\mathrm{R}+\mathrm{O}_{2} \rightarrow$ olefin $+\mathrm{HO}_{2}$ reaction is recommended until such time as RRKM or QRRK calculations for the alkylperoxy radical reactions are incorporated into detailed kinetic mechanisms.

Reports:

No. 1-2 in previous editions.

3. A Flow Reactor for the Study of Homogeneous Gas-Phase Oxidation of Hydrocarbons at Pressures Up to 20 Atmospheres (2MPa), by D.N. Koert and N.P. Cernansky, MS, $J$ Phys E: Sci Instrum.

4. Results of Reactivity Mapping Studies Through the Negative Temperature Coefficient Region for Propane at Pressures from 5 to 15 Atmospheres, by D.N. Koert et al., MS.

\section{THE STRUCTURE OF LAMINAR FLAMES OF $\mathrm{CH}_{4} / \mathrm{NO}_{2}, \mathrm{CH}_{2} / \mathrm{ONO}_{2}$, AND $\mathrm{HCN} / \mathrm{NO}_{2}$}

K. Seshadri

University of California, San Diego

\section{SL: ARL/WTD}

SC: ARDEC, MICOM

The principal objective of this research is to develop fundamentally sound models to describe the structure of diffusion flames and premixed flames burning $\mathrm{CH}_{4} / \mathrm{NO}_{2}, \mathrm{CH}_{2} \mathrm{O} / \mathrm{NO}_{2}$, and $\mathrm{HCN} / \mathrm{NO}_{2}$. Numerical calculations were performed to determine the structure of nonpremixed $\mathrm{CH}_{2} \mathrm{O}-\mathrm{NO}_{2}$ flames. The chemical kinetic mechanism employed in these calculations was the same as that used for calculating the structure of $\mathrm{CH}_{4}-\mathrm{NO}_{2}$ flames, with reactions leading from $\mathrm{CH}_{4}$ to $\mathrm{CH}_{2} \mathrm{O}$ removed from the mechanism. These results show that $\mathrm{CH}_{2} \mathrm{O}$ and $\mathrm{NO}_{2}$ do not directly react with each other; in fact the con- sumption of these species appears to occur in separate regions. The numerical calculations show that the structure of the reaction zone consists of three regions. $\mathrm{A} \mathrm{CH}_{2} \mathrm{O}$-consumption region where $\mathrm{CH}_{2} \mathrm{O}$ is attacked by radicals to form $\mathrm{CO}$ and $\mathrm{H}_{2}$, and a $\mathrm{NO}_{2}$-consumption region where $\mathrm{NO}_{2}$ is attacked by radicals to form $\mathrm{NO}$ and $\mathrm{O}_{2}$, and a product formation region where $\mathrm{NO}, \mathrm{CO}, \mathrm{O}_{2}$, and $\mathrm{H}_{2}$ participate in chemical reactions to form $\mathrm{N}_{2}, \mathrm{CO}_{2}$, and $\mathrm{H}_{2} \mathrm{O}$.

\section{BOOST-PHASE DISCRIMINATION RESEARCH}

David M. Cooper

George S. Deiwert

NASA Ames Research Center

\section{SC: ARO, MICOM}

Researchers have continued to investigate low density effects on flows at hypersonic speeds. Previous work involving compressed flow through a hypersonic shock wave has been updated and accepted for publication in the AIAA Joumal of Thermophysics and Heat Transfer. This work showed that the Burnett equations produce simulations of shock wave structure that are significantly more accurate than the Navier-Stokes equations and will therefore form a necessary basis for the representation of non-equilibrium chemical reactions. Accurate prediction of chemistry will be necessary for the estimation of radiation signatures arising from hypersonic shock waves. This work is now being extended to the expanding region behind a vehicle, to both the base flow and to the more highly expended portions of the plume. Comparisons are being made to NavierStokes calculations and will be summarized in a paper that is in preparation. The Burnett terms are also being investigated in conjunction with thermochemical modeling in order to understand the radiation signature of the BSUV experiment. Research efforts have continued to compute radiative intensity factors, transport properties, and reaction rates of air species that contribute to the radiation signatures of hard bodies and exhaust plumes. Considerable progress was made in the calculation of transport properties such as thermal conductivity, viscosity, and thermal diffusion. In particular, transport cross sections and collision integrals have been computed for all neutral-ion interactions involving nitrogen and oxygen. 


\section{HIGH-ALTITUDE HYPERSONIC FLOWFIELD RADIATION}

Robert W. MacCormack

D.R. Chapman

Stanford University

The primary objective of this research is to investigate the difference in high-altitude, hard-body, hypersonic flow radiation signatures as computed from the Burnett equations with rotational nonequilibrium instead of the more conventional Navier-Stokes equations with rotational/translational equilibrium. These differences are small at low flight velocities and low altitudes, but increase with both altitude and velocity to magnitudes that no longer are small. Comparison of computed radiation with the first SDIO/IST Bow-Shock-Ultra-Violet (BSUV) flight experiment launched at the relatively low hypersonic velocity of $3.5 \mathrm{~km} / \mathrm{sec}$, showed that the uncertainties in certain molecular physics aspects of the NEQAIR radiation code were much larger than were the effects of Burnett terms and rotational relaxation. Consequently, a serious revamping effect has been undertaken to improve the numerical efficiency of NEQAIR by more than an order of magnitude in order to make feasible the computation of very detailed radiation spectra; and to bring NEQAIR up to date with current progress in computational chemistry, as well as to extend its range of application to more species and broader flow-field regimes. The revamped code is termed NEQAIR 2. Thus far NEQAIR 2 has been compared with both the BSUV flight-1 results and with some new plasma torch radiation experiments conducted in the Department of Mechanical Engineering at Stanford. The agreement between computed intensities and radiation spectra is quite good except for a few isolated wavelength regions of the overall spectra. Currently, comparisons have begun between the NEQAIR 2 computations and the BSUV flight 2 results conducted at 5.1 $\mathrm{km} / \mathrm{sec}$.

\section{Reports:}

1. Effect of Rotational Relaxation and Approximate Burnett Terms on Hypersonic Flow-Field Radiation at High Altitudes, by Stephane Moreau et al., ALAA-91, ALAA-91-1702, 1991. AD A244 749

\section{IGNITION STUDIES IN NONPREMIXED HYDROCARBON/AIR COMBUSTION}

Chung K. Law

Princeton University

\section{BRADEC, TACOM}

This project investigates the detailed nature of the ignition process in counterflowing nonpremixed flames, in which a fuel jet impinges against an opposed jet of hot air. The ignition "kernel" structure and the dominant chemistry are examined as the system is brought to ignition by raising the air temperature. Experimental measurements of the ignition temperature are made as functions of both strain rate and fuel concentration. These are augmented by numerical modelling that elucidates the detailed structure and chemistry of the ignition kernel just prior to ignition, and its response to the effect of pressure, fuel concentration, and aerodynamic straining. This work represents the first step in a larger program to understand the ignition of complex fuel in realistic combustion environments such as diesel engines. The initial efforts have focused upon the ignition of $\mathrm{H}_{2} /$ air because of the relative simplicity and accuracy of the reaction mechanism as well as the central role that hydrogen oxidation plays in the combustion of all hydrocarbons.

\section{KINETICS AND ENERGY TRANSFER IN NONEQUILIBRIUM FLUID FLOWS}

D.R. Crosley

G.P.Smith

SRI International

The effort has concentrated on analyzing the data on the final states of quenching of $A^{2} \Sigma^{+}$NO. The goal of these experiments is an understanding of the distribution of vibrational, rotational, and translational energy following collisional quenching of the electronically excited nitric oxide. Such knowledge is needed for the proper application of microscopic reversibility to a situation where non-LTE conditions prevail. The experiment is a two laser, pump/probe determination of the populations of the ground state vibrational distributions following collisional selfquenching (NO by $\mathrm{NO}$ ) in a slowly flowing cell. A quantitative analysis of the population distribution has been hampered by a lack of knowledge of relative fluorescence efficiencies when probing different levels, and some uncertainties in vibrational band absorption coefficients. However, these results indicate comparable populations in each level over the entire range studied, $v=6$ to 23 . At the high end, over 80 percent of the electronic energy has been converted 
to vibrational energy, an extremely interesting dynamic effect. This distribution is many orders of magnitude different from predictions of FranckCondon overlap, demonstrating that the collision does not occur on a time scale faster than the diatomic vibrational frequency.

\section{DEVELOPMENT AND APPLICATION OF FLUORESCENT DIAGNOSTICS TO FUNDAMENTAL DROPLET AND SPRAY PROBLEMS}

Lynn A. Melton

University of Texas at Dallas

\section{SL: CRDEC}

SC: ARL/WTD, ARL/VPD, TACOM

Work on the interpretation of prior exciplex fluorescence thermometry results on hexadecane droplets has yielded significant results. Under a prior ARO program, Thomas Hanlon at UT-Dallas had used exciplex fluorescence thermometry to infer hexadecane droplet temperatures as a function of fall distance in quiescent heated nitrogen; his inferred temperatures showed a striking jump of about $100^{\circ} \mathrm{C}$ once the droplet reached a temperature of approximately $80^{\circ} \mathrm{C}$. Recent work by Dr. Zhang Jingyi has shown that a proper explanation of Hanlon's results requires inclusion of the optics of the droplet, the transient temperature field within the droplet, and the nonlinear characteristics of the exciplex fluorescence thermometry calibration curve. On the basis of Zhang's work, it appears that experiments such as those carried out by Hanlon can be (1) designed so that the optical weighting of fluorescence from within the droplet matches the geometrical weighting, i.e., unbiased transient volume-averaged temperatures are obtained, and (2) interpreted so that the anomalous temperature step yields directly the vapor-to-liquid heat transfer coefficient. Susan Eshelman has completed measurements of the quenching of naphthalene and $\mathrm{N}, \mathrm{N}, \mathrm{N}^{\prime}, \mathrm{N}^{\prime}$-tetramethyl-p-phenylenediamine (TMPD) fluorescence by nitrous oxide; the quenching of TMPD by $\mathrm{N}_{2} \mathrm{O}$, although twenty times less efficient than the quenching by $\mathrm{O}_{2}$, is still too efficient to allow quantitative use of naphthalene/TMPD exciplex-based vapor/liquid visualization systems under $\mathrm{N}_{2} \mathrm{O}$ supported combustion conditions.
Reports:

1. Interpretation of Droplet Temperatures Obtained by Fluorescence Methods, by Jingyi Zhang and Lynn A. Melton, MS, I Heat Transfer.

\section{A THERMOCHEMICAL} TRANSPORT MODEL FOR ANALYSIS OF HOT-SPOT FORMATION IN ENERGETIC MATERIALS

\section{P. Barry Butler \\ University of Iowa}

\section{SL: ARL/WTD}

Previous research has shown that a small fraction of voids in a condensed-phase energetic material can substantially increase its sensitivity to initiation by shock impact. Although the actual mechanisms for initiating reaction in an energetic material are not thoroughly understood, it is generally accepted that an excessive amount of energy is focused in a small fraction of the material. These localized high-energy sites are called "hot spots" and can be the result of a shock wave interacting with flaws or inhomogeneities in the grain matrix. Two key elements in understanding the detonation hazard associated with energetic materials are: (a) identification of the mechanisms(s) for generating hot spots in the grain, and $(b)$ identification of the mechanisms for the subsequent growth to detonation or in some cases, quenching of the reaction. This research focuses on the micromechanics of a single void to a specified stress history. To analyze the potential for hot spot formation under these conditions, a viscoplastic pore collapse model has been developed to model the dynamic behavior of a material void after it has been subjected to a specified stress-time profile. The model takes into account the effects of condensed-phase material viscosity, yield strength, and pore gas pressure on the time-evolving pore deformation and heating. In addition to treating pore dynamics, energy balances for the condensed-phase material and pore gas are introduced in order to track temporal variations of interface and gas temperatures. Important thermal processes such as viscoplastic heating, finite-rate chemical effects in the gas phase, surface reaction, phase change, and heat exchange between the pore gas and surrounding material are included in the model. 
Reports:

1. A Thermomechanical Analysis of Hot Spot Formation in Condensed-Phase, Energetic Materials, by J. Kang et al., Combust and Flame 89,117(1992).

\section{THE EFFECT OF TURBULENCE ON VAPORIZATION AND MIXING IN FUEL SPRAYS}

D.A. Santavicca

The Pennsylvania State University

$$
\text { SL: TACOM }
$$$$
\text { SC: BRADEC }
$$

A preliminary study of droplet dispersion was successfully conducted in the high pressure, high temperature turbulent flow system designed specifically for this research which demonstrated the feasibility of the planned study. The turbulent flow system was modified in order to achieve laminar flow conditions which would serve as a baseline or reference case for the turbulent measurements. A heated droplet generator and heated flow system for use in calibrating the exciplex based droplet thermometry technique was constructed and made operational. An automated image analysis program was developed for determining droplet position and velocity from droplet trajectory photographs. A two-dimensional Raman imaging system for use in characterizing the behavior of droplets injected into supercritical environments was specified, ordered and acquired.

\section{CONTROL OF SOOT FORMATION}

\section{R. A. Dobbins}

Brown University

\section{SL: ARLNPD, TACOM}

While it has been noted in the past that the addition of diluents can suppress soot formation, a systematic study of the role of inert additions to flames has not been undertaken. A number of diluents have been tested as additives to the fuel in a coannular diffusion flame configuration to determine their effectiveness in suppressing the yellow coloration that is associated with the formation of soot particles. Nitrogen, argon and carbon dioxide do suppress soot formation that is normally present in diffusion flames burning either methane, ethylene, or acetylene. The amount of particular diluent required to suppress soot formation increases with the theoretical adiabatic flame temperature of the fuel. Thus, the moles of nitrogen per mole of fuel required for soot suppression is 1.5 for methane, 6.0 for ethylene, and 10.5 for acetylene. The different diluents are effective in proportion to their molar specific heats with carbon dioxide being the most effective. An interesting result of these experiments is the discovery that the height of the flame for a given fuel flow is essentially unaffected by the addition of large amounts of diluents.

\section{STAR 63: MULTI-LASER TRANSMISSION EXPERIMENT}

K.S. Beale

Arnold Engineering and Development Center

SC: SDIO/IST

The research objective is to determine rocket exhaust plume temperatures and radiative characteristics. Ultraviolet and infrared spectral data, taken during the test firing of two STAR...63 solid propellant rocket motors in 1990, will be analyzed. Gas phase temperature will be determined from the observed aluminum chloride molecular absorption. This will be compared to temperature determined from the particulate continuum radiation. Temporal and spectral dependence of plume IR emission will be analyzed to determine the contribution of multiply scattered radiation to the total near-field radiation.

\section{ADVANCED DIESEL INJECTION STRATEGIES}

F.V. Bracco

Princeton University

SL: TACOM

SC: BRADEC, ARL/VPD

The research objective is to investigate novel approaches to increase combustion efficiency and reduce combustion-generated emissions in direct injection diesel engines. Using exciplex fluorescence visualization, relative (and perhaps absolute) equivalence ratio distributions will be determined for novel fuel injector designs. Candidate systems are impingement injectors, air-assist and air-entrainment injectors, electrostatic atomizers and spark-assisted, stratified charge designs. Measurements will be supported and analyzed using two-phase, unsteady reacting flow calculations. Routes to optimizing combustion chamber-injector design will also be investigated using 3-D calculations. 


\section{DIESEL SPRAY DYNAMICS, IGNITION, COMBUSTION AND SOOT FORMATION}

N.A. Henein

M.C. Lai

Wayne State University

Motoring tests of the transparent engine: Initial tests showed satisfactory compression ratio with Teflon piston ring. However, Teflon rings yielded easily and resulted in scuffing between piston and cylinder wall. Regrinding of cylinder wall and use of oil-impregnated piston ring assembly showed unsatisfactory compression ratio. Improvement has been in progress using both Teflon and oil-impregnated piston ring assembly, as well as smaller clearance between the piston and cylinder. Modification to the transparent test engine setup: Other modifications completed include optional four-valve and two-valve cylinder heads, optional flat and deep flat-bowl transparent pistons, and solenoid-controlled unit injector. On-going modifications include PC-based injection timing control and lighting access from the cylinder head. Analytical tool development: Further implementation of flamesheet model in KIVA-II program, including validation of the model with turbulent diffusion flames (hydrogen and methane) and $\mathrm{NO}_{x}$ data.

\section{IGNITION AND FLAME SPREADING SUSCEPTIBILITY OF GUN PROPELLANTS}

Kenneth K. Kuo

Stefan T. Thynell

The Pennsylvania State University

\section{SL: ARDEC, ARL/WTD}

The overall objective of this research program is to develop a more complete understanding of hot fragment conductive ignition of high energy low vulnerability ammunition within partially confined enclosures. Progress has been made related to the planned experiment and model improvements. A test rig has been designed and constructed. A comprehensive model was developed with funding from a previous ARL/WTD contract. Within this task, improvements to the existing model of the overall heat and mass transfer problem is underway. Another part of the research program is to study the effects of shock impact on the fracture and ignition time delay characteristics of various propellants using a shock tube facility. The test chamber required for conducting these experiments has been designed, fabricated and implemented. This chamber allows for high-speed visualization through two parallel side windows and photodetector response measurements through a smaller window which is directed toward the sample surface. Pressure versus time measurements are made at three separate locations along the test section. The sample can be mounted in a variety of ways, including complete or partial support to simulate propellant grain interactions. Another task is to examine the combustion behavior of layered propellants. Such propellants have been identified as suitable designs for tailoring the progressivity of the charge used in cartridges of large caliber guns. An increase of the muzzle velocity is expected without exceeding the maximum allowable pressure within the gun barrel. The composite rod layered propellants contain a slow burning outer layer and a fast burning inner layer (core). The thin, composite disk layered propellants contain slow burning outer layers and a fast burning middle layer. In the experimental program, the real-time $x$-ray radiography system has been employed to observe the burning behavior of the layered propellants.

\section{ELECTRON-MOLECULE COLLISIONS AND UV SIGNATURES IN FLOWFIELDS}

Vincent McKoy

California Institute of Technology

The research objective is to determine cross-sections for electron-molecule collisions necessary in modeling of ultraviolet emission from hypersonic flowfields. Recently developed computational methodology will be exploited to determine low-energy electron impact excitation cross-sections of electronic states of diatomic and triatomic molecules typical of hypersonic flowfields. Computations will be performed using the Schwinger multichannel variational principle implemented on a distributed memory computer. Cross-sections will be determined for excitation of ground state and excited state species.

\section{INVESTIGATION OF GASDYNAMIC PHENOMENA IN THE RAM ACCELERATOR}


A.P. Bruckner

University of Washington

\section{SC: ARDEC, ARL/WTD}

The research objective is to study fundamental gasdynamic phenomena associated with operating high speed ram accelerator projectiles. The existing ram accelerator test facility will be fitted with instrumentation to probe the reacting flowfield surrounding the ram projectile. Principal diagnostic will be high speed flow visualization, which will require extensive modification to provide optical access. Additional measurements will be temporally and spectrally resolved light emissions, as well as wall temperatures, and pressures. Flowfield properties will be studied as a function of reacting mixture composition and projectile geometry. Data will be compared to CFD model predictions.

\section{RESONANT HOLOGRAPHIC INTERFEROMETRY, AN INNOVATIVE TECHNIQUE FOR COMBUSTION DIAGNOSTICS}

Peter A. DeBarber

Cecil F. Hess

Metrol aser

\section{SC: TACOM}

The research objective is to develop analysis techniques for resonant holographic interferometry. The sensitivity and dynamic range of resonant holographic interferometry will be evaluated using holographic and spectrographic modeling. Measurement strategies will developed for monitoring species such as $\mathrm{C}_{2}, \mathrm{CH}, \mathrm{HCO}$, and $\mathrm{O}_{2}$. Experiments will be conducted with simple flat flame burners and propane/air and oxyacetylene flames. Emphasis will be placed on quantitatively measuring species concentration. Fourier transform holography will be modeled for the purpose of obtaining three dimensional spatial distributions of species concentrations.

\section{Structures and Dynamics}

\section{STABILITY OF ELASTICALLY TAILORED ROTOR SYSTEMS}

\section{D.H. Hodges}

L.W. Rehfield

Georgia Institute of Technology

Efforts to date have concentrated on achieving a better understanding of the design process and on development of analyses that will predict the aeroelastic stability of rotating composite blades. Researchers continued to develop and validate various aspects of the blade theories, especially for twisted blades, and stability analyses for rotors with composite blades. Three sectional analyses will be used in addition to NABSA which will be used and compared in the stability analysis: one is a new flexibilitybased method for a closed, thin-walled cell; the second is also a closed, thin-walled cell analysis; the third is a finite element analysis based on asymptotic theory which is able to account for the complex effects of the various elastic couplings without necessarily increasing the number of sectional coordinates beyond that of classical analyses. The stability analysis based on a mixed beam finite element method is now completed and coded. It treats general composite beams with variable spanwise geometry and accounts for all possible elastic couplings in the six by six sectional stiffness matrix which it can accept from any source, finite element or analytical. The aerodynamic model is similar to that of GRASP (developed at AFDD). Mathematica (a computerized symbolic manipulator) has been used to write extensive portions of the code. The analysis is capable of solving for the equilibrium deflections of a rotating blade in air and linearized dynamic aeroelastic stability of small motions about the equilibrium configuration. The structural and structural dynamics parts of the code have been validated for non-rotating beams for both static large deflections and linear dynamics about equilibrium by correlation with experimental data for both isotopic and composite beams; the results are excellent. Presently the stability analysis is being validated based on previously published results for equilibrium deflections and stability eigenvalues. So far, the results are also excellent.

\section{Reports:}

No. 1 in previous edition.

2. A First Order Nonlinear Theory for Thin-Walled Composite Beams with Closed Cross Sections, by Lawrence W. Rehfield, MS.

3. Analysis, Prediction, and Prevention of Edge Delamination in Rotor System Structures, by Wen S. Chan et al., J Am Helicopter Soc 36,44(1991).

4. Free-Vibration Analysis of Composite Beams, by Dewey $\mathrm{H}$. Hodges et al., J Am Helicopter Soc Ju1 91, p36. AD A245 403 


\section{ROTARY WING TECHNOLOGY CENTER}

Robert G. Loewy

Rensselaer Polytechnic Institute

\section{SL: AERO DIR, AP TEC DIR, ATCOM, ARL/VSD \\ SC: OASA (RDA)}

Areas of research currently under consideration include new materials for crashworthy structural concepts, analysis and design of composite fuselage frames, effects of hydrothermal environment and microcracking on tension-torsion coupled composites, design optimization and experimental studies of composite drive shafts, rotor optimization, helicopter maneuver loads, and air/ground resonance.

\section{Reports:}

No. $1-3$ in previous editions.

4. Unified Treatment for Dealing with Auxiliary Conditions in Blade Dynamics, by A. Rosen et al., AlAA J 29,968(1991). AD A244 632

5. Computer-Aided Conceptual Design of Rotorcraft, by Asa G. Trainer et al., MS.

6. Nonlinear Analysis of Anisotropic Rods Using Curvature Transformation and Including Warping, by A. Rosen et al., MS, J Comp and Struct.

7. Dynamic Analysis of Rotor Blades with Root Retention Design Variations, by R.G. Loewy et al., MS, ALAA J Aircraft.

8. Minimizing Hydrothermal Effects on the Dimensional Stability and Mechanical Properties of Composite Plates and Tubes, by Steven J. Winckler and Stephen C. Hill, MS.

9. A Multibody Formulation for Helicopter Nonlinear Dynamic Analysis, by O.A. Bauchau and N.K. Kang, MS.

10. Direct Integration of Helicopter Rotor Nonlinear Finite Element Dynamic Equations, by O.A. Bauchau et al., MS.

11. Influence of Pitch-Lag Coupling on Damping Requirements to Stabilize "Ground/Air Resonance", by Mark D. Zotto and Robert G. Loewy, MS, J Am Helicopter Soc.

12. An Adaptive Finite Element Methodology for Rotorcraft Aerodynamics, by Bruce E. Webster, MS.

\section{LARGE-AMPLITUDE FORCED RESPONSE OF DYNAMIC SYSTEMS}

Ali H. Nayfeh

Virginia Polytechnic Institute and State University

SL: AP TEC DIR

SC: TACOM

The following studies are being made: (a) Cyclic Motion Near a Hopf Bifurcation of a Four-Dimensional System, (b) Methods for Continuous Systems with Quadratic and Cubic Nonlinearities, (c) A Nonlinear Composite Plate Theory, $(d)$ Dynamic Characteristics of Slewing Metallic and Composite
Beams, (e) A Nonlinear Composite Beam Theory, $(f)$ Modal Interaction in the Response of Systems with Quadratic Nonlinerities to a Harmonic Excitation, (g) A Nonlinear Composite Shell Theory, (h) Modal Interaction in the Response of Composite Rectangular Plates, (i) On Identification of Structures with Internal Resonances, and (j) A Fully Nonlinear Model of Cables.

Reports:

No. $1-17$ in previous editions.

18. A Nonlinear Composite Beam Theory, by Perngiin F. Pai and Ali H. Nayfeh, Nonlinear Dynamics 2,445(1991).

19. Buckling of Shear-Deformable Cross-Ply Laminated Plates Using the State-Space Concept, by Jafar Hadian and Ali $\mathrm{H}$. Nayfeh, MS, Intl J Sol and Struct.

20. Dynamics of Three-Degree-of-Freedom Systems with Quadratic Nonlinearities, by Tariq Ali Nayfeh, MSThesis, 1991, $132 \mathrm{pp}$.

21. Nonlinear Dynamics of Composite Plates and Other Physical Systems, by Jamal Faris Nayfeh, PhD Thesis, 1990, 207 pp.

22. On Identification of Structures with Internal Resonances, by $\mathrm{B}$. Balachandran et al., MS, $A L A A J$.

23. A Fully Nonlinear Model of Cables, by Perngin F. Pai and A.H. Nayfeh, MS, $A L A A J$.

24. Modal Interaction in the Response of Composite Rectangular Plates, by J. Hadian et al., MS, Composites Eng.

25. A Nonlinear Composite Shell Theory, by Perngin F. Pai and Ali H. Nayfeh, MS, Nonlinear Dynamics.

26. Three-Mode Interactions in Harmonically Excited Systems with Quadratic Nonlinearities, by T.A. Nayfeh et al., MS, Nonlinear Dynamics.

27. A Nonlinear Composite Plate Theory, by Perngin F. Pai and Ali H. Nayfeh, MS, Nonlinear Dynamics.

28. Response of a Two-Degree-of-Freedom System Under Broad Band Random Excitation by Averaging and Stochastic Stability Methods, by A. Abourayan and A.H. Nayfeh, MS.

29. Observations of Nonlinear Interactions in a Flexible Cantilever Beam, by T.J. Anderson et al., MS.

30. A Refined Nonlinear Model of Piezoelectric Plates, by Perngjin F. Pai et al., MS, Intl J Sol and Struct.

31. On Identification of Nonlinear Interactions in Structures, by $B$. Balachandran et al., MS.

\section{A NEW TREATMENT OF PERIODIC SYSTEMS WITH APPLICATIONS TO HELICOPTER ROTOR BLADE DYNAMICS}

Subhash C. Sinha

Auburn University

\section{SL: AERO DIR, ARL/VSD \\ SC: AP TEC DIR}

A proposed technique has been extended to the application of analysis of nonlinear periodic systems 
and controller design of large order mechanical systems subjected to periodic loadings. Some important findings of the new scheme in the application of periodically varying systems include the following: (a) A simple picard type iteration scheme has been devised using Chebyshev polynomial expansions which is found to be remarkably accurate in the search for periodic, period doubling and chaotic solutions of nonlinear periodic systems. While the present method is as accurate as any conventional numerical integration procedure, it is also found to be less CPU time intensive compared to the latter method; (b) As an application of the proposed technique, controller designs of periodically varying large order mechanical systems have been achieved. Designs were undertaken using full state and observer based state feedbacks. The control problem is found to reduce to a set of algebraic equations suitable to parallel processing machines. The application of the technique to large order systems is found beneficial in terms of smaller computational time used; (c) As an offshoot of the solution of periodically varying systems in terms of Chebyshev polynomials, breakthrough has been achieved in obtaining the "Periodic Lyapunov-Floquet Transformations" in a symbolic form suitable for algebraic manipulations. It is anticipated that an application of such transformations would provide a remarkably superior technique in the analysis and control of nonlinear periodically varying systems.

Reports:

No. 1-7 in previous editions.

8. Development of an Efficient Computational Technique for the Analysis of Linear Dynamic Systems with Periodically Varying Parameters, by Der-Ho Wu, PhD Thesis, 1991, 203 pp.

9. A General Technique for Constructing Approximate Analytical Solutions for Linear Dynamic Systems with Periodic Coefficients, by Vikas Juneja, MS Thesis, 1991, 75 pp.

10. A New Computational Technique for the Stability Analysis of Slender Rods, by S.C. Sinha et al., MS, Arch Mech.

11. An Efficient Numerical Technique for the Analysis of Parametrically Excited Nonlinear Systems, by N.R. Senthilnathan and S.C. Sinha, MS, Nonlinear Dynamics.

12. Optimal Control of Large Scale Mechanical Systems Subjected to Periodic Loading via Chebyshev Polynomials, by Paul Joseph et al., MS.

\section{DYNAMICS OF DEFORMABLE MULTIBODY SYSTEMS USING RECURSIVE PROJECTION METHODS}

A.A. Shabana

University of Illinois at Chicago

\section{SL: AERO DIR, TACOM \\ SC: ARL/VSD}

The inertia projection schemes used in most existing recursive methods for the dynamic analysis of flexible robotics and mechanism systems lead to dense coefficient matrices in the acceleration equations and consequently there is a strong dynamic coupling between the joint and elastic coordinates. When the number of elastic degrees of freedom increases, the size of the coefficient matrix in the acceleration equations becomes large and consequently the use of these recursive methods for solving for the joint and elastic accelerations becomes less efficient. This investigation discusses the problems associated with the inertia projection schemes used in the existing recursive methods, and it is shown that decoupling the joint and elastic accelerations using these methods requires the factorization of nonlinear matrices whose dimensions depend on the number of elastic degrees of freedom of the system. An amalgamated formulation that can be used to decouple the elastic and joint accelerations is then proposed. In this amalgamated formulation, the relationships between the absolute, elastic and joint variables and the generalized Newton-Euler equations are used to develop systems of loosely coupled equations that have sparse matrix structure. Utilizing the structure of the inertia matrix of the deformable body and the fact that the joint reaction forces associated with the elastic coordinates do not represent independent variables, a reduced system of equations whose dimension is independent of the number of elastic degrees of freedom is obtained. This system can be solved for the joint accelerations as well as the joint reaction forces. The use of the procedure demonstrated using openloop robot manipulators and closed-loop flexible mechanisms.

\section{Reports:}

No. 1 in previous edition.

2. Constrained Motion of Deformable Bodies, by A.A. Shabana, Intl J Numer Meth Eng 32,1813(1991). AD A249 077

3. Use of the Generalized Impulse Momentum Equations in Analysis of Wave Propagation, by Wei-Hsin Gau and A.A. Shabana, $J$ Vibrtn and Acoust 113,533(1991).

4. Effect of the Order of the Finite Element and Selection of the Constrained Modes in Deformable Body Dynamics, by Frank Kirschner et al., Nonlinear Dynamics 3,57(1992). AD A249 672 
26995 THE FURTHER DEVELOPMENT AND REFINEMENT OF AN EXPERT-SYSTEMS APPROACH TO THE CREATIVE DESIGN OF MECHANISMS AND MECHANICAL SYSTEMS

F. Freudenstein

D. Hoeltzel

Columbia University

\section{SL: ARDEC, TACOM}

The research has continued actively with regard to the design analysis, synthesis and design automation of robotic end effectors including Coulomb friction. The nonlinearity of Coulomb friction makes the analysis challenging. The mechanical analysis has been applied to rather sophisticated end effectors, such as the Bendix design and others. With the many variables involved, it has nonetheless become possible to predict the extent of the frictional resistance, as well as to optimize the design geometry. An approach for the automatic generation and refinement of three dimensional finite element meshes subdivided by rigid body movable subdomains has been developed. A combination of computational geometry and geometric modeling techniques have been employed to implement parametric computer-aided design based on the finite element method. To demonstrate the utility of this approach to parametric redesign, a series of meshes that model a hip joint prosthesis and a reciprocating internal combustion engine have been generated.

Reports:

No. 1 in previous edition.

2. Automatic Finite Element Mesh Generation for the Automation of Parametric Conceptual Design, by Hyunik Yang and D.A. Hoeltzel, SAE Technical Paper Series 912219, 1991, p59. AD A249 338

\section{SYNTHESIS AND SIMULATION OF ROBOTIC SYSTEMS}

Bahram Ravani

University of California, Davis

\section{SL: BWL, TACOM}

SC: ARDEC

There have been three new basic developments. Two of these have been completed to a point that they are being submitted for publication. The last one has produced very promising results but needs additional work before it will be submitted for publication. The first development deals with automatic targeting and sensor based calibration of CAD models used in robotic simulation. Researchers have studied mechanics of automatic targeting using different sensor arrangements and have developed a method for reconstructing object or target locations based on redundant sensor measurements of different geometric features. Although the problem lends itself to a nonlinear least squares problem, it is possible to develop a formulation that reduces the problem into a linear problem suitable for fast computations. The results, in addition to their application in calibration of robotic and mechanical system simulation software packages have applications in automatic targeting for smart weapon systems. The second development is related to CAD based animation of three dimensional motion of mechanical systems. In computer simulation of large mechanical systems, generation of tightly spaced displacements of a moving body along its trajectory may not be cost effective. Instead, a series of positions are generated using the dynamical equations of motion and the in-between displacements are constructed using appropriate motion interpolants. In the past, smooth animation of three dimensional motions of mechanical systems were not possible when using motion interpolation since higher order continuity could not be achieved without loosing velocity scale. Researchers have combined studies in kinematic and computational geometry and have developed geometric algorithms that would allow motion interpolation with higher orders of continuity producing smooth animation. The third development has involved continued work on decoupling of the dynamical equations of motion of multibody systems.

Reports:

1. Kinematic Localization for World Model Calibration in Off-Line Robot Programming Using Clifford Algebra, by B. Ravani and Q.J. Ge,Proc 1991 IEEE Intl Confon Robotics and Automation, 1991, p584. AD A244 764

2. Kinematics of the Ball Screw Mechanism, by M.C. Lin et al., Adv in Design Automation 2,383(1991). AD A249 608

3. On the Analysis and Design of High Speed Thrust Ball Bearings, by M.C. Lin et al., Eighth World Congress on Theory of Machines \& Mechanics, Vol 1, 1991, p193. AD A249 416
27300 MODAL INTERACTIONS AND COMPLEX RESPONSES IN NONLINEAR MULTI-DEGREE-OF-FREEDOM MECHANICAL SYSTEMS 
A. Bajaj

P. Davies

Purdue University

Multiple mode solutions have been studied and equations generated through the method of averaging that allow one to study the multi-mode response of panels. Under certain conditions on the frequency of excitation, these equations reduce to those generated to examine the $(1,2)$ and $(3,1)$ coupled mode response. This two mode solution has been studied extensively. Second order averaging has been performed to yield a set of equations that are being used to study the behavior of the pendulum absorber. A careful bifurcation analysis is being performed. A study is being made of the response of a nonlinear single degree-of-freedom system to a harmonic excitation with a slowly changing frequency, commonly referred to as a swept sine excitation. The study involves the use of equations derived by using first order averaging theory. The excitation frequency is itself a slow function of time. The aim of this initial research investigation is to derive an algebraic expression that can be used to predict the jump phenomenon and the associated maximum amplitudes of the response. Experiments have been performed on the plate rig. To investigate under what excitation conditions there is observable nonlinear behavior, studies are being made of the excitation of single modes well separated from other modes of the plate. The plate has been excited close to the natural frequency of the mode of interest with a harmonic excitation. Three different types of excitation mechanism have been used: electromagnetic exciters, coil and magnet shakers (10lb and 50lb) and a loudspeaker.

Reports:

1. Non-Linear Vibrations and Chaos in Harmonically Excited Rectangular Plates with Internal Resonance, by S.I. Chang et al., MS, Nonlinear Dynamics.

2. Bifurcations in the Dynamics of an Orthogonal Double Pendulum, by S. Samaranayake, MS, Nonlinear Dynamics.

3. Amplitude Modulated Dynamics of a Resonantly Excited Autoparametric Two-Degree-Of-Freedom System, by A.K. Bajaj et al., MS, Nonlinear Dymamics.

\section{CLOSED LOOP VIBRATIONAL CONTROL: THEORY AND APPLICATIONS}

Semyon M. Meerkov

Pierre T. Kabamaba

University of Michigan

A paper has been prepared in which the zeros of linear systems that ensure $\mathbf{H}_{2}$-optimal transmission characteristics from inputs to outputs are analyzed, and qualitative features of the resulting behavior are investigated. In another paper, linear plants with control signals entering the open loop dynamics as amplitudes of periodic, zero average functions are considered. Stabilizability properties of such plants by state space and output feedback controllers are analyzed. Although the problem of optimal pole placement has received enormous attention during the last 30 years, that of optimal zero placement has been relatively neglected in control systems research. This latter problem could however be of substantial importance in many design problems, such as actuators and sensors positioning. In a third paper, researchers characterize the zeros that ensure $\mathrm{H}_{2}$-optimality of open and closed looped transfer functions and analyze qualitative properties of the resulting systems.

\section{HIGH AND LOW FREQUENCY DYNAMICS OF ISOLATED BLADES AND ROTORS WITH DYNAMIC STALL AND WAKE}

\section{G.H. Gaonkar}

Florida Atlantic University

\section{SL: AERO DIR}

SC: AP TECDIR

The influence of structural and aerodynamic modeling on the aeroelastic stability of isolated hingeless rotor blades is being investigated. The aerodynamic modeling is based on ONERA dynamic stall models of lift, drag and pitching moment. The study includes bending and torsional flexibility of blade and rootflexure under highly stalled conditions in forward flight. The predictions are correlated with the measured stability data of two experimental rotors. The rotors were tested at the U.S. Army Aeroflightdynamics Directorate, Ames Research Center. The nonlinear equations of blade motion and stall dynamics (typically involving 49 states: 12 from blade degrees of freedom and the remaining 37 from stall dynamics) are perturbed about a periodic orbit and the damping is evaluated by Floquet eigenanalysis. In hover, the substall drag effects and unsteady linear lift effects improve the correlation with the test data of the two-bladed experimental rotor. For the three-bladed experimental rotor in hover and forward flight, the predictions show increasing sensitivity to structural refinements in blade modeling and this sensitivity increases with increasing thrust levels and flight speed. Investigation showed that a correct 
structural simulation of blade root-flexure becomes increasingly crucial under high-speed and highthrust conditions. Further research is directed in this direction.

Reports:

1. Analysis of Dynamic Stall Effects on Isolated Rotor FlapLag Stability with Experimental Correlation, by Dinesh Barwey et al., MS, J Am Helicopter Soc.

2. Dynamic Stall Effects on Hingeless Rotor Stability with Experimental Correlation, by Dinesh Barwey, PhD Thesis, 1991, 203 pp.

3. Investigation of Dynamic Stall Effects on Isolated Rotor Flap-Lag Stability with Experimental Correlation, by Dinesh Barwey et al., J Am Helicopter Soc 36,12(1991). AD A249001

4. Effects of Dynamic Stall and Structural Modeling on Aeroelastic Stability of Elastic Hingeless Rotor Blades with Experimental Correlation, by Dinesh Barwey et al., MS.

5. Flap-Lag Damping in Hover and Forward Flight with a Three Dimensional Wake, by A.R. Manjunath et al., MS.

6. Trim Analysis by Shooting and Finite Elements and Floquet Eigenanalysis by $Q R$ and Subspace Iterations in Helicopter Dynamics, by Nagari S. Achar, PhD Thesis, 1992, 144 pp.

\section{NUMERICAL ANALYSIS OF THIRD AND HIGHER HARMONIC OVERTONES OF THICKNESS-SHEAR VIBRATIONS IN SC-CUT QUARTZ RESONATORS}

Yook-Kong Yong

Rutgers, The State University of New Jersey

\section{SL: ARL/EPSD}

A piezoelectric, laminated composite plate theory was developed and presented in a paper for the purpose of modeling and analyzing piezoelectric thin-film resonators and filters. The laminated plate equations are extensions of anisotropic composite plate theories by Yang, Norris and Stavsky and Whitney and Pagano to include piezoelectric effects and capabilities for modeling harmonic overtones of thickness-shear vibrations. Two-dimensional equations of motion of piezoelectric laminates were deduced from the three-dimensional equations of linear piezoelectricity by expanding mechanical displacement $u_{i}$ and electric potential $\phi$ in a series of trigonometric functions as proposed by Lee and Nikodem for elastic, isotropic plates and later extended to piezoelectric crystal plates by Syngellakis and Hou. The laminated plate equations are applied to a zinc oxide-silicon bilayer strip without electrodes and solved for straight crested waves. Dispersion relations and mode shapes of the fundamental thick- ness-shear are presented for different ratios of zincoxide to silicon thickness.

\section{Reports:}

1. Thickness-Shear Mode Shapes and Mass-Frequency Influence Surface of a Circular and Electroded AT-Cut Quartz Resonator, by Y-K Yong et al., MS, IEEE Trans on Ulrrason, Ferroelect, Freq Ctrl.

2. This number not used.

3. Piezoelectric, Laminated Composite Plate Theory for ThinFilm Resonators, by $Y-K$ Young et al,, MS.

4. Visualization Techniques for Bulk Wave Resonators, by $D$. Silver et al., MS.

\section{ADAPTIVE CONTROL OF NONLINEAR FLEXIBLE SYSTEMS}

\section{Robert L. Kosut}

Integrated Systems, Inc.

The sensitivity to parameter mismatch in feedback linearization based nonlinear tracking systems is investigated. The certainty-equivalence principle gives rise to four possible feedback configurations. One of these is the widely used case, where the original states are assumed to be available. Despite its implementability problem, the issue of the sensitivity of the design was addressed through a perturbation analysis. It is noted that the unacceptable transient behavior of the adaptive tracking scheme is closely related to the sensitivity of the underlying certaintyequivalence based control. Three other certaintyequivalence based controllers are proposed: two are observer-based; the third is a model-follower based on a feedforward/feedback implementation using a signal generator. Simulations are performed on an example to illustrate the points.

\section{SEVERE EDGE EFFECTS AND SIMPLE COMPLEMENTARY INTERIOR SOLUTIONS FOR ANISOTROPIC AND COMPOSITE STRUCTURES}

\section{C.O. Horgan}

J.G. Simmonds

University of Virginia

$$
\text { SC: ARL/WTD }
$$

A paper has been prepared which specializes to a semi-infinite tube Horgan's (1974) two-stress-function formulation of the equations for the axisymmetric deformation of a linearly elastic transversely isotropic cylindrical body free of surface tractions. The ratio of the tube's shear modulus to its radial (transverse) extensional modulus is taken to be of the 
order of magnitude of the square root of its thickness to its mean radius. The equations are solved by formal asymptotic expansion in fractional powers of the thickness to radius ratio for four canonical sets of end conditions, including (A) axisymmetric equilibrated tractions and (D) axisymmetric radial and axial displacements. The solutions exhibit interior (shell-like) parts and wide and narrow boundary (edge) layers, the latter containing components that vary extremely rapidly through the thickness of the tube. The analysis focuses on computing the lowest-order correction, both in the interior and in the boundary layers, to classical shell theory. It is shown that the interior correction to classical shell theory-that is, those effects so-called higher-order shell theories attempt to capture - cannot be computed without a consideration of three-dimensional boundary-layer effects, except in case A when there are edge tractions only.

\section{Reports:}

No. $1-2$ in previous editions.

3. Strain Energy Density Bounds for Linear Anisotropic Elastic Materials, by M.M. Mehrabadi et al., MS, J Elasticity.

4. An Asymptotic Analysis of End Effects in the Axisymmetric Deformation of Elastic Tubes Weak in Shear: HigherOrder Shell Theories are Inadequate and Unnecessary, by James G. Simmonds, MS, Intl J Sol and Struct.

5. The Eigenvalues for a Self-Equilibrated, Semi-Infinite, Elastically Anisotropic Strip, by E.C. Crafter et al., MS, $J$ Appl Mech.

6. The Effect of Constitutive Law Perturbations on Finite Anti-Plane Shear Deformations of a Semi-Infinite Strip, by C.O. Horgan and L.E. Payne, MS, Quart Appl Math .

\section{NONLINEAR DYNAMICS AND AEROELASTICITY OF ROTORCRAFT IN FORWARD FLIGHT}

Earl H. Dowell

Duke University

\section{SL: AERO DIR, ARL/VSD}

The theoretical and experimental behavior of articulated and hingeless rotor blades has been studied. The theoretical work included both rotating as well as non-rotating blades because of the limitations of costs and facilities. The work is described more fully in three papers. The first contains a theoretical study of the flutter and forced response of an articulated blade. Primary emphasis is on root restraint structural nonlinearities and aerodynamic stall nonlinearities. The second is an experimental-theoretical correlation study for flutter and forced response of an articulated blade. A good agreement between the- ory and experiment is shown for the general character, amplitude and frequency content of the nonlinear oscillations. The third paper describes a theoretical correlation study for a hingeless blade. Fair to good agreement between experiment and theory is shown. Given the complexity of the physical phenomena, the agreement between theory and experiment is generally encouraging, though clearly further work is needed.

\section{DESIGNING STRUCTURES FOR CONTROLLABILITY}

\section{R.E. Skelton}

Purdue University

\section{SL: ARL/MD \\ SC: ARDEC, TACOM}

The goal of this project is to show how to redesign a system to make it easier to control. For a given plant, a paper has been prepared which begins with the assumption that a controller is given which yields desired closed-loop properties. Both the controller and the original plant are redesigned so as to yield the same original closed-loop properties with a minimal active control effort. The initial linear controller before plant redesign is not restricted; it could be static or dynamic. Researchers call this the optimal mix problem since they optimally mix parameter changes of the plant with parameter changes of the controller. The problem requires matching the entire plant matrix of the original system. The problem is reduced to a standard mathematical quadratic program. Hence, the globally optimal answer is obtained in a finite number of steps. The applicability of the optimal mix theory to a general mechanical system is demonstrated by numerical examples of a springmass-damper system.

\section{E. Multidisciplinary Mechanics}

\section{ASSESSMENT OF CARTESIAN/CUBIC GRID SYSTEMS FOR COMPUTATIONAL FLUID DYNAMIC ANALYSIS OF COMPLEX GEOMETRIES}

\section{Francis X. Hurley}

North Carolina Agricultural and Technical State University

The research objective is to determine the potential, or not, of cartesian (cubic) grids in finite difference 
assaults on flow field problems featuring extremely difficult geometries. The theory of numerical analysis will be entered to identify methods of error estimation and of processor time prediction. Using the Navier-Stokes equations, and cartesian and body-fit- ted grid approaches, term-by-term counts, if necessary, will be made of error and processor time accumulations. Significant elements will include streamline curvature, gradients, and boundary conditions. Numerical stability will also be addressed. 


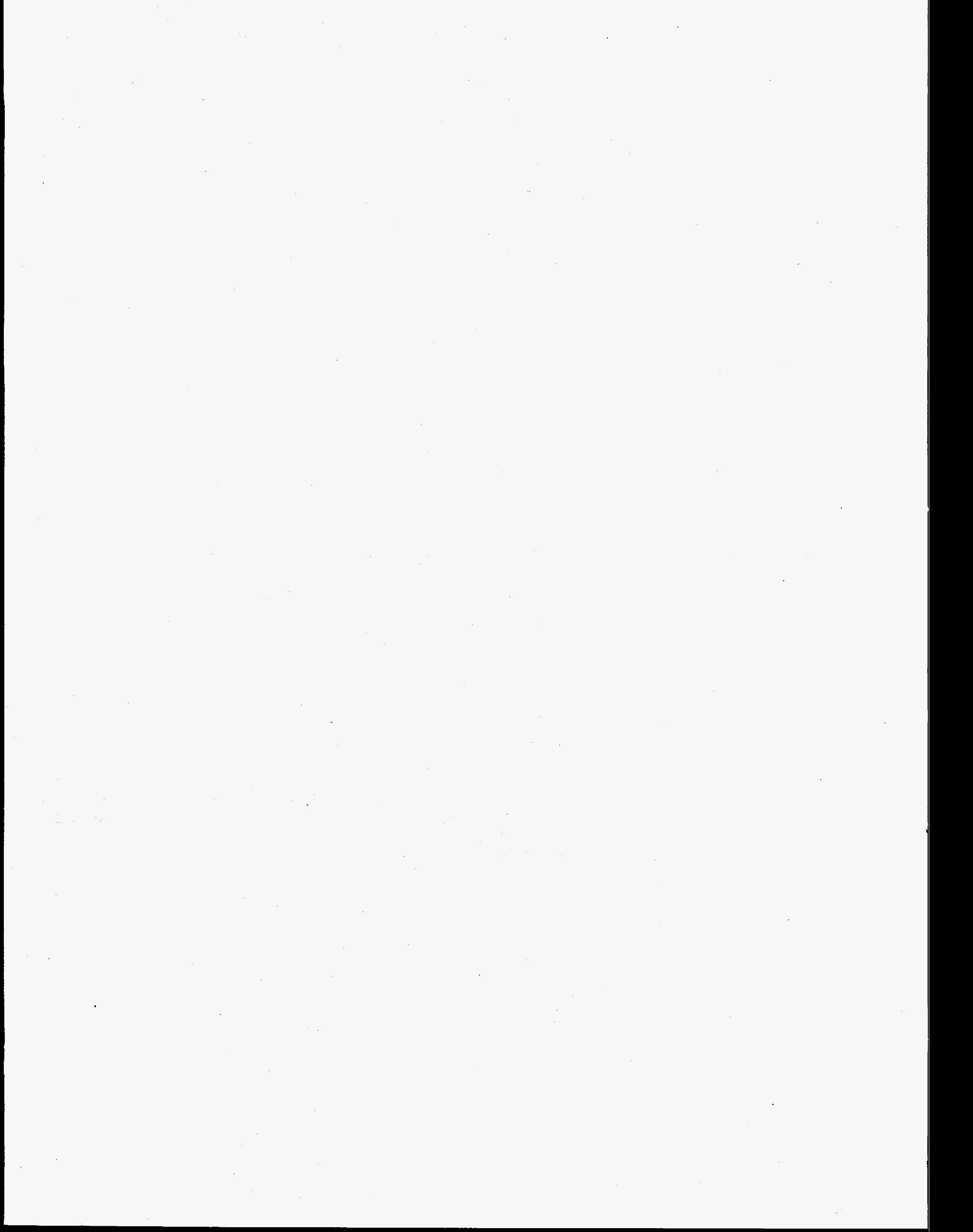




\section{METALLURGY AND MATERIALS SCIENCE}

\section{A. Degradation, Protection, and Reaction}

\section{LARGE SCALE SURFACE MODIFICATION BY HIGH CURRENT ION IMPLANTATION}

Ian G. Brown

University of California, Berkeley

SL: ARDEC, ARL/MD

SC: AVSCOM/DERSO, TACOM

Ion Source Development - Use of the Mevva V ion source for ongoing metal ion implantation research has continued routinely. This ion source, which was developed early in the ARO program, has proven to be highly reliable and to fulfill all the requirements for the surface modification research program. Ion Implantation Research - A wide range of metal ion implantation research programs are being carried out. These research topics all have in common that they require high doses of metal ions to be implanted, and thus make use of the unique capabilities of the Mevva implantation technology. Researchers have established an interaction with LRA Laboratories, Inc., and the Army Materials Testing Laboratory, Watertown, for fundamental research on the stress corrosion cracking behavior of different alloys. The fatigue behavior of $\mathrm{Fe}-14 \mathrm{Cr}-14 \mathrm{Ni}$ implanted with various species including $\mathrm{Ti}, \mathrm{Y}, \mathrm{Ta}, \mathrm{Ni}, \mathrm{Hf}$ and $\mathrm{Cr}$ is being investigated. Preliminary results are good. The work is continuing. Substantial improvement in the corrosion characteristics of the lead electrode material in lead/acid storage batteries by implantation of titanium into the $\mathrm{Pb}$ has now been confirmed. Work is now centered on systematically exploring the effect of implantation dose and comparison of Ti implantation with implantation of other metal ion species. The work is continuing. The effect of metal ion implantation on the oxidation resistance of titanium aluminide is being investigated jointly with a researcher from Argonne National Laboratory. The species $\mathrm{Si}, \mathrm{Ca}$,
$\mathrm{Cr}, \mathrm{Y}, \mathrm{Nb}, \mathrm{W}$ and $\mathrm{Pt}$ were implanted into TizAl substrates at surface concentrations from 1 to 20 atomic percent. Oxidation testing and characterization of these samples is now being carried out.

Reports:

No. $1-4$ in previous editions.

5. Development of a DC, Broad Beam, Mevva Ion Source, by I.G. Brown et al., MS, Surface and Coatings Tech.

6. Versatile High Current Metal Ion Implantation Facility, by I.G. Brown et al., MS, Surface and Coatings Tech.

7. Some Novel Surface Modification Applications of a New Kind of High Current Metal Ion Implantation Facility, by I.G. Brown et a1., J Mater Eng 13,217(1991). AD A249 781

\section{IN SITU SURFACE STUDIES OF CONVERSION COATINGS AND PASSIVE FILMS ON STEEL AND ALUMINUM BASED METAL MATRIX COMPOSITES}

Henry W. White

University of Missouri at Columbia

SC: ARDEC, TACOM

Several experimental tools - Raman spectroscopy, tunable diode laser infrared reflection-absorption spectroscopy (IRRAS), scanning tunneling microscopy (STM), atomic force microscopy (AFM), and electrochemical impedance spectroscopy (EIS) are being used to investigate several protective coating systems. These protective systems are (1) polymer modified zinc phosphate $(\mathrm{Zn}: \mathrm{Ph})$ based protective coatings on steel, (2) protective films formed on $\mathrm{Al}$ and $\mathrm{Al}$-based metal matrix composite (MMC) materials formed by immersion in rare earth chloride solutions, and (3) passive films formed on iron in aqueous solutions. Researchers are also investigating other films with potential applications for surface modification and protection. They are (1) plasma deposited carbon polymer films, (2) the conducting polymer polyaniline, and (3) aluminum nitride and polycrystalline diamond films. 
Reports:

No. $1-3$ in previous editions.

4. In Situ Infrared Spectroscopy, by Jeffrey L. Wragg and Henry W. White, MS.

5. Scanning Tunnelling Microscopy of Solid $\mathrm{C}_{60} / \mathrm{C}_{70}$, by J.L. Wragg et al., Nature 348,623(1990). AD A239 697

6. Large-Scale Periodic Features Associated with Surface Boundaries in Scanning Tunneling Microscope Images of Graphite, by J.E. Buckley et al., J Vac Sci Tech B9,1079(1991). AD A239 698

\section{PROCESS PARAMETER/GROWTH ENVIRONMENT/FILM PROPERTY RELATIONSHIPS FOR REACTIVE SPUTTER DEPOSITED METAL OXIDE, NITRIDE, AND OXYNITRIDE FILMS}

Carolyn R. Aita

University of Wisconsin - Milwaukee

SL: ARDEC, BWL, ARL/MD

SC: ARO, BRADEC, CERL, ARL/MD, NVESD, TACOM

Researchers continued work on the phase map for the $\mathrm{V}-\mathrm{O}$ system, relating process parameters, the growth environment, and metallurgical phase in the growth regime of near-room substrate temperature, low surface diffusion, and unity sticking coefficient. Data from $\mathrm{Ne}-\mathrm{O}_{2}$ and $\mathrm{Kr}-\mathrm{O}_{2}$ discharges were compared to that from $\mathrm{Ar}-\mathrm{O}_{2}$ discharges. Highlights include: Working out the details for producing single orientation crystalline vanadia with tailorable interlayer spacing and defining the conditions under which dense, amorphous stoichiometric vanadia was produced (for optical windows). In addition, data for the V-O system further verified important generalizations concerning the effect of fractional flux of metal, metal-oxide, and oxygen species in the growth environment on the metal-oxide metallurgical phase boundaries. These generalizations were based on data from the $\mathrm{Nb}-\mathrm{O}, \mathrm{Y}-\mathrm{O}$, and $\mathrm{Zr}-\mathrm{O}$ systems. Phase mapping the $\mathrm{Nb}-\mathrm{N}$ system was initiated, with a study of the effect of rare gas type and cathode voltage on the metallurgical phase produced in the film. $\mathrm{NbN}$, sometimes with an excess of nitrogen, was produced under a wide range of deposition conditions. Amorphous subnitrides with resistivity lower than $\mathrm{Nb}$ were also produced. These may be new materials. A generalized study of the effect of incident ion energy (E) on sputter yield (S) was carried out, using optical emission spectrometry as the detection tool for both the incident ion and the sputtered species.
Reports:

No. $1-8$ in previous editions.

9. Annealing Response of Disordered Sputter Deposited Vanadium Pentoxide ( $\left.\mathrm{V}_{2} \mathrm{O}_{5}\right)$, by John Luksich and Carolyn Rubin Aita, J Vac Sci Tech A9,542(1991). AD A249 568

10. Core Level and Valence Band X-Ray Photoelectron Spectroscopy of Gold Oxide, by Carolyn Rubin Aita and N.C. Tran, JVac Sci Tech A9,2498(1991). AD A250 230

11. A Phase Map for Sputter Deposited Niobium Oxides, by Ray C. Lee and Carolyn Rubin Aita, $J$ Appl Phys 70,2094(1991). AD A250 195

\section{INHIBITION OF CORROSION BY ELECTRODEPOSITED CONDUCTIVE-POLYMER FILMS}

Dale P. Barkey

University of New Hampshire

\section{SC: ARDEC, BRADEC, ARL/NPD}

Efforts focused on two areas: deposition of poly(3-methyl thiophene) films on titanium and studies on anion effects on film behavior. A two-step procedure was developed for deposition of adherent films on titanium. In the first step, a very thin film is deposited from a dilute monomer solution in the presence of chloride. SEM images of the surface after this treatment show pits and small islands of polymer deposit. A thick film is then built up by deposition from a concentrated monomer solution without chloride. This process is novel. Other studies on anion effects have concerned the rest potential of the polymer. Chloride and hydroxide may reduce the polymer to a nonconductive state and prevent its function of maintaining substrate passivity: Further investigation of chloride ion in the presence of other anions shows more complicated behavior. In particular, sulfate may prevent polymer reduction even when chloride is added. The mechanisms at work are probably doping and sieving. Doping is the process by which anions affect the electronic or physical structure of the polymer resulting in changes in conductivity and rest potential. Sieving refers to relative permeabilities of anions in the polymer and their dependence on potential or the presence of other anions. Chloride and hydroxide appear to shift the polymer potential in the negative direction, while nitrate and sulfate have the opposite effect. The intercalation of sulfate evidently reduces the permeability of chloride and to some extent protects the film. 


\section{ADHESION AND POLYMER-MODIFIED PRECOATINGS}

Toshifumi Sugama

Brookhaven National Laboratory

SL: ARL/EPSD, TACOM

SC: ARDEC, BRADEC, ARL/VPD

Fractal polyzirconosiloxane (PZS) cluster films were prepared through the hydrolysis-polycondensationpyrolysis synthesis of two-step $\mathrm{HCl}$ acid- $\mathrm{NaOH}$ base catalyzed sol precursors consisting of $\mathrm{N}$-[3-(triethoxysilyl)propyl] - 4,5-dihydroimidazole, $\mathrm{Zr}\left(\mathrm{OC}_{3} \mathrm{H}_{7}\right)_{4}$, methanol, and water. When amorphous PZSs were applied to aluminum as protective coatings against $\mathrm{NaCl}$-induced corrosion, the most effective film was derived from a sol having a $\mathrm{pH}$ near the isoelectric point in the positive zeta potential region. Researchers investigated the characteristics of melt-crystallized polyphenyletheretherketone (PEEK) as an adhesive for steel-to-steel joints. Interfacial chemistry, which plays an important role in determining whether adhesion will be poor or good at PEEK/steel interfaces involved the following reactions. Oxygen-catalyzed deformation of PEEK in air led to a low rate of crystallinity, which gave poor adhesion to the steel surfaces. The enrichment of $\mathrm{Fe}_{2} \mathrm{O}_{3}$ by extensive oxidation of the interface also created an undesirable boundary region, thereby indicating that failure occurs cohesively through weak $\mathrm{Fe}_{2} \mathrm{O}_{3}$ layer. Crystallizable polyphenyletheretherketone (PEEK) mortar specimens were prepared by melting-cooling processes of packed $P E E K$ powdersilica sand mixtures at temperatures ranging from 25 to $400^{\circ} \mathrm{C}$, in air or $\mathrm{N}_{2}$ gas. The specimens made in air had a low degree of PEEK crystallinity. This was caused by oxidation-induced deformation of the polymer matrix, and resulted in a lack of thermal stability. The formation of well-crystallized PEEK in the vicinity of silica aggregate was found in the molded body made in $\mathrm{N}_{2}$. Such a PEEK crystalline structure contributes significantly to the thermal and hydrothermal stability at temperatures up to $200^{\circ} \mathrm{C}$, and to the resistance to a 5 weight percent $\mathrm{H}_{2} \mathrm{SO}_{4}$ solution at $80^{\circ} \mathrm{C}$.

Reports:

No. 1-11 in previous editions.

12. Durabilities of Crystalline Polyphenyletheretherketone (PEEK) Polymer Mortars, by T. Sugama and N.R. Carciello, MS, Cement Concrete Res.

13. Adhesion of Crystalline Polyphenyletheretherketone (PEEK) in Metal-to-Metal Joints, by T. Sugama et al., MS, Intl $J$ Adhesion and Adhesives.
14. Fractal Polyzirconosiloxane Cluster Coatings, by T. Sugama, MS.

\section{CORROSION INHIBITION BY PLASMA ION IMPLANTATION}

J. Reece Roth

Raymond A. Buchanan

University of Tennessee, Knoxville

\section{SL: ARL/MD}

SC: BRADEC

Promising electrochemical results were found for nitrogen-implanted commercially-pure aluminum. In a $\mathrm{N}_{2}$-deaerated 1.0 wt percent $\mathrm{NaCl}$ solution, the anodic polarization behaviors indicated similar pitting potentials but lower pit-propagation rates for the nitrogen-implanted aluminum as compared to unimplanted aluminum. Measurements of anodic charge transferred at a constant potential in the deaerated solution confirmed these results, with the anodic charge transferred (and hence the amount of corrosion) being 30-60 times less for the nitrogenimplanted aluminum. Electrochemical tests have been performed with the electrolyte in the aerated (air-saturated) condition. The results of these tests were not encouraging. Under aerated conditions, nitrogen implantation did not render the aluminum more resistant to pitting corrosion. Indeed, in the polarization tests, the anodic current densities above the pitting potential were somewhat higher for the implanted material; and in the constant-potential tests, the anodic charge transferred was again somewhat higher for the implanted material. Both of these results indicated detrimental effects due to nitrogen implantation. Thus, when the electrolyte was in the deaerated condition, nitrogen implantation was beneficial, but when in the aerated condition, nitrogen implantation was somewhat detrimental. At least a partial explanation for the detrimental results with the aerated electrolyte involves the oxidizing power associated with the dissolved oxygen. It is reasonable to suspect that the dissolved-oxygen reduction reaction induced an initial corrosion potential sufficiently high to cause severe pitting even on the nitrogen-implanted aluminum samples, and certainly on the unimplanted aluminum samples. The subsequent anodic polarization and constant-potential tests were then being conducted on samples that were already severely pitted. Since most practical chloride-containing electrolytes will be in the naturally-aerated condition, these latest results indicate that nitrogen 
implantation of aluminum would not be an effective corrosion inhibition treatment. Implantation of certain metallic elements ( $\mathrm{W}, \mathrm{Cr}, \mathrm{Mo}$ ), which will allow significant chemical modification of the normal $\mathrm{Al}(\mathrm{OH})_{3} / \mathrm{Al}_{2} \mathrm{O}_{3}$ passive film, appears to be a more promising approach.

Reports:

No. $1-4$ in previous editions.

5. A Large-Volume Microwave Plasma Facility (MPF) for Industrial Plasma Processing, by Paul D. Spence et al., MS.

6. Performance of a Large-Volume, Unmagnetized Microwave Plasma Facility (MPF) for Plasma Ion Implantation Applications, by Paul D. Spence et al., MS.

7. A Large-Volume, Uniform, Unmagnetized Microwave Plasma Facility (MPF) for Industrial Plasma Processing Applications, by Paul D. Spence et al., MS

\section{ION BEAM MODIFICATION OF CERAMICS: MECHANICAL PROPERTIES AND STRUCTURE}

James W. Mayer

Cornell University

SL: ARL/MD

SC: ARDEC, ARL/MD

Researchers have continued to investigate the use of ion beams and lasers to modify the surface mechanical properties of zirconia. The latest thrust has involved the use of excimer laser processing to lower the sliding friction coefficient of zirconia. Most work with excimer processing has involved the titanium/zirconia system although other metal/zirconia systems are being examined. Extensive pin-on-disc friction and wear testing has been performed on laser processed layers of titanium on zirconia. Irradiation fluences have been varied from 0.1 to $1.0 \mathrm{~J} / \mathrm{cm}^{2}$ with optimal results obtained at a fluence of approximately $0.4 \mathrm{~J} / \mathrm{cm}^{2}$. No significant load or velocity dependencies have been found but the friction coefficient may be sensitive to relative humidity. Rutherford backscattering analysis was used to determine the amount of titanium lost during processing. At the optimal fluence of $0.4 \mathrm{~J} / \mathrm{cm}^{2}$ about 20 to 30 percent of the titanium is lost; at fluences above $0.6 \mathrm{~J} / \mathrm{cm}^{2}$ almost all of the titanium is lost due to ablation. X-ray diffraction shows that no phase formation is occurring during the irradiation process since only peaks due to the zirconia substrate and titanium metal are seen both before and after processing. This is probably due to the very high cooling rates achieved which prevent the titanium from oxi- dizing even though the processing was done in air. Both optical and scanning electron microscopy were used to determine the surface structure following processing and wear testing. The surface does not remain completely flat after resolidification. The surface exhibits cracking that can be related to the crystallographic orientation of the cubic zirconia substrate. This cracking is probably due to the differences in thermal expansion coefficients of titanium and zirconia.

Reports:

No. 1-2 in previous editions.

3. Occurrence of Solid Noble-Gas Inclusions in Ion-Beam-Implanted Magnesium Oxide, by M. Grant Norton et al., Phys Rev B43,9291(1991). AD A239 756

4. Microstructure of Hardened and Softened Zirconia after Xenon Implantation, by Elizabeth L. Fleischer et al., JMater Res 6,1905(1991). AD A244 791

\section{MOLECULAR DYNAMICS OF PHYSICAL AGING PROCESSES IN POLYMER GLASSES: EXPERIMENT AND THEORY}

John D. McGervey

Case-Western Reserve University

\section{SL: ARL/MD \\ SC: ARDEC, ARL/MD, ARL/VPD}

Studies are being made of the temperature dependence of the ortho-Ps intensity $I_{3}$ and mean lifetime $t_{3}$ for polymer glasses, and of the changes in these parameters with aging. The changes in $I_{3}$ with temperature appear to be an artifact of the fitting procedure, resulting from the fact that the $o$-Ps component is really a composite of several components. Simulated curves on the computer revealed that when there are more than three components in the input data, the fitting program can still fit the data to three components, but the intensity of the third component is not equal to the sum of the intensities of the third and longer components in the input data, as had been suspected. By using as input data the theoretical size distribution of free volume elements (Simha theory, applied by $\mathrm{S}$. Vleeshouwers, on the assumption of spherical free volume elements) and the known lifetimes in holes of these sizes, one finds that the difference between the observed intensity and the theoretical intensity is explained as an artifact of the fitting procedure. The temperature dependence of $t_{3}$ does not display this sort of problem; experiment and theory agree well. Three papers are being written to 
cover the various aspects of the problem: one on the measurements, one on the Monte Carlo calculations to find the hole size distributions, and one on the computer simulations of experimental data with multiple lifetimes. All of this work, for temperature effects in polycarbonate, is now complete. On the other hand, aging leads to considerable changes in $I_{3}$ but virtually no change in $\mathrm{t} 3$ over a period of hundreds of hours. Work on positron lifetimes in amorphous bisphenol $A$ polycarbonate under applied tensile strains was presented at the Ninth International Conference on Positron Annihilation.

Reports:

1. Positron Annihilation Lifetime Studies of Free Volume Changes in Polycarbonate Under Static Tensile Deformation, by M.Y. Ruan et al., MS, Macromol.

2. Probe Spectroscopy, Free Volume Concepts, and Physical Aging of Polymer Glasses, by M.Y. Ruan et al., MS, Macromol.

3. Temperature and Time Dependence of Free Volume in Bisphenol-A Polycarbonate Studied by Positron Lifetime Spectroscopy, by J.-E. Kluin et al., MS, Macromol.

4. Monte Carlo Calculations of Hole Size Distributions; Simulation of Positron Annihilation Spectroscopy, by S. Vleeshouwers et al., MS, J Polym Sci Polym Phys.

\section{FORMATION OF TRANSITION ELEMENT NITRIDE COATINGS ON STEELS USING ELEVATED-TEMPERATURE PLASMA SOURCE ION BEAM ENHANCED DEPOSITION}

John R. Conrad

Richard A. Dodd

University of Wisconsin - Madison

\section{SL: ARDEC, ARL/MD}

SC: ARDEC, TACOM

(a) Researchers have developed an experimental procedure to measure the secondary electron emission coefficient, the average number of electrons emitted per incident ion, in PSII. (b) A scratch tester has been designed and constructed to provide a qualitative measurement of the adhesion of PSII-IBED coatings to the substrate. $(c)$ The term fretting refers to small-amplitude, high frequency oscillating motion between two solid surfaces. While fretting wear occurs in localized and almost microscopic regions, it can become a precursor to expensive and catastrophic failures. Fretting wear is a source of major concern in a number of defense related applications, and particularly in strategic helicopter components.
Sea water, when present, augments fretting wear. Researchers have designed and constructed a fretting wear tester. The machine uses an electro-mechanical actuator to provide a linear displacement of the stylus or flat against a test coupon. The design provides for wide range adjustment of the fretting parameters of loading force, frequency, and displacement. Furthermore, the fretting wear process can be carried out in the presence of a corrosive medium. These options allow simulation of a wide variety of fretting wear applications. (d) Aggressive tests of PSII-nitrogen implanted 17-7 PH stainless steel showed that implanted samples had better erosion and corrosion resistance as compared to untreated samples. (e) A corrosion resistant $\mathrm{Cr}-12 \mathrm{Mo}$ layer, one micron thick, has been deposited on 304 stainless steel using independent $\mathrm{Cr}$ and Mo sputter sources. The corrosion rate relative to stainless steel in $0.1 \mathrm{~N}$ sulfuric acid was reduced by three orders of magnitude after the IBED process. The corrosion properties have been correlated with the microstructure of the film, which was evaluated using electron microscopy and grazing incidence diffractometry. $(f)$ A lumped capacitance model was developed for predicting the target temperature during implantation. The model incorporates both conducting and radiating modes of heat transfer, while assuming a uniform target temperature. Preliminary calculations show that the cooling of the target in the PSII chamber is dominated by radiation.

Reports:

No. 1 in previous edition.

2. This number not used.

3. Measurements of Spatial and Temporal Sheath Evolution for Spherical and Cylindrical Geometries in Plasma Source Ion Implantation, by M. Shamim et al., J Appl Phys 69,2904(1991). AD A239 589

4. Measurement of Electron Emission Due to Energetic Ion Bombardment in Plasma Source Ion Implantation, by M.M. Shamim et al.,J Appl Phys 70,4756(1991). AD A249 495

5. Measurements of Spatial and Temporal Sheath Evolution for Spherical and Cylindrical Geometries in Plasma Source Ion Implantation, by M. Shamim et al., J Appl Phys 69,2904(1991).

6. Comparison Between Conventional and Plasma Source Ion-Implanted Femoral Knee Components, by A. Chen et al., J Appl Phys 70,6757(1991). AD A249 493

7. Influence of Temperature on Nitrogen Ion Implantation of Incoloy Alloys 908 and 909 , by L. Xie et al., Mater Sci and Eng A139,179(1991). AD A249 273 
27624 TAILORED

POLYMER/NONPOLYMER

INTERFACES FOR CONTROLLED ADHESION

Edward J. Kramer

Cornell University

SL: ARL/MD

SC: ARDEC, BRADEC, CRDEC, ARL/MD, ARL/NPD

Common tests for measuring polymer-nonpolymer adhesion are riddled with problems which are intrinsic to the types of materials being measured and are thus difficult to circumvent. A fracture mechanics approach, modified to eliminate some of the difficulties in testing, is the most useful approach to the problem. A double cantilever beam fracture specimen has been developed and used to make fracture toughness measurements on interfaces between polystyrene and glass that have been strengthened by the addition of deuterated polystyrenepoly(2vinylpyridine) block copolymers. The PVP hydrogen bonds to the silanol groups on the glass and the DPS block, if long enough, will entangle with the polystyrene homopolymer. Glass slabs of unequal thickness form the two beams. A thin layer of the block copolymer is spun onto the thinner beam from solution. A much thicker polystyrene film is spun onto a glass plate, floated off onto water, and then transferred onto the copolymer coated glass slab. These coated slabs were then annealed for three hours at $160^{\circ} \mathrm{C}$ in vacuum to produce an equilibrium interface. The top surface of the polystyrene layer is bonded to the thicker glass slab using a thin layer of an epoxy resin, after first etching the polystyrene surface with oxygen plasma to produce suitable polar surface groups which can react with the epoxy. The glass/copolymer/polystyrene/epoxy/glass sandwich is then annealed at $100^{\circ} \mathrm{C}$ for two hours to cure the epoxy. From each glass slab several $7.5 \mathrm{~mm}$ wide samples were cut using a water lubricated diamond saw. After polishing the edges of these samples to remove cracks in the glass, they were left in a vacuum desiccator overnight to remove moisture from the cutting and polishing procedure. The test itself involves forcing a razor blade between the two beams causing the interface between the polystyrene and the thinner glass beam to crack ahead of the blade. A small testing jig has been constructed which drives the razor blade at a constant rate and the length of the crack ahead of the razor blade is measured at many points along the specimen using a television camera and VCR system. From each measurement of the crack length one can compute one value of fracture energy, allowing one to make many measurements on the same specimen and to determine the statistical spread of these measurements. After the fracture experiment has been terminated, forward recoil spectrometry is used to measure the DPS on each fracture surface and thus determine the real density of block copolymer at the interface. Two papers were being prepared, one on the test itself and the other on the effects of block copolymer additions.

\section{MOLECULAR ENGINEERING OF THIN POLYMER FILMS PREPARED FROM FUNCTIONALLY TERMINATED OLIGOMERS}

Jeffrey $\mathbf{T}$. Koberstein

University of Connecticut

\section{SL: ARL/MD \\ SC: ARDEC}

The university recently received an ISC 200 pin-ondisk friction tester. This is a computerized continuous motion apparatus which has the capability of testing materials up to temperatures of $600^{\circ} \mathrm{C}$ either in air or in an inert atmosphere. It has been designed to be used as part of the ASTM G99 pin-on-disk friction testing protocol. A computer program allows for data storage and retrieval. The data can be output in several different modes; e.g., friction coefficient versus time or number of sliding cycles. Another program computes the contact pressure of the normal load based on the pin contact area, the elastic modulus, and Poisson's ratio for the pin and disk materials. The contact pressure is helpful when trying to compare friction data for different lubricants or sliding materials. Synthesis of di-carboxylic acid-terminated polydimethylsiloxane has been carried out via an equilibration reaction. Basically this is a bulk ring-opening polymerization where a cyclic dimethyl siloxane monomer is first opened using a very strong acid catalyst. It is then inserted by an equilibration reaction into a siloxane molecule that contains the carboxylic acid end groups. Since this type of polymerization gives fairly large polydispersities, the material is being fractionated by supercritical fluid extraction. This should give fairly narrow fractions with number average molecular weights in the range of $900-3000 \mathrm{~g} / \mathrm{gmole}$. This material will be tested to determine its high temperature lubricating properties. Synthesis of a mono-acid ter- 
minated 1,2-polyisoprene is also being completed. This material is being synthesized anionically using sec-butyl lithium as the initiator.

Reports:

1. Tribology of Langmuir-Blodgett Films, by C.L. Mirley and J.T. Koberstein, TR, Mar 92, 25 pp. AD A250 075

2. Tribology of Functionally-Terminated Oligomer Films, by C.L. Mirley and J.T. Koberstein, TR, Mar 92, 13 pp. AD A250076

28369 REAL TIME X-RAY SCATTERING STUDY OF PROCESSING OF HIGH PERFORMANCE THERMOPLASTICS

Peggy Cebe

Massachusetts Institute of Technology

\section{SL: ARL/MD, NRDEC}

SC: BRADEC, TACOM

The polymers to be investigated are two homopolymers, poly(etheretherketone), PEEK, and poly(butylene terephthalate), PBT, both of which are used as composite materials. To exercise control over crystallization kinetics, these will be blended with an amorphous component, creating the blends PEEK/PEI (polyetherimide) and PBT/PAR (poly arylate). These are blends of a semicrystalline polymer with an amorphous polymer, the latter of which will serve to alter the processing characteristics of the crystallizable component. Films of the homopolymers and blends in four composition ratios, 80/20, 60/40, 40/60, 20/80, and preliminary characterization has begun using FTir, thermal analysis, and $x$-ray diffraction. The first real time $x$-ray scattering studies were conducted using the Brookhaven National Synchrotron Light Source. Homopolymer PBT and PBT/PAR materials were examined under isothermal conditions in the temperature range from $50{ }^{\circ} \mathrm{C}$ to $300{ }^{\circ} \mathrm{C}$ using wide angle diffraction. PBT proved to be an excellent choice for this study because it is a strong scatterer and presents six very sharp reflections in the angular range from $7^{\circ}$ to $35^{\circ}$. PBT has a triclinic unit cell structure with lattice parameters known from previous research. The coefficient of thermal expansion (CTE) was determined for the crystal lattice using a nonlinear least squares fit to the peak positions of six major wide angle reflections. CTE values for the blends were the same as for homopolymer PBT.

\section{GROWTH AND}

\section{CHARACTERIZATION OF \\ REFRACTORY METAL \\ MULTILAYERS: POTENTIAL ULTRAHIGH STRENGTH HIGH \\ TEMPERATURE SURFACE COATINGS}

John C. Bilello

Steven M. Yalisove

University of Michigan

SL: ARL/MD

SC: BWL

Preliminary work has focused on the first layer with the view of defining optimal coating parameters and refining characterization techniques. The prototypical material has been high temperature refractory metal Mo on Si substrates. The stress evolution in thin films of sputtered $\mathrm{Mo}$ on $\mathrm{Si}(100)$ substrates has been studied, as a function of microstructure, byx-ray diffraction, transmission electron microscopy (TEM), and Rutherford backscattering spectroscopy. Double crystal $\mathrm{x}$-ray diffraction topography has been employed to determine film stress as a function of thickness. High compressive stress, about $1 \mathrm{GPa}$, is found for the thinnest Mo film. With increasing film thickness a minimal residual stress level is reached. Low angle incidence $x$-ray diffraction patterns indicated that crystalline Mo is present in the thinnest films. Line broadening of the $\mathrm{Mo}(110)$ diffraction peak has shown that the grain dimension is comparable to the film thickness over the range studied. Plane view TEM observations of films less than $20 \mathrm{~nm}$ demonstrated the presence of discontinuous films with island dimensions on the order of $80 \AA$. This is in good agreement with $\mathrm{x}$-ray mosaic spread results. These results are discussed in a paper in the context of mechanisms for the development of ultrahigh strength metal matrix composite thin film coatings.

\section{SCANNING TUNNELING MICROSCOPY AND SPECTROSCOPY OF BONDING MECHANISMS BETWEEN ADVANCED CERAMICS AND THIN METAL FILMS}

\section{S.T. Tsong}

W.T. Petuskey

Arizona State University

SL: ARDEC

SC: BWL, CERL, ARL/MD, NVESD, TACOM 
Researchers have interpreted the dislocation line of atoms on the $\mathrm{Si}(111)-(7 \times 7)$ surface as an antiphase boundary. A detailed analysis of the surface structure shows that the observed line defect does not involve an extra plane of atoms emerging from the bulk to intersect the surface. Hence it is not a true dislocation, but merely an antiphase boundary. STM observations were performed on the effects of applying an external stress and an electrical current to a Si(100) surface. During the passage of the current, the temperature of the surface reaches $650^{\circ} \mathrm{C}$. When the current is passed in the opposite direction, however, the terrace widths change dramatically. The current in the step-up direction renders the large terraces even larger. Obviously, the Si atoms on the surface are mobile under the influence of the applied current or the electric field. Although the observed phenomenon bears a certain similarity to the "electromigration effect", in which thin metal films deposited on silicon are observed to move when an electric field is applied, the exact mechanism for the movement of steps on the $\mathrm{Si}(100)$ surface is unknown. A new UHV chamber for STM is presently under construction. This chamber will accommodate a new STM with a levitate-and-damp system using high $\mathrm{T}_{\boldsymbol{c}}$ superconducting thin films.

\section{NONDESTRUCTIVE CHARACTERIZATION OF TWO-PHASE METAL-MATRIX MATERIALS}

Kamel Salama

University of Houston

\section{SC: BRADEC}

The pulse-echo overlap and an automated time-offlight acquisition system have been used to determine the second-order Lamé constants and longitudinal ulltrasonic velocity dependence on temperature in two aluminum-silicon carbide metal matrix composites, namely Al-7064 and Al-8091, and in monolithic silicon carbide. The third-order Murnaghan constants were determined as a function of temperature for the Al-7064 MMC alloy for silicon carbide.

\section{THE ANALYSIS OF HYDROGEN IN MATERIALS USING LOW ENERGY ION BEAMS}

Robert A. Weller

Marcus H. Mendenhall

Vanderbilt University

\section{SL: CECOM \\ SC: BRADEC, CERL, ARL/MD, TACOM}

A faraday cage has been designed and is being fabricated. Theoretical studies are under way to determine whether or not it is possible to predict time-of-flight detector quantum efficiency from a knowledge of quantities such as stopping power, secondary electron yield and multiple scattering angular distributions. Work during this period has demonstrated the usefulness of multiply charged ions. Researchers are currently focusing their attention on multiply-charged $\mathrm{Ne}$ and $\mathrm{Ar}$. The polymer formvar is especially useful for measurements since it can be prepared in self-supporting films of only a few $\mathrm{nm}$ and can, of course, also be used to coat other samples with a uniform hydrogen bearing layer. Several experiments using this film are under way, including ones that compare transmission and grazing incidence forward scattering and others that assess the effects of the composition of the underlying substrate material on the apparent spatial resolution for surface hydrogen.

\section{MODIFICATION OF PALLADIUM ELECTROLYTICALLY CHARGED WITH DEUTERIUM}

James $T$. Waber

Michigan Technological University

The objective of the research is to investigate heatproducing and non-heat producing palladium electrodes with positron annihilation spectroscopy, and characterize defects and their size distribution, carry out $\mathrm{X}$-ray diffraction studies to detect phase changes and line broadening for the palladium samples, and evaluate the Bush-Eagleton model as extended by the Chubb BBC concept in terms of the types of radiation damage expected and found experimentally. 


\section{B. Mechanical Behavior}

\section{FRACTURE BEHAVIOR OF IONOMERS AND IONOMERIC BLENDS}

Masanori Hara

John A. Sauer

Rutgers, The State University of New Jersey

SL: ARL/MD

SC: MICOM, ARL/VSD, TACOM

Two types of plasticizer have been considered for morphological studies: polar plasticizer that preferentially interacts with ion-rich phase, and nonpolar plasticizer that preferentially interacts with ion-poor phase. Researchers have conducted tensile testing for plasticized ionomers and found the following: nonpolar plasticizer just reduces modulus and strength without improving toughness, while polar plasticizer enhances toughness and elongation with some reduction in modulus. In short, polar plasticizer is more effective in improving fracture properties of PS ionomers. A morphological study by using dynamic mechanical testing was conducted for a new type of amorphous ionomer, i.e, PMMA-based ionomers. It was found that the polar plasticizer, glycerol, interacts not only with ion-rich phase of PMMA ionomers but also with ion-poor PMMA phase. Therefore, glycerol works as a dual plasticizer for PMMA ionomers. This is new to the study of plasticization of ionomers. Dynamic mechanical testing and deformation were conducted for PMMA ionomers with divalent $\mathrm{Ca}$ ions and were compared with the results on monovalent Na salts. Ionic crosslinking due to Ca salt is much stronger than Na salt; therefore, the rubbery plateau of $\mathrm{Ca}$ salt is much more extended than $\mathrm{Na}$ salt. Also, the deformation mode of Ca salt is crazing plus shear deformation, while that of $\mathrm{Na}$ salt is only crazing. In short, divalent $\mathrm{Ca}$ ion is much more effective than monovalent $\mathrm{Na}$ ion to create strong ionic crosslinkings, and, thus, to improve fracture properties. This finding is completely consistent with findings on PS-based ionomers and is used as a general guide to develop ionomers for engineering applications.

Reports:

No. $1-6$ in previous editions.

7. Fatigue Behavior of Ionomers. 3. Effect of Excess Neutralizing Agent on Sulfonated Polystyrene Ionomers, by $M$. Hara et al., Macromol 23,4964(1990). AD A245 294
26751 SUPERPLASTICITY IN

FINE-GRAINED CERAMIC

COMPOSITES

T.G. Nieh

Lockheed Missiles \& Space Co., Inc.

SC: BRADEC, ARL/VPD

1. Superplastic 20 percent $\mathrm{Al}_{2} \mathrm{O}_{3} / \mathrm{YTZ}$ composite has for the first time been successfully deformed into hemispherical caps at $1475{ }^{\circ} \mathrm{C}$ via biaxial gas-pressure forming using Ar at shell stresses in the range of 3.45-13.8 MPa. The hemispherical caps exhibited a thickness variation from dome apex to dome base. The observed variations in thickness agree well with predictions from the Cornfield-Johnson model. An analytical model based upon the Cornfield-Johnson model but incorporating additional material parameters such as variations during forming in the strain rate sensitivity exponent and grain growth-induced strain hardening is needed to accurately model and precisely control the biaxial gas-pressure forming of superplastic ceramics. No experimental difficulty was encountered in applying the required gas pressures and temperatures to achieve the results presented in the study. 2. Both superplastic 3Y-TZP and 20 percent $\mathrm{Al}_{2} \mathrm{O}_{3} / \mathrm{YTZ}$ composite have been successfully deformed into hemispherical caps via a biaxial gas-pressure forming technique. Based on the results of this study, the PI proposes to fabricate zirconia insulation tubes by superplastic extrusion of zirconia polycrystal. This would not only reduce the cost, but also improve the reliability of the tube products. An analytical model incorporating material parameters such as variations during forming in the strain rate sensitivity exponent and grain growth-induced strain hardening is currently under development in order to model accurately and therefore precisely control the biaxial gas-pressure forming of superplastic $3 \mathrm{Y}-\mathrm{TZP}$ and 20 percent $\mathrm{Al}_{2} \mathrm{O}_{3} / \mathrm{YTZ}$ composite.

26825 A STUDY INTO THE EFFECTS OF ELECTRIC FIELDS AND CURRENTS ON THE AGING AND HARDENING OF STEELS

Hans Conrad

North Carolina State University

SL: ARDEC

SC: BWL 
An externally applied electric field reduced both the volume fraction and the density of cavities which developed during superplastic deformation of aluminum alloy 7475 . The decrease in cavitation resulted from a reduction in the nucleation rate of cavities at small strains; their subsequent growth rate was increased by the electric field. The principal nucleation sites for cavities without an electric field were triple junctions of grain boundaries, whereas with an electric field nucleation around second-phase particles also became important. The reduced nucleation rate of cavities in an electric field was attributed to a field-enhanced accommodation of grain boundary sliding. The lower cavitation of aluminum alloy 7475 deformed in an electric field resulted in improved post-deformation strength and ductility.

Reports:

No. 1-14 in previous editions.

15. Effect of Electric Field on Cavitation in Superplastic Aluminum Alloy 7475 , by H. Conrad et al., Mater Sci and Eng A138,247(1991). AD A245 159

16. Effect of Electric Current Pulses on Fatigue Characteristics of Polycrystalline Copper, by H. Conrad et al., Mater Sci and Eng A145,1(1991). AD A244 801

17. Influence of an Electric Charge During Quench Aging of a Low-Carbon Steel, by Hao An Lu and Hans Conrad, Appl Phys Let 59,1847(1991). AD A244 157

18. On the Effect of Persistent Slip Band (PSB) Parameters on Fatigue Life, by Wei-di Cao and Hans Conrad, Fatigue Fract Engng Mater Struct 15,573(1992).

19. Evaluation of the Parameters Which Govern Local Necking in 3003-0 Al Sheet, by A. Zayed and H. Conrad, MS.

20. Cyclic Softening by High Density Electric Current Pulses During Low Cycle Fatigue of a-Ti, by Z.H. Lai et al., MS, Scripta Met.

\section{TOUGHENING MECHANISMS IN ULTRAHIGH-STRENGTH STEELS}

Gregory B. Olson

Northwestern University

SL: BWL, ARL/MD

SC: CERL, ARL/MD

A paper by J.S. Montgomery and G.B. Olson detailing a study of $\mathrm{M}_{2} \mathrm{C}$ carbide precipitation in AF1410 steel was presented at the G.R. Speich Symposium of ISS-AIME in Montreal on October 27, 1992 and has been published in the Symposium Proceedings. The paper represents a synthesis of Montgomery's electron microscopy with results of collaborative APFIM microanalysis and SANS studies and applies a theoretical analyses of coherent precipitation to account for initial carbide nucleation on dislocations. The analysis could not account for an observed second stage of nucleation occurring at half-completion of precipitation. Elastic calculations have now satisfactorily accounted for the second stage as autocatalytic nucleation in the stress fields of the first generation carbides when they reach a critical size. Average size particles at this stage are more than sufficient to give barrierless heterogeneous nucleation of second-generation carbides. As an aid to control of the diffusion time scale of precipitation, a theory of coarsening kinetics in a general multicomponent system has been completed and will next be tested against recent SANS Data on $\mathrm{M}_{2} \mathrm{C}$ coarsening in model Fe-Ni-Cobased alloys.

Reports:

No. 1 in previous edition.

2. Materials Design: An Undergraduate Course, by G.B. Olson, MS.

\section{THE ROLE OF SECOND PHASE PARTICLES IN THE DESIGN OF ULTRAHIGH STRENGTH STEELS OF IMPROVED TOUGHNESS}

Warren M. Garrison, Jr.

Carnegie-Mellon University

\section{SL: BWL, ARL/MD}

SC: ARL/VSD

The emphasis has been on the morphologies of the $\mathrm{Ti}_{2} \mathrm{CS}$ particles in the HY180 and AF1410 steels. Particles have been extracted from fracture surfaces of both steels. The $\mathrm{Ti}_{2} \mathrm{CS}$ particles in these experimental heats were typically thin plates with a very broad size distribution. The plates varied from about $2 \mu \mathrm{m}$ in diameter to as small as $500 \AA$ in diameter. The larger plates had fractured and these larger, fractured plates were about 20 percent of the $\mathrm{Ti}_{2} \mathrm{CS}$ particles observed in both steels. The $\mathrm{Ti}_{2} \mathrm{CS}$ particles are rather stable and do not dissolve in AF1410 steel until the steel is held at about $1300^{\circ} \mathrm{C}$. The goal with AF1410 was to dissolve these larger plates by forging at an initial temperature of $1300^{\circ}$ and reprecipitating smaller $\mathrm{Ti}_{2} \mathrm{CS}$ on cooling during forging. After forging at initial temperatures of $1150^{\circ} \mathrm{C}, 1225^{\circ} \mathrm{C}$ and $1300^{\circ} \mathrm{C}$ the Charpy impact energies were $207 \mathrm{~J}, 221 \mathrm{~J}$ and $152 \mathrm{~J}$ respectively. Thus, while it is anticipated the larger plates dissolved at $1300^{\circ} \mathrm{C}$, forging at an initial temperature of $1300^{\circ} \mathrm{C}$ does not seem to improve toughness. Fracture surfaces are presently being analyzed to determine $\mathrm{Ti}_{2} \mathrm{CS}$ and other particle sizes after forging at $1150^{\circ} \mathrm{C}, 1225^{\circ} \mathrm{C}$ and $1300^{\circ} \mathrm{C}$. 


\section{MEASUREMENT OF INTERFACE STRENGTH, INTRINSIC TOUGHNESS, AND THEIR DEPENDENCE ON INTERFACIAL SEGREGANTS}

\author{
Vijay Gupta \\ Dartmouth College

$$
\text { SC: ARDEC, CERL }
$$

The laser Doppler displacement and velocimeter have been installed to record directly the free surface displacement and velocities, respectively, of the test coating as the stress pulse rebounds from the coating free surface in the laser spallation experiment. This allows one to determine the interface stress directly, without resorting to a computer program as was done previously. This permits the use of the laser spallation experiment for interface systems involving ductile components. Other work includes the assembly and construction of an $r f$-magnetron sputter coating system capable of depositing both metals and dielectrics. Furthermore, for control of interface strength through segregants, the chamber is equipped with two guns capable of depositing both the coating and segregants without breaking the vacuum. Chromium was used as a strength enhancing segregant for the niobium/alumina system. It was recently discovered that if a liquid is used to sandwich the laser-absorbing gold film instead of a fused quartz disc as was used previously, one obtains unusual results. Although the stress pulse rise time and the amplitude are exactly the same in both cases, for the liquid, the stress pulse has a much sharper post-peak fall. If properly understood, this can revolutionize the field of interface strength measurement for very thin coatings $(<0.1$ $\mathrm{mm}$ ), which are of significant importance to the microelectronic device industry.

\section{LASER-INDUCED CONTROLLED FLAW TESTING IN CERAMICS}

Y.T. Chou

M.P. Harmer

Lehigh University

\section{SC: ARDEC}

In earlier studies, it was difficult to produce a single crack in a specimen by laser irradiation; cracking always took place at various spots along the path of the laser beam. This difficulty has been overcome by using a modified irradiation system in which the laser pulse energy and the shape of the beam focal spot were precisely measured and controlled. Fused quartz specimens were laser-irradiated to produce internal flaws. The specimens were fractured at room temperature under four-point bending, and the flaw size was measured. The results were: $(a)$ above a threshold value, the irradiation energy is linearly related to flaw size, and $(b)$ the relationship between the bending stress and the flaw size obeys the Griffith criterion of fracture. The experimental investigation of internal cracks will provide new information on the fracture process in ceramic materials.

\section{HIGH-TOUGHNESS GLASS-CERAMICS}

Minoru Tomozawa

Rensselaer Polytechnic Institute

SL: ARL/WTD

SC: ARL/MD

Fracture toughness of $\mathrm{Li}_{2} \mathrm{O}_{3}-\mathrm{Al}_{2} \mathrm{O}_{3}-\mathrm{SiO}_{2}$ system glass-ceramics containing $\mathrm{ZrO}_{2}$ was measured as a function of crystal growth temperature. The toughness increased even though a large fraction of $\mathrm{ZrO}_{2}$ transformed during the heat treatment. After prolonged heat treatment the fracture toughness decreased. Thus this phenomenon appears similar to precipitation hardening of metal alloys. Melting of $\mathrm{ZrO}_{2}$ containing glasses requires an extremely high temperature. Alternatively, $\mathrm{ZrO}_{2}$ can be incorporated into glass-ceramics by sintering. As a preliminary experiment, sintering characteristics of crystallizable glasses were investigated. The sintering appears controlled by the viscosity at the crystallization start temperature. Compositions having a lower viscosity at the crystallization start temperature gave a greater densification.

\section{SMART MATERIALS/STRUCTURES TECHNICAL ANALYSIS PROPOSAL}

Phillip Parrish

BDM Corp.

BDM developed a smart armor conceptual design for a multi-hit, survivable ceramic armor system which utilizes piezo-electric ceramic composites to impedance match and defeat shock waves from ballistic impact of long rod, high density penetrators. Based upon initial analytical studies involving cur- 
rent ballistic codes, it is estimated that a $1-2$ orders of magnitude improvement in ballistic resistance to penetration by a K.E. projectile is possible with an armor system utilizing PLZT composites. BDM is developing a conceptual design for a micromechanical magnetostrictive actuator. The concept involves using layered material to produce a magnetostrictive structure with improved crystal orientation in order to achieve $30-40$ percent increase in strain due to an applied electromagnetic field. The actuator can then be used to improve the operating envelopes of micromechanical devices such as a micromechanical tuning fork gyroscope. The gyroscopic response of the micromechanical tuning fork to an input rate about the longitudinal axis between the tines has substantial stability problems at high frequency due to time periodic dynamics. Implementation of a micromechanical magnetostrictive actuator, with a suitable control algorithm, will allow the gyroscope to operate at higher frequencies than is currently possible. BDM is continuing to develop this concept.

\section{SUPERPLASTIC CERAMICS}

Oleg D. Sherby

Stanford University

\section{SC: ARDEC, ARL/WTD}

Fine-grained iron carbide material ( $80 \mathrm{v} / \mathrm{o}$ iron carbide and $20 \mathrm{v} / \mathrm{o}$ of an iron-base second phase) was prepared using two different powder metallurgy procedures: $(a)$ hot isostatic pressing followed by uniaxial pressing and $(b)$ hot extrusion followed by uniaxial pressing. Both procedures yield materials that are superplastic at elevated temperature with low values of the stress exponent ( $n=2$ to 1$)$ and tensile elongations as high as 600 percent. The strain rate in the $n=2$ region is inversely proportional to approximately the cube of the grain size with an activation energy for superplastic flow between 200 and 240 $\mathrm{kJ} / \mathrm{mol}$. It was postulated that superplastic flow in the iron carbide material, in the $n=2$ region, is grain-boundary sliding accommodated by slip controlled by iron diffusion along iron carbide grain boundaries. The flow stress in compression is about 2 times higher than in tension in the region where grain-boundary sliding is the rate-controlling process. It is believed that the difference in flow stress is a result of the greater ease of grain-boundary sliding in tension than in compression. Tensile elongations were observed to increase with a decrease in stress and a decrease in grain size. These effects are quan- titatively explained by a fracture mechanics model that was developed to predict the tensile ductility of superplastic ceramics.

\section{THERMAL WAVE IMAGING AND CHARACTERIZATION OF SOLIDS}

Robert L. Thomas

Lawrence D. Favro

Wayne State University

\section{SL: ARL/MD, TACOM}

SC: ARDEC, ARL/EPSD

Good progress has been made on a new experiment aimed at real time thermal-wave imaging of dynamically propagating cracks in polymeric materials. It is being carried out by using an advanced infrared (IR) focal plane array imaging system. With 16,384 high speed InSb IR detectors arranged in a $128 \times 128$ format, the system is demonstrated to be capable of obtaining full-field information on the temperature distribution around the crack tips. Samples investigated in this work are edge-notched thin plates of polycarbonate, epoxy, and rubber-modified, highimpact polystyrene. Experiments are performed by cold drawing the samples with dynamic loading, the speed of which ranges from $10 \mathrm{~mm}$ to $99 \mathrm{~mm}$ per minute, producing crack speeds ranging from about $0.5 \mathrm{~cm} / \mathrm{s}$ to more than $2.5 \mathrm{~m} / \mathrm{s}$. With the image IR having the ability to store 192 frames of image in the buffer and an adjustable frame rate in the range of 15 $\mathrm{Hz}$ to $244 \mathrm{~Hz}$, the technique is able to record the temperature field on the surface of the specimens during the whole process. The full-field picture has the potential to provide detailed information about the local energy dissipation as a function of time in the vicinity of the propagating crack, and thereby to study the fracture toughness of various polymers and polymer blends.

\section{EFFECT OF PROCESSING PARAMETERS ON THE HIGH TEMPERATURE CREEP OF SiC WHISKER-REINFORCED ALUMINA}

Terence G. Langdon

University of Southern California

SL: ARL/MD

SC: BRADEC, TACOM 


\section{Processing}

This research program is designed to investigate the influence of processing parameters on the high temperature creep properties of polycrystalline alumina reinforced with silicon carbide whiskers. Some preliminary results suggested that the whisker aspect ratio is an important, but hitherto overlooked, parameter. This program will investigate the effect of different whisker morphologies and it will be used to develop guidelines for processing which will lead to an optimization of the creep resistance. Attention has been devoted primarily to the fabrication of the alumina composites. Silicon carbide whiskers have been incorporated into the alumina matrix under three different conditions: $15 \mathrm{v} / \mathrm{o}$ of unmilled whiskers, $15 \mathrm{v} / \mathrm{o}$ of whiskers after ball milling for $30 \mathrm{~min}$ utes, and $15 \mathrm{v} / \mathrm{o}$ of whiskers after ball milling for four hours. Examination by scanning electron microscopy shows that the average whisker length is $16.6 \mathrm{~mm}$ without milling, $15.6 \mathrm{~mm}$ after ball milling for 30 minutes, and $8.9 \mathrm{~mm}$ after ball milling for four hours, respectively. A four-point bending apparatus has been set up for creep testing at high temperatures. The creep data will be recorded continuously using a computerized acquisition system which was being established.

\section{Processing}

\section{PROCESSING AND PROPERTIES OF SILICON CARBIDE REINFORCED REACTION BONDED SILICON NITRIDE COMPOSITES}

John S. Haggerty

Yet-Ming Chiang

Massachusetts Institute of Technology

\section{SC: BRADEC, MICOM, ARL/VPD}

MIT has begun initial processing experiments designed to define procedures by which parts with large dimensions and complex shapes can be made which have the same high quality as the small cylindrical parts shaped by colloidal pressing. Samples made using the warm forming technique and the polystyrene/xylene binder/solvent system have been characterized with respect to phase content, room temperature mechanical properties and room tem- perature dielectric properties. Initial results indicate that the new forming technique and binder/solvent permit the same quality as has been achieved by colloidal pressing.

\section{A FUNDAMENTAL UNDERSTANDING OF THE EFFECTS OF CERAMIC PROCESSING ON PRODUCT MICROSTRUCTURE}

\section{G.G. Long}

National Institute of Standards and Technology

\section{SL: ARL/MD \\ SC: ARL/MD, ARL/VPD, TACOM}

Small-angle $x$-ray scattering was used to follow the evolution of the pore size distribution during final stage sintering of alumina and of alumina doped with 0.25 wt. percent magnesia. The volume-weighted (Guinier) results indicate that the effective size of the largest pores increases as the body goes from 97 percent to more than 99 percent dense. The surfacearea-weighted (Porod) results show that the median size of the smallest pores decreases slightly over the same density range. Taken together, these data indicate that the pore size distribution becomes broader as final stage densification proceeds. This was confirmed by a maximum entropy analysis, which was used to derive pore size distribution directly from the data. Finally, the evolution of the pore size distributions in alumina, with and without sintering aid, were compared.

Reports:

No. $1-5$ in previous editions.

6. A Fundamental Understanding of the Effects of Ceramic Processing on Product Microstructure, by Gabriell Long et al., FR, Feb 92, 55 pp.

\section{PRODUCTION AND EVALUATION OF DENSE CERAMIC COMPOUNDS BY COMBUSTION SYNTHESIS AND DYNAMIC COMPACTION}

Marc A. Meyers

University of California, San Diego

SL: ARL/WTD, ARL/MD
SC: ARL/WTD, ARL/MD 
A researcher continued his experiments on titanium carbide and improved the consolidation procedure, obtaining densities in excess of 97 percent. Nickel was added to the $\mathrm{Ti}+\mathrm{C}$ mixtures at 5 and $25 \mathrm{wt}$. percent, yielding microstructures characterized by a continuous metallic phase with spheroidal TiC particles. This microstructure was characterized by scanning and transmission electron microscopy, and it was found that $\mathrm{TiC}$ contained arrays of dislocations while nickel was annealed. The Ni-TiC interface was clean of impurities and highly cohesive, as could be gathered from TEM observations. He performed a comprehensive and systematic survey of constitutive models for porous materials and performed calculations on his material. He developed a novel quasi-static testing technique to determine the constitutive behavior of the hot and porous TiC. Another researcher carried out quasi-static as well as dynamic loading experiments on a $\mathrm{TiB}_{2}+\mathrm{Al}_{2} \mathrm{O}_{3}$ mixture to obtain fully dense compacts. He also conducted a series of quasi-static experiments at varying strain rates and with specimens of varying volumes. He was able to deconvolute the temperature effect and to obtain the flow stress of the porous material, modeling according to a Kuhn-Downey analysis. This study was complemented by $\mathrm{x}$-ray diffraction, SEM, and TEM.

\section{Reports:}

No. $1-2$ in previous editions.

3. Reaction Synthesis/Dynamic Compaction of Titanium Diboride, by D.A. Hoke et al., Met Trans 23A,77(1992). AD A249 412

4. Microstructural Characterization of Self-Propagating High-Temperature Synthesis/Dynamically Compacted and Hot-Pressed Titanium Carbides, by Kenneth S. Vecchio et al., Met Trans 23A,87(1992). AD A249540

5. Combustion Synthesis/Densification of an $\mathrm{Al}_{2} \mathrm{O}_{3}-\mathrm{TiB}_{2}$ Composite, by J.M. Jamet and M.A. Meyers, MS, Met Trans.

\section{TO STUDY AND EVALUATE SPECIAL REACTIVE ARMOR CONCEPTS}

Louis Zernow

Zernow Technical Services, Inc.

\section{SL: ARL/WTD, ARL/MD}

SC: ARL/WTD

The experimental activities at Aerojet-Chino Hills have been used in the Phase III program and all 12 of the special material holders filled at Los Alamos, have now been fired. These firings included some modifications of the targets which introduced addi- tional desirable mechanisms. There are a few more shots that remain to be fired, which are essentially calibration shots intended to separate out the contribution of the container material.

\section{IN-PROCESS CURE MONITORING OF COMPOSITES VIA FIBER-OPTIC FLUORESCENCE}

C.S.P. Sung

University of Connecticut

SL: ARL/MD

SC: ARDEC, BRADEC, ARL/VSD

An analysis was made of the kinetics parameters for thermal imidization of polyamic acid made from 1.5 diaminonaphthalene and a nonconjugated anhydride. In dilute solution, the dissociation rate was about three times greater than that of imidization with activation energies of about $15 \mathrm{Kcal} / \mathrm{Mole}$. In solid state reactions, much less dissociation was observed. Activation energies of about $24 \mathrm{Kcal} / \mathrm{mole}$ were observed for imidization whose rate is slightly faster than those in dilute solution. An investigation was made of the possibility of using intrinsic fluorescence due to the curing agent itself during cure. A common curing agent for high temperature epoxy matrix resins, diamino diphenyl sulfone shows a progressive red shift of about $20 \mathrm{~nm}$ when it is reacted with epoxide both in UV absorption and fluorescence spectra, due to the conversion of primary amines to secondary and finally to tertiary amines. The excitation spectra exhibits an easily measurable peak shift, which has also been observed by fiberoptic fluorimeter.

\section{USE OF BLOCK COPOLYMER AS POLYMER BLEND COMPATIBILIZER}

R.J. Roe

University of Cincinnati

\section{SC: ARDEC, NRDEC}

Block copolymer is often added to incompatible polymer blends to reduce the phase-separated domain sizes and to improve the adhesion between the phases. The domain size reduction probably arises as a result of the lowered interfacial tension due to accumulation of the block copolymer there, but experimental evidence on the detailed mechanism has 
been lacking, especially on the question of whether this is an equilibrium or kinetic effect. Experimental results show that the added block copolymer retards Ostwals ripening of phase-separated domains, thus producing smaller average domain sizes when a fixed time period is allowed for the domain growth. The Ostwald ripening process has been studied, by direct optical microscopic observations and by light scattering, with a blend of low molecular weight polystyrene and polybutadiene to which styrene-butadiene block copolymer has been added. The dependence of the retardation effect on the amount and molecular weight of the block copolymer has been studied.

\section{INTEGRATED SYNTHESIS AND POST-PROCESSING OF SILICON CARBIDE AND ALUMINUM NITRIDE}

Angus I. Kingon

North Carolina State University

SL: ARL/VPD

SC: ARL/WTD, ARL/MD, TACOM

Synthesis of nanocrystalline powders of $\mathrm{SiC}$, TiN, $\mathrm{TiC}, \mathrm{Si}_{1} \mathrm{Si}_{3} \mathrm{~N}_{4}$, diamond and $\mathrm{TiO}_{2}$ has been achieved by a nonthermal microwave plasma reactor. In the case of $\mathrm{TiO}_{2}$ the phase can be a metastable high pressure plasma $\left(\mathrm{B}-\mathrm{TiO}_{2}\right)$. The nanocrystalline $\mathrm{Si}$ particles displayed photoluminescence. This is being studied to determine whether this is consistent with 3-D quantum confinement. Creep of finegrained $(0.7 \mu \mathrm{m})$ AIN has been studied for the first time. The mechanism is diffusion-accommodated grainboundary sliding.

Reports:

No. $1-4$ in previous editions.

5. Synthesis of Nonoxide Ceramic Powders by Nonthermal Microwave Plasma, by A.K. Singh et al., MS.

6. Kinetics and Mechanisms of High Temperature Creep in Polycrystalline Aluminum Nitride, by A. Vasudev et al., MS, JMater Res.

7. Nonthermal Synthesis of Ultrafine Crystalline Titanium Oxide by Microwave Plasma, by A.K. Singh et al., MS.

8. Synthesis of Ultra-fine Crystalline Titanium Nitride by Nonthermal Microwave Plasma, by A.K. Singh et al., MS.

\section{POLYMER GELS AS PRECURSORS TO HIGH-PERFORMANCE MATERIALS}

James E. Mark

University of Cincinnati

$$
\begin{array}{ll}
\text { SL: } & \text { NRDEC } \\
\text { SC: } & \text { BRADEC, ARL/MD }
\end{array}
$$

Microcellular foams were prepared by the thermallyinduced phase separation technique, which yields materials having very small cell dimensions $(0.1-20$ $\mu \mathrm{m})$. The polymers employed were isotactic polystyrene, polyacrylonitrile, poly(4-methyl-1-pentane), polyurethane, and Lycra $($ and the resulting foams all had densities in the range $0.004-0.27 \mathrm{~g} \mathrm{~cm}^{-3}$. Values of Young's modulus and the collapse stress for these foams were measured and compared with predictions for conventional foams containing defects. Also investigated were plastic deformations, some timedependent behavior, and Poisson's ratio.

\section{Reports:}

No. 1-2 in previous editions.

3. The Use of Mechanical Property Measurements to Characterize Gels and Gelation Processes, by Y. Yang et al., MS.

4. Novel Reinforcement Techniques for Elastomers, by J.E. Mark, J Appl Polymer Sci 50,273(1992).

5. The Elastic and Plastic Mechanical Responses of Microcellular Foams, by M.H. Ozkul et al., MS, $J$ Appl Polymer Sci.

\section{SOLIDIFICATION STRUCTURE SYNTHESIS IN UNDERCOOLED LIQUIDS}

John H. Perepezko

University of Wisconsin - Madison

\section{SL: ARDEC, ARL/MD \\ SC: ARDEC, ARL/VPD}

One component of the current studies involves the examinations of grain refinement where powder samples of master alloys have been produced with the droplet emulsion technique to contain isolated inoculant particles of variable catalytic potency. With controlled quenching experiments on thermally cycled droplet samples, a post-solidification microstructural evaluation allows for the identification of the active inoculant particles. Currently, two common commercial master alloys, $\mathrm{Al}-5 \mathrm{Ti}-0.2 \mathrm{~B}$ and $\mathrm{Al}-$ 5Ti-1B, are under study. In other work on the microstructural analysis of Al-Y alloys following solidification at high undercooling several new features have been identified that impact the alloy design and processing analysis. For compositions beyond $15 \mathrm{w} / \mathrm{o}$ $\mathrm{Y}, \mathrm{x}$-ray diffraction results suggest the development of an $\mathrm{L}_{2}$ phase which may be coherent with the $\mathrm{Al}$ 
matrix. The identity of the $\mathrm{L}_{2}$ phase has now been confirmed by TEM analysis. With this confirmation a new class of high temperature RSP Al alloys has been established. It has recently been shown that small additions of Er dramatically increase the yield of the $\mathrm{L1}_{2}$ phase in Al-Y alloys. The microstructural development during solidification of undercooled $\mathrm{Fe}-\mathrm{Ni}$ alloys has been reported in numerous past studies, but a review of these studies reveals conflicting interpretation as a function of sample size and processing conditions. In order to identify the hierarchy of microstructural pathways and transitions, a systematic evaluation was performed on uniformly processed samples covering more than seven orders of magnitude in volume. The results yielded the identification of microstructural transitions with controlled variations in sample size and composition and were used to develop a microstructure map which delineates regimes of structural evolutions. This study has also provided a unified analysis of all previous experimental observations in the $\mathrm{Fe}-\mathrm{Ni}$ system.

\section{MATERIALS PROCESSING AND MICROSTRUCTURE CONTROL IN HIGH TEMPERATURE ORDERED INTERMETALLICS}

John H. Perepezko

University of Wisconsin - Madison

\section{SL: ARDEC, ARL/MD \\ SC: ARL/VPD, TACOM}

A recurring issue in the development of high temperature intermetallic systems for structural applications is the formulation of effective materials processing strategies in order to achieve controlled microstructures that are designed to provide for the required mechanical performance in elevated temperature, aggressive environments. In the current research an integrated approach, coupling processing with microstructure control as guided by the operative phase equilibria is being used to identify several intermetallics that show promise for further development. Based upon the experience that is currently developing, it appears that intermetallic alloys that are multiphase either in monolithic form or as composites are expected to offer the most attractive combination of properties. In a continuing development of $\mathrm{MoSi}_{2}$-based systems, a systematic overview of the ternary phase diagram data for Mo-Si-X systems has been completed to provide a basis for a rational approach to alloy design and to the interpre- tation of microstructural evolution during processing. In the studies on Ti-Al base systems a further analysis has been completed on the structures developed in a $\mathrm{Ti}_{45} \mathrm{Al}_{50} \mathrm{Mo5}$ alloy. In as-cast ingots the primary phase to solidify from the melt is $b c c$. For the $\mathrm{NbCr}_{2} \mathrm{C} 15$ Laves phase, the solubility behavior and distinct change in the composition dependence of the lattice parameter indicate a different defect structure on either side of the stoichiometric composition in the intermetallic phase field. Due to the strict geometrical relation of atom sizes in Laves phases it appears that anti-site defects involving the substitution of smaller $\mathrm{Cr}$ atoms on $\mathrm{Nb}$ sites is possible, but the reverse substitution of $\mathrm{Nb}$ on $\mathrm{Cr}$ sites is unlikely. Instead, excess vacancy formation on $\mathrm{Cr}$-sites may be developed for the $\mathrm{Nb}$-rich side of stoichiometry. It seems likely that these defects will affect the deformation behavior of the $\mathrm{NbCr}_{2}$ phase. In order to define composite thermal stability diffusion couples between $\mathrm{b}-21 s$ and sapphire $\left(\mathrm{Al}_{2} \mathrm{O}_{3}\right)$ have been prepared by hot-pressing at $900^{\circ} \mathrm{C}$ for one hour under high vacuum. After annealing at $1100^{\circ} \mathrm{C}$ for 69 hours and $750{ }^{\circ} \mathrm{C}$ for 864 hours several reaction layers developed at the initial interface.

\section{SYNTHESIS AND STRUCTURAL PROPERTIES OF NEW METALLIC PHASES EXHIBITING NONCRYSTALLOGRAPHIC SYMMETRIES}

S. Joseph Poon

Gary J. Shiflet

University of Virginia

SL: ARL/MD

SC: ARL/EPSD, ARL/VPD

The objectives of this research are to synthesize new aluminum-based metallic glasses and quasi-crystalline alloys, to investigate their atomic-scale structures, and to study their thermal stability and formation in order to shed light on the unusual formability of the new materials. Structures of the new materials are studied by $x$-ray, electron, and neutron diffraction, including that of high-resolution diffraction. The structure-property relationships in systems of technological importance are also being investigated. In the course of exploring the means by which the mechanical properties of aluminum glasses can be enhanced, new compositions with enhanced glass formability were discovered. Bulk melt-spun ribbons of thickness up to about 
$250 \mathrm{~mm}$ have been prepared. The new alloys are of the quaternary type AlNiFeGd and they contain about $85 \mathrm{a} / \mathrm{o} \mathrm{Al}$. This retention of bulk glassy samples is accomplished without a loss in strength; and their formability is unique. A better understanding of the structure of glassy $\mathrm{Al}$ has been acquired by a diffraction method using $\mathrm{x}$-rays from a synchrotron source. This is one of the series of structural studies for investigating structure-property relationships. It is found that both $\mathrm{Fe}$ and $\mathrm{Ce}$ atoms in the $\mathrm{Al} \mathrm{l}_{0} \mathrm{Fe}_{x} \mathrm{Ce}_{10}$ $x(x=3,5,7)$ show strong compositional short range ordering. Meanwhile, the $\mathrm{Ce}$ atoms form a dilute dense random packing substructure. On the other hand, the substructure of $\mathrm{Fe}$ atoms and the surrounding $\mathrm{Al}$ is found to be substantially different from the random packing, and may resemble that of quasi-crystals. It is thus clear that Ce plays an important role in the stabilization of the glassy structure. The Al-Fe distance of only about $2.49 \AA$ is unusually short, indicating strong interactions between $\mathrm{Al}$ and $\mathrm{Fe}$ atoms. These results have thus provided some clues to the unusual formability of Al glasses.

Reports:

1. Stability Investigation of a Decagonal Al-Cu-Co Quasicrystal, by Y. He et al., Phil MagLet 63,211(1991). AD A244 314

2. Mechanical Properties of Partially Crystallized Aluminum Based Metallic Glasses, by H. Chen et al., Scripta Met 25,1421(1991). AD A244 443

3. The Effect of Temperature on Stability of Al-Cu-Co Decagonal Phase, by Yi He et al,, Phil Mag Let 64,307(1991). AD A249 670

4. Short Range Ordering in Amorphous $\mathrm{Al}_{90} \mathrm{Fe}_{\mathbf{x}} \mathrm{Ce}_{10-\mathrm{x}}$, by H.Y. Hsieh et al., J Non-Cnyst Sol 135,248(1991).

\section{PRODUCTION AND EVALUATION OF DENSE CERAMIC COMPOUNDS BY COMBUSTION SYNTHESIS AND DYNAMIC COMPACTION}

Marc Andre Meyers

University of California, San Diego

SL: ARL/WTD

SC: ARL/WTD, ARL/MD

Considerable progress was made both with the titanium carbide and diboride compactions. Characterization of TiC produced by SHS and conventional hot pressing by transmission electron microscopy was performed. Quasistatic and dynamic testing of the TiC specimens was performed. The specimens exhibited a compression strength above $1500 \mathrm{MPa}$. The addition of controlled amounts of nickel to TiC yielded a decrease in cracking. Systematic experiments are planned.

\section{PROCESSING STUDIES ON COMPOSITES BASED ON BLENDS OF THERMOTROPIC LIOUID CRYSTALLINE POLYMERS WITH THERMOPLASTICS}

Donald G. Baird

Virginia Polytechnic Institute and State University

\section{SL: ARL/MD, NRDEC}

SC: ARDEC, BRADEC

(1) The enhancement of physical properties of thermoplastics by the blending of minor amounts of a thermotropic liquid crystalline polymer (LCP) is well known. However, the compatibility of these blends can be marginal and thus limited mechanical properties are exhibited. Hence, the ability to make a blend compatible is desired. While often the compatibility of blends can be enhanced by the addition of block copolymers, a suitable copolymer for thermoplastics with LCPs does not exist. Hence, in the laboratory this compatibility is achieved through a reactive extrusion process. This process consists of adding a functionalized polypropylene to the polymers prior to blending. This functionalized polypropylene then forms an in situ graft copolymer with the LCP during extrusion of the polymers. The effect of this compatibilizer on tensile strength, modulus, toughness, and surface finish for polypropylene/LCP blends is presented in a paper. (2) Polypropylene is of considerable interest to the automotive industry for interior parts because of its low cost, recyclability, and relatively desirable properties. However, it lacks the stiffness and strength to be used in most exterior applications. Less reinforcement gives adequate properties but the surface appearance is poor and glass/polypropylene systems can usually be processed only by means of injection molding. Blends of liquid crystalline polymers with polypropylene lead to improvements in stiffness but the surfaces of parts are poor and the strength of the blend is lower than that of polypropylene. Compatibilization involving a reactive extrusion process leads to blends with significantly improved stiffness, strength, and surface appearance. The nature of the compatibilization process as well as its effect on physical properties is discussed in detail in another paper. 
Reports:

1. The Effect of Compatibilization on the Properties of Blends of Thermotropic Liquid Crystals with Polypropylene, by H.J. O'Donnell et al., MS.

2. Thermoplastic Composites Based on Blends of Polypropylene with Liquid Crystalline Polymers, by H.J. O'Donnell, MS.

3. Processing Studies of In Situ Composites Based on Blends of Liquid Crystalline Polymers with Engineering Thermoplastics, by J.P. de Souza et al., MS.

4. Effect of Deformation History on the Morphology of Blends of Liquid Crystalline Polymers with Thermoplastics, by A.M. Sukhadia and D.G. Baird, MS.

5. The Effect of Miscibility on the Morphology and Properties of Blends of Polyetherimide with Liquid Crystalline Polymers, by S.S. Bafna et al., MS, Polymer.

6. Miscibility and Mechanical Properties of Polyetherimide/Polyetheretherketone/Liquid Crystalline Polymer Ternary Blends, by R.E.S. Bretas and D.G. Baird, MS, Polymer.

28639 MECHANICAL ALLOYING, PROCESSING, AND APPLICATION TO DEVELOPMENT OF STRUCTURAL MATERIALS

Thomas H. Courtney

University of Virginia

\section{SL: ARDEC, ARL/MD}

SC: BWL, ARL/VPD

A thesis was completed. It involved determination of the kinetics of fracture and welding during the initial stages of mechanical alloying, with specific reference to mechanical alloying in an attritor. Material systems studied were copper in combination with several $b c c$ refractory metals ( $\mathrm{Cr}, \mathrm{Fe}$, and $\mathrm{Nb}$ ) and system parameters varied were mill power and charge ratio (mass of grinding media/mass of powder). From a technological perspective, perhaps the most important of the results are the results of the effect of charge ratio and mill power on milling. In particular, over a limited range of these variables the respective fracture and welding probabilities (and thus reaction "time") scale linearly with the inverse of the charge ratio and nearly linearly with mill power. Another thesis extended earlier studies on mechanical alloying induced amorphization of $\mathrm{Ni}-\mathrm{W}$ alloys in a Spex mill to a much broader (and higher) range of tungsten contents. When alloy compositions less thian about 28 percent nickel are milled, a metastable solid solution of crystalline nickel (with all of the tungsten being dissolved in it) is formed. For tungsten contents greater than this critical one, a two phase mixture of crystalline tungsten and amorphous nickel is found. These alloys cannot be consolidated to full density by hot isostatic pressing (HIP). Moreover, the amorphous phase is not maintained at the high temperatures needed to approach full density. Full density can be achieved if a "binder phase" of approximately $10 \mathrm{v} / \mathrm{o}$ nickel powder is added to the milled product. The crystallization products formed from the amorphous phase during HIP are very fine. Phase scales are on the order of tenths of micrometers. This size can be compared with liquid phase sintered heavy metal alloys having microstructural scales on the order of tens of micrometers.

\section{Reports:}

1. Hot Isostatic Pressing of Mechanically Alloyed Cu-Nb Powders, by R.R. Vance and T.H. Courtney, Scripta Met 26,1435(1992).

\section{THERMOPLASTIC AND THERMOSETTING HIGH GLASS TRANSITION TEMPERATURE MATRIX RESINS}

James E. McGrath

Virginia Polytechnic Institute and State University

SL: BRADEC, ARL/MD, NRDEC

SC: ARL

This research is focused toward the development of high temperature matrix resins and structural adhesives, which are applicable at $700 \mathrm{~F}$. The effort has included the generation of high temperature polyimides, polybenzoxazoles and poly(arylene ether)s, with the first priority being focused on the aromatic polyimides. A major thrust of the effort has been to generate so-called $3 \mathrm{~F}$ based materials. The approach has been to generate both thermoplastic materials with nonreactive end groups, wherein the molecular weight is controlled in the range of 15,000 to 30,000 grams/mole, or, reactive oligomeric species which contain phenyl acetylene or acetylene reactive end groups. The purpose of the latter, then, is to provide a thermosetting curing mechanism. These materials also show very high temperature capability. The generation of the fluorinated system required extensive studies of the precursor monomers, including trifluoroacetophenone. The trifluoroacetophenone was generated by forming derivatives of trifluoroacetic acid. This particularly emphasized the acid chloride in early studies, and more recently the anhydride has become a preferred intermediate. The resulting reaction to generate the critical $3 F$ diamine was known in the literature, but a number of impurit- 
ies were not well characterized in earlier publications by others.

Reports:

1. Molecular Design of Processable High $T_{\mathrm{g}}$ Thermoplastic Matrix Resins and Structural Adhesives, by J.E. McGrath et al., MS

2. Synthesis of Soluble-Polybenzoxazoles via Acid Catalyzed Solution Cyclization, by W.D. Joseph et al., MS.

3. Very High $T_{\mathrm{z}}$, Soluble Polyimides Based on the " $3 \mathrm{~F}$ " Monomers, by M.E. Rogers et al., MS.

4. Synthesis of Novel Thermosets From Pendent Amine Groups on Poly(Arylene Ether)s, by S.J. Pak et al., MS.

5. Synthesis of Bis-A Based Polybenzoxazoles via Acid-Catalyzed Solution Cyclization, by W.D. Joseph et al., MS, Polymer Preprints.

6. Synthesis of Soluble Polyimides and Functionalized Imide Oligomers via Solution Imidization of Aromatic DiesterDiacids and Aromatic Diamines, by T.M. Moy et al., MS, Polymer Preprints.

7. Polymeric Materials Systems Designed for $700^{\circ} \mathrm{F}$ Applications, by James E. McGrath et al., MS, Polymer Preprints.

8. Semicrystalline and Amorphous Fluorine Containing Polyimides, by M.E. Rogers et al., MS.

28900 INTELLIGENT SYNTHETIC POLYMERS: A BLEND OF POLYMER SYNTHESIS AND BIOTECHNOLOGY

Paul Calvert

University of Arizona

\section{SL: NRDEC \\ SC: ARL/MD, ARL/VSD}

This project has the overall aim of making highly structured polymers to modify crystal growth and selectively to transport water-soluble compounds through polymer or lipid membranes. The active polymers are to have a globular form induced by having highly soluble extensions at either end of an insoluble core. This core-and-shell form resembles that of enzymes and the activity will arise from structures in the core. Two approaches are being followed. The first is to use group transfer polymerization to make acrylate block copolymers. First, simple free radical copolymerization was used to make copolymers of methacrylic acid (water soluble) and ethylhexylmethacrylate (water insoluble). Tests showed that about $50 \mathrm{~mol}$ percent methacrylic acid was needed to produce water solubility in the random copolymer. Group transfer polymerization of polymethylmethacrylate, a standard system, has been carried out to establish conditions for living polymerization. The second approach is to form the soluble polymer sections synthetically and then couple these chains to a polypeptide chain. For this purpose acrylic polymers are being made by anionic polymerization following the methods recently described by Teyssie and coworkers from Liege. The protocol uses butyllithium as an initiator with lithium chloride to stabilize the living chain end. Polymerization of $t$-butylacrylate was chosen as the polymer can be readily hydrolyzed to release the acid. A molecular weight of 1200 has been obtained. More work is needed to make this reaction reproducible.

\section{ADVANCED MANUFACTURING INSTITUTE}

Donald Sebastian

Stephen Tricamo

Stevens Institute of Technology

\section{SC: CECOM, ARL/EPSD}

The research will involve a multi-disciplinary program of research, development education and technology transfer addressing the use of concurrent engineering practices. Such practices will be applied to the product development of polymer and polymer matrix composites wherein manufacturing techniques, performance specifications, material properties, reliability and cost are simultaneously considered at the initial stage of design. Activities under this effort will include (1) development of processing methods which offer unusually strong for improved quality, reproducibility and yield of product; (2) in-process nondestructive evaluation techniques for real time inspection and closed loop computer control of critical parameters in the manufacturing process; (3) determination of computer integrated manufacturing models incorporating a common data base and compatible software to relate stage of manufacture; (4) incorporation of opportunities for automation and (5) integrated manufacturing concepts.

\section{FUNCTIONALLY GRADIENT REFRACTORY MATERIALS FABRICATION BY THE SELF-PROPAGATING HIGH-TEMPERATURE SYNTHESIS PROCESS}


Gregory C. Stangle

James W. McCauley

New York State College of Ceramics at Alfred University

This research is concerned primarily with the fabrication of advanced materials by the self-propagating high-temperature synthesis (SHS) process, such that reliable, low-cost and reproducible materials can be produced. Two overall goals are currently being pursued. First, a combined experimental and theoretical study is being conducted that focuses on the fundamental chemical and physical processes that occur during the SHS process. This work is expected to lead to the development of routine processes for producing materials with a controlled, tailored microstructure. Second, an SHS-based route to preparing functionally gradient materials (FGMs) for use in joining high-temperature, corrosion-resistant and strong materials is being studied. These FGMs are expected to possess desirable and useful mechanical, thermomechanical, and electromechanical properties, and lead to materials with attractive heat-, corrosion-, thermal shock- and wear- (or abrasion-) resistance, as well as excellent intermaterial adhesion capabilities. The overall outcome is expected to be an understanding of, and a procedure for, the fabrication of high-performance materials with improved and precisely controlled microstructures and properties.

\section{HIGH PERFORMANCE HEAVY ALLOYS BY ALLOYING AND PROCESS CONTROL}

Randall M. German

The Pennsylvania State University

\section{SL: ARL/WTD, ARL/MD}

Conventional tungsten heavy alloys, based on nickel and iron, show good wetting and solubility of tungsten in the matrix. This results in high grain growth rate and accordingly a coarse microstructure consisting of tungsten mean grain sizes ranging from 20 to 60 micrometers at liquid phase sintering temperatures. The addition of molybdenum to this system proved beneficial in the refinement of the microstructure through decreasing the solubility of tungsten in the nickel-iron matrix and hence slowing the solution reprecipitation process. As a consequence the strengths of these alloys have increased substantially. However, the processing of these alloys proceeds at temperatures as high as $1500^{\circ} \mathrm{C}$.
Accordingly, different processing routes have been under study recently to achieve full densification at lower sintering temperatures and different matrices with lower liquid formation temperatures. Among the systems under study, the nickel-manganese system is a good candidate for applications such as kinetic energy penetrators. In addition, research is under way to further improve the mechanical properties and densification at lower temperatures for the heavy alloys based on tungsten and nickel with iron and/or cobalt additions.

\section{Reports:}

1. Grain Growth in Liquid-Phase-Sintered W-Mo-Ni-Fe Alloys, by P.B. Kemp and R.M. German, $J$ Less-Common Metals 175,353(1991). AD A249 743

2. The Effects of Powder Pretreatment on the Microstructure and Mechanical Properties of Tungsten Heavy Alloys, by A. Belhadjhamida and R.M. German, Advances in Powder Metallurgy - 1991, Volume 6, 1991, p407. AD A249 402

3. Development of a New W-Ni-Mn Heavy Alloy, by A. Bose et al., Advances in Powder Metallurgy - 1991, Volume 6, 1991, p425. AD A249 508

4. Sintering Time and Atmosphere Influences on the Microstructure and Mechanical Properties of Tungsten Heavy Alloys, by R.M. German et al., Met Trans 23A,211(1992). AD A249 159

\section{SOLIDIFICATION PROCESSING AND MECHANICAL BEHAVIOR OF TaW/Wp REFRACTORY METAL BASE COMPOSITES}

E. J. Lavernia

University of California, Irvine

$$
\begin{array}{ll}
\text { SL: } & \text { ARL/MD } \\
\text { SC: } & \text { BRADEC, ARL/MD }
\end{array}
$$

To accomplish the objectives of the present research program, an ultra-elevated temperature spray facility is presently being constructed. This facility is designed to handle the melting and spraying of refractory metals $\left(\mathrm{T}=3000^{\circ} \mathrm{C}\right)$. The creep behavior of powder metallurgy (PM) $6061 \mathrm{Al}$, which has been used as a metal matrix alloy in the development of discontinuous silicon carbide reinforced aluminum (SiC-Al) composites, has been studied over six orders of magnitude of strain rate. The experimental data show that the steady-state stage of the creep curve is of short duration; that the stress dependence of creep rate is high and variable; and that the temperature dependence of creep rate is much higher than that for self-diffusion in aluminum. In recent years, refractory intermetallics, especially molybde- 
num disilicide ( $\mathrm{MoSi}$ ), have attracted considerable attention as an elevated temperature structural material to be used in gas turbine engines expected to operate at temperatures of up to $1600^{\circ} \mathrm{C}$ as a result of its unique combination of physical attributes, which include: moderate density of $6.31 \mathrm{~g} / \mathrm{cm}^{3}$, high melting point of approximately $2030^{\circ} \mathrm{C}$, excellent oxidation resistance, and high modulus at elevated temperatures. To provide preliminary guiding information useful for the Ta-W experiments, a series of plasma runs were conducted with $\mathrm{MoSi}_{2}-\mathrm{SiC}$. A series of plasma deposition experiments were conducted with pure tungsten. The microstructure of plasma-sprayed tungsten on a molybdenum substrate was studied in detail.

\section{JOINING OF MONOLITHIC AND PARTICULATE REINFORCED LIGHT ALLOYS}

William A. Baeslack

Laszlo Adler

Ohio State University

\section{SC: ARDEC, BRADEC, ARL/MD, RIA}

The objective of the research is to investigate the metallurgical aspects of joining high performance lightweight alloys. The approach will involve research in the following four areas: (1) The theoretical and experimental analyses of weld microstructure evolution in rapidly-solidified dispersion-strengthened aluminum alloys; (2) The joining of alumina and SiC particulate-reinforced aluminum alloys; (3) Phase transformation effects on the properties and weldability of advanced $\mathrm{Al}-\mathrm{Cu}-\mathrm{Li}-\mathrm{Ag}-\mathrm{Mg}-\mathrm{Zr}$ alloys; (4) Ultrasonic spectroscopy analysis of solid-phase welds in advanced light alloys.

\section{PYROMETALLURGICAL, PHYSICAL AND MECHANICAL BEHAVIOR OF WELDMENTS}

David L. Olson

Colorado School of Mines

SL: ARL/MD

SC: ARDEC, TACOM

The project is being carried out in three phases. Phase 1 is evaluating various thermite assisted submerged arc welding flux formulations: $\mathrm{Fe}_{2} \mathrm{O}_{3}$ to supply oxygen plus $\mathrm{Mg}, \mathrm{Al}, \mathrm{Fe}-\mathrm{Al}, \mathrm{Al}-\mathrm{Mg}$ metallothermic additions. The increase heat input is being evaluated by measuring the increase in the size of weld bead (nugget area) for constant welding parameters. Previous investigators have related cross sectional bead area directly to heat input. This work is in progress. Phase 2 will use the most favorable submerged arc exothermic combinations to formulate exothermically assisted shielded metal arc welding electrodes. This work will be based on that done for submerged arc welding. Phase 3 will evaluate the most successful exothermic additions in flux cored arc welding consumables.

\section{SUPERCRITICAL FLUID EXTRACTION: PREPARING A SUPERIOR MESOPHASE PRECURSOR FOR CARBON FIBERS}

Mark C. Thies

Clemson University

$$
\begin{aligned}
& \text { SL: ARL/MD, NRDEC } \\
& \text { SC: ARDEC, BRADEC }
\end{aligned}
$$

Supercritical fluid extraction is being investigated for the production of mesophase pitch, a liquid crystalline precursor the the manufacture of high-performance carbon fibers. A continuous flow apparatus is being used to fractionate an isotropic petroleum pitch feedstock with supercritical toluene at temperatures to $400^{\circ} \mathrm{C}$ and pressures to $200 \mathrm{bar}$. This pitch was separated into isotropic and liquid crystalline, or mesophase, portions in a region of liquid-liquid equilibrium. Since the phases are opaque, an electrical impedance technique was used to monitor the location of the liquid-liquid interface. A 100 percent mesophase pitch was produced at $340^{\circ} \mathrm{C}$ and $70 \mathrm{bar}$ using a solvent to feed ratio of 3:1. This material was spun into $10 \mu \mathrm{m}$-diameter fibers which were oxidized, carbonized and tested for physical properties. The final fibers exhibit tensile strengths and moduli comparable to the best pitch-based fibers currently available. With these fiber properties and the continuous nature of this process, supercritical extraction is an attractive alternative to existing pitch treatment processes. 


\section{Physical Behavior}

\section{DIAMOND EPITAXY AND DIAMOND-LIKE COATINGS BY LASER ASSISTED AND ELECTRON BEAM ENHANCED TECHNIQUES}

J. Narayan

J. Krishnaswamy

North Carolina State University

\section{SC: MICOM, NVESD, TACOM}

1. The physical properties of the ablated vapor plume, including the type and temperature of the species, were characterized using an optical multichannel analyzer. It was also shown from ellipsometric measurements that an accurate assessment of the percentage of $\mathrm{sp}^{3}$ bonds relative to $\mathrm{sp}^{2}$ bonds in the DLC films could be obtained by evaluating the effective number of valence electrons. 2 . Significant progress has been made in understanding the nucleation and growth of diamond films. Controlled experiments on copper and silicon substrates with and without diamond and alumina abrasion treatments show that damage in the form of plastic deformation and microcracks plays a minor role in diamond nucleation, whereas residual diamond particles provide effective sites for nucleation and growth of diamond. Pulsed laser treatment can be used to selectively remove these nuclei and also obtain selective deposition of diamond films. 3. An investigation was made of the characteristics of diamond nucleation on silicon substrates alloyed with iron during hot filament chemical vapor deposition of diamond film. It was found that the presence of $\mathrm{FeSi}_{2}$ on silicon enhances nucleation of diamond by more than an order of magnitude compared to that on silicon substrates with laser deposition of iron followed by thermal annealing at $700^{\circ} \mathrm{C}$. The deposition characteristics of diamond and reaction of iron with silicon substrates were investigated as a function of annealing treatments using Rutherford backscattering, x-ray diffraction, auger electron spectroscopy, and transmission electron microscopy. 4. Novel laser assisted methods were developed for deposition of diamond films.

\section{SUPERLATTICE DISORDERING AND DIFFUSION MECHANISMS IN GaAs}

Teh Y. Tan

Ulrich M. Gosele

Duke University

SC: ARL/EPSD, ARL

1. The diffusion of the substitutional chromium atoms, $\mathrm{Cr}_{s}$, in GaAs results from the rapid migration of the interstitial atoms, $\mathrm{Cr}_{i}$, and their subsequent changeover to occupy $\mathrm{Ga}$ sites (or vice versa), a typical substitutional-interstitial diffusion (SID) process. There are two possible ways for the $\mathrm{Cr}_{i}-\mathrm{Cr}_{s}$ changeover to occur: the kick-out mechanism in which Ga self-interstitials are involved, and the dissociative mechanism in which Ga vacancies are involved. The $\mathrm{Cr}_{s}$ in-diffusion profiles are of characteristic shapes, indicating the dominance of the kick-out mechanism, while the $\mathrm{Cr}_{s}$ out-diffusion profiles are error-function shaped, indicating the dominance of the dissociative mechanism. In this study, an integrated SID mechanism, which takes into account both the kick-out and dissociative mechanisms, is used to analyze $\mathrm{Cr}$ diffusion results. Going beyond just qualitative consistency, the $\mathrm{Cr}$ in- and out-diffusion features in GaAs were explained on a quantitative basis. It was confirmed that the kick-out mechanism dominates $\mathrm{Cr}$ in-diffusion while the dissociative mechanism dominates $\mathrm{Cr}$ out-diffusion. $\mathrm{Pa}$ rameters used to fit existing experimental results provided quantitative information on the $\mathrm{Ga}$ self-interstitial contribution to the $\mathrm{Ga}$ self-diffusion coefficient. The values obtained are consistent with those obtained from a study of zinc diffusion in GaAs, and with available experimentally determined $\mathrm{Al}-\mathrm{Ga}$ interdiffusion coefficients. 2. A quantitative determination of the contributions of the triply negatively charged $G a$ vacancies and of the doubly-positively charged Ga self-interstitials to Ga self-diffusion coefficient in GaAs has been carried out.

Reports:

No. $1-6$ in previous editions.

7. Diffusion Mechanism of Chromium in GaAs, by $\mathrm{S}$. Yu et al., J Appl Phys 70,4827(1991). AD A249 272

8. Determination of Vacancy and Self-Interstitial Contributions to Gallium Self-Diffusion in Gallium Arsenide, by T.Y. Tan et al., MS; J Appl Phys.

9. Studies of Point Defects Equilibria and Impurity Diffusion Mechanisms in Gallium Arsenide, by Shaofeng Yu, PhD Thesis, 1991, 173 pp. 
10. Diffusion Mechanism of Zinc and Beryllium in Gallium Arsenide, by S. Yu et al., J Appl Phys 69,3547(1991). AD A244 521

11. Atomistic Mechanisms of Dopant-Induced Multiple Quantum Well Mixing and Related Phenomena, by T.Y. Tan et al., Opt and Quant Electron 23,S863(1991). AD A244 442

12. Distribution Mechanism of Voids in Si-Implanted GaAs, by Samuel Chen et al., J Appl Phys 70,656(1991). AD A249 190

13. Point Defect Thermal Equilibria in GaAs, by T.Y. Tan, Mater Sci and Eng B10,227(1991). AD A249 271

\section{POLING OF THIN POLYMER FILMS IN ELECTRO-OPTIC APPLICATIONS}

Gerald Fuller

Stanford University

\section{SC: MICOM, NVESD}

In this study materials suitable for nonlinear optical applications were considered. In particular, thin polymer films, either doped with nonlinear optical chromophores or films with the chromophores covalently bonded to side groups were studied. Orientation of these chromophores was achieved by the birefringence resulting from chromophore concentration, spacer length, and molecular dominant factor determining the steady state and transient behaviors. The relationship between monomer and polymer structure and the resulting effect on the orientation dynamics was studied. Qualitative agreement with theoretical predictions was obtained for the simpler guest/host systems studied. The results have provided valuable insight into the molecular processes that occur during the chromophore orientation and relaxation, and the effects of structural characteristics of the polymers on these mechanisms. In addition to orientation of thin polymer films by electric field poling, research was commenced on the problem of flow field orientation of polymer monolayers during Langmuir-Blodgett deposition. In this work, a stagnation point extensional flow was created by simultaneously immersing two identical glass substrates into a Langmuir through supporting a monolayer of "hairy" rod-like macromolecules. The materials that were studied were polyglutamates with alkyl side chains distributed along the backbone. It was observed that very well characterized, homogeneous extensional flows could be generated between the two substrates as evidenced by tracking the trajectories of tracer particles residing on top of the polymer film. Consequently, very high degrees of planar polymer orientations could be achieved, which could potentially yield products with large nonlinear optical properties.

\section{INVESTIGATION OF QUANTUM EFFECTS IN HETEROSTRUCTURES}

Leroy Chang

Emilio Mendez

IBM Research Center

Researchers have studied in detail the magnetotransport properties of $p$-type InMnAs, the new diluted magnetic semiconductor made of III-V compounds. The anomalous Hall effect dominates the Hall resistivity throughout the entire temperature range of $0.4 \mathrm{~K}$ to room temperature, and it plays a key role in the formation of partial ferromagnetic order below 7.5. Pronounced many-body exciton effects have been observed through photoluminescence in modulation-doped $n$-type GaAs-GaAlAs single quantum wells. The structure is designed to achieve a near-resonance coincidence between a transition involving two-dimensional electrons at the Fermi level in the first conduction subband and an exciton transition from the second conduction subband. Under these conditions, strong and distinct Fermiedge singularities are observed. By optically pumping a laser heterostructure whose active region consists of coupled quantum wells, researchers have succeeded in tuning the stimulated emission by external electric fields. This was made possible by the relatively large energy shifts of the quantum states with the field in such coupled structures. Researchers have investigated the dependence of the dislocation characteristics on the initial growth mode of $\mathrm{ZnSe}$ epilayers on $\mathrm{GaAs}(100)$ by molecular beam epitaxy. For the case where the initial growth proceeds by the formation and coalescence of three-dimensional islands, the threading dislocation density has been found to be an order of magnitude higher and the misfit dislocation length much shorter than that for the case where the initial growth is by a two-dimensional layer-by-layer mode.

\section{RELIABILITY AND REPRODUCIBILITY ACHIEVED VIA GRAIN BOUNDARY ENGINEERING OF HIGH PERFORMANCE · ELECTRONIC CERAMICS}


Vasantha Amarakoon

Robert Condrate

Alfred University

$$
\text { SL: ARL/EPSD }
$$$$
\text { SC: ARL/MD }
$$

1. Lithium niobate powders were synthesized through the gelation of organometallic species of lithium and niobium. Single phase $\mathrm{LiNbO}_{3}$ was successfully formed through calcination of the gelation product. The powders were characterized using FTIR spectroscopy at various stages of the thermal treatment. 2. PTCR $\mathrm{BaTiO}_{3}$ thermistors prepared by sol-gel coating of powders were characterized using resistivity v temperature measurements, SEM (scanning electron microscopy) microstructural analysis, and degradation measurements under reducing conditions such as $\mathrm{N}_{2}$ atmosphere and vegetable oil vapor. A strong relationship between the degradation behavior and microstructure characteristics has been established. Experiments are being done on the analysis of microstructural changes during processing of PTCR $\mathrm{BaTiO}_{3}$. Changes in the microstructure and liquid phase distribution during the firing procedure of $\mathrm{BaTiO}_{3}$ are being characterized using SEM and TEM (transmission electron microscopy). These microstructural changes will then be related to the electrical properties to yield important information on the role of the liquid phase and microstructure, and the reliability of the process in the formation of the PTC effect. Studies are also being carried out on the role of the liquid phase and microstructure on the oxidation behavior of PTCR $\mathrm{BaTiO}_{3}$. Semiconducting powder was first prepared by an oxalate sol-gel method developed recently. Silica was then added to the surface of the powder via a silica sol-gel method. Evaluation of the liquid phase additives (Si) was done with TEM.

\section{IONIZED-CLUSTER-BEAM/PARTIALLY IONIZED-BEAM DEPOSITION OF ELECTROOPTICAL AND NONLINEAR OPTICAL ORGANIC MATERIALS AND DEVICE DEVELOPMENT}

Toh-Ming Lu

Nickolas P. Vlannes

Rensselaer Polytechnic Institute

SL: NVESD

SC: ARL/EPSD
Progress has been made on the partially ionized beam (PIB) vacuum deposition of thin oriented films of nonlinear optical organic materials. Following previously reported work on oriented 2-methyl-4-nitroaniline (MNA) films by PIB deposition, depositions of 4-dimethylamino-4'-nitrostilbene (DANS) thin films were carried out by the PIB method. Unlike MNA, DANS did not yield to orientational control via variation of substrate bias during PIB deposition. Although it has one of the largest known second order molecular hyperpolarizabilities (b), DANS possesses a null electrooptic (EO) effect in the bulk because of inversion symmetry in the crystal structure. However, also unlike MNA, DANS forms highly transparent films by vacuum evaporation. Researchers therefore undertook a series of binary codepositions with other vapor-depositable organic materials aimed at disrupting this crystal structure and enabling the DANS molecule to contribute to second order effects. Although the orientation could in fact be influenced by this method, this failed to produce novel DANS crystal phases. Films of 3-methyl-4-methoxy-nitrostilbene (MMONs) were also grown by vacuum evaporation and PIB. Although MMONs possesses a lower molecular $\beta$ than DANS, its crystal structure is noncentrosymmetric so that the bulk EO is high. However, films of this material deposited under a variety of conditions remained amorphous excepting surface crystallization. In the course of other work it was discovered that Teflon AF (a copolymer of tetrafluoroethylene and 2,2-bis-trifluoromethyl-4,5-difluoro-1,3-dioxole) could be pyrolized in vacuum leading to highly transparent polymer film. Given the feasibility of depositing a polymer host such as this, codepositions with a suitable organic chromophore are being pursued. This work includes the evaluation of the glassy properties of the host and the use of several different chromophores as dopants.

\section{FUNDAMENTAL AND APPLIED ASPECTS OF DEFECT ENGINEERING IN GaAs}

Jacek Lagowski

University of South Florida

Work on the non-contact, wafer-scale SPV-DLTS study of defects in GaAs has generated a great deal of interest. New defect monitoring possibilities were demonstrated by a surface charge imaging technique. A representation of these results are shown which 
compares surface charge maps on state-of-the art wafers of GaAs, Si and InP. All wafers are (100) grown by the Czochralski method. Results demonstrate that GaAs exhibits an extremely uniform surface charge, which is apparently a consequence of very strong Fermi level pinning at the GaAs surface caused by the surface defects. On the other hand, InP, which is known for much weaker Fermi level pinning, exhibits a characteristic charge pattern with symmetry axis representative for LEC-grown (100) wafers. Silicon uniformity is between that of GaAs and InP. Researchers believe that non-contact, no preparation, wafer-scale characterization of defects is a critical task in the implementation of defect engineering in the semiconductor wafer processing cycle.

\section{INDIUM-VACANCY COMPLEXES IN MERCURY CADMIUM TELLURIDE}

M.L. Swanson

University of North Carolina at Chapel Hill

\section{SL: NVESD}

Researchers have used the perturbed angular correlation (PAC) method, employing the isotope ${ }^{111}$ In, to study point defects in mercury cadmium telluride (MCT), and have identified ${ }^{111}$ In-vacancy complexes with characteristic PAC frequencies $83 \mathrm{MHz}$ and 91 MHz. These defects are created by annealing in a saturated mercury atmosphere. These experiments will be extended to study the influence of both stoichiometry and indium content on the defect structure and concentration. The defects have also been produced by low temperature implantation of the indium isotope. Researchers have also obtained evidence that hydrogen atoms, introduced by heating the sample in water, are trapped by the ${ }^{111}$ In-vacancy complexes. This is the first atomic-scale characterization of point defects in MCT. The interaction of these defects with impurities can be studied, since the characteristic PAC frequency will be affected by the trapping of impurities. In addition, the PAC data can provide basic information about mobilities of vacancies, self-interstitials and impurities in MCT. The data indicate a migration energy of $0.6 \mathrm{eV}$ for vacancies.

\section{UNCOMPENSATED GARNETS: A MAGNETIC SEMICONDUCTOR}

Philip E. Wigen

Ohio State University

$$
\begin{array}{ll}
\text { SL: } & \text { ARL/MD } \\
\text { SC: } & \text { ARL/EPSD }
\end{array}
$$

In charge uncompensated Cax:YIG, magnetic anomalies and a photoinduced absorption spectrum have been reported. The temperature dependence of the photoinduced peak observed at $1.85 \mathrm{eV}$ (width 0.5 $\mathrm{eV}$ ) has the same temperature dependence as the low-temperature anomalies observed in the magnetizations. A model to explain the low-temperature magnetic anomaly assumes that a non- $S$ state iron ion, $\mathrm{Fe}^{4+}$, is created in the tetrahedral site that is ferromagnetically coupled to the octahedral $\mathrm{Fe}^{3+}$ ion. An alternative model recently proposed assumes that $\mathrm{O}^{-}$ions are formed which would influence the magnetic properties in similar fashion. The broad nature of the absorption at $1.85 \mathrm{eV}$ is characteristic of a resonant transition of photocarriers. In a paper the absorption band is discussed in terms of a polaron hopping model.

\section{STUDIES DIRECTED TOWARD NEW AND IMPROVED PERMANENT MAGNET MATERIALS}

W.E. Wallace

Carnegie-Mellon University

\section{SL: ARL/EPSD}

The microstructure, crystallography, and magnetic properties of the $\mathrm{RTiFe}_{11-x} \mathrm{Co} x$ alloys were examined. It was found that $\mathrm{Fe}$ and $\mathrm{Co}$ are completely miscible in this system and crystallize in the $\mathrm{ThMn}_{12}$ structure. The initial replacement of $\mathrm{Fe}$ by $\mathrm{Co}$ increases the Curie temperature significantly and then more slowly with further Co substitution. The magnetic moment exhibits a maximum value at $x \sim 4$. The magnetic anisotropy of the rare earth sublattice appears to be planar for the rare earth with positive second order Stevens factor (i.e., Tb, Dy, and $\mathrm{Ho}$ ) in the RTiCo11 alloy system. This behavior is the opposite of that observed in RTiFe11. The magnetic anisotropy phase diagrams of the $\mathrm{RTiFe}_{11-x} \mathrm{Co} x$ systems were determined. 


\section{MISFIT DISLOCATIONS AND MAGNETIC ANISOTROPY IN EPITAXIAL FILMS}

Carl V. Thompson

R.C. O'Handley

Massachusetts Institute of Technology

\section{SC: ARL/WTD, ARL/MD}

1. Optimal conditions have been determined for deposition of continuous, single crystal films of copper on $\mathrm{NaCl}(001)$. 2. Conditions have been determined for deposition of nickel on these copper films. Researchers are consistently able to achieve epitaxial $\mathrm{Ni}$ layers and have observed misfit dislocations in many of these samples of $\mathrm{Ni} / \mathrm{Cu}(001)$ on $\mathrm{NaCl}$. Transmission electron micrographs clearly show dark lines corresponding to misfit dislocations. The spacing between these dislocations is irregular and averages about $600 \AA$. These observations are consistent with those of other researchers studying deposition of $\mathrm{Ni}$ films on bulk $\mathrm{Cu}$. 3. Vacuum $\mathrm{NaCl}$ is too rough to allow magnetooptic Kerr measurements on the $\mathrm{Ni}$ films. Researchers are therefore investigating growth on polished $\mathrm{NaCl}$. They will also investigate growth on other substances such as $\mathrm{Si}$. 4. In order to control the MD spacing through the misfit, plans have been made to use CuNi alloys as substrates for some of the $\mathrm{Ni}$ depositions. A series of experiments has determined that alloys of nickel and copper cannot readily be formed through annealing of multilayer copper-nickel structures. A deposition system has been adapted for controlled co-deposition of $\mathrm{Ni}$ and $\mathrm{Cu}$ to form alloys. 5. The magnetooptic Kerr apparatus has been purchased and the necessary ports, electromagnet and sample holder were being fabricated.

\section{NEW SOURCES FOR CHEMICAL BEAM EPITAXY}

L.P. Sadwick

G.B. Stringfellow

University of Utah

SL: NVESD

SC: ARL, ARL/EPSD

Researchers have completed the design and implementation of a specially designed UHV system for CBE growth and characterization of existing and novel precursors in the high vacuum and ultra high vacuum gas phase growth regime. The system consists of two main parts which are interconnected via an UHV gate valve. The first subsystem is designed for fundamental experiments on the kinetics, reaction rates and pyrolysis of precursors. It consists of a small UHV chamber with mulitple $\mathrm{LN}_{2}$ cooled cyroshrouded diffusion pumps, two computer-controlled residual gas analyzers, various UHV valves, and a modular and highly flexible gas injection system. A heated/cooled sample manipulator with three axis movement and rotation can also be mounted in the chamber along with a chopper wheel to utilized enhanced measurement techniques such as thermally programmed desorption (TPD) and modulated beam mass spectroscopy (MBMS). The second subsystem consists of a homemade CBE system capable of growing on two inch substrates. The CBE system can be operated from near atmosphere (i.e., 100 torr) down to UHV (i.e., $10^{-10}$ torr) conditions. System confidence/verification experiments have been performedon the highly studied and well characterized group III precursor, trimethylgallium (TMG). The results on the temperature behavior, pyrolysis and kinetics of TMG are in good agreement with the collective published works on TMG.

\section{AUTOMATED FINITE ELEMENT MODELING OF ELECTRONIC CARD ASSEMBLIES}

Nasser H. Paydar

Indiana University - Purdue University at Indianapolis

SL: ARI,

$\mathrm{Pb}-\mathrm{Sn}$ alloys are used frequently as joining media in electronic circuitry. When a solder joint is subjected to thermally induced mechanical strains resulting from cyclic loading environment, it will ultimately fail due to fatigue. The long-term fatigue life of a solder joint under thermal cyclic condition is an important issue in assessing the reliability of electronic assemblies. In this study, an eutectic $\mathrm{Pb}-\mathrm{Sn}$ solder joint of a resistor carrier subjected to thermal cycling between $125^{\circ} \mathrm{C}$ and $-55^{\circ} \mathrm{C}$ is studied. The existing creepplastic models for the eutectic solder alloys are implemented by the use of user defined material subroutine, available in general purpose nonlinear finite element code ABAQUS. The creep characteristics of the solder alloy are modeled by two creep relations: the power law creep relation and the hyperbolic sine creep relation. The elastoplastic behavior of the solder joint is described by temperature-dependent bilinear stress/strain curves. The strain range per cycle obtained from finite ele- 
ment analysis is then used in the Engelmaier's version of the Coffin-Manson equation to estimate the number of cycles to failure. The results as compared with linear elastic case indicate that creep and plasticity have a substantial effect on thermal fatigue life of solder joints. Parameter studies include ramp time, hold time, grain size of solder, and initial temperature.

\section{THIN FILM GROWTH WITH IONS, CLUSTERS, AND METAL ORGANIC COMPOUNDS}

John $H$. Weaver

University of Minnesota, Minneapolis

$$
\begin{aligned}
& \text { SL: ARL/EPSD } \\
& \text { SC: ARL }
\end{aligned}
$$

Researchers have completed the construction of the cluster source, associated electronics, computerized data acquisition and control system, and the time-offlight mass spectrometer. It was demonstrated that the source produces ionized clusters. Current efforts involve the optimization of the cluster beam intensity by adjusting parameters and conditions in the cluster growth chamber. Design and construction of the electrostatic ion separation system is complete and installation has begun.

\section{AN EXTENDED APPROACH TO OXIDATIONS AND NITRIDATIONS OF $\mathrm{Si}$ AND $\mathrm{Ge}_{x} \mathrm{Si}_{1-x}$ MATERIALS}

Ralph J. Jaccodine

Donald R. Young

Lehigh University

\section{SC: ARL, NVESD}

The work on a previous approach to chemically enhanced oxidation using fluorine additions to the oxidation stream was extended to the oxidation of $\mathrm{SiGe}$ alloy material. The addition of fluorine additive allows one to oxidize this material at a temperature which will not cause a relaxation of the built-in strain. At the same time, in a dry oxidation process a reasonably thick oxide is achieved. Early indication from the work on these oxides show that the $\mathrm{Ge}$ is not piled up at the interfaces. This is an important problem for any surface active device. Additional profile analyses were done using the Scientia XPS and sequential etching. These results indicate that the surface con- centration of suboxides are related to the composition of the Si-Ge alloy that is oxidized. This correlation, along with work on the Ge oxide structure (or composition in the oxide) needs additional detailed work. A paper has been prepared which reviews the effect of small additions (ppm) of fluorine to the dry oxidation process of silicon. It deals with the various critical areas of the oxidation growth such as (a) growth kinetics, $(b)$ interfacial strain, $(c)$ stacking fault shrinkage, $(d)$ effects on oxidation enhanced or retarded diffusion (OED/ORD) and electrical characterization.

\section{INVESTIGATION OF THERMOELECTRIC PROPERTIES OF SUPERLATTICES GROWN BY MOLECULAR BEAM EPITAXY}

Theodore C. Harman

MIT Lincoln Laboratory

SC: NVESD

This research is on the molecular beam epitaxial (MBE) growth of superlattices from two alloys with features that may be necessary for a potential breakthrough in the development of higher performance thermoelectric cooling materials. It was earlier shown that bulk thermoelectric properties at $300 \mathrm{~K}$ can be obtained in Bio.9Sb0.1 MBE-grown films. Epitaxial Bi $0.9 \mathrm{Sb}_{0.1}$ films with low carrier concentrations and record high electron mobilities at $77 \mathrm{~K}$ were grown. Their electrical properties represent not only a substantial improvement over the literature values for epitaxial BiSb alloys, but more importantly, nearly ideal properties for the small-energy-gap component of the thermoelectric superlattices. In addition to upgrading the quality of the Bio. $\mathrm{Sb}_{0.1}$ layers and the MBE system, high priority was given to the measurement of the thermoelectric figure of merit $(\mathrm{ZT})$ on the MBE-grown films. For the film measurements, a modified ZT-meter has been designed and built.

\section{STABLE CONTACTS TO SEMICONDUCTORS}

Marc-A. Nicolet

Elzbieta Kolawa

California Institute of Technology

SL: ARL/EPSD

SC: NVESD 
A simple thermodynamic analysis with available data, supplemented by modelled free energies where necessary, has led to the realization that $\mathrm{Cu}$ is thermodynamically stable with the silicides of the VB and VIB transition metals $(\mathrm{M}=\mathrm{V}, \mathrm{Nb}, \mathrm{Ta}$ and $\mathrm{Cr}, \mathrm{Mo}$, $\mathrm{W})$ as long as the silicide is metal-rich. Since nitrogen does not form compounds with $\mathrm{Cu}$, the results will continue to hold for MSiN alloys, barring the existence of ternary $\mathrm{Cu}$ compounds. The unparalleled performance of amorphous $\mathrm{TaSiN}$ as a barrier for $\mathrm{Cu}$ then becomes manifest: the thermodynamic stability prevents the formation of new compounds. A next step will be to characterize the diffusivity of $\mathrm{Cu}$ in these materials. To experimentally verify these thermodynamic predictions, plans are to synthesize WSiN and investigate its properties as researchers have for TaSiN. Initial results show that the as-deposited compound is amorphous. Amorphous Ta36 $\mathrm{Si}_{14} \mathrm{~N}_{50}$ films have shown their superior qualifications in yet another application. As caps to suppress the evolution of $P$ when InP is thermally annealed, these films are comparable with $\mathrm{Si}_{3} \mathrm{~N}_{4}$, but offer the advantage of an electrically conducting layer.

\section{MAGNETIC HARDENING STUDIES AND NOVEL TECHNIQUES FOR PREPARATION OF HIGH PERFORMANCE MAGNETS}

George C. Hadjipanayis

University of Delaware

\section{SL: ARL/EPSD}

The research will investigate the magnetic behavior of several promising new alloy systems and novel processing approaches for possible permanent magnet development. Melt spinning, mechanical alloying, sputter deposition and gas atomization synthesis techniques will be used to prepare transition metal rare earth intermetallic alloys, including $\mathrm{R}(\mathrm{Fe}, \mathrm{Co})_{2}$, $\mathrm{R}(\mathrm{Fe}, \mathrm{Co})_{3}, \mathrm{R}_{2}(\mathrm{Fe}, \mathrm{Co})_{7}, \mathrm{R}(\mathrm{Fe}, \mathrm{Co})_{17}$. Powders of these alloys will then be subjected to gas-phase interstitial modification processing to introduce interstitial borides, carbides and nitrides. In addition, the origin of magnetic hardness in substituted Nd-Fe-B magnets with $\mathrm{Al}, \mathrm{Cu}$, and $\mathrm{Zn}$ will be investigated. The magnetic properties of the alloys will be determined using standard magnetometer techniques, and will be correlated with the microstructure determined by TEM and SEM. This approach will provide an en- hanced understanding of the operative coercivity mechanisms and thereby suggest improved alloying and processing approaches that can actually convert the outstanding intrinsic characteristics of some of the more promising alloy systems into useful magnets.

\section{METAL-ORGANIC VAPOR PHASE EPITAXY OF CONTROLLED DEEP LEVEL STRUCTURES: MATERIALS, DEFECT STRUCTURE AND DEVICE APPLICATIONS}

T.F. Kuech

Kevin L. Bray

University of Wisconsin - Madison

The research will investigate the controlled introduction of oxygen, boron and related impurities during the metal-organic chemical vapor phase epitaxy (MOVPE) of GaAs, AlGaAs, InGaAs and InP. The microscopic nature of the defects and a comprehensive model of their effect on the optical and electrical properties of the epitaxial layers will be established. High pressure measurements will be used in combination with conventional, ambient pressure measurements (e.g. Hall effect, DLTS and PL) to determine the physical and electronic structure of the deeplevel defects. The basic relationships linking the growth process, defect structure and materials properties will be established.

\section{NOVEL ACTUATOR MATERIALS: MONOMORPHS AND PHOTOSTRICTIVE ACTUATORS}

Kenji Uchino

The Pennsylvania State University

\section{SL: ARL/EPSD}

The theme connecting the studies in this project is the fundamental examination and the application development of newly discovered phenomena: monomorph and photostrictive effect type actuators. The monomorph mode of a semiconductive piezoelectric ceramic causes non-uniform distribution of the electric field when metal electrodes are coated on the ceramic faces, and exhibits a bending deformation under an electric field. Photostriction arises from a superposition of the phenomena of the pho- 
tovoltaic and inverse piezoelectric effects, and is applicable to fabricate photo-driven (remote control) actuators.

\section{THEORIES OF NONLINEAR OPTICAL PROPERTIES OF COMPOSITE MATERIALS: APPLICATION OF PHTHALOCYANINE DYES}

David G. Stroud

Ohio State University

$$
\text { SL: VAL }
$$

SC: NRDEC

The research will theoretically investigate the nonlinear optical properties of composite materials using existing theories such as effective-medium theory. New theories will be developed as appropriate. A wide variety of particle shapes, particle concentrations, grain arrangements, nonlinear particle coefficients, and nonlinear coatings on the particles will be considered to identify parameters that will lead to enhanced nonlinear responses.

\section{E. High Strain Rate Behavior of Materials}

\section{LOCALIZATION IN TUNGSTEN HEAVY ALLOYS SUBJECTED TO SHEARING DEFORMATIONS UNDER SUPERIMPOSED HIGH PRESSURES}

\section{K.T. Ramesh}

The Johns Hopkins University

\section{SL: ARDEC, ARL/WTD}

The first phase of this project involved the construction of a new plate impact facility, and that is proceeding. A $60 \mathrm{~mm}$ diameter barrel has been procured for the gas gun, and a keyway cut into it for controlling oblique impact. The target chamber and breech are under construction. An optic table and facilities for laser interferometry have been installed. Simultaneously, some work has been conducted on the response of tungsten heavy alloys to high rates of deformation using compression and torsional Kolsky (split-Hopkinson) bars. In particular, previous work has been expanded by showing conclusively that there is a strong influence of prior plastic deformation (swaging) on the rate-sensitivity of these alloys, and that increasing tungsten contents appears to effect increases not only in the observed flow stresses but also in the observed rate sensitivities for these alloys. Work is also in progress on investigating dynamic shearing deformations of a tungsten heavy alloy using the torsional Kolsky bar. Adiabatic shear localization occurs during high-rate shearing of these alloys. SEM micrographs of the failed specimens show evidence of large but heterogeneous shearing, a large number of tensile microcracks within tungsten grains close to the shear band, and what appears to be melting within the actual shear band. It appears that the ability of the matrix material to accommodate the shearing deformations is crucial to the capacity of the alloy to sustain large shear.

\section{DYNAMIC MATERIAL RESPONSE FOR MODEL DEVELOPMENT}

\section{R.J. Clifton}

Brown University

$$
\text { SL: ARL/W̄TD }
$$

SC: ARL/WTD

A thin foil of a W-Ni-Fe alloy, $50-200 \mu \mathrm{m}$ in thickness, was sheared for $2 \mu$ s under plane wave conditions by plate impact. Shear bands observed show more intensely localized deformations than obtained in other experiments. The responses of the constituent phases, the tungsten grains and the matrix, as well as that of the composite alloy over a strain rate range of $10^{-4} \mathrm{~s}^{-1}$ to $7 \times 10^{5} \mathrm{~s}^{-1}$ were experimentally obtained and characterized by a viscoplastic model. The effects of strain hardening, thermal softening, material inertia, rate sensitivity, heat conduction and material microscopic inhomogeneity, on dynamic shear localization, are investigated through finite element simulations.

\section{DYNAMIC CONSOLIDATION OF CRYSTALLINE AND CVD DIAMOND}


Thomas J. Ahrens

California Institute of Technology

SL: ARL/MD

SC: ARL/WTD, ARL/MD, TACOM

Shock consolidation of powders with 50 to 60 percent of crystal density of diamond and boron nitride, both pure and admixed with $\mathrm{SiC}$ and $\mathrm{Si}_{3} \mathrm{~N}_{4}$ whiskers and graphite, occurs upon application of $<1 \mu$ s duration shock pulses of 15 to $20 \mathrm{GPa}$. For powders, consolidation gives rise to surface heating to temperatures of $\sim 400 \mathrm{~K}$ and results in a molten layer several $\mathrm{mm}$ thick. This molten layer freezes via heat conduction into the interior of the grains on a time scale short compared to the shock pulse duration. Using the geometric model of compaction of Kaker and Chaklader for spherical or cubic grains, researchers estimate that the irreversible work carried out against materials strength in the sample is $\sim 0.4$ percent of the total shock energy. Therefore, most shock energy goes into surface heating. The recently reported consolidation of $<1 \mathrm{~mm}$ sized diamond powders is attributed to shock induced nearly uniform heating and resulting plastic deformation above $10 \mathrm{GPa}$ and $1300 \mathrm{~K}$. Consolidation of diamond admixed with graphite is assisted by the transformation of graphite to amorphous carbon which occurs above $\sim 20 \mathrm{GPa}$ as a result of graphite in the diamond field crossing the metastable extension of its melting line.

\section{STRENGTH AND INELASTIC DEFORMATION OF SHOCKED CERAMICS}

Yogendra M. Gupta

Washington State University

\section{SL: ARL/WTD}

SC: ARL/MD

An extensive set of controlled oblique as well as normal plate impact experiments on well-characterized ceramic materials will be conducted to permit a detailed examination of material strength and inelastic deformation under shock loading. Redundant instrumentation techniques (particle velocity and stress measurements) with high time resolution will be employed to make reliable quantitative measurements during loading and unloading at different peak stress levels. In the pressure-shear experiments, appropriate separation between the compression and the shear waves will be used to effectively broaden the loading paths. Recovery experiments will be per- formed to characterize the microstructural damage under different loading conditions. Wave profile measurements will be correlated with damage features to arrive at descriptions of inelastic deformation under shock loading.

\section{DYNAMIC BEHAVIOR OF BRITTLE MATERIALS}

Thomas J. Ahrens

California Institute of Technology

The research objectives are to develop an experimental technique for the bi-axial testing of ceramic materials at high strain rates; to develop micromechanical models of damage evolution in brittle materials under multi-axial stresses; to study shock wave propagation and flow of material in compatible rubble; and to develop analytical modelling of phase transitions to describe the kinetics of phase transformations in hard ceramics.

\section{ASSESSMENT OF DAMAGE TO CERAMIC TARGETS}

Donald A. Shockey

Donald R. Curran

SRI International

\section{SC: ARL/WTD, ARL/MD, TACOM}

Researchers designed and constructed fixtures for symmetric pressure/shear experiments to reveal whether slip occurs at the impact plane of the colliding slanted plates and to reveal whether shear wave propagation through an unloaded region invalidates strength measurements. These two experiments will use in-material magnetic particle velocity gages in Coors AD-85 and AD-995 alumina. To further develop a model (called FRAGBED) that describes the failure process of a ceramic target in the region ahead of an advancing penetrator, workers are constructing a comminution relation that contains the current particle geometry, crack sizes, fracture toughness, and stress state, plus the crack growth velocity. Initial exercises with the model show approximately the variations in particle sizes seen in the $\mathrm{TiB}_{2}$ test. After further testing, this comminution relation will be added to the treatment of fragment sliding now in the FRAGBED model, and simulations of long rod penetration experiments will be performed to test the model. 


\section{F. Miscellaneous}

\section{ADVANCED ARMOR MATERIALS DEVELOPMENT}

Kathryn V. Logan

Georgia Institute of Technology

\section{SL: ARL/WTD, ARL/MD}

SC: ARL/WTD, ARL/MD

The main progress was focussed on trying to identify possible justifications for the order of magnitude difference in the depth of penetration results of the two composite samples. Automation of the hot pressing system was begun. There are direct relationships between ram movement and densification mechanisms of the hot pressed powders. The ram movement can be monitored by making relatively simple modifications to the existing hot press. The new $50 \mathrm{KW}$ induction generator was added to the hot press system which will now allow pressing of up to 6 inch diameter disks with the present load frame. Efforts are being made to determine the types and sensitivity of various transducers necessary to automate the hot press. Automation will improve the ability to finely control the pressing parameters in a reproducible fashion. The system will ultimately be computer controlled during all stages of the pressing run and subsequent cool down. Other progress during the interval was focussed on trying to identify possible justifications for the order of magnitude difference in the depth of penetration results of the two composite samples.

Reports:

No. 1 in previous edition.

2. A Thermoanalytical Study of the $\mathrm{Al}-\mathrm{B}_{2} \mathrm{O}_{3}$ System, by William Jesse Stuart McLemore, MS Thesis, 1991, 140 pp.

\section{CRYSTAL CHEMISTRY OF CERAMIC/MINERAL SYSTEMS}

Robert R. Reeber

University of North Carolina at Chapel Hill

Crystal growth furnaces have been completed and instrumented. Four crystal growth runs of aluminum diboride in aluminum melts were carried out during Professor Klapper's visit over the February-March timeframe. Two and perhaps three types of crystals were grown. Several methods have been described in the literature for removing similarly grown crystals from the solid aluminum. These and several variants of them had limited success. Very dilute $\mathrm{HCl}$ ( 1 to 5 percent by volume) in anhydrous ether cooled with ice allowed recovery of some small crystals. The grey metallic material was identified as $\mathrm{AlB}_{2}$ by powder $\mathrm{x}$-ray diffraction. One of the boules was cross-sectioned without etching to reveal at least two and possibly three varieties of crystals present. One type, light yellow in color, was apparently destroyed by the etching treatments. Another, grey in color but not metallic, had a different morphology than the material tentatively identified as $\mathrm{AlB}_{2}$. The results were extremely interesting from several perspectives. (1) The crystals apparently have very similar chemical reactivity to the aluminum base metal and have a platy morphology. One would expect that metal matrix composites made from such material would be extremely strong and relatively unreactive to corrosion. (2) The presence of several different crystals in the boule where only $\mathrm{AlB}_{2}$ is expected indicate that there may be a problem with the known phase diagram for this system.

\section{SHOCK WAVE INTERACTIONS WITH EXOTHERMIC MIXTURES}

K.R. lyer

North Carolina State University

\section{SL: ARL/MD}

Two series of successful high velocity impact experiments (in addition to a large number of shots in which either the impact was not planar and/or the significant measurements were not satisfactory) were performed to quantitatively monitor the shockwave interactions with green compacts (55 percent theoretical density) of $\mathrm{Ni}$-Al powder mixture in the ratio of 2.6 to 1 . The ratio was chosen to have slightly lower impedence in the fully dense specimen than that of the backing plate. The difference in the two series was the particle size of the $\mathrm{Ni}$ powder $-3 \mu \mathrm{m}$ and 7 $\mu \mathrm{m}$. The pressure recorded is the pressure at the reflecting surface behind the specimen as the reflected shockwave sweeps through the specimen which has been agitated and densified by the forward passage of the incident wave. From calculated values of shock velocities and assumptions about the equation of state of powder mixtures, peak pressures for inert mixtures were also calculated. For values of impact velocity higher than a threshold, the peak values of pressures recorded in these experiments were significantly higher than the calculated values 
for corresponding cases. The excess pressure is attributed to the exothermic reaction in the shock front. Solid state exothermic reaction induced by the sweep of a strong shock wave through freshly agitated and densified mixture was proposed.

\section{CHARACTERIZATION OF DIAMOND FILM GROWTH IN A COMBUSTION FLAME}

John T. Prater

Jeffrey T. Glass

North Carolina State University

$$
\begin{array}{ll}
\text { SL: } & \text { NVESD } \\
\text { SC: } & \text { ARL/EPSD, MICOM }
\end{array}
$$

Excellent progress is being realized assembling a combustion growth system which will be contained inside a controlled environmental chamber. This will allow extensions of combustion growth experiments to permit substrate biasing at low pressure and growth in inert gas environments. It is felt that this capability will be of great importance in controlling the nucleation process. Recent developments suggest that it may now be possible to grow high-quality heteroepitaxial diamond films. Program emphasis has shifted to exploring this possibility. The working premise for heteroepitaxial growth is that a high density of nucleation sites with direct registry to the underlying substrate can be formed, and that lateral growth along the surface can be encouraged, such that the islands can merge into a single epitaxial layer early in the growth process. Recently, formation of a high density of nuclei with direct registry to $\mathrm{Si}(100)$ substrates has been demonstrated using microwave plasma chemical vapor deposition techniques. The films are prepared using a two-step process: (1) carburization followed by (2) biasing. The carburization and biasing steps can be considered as in-situ pretreatments to first deposit an epitaxial $\beta$-SiC layer and then promote the formation of stable oriented nuclei. Researchers are now identifying the optimium combustion flame growth conditons which will provide selective growth of correctly oriented nuclei such that they coalesce into a single-crystal epitaxial film.

\section{Reports:}

1. Microstructural Studies by TEM of Diamond Films Grown by Combustion Flame, by G.-H. Ma et al., New Diamond Science and Technology, 1991, p587. AD A244 354

2. Analysis via Transmission Electron Microscopy of the Quality of Diamond Films Deposited From the Vapor
Phase, by G.-H. M. Ma et al,, Diamond and Related Mater 1,25(1991). AD A244 795

\section{SCALE MODELING ISSUES IN ARMOR PENETRATION EXPERIMENTS}

Charles E. Anderson, Jr.

Southwest Research Institute

A computational study has been performed to quantify the effects of strain rate on replica-model (scaled) experiments of penetration and perforation. The impact of a tungsten-alloy, long-rod projectile into an armor steel target at $1.5 \mathrm{~km} / \mathrm{s}$ was investigated. It was found that over a scale factor of 10 , strain-rate effects change the depth of penetration, for semi-infinite targets, and the residual velocity and length of the projectile, for finite-thickness targets, on the order of 5 percent. Although not modeled explicitly in the present study, the time-dependence of damage was examined. Damage accumulation is a strong function of absolute time, not scaled time. At homologous time, a smaller scale will have less accumulated damage than a larger scale; therefore, the smaller scale will appear stronger, particularly in situations where the details of damage evolution are important.

\section{POSITRON ANNIHILATION STUDIES OF THERMOPLASTIC LCP COMPOSITES}

Andrew Crowson

Phillip L. Jones

Duke University

\section{SC: ARL/MD}

The majority of the experimentation involved reconfiguring the positron annihilation lifetime systems. Both lifetime systems were completely recabled to ensure proper electrical contacts. The computer control system of the positron lifetime spectroscopy (PALS) set-up was converted to a new system. This conversion has reduced that modeling time of PALS data in half. In addition, both PALS systems were recalibrated. The operation of the system was verified through a partial repetition of work done under a previous contract and through the use of ${ }^{60} \mathrm{Co}$ to verify system resolution. A new PALS sample chamber was designed to enable thermal cycling of PALS samples. Positron annihilation lifetime spectroscopy (PALS) was employed to obtain isothermal data for 
the following thermotropic liquid crystalline polymer (TLCP)/thermoplastic composites: (1) Pure hydrobenzoic acid and 2-hydroxy 6-naphthoic acid TLCP (Vectra), (2) pure isotactic polypropylene (PP), and (3) blends of 5, 10, 20, and 30 percent Vectra with PP. Isothermal data were collected for all blend systems at $20^{\circ} \mathrm{C}$ and $25^{\circ} \mathrm{C}$. The data indicates a relaxation in the ortho-positronium intensity component for the pure $\mathrm{PP}$ and all blends. The pure Vectra TLCP does not show the same behavior. The ortho-positronium lifetime of all samples is constant and shows no sign of relaxation. A decrease in the initial ortho-positronium intensity value is exhibited with increasing concentration of TLCP reinforcements. Currently, this decrease is being attributed to the rather recent development of $\mathrm{e}^{+}$irradiation damage. Differential scanning calorimetry was used as a complimentary technique to PALS to determine thermal properties (i.e., glass transition, melting transition, etc.) of the TLCP, matrix, and blends. In addition, DSC measurements were used to investigate any possible miscibility effects in the melts. 


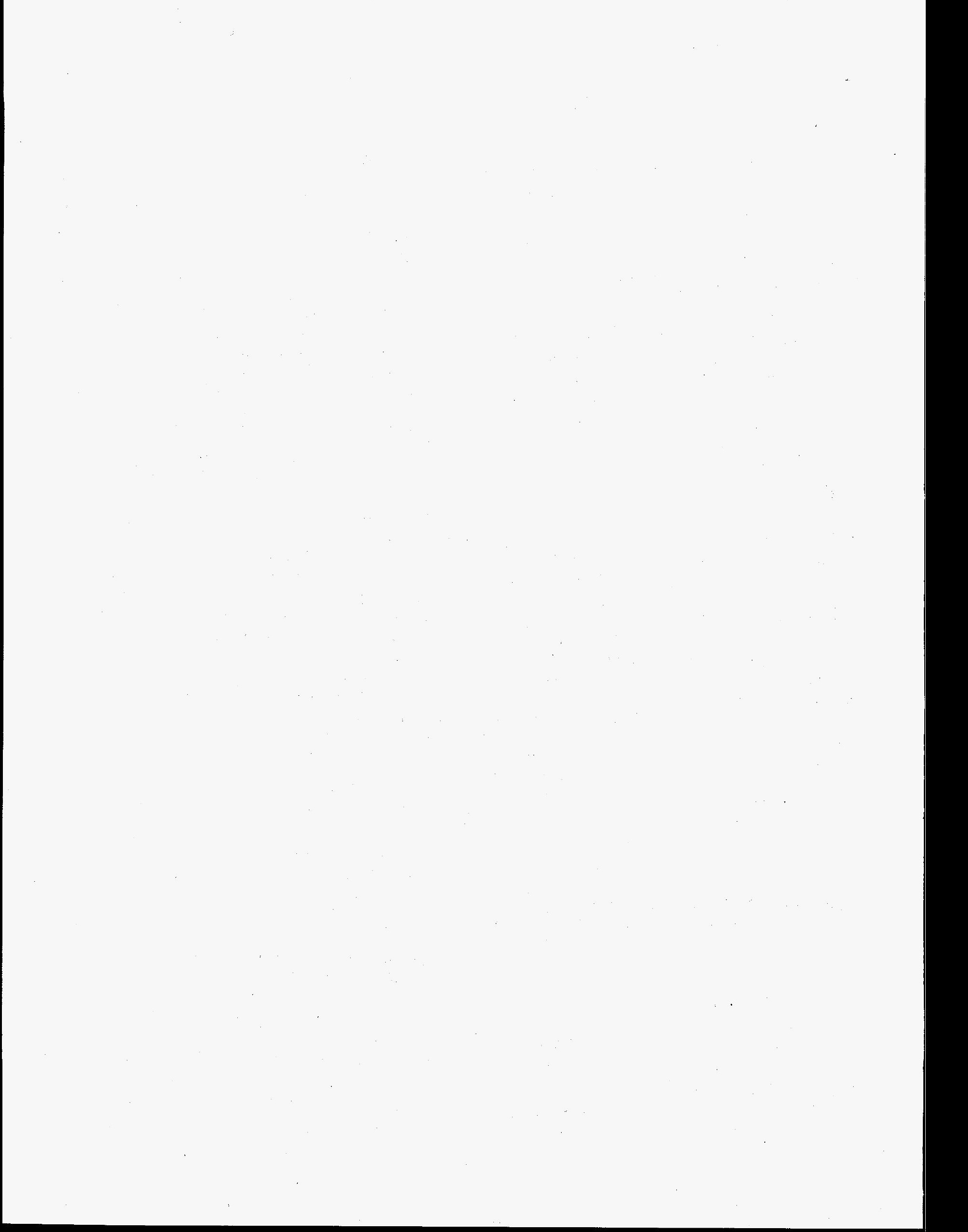




\section{GEOSCIENCES}

\section{A. Geomorphology/Hydrology}

\section{DIGITAL ELEVATION MAPS AND WEATHER RADAR IN FLOOD FORECASTING: THE ARNO RIVER BASIN AS A CASE STUDY}

Rafael L. Bras

Massachusetts Institute of 'Technology

SL: WES

SC: CRREL

A digital elevation map-based distributed rainfallrunoff model is used to study the value of the sensitivity to inputs from meteorological data. It is found that use of the proper radar reflectivity - rainfall intensity (Z-R) relationship is the most crucial factor in obtaining correct flood hydrographs. Errors resulting from spatially averaging radar rainfall are acceptable but the use of discrete point information can lead to serious problems. Reducing the resolution of the 5-min radar signal by temporally averaging over 15 and $30 \mathrm{~min}$ does not lead to major errors. Using 3-bit radar (rather than the usual 8-bit) to represent intensities results in significant operational savings without serious problems in hydrograph accuracy.

\section{COMPUTER SIMULATION OF SUBAQUEOUS SEDIMENT TRANSPORT}

Peter K. Haff

Duke University

\section{SL: CRREL, WES}

Studies of mixing are now nearing completion and are being written up. The basic result is that, for a modest applied traction, mixing depth is proportional to applied stress and is relatively independent of particle size. Further, vertical diffusion of tagged sediment particles can occur over this depth on time scales of only a few seconds. This is important if the tagged particles represent contaminated sediment of some kind. Conversely, buried particles can be exhumed in only a matter of seconds if they lie within the shear depth of the sediment. In the area of swash mechanics, a multiple-slab fluid model has been implemented in which the fluid is viewed as composed of multiple parallel slabs which can move independently of one another; each of these slabs exerts an eddy-viscosity type of friction on its neighbors. At the bottom of the water column, the slabs exert a drag on an embedded sediment particle. Each slab, like each sediment particle, also obeys its own equation of motion. The bed is initially tilted, and a layer of water is set in motion upslop (run-up). Gravity and friction with the bed slow the fluid, which eventually reverses direction and runs back out (backwash). Simulation studies of the stability of a 3D surface are continuing. Detailed studies of the pivot angles of individual grains are averaged over many test grains to obtain a mean pivot angle; the distribution of angles is also obtained, for any given flow direction. The mean angle need not be independent of flow direction if a previous flow regime has resulted in non-isotropic surface fabric.

\footnotetext{
Reports:

1. Transport of Granules by Wind and Water: Micromechanics to Macromechanics in Geology and Engineering, by Peter K. Haff et al., MS.

2. Grain-Scale Simulations of Loose Sedimentary Beds, with Examples from Aeolian Saltation, by Peter K. Haff, MS.

3. Basic Physical Models in Sediment Transport, by Peter $K$. Haff, MS.

4. Application of Multi-Particle Simulation Methods to the Study of the Micromechanics of Bedload Transport, by $\mathbf{Z}$. Jiang and P.I. Haff, MS, Water Resources Res.
}

\section{SEMI-AFFINE TOPOGRAPHY AS A FRAMEWORK FOR SUBSURFACE FLOW AND SOIL MOISTURE MODELING IN RUGGED TERRAIN}


Christopher J. Duffy

The Pennsylvania State University

SL: ETL

The hypothesis of this research is that topographic structure exerts a fundamental control on the space and time distribution of hydrologic processes occurring on a landscape. A multi-scale approach to modeling is to use the texture of the topography as a framework to conduct hydrologic experiments and to develop quantitative hydrologic models for soil moisture, storage, and runoff resulting from space and time variable precipitation and evapotranspiration. It has been proposed that the characteristic topography of a physiographic region is self-similar, or more correctly self-affine, where increments of altitude and map distance maintain a constant proportionality over some subregion of the terrain. Hwang and Turcotte (1990) propose that this self-similarity is a fundamental geomorphic property, unique to a particular physiographic region (Colorado Plateau, Basin and Range). In this research, this notion was examined for portions of the Appalachian Plateau and the Colorado Plateau for the purpose of finding a geometric framework for hydrologic modeling. The parameters appear to be most consistent in the upland portions of the terrain. In the upland regions, researchers have examined in detail the concept of self-affine slopes and have constructed "shape functions" for hillslopes draining to streams. These shape functions are then used to rescale correlated and spatially scattered hydrologic information (depth to bedrock, water table, soil moisture) onto the unit cube. This rescaling of the hydrologic observations with respect to a "unit cube" allows simplification of the flow problem, particularly useful for studying nonlinear rainfall-storage-runoff. Spectral/harmonic analysis has been used to study $40-100$ year records of monthly precipitation-temperature time series for 21 stations in the Wasatch Range, Utah. The emphasis is on seasonal and stochastic fluctuations in the orographic effect for predicting the hydrologic inputs/outputs for a mountainous region.

\section{SCALE AND HETEROGENEITY EFFECTS ON FLOW AND TRANSPORT IN MULTIPHASE SYSTEMS}

Cass T. Miller

University of North Carolina at Chapel Hill

$$
\begin{aligned}
& \text { SL: CRREL, WES } \\
& \text { SC: ATHAMA }
\end{aligned}
$$

The key objective of the project was to determine the effect of scale on flow and transport phenomena in multiphase systems. Good progress toward meeting this objective has been realized. This progress includes the development of a novel in situ polymerization technique, which has been applied to a set of similar media to measure the effect of media characteristics on residual saturation. This work shows that scale effects are a valid concern, which may require very large sample sizes of the continuum hypothesis to hold for typical porous media. An important component of the work is the development and use of mathematical models to describe flow and transport phenomena in multiphase systems. The existing general one-dimensional model has been extended to two-dimensions and work is currently underway to perform simulations for heterogeneous systems. Complementary modeling work has led to new developments in the solution of flow and transport in unsaturated media, a subset of the systems of concern in this work. The complementary modeling work has centered around the development of Petrov-Galerkin methods and adaptive methods for the solution of fluid flow and solute transport in two-phase systems. These techniques are being developed and used for the general multiphase flow and transport problems of concern in this work.

\section{Reports:}

1. The Influence of Porous Media Characteristics and Measurement Scale on Pore-Scale Distributions of Residual Nonaqueous Phase Liquids, by Alex S. Mayer and Cass T. Miller, MS, $J$ Contaminant Hydrology.

2. Modeling Long-Term Solute Transport in Drained Unsaturated Zones, by Hesham Kandit et al., MS, Water Resources Res.

\section{SPATIAL MODELS OF HYDROLOGIC RESPONSE FOR REGIONS OF DIVERSE PHYSIOGRAPHY AND CLIMATE}

James A. Smith

Princeton University

The major research result obtained in this study is that central Appalachian flood peaks exhibit a peak in variability for basin scales ranging from $10-100$ square miles. The most surprising element of the results is the small variability of flood peaks in basins 
less than 10 square miles in drainage area. Several hypotheses have been developed for explaining the empirical results. Radar is a principal tool that is being used to examine the spatial organization of extreme storm rainfall. An extensive radar data base has been assembled for the southern plains study region and preliminary analyses of spatial and temporal variability of extreme storm rainfall have been carried out. A paper has been prepared which shows that the spatial correlation function for radar derived rainfall rate fields decays from slightly larger than 0.8 for a separation distance of approximately $2 \mathrm{~km}$ to less than 0.2 for separation distances greater than 25 $\mathrm{km}$. These results are generally consistent with the hypothesis, given above, that spatial organization of extreme storm rainfall can control scale properties of extreme rainfall.

\section{B. Snow, Ice and Frozen Ground}

\section{FATIGUE CRACK PROPAGATION IN FRESHWATER ICE}

Wilfred A. Nixon

University of Iowa

\section{SL: CRREL}

Work has concentrated on gathering fatigue crack growth data for polycrystalline fresh water ice, using the crack growth techniques developed previously. In addition, and by way of providing quality control on the ice being tested, a series of fracture toughness tests have been conducted on the ice. Specifically, from each manufactured piece of ice, two test samples can be machined. One of these is tested under fatigue loading, the other is used for a fracture toughness test. In this way, for each fatigue test the $K_{\max }$ and $\Delta K$ levels can be expressed both as absolute values and relative to the measured $\mathrm{K}_{I C}$ value for that ice. The fracture toughness results to date appear to show very little dependence on grain size, though the range of grain size stacked is currently very limited. While the two gauge method of crack tip measurement gives good results, there is still considerable scatter. To examine possible causes of this scatter, a finite element analysis was performed for the specimen geometry. From this, it became clear that placement of the gauges plays a critical role in determining the location of the center of rotation for the sample. At present, appropriate corrections are being included in the data analysis to take this fact into account and it appears that, by doing so, much of the scatter is reduced. Much work has been done to preform a scanning electron microscopy study of the fracture surface after the test. This has involved developing an accurate and reliable replication method, which has proved to be a rather difficult task. Nonetheless, this part of the study is now bearing fruit and interesting, striation-like, features have been observed on the fatigued fracture surfaces.

\section{Reports:}

No. 1 in previous edition.

2. Fracture Toughness of Granular Freshwater Ice, by L.J. Weber and W.A. Nixon, MS.

\section{A FIELD INVESTIGATION OF WATER AND SALT MOVEMENT IN PERMAFROST AND THE ACTIVE LAYER}

T.E. Osterkamp

J.P. Gosink

University of Alaska Geophysical Institute

\section{SL: CRREL}

Field measurements were made during break-up, although unseasonably cold weather hampered efforts. A result of the modeling work is the role that temperature-dependent thermal parameters and distributed latent heat play in permafrost that is responding to surface temperature changes. The temperature-dependence and latent heat effects are caused by changing unfrozen water contents at temperatures near $0^{\circ} \mathrm{C}$. These substantially slow the thermal response of the permafrost causing the time scales for this response to lengthen dramatically. These results will be important in developing an understanding of heat flow in the active layer and near-surface permafrost.

\section{TOWARD A MOLECULAR-SCALE UNDERSTANDING OF FROST HEAVING: PHASE I}

John H. Cushman

Purdue University

\section{SC: CRREL}

Molecular relaxation in ultra-thin films has been studied by computer simulation of diffuision in a monolayer of atomic fluid constrained between two structured plane-parallel solid walls. The transverse alignment of the walls is varied to alter the micro- 
scopic environment in which the confined atoms move. Log-log plots of mean square displacement versus time yield straight lines of slope $d<1$, where $d$ is a function of alignment. That is the fluid evinces fractional (fractal) Brownian motion. The fractal character is rationalized qualitatively in terms of the constrictive structure of the "tunnels" through which atoms must diffuse. Diffusion is in general anisotropic.

\section{Reports:}

No. 1 in previous edition.

2. Fractional Brownian Diffusion in Molecularly-Thin Films, by John H. Cushman et al., MS, Science.

3. Monte Carlo Calculation of Chemical Potential for the Stockmayer Fluid, by K.-K. Han et al., MS, J Chem Phys.

4. Shear Melting of Molecularly Thin Confined Films, by Martin Schoen et al., MS, Phys Rev.

5. Isostress-Isostrain Ensemble Monte Carlo Simulation of Shear Induced Melting in Confined Solid Monolayers, by Martin Schoen et al., MS, Phys Rev.

6. Grand Canonical Monte Carlo Simulations of Stockmayer Fluids in Micropores, by K.-K. Han et al., MS, Mol Phys.

\section{THE DUCTILE TO BRITTLE TRANSITION IN POLYCRYSTALLINE ICE UNDER COMPRESSION}

Erland M. Schulson

Dartmouth College

\section{SL: CRREL}

Additional experiments were performed to examine wing crack behavior on either side of the D/B transition, to determine the effect of crack-tip creep deformation on crack growth, and to measure the effect of grain size on the transition strain rate. Again, all observations/measurements were made at $-10^{\circ} \mathrm{C}$ using fresh-water columnar ice compressed uniaxially along a direction perpendicular to the long axis of the columnar grains. Upon adopting the modified loading procedure, it was found that short wing cracks, which were initiated at the tips of the inclined "Teflon" cracks by high-rate pulse loading and then held under zero load for a few minutes, did not propagate when they were subsequently loaded slowly; that is on the ductile side of the transition. This observation confirms that wing cracks which form naturally do not lengthen when the ice is strained slowly enough to be macroscopically ductile. A final set of experiments is now underway to obtain better observations of wing crack initiation but resistance to growth within ductile ice. Upon initiating wing cracks at the tips of inclined "Teflon" cracks, holding under a reduced load and then loading again at a high rate, it was found that whether the wings propagated or not did not correlate well over the whole range of hold times that were examined.

\section{Reports:}

1. The Kinetic Friction of Saline Ice Against Itself at Low Sliding Velocities, by D.E. Jones et al., Ann Glaciology 15,242(1991).

2. The Growth of Wing Cracks and the Brittle Compressive Failure of Ice, by E.M. Schulson et al., Acta Metall Mater 39,2651(1991). AD A249 574

\section{CHANGES IN MICROSTRUCTURAL PARAMETERS IN SNOW DURING DEFORMATION}

Robert L. Brown

Montana State University

\section{SC: CRREL}

Most of the work effort was directed at acquisition of equipment, review of literature and production of artificial snow for stereology analysis. A computer, data acquisition/image analysis board and, a high resolution video camera were all acquired. Difficulties with the camera-board compatibility were encountered, and the board has been returned to the manufacturer for correction of the problem. In the meantime, software and a robust interface for executing the analysis of the snow microstructure are being developed. This software will provide the user with the capability of quickly and accurately analyzing surface sections of snow or firn. Artificial snow with spherical particles was produced. This model snow will be used for calibrating and verifying the software and theory used to analyze surface sections for quantifying the material microstructure.

\section{PHYSICAL PROCESSES IN SNOWPACK DURING RAIN OR MELT EVENTS}

\author{
Charles F. Raymond \\ University of Washington
}

\section{SL: CRREL}

Measurements of the thermal distribution of a maritime snowpack were made using a two-dimensional array of thermistors. Measurements are used to track the progress of the zero degree isotherm during two rain on snow events and one cooling event during the winter of 1991-92. During water infiltration, the zero degree isotherm is interpreted as the position of the 
wetting front and the data illustrate clearly the nonhomogeneous percolation pattern. The rate of water infiltration is controlled by snow properties such as temperature, texture, and stratigraphy as well as the temperature and rate of precipitation. In one of the rain on snow events, water penetrated less than $10 \mathrm{~cm}$ into the snow during the first hour of rain, but in the other event it had penetrated over $50 \mathrm{~cm}$ in the same time. The measured temperature profiles are compared with those calculated numerically using a onedimensional model based on heat conduction only. Deviations between model and measured temperature profiles occur because of heat transfer processes that are nonconductive such as radiative heating and the latent heat associated with phase changes.

\section{A BOOK AND A SERIES OF MONOGRAPHS ON ICE PHYSICS}

Victor F. Petrenko

Dartmouth College

\section{SL: CRREL}

This project is dedicated to producing a series of monographs on ice physics. Monographs will cover all of the important physical properties of ice and will later be unified in a book. A monograph on "Electrical Properties of Ice" was prepared. This monograph is the largest of the series and consists of two parts: Part I: Conductivity and Dielectric Permittivity of Ice, and Part II: Advance Topics and New Physical Phenomena. Part $I$ is written in textbook format for college students. Almost no special background is necessary for its readers. The description of materials in this part is based on Jaccard's theory. Concluding this part of the monograph is a critical review of the experimental results of measurements of conductivity and dielectric permittivity of pure and doped ice and of mobilities of protonic charge carriers. The second part of the monograph contains information about more advanced and specific optics associated with the electrical properties of ice and also some recently discovered physical phenomena.

\section{Reports:}

1. Electrical Properties of Ice, by Victor F. Petrenko, TR, Dec 91, $143 \mathrm{pp}$.

\section{Other (Terrestrial)}

\section{HORIZONTAL STRESS IN SITU BY CONE PENETROMETER AND RELATED STUDIES}

James K. Mitchell

University of California, Berkeley

SL: WES

SC: CRREL

A greatly improved theory has been developed for prediction of the cone penetration resistance of sands both in the free field and in a calibration chamber. Evaluation of this theory has been possible using the results of 222 tests done over several years that were made available by Italian colleagues of the P.I. The results of the analyses so far are very encouraging, and it appears that it will be possible to make reliable predictions of penetration resistance using modified cavity expansion theory. The Italian data are useful also for the further evaluation of the method for determination of in situ lateral stress on the basis of sleeve friction during cone penetration. The research on the aging of sands has continued with additional measurements of the small strain shear wave velocity in triaxial specimens. While a change in the shear wave velocity with time for saturated sand samples has still not been observed, a consistent increase in the shear wave velocity of dry samples has been measured. Many tests have been conducted to characterize the material in its dry state, and to investigate the effect of the initial fabric on the change in shear wave velocity with time. More tests are planned to help to understand the difference between the behavior of dry and saturated samples.

\section{Propagation}

\section{BACKSCATTERING FROM ROUGH SURFACES}

Zu-Han Gu

Surface Optics Corp.

\section{SL: CECOM, CRDEC}

Work has been concerned with an experimental study of light scattering from a gold-coated quasi1-D random grating with RMS Height $\sigma=1.5 \mu \mathrm{m}$, and a correlation length $a=4.0 \mu \mathrm{m}$. This plate was fabricated using a combination of the scanning and speckle techniques in attempts to make the surface 
more one-dimensional. Studies have been made of the problem of light scattering in double passage configurations. Of particular interest are the mean scattered intensity and the motion of the speckle produced around the backscattering direction as the source and detector are moved. As is usual with this problem, the analysis is based on scaler wave theory and the thin phase screen model. However, with the additional assumption that the mirror is illuminated with Gaussian speckle one obtains analytical results without further approximations. As expected, the results show that in some cases there is an enhancement in the backscattering direction. Also, in normal circumstances, the speckle pattern moves in a direction opposite to that of the source, and decorrelates very rapidly. However, some special circumstances are found in which the speckle pattern seems to track the backscattering direction. This unusual behavior has been interpreted and, based on this a new configuration is proposed for which the speckle pattern is highly symmetric and tracks the backscattering direction under very general conditions. Enhanced backscattering of light from the surface of a liquid metal was calculated analytically. Enhanced backscattering is displayed in the angular dependence of the intensity of the incoherent component of the scattered light for both polarizations of the incident light. $\mathrm{Nu}$ merical estimates of the size of the effect are available for liquid mercury.

Reports:

No. 1-11 in previous editions.

12. Light Scattering From Gold-Coated Ground Glass and Chemically Etched Surfaces, by V. Ruiz-Cortes et al., MS, SPIE Proc.

13. Coherence in Single and Multiple Scattering of Light From Randomly Rough Surfaces, by Zu-Han Gu et al., MS, SPIE Proc.

14. Enhanced Backscattering from One- and Two-Dimensional Random Surfaces, by A.A. Maradudin et al., MS.

15. Enhanced Backscattering and Transmission of Light From Random Surfaces on Semi-Infinite Substrates and Thin Films, by A.A. Maradudin et al., Waves in Random Media 3,S129(1991). AD A249 345

16. Enhanced Backscattering of Light From a Random Rough Surface Nearly Perpendicular to a Mirror, by E.R. Mendez et al., MS, Opt Commun.

\section{ELECTROMAGNETIC WAVE SCATTERING FROM CHARACTERIZED ROUGH SURFACES}

Kenin O'Donnell

Georgia Institute of Technology

\section{SL: CECOM}

A theoretical paper has been prepared on the angular correlation functions of amplitude scattered from a one-dimensional rough surface. Another paper is being prepared. It is based on very recent experimental measurements of the angular correlation functions of intensity scattered from a rough surface. These are analogous to theoretical results discussed in the theoretical paper. In this work, some radically different experimental techniques are used to measure correlation functions analogous to those studied in the so-called memory effect in volume scattering. In particular, researchers find experimentally that the far-field scattered intensity may be nearly perfectly correlated for quite different angles of illumination, as long as the two scattering angles involved satisfy a reciprocity condition. At other angles, one finds a smaller but generally non-zero correlation between the two intensities, if the four incident and scattering angles involved satisfy a condition that conserves momentum parallel to the mean surface. Further, depending on the polarization component of the scattered field that is measured, different forms of the intensity correlation functions are found. Intensity correlation functions consistent with either single or multiple scattering are observed, and these correlation functions provide considerable insight into the scattering processes occurring on a rough surface. Further work continues with a study of the Stokes matrix elements of the set of rough surfaces with increasing roughnesses. Researchers have refined the profilometric techniques and recalibrated the Talystep profilometer so as to get more accurate surface statistics. Some of the scattering measurements on this series of surfaces have been performed.

\section{COMPARATIVE EVALUATION OF KIRCHHOFF, PERTURBATION AND FULL WAVE SOLUTIONS FOR ROUGH SURFACE SCATTERING}

R.E. Collin

Case-Western Reserve University

SL: CECOM

SC: ARDEC

In numerical calculations of scattering from a rough surface it is common practice to use a tapered field 
for the illumination in order to limit the area of the rough surface from which significant scattering occurs. A paper has been prepared which develops a full wave theory for the scattering of a gaussian beam by a perfectly conducting rough surface. The incident field is assumed to be produced by a large aperture antenna having a gaussian electric field amplitude distribution. The field from this antenna is expressed as a spectrum of incident plane waves. The full wave theory described previously is used to determine the scattered field for each incident plane wave. The total scattered field is synthesized by integrating over the spectrum of incident plane waves. The theory developed will show that the total scattered field consists of a specular reflected gaussian beam with a local phase correction plus an additional scattered field that is given by an integral which has the same scattering coefficient and other factors as occurs for an incident plane wave but which also includes a gaussian tapered illumination function. It is also shown that the small perturbation results are only obtained when the integrated term that results from integration by parts to eliminate the explicit dependence on the surface slopes is retained.

\section{A FULL WAVE STUDY OF RADAR CROSS SECTIONS OF ROUGH TERRAIN AND FOLIAGE COVERED TERRAIN}

\section{E. Bahar}

University of Nebraska

SL: CECOM

SC: CRREL, ARL, MICOM

In the paper, "Full Wave Analysis for Rough Surface Diffuse Incoherent Radar Cross Sections with Height-Slope Correlations Included" one dimensionally rough surfaces are characterized by four dimensional joint probability density functions for the heights and slopes at two points on the surface at a distance $x_{d}$ apart. It is shown that the expression for the full wave incoherent diffuse scatter cross section reduces to the low frequency small perturbation solution when the surface heights and slopes are small and to the high frequency physical optics solution when the major contributions to the scattered fields come from the neighborhoods of the stationary phase points. The full wave approach has also been used to evaluate singly and multiply scattered electromagnetic fields from rough surfaces.
Reports:

1. Full Wave Solutions for Rough Surface Bistatic Radar Cross Sections - Comparison with Small Perturbation and Physical Optics Solutions, by Ezekiel Bahar and Bom Son Lee, MS.

2. Examination of Full-Wave Solutions and "Exact Numerical Results" for One-Dimensional Slightly Rough Surfaces, by Ezekiel Bahar, J Geophys Res 96,17,123(1991). AD A251 701

3. Full Wave Analysis for Rough Surface Diffuse, Incoherent Radar Cross Sections with Height-Slope Correlations Included, by Ezekiel Bahar, IEEE Trans on Ant and Prop 39,1293(1991). AD A250 079

4. Scattering Cross Sections for Non-Gaussian Rough Surfaces: Unified Full Wave Approach, by Ezekiel Bahar and Yan-Feng Li, IEEE Trans on Ant and Prop 39,1777(1991). AD A251 489

5. Use of Supercomputers to Evaluate Singly and Multiply Scattered Electromagnetic Fields From Rough Surfaces, by Ezekiel Bahar and M. El-Shenawee, IEEE Trans on Magnetics 27,4287(1991). AD A251 511

\section{INTERACTION OF AEROSOLS AND DROPLETS WITH ELECTROMAGNETIC RADIATION}

Petr Chylek

State University of New York at Albany

\section{SL: ARL/BED, CRDEC}

Researchers have developed an accurate approximation of the absorption and extinction coefficients, single scattering albedo and asymmetry factor in the 8 to $12 \mu \mathrm{m}$ spectral region. The extinction and absorption efficiencies are approximated by a polynomial expansion. No Mie calculations are required and the computer time is reduced by a factor of 100 to 1000 . The accuracy was checked for all cloud types used in the LOWTRAN codes. Consideration has been given to a spherical particle illuminated by two plane waves propagating in opposite directions along the same axis. This situation may describe a particle within a laser cavity, or a particle located close to a plane mirror, or a particle illuminated by two beams which were produced from the same laser source using a beam splitter. Some of the ordinary Mie resonances are suppressed and others are enhanced, depending on the relative phase between two beams.

Reports:

1. Resonance Structure of Composite and Slightly Absorbing Spheres, by Petr Chylek, J Opt Soc Am 9,775(1992).

2. Simple Approximation for Optical Properties of Water Clouds in the 8 to $12 \mathrm{~mm}$ Spectral Region, by Petr Chylek et al., MS, J Appl Meteor.

3. Absorption and Scattering of Electromagnetic Radiation by Prismatic Columns: Anomalous Diffraction Approxima- 
tion, by Petr Chylek and James D. Klett, $J$ Opt Soc Am A8,1713(1991). AD A249 280

\section{MULTIPLE SCATTERING STUDY USING SUPERCOMPUTERS}

\author{
Weng Cho Chew \\ University of lllinois \\ SL: CECOM \\ SC: CRDEC
}

Work has concentrated on studying the three dimensional scattering of waves from a cluster of spheres. A solution was developed for the low frequency regime of the scattering problem by a cluster of spheres for the scalar wave equation. Now, the solution has been generalized to higher frequencies for the scalar wave equation, and a cluster of 1,000 spheres can be solved. The diameter of the cluster is about one wavelength. Other work has been concerned with developing the electromagnetic scattering solution. A bottleneck in calculating the scattering solution has been the vector addition theorem which is very time consuming to calculate. A new way was developed to calculate the vector addition theorem that is much more efficient compared to the conventional method. With the availability of the vector addition theorem, the vector electromagnetic wave scattering solution is developed from a cluster of spheres.

\section{MEASUREMENTS OF NATURAL SURFACES AT MILLIMETER WAVELENGTHS}

Robert E. McIntosh

University of Massachusetts

SL: ARL

SC: WES

This research objective is to extend the range of MMW fully polarimetric measurements of clutter environments and to further investigate simplifications to the Mueller matrix of extended targets. The principal investigator's plan is to operate the equipment he previously constructed to obtain extended measurements of the scattering amplitude matrix and the Mueller matrix for clutter environments. $\mathrm{He}$ plans to compare Mueller matrices directly measured with those deduced from concurrent scattering amplitude matrices.

\section{NUMERICAL AND THEORETICAL STUDIES OF ROUGH SURFACE SCATTERING}

Eric I. Thorsos

University of Washington

\section{SL: CECOM}

This research objective is to explore the limits of the oft-used perturbation theory with special emphasis on the case of small grazing angles and to develop new theory to overcome the deficiencies of standard perturbation theory. Standard and higher order perturbation theory solutions will be compared to integral equation numerical solutions and the limits of perturbation theory will be carefully established. The insight obtained will be applied to the development of new theory to overcome the difficulties of standard perturbation theory. All results will be compared to the numerical solutions of the appropriate exact integral equations.

\section{ROUGH SURFACE SCATTERING STUDIES USING THE METHOD OF SMOOTHING}

Gary S. Brown

Virginia Polytechnic Institute and State University

\section{SL: CRDEC}

This research objective is to improve analytical techniques for the prediction of backscattering from random rough surfaces, establishing the range of validity of the approximations which will undoubtedly be necessary. Rough surface scattering computations using variations of the method of smoothing will be compared with full numerical calculations for 1-D surfaces. The analysis will be extended to the coupled integral equations appropriate to the problem of lossy dielectrics.

\section{STATISTICAL BEHAVIOR OF THE POLARIMETRIC RADAR RESPONSE OF TERRAIN WITH EMPHASIS ON THE MILLIMETER-WAVE REGION}

Fawwaz T. Ulaby

University of Michigan

\section{SL: ARL/WTD, CECOM \\ SC: CRREL}

This research objective is to investigate the relationship between the statistical properties of the phase 
difference between the co-polarized radar returns and the terrain and clutter scenes which give rise to these statistics. The program will include polarimetric measurements at 1.5, 4.74, 9.5,35, 94 and $140 \mathrm{GHz}$ of a variety of clutter scenes under a variety of weather conditions. Theoretical or empirical models will be developed to explain the polarimetric behavior.

\section{E. Atmospheric Remote Sensing}

\section{MULTIPARAMETER RADAR \\ MEASUREMENTS OF \\ PRECIPITATION IN COMPLEX \\ TERRAIN: METEOROLOGICAL \\ AND HYDROLOGICAL APPLICATION}

Kultegin Aydin

The Pennsylvania State University

\section{SC: CRREL, WES}

Both modeling and experimental studies on the polarimetric signatures of the stratiform region of mesoscale convective systems were continued. The experimental data were obtained from the S-band Cimarron radar at NSSL (National Severe Storm Laboratory) in Oklahoma. Aircraft observations and a one dimensional cloud model were used to aid the interpretations of polarimetric measurements (differential phase, differential reflectivity, correlation coefficient and reflectvity factors) and to infer the presence of aggregates, graupel and supercooled cloud water in the stratiform region. The differential phase (propagation and backscattering) together with the other measurands proved to be very useful in estimating the maximum sizes of the particles in the bright band. A significant feature was an abrupt notch in the correlation coefficient that occurred towards the bottom of the bright band, very close to the enhanced positive $Z_{D R}$ region. At the University of Washington, studies on tomographic imaging of rainfall fields from multiparameter microwave observations were continued. Storm simulations for various combinations of network geometry, propagation parameters, and basic functions were evaluated in terms of the the total accumulated rainfall. Differences were minimal between reconstructed accumulated-rain fields resulting from three observables $K_{A}$ (specific attenuation at $K_{a}$-band at horizontal polarization), $K_{D A}$ (specific differential attenuation at $K_{a-}$ band) and $K_{D P}$ (specific differential phase shift at
$\mathrm{X}$-band). The simulated storm types varied from intense rain events to stratiform rain. Overall, the tomographic imaging method was able to identify the main underlying structures of the rain events, with better results as integration time increased.

\section{HIGH-RESOLUTION PROBING OF BOUNDARY LAYER WIND AND MOISTURE FIELDS}

Madison J. Post

Robert M. Banta

National Oceanic and Atmospheric Administration

A great deal of effort was spent on acquiring the major lidar components. Major hardware components are costing considerably more than anticipated, and taking longer to acquire. In order to specify the wavelength of the new lidar optimally, existing NOAA software was enhanced to compute detailed atmospheric absorption spectra in the 2.0 $2.1 \mu \mathrm{m}$ region. By deciding to buy only the pump assembly and reference oscillator portions of the complete transceiver, NOAA will have to develop the power oscillator, the Q-switch, the injection seeding, and the electronic lock loops. Conceptual designs have been developed for these subsystems.

\section{PATH RESOLVED OPTICAL REMOTE SENSING OF ATMOSPHERIC WINDS UTILIZING SPECKLE-TURBULENCE INTERACTION AND PSEUDO RANDOM CODE MODULATION}

\section{J. Fred Holmes}

Oregon Graduate Institute of Science and Technology

\section{SC: CRDEC, TECOM}

Work has been completed on analyzing and optimizing the processing technique. It should be possible to operate down to a backscatter coefficient of $5 \times 10^{-10}$ $\mathrm{m}^{-1} \mathrm{sr}^{-1}$ and ranges out to $2,000 \mathrm{~m}$ with the current optical receiver size ( $\sim 20 \mathrm{~mm}$ diameter). It has been experimentally verified that an acoustooptic frequency shifter (AFS) can be used to diphase modulate the output of the $\mathrm{CO}_{2}$ laser. After verification, a new wide band AFS was installed in the $\mathrm{cw}, \mathrm{CO}_{2}$ coherent lidar. The lidar is now ready for the planned atmospheric experimental work to verify the proposed concepts. New receiver electronics were designed and tested. In order to verify the processing 
techniques, range resolution capability, etc., plans have been made to use hard targets and distributed hard targets before concentrating on the much more difficult problem of using aerosols for backscattering.

\section{F. Small-Scale Atmospheric Processes}

\section{TURBULENT MICROFRONTS}

Larry Mahrt

Oregon State University

SL: ARL/BED

SC: ARL/BED, CRDEC, DPG

A manuscript on decomposition of variances and momentum fluxes has been accepted for publication. This study analyzes LAMEX data by decomposing the momentum flux and variances into coherent structures containing gust microfronts and less coherent turbulence. The microfronts and parent eddies account for the majority of the momentum flux in the windy boundary layer. Another manuscript comparing the ability of different transforms to identify the scale of principal activity in the record is being revised. The Fourier spectrum identifies the principal scale of any periodicity. This scale includes the spacing between the vents (microfronts). The Haar spectrum identifies the width of the events (parent eddies containing the microfronts). Work on comparison between wavelet, Fourier and eigenvector decompositions has been extended to include additional data. The basic analysis has been improved by converting to dyadic energy-conserving plots. The various spectrums with different basis sets are computed for both samples "phase-locked" about gust microfronts and for the records as a whole. The work is now moving from the methodology phase to the physical interpretation phase. An extensive literature survey has been completed as an introduction to this work. Work has started using the Haar inverse transform to analyze turbulence data. This inverse transform filters out noise and fine scale fluctuations yet preserves the sharp edges of thermals and preserves sharp gradients associated with wind gusts.

Reports:

No. 1 in previous edition.

2. Detection and Analysis of Microfronts and Associated Coherent Events Using Localized Transforms, by Nimal Gamage and Carl Hagelberg, MS, J Atmos Sci.
3. Flux Decomposition Into Coherent Structures, by L. Mahrt and Wayne Gibson, MS, Boundany-Layer Meteor.

\section{LAGRANGIAN STOCHASTIC MODELING OF DISPERSION IN THE PLANETARY BOUNDARY LAYER}

Jeffrey C. Weil

University of Colorado

\section{SL: ARL/BED}

SC: CRDEC, DPG, MICOM

This research program is aimed at improving the understanding and predictive capability of dispersion in the planetary boundary layer (PBL) with focus on three problems: $(a)$ the mean $(C)$ concentration field due to a continuous source of passive tracer in a rapidly evolving convective boundary layer (CBL), (b) the root-mean-square $\left(r m s, \sigma_{c}\right)$ concentration field due to a continuous, passive scalar source in a quasi-steady CBL, and (c) the mean concentration field due to a passive scalar source in the stable boundary layer. For problem $b$, researchers are using the Lagrangian two-particle approach to compute relative dispersion and $\sigma_{c}$ for sources at various heights in the CBL. This is being done with: (a) the resolvable velocity fields from large-eddy simulations (LES: Moeng and Wyngaard, 1989) to determine the particle-pair motion due to the large eddies, and (b) the stochastic two-particle model tofreat the random velocities of the subgrid-scale eddies. The vertical inhomogeneity and skewness of the CBL turbulence exist mostly on the large scales and thus appears in the resolvable eddies, whereas the subrid-scale eddies are closer to being homogeneous and isotropic. The stochastic model approximates the latter. A numerical code is currently being developed to access and use the LES data for the trajectory calculations; the principal data are the three components of the resolved velocity field, temperature, and the subgridscale kinetic energy, es. The $e_{s}$ and LES grid size $l$ are used to parameterize the dissipation rate and time scale of the small eddies for the stochastic model. The existing stochastic two-particle code is packaged as a subroutine, but is used only when the two-particle separation, $\Delta$, is $\leq 2 l$; i.e., particle velocities are sufficiently correlated only for $\Delta \leq 2 l$. For larger $\Delta$, the velocities of the two particles are treated independently with each modeled by the existing oneparticle model, also packaged as a subroutine. In each time step, the resolved and random velocities 
are superposed and integrated over $t$ to yield the particle displacements. Currently, four basic LES data sets exist for the PBL and are available as input.

\section{WAVES AND STABILITY IN THE PLANETARY BOUNDARY LAYER}

Carmen J. Nappo

Richard M. Eckman

National Oceanic and Atmospheric Administration

\section{SL: ARL/BED}

SC: CRREL, DPG

The analyses of large pressure perturbations, i.e., mesocale gravity waves, continues. A report of this work is in preparation. A key element in this analysis is the use of the wavelet transform technique to isolate episodes of instability in the stable PBL. The wavelet transform is a recent mathematical development that can be used as an alternative to the Fourier transform. The Fourier transform decomposes a signal into a set of sinusoidal waves having different frequencies. This localization in frequency makes the Fourier transform useful for investigating phenomena that produce continuous oscillations in a signal. But the Fourier transform is not localized in time, since it cannot provide information about the locations of the oscillations in the signal. In contrast, the wavelet transform decomposes a signal into a set of wavelike oscillations that damp out after a finite number of cycles. The damping of the oscillations allows the wavelet transform to localize signals both in time and in frequency. Hence, the wavelet transform is well suited for investigating phenomena that produce intermittent bursts of activity in a signal. Work has begun on a numerical study of gravity-wave modulated PBL flow. PBL flow and stratification fields are predicted using Blackadar's one-dimensional numerical model. These fields are then used in a linear-wave theory model to evaluate the flow and temperature perturbations generated by terrain features. These perturbations are then used to modify the PBL fields, and the cycle is repeated.

\section{TURBULENCE IN THE ATMOSPHERIC SURFACE LAYER: ANALYSIS OF THE FLAT DATA SET}

Steven P. Oncley

National Center for Atmospheric Research

SL: CRREL

SC: DPG
The focus of this research has been to make a first data reduction pass through the data from the FLAT (Full Look at TKE [turbulent kinetic energy]) experiment. The results from the reduced data are being used to investigate instrument performance and to sort the two-month data set by conditions (stability, fetch). All of the first, second, and third-order moments have been calculated on the fluctuation data so that the TKE budgets can be computed directly. Research into the "ogive" technique to determine averaging times, which should yield the maximum number of statistically-independent data points, has continued. The averaging times so obtained appear to be generally smaller than those predicted by Lumley and Panofsky. Finally, software has been generated to apply most of the corrections to the data. These include: sonic anemometer leveling, Webb correction for path absorption measurements, and oxygen response correction for the P.I.'s krypton hygrometer. Other software tools such as data averaging and plotting have also been updated.

\section{REFRACTIVE INDEX STRUCTURE IN THE LOWER ATMOSPHERE}

John C. Wyngaard

The Pennsylvania State University

\section{SL: ARL/BED, CRREL}

SC: CRDEC, MICOM

Work has concentrated on developing and testing a similarity theory for the temperature structure parameter $C^{2} T$ in the stably stratified atmospheric boundary layer. This theory uses the notion of "local similarity" in stably stratified flow. Thus, if local similarity exists researchers expect the dimensionless $C^{2} T$ to be constant at a magnitude of about 12 throughout the stable boundary layer. This prediction is being tested with the results of three stable boundary layer simulations. One, due to Coleman, is a direct numerical simulation (DNS) of the stably stratified Ekman layer at relatively low Reynolds number; in geophysical terms, it corresponds to a midlatitude, nocturnal boundary layer over land with a geostrophic wind magnitude of about $0.5 \mathrm{~cm} \mathrm{~s}^{-1}$. The low Reynolds number makes the surface layer in this simulation fairly viscous, so that there is no appreciable region that satisfies $\mathrm{M}-\mathrm{O}$ similarity. For the second test, researchers used the results of the Mason and Derbyshire (1990) large-eddy simulation (LES). In this case the domain was $500 \mathrm{~m}$ long in the direction of geostrophic wind, $300 \mathrm{~m}$ wide and boundary layer was $1000 \mathrm{~m}$ deep. This domain has $\mathbf{4 0}$ 
$\times 32 \times 62$ grid points. The third test uses the results of the Moeng-Sullivan LES of a PBL made stably stratified by an overlying inversion rather than by surface cooling. Here the computational domain is $3000 \mathrm{~m}$ long, $3000 \mathrm{~m}$ wide and $1000 \mathrm{~m}$ deep with $96 \times 96 \times 48$ grid points. For analysis of these numerical data, researchers used the Cray-YMP computer facility at the National Center for Atmospheric Research (NCAR). The LES data for the inversion-capped neutral atmospheric boundary layer were available on massive memory storage at NCAR; with Dr. Coleman's cooperation his DNS data files were transferred there as well. From the DNS, velocity and temperature fields provided by Dr. Coleman, researchers computed first, second, and third moments and used them to compute the budgets of turbulent kinetic energy and temperature variance. Over most of the stably stratified layer the dimensionless $C^{2} T$ is in the range 10-20, in fairly good agreement with the Kansas values. Results from the Mason-Derbyshire LES are even better: the dimensionless $C_{T}^{2}$ is in the range 12-16 over the stable PBL. Analysis of the Moeng-Sullivan LES data is still underway.

\section{THE SMALL-SCALE STRUCTURE OF DISPERSING CLOUDS IN THE ATMOSPHERE}

R.I. Sykes

Titan Systems, Inc.

\section{SL: ARL/BED, DPG}

This research objective is to develop techniques for generating realistic representations of instantaneous clouds or plumes in the atmospheric boundary layer. Large eddy simulations of the dispersion of a tracer material have been conducted. Analysis of the mean and fluctuation components of concentration fields will be examined for structural measures of their distributions in space and time. Analyses will use structure functions, fractal representations, pdf's and other appropriate techniques. Practical methods for generating ensemble average statistics will be developed on the basis of appropriate techniques.

\section{G. Aerosol Research}

\section{COMPUTATION OF NONLINEAR OPTICAL SCATTERING BY DROPLETS}

Steven C. Hill

Peter Barber

Clarkson University

\section{SL: CRDEC}

SC: ARL/BED

To model the time-dependent nonlinear optical fields, one needs to understand the time-dependent linear internal fields, both on and near resonance. A paper describing the build-up and decay of the fields for various pulse durations and trains of pulses was prepared. In a previous report, researchers state that modes having high- $\mathrm{Q}$ MDRs can dominate the internal energy distribution in a sphere even when excited many linewidths from the resonance location. The near-resonance dominance is more pronounced when the incident beam is focused outside the droplet.

\section{LASER BEAM-AEROSOL INTERACTIONS}

Robert L. Armstrong

New Mexico State University

$$
\begin{array}{ll}
\text { SL: } & \text { ARL/BED, CRDEC } \\
\text { SC: } & \text { CRDEC }
\end{array}
$$

Stimulated Raman Scattering (SRS) from laser-irradiated microdroplets was observed from two distinct spatial regions, the droplet rim and a ringlike region encircling the laser beam axis on the droplet shadow face (the Descartes ring). With the use of two pulsed laser beams, a perturbing beam and a delayed SRS pump beam, the physical mechanism for Descartes ring scattering was investigated. Evidence was obtained for a persistent mechanism that continues even after the perturbing laser pulse is turned off. This mechanism is tentatively identified as electrostriction. At shorter times, where the perturbing laser beam and the scattered SRS light overlap, electrostriction, nonlinear refractive index changes $\left(\mathrm{N}_{2}\right.$ gradient), and plasma effects remain candidates for the existence of prompt Descartes ring scattering. Sol lasing and fluorescence was observed from microdroplets containing fluorescine 548 (F548) dye and seeded with $\mathrm{nm}$-sized fluorescent sol in ethanol. Sol lasing emission offers an example of a lasing-lasing double resonance, analogous to previously reported elastic-SRS double resonance emission. An investigation was made of the dependence of lasing from Rhodamine 6G (R6G)-doped microdroplets seeded with $\mathrm{nm}$-sized latex or sol particles. Small overlap between R6G emission and sol-dye absorption bands prevents the occurrence of a lasing-lasing double resonance, and particle seeding serves only to 
modify the cavity quality factor $\mathrm{Q}$ of the lasing MDR's. When the number density of the latex or sol particles reaches a well-defined threshold value, the R6G lasing can be quenched by the seeded latex or sol particles. Detailed measurements of the threshold number densities for different size latex or sol particles have been performed.

\section{HIGH-INTENSITY AND HIGH-ENERGY LASER INTERACTIONS}

Richard K. Chang

Yale University

$$
\begin{array}{ll}
\text { SL: } & \text { CRDEC, MICOM } \\
\text { SC: } & \text { ARL/BED, CRDEC }
\end{array}
$$

A series of five papers were being prepared, all of which are in various stages of completion. Two of the papers summarize observations of two new experiments involving multiple-short pulse irradiation of micrometer-sized droplets. Several types of droplets are chosen to emphasize particular aspects of nonlinear optical interactions within the droplet media. Some experiments related to laser-induced perturbations associated with the Descartes ring on the droplet surface are being finished. The papers in preparation are: $(a)$ The role of two-photon absorption (TPA) on stimulated Raman scattering (SRS) in microdroplets; $(b)$ Experimental results of thirdorder sum frequency generation (TSFG) in $\mathrm{D}_{2} \mathrm{O}$ and $\mathrm{CCl}_{4}$ droplets; (c) Numerical results on TSFG in microdroplets; (d) Stimulated Kerr scattering (SKS) and stimulated Raman Kerr scattering (SRKS) from droplets which are irradiated with multiple series of picosecond pulses with high intensity and small energy; (e) Phonon and photon retention affects the nonlinear scattering of droplets which are irradiated with a series of short pulses.

Reports:

1. Nonlinear Optical Processes in Droplets with Single-Mode Laser Excitation, by Richard $\mathrm{K}$. Chang and Gang Chen, SPIE Proc 1497,1(1991). AD A249 535 


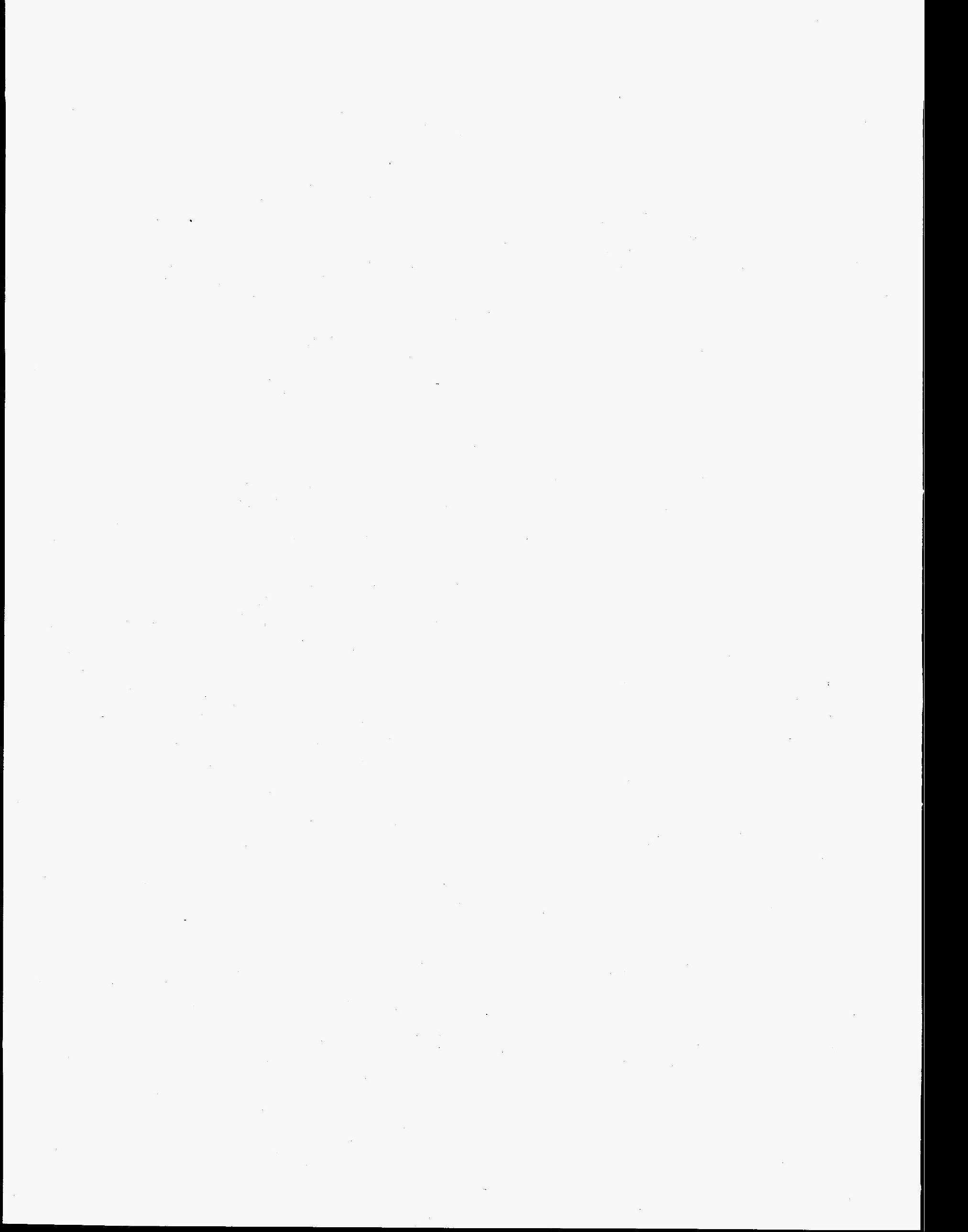




\section{ELECTRONICS}

\section{A. Physical Electronics}

\section{MEASUREMENT OF OPTICAL PROPERTIES OF INFRARED BANDGAP SUPERLATTICES}

Donald F. Nelson

Worcester Polytechnic Institute

SL: ARI

A paper has been prepared in which Raman light scattering experiments in lattice-matched InGaAsP/InP multiple quantum wells are reported. Use of laser light below the InP band gap permitted study of right-angle scattering with the scattered light emerging parallel to the layers. Scattering by the binary InP longitudinal optic (LO) and transverse optic (TO) phonons dominated the spectra. The polarization selection rules for the TO and LO scattering were found to differ strongly compared to those operative in bulk InP. This is interpreted as arising from the interchange of the LO and TO phonon frequencies as expected in a dielectric continuum slab whose thickness is small compared to the wavelength of the incident radiation. In addition, weak Raman scattering by a mixture of InGaAs and InP vibrations in the quaternary well material is reported. In another paper, researchers develop a multiband $k$ $\mathbf{P}$ transfer-matrix algorithm by defining a "diagonal representation" that provides a unified way of calculating energy levels and wave functions for superlattices as well as for quantum wells and arbitrarily shaped band-edge profiles. Numerical results of transfer-matrix calculations are presented for specific heterostructures. Contrary to expectations, the various versions of the boundary conditions used in the literature lead to results for the subband energies that agree with one another reasonably well for the wide-band-gap materials.

\section{SHORT-PERIOD SUPERLATTICE MATERIALS FOR HETEROJUNCTION BIPOLAR TRANSISTORS}

David L. Miller

The Pennsylvania State University

\section{SC: ARL/EPSD}

Work has continued to concentrate on the investigation of the migration-enhanced epitaxy technique on the electrical properties of material grown at low temperatures and the development of processing for heterojunction bipolar transistors (HBTs). Low temperature Be-doped material has been incorporated in a second set of HBT devices, with conventional devices (base grown at conventional temperatures by MBE techniques) grown at the same time for comparison. Recently processed HBTs have resulted in dc gains of 13 for low temperature base GaAs material doped to $2-3 \times 10^{19} / \mathrm{cm}^{3}$ in $8 \mu \mathrm{m} \times 8 \mu \mathrm{m}$ emitter HBTs. This is much higher than the gain achieved for devices in which the base material was grown at 550 ${ }^{\circ} \mathrm{C}$ substrate temperatures at the same doping density. SIMS showed that the conventional MBE-grown devices exhibited diffusion of $\mathrm{Be}$ about $400 \AA$ into the AlGaAs emitter material. This resulted in a dc current gain much below one. In contrast, the HBTs grown with MEE low temperature base material at the same doping density had dc gains of 13 . This is somewhat lower than expected for the base thickness and doping density used. Photoreflectance has continued to be a useful tool in characterizing low temperature material and HBTs. The large degree of diffusion in the conventional MBE-grown devices described above had clearly different spectra than the low temperature devices. This is primarily because of $\mathrm{Be}$ diffusion in the conventional devices, which shows up in the Franz-Keldysh oscillations from the AlGaAs emitters.

\section{Reports:}

1. Be-Doped GaAs Grown by Migration Enhanced Epitaxy at Low Substrate Temperature, by $\mathbf{K}$. Zhang et al., J Electron Mater 21,187(1992). AD A249075 
2. Migration-Enhanced Epitaxy of Doped GaAs on (111)B and (100)GaAs Substrates, by Jianming Fu et al., J Vac Sci Tech B10,779(1992).

3. Study of (InAs) m(GaAs) $\mathbf{n}$ Short-Period Superlattice Layers Grown on GaAs Substrates by Molecular-Beam Epitaxy, by Jung-Geau Jang et al., J Vac Sci Tech B10,772(1992).

\section{MODIFICATION AND CHARACTERIZATION OF II-VI, III-V AND $T_{c}$ USING ION BEAMS}

Robert G. Wilson

Hughes Research Laboratories

\section{SL: NVESD}

Work has been concerned with measuring and plotting the dependence of secondary-ion mass spectrometry (SIMS) relative sensitivity factor (RSF) on electron affinity and ionization potential of up to 74 elements implanted into 23 materials, measured using oxygen-ion bombardment and positive SIMS, and cesium-ion bombardment and negative SIMS. Reproducibility of \pm 60 percent in RSF has been demonstrated for many of these implanted standards over a period of 8 years in several Cameca magnetic sector instruments, especially in $\mathrm{Si}, \mathrm{GaAs}$, and $\mathrm{HgCdTe}$ matrices. More recently, reproducibilities of \pm 20 percent -30 percent have been achieved using more controlled experimental techniques. Similarities among plots of $\log$ RSF versus ionization potential or electron affinity have been found for a variety of semiconductor, metal, and insulator matrices. Universal patterns and trends are observed for oxygen bombardment and positive SIMS, and for cesium bombardment and negative SIMS. Four major regions are seen in the dependence of log RSF on ionization potential, one with possibly three branches with slightly differing slopes, that are characterized by elements from various groups of the periodic table. There are two major regions in the plots of RSF versus electron affinity, one of which is a region of constant RSF versus electron affinity, which includes the more electronegative elements, and meaning that the RSF is a constant for most of the elements commonly analyzed using negative (cesium) SIMS. This constant RSF has been measured for 20 matrices.

\section{Reports:}

1. High Resistivity GaSb Grown by Molecular Beam Epitaxy, by A.Y. Polyakov et al., MS, J Appl Phys.

2. SIMS Depth Profile Measurements of $\mathrm{H}, \mathrm{Li}, \mathrm{Nd}$, and $\mathrm{Er}$ in $\mathrm{LiNbO}_{3}$ and $\mathrm{LiTaO}_{3}$, by Steven W. Novak, MS.

3. Effects of Hydrogen on the Photoluminescence of SiDoped GaAs/AlGaAs Multiple Quantum Welis, by F. Voillot et al., MS.
4. Hydrogen Stability and Passivation of Shallow and Deep Centers in GaSb and InSb, by A.Y. Polyakov et al., MS.

5. High Resistivity GaAs Grown by High Temperature Molecular Beam Epitaxy, by A.Y. Polyakov et al., MS, J Appl Phys.

6. Implantation Range Statistics in III-V Materials, by R.G. Wilson, JElectrochem Soc 138,718(1991). AD A249 086

7. Systematics of Secondary-Ion-Mass Spectrometry Relative Sensitivity Factors Versus Electron Affinity and Ionization Potential for a Variety of Matrices Determined From Implanted Standards of More Than 70 Elements, by R.G. Wilson and S.W. Novak, J Appl Phys 69,466(1991). AD A249 085

8. Direct Comparison of Hydrogen Depth Distributions and Refractive Index Profiles in Annealed Proton-Exchanged Z-Cut $\mathrm{LiNbO}_{3}$ Waveguides, by A. Loni et al., Electron Let 27,1245(1991).

\section{SEMICONDUCTOR HETEROJUNCTION ENGINEERING}

\section{A. Franciosi}

University of Minnesota, Minneapolis

\section{SL: MICOM}

AlAs-Si-GaAs(001) and GaAs-Si-AlAs(001) heterostructures were synthesized by molecularbeam epitaxy. Transmission electron microscopy and in situ $x$-ray photoemission spectroscopy, together with $x$-ray interference measurements of model GaAs-Si-GaAs(001) structures and SiGaAs(001) superlattices indicate that pseudomorphic $\mathrm{Si}$ layers can be grown at the interface for layer thickness $\leq 4-8$ monolayers with no detectable dislocation or twin formation. While the band offsets for isovalent AlAs$\mathrm{GaAs}$ heterostructures follow the commutativity rule, the presence of $\mathrm{Si}$ at the interface is found to give rise to deviations from the rule as large as \pm 0.4 $\mathrm{eV}$. Such deviations are associated with a Si-induced local dipole that can be established with high reproducibility within the interface region. ZnSe-GaAs heterostructures were synthesized by molecular beam epitaxy at room temperature on $\mathrm{GaAs}(110)$ substrates cleaved in situ, and at $290-320^{\circ} \mathrm{C}(\mathrm{Zn} / \mathrm{Se}$ beam pressure ratio $=1$ ) on epitaxial $\operatorname{GaAs}(100)$ layers grown on $\mathrm{GaAs}(100)$ wafers. The epitaxial structures were characterized in situ by photoemission spectroscopy and reflection high energy electron diffraction. Phonon dispersion in strained heterovalent $(\mathrm{Si})_{n}(\mathrm{GaAs})_{m}$ (001) superlattices has been calculated using first principles force constants. Raman scattering experiments have been conducted on fifteen period ( $\mathrm{Si})_{2}(\mathrm{GaAs})_{28}$ and $(\mathrm{Si})_{3}(\mathrm{GaAs})_{50}$ superlattices synthesized by molecular beam epitaxy. 
Reports:

No. 1-8 in previous editions.

9. A Novel Facility for MBE Growth and In-Situ High Resolution XPS Characterization of III-V and II-VI Semiconductor Heterostructures, by G. Bratina et al., Vuoto 20,565(1990). AD A239 763

10. Engineering ZnSe-GaAs Band Offsets, by L. Vanzetti et al., MS, J Cyst Growth.

11. AlAs-GaAs Heterojunction Engineering by Means of Group IV Elemental Interface Layers, by G. Bratina et al., MS, Phys Rev.

\section{QUANTUM DEVICES USING Si-BASED SUPERLATTICES AND SUPERSTRUCTURES}

KL. Wang

University of California, Los Angeles

SL: ARL

Normal incident infrared detector was demonstrated using Si ${ }_{1-x} \mathrm{Ge}_{x} / \mathrm{Si}$ multiple quantum wells for the first time. A broad peak in the photoresponse is observed near $7.2 \mu \mathrm{m}$ with a full width at half maximum of about $80 \mathrm{meV}$. The mechanism of infrared absorption at normal incidence is found to be due to internal photoemission from the 2-dimensional hole gas in the quantum well. The infrared absorption due to 2-D free carriers is found to be strong in the case of photon electric field parallel to the layers (i.e., normal incidence). The responsivity of the detector is determined to be comparable with that typically observed in quantum well detectors based on intersubband transition. This suggests the application $\mathrm{Si} 1-x \mathrm{Ge} / \mathrm{Si}$ multiple quantum well detectors in focal plane arrays without grating couplers that are required in the case of intersubband detectors.

Reports:

No. $1-4$ in previous editions.

5. Effect of Hydrogenation on Hole Intersubband Absorption in D-Doped Si Layers, by V. Arbet-Engels et al., MS.

6. Effect of Hydrogenation on Intersubband Absorption in D-Doped SiGe Layers, by V. Arbet-Engels et al., MS.

7. Long Wavelength $(10 \mathrm{~mm})$ Infrared Detector Using $\mathrm{Si}_{1-\mathrm{x}} \mathrm{Ge} \times \mathrm{Si}$ Multiple Quantum Wells, by R.P.G. Karunasiri et al., MS.

8. Photoluminescence of Hydrogenated SimGen Superlattices, by V. Arbet-Engels et al., MS.

27298 OPTICAL STUDIES OF LATERALLY CONFINED QUANTUM WELL STRUCTURES

GROWN ON EX-SITU AND

IN-SITU PATTERNED SUBSTRATES
Anupam Madhukar

University of Southern California

\section{SL: ARL/EPSD, NVESD}

The research objective is to advance the understanding of compound-semiconductor structures suited for ultrafast electronics and optoelectronics. Realtime diagnostics will be performed on electronic and optoelectronic materials as they are grown by molecular beam epitaxy (MBE); to characterize these MBE-grown materials both electrically and optically.

\section{DOUBLE SUPERLATTICE GaAs IR TRANSISTORS}

D.C. Tsui

Princeton University

\section{SL: ARL/EPSD}

A superlattice structure can be incorporated into a quantum-well infrared detector as an electron bandpass filter to block electrons with energies higher or lower than the miniband energy. Electrons with energies inside the miniband can relax their energies to the lower edge of the miniband as they pass through the superlattice. Therefore, the superlattice not only filters the electron energy but also keeps the filtered electrons to a minimum energy. A double superlattice IR transistor has been designed, built and tested to realize these expected advantages.

\section{MONTE CARLO SIMULATION OF MERCURY CADMIUM TELLURIDE}

Michael Shur

University of Virginia

$$
\begin{array}{ll}
\text { SL: NVESD } \\
\text { SC: SDC }
\end{array}
$$

Expressions have been derived for scattering rates for electrons in MCT which account for correct wave functions for narrow band gap materials. These scattering rates differ slightly from the rates obtained from standard expressions for wide band gap materials. The difference is related to spin-flip processes and has a relatively small effect on the transport properties. However, it is very important for spin-orientation phenomena. Monte Carlo simulations have been performed in order to investigate the mobility and steady state velocity-field characteristics of electron transport in MCT with $x=0.205$ at a lattice temperature of $77 \mathrm{~K}$. The simulations include scattering on polar optical phonons, ionized impurities, 
and alloy scattering. The Pauli exclusion principle as well as the dependence of the screening length on the distribution function have been accounted for. The distribution function, the screening length, and the scattering rate table were updated with a period of $140 \mathrm{fs}$ through the simulations. The simulations show that the screening length increases with increasing electric field with this dependence being the strongest for low carrier concentrations. For $n=5.4 \times 10^{15}$ $\mathrm{cm}^{-3}$, the inclusion of the Pauli exclusion principle and varying screening length reduces the electron velocity by 5-10 percent depending on the electric field. Velocity-field curves are in good agreement with experimental data up to $160 \mathrm{~V} / \mathrm{cm}$. The dependence of low-field mobility on the electron concentration was also investigated.

\section{MATERIALS AND DEVICE RESEARCH FOR HIGH-SPEED INTEGRATED OPTOELECTRONIC TRANSMITTERS USING VERTICAL-CAVITY SURFACE EMITTING LASERS}

Russell D. Dupuis

University of Texas at Austin

\section{SC: ARL/EPSD}

SC: NVESD

The device structures being studied are grown by low-pressure metalorganic chemical vapor deposition (LP-MOCVD). The materials growth work was devoted to the accomplishment of two goals: (a) the variation of materials growth parameters and the characterization of epitaxial films to establish the conditions required for the growth of latticematched epitaxial layers; and (b) the growth of heterostructure devices using the results of these studies. The materials studied were InP, InGaAs, and InGaAsP. The growth conditions for the optimized growth of undoped and doped lattice-matched epitaxial films were established. The doping characteristics for both $p$-type ( $\mathrm{Zn}$-doped) and $n$-type (Sn-doped) epitaxial layers of these materials were studied. After these initial experiments, the growth of "test" structures for HBTs and laser heterostructures was performed. The growth experiments were then devoted to the growth of complete HBT and injection laser structures. InGaAs-InP heterojunction bipolar transistor structures were grown and working devices were successfully fabricated from these wafers. Other efforts have been to develop a baseline InGaAs/InP HBT process which gives reproducible and consistent results. Several sets of MOCVD InGaAs/InP HBT structures have been processed. The films have demonstrated solid HBT characteristics that are consistent with calculated estimates of device performance and indicated that both the crystal growth and the device processing are at a level of development which can now support efforts in device design and refinement. Double-heterojunction InGaAs-InP injection lasers are also currently under study. At this time, edgeemitting broad-area lasers of dimension $\sim 60 \mu \mathrm{m} x$ $500 \mu \mathrm{m}$ have been fabricated. These devices will initially be tested under pulsed currents at room temperature.

\section{TIME RESOLVED STUDIES OF IN-WELL AND VERTICAL CARRIER TRANSPORT IN MOW SEMICONDUCTOR STRUCTURES}

Alan Miller

University of Central Florida

\section{SL: ARL/EPSD}

Picosecond time resolution, laser pump-probe measurements of cross-well transport in GaAs/AlGaAs MQW SEED-type optical modulator structure were carried out at higher optical excitation levels than previously studied. The effect of significant space charge build-up and cancellation of the applied field was identified via the longer response time of the device at higher photo-induced carrier densities. These results are important for the design of high power, high frequency optical modulators currently being developed which employ the quantum confined Stark effect and fast optical switching SEED optical logic devices. Researchers have carried out extensive theoretical modeling of the optically injected carrier dynamics in MOW SEED structures. The model both spatially and temporally resolves the carriers as they are swept from the quantum wells by an applied electric field. The effect of resonant tunneling on the carrier dynamics and consequent distribution is significant. The microscopic carrier motion within the $n$ - and $p$-doped electrode regions is found to effectively cancel the field perturbation at longer timescales while significant numbers of carriers are still within the wells but the optical response has recovered. Initial time resolved measurements of carrier sweep-out in a single quantum well waveguide structure show a faster recovery, $\sim 110$ psec of the nonlinear optical response than in electrically biased multiple well structures. 
Reports:

1. Modeling of Cross-Well Carrier Transport in a Multiple Quantum Well Modulator, by D.C. Hutchings et al., Appl Phys Let 59,3009(1991). AD A249 191

2. Time Resolved Measurements of Cross-Well Transport in a Multiple Quantum Well p-i-n Modulator at High Photogenerated Carrier Densities, by A. Miller et al.,Appl Phys Let 60,97(1992). AD A249615

3. Uttrafast Charge Transfer and Optical Switching in MQWs, by A. Miller et al., MS.

4. Optical Switching and Transient Carrier Transport Phenomena in MQW Structures, by A. Miller et al., MS.

\section{SCATTERING AND GUIDING OF WAVES BY METAL STRIP GRATINGS}

Song-Tsuen Peng

New York Institute of Technology

\section{SL: ARDEC, CECOM}

A new approach has been developed that provides two distinct sets of air and metal modes; thus, it is necessary to determine the number of modes to be chosen for each set, so that the results will converge to a correct value. This is what has been known in the literature as the problem of the relative convergence. Researchers have systematically investigated the proper ratio of the numbers of air and metal modes from two different viewpoints. One is the conservation of power in the scattering process, and the other is the continuity of the tangential components of the electric and magnetic fields. It is found that the ratio of the numbers of air and metal modes should be roughly equal to the ratio of the widths of the air and metal regions, as is generally true in the case of bifurcated waveguides. With the proper ratio of the numbers of air and metal modes determined, researchers then have to determine the total number of modes to be included in an analysis. To do this, it is necessary to have reliable data to be used as a reference. In the special case of an isotropic metal grating in a uniform medium, numerical results were obtained that agree exceedingly well with published data that were obtained by the exact Wiener-Hopf technique. The results check fairly well with those obtained from the method of point matching, except in the vicinity of the cutoff of a mode involved. Based on the results of the Wiener-Hopf technique, researchers are convinced that results are more accurate than the method of point matching.

\section{Al-BASED NATIVE OXIDES ON III-V SEMICONDUCTOR QUANTUM WELL LASERS AND OPTOELECTRONIC DEVICES}

N. Holonyak

University of Illinois

\section{SL: ARL/EPSD, NVESD \\ SC: ARL}

The objective of the research is to investigate the properties of Al-based native oxides on III-V semiconductors and to fabricate novel optoelectronic structures using this technique. This research involves growth of Al-based native oxides on III-V semiconductors and the fabrication of optoelectronic devices incorporating such oxides. In particular, the research will study oxides on quantum well heterostructures, field effect transistors, lasers defined by native oxides, and individually addressable laser arrays. The research will also be directed towards the integration of lasers and transistors into optoelectronic circuits.

\section{OPTOELECTRONIC TECHNOLOGY ROADMAP}

Ron Nelson

Optoelectronics Industry Development Association

\section{SL: DARPA, ARL/EPSD}

The research objective is to conduct a series of market reviews and technology assessments in order that a critical path for the development of optoelectronic technologies in the US can be established. A series of working groups and technology workshops will be held to develop a strategy for US optoelectronics in the next two decades. The program consists of: seven (7) market sector working groups, two (2) review forums, six (6) technology workshops, two (2) foreign study programs, and a critical assessment. In particular, draft "strawman" technology roadmaps will be prepared to identify the critical technology vehicles for each relevant sector. A final report will be prepared which summarizes the conclusions and recommendations from the program.

\section{B. Electron Devices}

\section{ISOLATION MECHANISMS IN III-V DEVICES}


George N. Maracas

Arizona State University

SL: ARI

SC: ARL/EPSD

Work proceeded on understanding the electrical and optical properties of InP grown at low temperatures by GSMBE. The chemical composition of the observed precipitates needs to be understood in order to model the conduction in the layers. In attempts to understand the role of precipitates on the conductivity of materials grown at low temperatures (LT) by MBE, the growth of InP by GSMBE was undertaken to compare with LT GaAs. LT GaAs has distinct As precipitates having different crystal phases which, in one theory, act as imbedded Schottky barriers. These barriers deplete the surrounding material rendering it highly resistive. In another theory, the high resistivity is attributed to a high concentration of As antisite defects (EL2) induced by the 1 percent excess As in the lattice. The motivation for examining the LT InP system was to see if high resistivity could be obtained in material containing precipitates. Since the concentration of antisite defects in InP is much less than in GaAs, highly resistive precipitate filled LT InP would give credence to the precipitate model. A series of LT InP samples were grown at different temperatures and $\mathrm{P} / \mathrm{In}$ ratios to explore growth parameter space. Conductivity, photoluminescence (PL) and high resolution transmission electron microscopy (TEM) were performed on all samples. The resistivity of the material was found to be higher than the equivalent LT GaAs most likely because indium has a tendency to enter substitutionally into $P$ sites rather than the reverse. The photoluminescence intensity of the LT InP layers was considerably higher than that in LT GaAs and had much narrower linewidths. This is in part because of the higher (by a factor of $10^{7}$ ) than the LT GaAs. Precipitates of dimensions $100 \AA$ to $500 \AA$ were observed for the first time in the LT InP. These are larger than those found in LT GaAs.

Reports:

No. 1-6 in previous editions.

7. Optical Properties of Quantum Wells Grown Upon Gas Source Molecular Beam Epitaxy Low Temperature Buffers, by K.T. Shiralagi et al., J Appl Phys 69,7942(1991). AD A249 008

8. Optical Properties of Strained Asymmetric Triangular InGaAs/GaAs Multiple Quantum Wells, by R. Droopad et al., MS, Appl Phys Let.

9. Narrow Photoluminescence Linewidth of Quantum Wells Grown by Gas Source Molecular Beam Epitaxy, by K.T. Shiralagi et al., MS, J Cryst Growth.

10. Transition from Longitudinal-Optical Phonon Scattering to Surface-Optical Phonon Scattering in Polar Semiconduc- tor Superlattices, by Michael A. Stroscio et al., MS, Appl Phys Let.

11. Growth and Characterization of Low Temperature InP by Gas Source MBE, by G.N. Maracas et al., MS.

12. Low Temperature GaAs Grown by Gas Source Molecular Beam Epitaxy, by R. Droopad et al., J Cyst Growth 120,200(1992).

\section{SIMULATION OF ELECTRONIC TRANSPORT IN SEMICONDUCTOR HETEROLAYER DEVICES}

K. Hess

University of Illinois

\section{SL: ARO, ARL/EPSD}

The numerical code which was developed over the past three years has been applied to InP, GaAs and InAs and work is in progress on ternary III-V compound materials. For the first time, a Monte Carlo approach including a precise theory of the ionization events is in hand. The ionization probability has previously only been treated by an approximate formula derived by Keldysh. This formula is incorrect by orders of magnitude and it has been replaced by an approach pioneered by Kane. The puzzle was also solved that two totally different results for the ionization rate can lead to agreement of the theory with experiments for the ionization coefficient $\alpha$. This coefficient is the convoluted product of the energy distribution and the ionization rate. In case of very large ionization rates (Keldysh theory), the tail of the distribution is depleted because of the intense scattering of electrons to small energies and $\alpha$ is therefore small. In case of small ionization rate (Kane like theories) the high energy tail of the distribution contains a significant number of electrons and is much larger. However the convolution with the now small ionization rate give again a small value of $\alpha$. The new theory of impact ionization and the coefficient $\alpha$ should be particularly important for situations when the electric field varies rapidly (in space or time) since in such situations one cannot accidentally find the same solution for $\alpha$ for both weak and strong ionization rate and the true form of the ionization rate, which in this researchers opinion is the rate obtained in the Kane-like formalism, must then be used. The goal of the two-electron simulation work is the modeling of the essential physics of two-electron systems in prototypical structures. Currently capabilities are in hand for calculating transient wave functions, propagating eigenstates and bound eigenstates for two-electron systems. All of the numerical algo- 
rithms are based on a tight-binding formalism, and while the number $n$ of tight-binding lattice sites considered is restricted because the memory and cpu time requirements vary as $n^{2}$ (currently $n-100$ ), the coupling among these sites as well as the site potentials can now be defined arbitrarily allowing great flexibility in defining the simulation geometries. Prototypical structures varying in three spatial dimensions can be defined.) Transient wave functions and propagating eigenstates are calculated by solving the time-dependent Schrödinger equation for the twoelectron system, with open boundary conditions. Two-electron bound eigenstates are calculated again with the time-dependent Schrödinger equation, but this time in imaginary-time, combined with the Schmidt orthogonalization method to sequentially isolate eigenstates of increasing energy.

Reports:

No. $1-10$ in previous editions.

11. Impact Ionization: Beyond the Golden Rule, by J. Bude et al., Semicond Sci Technol 7,B506(1992).

12. Impact Ionization in Semiconductors: Beyond the Golden Rule, by J. Bude et al., MS, Phys Rev.

13. Monte Carlo Simulation of Hot Electron Transport in Si Using a Unified Pseudopotential Description of the Crystal, by P.D. Yoder et al., MS.

14. Monte Carlo Simulations of Electronic Transport in Semiconductors at High Energies, by P.D. Yoder et al., MS.

15. A Two-Particle Model of Electron-Electron Interactions in Quantum Structures, by Leonard F. Register and Karl Hess, MS.

16. Dissipative Scattering and Transport in Mesoscopic Systems, by Fernando Sola, MS.

17. Scattering Mechanisms for Semiconductor Transport Calculations, by J. Bude, Monte Carlo Device Simulation: Full Band and Beyond, p27. AD A249 260

\section{SUPPRESSED ELECTRON-PHONON SCATTERING IN SUPERLATTICES}

Lester F. Eastman

Cornell University

\section{SL: ARO, ARI/EPSD}

A continuum theory of optical vibrations in quantum wells was developed based on the idea originating in the consultancy at Cornell last winter, some of which has been published (report 1, below). Basically, this consists of a scheme of triple hybridization of LO, TO and IP (interface polariton) modes, chosen to satisfy both elastic and electromagnetic boundary conditions. It has been shown that this theory produces the same results as microscopic theory in the case of the AlAs/GaAs system, and a calculation of the electron"hybridon" interaction in the GaAs quantum well agrees with the Huang-Zhu result. There is thus some optimism that a consistent continuum theory is now available, and the question of suppression of phononscattering can be tackled with confidence. Time was spent in repeating the theory for the case of a superlattice. Although no new point of principle was involved, the task involved a lot of laborious algebra. Except for a couple of simple cases the full disperion has not been explored - this will be left for a later period. However, it is shown that for propagation directions perpendicular or parallel to the superlattice axis the mode patterns are identical with those for a single quantum well, and hence the single-well results for the electron-hybridon interaction can be used. This work will be suitable for publication as soon as the rather complicated dispersion has been worked out and illustrated.

Reports:

1. Free Optical Vibrations of an Infinite Plate of Homogeneous Isotropic Elastic Matter, by B.K. Ridley, Phys Rev B44,9002(1991).

\section{THEORETICAL AND EXPERIMENTAL STUDIES OF QUANTUM WELLS}

\section{Raphael Tsu}

University of North Carolina at Charlotte

\section{SL: ARL/EPSD}

The major research findings involve the observations of quantum confinement in nanocrystalline silicon of the size about $100 \AA$ embedded in an amorphous silicon dioxide matrix. This medium is sandwiched between an aluminum contact and silicon wafer for transport measurements. Distinct peaks reflecting the energy density of states of quantum confinement affected by the presence of electrons were observed in resonant tunneling experiments. These results prompted an intensive theoretical calculation of quantum levels taking into account of electron-electron Coulomb term. Two ends have been calculated: small enough so that the usual approximation for the calculations of a helium atom may be valid; large enough where classical electrostatic approximation is valid. Researchers are extending the present theories to cases where effective mass is no longer valid and are designing a means to control the substrate temperature of the silicon deposition from $77 \mathrm{~K}$ to $700 \mathrm{~K}$. 
27578 DEVELOPMENT OF NANOSTRUCTURE FABRICATION TECHNOLOGY AND NEW ELECTRON-QUANTUM WAVE DEVICES

Stephen Y. Chou

University of Minnesota, Minneapolis

$$
\text { SC: ARL/EPSD, ARL, SDC }
$$

Using high resolution electron beam lithography and RIE with $\mathrm{Cl}_{2}$ and $\mathrm{SiCl}_{4}$ gases, researchers have etched sub-50 $\mathrm{nm}$ Si pillars, trenches, and ridges with aspect ratios greater than 10 . These are among the smallest features ever fabricated using these techniques. They believe that the size of these Si features etched with the recipe described here is limited by the size of the etching mask, rather than by the etching process itself. Furthermore, they found that the RIE etching is crystallographically dependent and that the nanoscale structures can be further reduced by wet HF etching. No photoluminescence has been detected from arrays of Si pillars with feature sizes of the order of $10 \mathrm{~nm}$ and passivated using HF acid. An extensive investigation has been made of the new single electron transistors. Researchers have found that the periodic oscillation in drain current is much stronger when the device is measured using a HP4145 parameter analyzer than when using a lockin amplifier. The reason is that the source-drain bias researchers used in all measurements is much greater than the ambient temperature and therefore heated up the sample. The heating effect is much more severe in lockin amplifier measurement than with HP-4145 because its measuring time is much longer. The investigation also shows that the smaller the gate gap, the larger the oscillation period, indicating that higher operation temperature can be achieved in smaller devices.

\section{AlAs/InAs/InGaAs QUANTUM WELL BASE N-N-N TRANSISTORS}

Clifton G. Fonstad

Massachusetts Institute of Technology

$$
\text { SL: ARL/EPSD }
$$$$
\text { SC: ARL }
$$

In earlier work in this effort, several organic acids were identified that enable the etching of indium compounds while maintaining selectivity with respect to AlAs, and these acids have been used to develop InGaAlAs etching solutions that allow the selective etching of InP lattice-matched InGaAlAs heterostructures using pseudomorphic AlAs etchstop layers. A broad array of related organic acids were examined to insure that the optimum choices had been identified, and of the organic acids tested, the non-aromatic, polycarboxylic acids were found to be the most effective. The best results continue to be obtained for etchants consisting of succinic acid, ammonia, and hydrogen peroxide. It was found that the etch rate of In $\mathrm{GaAs}$ with this solution can be as much as 1000 times the etch rate of AlAs, while the etch rate of InAlAs can be 500 times as large. Work continued on improving growth techniques as applied to the quantum-well, tunnel-barrier structures of interest in this work. While most of the improvements seen are incremental and produce subtle changes, their cumulative effect can at times result in spectacular advances in the state-of-the-art. The intersubband relaxation rate due to electron-electron interaction has been calculated for the first time and compared to the same rates for polar optical phonon emission, for photon emission, and for impurity scattering. It is found that this process can be comparable to phonon emission and can involve subpicosecond relaxation times. Taken together, these intersubband relaxation processes have a major negative impact on the efficiency of electronic and optoelectronic devices relying on a long upper level lifetime. The solution to this problem appears to lie in new device concepts.

\section{MODELING FAILURE AND RELIABILITY IN NEW-GENERATION DEVICES}

Jeffrey Frey

University of Maryland

SL: ARL

SC: ARL/EPSD, SDC

Researchers have moved toward an understanding of why, and by how much, silicon MOSFETS can be expected to become relatively more reliable as their gate lengths are shrunk below about .15 $\mu$. The average electron energy at any point in a device is a measure of how many electrons are hot enough to cause such reliability problems as gate and substrate currents in MOSFETs, or real-space transfer in HEMTs. By calculating this quantity for sample MOSFETs designed to have equal peak electric fields but with channel lengths from $.05 \mu$ to $.20 \mu$, the peak value of energy decreased as gate lengths de-

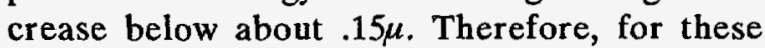
MOSFET channel lengths, fewer troublesome hot 
electrons exist in the channel, as channel length decreases. This phenomenon is explained by a reduction in the number of thermalizing collisions that any electron can undergo, as the channel length is shortened. Studies of the reliability of compound semiconductor MESFET and HEMT devices continued, and additional progress was made on developing models for the simulation of quantum effects in the potential well created at the heterostructure, and the interaction of these effects with the distribution of electrons in both momentum and real spaces. An effort to produce color plots of the two-dimensional distributions of such quantities as particle densities, energies, and current densities, has begun.

\section{HIGH PERFORMANCE GaAs MICROCOMPUTER SYSTEM}

W.P. Birmingham

R.B. Brown

University of Michigan

\section{SL: ARO, ARL/EPSD, MICOM, NVESD, SDC \\ SC: ARO, SDC}

The major accomplishments have been: design of a GaAs DCFL microprocessor with 60,500 transistors; set up of HP82000 tester and verification of correct operation of GaAs microprocessor at over $100 \mathrm{MHz}$; development of a GaAs circuit compiler, including generation of layout and simulation models; an interface to allow input to the compiler from Verilog and output (for simulation) in Verilog format; development of a timing model for GaAs gates and on-chip interconnect; instrumentation of DEC 3100 for operating system studies; and design of an MCM test structure.

\section{Reports:}

1. The Design of a Micro-Supercomputer, by T.N. Mudge et al., Computer p57, Jan(1991). AD A239 166

2. The Design of a GaAs Micro-Supercomputer, by T.N. Mudge et al., Systems Sciences, 1991, p421. AD A239 904

3. Multilevel Optimization in the Design of a High-Performance GaAs Microcomputer, by O.A. Olukotun et al., IEEE J Sol St Circuits 26,763(1991). AD A239 903

5. Impact of MCM's on System Performance, by Ayman I. Kayssi et al., MS.

6. The Silicon Compiler as a Tool for Teaching Microelectronic System Design, by Richard B. Brown, MS.

7. Module Generation for a GaAs Microprocessor, by Michael Upton et al., MS.

8. Implementing a Cache for a High-Performance GaAs Microprocessor, by O.A. Olukotun et al.,ACM 0-89791-394-9, 1991, p138.

9. Delay Macromodels for the Timing Analysis of GaAs DCFL, by Ayman I. Kayssi and Karem A. Sakallah, MS.
10. A Fine Grained Analysis of Monolithic and Micro-Kernel Contex Switch Performance (Extended Abstract), by David Nagle et al., MS.

11. Impact of MCMs on System Pewrformance Optimization, by A.I. Kayssi et al., MS.

12. Performance Optimization of Pipelined Primary Caches, by Kunle Olukotun et al, MS.

13. A High-Performance GaAs Microprocessor, by R.B. Brown et al, MS.

14. Delay Macromodels for Point-to-Point MCM Interconnections, by Ayman I. Kayssi and Karem A. Sakallah, MS.

15. A Fine Grained Analysis of Monolithic and Micro-Kernel Context Switch Performance (Outline), by David Nagle et al, $M S$.

16. Synthesis and Verification of a GaAs Microprocessor From a Verilog Hardware Description, by R. Brown et al., Proc of the Open Verilog Int'l User Group Meeting, 1992, p85. AD A249 162

17. Monster: A Tool for Analyzing the Interaction Between Operating Systems and Computer Architectures, by Richard Uhlig et al., MS.

18. Compound Semiconductor Device Requirements for VLSI, by R.B. Brown et al., MS

19. GaAs RISC Processors, by R.B. Brown et al., MS.

20. The Impact of Signal Transition Time on Path Delay Computation, by Ayman I. Kayssi et al., MS, Trans Circuits and Systs.

\section{RISC PROCESSOR FOR SUPERWORKSTATION AND TERAOPS PARALLEL PROCESSING APPLICATIONS}

John F. McDonald

Rensselaer Polytechnic Institute

\section{SL: ARO, ARL/EPSD, MICOM, NVESD, SDC \\ SC: ARO, SDC}

The goal of the F-RISC/G (Fast Reduced Instruction Set Computer-version G) project is to develop a 32-bit GaAs/AlGaAs processor with a cycle time of 1.0ns. The processor will be fabricated with Rockwell's Heterojunction Bipolar Transistor (HBT) process. Because of the high power dissipation and low device count available with this technology, F-RISC/G must be partitioned into several chips. The system consists of an Instruction Decoder, a Clock Distribution chip, eleven primary cache chips in various configurations, and four copies of the Datapath chip. These are to be implemented with four distinct, reconfigurable chip types. The standard cell library has been completed. Artwork for the Datapath chip has been generated. A test chip has recently been designed and fabricated by Rockwell, in order to verify the proper performance of the register file and ALU carry chain that will be used in the Datapath chip. It will also give critical informa- 
tion about the delays of the pad drivers and receivers which thus far have been available primarily only from SPICE simulation. In the course of producing the artwork for the test chip the CAD tools under development for performing differential wiring were completed and given a full exercise under typical situations likely to arise with the other chips. An additional CAD tool suite was developed for Power analysis, including power supply distribution and power dissipation.

\section{FOCUSED ION BEAM FABRICATION OF MICROELECTRONIC STRUCTURES}

John Melngailis

Massachusetts Institute of Technology

$$
\begin{aligned}
& \text { SL: ARI/EPSD } \\
& \text { SC: SDC }
\end{aligned}
$$

The microstructure of the gold films is linked to their purity and to the growth conditions. Using transmission electron microscopy researchers have examined many gold films grown by ion induced deposition using both broad beams of $\mathrm{Ar}^{+}$ions and scanned beams of $\mathrm{Ga}^{+}$ions from an FIB system. The ion species and the scanned vs. continuous beam did not appear to significantly affect the microstructure. The conditions that affect the microstructure are average current density substrate temperature during deposition, and ion energy. Three types of film growth can be identified: granular growth which correlates with higher current density and with high carbon content (more then 50 percent) in the film; columnar growth with 30-50 percent carbon, observed at low-current room-temperature growth or at high current growth at $100^{\circ} \mathrm{C}$, and polycrystalline growth, with less than 30 percent carbon observed either for high-temperature, low-current conditions or for low voltage (2-10 $\mathrm{kV}$ ) ion induced deposition. Clearly for most applications the low-carbon-content, denser films are desirable. The rate of deposition of gold was measured from a precursor gas of dimethylgold hexafluoroacetylacetonate as a function of the angle of incidence. The measurements were done in one step by depositing over a Pyrex fiber $30-40 \mu \mathrm{m}$ in diameter. The thickness as a function of angle is then measured in an SEM. The milling yield is found to increase by a factor of at least 4 in going from normal incidence to near grazing incidence. In addition for angles of incidence greater than $50^{\circ}\left(0\right.$ is normal, $90^{\circ}$ is grazing) the deposited film takes on a ripply ap- pearance, which becomes quite severe at grazing incidence. This effect can be reduced by reducing the gas pressure; i.e., raising the gas feed tube in the apparatus. The results of this work are very much of interest for the fundamental understanding of the ion surface interaction and for the repair processes, since complex topography is often encountered.

Reports:

1. Microstructure and Mechanism of Gold Films Grown by Ion Beam Induced Deposition, by Jae-Sang Ro, PhD Thesis, $1991,199 \mathrm{pp}$.

\section{TRANSPORT SIMULATION IN SPATIALLY MODULATED LOW-DIMENSIONALITY SYSTEMS}

Jean Leburton

University of Illinois

SC: SDC

An investigation has been initiated on transport in spatially non-uniform quantum wires by considering two different problems: (a) Work has started on an analysis of transport in a quantum chicane and a numerical model was developed for the calculation of the electronic states and transmission coefficients through the quantum cavity by a mode matching technique. The existence of a bound state in the chicane has already been confirmed. (b) The analysis of 1D transport in a periodic potential has been initiated. A model for acoustic phonon scattering including intra- and inter-subbands, as well as umklapp process, has been formulated for a two-subbands model.

\section{QUANTUM TRANSPORT IN SEMICONDUCTOR DEVICES}

\section{David Ferry}

Arizona State University

SC: ARL/EPSD

Studies are being made of interface phonons in heterostructures with magnetophonon resonance. The configuration of the experiment is to make measurements on a standard Hall bar with a constant current applied to the source and drain and the voltage measured between two of the arms sidearms. The magnetic field is applied perpendicular to the current flow. The materials that are being studied are GaAs/AlGaAs heterojunctions, a pseudomorphic InGaAs/GaAs single quantum well, and GaAs/AlAs 
multiple quantum wells. The oscillations due to magnetophonon resonance are weak compared to the background magnetoresistance and are seldom seen in the signal as measured, so some manipulation of the data is necessary. The resistance can be normalized by subtracting, and then dividing, by the zero magnetic field resistance. To further enhance the magnetoresistance a signal proportional to the magnetic field squared is then subtracted. The weak oscillations due to resonant scattering with phonons can be discerned. Gallium arsenide high electron mobility transistors have been fabricated to investigate the quantum effect. As the gatelength of an FET approaches the sub- $100 \mathrm{~nm}$ regime, quantum effects begin to dominate the device properties. Evidence of velocity overshoot in this regime was previously found to occur near $35 \mathrm{~nm}$. Below this gatelength, the transconductance decreases as a result of the existence of a minimum acceleration length required to attain the peak velocity. Furthermore, single electron tunneling and inter-device coupling are also possible. The present research is a continuing effort, where emphasis will be placed on the study of low temperature tunneling effects and improvement of microwave performance at room temperature. Finite difference methods are being used to discretize quantum moment equations and the Poisson equation in a two-dimensional HEMT structure. The typical doping in the channel is $1.5 \times 10^{18} \mathrm{~cm}^{-3}$, and a semi-insulating substrate is included. The lattice temperature is taken to be $300 \mathrm{~K}$. The I-V characteristics of a $24 \mathrm{~nm}$ gate length HEMT device have now been calculated. The gate voltage runs from $0 \mathrm{~V}$ to $-2.5 \mathrm{~V}$ with largest current for the $0 \mathrm{~V}$ gate bias, with increment gate voltage of $-0.5 \mathrm{~V}$. The characteristics suggest that the device has normal working performance.

Reports:

1. Some Fundamental Issues on Metallization in VLSI, by D.K. Ferry et al., SPIE Proc 1596,1(1991). AD A249549

2. Quantized Conductance Through a Potential Barrier, by Qin Li an D.K. Ferry, Superlattices and Microstructures 11,245(1992). AD A249 539

3. Simulation of Ultra-Small GaAs MESFET Using Quantum Moment Equations, by Jing-Rong Zhou and David $\mathrm{K}$. Ferry, IEEE Trans on Electron Devices 39,473(1992). AD A249 219

4. Ballistic Phenomena in GaAs MESFETS: Modelling with Quantum Moment Equations, by J.R. Zhou and D.K. Ferry, Semicond Sci Technol 7,BS46(1992).

5. Simulation of Ultra-Small GaAs MESFETs Using Quantum Moment Equations: II. Velocity Overshoot, by JingRong Zhou and David K. Ferry, MS, IEEE Trans on Electron Devices.
6. Modeling of Quantum Effects in Ultrasmall HEMT Devices, by Jing-Rong Zhou and David K. Ferry, MS, IEEE Trans on Electron Devices.

7. Modeling of Quantum Barrier Devices Using Quantum Moment Equations, by Jing-Rong Zhou and David $\mathrm{K}$. Ferry, MS.

\section{SCANNING TUNNELING MICROSCOPY OF III-V SEMICONDUCTORS}

John D. Dow

William E. Packard

Arizona State University

\section{SC: ARL/EPSD}

Ethereal "interstitials" were observed on the (110) surface of InSb at very small negative biases only (tunneling out of the valence band). These "interstitial" atoms, for some biases, are brighter in the STM image than regular surface atoms, and appear to lie above the surface. A theory has been worked out that shows that these ethereal interstitials are in fact second-layer $\mathrm{Sb}$ atoms, that appear to lie above the surface because the relevant wavefunction is mostly from $k=0$ of the Brillouin zone. An interpretation has been made of the $\beta$-SiC (III) surface STM data as due to a graphitic overlayer masking the SiC STM spectrum. This results in an STM image that has a superperiodicity of many times a lattice constant, and contrast reversal that was unanticipated. Steps on the (III) surfaces of InAs, InP, and InSb were investigated and surface reconstruction models were developed to explain the STM data. STM measurements were made on GaAs (001), on InSb (001) with and without radiation damage. No steps were observed.

\section{Repons:}

1. Relaxation of the $\mathrm{ZnTe}$ and $\mathrm{CuCl}$ (110) Surfaces, by M.-H. Tsai et al., MS.

2. Scanning Tunneling Microscopy Study of the Cleaved InSb (110) Surface, by Yong Liang et al., J Vac Sci Tech B9,730(1991).

28646 DENSITY MATRIX, SPACE CHARGE DEPENDENT, STUDIES OF CAPACITANCE VERSUS VOLTAGE OF QUANTUM WELL STRUCTURES 
Harold L. Grubin

Scientific Research Associates, Inc.

\section{SC: ARL/EPSD, ARL}

This study involves analytical and numerical studies of solutions to the Liouville equation in the coordinate representation. A comparison of the analytical and numerical studies indicates that the qualitative distribution of carriers within and around the vicinity of single and multiple barriers can be well described through a modification of the classical potential energy of the carriers. This modification includes a term that is proportional to the quantum potential. Numerical studies have also included calculations of the capacitance of a wide band gap/narrow band gap heterostructure, by varying the bias at the surface of the wide band gap material from a condition of depletion to that of charge accumulation. The resulting slope of the charge density versus the square root of the voltage shows an intersection that yields the offset voltage of the structure. This is the first density matrix calculation to confirm the analytical predictions of this approach to determining capacitance.

\section{MBE GROWTH FOR ELECTRONICS AND PHOTONIC DEVICE APPLICATIONS}

Ben G. Streetman

University of Texas at Austin

$$
\text { SC: ARL }
$$

This research has concentrated on advanced growth of multilayer heterostructures by Molecular Beam Epitaxy (MBE) and applications to electronic and photonic devices. In the area of advanced crystal growth, researchers have continued efforts previously sponsored by ARO in RHEED studies and low temperature photoluminescence (PL) of layers grown with accuracies of a single monolayer. Using PL studies of GaAs/AlGaAs quantum wells grown with various $Q W$ widths, it was possible to show that growth of half-monolayer (ML) well thickness (e.g., $3.5,4.5, \ldots, 10.5 \mathrm{ML}$ ) results in PL lines corresponding to both integer ML thickness and half-ML thicknesses. Presumably this is due to the exciton sampling an average of the microroughness in an integer plus half-monolayer thick well. Using 60 -second growth interruptions between layers, only inter-multiple ML well thicknesses were observed. In studies of high-resistivity $\mathrm{GaAs}$ and $\mathrm{AlGaAs}$ layers resulting from MBE growth at low temperatures researchers have studied the degradation of PL for quantum well structures grown at normal growth temperatures on top of the low-temperature grown (LTG) buffer layers. Experiments give information regarding the scale of interface roughness and the presence of nonradiative recombination centers in the near region of material grown on these LTG buffers. In the photonic applications of MBE-grown multilayer heterostructures, attention was concentrated on the application of Bragg reflectors and optical cavities to both emmitters (VCSELs) and detectors. A new type of photodetector was developed which incorporates an active cavity in which light is confined by Bragg reflectors to enhance detectivity while maintaining the response speed of a short absorption region. A VCSEL structure was developed which used MBE regrowth to define a current funneling region to the active part of the laser.

\section{Reports:}

1. Degradation of Photoluminescence from Quantum Wells Grown on Top of Low-Temperature Buffers, by A. Srinivasan et al., J Vac Sci Tech B10,835(1992).

2. Enhancement of Carrier Concentration and Spatial Confinement in Molecular-Beam Epitaxial Si and Be D-Doped GaAs by Increasing As4/As2 Flux Ratio, by Y.C. Shih et al., J Vac Sci Tech B10,863(1992).

3. Optical Memory Using a Vertical-Cavity Surface-Emitting Laser, by D.L. Huffaker et al., Trans Photonics Tech Let 3,1064(1991). AD A249 277

4. Photoluminescence Study of the Effects of Growth Interruption on Integer and Fractional Monolayer AlGaAs/GaAs Quantum Wells, by T.R. Block et al., MS, $J$ Vac Sci Tech.

5. Photoluminescence Study of the Effects of Growth Interruption on Integer and Fractional Monolayer AlGaAs/GaAs Quantum Wells, by T.R. Block et al., J Vac Sci Tech B10,832(1992).

\section{PHOTOREFLECTANCE FOR THE IN-SITU STUDY AND CHARACTERIZATION OF SEMICONDUCTOR GROWTH/PROCESSING}

Fred H. Pollak

Brooklyn College

The research objective is to improve the understanding of the electronic properties of semiconductors through in situ photoreflectance spectroscopy. An experimental study will be made of electronic properties of both unstrained and strained III-V heterostructures suitable for future uses in field-effect transistors, bipolar transistors and modulators. Heterostructures grown by molecular beam epitaxy will be characterized by photoreflectance to provide 
accurate determinations of the electronic properties of these semiconductor structures.

\section{NANOFABRICATION AND HIGH-SPEED NANOSCALE ELECTRONIC AND OPTOELECTRONIC DEVICES}

Stephen Y. Chou

University of Minnesota, Minneapolis

SC: ARL/EPSD, ARL

The research is directed toward increasing the understanding of charge transport in nanoscale electronic structures. Electron-beam lithography will be used to fabricate a series of electronic structures with feature sizes of approximately 100 Angstroms; charge transport will be studied experimentally in these structures.

\section{TRANSPORT PHENOMENA AND NOISE IN REAL QUANTUM WIRES}

V. Mitin

Wayne State University

\section{SL: ARL/EPSD}

SC: NVESD

Work has concentrated in three main directions: $(a)$ electron scattering in real quantum wire structures, (b) semi-classical electron transport and hot electron effects in real quantum wires, and (c) warm and hot electron diffusion and noise in quantum wires. In the first direction, it has been possible to calculate electron intra-subband and inter-subband scattering rates by confined longitudinal optical (LO) phonons and localized surface (interface) optical phonons in 1D structures with constant cross section (ideal wires) and with cross section variable along quantum wire (real wires). For ideal quantum wires electron inter-subband scattering rate exhibits multiple peaks associated with transitions to zero final kinetic energy. In real quantum wires the peaks on the scattering rate are essentially broadened and even completely washed out at higher energies. This fact resolves the problem of unphysical behavior of $1 D$ density of states obtained within Fermi golden rule approach. In both ideal and real quantum wires electrons are primarily scattered by LO phonons. In the second direction, researchers have calculated lowfield electron mobility controled by optical phonon scattering. This mobility tends to decrease dramati- cally with the increase in either electric field or temperature. At moderate fields and temperatures electron mobility and drift velocity are close to corresponding bulk material values. In the third direction, researchers are studying electron diffusion and noise in 1D systems under different conditions. Electron noise in cooling and streaming regimes, and within transition from cooling to streaming is under particular attention.

\section{STRAIN INDUCED POLARIZATION EFFECTS FOR III-V HETEROSTRUCTURE DEVICE APPLICATION}

P. Paul Ruden

Marshall I. Nathan

University of Minnesota, Minneapolis

\section{SC: ARL/EPSD, ARL, NVESD}

The research objective is to improve the understanding of strain-induced polarization effects in III-V heterostructures. An experimental and theoretical study will be made of piezoelectric effects in strained III-V heterostructures suitable for future uses in field-effect transistors, bipolar transistors and modulators. Heterostructures will be grown by gas source molecular beam epitaxy and will incorporate a wide range of phosphorus-based and arsenic-based polar semiconductors. Piezoelectric fields calculated for pseudomorphic III-V structures will be compared with experimental values of fields.

\section{NANOFABRICATION BY FOCUSED ION BEAMS}

John Melngailis

Dimitri A. Antoniadis

Massachusetts Institute of Technology

\section{SC: DARPA, ARL/EPSD}

The research is directed toward scientific applications of the currently-developing focused-ion-beam fabrication technology. New focused-ion-beam techniques will be used to fabricate novel and improved microelectronic structures. Attempts will be made to advance focused-ion-beam technology to a level that will facilitate the fabrication of novel electronic and optoelectronic devices including quantum-effect devices. The MIT team will emphasize the use of inorganic resists and limited lateral ion straggle to facilitate the nanofabrication of quantum-effect devices. 


\section{Antennas and EM Detection}

\section{GUIDED WAVE PHENOMENA IN MILLIMETER WAVE INTEGRATED CIRCUITS AND COMPONENTS}

Tatsuo Itoh

University of Texas at Austin

\section{SL: CECOM}

Slot radiators on one side of the substrate coupled with oscillators on the other side to form active antennas for quasi-optical applications have been studied. Five different configurations have been tested. The first is to use a high impedance section of the slot to weakly couple two slot oscillator/radiators. In the second configuration, a $40 \mathrm{GHz}$ radiation was generated by spatial power combing from either 2 or 4 slot radiations excited by 2 HEMT frequency doubling oscillators. In the third configuration, the frequency multiplying oscillator-power combiner was worked out by slot arranged in the $H$ plane. A $2 \times 2$ array was configured in the fourth structure. A chirped 6 element oscillator power combining was built which exhibited a broader bandwidth than the one from a uniform array. The optically tunable active filter based on the laser illumination of a gate-source capacitance was modified. The negative resistance generated by an active device enhances the $Q$ of the filter. Another active device is used for frequency tuning. Instead of the two-terminal operation of a tuning FET, a reactance generated between the drain and source by optical illumination of the junction area was used. The FET was now used as a three terminal device. Hence, in addition to the optical control, an additional control by the dc bias can be used. In addition, the functions of tuning and generation of negative resistance are combined into a single FET. In this version, the optical control was found not effective. However, the electronic tuning is still possible. A trapezoidal transmission line was analyzed. The line consists of a dielectric material of a trapezoidal cross section placed on a ground plane and a strip conductor on a top surface of the trapezoid. The boundary element method is being applied. A quasiTEM analysis has been completed. The frequency dependent dynamic solutions are being obtained. A simple analysis based on the small signal theory is being developed for inspecting the stability of mutually locked radiating oscillators for quasi-optical applications. As a first step, a perturbation analysis is carried out for two-device oscillator to test its stability for the push-pull and push-push operations.

Reports:

No. $1-57$ in previous editions.

58. Two-Port FET Oscillators with Applications to Active Arrays, by Joel Birkeland and Tatsuo Itoh, IEEE Microwave and Guided Wave Let 1,112(1991). AD A245 122

59. Characterization of Shielded Coplanar Type Transmission Line Junction Discontinuities Incorporating the Finite Metallization Thickness Effect, by Chih-Wen Kuo and Tatsuo Itoh, IEEE Trans on Microwave Theory and Tech 40,73(1992). AD A249 188

60. A 16 Element Quasi-Optical FET Oscillator Power Combining Array with External Injection Locking, by Joel Birkeland and Tatsuo Itoh, IEEE Trans on Microwave Theory and Tech 40,475(1992).

61. Fullwave Analysis of the Effect of Ground Plane Width on Radiation Loss in Open-End Discontinuities in Finite Ground Plane Coplanar Waveguide, by J.S. McLean et al., MS, IEEE Trans on Microwave Theory and Tech.

62. Analysis of a New Configuration of Coplanar Stripline, by J.S. McLean and Tatsuo Itoh, IEEE Trans on Microwave Theony and Tech 40,772(1992).

63. Boundary Element Analysis of a Trapezoidal Transmission Line, by B. Toland and T. Itoh, MS, IEEE Microwave and Guided Wave Let

64. Tunable Active Bandpass Filters Using Three-Terminal MESFET Varactors, by Jenshan Lin and Tatsuo Itoh, MS.

65. $40 \mathrm{GHz}$ Quasi-Optical Second Harmonic Spatial Power Combiner Using FETs and Slots, by Shigeo Kawasaki and Tatsuo Itoh, MS.

66. Active Integrated Antenna Based on Slots with FETs, by Shigeo Kawasaki and Tatsuo Itoh, MS.

67. Tunable Active Microwave Bandpass Filters Using ThreeTerminal MESFET Varactors, by Jenshan Lin and Tatsuo Itoh, TR, Jan 92,57 pp.

\section{MILLIMETER-WAVE APPLICATION OF SEMICONDUCTOR DIELECTRIC WAVEGUIDES WITH PLASMA LAYERS (SURFACE OR BURIED) GENERATED FROM SEMICONDUCTOR LASER}

Jerome K. Butler

Southern Methodist University

\section{SL: CECOM}

Experimental and theoretical progress has advanced at an excellent rate. Researchers are extremely happy with the correlation of theoretical calculations with experimental measurements of the periodic waveguides fabricated on the $\mathrm{Al}_{2} \mathrm{O}_{3}$ material. In addition to the improvement of the design program of the periodic waveguides, simulation experiments of waveguide structures that have excited plasma layers have been performed. These new periodic structures 
have a fixed grating waveguide coupled with a separate confined plasma layer in the opposed waveguide surface. Typically, the new structures would be fabricated on wafers composed of either $(a)$ silicon on sapphire (the grating formed in the sapphire layer and the plasma layer excited in the silicon layer), or (b) gallium arsenide on aluminum gallium arsenide (the grating formed in the aluminum gallium arsenide layer and the plasma layer excited in the gallium arsenide layer). In these experiments, researchers started with an $\mathrm{Al}_{2} \mathrm{O}_{3}$ waveguide with a grating formed on one surface. The waveguide was designed to radiate broadside. Next, a layer of metal foil was attached to the waveguide on the side opposite the grating layer. (The metal foil was to simulate an excited plasma layer with high electron/hole concentrations.) The radiation pattern measured with the foil attached produced a beam at about $30^{\circ}$ to the broadside direction. This implies that a corresponding silicon on sapphire structure would electronically scan about $30^{\circ}$.

Reports:

No. $1-2$ in previous editions.

3. A Boundary Element Technique Applied to the Analysis of Waveguides with Periodic Surface Corrugations, by J.K. Butler et al., IEEE J Quant Electron 28,1701(1992).

\section{ELECTRO-OPTICS DISPLAY RESEARCH, TEST AND EVALUATION LABORATORY PROGRAM}

Donald L. Moon

University of Dayton

\section{SL: NVESD}

Discrepancies between the predicted minimum resolvable temperature difference and field performance are indicative of the fact that the modeling of MRTD has certain inherent problems. Several sources for MRTD error have been identified. MRTD was also shown to be a special case of a more general minimum resolvable luminance difference (MRLD) measure. The MRLD measure involves absolute temperature and can be used to describe more generic targets. A device nonspecific dynamic minimum resolvable temperature difference performance model was developed for characterizing thermal imaging systems capable of displaying moving targets. Experiments have been set-up to collect data pertaining to the overlap of rectangular displays at close range. The effect of the parameters such as field-of-view, motion of scene, distance between the eyes and display, and degree of overlap are being studied.

\section{Reports:}

No. 1-7 in previous editions.

8. Device Nonspecific Dynamic Performance Model for Thermal Imaging Systems, by Ming L. Gao et al., MS, Opt Eng.

9. Minimum Resolvable Temperature Difference Model: A Critical Evaluation, by Mohammad A. Karim et al., MS, Opt Eng.

\section{INTERINJECTION-LOCKED QUASIOPTICAL POWER COMBINERS AND PHASED ARRAYS}

Karl D. Stephan

University of Massachusetts

\section{SL: CECOM}

A semiconfocal open-cavity resonator has been used to stabilize a resonant-tunneling-diode waveguide oscillator at frequencies near $100 \mathrm{GHz}$. The high quality factor of the open cavity resulted in a linewidth of approximately $10 \mathrm{kHz}$ at $10 \mathrm{~dB}$ below the peak, which is about 100 times narrower than the linewidth of an unstabilized waveguide oscillator. This technique is well suited for resonant-tunnelingdiode oscillators in the submillimeter-wave region. An oscillator using an array of 25 resonant-tunneling diodes in parallel has delivered an output power of $5 \mathrm{~mW}$ at $1.18 \mathrm{GHz}$, which is the highest microwave power ever obtained from a quantum-well-device oscillator.

Reports:

No. 1-3 in previous editions.

4. Resonant-Tunneling Diode Oscillator Using a Slot-Coupled Quasioptical Open Resonator, by K.D. Stephan et al., Electron Let 27,647(1991).

5. Lossy-Line Stabilization of Negative-Resistance Diodes for Integrated-Circuit Oscillators, by Karl D. Stephan and SaiChu Wong, MS.

6. A Quasioptically Stabilized Resonant-Tunneling-Diode Oscillator for the Millimeter-and Submillimeter-Wave Regions, by E.R. Brown et al., MS.

\section{RESEARCH ON LEAD TITANATE FILMS FOR RADIATION DETECTION}

Paul Kruse

Barry Cole

Honeywell, Inc.
SL: CECOM, NVESD
SC: ARL/MD 
A statistically designed experiment on Honeywell's dual target ion beam sputtering technique for $\mathrm{PbTiOB}_{3}$ films was completed. The analysis showed the need for a higher level of control in several key areas. While stoichiometry control and film tetragonality are excellent using this technique, three other critical parameters - the preferred orientation, loss tangent and remanent polarization - depend strongly on control of the substrate temperature, electrode metal preparation and oxygen pressure during deposition. For improved temperature control, an IR pyrometer was added to monitor the wafer temperature. The output of the pyrometer feeds back to the heat lamp controller, providing closed loop temperature control that is stable to within about $2^{\circ} \mathrm{C}$. To ensure that the pyrometer reading is not affected by the doping density of the silicon substrate, or the films on the front side of the wafer, a high emissivity coating is applied to the back surface of the wafer prior to $\mathrm{PbTiO}_{3}$ deposition. These changes have resulted in increased repeatability of the crystallographic orientation. Some of the material has been almost entirely oriented in the highly desirable (001) direction. A simple, single pixel mask set was made for development of the basic fabrication sequence, and for initial testing of the pyroelectric portion of pixels for IR and mm-wave detection. Pixels can be fully fabricated in less than a week with this mask set. This has led to improved processes for the patterning of the absorber/top electrode layer and for the etching step that creates the thermally isolated structure. Depositing $\mathrm{PbTiO}_{3}$ on patterned Pt electrodes, rather than on a solid Pt film has resulted in greatly improved film morphology. One wafer has completed fabrication, but results were poor due to a high loss tangent. A more complex mask has also been designed. A new concept has been developed for an improved pyroelectric pixel structure. This has the potential of increased performance by lowering the thermal mass and thermal conductivity of the structure and by improving the film orientation. This is done by depositing oriented $\mathrm{PbTiO}_{3}$ on an epitaxial buffer layer, probably $\mathrm{MgO}$. After processing is complete the buffer layer is sacrificially etched away.

Reports:

1. $\mathrm{PbTiO}_{3}$ Films Deposited by an Alternating Dual-Target Ion Beam Sputtering Technique, by B.E. Cole et al., MS.

\section{IMPROVEMENTS IN OSCILLATORS FOR PLANAR MILLIMETER-WAVE CIRCUITS}

Steven E. Schwarz

University of California, Berkeley

\section{SL: CECOM, ARL/EPSD}

Optimal design of a non-contacting magnetic probe for measurements on the interior of planar high-frequency circuits has been studied, and performance of the probe has been determined. The probe is a relatively simple device that may find uses in circuit design and optimization, troubleshooting, and production testing. In the present work, its design has been studied by means of enlarged models tested at frequencies 100 times lower than those of the actual intended use. The nature of its errors has been investigated, and some techniques for error reduction have been found. S-parameter measurements on general 2-ports can also be made by using the probe at several different positions on the associated transmission lines. This technique effectively eliminates the problem of de-embedding that arises in other kinds of S-parameter measurements. Examples of measurements with the large model probe are presented and compared with theory. Performance appears to be acceptable for the intended applications. The probe has been designed with eventual microfabrication in mind, but difficulties in this final step remain to be resolved.

Reports:

1. Design and Performance of a Non-Contacting Probe for Measurements on High-Frequency Planar Circuits, by Samuel S. Osofsky and S.E. Schwarz, MS.

\section{MONOLITHIC MILLIMETER WAVE RADIATING SYSTEMS AND FEED NETWORKS}

David R. Jackson

University of Houston

\section{SL: CECOM, ARL, MICOM}

A paper has been prepared which describes an exact solution for the problem of transverse electric or transverse magnetic plane-wave scattering from a periodic, planar double-strip grating at a dielectric interface. The metal-strip grating is assumed to be perfectly conductive and infinite in length, with two different strips within a unit-cell. The formulation is based on a multimode equivalent network representation, and uses a rigorous solution for the relevant integral equation that extends the novel solution developed previously for the single-strip grating. Expressions for the elements of the multimode coupling matrices are given, together with a comparison of 
results for power transmitted through the grating, obtained by using the networks developed with the present method and a simple point-matching solution. Results are also presented to illustrate the differences between single and double-strip gratings. It is seen that the addition of the extra strip within the unit cell can have a significant effect on the scattering behavior. One interesting feature that follows from the formulation is that double-strip structures maintain the symmetry of the scattered power with respect to the angle of incidence of the excitation, even if they are asymmetric.

Reports:

1. Scattering From a Double-Strip Grating: Rigorous Equivalent Network Formulation, by Marco Guglielmi and David R. Jackson, IEEE Trans on Ant and Prop 39,1479(1991). AD A249492

2. Leaky-Wave Propagation Characteristics of Strip-Grating and EMC Dipole Array, by Pavan K. Potharazu, MS Thesis, $1991,119 \mathrm{pp}$

3. Analysis and Design of a Leaky-Wave EMC Dipole Array, by Pavan K. Potharazu and David R. Jackson, MS, IEEE Trans on Ant and Prop.

4. Broadside Radiation From Periodic Leaky-Wave Antennas, by M. Guglielmi and D.R. Jackson, MS.

\section{PRINTED CIRCUIT ELEMENTS WITH APPLICATIONS TO ANTENNAS, SCATTERING AND CIRCUITS}

N.G. Alexopoulos

University of California, Los Angeles

\section{SL: CECOM}

Two configurations of broadband microstrip antennas were proposed and analyzed numerically by the full-wave spectral-domain method. One is a square Archimedean spiral microstrip antenna which yields circular polarization; the second configuration is a log-periodic patch microstrip antenna, which is a linearly polarized antenna. In a paper, the definition of bandwidth of an antenna is redefined and is based on both VSWR and radiation pattern behavior. A 25 percent bandwidth for a square Archimedean spiral microstrip antenna and a 2:1 bandwidth for a log-periodic patch microstrip antenna are achieved using electrically thick $(\lambda / 8$ to $\lambda / 4)$ substrates. In a dissertation, a full-wave spectral-domain analysis combined with the method of moments is used to accurately characterize arbitrarily-shaped microstrip discontinuities in an open structure. The spectral-domain dyadic Green's function, which takes into account both radiation and surface waves, is used to formulate an electric field integral equation. The method of moments is then employed to obtain the current distribution on microstrips, and subsequently the scattering parameters of the junctions. Since all field components can be expressed in terms of the dyadic Green's function and the current distribution, the losses due to both radiation and surface waves are further determined through a rigorous Poynting vector analysis. To model the discontinuities of arbitrary shape, both rectangular and triangular subdomain functions are flexibly used as the current expansion functions in the moment method procedure. In addition, the semi-infinite traveling wave functions are applied to simulate the feeding structure and isolate individual junction effects. Many examples are given to illustrate the utility of each technique. Comparison of some numerical results with experimental data shows excellent agreement. A method of analysis is presented in another dissertation for investigating the radiation and scattering properties of arbitrarily-shaped, planar, printed apertures. This full-wave space domain analysis solves the vector aperture integral equation for the unknown electric field by using a moment method technique and vector-valued triangular-domain expansion functions.

Reports:

No. 1-2 in previous editions.

3. Characterization of a Microstrip Patch Antenna Including a Coplanar Feed, by Shih-Chang Wu and N.G. Alexopoulos, MS.

4. Microstrip Linear Array of EMC Dipoles with a Corporate Feed, by Tzyy-Sheng Horng and N.G. Alexopoulos, MS.

5. Feeding Structure Contribution to Radiation by Patch Antennas with Arbitrarily Rectangular Boundaries, by Shihchang $\mathrm{Wu}, \mathrm{MS}$, IEEE Antennas and Propagation Trans.

6. Corporate Feed Design for Microstrip Arrays, by T. S. Horng et al., MS.

7. Radiation From Aperture Antennas with a Coplanar Waveguide Feed, by Huang-Chang Liu et al., MS.

8. Full-Wave Analysis of Superstrate Cavity-Backed Aperture Antennas, by Jind-Yeh Lee et al., MS.

9. A Rigorous Full-Wave Analysis for Microstrip Power Dividers and Corporate Feeds, by Tzyy-Sheng Horng and Nicolaos G. Alexopoulos, MS.

10. Full-Wave Spectral-Domain Analysis for Open Microstrip Discontinuities of Arbitrary Shape Including Radiation and Surface-Wave Losses, by Tzyy-Sheng Horng et al., MS.

\section{DEVELOPMENT OF THE MODIFIED DIAKOPTIC THEORY: ANALYSIS}


Chalmers M. Butler

Clemson University

\section{SL: CECOM}

Work has continued on the development of the modified diakoptic theory and its applications to the analysis of microstrip and stripline array antennas. The theoretical and experimental results are in close agreement in the case of the antenna array structure comprising a microstrip feed line coupled to tracetype radiating elements. In the case of the array of slot radiators fed by striplines, agreement is excellent except in frequency ranges near resonances of the open cavity formed by the parallel-plate ground planes of the microstrip. At these frequencies, the experimental model used in the measurements does not adequately replicate the theoretical model due to the finite extent of the parallel plates. In the most recent theoretical models of the microstrip-fed array of traces, conductor losses are accounted for which has enabled researchers to obtain far closer agreement between measured and calculated data. With this improved model, theoretical input admittance at the antenna feed now has a significant real part in agreement with the measured real part even in the ranges of frequency where radiation losses are not particularly strong. In all cases for both the microstrip-fed array of traces and the stripline-fed array of slots, the computed data obtained by means of the modified diakoptic theory and those obtained by means of the method of moments are in excellent agreement.

\section{Reports:}

1. Flanged Parallel-Plate Waveguide Coupled to a Conducting Cylinder, by Chalmers M. Butler et al., IEE Proc H138,549(1991).

2. Slotted Parallel-Plate Waveguide Coupled to a Conducting Cylinder, by Paul D. Mannikko et al., IEE Proc H139,193(1992).

3. The Modified Diakoptic Theory for Slots Coupled to Strips and the Analysis of a Slotted Stripline, by Paul Douglas Mannikko, PhD Thesis, 1992, 189 pp.

4. The Analysis of Microstrip Transmission Lines Coupled to Microstrip Dipoles by the Modified Diakoptic Theory, by Clifton Carthelle Courtney, PhD Thesis, 1992, 178 pp.

28483 THEORETICAL AND EXPERIMENTAL STUDY OF LOW-LOSS, HIGH EFFICIENCY MONOLITHIC ANTENNA STRUCTURES AT $94 \mathrm{GHz}$
Linda P.B. Katehi

Gabriel M. Rebeiz

University of Michigan

\section{SL: ARDEC, CECOM, MICOM}

A study is being made to develop a monolithic array operating at $94 \mathrm{GHz}$ which is fed by a dielectric network exhibiting low-losses. The most critical part of this effort is the successful development of the feeding network. Towards achieving this goal the following has been accomplished: A novel formulation has been completed for the accurate very effective characterization of two dimensional open waveguide problems. This powerful method transforms complex two-dimensional dielectric waveguide problems to simplified equivalent planar ones which are then solved using known planar integral equation techniques. At first, the electric field in the dielectric waveguide is expressed in terms of volume polarization currents from which a planar polarization dipole moment is defined. Further, generalized boundary conditions on the surface of the dielectric waveguide are enforced to make the introduced planar dipole moment equivalent to the original volume polarization dipole moment. In this manner, a simplified planar integral equation is derived which is then solved in the spectral domain to provide very satisfactory results. In parallel to the above analysis, researchers have applied a mode-matching/integral equation method to theoretically characterize various transitions from microstrip to dielectric ridge waveguide. In parallel with the theoretical study, it has been possible to fabricate low-loss dielectricridge waveguides and measure their attenuat:on and phase constant at lower frequencies using scaled models.

Reports:

1. Design and Analysis of Quasi-Integrated Horn Antennas for Millimeter and Submillimeter-Wave Applications, by George V. Eleftheriades and Gabriel M. Rebeiz, MS, IEEE Trans on Microwave Theory and Tech

2. Double-Slot Antennas on Hyperhemispherical, Elliptical, and Extended Hemispherical Dielectric Lenses, by Daniel F. Filipovic et al., MS, IEEE Trans on Microwave Theory and Tech.

3. Large Area Bolometers for $\mathrm{THz}$ Power Measurements, by Curtis C. Ling et al., MS, IEEE Microwave and Guided Wave Let.

4. An 86-106 GHz Quasi-Integrated Low Noise Schottky Receiver, by Walid Y. Ali-Ahmad et al., MS, IEEE Trans on Microwave Theory and Tech.

5. A 26-GHz Harmonic-Mixer Receiver, by Brian K. Kormanyos and Gabriel Rebeiz, MS, Microwave J. 


\section{PERFORMANCE CHARACTERISTICS OF PHASE-CORRELATING FRESNEL ZONE PLATES}

James C. Wiltse

Georgia Institute of Technology

\section{SL: CECOM}

The primary effort has been concentrated on the investigation of Fresnel zone plate antennas. The secondary activity has dealt with the characteristics of propagation in elliptical waveguides. The zone plate work has dealt with several aspects of the problem. An experimental design was carried out at 35 $\mathrm{GHz}$ for both a transmitting and a reflecting zone plate. A reflecting zone plate was fabricated using the same design parameters. The physical measurements for the reflecting case are the same as for the transmission zone plate, except the depth of cut is half as thick since the incident rays on a reflecting plate travel through the zone plate twice. The design of the reflecting zone plate is based on the feed being directly in front of the center of the zone plate, but this causes unwanted feed blockage and increases sidelobe levels. Previous work by Garrett and Wiltse has shown that the intensity at the focal point does not significantly diminish for feeds off-axis by up to $20^{\circ}$ for the transmission zone plate. If it is desired to move the feed of a reflecting zone plate to an angle greater than $20^{\circ}$, a redesign of the zone plate is needed. For the problem of propagation in an elliptical waveguide, an error was detected in a paper published in late 1990 , and a correction was developed and has now appeared in print. In addition, a compilation has been made of all published articles dealing with propagation in or on waveguides of elliptical crosssection, and a bibliography of about 90 articles has been prepared.

Reporis:

1. Errors in Solutions for Waveguides of Elliptical Cross-Section, by J.C. Wiltse and T.H. Gfroerer, MS.

2. Recent Developments in Fresnel Zone Plate Antennas at Millimeter Wavelengths, by James C. Wiltse, MS.

3. Further Comments on "Modes of Elliptical Waveguides: A Correction", by J.C. Wiltse and T.H. Gfroerer, MS, IEEE Trans on Microwave Theory and Tech.

4. Design Parameters for Zone Plate Antennas, by C.A. Barrett and J.C. Wiltse, MS.

\section{GRID AMPLIFIERS}

David Rutledge

California Institute of Technology

\section{SL: CECOM, ARL/EPSD, MICOM}

A paper has been prepared which presents a Schottky diode grid mixer suitable for mixing or detecting quasi-optical signals. The mixer is a planar bow-tie grid structure periodically loaded with diodes. A simple transmission line model is used to predict the reflection coefficient of the grid to a normally incident plane wave. The grid mixer power handling and dynamic range scales as the number of devices in the grid. A $10 \mathrm{GHz}$ 100-element grid mixer has shown an improvement in dynamic range of 16.3 to $19.8 \mathrm{~dB}$ over an equivalent single-diode mixer. The conversion loss and noise figure of the grid are equal to that of a conventional mixer. The quasi-optical coupling of the input signals makes the grid mixer suitable for millimeter-wave and submillimeter-wave applications by eliminating waveguide sidewall losses and machining difficulties. The planar property of the grid potentially allows thousands of devices to be integrated monolithically.

\section{MESFET GRID OSCILLATORS - INTERJECTION-LOCKING POWER DESIGN AND MODULATION}

Zoya B. Popovic

Alan R. Mickelson

University of Colorado

\section{SL: ARL/EPSD, CECOM, MICOM}

A 25-HEMT grid oscillator was fabricated on an electrooptic GaAs substrate and the potential distribution across the oscillator was measured using a unique electrooptic sampling technique. From the measured results, the current distribution across a grid oscillator can be found. The existence of a unit cell was confirmed experimentally. A new design-oriented theory for a generalized unit ce was developed. This theory is based on a moment method solution for the current distribution on the grid structure. The driving point impedance can be found and an $n$-port formulated as an imbedding circuit for an active device. This theory will enable optimization of grid oscillators and amplifiers with respect to power, bandwidth or any other parameter of interest. 


\section{Circuits, Networks and Related Systems}

\section{CARRIER COLLECTION AND SCATTERING IN QUANTUM WELL AND SUPERLATTICE DEVICES}

Robert M. Kolbas

North Carolina State University

Researchers have reported the photopumped operation of a vertical cavity surface emitting laser where the active region consists of a single pseudomorphic InAs-GaAs quantum well that is less than one monolayer thick. This represents the thinnest active layer to support stimulated emission when the optical feedback is perpendicular to the layer. Lasing action supported across a submonolayer thick quantum well can be understood by considering the effects on the carrier collection process and the gain across an ultra thin quantum well due to the spreading out of the electron and hole wavefunctions. Pulsed lasing due to gain across the InAs quantum well was confirmed for photoexcitation energies above and below the band edges of the GaAs confining layers at 17 and $77 \mathrm{~K}$.

\section{OUASI-OPTICAL MILLIMETER WAVE POWER COMBINING}

Christopher J. Summers

James Wiltse

Georgia Institute of Technology

SL: ARO, CECOM, SDC

SC: ARL/EPSD, MICOM, SDC

Work continued into the development of a quasi-optical power combiner for $35 \mathrm{GHz}$ MESFET and HEMT arrays. The design features for the optical combining cavity were completed and a preliminary design was generated and implemented for the grid. Also, commercially available MESFET and HEMT devices were characterized and incorporated into the grid designs. The best performance was achieved for a $3 \times 3$ array of HEMT devices which oscillated at 37.1 GHz.

Reports:

1. Quasi-Optical Power Combining of Solid State Sources, by James C. Wiltse and James W. Mink, MS, Microwave J.

\section{E. Signal Processing, Communications and Related Systems}

\section{REDUCING DATA DIMENSION TO LOWER SIGNAL COMPUTATIONAL REQUIREMENTS AND MAXIMIZE PERFORMANCE}

Barry Van Veen

University of Wisconsin - Madison

$$
\text { SC: } \mathrm{SMO}
$$

A paper has been completed which describes work on subspace based generalized likelihood ratio adaptive detection of signals in noise. In addition, the cases for which dimension reduction reduces the detector's sensitivity to mismatch between the actual and assumed signal model have been characterized. While one cannot guarantee that sensitivity is always reduced, it is reduced for the most probable classes of mismatch. Furthermore, a computationally efficient form was derived for evaluating the detection statistic. Lastly, an alternate approximate method for designing the subspace to maximize the detection probability was developed. In adaptive beamforming, work has been refocussed on the transformation design problem with an emphasis on approaches that incorporate a specification on the worst case performance loss over a likely set of interference scenarios. A quadratic constraint approach has been taken to reducing degrees of freedom in beamformers that are faced with coherent interference.

Reports:

No. 1-9 in previous editions.

10. Convergence of the SMI AJgorithm in Partially Adaptive Linearly Constrained Beamformers, by Barry D. Van Veen, Proc IEEE Jul91, p1373. AD A239 694

11. Modular Implementations of Linearly Constrained Beamformers, by Tsung-Ching Liu and Barry Van Veen, SPIE Proc 1566,419(1991).

12. Coherent Interference Suppression via Partially Adaptive Beamforming, by Feng Qian and Barry D. Van Veen, MS IEEE Trans on Acoust, Speech, and Signal Proc.

13. Improved Adaptive Detection Performance via Subspace Processing, by Keith A. Burgess and Barry D. Van Veen, MS, IEEE Trans on Acoust, Speech, and Signal Proc.

14. Subspace Based Adaptive Detection, by Keith A. Burgess et al., MS, IEEE Trans on Signal Proc.

15. A Modular Structure for Implementation of Linearly Constrained Minimum Variance Beamformers, by TsungChing Liu and Barry Van Veen, IEEE Trans on Signal Proc 39,2343 (1991). AD A249 536 


\section{THE GAUSS MACHINE}

Fred J. Taylor

University of Florida

\section{SL: WSMR}

The overwhelming majority of defense signal and image processing (SP/IP) operations can be accelerated with faster arithmetic. In addition, in real-time $S P / I P$ applications, there is a desire to move the data processing capability as close to the sensor groups as possible (i.e., sensor fusion). The rationale for such action is one of maximizing the potential bandwidth, promoting modularity, improving reliability, and reducing system power and packaging requirements. These attributes will undoubtedly be required in the next generation of SP/IP systems. The objective of this study has been to develop a new technology which achieves these desired attributes. More specifically, researchers are developing a technology they call the Gauss Machine. The Gauss Machine is, in fact, a technology family consisting of a new class of DSP processor, architecture, and development environment. The research program will add new basic knowledge to this field as well as provide the SP/IP community with an innovative and viable research tool. Efforts have resulted in: (a) The research and design of a new class of processor cell which functions as an "array processor on a chip." The target complex multiply-accumulate (CMAC) rate is on the order of $1 \mathrm{G} \mathrm{CMAC/sec.} \mathrm{(b)} \mathrm{A} \mathrm{design} \mathrm{for} \mathrm{the} \mathrm{new}$ single chip array processor, a multi-SIMD (MSIMD) DSP architecture having a maximum peak CMAC rate of $8 \mathrm{GHz}$ and a core processor area of 8 chips (fewer if multi-chip module (MCM) integration techniques are used), along with optimizing the architecture for a suite of applications and missions. (c) The development of an experimental Gauss Machine which is suitable for conducting basic and SP/IP applied research. (d) Providing a technology transfer distribution pathway of the Gauss technology to other academic and federal $R \& D$ centers, laboratories, and programs.

\section{ROUTING FOR TACTICAL AND STRATEGIC COMMUNICATION IN RADIO NETWORKS}

Imrich Chlamtac

University of Massachusetts

\section{SC: AIRMICS}

In a CDMA/TDMA data-link protocol of radio networks collision/conflict free transmission schedules are created using a cyclic, time oriented scheduling of transmissions (report 2, below). The number of times a link can be activated in a cycle determines a virtual capacity of each link. Transmission scheduling has so far been considered in the context of data link control as a means to maximize spatial reuse of the available bandwidth and to optimize link capacity allocation according to traffic requirements. Report 2 shows how routing consideration can be incorporated in determining data-link schedules. By setting these schedules in a way that accounts for a given set of routes, it is possible to use shortest path routing in a radio network while avoiding congestion on heavily occupied links. This procedure is shown to significantly reduce end to end delays and increase network capacity in radio networks. In addition, no analytical models are currently available for analyzing multihop CDMA/TDMA networks. A new analytical approach is introduced which allows the performance of such networks to be derived analytically. Using recursion relation the authors develop an approximate solution for analyzing general topology radio networks with finite number of buffers, using fixed routes and arbitrary datalink schedules.

\section{Reports:}

1. Nearly Optimum Scheduling in CDMA Packet Radio Networks, by Andras Farago et al., MS, IEEE Trans on Commun.

2. Congestion Free Routing in Multi-Hop Radio CDMA/TDMA Networks by Virtual Capacity Assignment, by I. Chlamtac et al., MS.

3. Making Transmission Schedules Immune to Topology Changes in Multi-Hop Packet Radio Networks, by Imrich Chlamtac and Andras Farago, MS.

4. An Optimal CDMA Channel Access Protocol, by Imrich Chlamtac and Andras Farago, MS.

\section{COMMUNICATION IN THE PRESENCE OF UNKNOWN INTERFERENCE}

Brian L. Hughes

The Johns Hopkins University

SL: ARL/WTD

SC: SMO

Efforts have continued to develop new codes for asynchronous packet-switched networks (such as frequency-hopped and satellite networks) that perform well in an environment of strong multiple-access interference. Work has continued on the problem of assigning codes to $M$ potential users of a common channel in a way that permits any collection of up to $T(\leq M)$ of these users to transmit data 
reliably at a time. Previously some preliminary results were obtained on this problem for the collision channel without feedback, which models a multiple-access packet-switched channel. These results were as follows: for every $T$ and $M$, researchers determined the capacity region (i.e., the maximum user data rates that can be sustained with a small error) of the collision channel for synchronous, slot-synchronous and asynchronous users. Moreover, optimal codes were found for the $M$ users that approach the boundary of the capacity region. A more explicit characierization of the capacity region was found which greatly simplifies its calculation. Also, shorter (but still optimal) codes to use on this channel were found. These results were applied to determine the capacity and optimal codes for packet-switched frequencyhopped communications systems in the case of synchronous transmitters.

\section{CONCURRENT ARCHITECTURES FOR VLSI SIGNAL AND IMAGE PROCESSING}

Keshab K. Parhi

University of Minnesota, Minneapolis

SL: NVESD

SC: SDC

The stochastic gradient adaptive lattice filter is pipelined by the application of the relaxed lookahead. This form of look-ahead maintains the functional behavoir instead of the input-output mapping. The sum and product relaxations are employed to pipeline the lattice filter. The hardware complexity of the proposed pipelined filter is of the same order as the sequential filter. Thus, the new architecture is attractive from an implementation point of view. Convergence analysis results are presented to illustrate the trade-off offered by relaxed look-ahead. Application of the lattice filter to pipelined video predictive coding is demonstrated in publications.

Reports:

No. 1-15 in previous editions.

16. A Pipelined LMS Adaptive Filter Architecture, by Naresh R. Shanbhag and Keshab K. Parhi, Proc of Twenty-Fifith Asilomar Conference on Signals, Systems, Computers, 1991, p5. AD A244 323

17. A Pipelined Adaptive Lattice Filter Architecture, by Naresh R. Shanbhag and Keshab K. Parhi, MS, IEEE Trans on Signal Proc.

18. Synthesis of Pipelined Lattice IIR Digital Filters, by JinGyun Chung and Keshab K. Parhi, MS, IEEE Trans on Signal Proc.
19. High-Speed Huffman Decoder Architectures, by Keshab K. Parhi, Proc of Twenty-Fifth Asilomar Conference on Signals, Systerns, Computers, 1991. AD A244 309

20. Design of Pipelined Lattice IIR Digital Filters, by Jun-Gyun Chung and Keshab K. Parhi, Proc of Twenty-Fifth Asilomar Conference on Signals, Systems, Computers, 1991. AD A244 545

21. A High-Speed Architecture for ADPCM Codec, by Naresh R. Shanbhag and Keshab K. Parhi, MS.

22. High Level DSP Synthesis Using the MARS Design System, by Ching-Yi Wang and Keshab K. Parhi, MS.

23. Pipelining of Adaptive Filters with Relaxed Look-Ahead. Part I: Theory, by Naresh R. Shanbhag and Keshab K. Parhi, MS, IEEE Trans on Circuits and Systs.

24. Pipelining of Adaptive Filters with Relaxed Look-Ahead. Part II: Applications to Speech and Video Predictive Coding, by Naresh R Shanbhag and Keshab K. Parhi, MS, IEEE Trans on Circuits and Systs.

\section{AUTOMATIC TARGET RECOGNITION}

Avi C. Kak

Purdue University

SL: ARDEC

SC: MICOM, SDC, VAL, WSMR

The objective of this research is to investigate machine vision algorithms and architectures for low resolution LADAR imagery with real-time performance requirements. Particulary of interest will be methods for target detection that do not require the acquisition of a full scene buffer, thus improving the system response time.

\section{SEQUENTIAL ACQUISITION SCHEMES FOR SSMA SYSTEMS WITH GENERALIZED SIGNATURE SEQUENCES}

Sawasd Tantaratana

University of Massachusetts

SL: ARL/WTD

SC: SMO

Noncoherent sequential acquisiton schemes have been studied with and without channel fading. The sequential schemes under study differ from those of other authors, where independent and identically distributed samples were customarily assumed. The i.i.d assumption requires the integrators of the correlators in the system to be periodically reset, creating noncoherent combining loss in the effective signal-to-noise ratio (SNR). In the approaches used here, the integrators are not reset, creating non-i.i.d samples. It is found that such acquisition schemes 
perform much better than those with i.i.d samples. The effect of Ricean channel fading was studied. Results show that severe fading significantly affects the probability of detection when the local and incoming PN sequences are in synchronization. However, it does not affect the false alarm probability when the two sequences are out of synchronization. Acquisition with the presence of data modulation was studied. Most works in acquisition of PN sequences assume that the data is not transmitted during acquisition. However, in some situations such as when the system loses synchronization and it has to re-establish the synchronization, acquisition must be performed while data is being transmitted. It is found that conventional acquisition systems degrade significantly when there is data modulation. Therefore, better schemes are needed. The optimum acquisition scheme is derived using the principle of maximum likelihood estimate. However, the optimum scheme is impractical to implement. Therefore, suboptimum systems are obtained. The performances of these optimum and suboptimum schemes are currently being studied. Upper and lower bounds have been derived for the probability of bit error $\left(\mathrm{P}_{\mathrm{be}}\right)$ for DS/SSMA systems with complex signature sequences using MPSK signaling. This is in contrast to previous works, which obtained either bounds for $\left.P_{b e}\right)$ with binary sequences or bounds for probability of symbol error. The resulting bounds can be numerically computed. They can be made arbitrary tight with the expense of longer computation time.

Reports:

1. A 1.8-GHz Monolithic LC Voltage-Controlled Oscillator, by Nhat M. Nguyen and Robert G. Meyer, IEEE J Sol St Circuits 27,444(1992).

\section{STUDY OF VIDEO TRANSMISSION TECHNIQUES IN TACTICAL SWITCHED NETWORK SYSTEMS}

Donald L. Schilling

Tuvia Apelewicz

SCS Telecom, Inc.

\section{SL: CECOM}

SC: CECOM

While the functions of the VPAD are not currently established and approved requirements, the need for video surveillance in the battle field is considered an unstated requirement. Although such a requirement could evolve as a weapon system with dedicated communications, this study is considered a necessary step to determine if the system can be integrated into the common user communications network which is now depicted as the MSE/TRITAC tactical communications system. The first study established the basis for further study of the difficulties in integrating the services into the common user network. A continued study of the effects of the requirements on the intended network has yielded a promising approach to achieve the service within the confines of the voice switched network. The apparent effects of the data transmission within real time communications on the voice network which has a primary role in satisfying communications for the tactical commanders is not considered detrimental to that system's mission. As a condition of establishment of requirements on fielded systems, the study of the effects on the systems must be undertaken. To this end, CECOM has taken the appropriate steps to ensure that the added requirements do not interfere or jeopardize the mission of the fielded system. Factors influencing the introduction of a new requirement on the present system are the primary goal of this study. The effect of the new data transmission requirements on the current MSE/TRITAC network was discussed in a report. While further study is necessary to insure that the new requirements are possible and cost effective, it appears that the voice common user network can sustain the data requirements. Further study of the effects of the normal activities of the switched network is justified before hardware design is implemented. These study objectives include the effects of the network requirements as well as new requirements on the data transmission.

\section{IMPROVED INTERCEPTION CAPABILITY THROUGH THE USE OF TRANSFORM DOMAIN PROCESSING}

\author{
L.B. Milstein \\ University of California, San Diego
}

$$
\text { SC: SMO }
$$

A paper has been prepared in which the coherent reception of direct sequence-code division multiple access (DS - CDMA) signals in a multipath fading channel is considered. The channel model assumes independent paths with Nakagami fading statistics; this model includes the Rayleigh channel as a special case and is a reasonable model for a frequency-selective fading channel. The bit error rate (BER) performance of a RAKE receiver under various multipath fading conditions is derived and evaluated. The results indicate that, when channel coding is employed, 
over 40 users may access the channel (assuming a processing gain of 127 ) simultaneously under typical fading conditions, and can achieve a BER of 0.001 with an average received signal-to-noise ratio per bit of $5 \mathrm{~dB}$.

\section{.27834 CONGESTION/FLOW CONTROL AND ROUTING IN DATA NETWORKS: A CONTROL-THEORETIC APPROACH}

Semyon M. Meerkov

University of Michigan

\section{SL: CECOM}

SC: AMSAA

A model for a datagram network with a feedback congestion control architecture has been introduced and analyzed. It has been shown that in the case of a single congested node there exists a set of gains that prevents congestion and ensures stabilization of the network in a steady state with certain fairness properties. A method for choosing the control gains that guarantee congestion prevention is given.

\section{HIGH-FREQUENCY SIGNAL-PROCESSING INTEGRATED CIRCUITS}

Robert G. Meyer

Donald O. Pederson

University of California, Berkeley

SC: ARL

Research in high-frequency monolithic receivers is proceeding on several fronts. A new monolithic widerange voltage-controlled $\mathrm{LC}$ oscillator (VCO) topology suitable for use in any integrated-circuit technology was devised. The topology is based on the new concept of steering the oscillator loop signal continuously between two offset on-chip LC tank circuits via an on-chip linear multiplier. A test chip has been fabricated in a $15 \mathrm{GHz}$ BiCMOS process and extensive evaluation performed. The circuit displayed a tuning range close to the predicted value of 1.6-1.9 GHz using on-chip inductors of $6.5 \mathrm{nH}$ and $3.7 \mathrm{nH}$. Active die area was $315 \mu \mathrm{m}$ and quiescent power dissipation (including an on-chip measurement buffer) was $70 \mathrm{~mW}$ from a single $5 \mathrm{~V}$ supply. The VCO was simulated assuming $3 \sigma$ limits of \pm 10 percent on the on-chip tuning capacitors. These process variations caused only a 3 percent change in tuning range, indicating insensitivity to process vari- ations. As temperature was varied from $-40^{\circ} \mathrm{C}$ to $+85^{\circ} \mathrm{C}$ the measured oscillation frequency only varied \pm 1.5 percent, again indicating insensitivity of the topology to environment variation. Measured output power varied from $-23 \mathrm{dBm}$ to $-27 \mathrm{dBm}$ across the band. Research in high-frequency oscillators uncovered serious defficiencies in existing accepted theories for oscillator design. In particular, parasitic elements increase the order of the system and can result in the creation of phenomena not predicted by conventional first-order theories. New design method were derived that allow synthesis of reliable high-frequency oscillations with well-defined startup behavior and freedom from the possibility of simultaneous oscillation at multiple frequencies. This work was verified by the design, fabrication and testing of several microwave monolithic oscillations.

\section{Reports:}

1. A 1.8-GHz Monolithic LC Voltage-Controlled Oscillator, by Nhat M. Nguyen and Robert G. Meyer, IEEE J Sol St Circuits 27,444(1992).

\section{ADVANCED ARRAY PROCESSING FOR COMMUNICATION SYSTEMS}

Benjamin Friedlander

Signal Processing Technology, Ltd.

\section{SL: SWC}

The main thrust of the research effort has been in the general area of direction finding algorithms and their performance analysis, with emphasis on the following problems: direction of arrival estimation in a multipath environment; direction finding for broadband signals; and direction finding using diversely polarized arrays. In a series of papers, a detailed performance analysis was carried out for each of these algorithms. Closed form expressions for the covariance matrix of the DOA estimation errors were derived using a perturbation analysis. Evaluating these expressions for specific cases and comparing them to the Cramer Rao lower bound for the DOA estimates, provides insight into the statistical efficiency of these algorithms. The formulas for the error covariance are quite general, and can be specialized to provide results for other DOA estimation algorithms as well. More recently work has focused on the exploitation of polarization diversity for direction finding problems. It was possible to develop a number of computationally efficient algorithms for the simultaneous estimation of the direction of arrival and polarization parameters of multiple signals. By studying the performance of these techniques, it was possible to 
quantify the advantages of utilizing polarization diversity for both uncorrelated and correlated sources. It is quite clear that polarization diverse systems should be preferred in future direction systems. Such systems are not significantly more complex than systems which do not use polarization diversity, but are capable of improved performance if the signals of interest have different polarization characteristics. As expected, when the signals of interest have identical polarizations, the performance of a polarization diverse array is essentially the same as that of a corresponding uniformly polarized array.

\section{CYCLIC FEATURE ANALYSIS, DESIGN AND EXPLOITATION}

William A. Gardner

Statistical Signal Processing, Inc.

SC: ARL

An investigation is in progress on the problem of characterizing the sine-wave components in the output of polynomial nonlinear systems with cyclostationary time-series inputs. Researchers have introduced the concept of a pure $n$ th-order sine wave, and have shown that pure sine wave strengths in the output time-series are given by the Fourier coefficients of the temporal cumulant of the input time-series. The moments and cumulants of narrowband spectral components of the input time-series have also been defined and idealized to the case of infinitesimal bandwidth. Such spectral moments and cumulants have been shown to be characterized by the Fourier transforms of the temporal moments and cumulants of the input time-series and to, therefore, provide a frequency-domain characterization of sine wave generation with polynomial nonlinear transformations. Consideration has been given to the problem of estimating cyclic polyspectra, which are higher-order statistics of cyclostationary time-series. The cyclic polyspectrum can be viewed as the Fourier transform of a higher-order cyclic temporal cumulant, or as a cumulant of spectral components of the time-series. In the latter case, the cyclic polyspectrum is seen to be a linear combination of products of spectral moment functions which include Dirac deltas, all of which cancel out in the cyclic polyspectrum. Because of the impracticality of this cancellation using finite precision arithmetic, it is concluded that the cyclic polyspectrum is best measured by first measuring the cyclic temporal cumulant, tapering it, and then transforming it. The noise tolerance of the cyclic polyspectrum, which results from the tolerance to Gaussian noise exhibited by any stationary noise exhibited by any cyclic statistic has been demonstrated by simulation, as has the tolerance to cyclostationary interference with cycle frequencies unequal to those of the signal of interest. In addition a new approach has been developed to obtaining sets of operators that are orthogonal to the Volterra operators by exploiting a class of cyclostationary input time-series.

Reports:

1. Exploitation of Cyclostationarity for Identifying the Volterra Kernels of Nonlinear Systems, by William A. Gardner and Teri L. Archer, MS, IEEE Trans on Info Theory.

2. An Overview of the Theory of Higher-Order Cyclostationarity, by Chad M. Spooner and William A. Gardner, MS

3. Estimation of Cyclic Polyspectra, by C.M. Spooner and W.A. Gardner, MS.

4. Theory and Application of Higher-Order Cyclostationarity, by Chad M. Spooner, PhD Thesis, 1992, 191 pp.

\section{SPREAD-SPECTRUM RADIO SYSTEMS AND NETWORKS}

Michael B. Pursley

D.V. Sarwate

University of Illinois

\section{SC: ARL/WTD}

In work on concatenated coding, researchers have developed a set of bounds on the packet, codeword, and bit error probabilities for frequency-hop signaling, convolutional coding and maximum-likelihood decoding within the dwell intervals, and Reed-Solomon coding across the dwell intervals. It has also been observed that tighter bounds are possible, and work is in progress to refine the bounds and improve the results. In addition, a simulation program has been written, and the simulation is being used to determine the actual performance of the concatenated coding scheme. Work on coding and combined coding and equalization for channels with intersymbol interference continues. Several different methods are available for computing the power spectral density (PSD) of a general continuous phase modulated (CPM) signal. The most commonly used method required the evaluation of a two dimensional integral. In simple cases such as that of an M-ary 1REC CPM signal with equally likely data symbols, it is possible to evaluate this integral analytically, and to obtain closed-form expressions for the PSD. In general, however, it is necessary to use numerical integration in order to determine the PSD. Unfortu- 
nately, the integrands become more oscillatory as the frequency increases and maintaining accuracy in the numerical integration becomes quite difficult. An analytic expression was derived for the PSD of an M-ary $L R E C$ CPM signal where $L$ is an arbitrary positive integer and the data symbols are not necessarily equally likely. This analytical expression is obtained by using simple properties of the convolution integral and the Fourier transform, and it specifies the PSD as a sum of elementary functions. Although the value of the PSD at any particular frequency must be obtained by computing the sum, the time required is far less than that required for numerical integration. Furthermore, even for very large values of the frequency variable, there is no difficulty in maintaining numerical accuracy. Thus, it is possible to use the result to compute the PSD quickly and accurately over a wide range of frequencies.

Reports:

1. Concatenated Coding for Frequency-Hop Packet Radio, by Greg M. Chiasson and Michael B. Pursley, MS.

2. An Analytic Expression for Power Spectra of M-ary LREC Continuous Phase Modulated Signals, by Richard A. Korkosz and Dilip V. Sarwate, MS, IEEE Trans on Commun.

3. Bounds on the Performance of Maximum-Likelihood and Reduced-State Decoding of Trellis Codes on Channels with Intersymbol Interference, by Colin D. Frank and Michael B. Pursley, MS.

4. A Spread-Spectrum Signaling Scheme with Continuous Phase Modulation, by R.A. Korkosz and D.V. Sarwate, MS.

\section{MEGA-SCALE SIMULATION OF MULTI-LAYER DEVICES - FORMULATION, KINETICS AND VISUALIZATION}

Robert W. Dutton

Stanford University

SC: AMSAA, NRL

A paper has been prepared which proposes a modularized, mixed-mode device/circuit simulation system to expand the modeling capability of a circuit simulator by including numerical device models and to equip a device simulator with a generalized way to implement circuit boundary conditions (BCs). With such a system the device characteristics can be evaluated under a real circuit environment and the circuit performance can be simulated by using numerical models for critical devices. The system is highly modularized, meaning that each simulator can easily be replaced with minor code changes. Moreover, the quadratic convergence rate of member simulators is preserved for the overall system performance. This modularized approach is particularly attractive considering the rapid development of advanced device simulators with increasingly complex physics involved. For example, new opto-electronic circuits use both silicon and GaAs device technologies. Numerical techniques necessary for realizing of the system and simulation example for a three-stage ring oscillator are provided. Another paper investigates the detailed physical and mathematical causes of the spurious velocity overshoot spike, observed in hydrodynamic simulations of $n$ in diodes. The magnitude of the spike is related to the variation of the heat conduction coefficient in the conventional formulation. Results obtained using a recently proposed energy transport model are presented which show virtually no spurious spike without any adhoc parameter variation.

Reports:

1. Steady-State Macroscopic Transport Equations and Coefficients for Submicron Device Modeling, by Datong Chen et al., MS, IEEE Trans on Electron Devices.

2. A Modularized, Mixed IC Device/Circuit Simulation System, by Zhiping Yu et al., MS.

3. Analysis of Spurious Velocity Overshoot in Hydrodynamic Simulations, by D. Chen et al., MS.

\section{VLSI CIRCUITS FOR HIGH-SPEED} DATA CONVERSION

Bruce A. Wooley

Stanford University

The principal accomplishments include the completion of research into the design, integration, and testing of a 12-bit, 5-MHz CMOS A/D converter, the completion of work on a monolithic fiber optic receiver front end implemented in a GaAs-on-silicon technology, and the design of a novel CMOS preamplifier suitable for use in fiber optic receivers. Owing to constraints on area and power dissipation, fully parallel (flash) A/D converter architectures are impractical for applications demanding a resolution greater than 8 bits. In order to achieve a resolution of 12 bits or more, some form of two-step architecture is generally used. Within this program, research into precision, high-speed data conversion circuits employing such an architecture has led to the successful design and integration of a 12-bit, $5-\mathrm{MHz}$ analog-to-digital converter in a $1-\mu \mathrm{m}$ CMOS technology. The CMOS A/D Converter employs a two-step flash topology consisting of a 7-bit course flash stage, a 7-bit capacitive D/A converter, a unity-grain sub- 
tractor, and a 6-bit fine flash stage, together with decoding logic and memory. One bit of overlap between the two stages is used to relax the performance required of the first-stage comparators, and offsets are cancelled in both the first and second stage comparators on every comparison cycle. The use of offset cancellation on every cycle also served to attenuate flocker noise at the comparator inputs. In the architecture used for the two-step converter, only the comparators in the second stage need resolve differences as small as 0.5 LSB of the overall converter. These comparators have been implemented by means of a novel self-calibrating topology wherein offsets are cancelled in both the preamplifier and subsequent latch. The use of offset cancellation in the latch significantly reduces the gain required of the preamplifier and thereby improves the bandwidth. A fully-differential implementation of the A/D converter has been successfully integrated in a $1-\mu \mathrm{m}$ CMOS technology at National Semiconductor. Experimental measurements confirm that the comparator provides a resolution of 12 bits at sampling rates as high as $5 \mathrm{MHz}$, while dissipating only $200 \mathrm{~mW}$ from a single $5 \mathrm{~V}$ supply.

\section{Reports:}

No. 1 in previous edition.

2. Monolithic Integration of $\mathrm{GaAs}$ and $\mathrm{Si}$ Bipolar Devices for Optical Interconnect Systems, by Gitty N. Nasserbakht et a1., Proc 1992 IEEE Custom Integrated Circuits Conference, 1992.

3. High-Speed, High-Resolution Analog-to-Digital Conversion in VLSI Technologies, by Behzad Razavi, PhD Thesis, 1991, $142 \mathrm{pp}$.

4. A 12-Bit 10-MHz BiCMOS Comparator, by Behzad Raxavi and Bruce A. Wooley, Proc of 1991 Bipolar Circuits and Technology Meeting, 1991, p 289. AD A249 660

5. Design Techniques for High-Speed, High-Resolution Comparators, by Behzad Razavi and Bruce A. Wooley, MS, IEEE J Sol St Circuits.

6. Monolithic Integration of $\mathrm{GaAs}$ and $\mathrm{Si}$ for Receiver FrontEnds in Optical Interconnect Systems, by Gitty $\mathrm{N}$. Nasserbakht et al., MS, IEEE J Sol St Circuits.

7. A 12b 5MS/s Two-Step CMOS AD Converter, by Behzad Razavi and Bruce A. Wooley, Proc 1992 IEEE Intl Sol St Circuits Conf, Paper WP 2.4, 92CH3128-6, 1992.

\section{PROTOCOL ENGINEERING FOR BROADBAND NETWORKS}

Ming-Tsan Liu

Ohio State University

\section{SL: CECOM}

SC: AIRMICS

Protocol interworking is concerned with building an internet consisting of multiple networks that were not originally designed to work together, protocol validation and protocol synthesis are to ensure the syntax correctness of a single protocol. A dissertation presents several approaches to the protocol interworking problem, while applying the approaches to the protocol validation and protocol synthesis problems as efficient tools. With the proliferation of network architectures and communication protocols, assuring the correctness of the communication between users on different networks becomes increasingly difficult. The current approaches to the protocol internetworking problem can be divided into two categories: protocol conversion and protoccol complementation. Protocol conversion approaches solve the problem by placing an entity, the so-called converter, at the gateway to translate transition sequences in one network to those in another network, while protocol complementation approaches implement the translation by building a virtual layer at the gateway and end-nodes. The first part of the dissertation investigates the common properties of protocol conversion and protocol validation. The second part of the dissertation presents a protocol complementation approach to the protocol interworking problem. In contrast to the first two parts of the dissertation, the third part of the dissertation discusses the protocol interworking problem in the EFSM model and presents a protocol conversion approach to it.

\section{Reports:}

1. Distributed Rule Processing in Active Databases, by IngMiin Hsu et al., MS.

2. A New Protocol Test Sequence Generation Method Based on UIOS, by Shwuhwa S. Yu and Ming T. Liu, MS.

3. Constructing Protocol Converters From Service Specifications, by Ming T. Liu and Yow-Wei Yao, MS.

4. Axiomatic Test Sequence Generation for Extended Finite State Machines, by Chang-Jia Wang, MS.

5. Utilizing Multiple UIO Sequences and Segment Overlap to Shorten Test Sequences, by Shwuhwa S. Yu and Ming T. Liu, MS.

6. An Incremental Protocol Test Method: Formal Modeling and Architectures, by Chung-Ming Huang and Ming T. Liu, MS.

\author{
28506 FORMAL DESIGN OF \\ COMMUNICATION PROTOCOLS \\ BASED ON THE ESTELLE ISD \\ FORMAL DESCRIPTION \\ TECHNIQUE
}


Paul D. Amer

University of Delaware

\section{SL: AIRMICS, CECOM}

Two necessary conditions were derived for determining a shortest Unique Input Output (UIO) sequence. Using these conditions, Sabnani and Dahbura's original algorithm (SD88) for UIO sequence generation in test case generation is made more efficient. For states having no UIO sequence, an algorithm for generating Partially Unique Input Output (PUIO) sequences is introduced and analyzed. While a UIO sequence distinguishes a state from all other states, a PUIO sequence distinguishes a state from only a subset of other states. A test case generation technique combining UIO and PUIO sequences is explained. A strict real-time delay mechanism allows the formal specification of maximum execution times for transitions once they are enabled. This is somewhat contrary to a basic tenet of Estelle; that is, that the execution time of a transition is unknown and unpredictable. A real-time constraint mechanism is commonly required to specify distributed system; Estelle's current delay clause does not serve this purpose. Thus, a new clause, called the doby clause, is introduced. The doby clause simply toggles the independent time process on and off, thus providing a non-interfering, formal interpretation of real-time constraints on the elapsed execution time of transitions. Using terminology consistent with that used in the current Estelle International Standard, a paper has been prepared which presents the formal syntax and semantics of a doby clause for use in formally specifying real-time constraints while still preserving backward compatibility with existing Estelle specifications.

\section{PROTOCOL ENGINEERING FOR MULTIMEDIA COMMUNICATIONS}

Ming-Tsan Liu

Ohio State University

\section{SL: AIRMICS, CECOM}

The research objective is to investigate the design, specification and verification of protocols in a multimedia computer communication network environment. The work is organized into four areas: investigate multimedia protocol composition; test sequence generation for context sensitive protocols; conformance testing for broadcast/multicast protocols; and context sensitive protocol conversions.

\section{F. External Program}

\section{OPTOELECTRONIC INTEGRATED CIRCUITS FABRICATED USING ATOMIC LAYER EPITAXY}

P. Daniel Dapkus

University of Southern California

SL: ARL

The atomic layer epitaxy growth mechanisms have been investigated in the temperature regime where researchers have previously observed enhanced growth due to laser photoexcitation. Two major conclusions are forthcoming from the measurements: $(a)$ The thermal decomposition of trimethylgallium (TMGa) on a GaAs surface is very slow below a temperature of $400^{\circ} \mathrm{C}$. The growth rate is limited by the surface decomposition rate and by steric hindrance effects of the adsorbed species. When TMGa is present on the surface, the large physical size of the adsorbate inhibits adsorption on neighboring sites. As a result, the deposition of one monolayer cannot occur until the TMGa adsorbate decomposes and molecules are adsorbed on adjacent sites. (b) The saturation of the growth rate at one monolayer results from the differential reaction rates of TMGa on $\mathbf{G a}$ and As sites. On an arsenic rich surface, TMGa decomposes to atomic $\mathrm{Ga}$ to produce gallium stabilized surface. TMGa does not react with this surface in the temperature range up to $570^{\circ} \mathrm{C}$. These observations have been made by observing the adsorption kinetics of TMGa on GaAs (100) surfaces using RHEED and XPS studies. Below $400^{\circ} \mathrm{C}$, the investigators observed that the decomposition of TMGa on $\mathrm{GaAs}$ surfaces in UHV is very slow in agreement with higher pressure growth studies. In the range $400-$ $550^{\circ} \mathrm{C}$, the surface reaction rate of TMGa on GaAs surfaces increases with increasing temperature.

\section{INTELLIGENT PROCESSING OF GaAs FOR LARGE SINGLE CRYSTAL GROWTH}

H.S. Goldberg

General Electric Company R \& D Center

SL: NVESD

SC: ARL/EPSD

Work has focused on three areas: the acquisition of process understanding of the $8 \mathrm{~kg}$ process; the demonstration of model guided improvement of the $8 \mathrm{~kg}$ process; and the tooling for information gathering 
with Airtron's $6 \mathrm{~kg}$ process. The $8 \mathrm{~kg} \mathrm{GaAs}$ growth process running in GE laboratories is the first test case for the IPM concept. Both the methods and the equipment are patterned on the high pressure Czrokalski process. GE completed the development of process instruments this year. The puller was fitted with a suite of thermocouples and a melt height sensor to characterize crystal growth. Five design generations of thermocouples were required to create practical sensors. The final temperature sensors are multi-run durable in all locations except in the molten mass. The melt thermocouples last one complete growth cycle and are sufficient for process characterization. Process insights gathered in the previous phases of this project were used to guide improvement of the $8 \mathrm{~kg}$ process in both control strategy and interal design. Both changes resulted in significantly better crystals than are possible with an unmodified Cambridge 358 puller like the one at GE. Airtron dedicated one of their production pullers for full-time use as an IPM testbed. During the period, the puller was instrumented with a set of thermocouples and a crystal weight sensor to collect process information. A computer and IPM software was installed in their plant to acquire the process data in a compatible form.

\section{FUSION OF RANGE AND LUMINANCE DATA FROM LASER RADAR SYSTEMS}

Wesley E. Snyder

James W. Mink

North Carolina State University

An additional tack has been taken in the research in an attempt to exploit the data available at the Bowman Gray School of Medicine Magnetic Resonance Imaging (MRI) Unit. Researchers are investigating the applicability of the models and methods developed for the tri-Services Lasar Radar (TSLR) to these signal sources. An advantage of this direction of investigation is the control over data for experiments; synthetic, phantom as well as real data taken in the MRI environment. There are interesting similarities between the TSLR problem and the MRI problem. Sensor fusion techniques are applicable to both problems since signals may be restored using mathematical relationships among separate data channels (TSLR) or a known functional relationship among the data channels (MRI) and imbedded signals. Applying the models and methods developed for the TSLR or multiple channels of MRI data, researchers have developed maximum likelihood restorations for multiple signals which are nonlinearly imbedded within noisy data, using constrained optimization techniques. These signals constitute a basis for the MRI system, and as such, one can synthesize data by adjusting parameters within the forward transform of the nonliner sensor mode. This gives a radiologist the ability to view a typical MR image with parameter settings different from those used to gather the original data without obtaining additional MRI data. It has also been possible to reduce the noise inherent in the imbedded signals with linear-smoothing (regularization) techniques. These signals should prove to be very useful for tissue/object classification, since they should provide a radiologist with the ability to more accurately view the pathology of imaged tissues. Furthermore, the regularized signals can also be used in the forward transform, resulting in a reduced-noise MR image.

\section{SOLID-STATE DYNAMICS IN NOVEL SEMICONDUCTOR NANOSTRUCTURES}

\author{
M.A. Stroscio \\ K.W. Kim \\ North Carolina State University
}

The investigators made a number of significant findings including: the fact that the imposition of metalsemiconductor boundary conditions on interface-optical phonons in quantum wells and quantum wires may be used to eliminate or reduce inelastic scattering due to interface optical phonons; the fact that irregular cross sections in quantum wires can cause 1D electron scattering rates to reduce to the 3D results; the fact phonon lifetime effects dominate over collective effects in carrier energy loss due to LO phonon emission in quantum wires; and the finding that phonon potentials for double barrier quantum wells approximate that for two-heterojunction structures for a wide range of dimensional parameters.

\section{LIMITED REACTION PROCESSING: HETEROSTRUCTURE AND NOVEL DEVICE FABRICATION}

James F. Gibbons

Stanford University

SL: ARO, DARPA, ARL/EPSD
SC: ARL/EPSD, ARL 
The characteristics of diodes fabricated in thick $\mathrm{Si}_{1-x} \mathrm{Ge}_{x}$ layers formed by selective epitaxial deposition have been examined by dc electrical measurements, transmission electron microscopy, and x-ray topography. Because depositing in restricted areas limits the propagation of misfit dislocations in thick layers, a lower misfit dislocation density is found in small-area deposited regions. Similarly, diodes fabricated in small deposited regions have more ideal forward characteristics than diodes fabricated in large regions. An investigation has been made of boron diffusion in $\mathrm{Si}$ and strained $\mathrm{Si}_{1-x} \mathrm{Ge}_{x}$, in-situ doped, epitaxial layers. During inert ambient annealing at $860^{\circ} \mathrm{C}$, boron diffusion is observed to be slower in $\mathrm{Si}_{0.83} \mathrm{Ge}_{0.17}$ than in $\mathrm{Si}$ for doping levels between $1 \times 10^{18}$ and $2 \times 10^{19} \mathrm{~cm}^{-3}$. Computer simulations of the measured boron diffusion profiles indicate that the ratio of the effective boron diffusivity $D_{\text {eff }}$ in Si to that in $\mathrm{Si}_{0.83} \mathrm{Ge}_{0.17}$ is approximately $6: 1$ for the intrinsic doping case $\left(p=1 \times 10^{18} \mathrm{~cm}^{-3}\right)$, and 17:1 for the extrinsic doping case $\left(p=2 \times 10^{19} \mathrm{~cm}^{-3}\right)$.

\section{IN SITU GROWN QUANTUM-WIRE LASERS}

Larry Coldren

University of California, Santa Barbara

Substrate cleaning and etching in preparation for regrowth experiments is now being carried out in the in situ etching chamber. The field-emission E-gun has been received and debugged; however, it has not been installed yet. The etch rates and surface morphologies for various etching conditions, including ion-beam etching, chemically assisted ion-beam etching, radical beam ion beam etching, and radical beam etching has been characterized. Regrowth following chlorine radical beam etching and hydrogen cleaning has resulted in charge-free interfaces as measured by CV. Several different quantum-wire growth techniques are being explored. Quantumwire lasers have been formed with the in situ grown serpentine-superlattice (SSL) structures. Gain anisotropy in good agreement with theory has been measured at low temperatures using the fringe visibility technique. Ridge lasers with their axial directions both parallel and perpendicular to the wire axes have been tested. The gain anisotropy corroborates the small lateral confinement energy first predicted from photoluminescence. Continued efforts in modeling and VCSEL growth have led to useful advances. Results from a two-dimensional VCSEL model have suggested that higher $\mathrm{Al}$ content in the quantum-well barriers should be used. The first growth has verified that higher powers and temperatures are possible with this change. Top-emitting VCSEL structures with intra-cavity contacts accessed by side ion implants have shown good I-Vs, but the gain characteristics seem to have been compromised in the implant/anneal cycle.

Reports:

1. A Tanh Substitution Technique for the Analysis of Abrupt and Graded Interface Multilayer Dielectric Stacks, by Scott W. Corzine et al., IEEE J Quant Electron 27,2085(1991). AD A249563

2. Investigation of Tilted Superlattices for Quantum-Wire Laser Applications, by Jong Chang Yi et al., Appl Phys Let 59,3015(1991). AD A250 247

3. Theoretical Gain in Strained InGaAs/AlGaAs Quantum Wells Including Valence-Band Mixing Effects, by S.W. Corzine et al., Appl Phys Let 57,2834(1990).

\section{PARALLEL ADAPTIVE FINITE ELEMENT SOFTWARE FOR SEMICONDUCTOR DEVICE SIMULATION}

Robert W. Dutton

Stanford University

\section{SL: ARO, ARL/EPSD, ARL}

The objective of this research is the development of a general, portable and reliable parallel adaptive finite element analysis software system that can be used for effective simulation of semiconductor devices. The research has two main approaches. First, the work deals with the design of a parallel, adaptive finite element program. Second, the work deals with the simulation of semiconductor devices. Thus, in this work, a three-dimensional finite element method will be developed which exploits new parallel computer environments to specifically support semiconductor device modeling applications.

\section{OPTOELECTRONIC INTEGRATED CIRCUITS FABRICATED USING ATOMIC LAYER EPITAXY}

\section{P.D. Dapkus}

University of Southern California

The research objective is to explore and understand the integration of a wide variety of III-V compound semiconductor heterojunction devices fabricated by atomic layer epitaxy (ALE) and photoassisted 
atomic layer epitaxy (PALE). Atomic layer epitaxy (ALE and PALE) will be used to fabricate monolithically integrated structures consisting of a variety of heterojunction devices. This program will use PALE to fabricate selectively-grown structures in order to determine the materials processing technology required to merge electronic and optical devices on the same substrate.

\section{QUANTUM TRANSPORT IN SOLIDS: TWO-ELECTRON PROCESSES}

Gerald J. Iafrate

North Carolina State University

\section{SC: ARL/EPSD}

The research objective is to understand the role of two-electron processes in dynamical quantum transport theory. Attempts will be made to theoretically model two-electron processes by analytical techniques for inclusion in full dynamical, high-field quantum transport theories.

\section{NOVEL ENGINEERING COMPOUND SEMICONDUCTOR HETEROSTRUCTURES FOR ADVANCED ELECTRONIC AND OPTOELECTRONIC INTEGRATED CIRCUIT APPLICATIONS}

\section{G.E. Stillman}

University of Illinois

The research objective is to advance the technology base required to realize high-performance phased array lasers, superlattice and quantum-well avalanche photodiodes, high-speed electronic amplifiers and laser or modulator drivers. An integrated research program will be conducted for the study of the crystal growth and characterization, as well as device design, fabrication and characterization of electronic and optoelectronic structures made of III$\mathrm{V}$ compound semiconductors, with a particular emphasis on the study of heteroepitaxial systems and the utilization of heterojunction device structures.

\section{G. Joint Services Electronics Program}

\section{JOINT SERVICES ELECTRONICS PROGRAM}

James S. Harris

Stanford University

$$
\text { SC: OASA (RDA) }
$$

Resonant tunneling devices are based on quantum transport of electrons through ultra-thin heterostructure layers. These devices have the potential to become the foundation for the next generation of ultra-high speed electronics and multi-valued logic devices. Resonant Tunneling Diodes (RTD) provide an excellent research vehicle to study the fundamentals of electron transport in ultra-thin layered semiconductors. Effects of X-valley transport of electrons were studied in triple barrier RTDs with significantly different AlAs barrier thickness. Researchers have found that the electron tunneling through GaAs/AlAs quantum well structures is dominated by $\Gamma$ valley tunneling when AlAs layer is thin (less than $30 \AA$ ) and bias is low. Electron tunneling through $X$ valley dominates when AlAs barrier is thick (more than $130 \AA$ ). The $\mathrm{X}$ valley tunneling is significant when bias is sufficiently high so that the Fermi level in front of the tunnel barrier is equal to or higher than the $\mathrm{X}$ barrier height in AlAs barrier. The effects of Si impurity scattering on double barrier resonant tunneling diode (DBRTD) performance were studied by systematically varying the Si doping levels in the AlAs barriers and measuring the current-voltage characteristics of the DBRTDs. The results show that the resonant tunneling diode performance is virtually unaffected by up to $1.2 \times 10^{17} \mathrm{~cm}^{-3} \mathrm{Si}$ doping in the AlAs barrier. Reasonably good peak-to-valley current ratio (PVCR) is observed even with $3 \times 10^{18} \mathrm{~cm}^{-3} \mathrm{Si}$ doped AlAs barrier. Improvement in PVCR of DBRTDs by using two step spacer layers has been attributed to the band bending in the spacer layers.

\section{Reports:}

No. $1-3$ in previous editions.

4. PMOS Transistors in LPCVD Polycrystalline Silicon-Germanium Films, by Tsu-Jae King et al., IEEE Electron Device Let 12,584(1991).

5. Depth-Size Tradeoffs for Neural Computation, by KaiYeung Siu et al., IEEE Trans on Comp 40,1402(1991).

6. On the Power of Threshold Circuits with Small Weights, by Kai-Yeung Siu and Jehoshua Bruck, SLAM J Disc Math 4,423(1991). 
7. Subspace Based Detection for Linear Structural Relations, by $M$. Viberg et al., $J$ Combinatorics Inform \& Syst Sci $16,170(1991)$.

8. Detection and Estimation in Sensor Arrays Using Weighted Subspace Fitting, by Mats Viberg et al., IEEE Trans on Signal Proc 39,2436(1991).

9. A General Weight Matrix Formulation Using Optimal Control, by $\mathrm{O}$. Farotimi et al., MS.

10. On the Competitive Optimalilty of Huffman Codes, by Thomas M. Cover, IEEE Trans on Info Theory 37,172(1991).

11. Low-Frequency Noise Characterization of npn AlGaAs/GaAs Heterojunction Bipolar Transistors, by Damian Costa and J.S. Harris, Jr., MS.

12. Evidence of Chemical-Potential Shift with Hole Doping in $\mathrm{Bi}_{2} \mathrm{SrCaCu}_{2} \mathrm{O}_{8}+\mathrm{D}$, by Z.-X. Shen et al., Phys Rev B44,12 098(1991).

13. A Variable-Work-Function Polycrystalline-Si1-x $\mathrm{Ge}_{\mathbf{x}}$ Gate Material for Submicrometer CMOS Technologies, by TsuJae King et al., IEEE Electron Device Let 12,533(1991).

14. Formation of Single-Phase MAsx Films on GaAs by Selective Wet-Hydrogen Oxidation and Etching, by Eliezer Weiss et al., J Appl Phys 69,2623(1991).

15. Novel Tunneling Barrier Designs for Resonant Tunneling Diodes, by Peng Cheng, PhD Thesis, 1991, 136 pp.

\section{TWO-DIMENSIONAL SIGNAL PROCESSING, OPTICAL INFORMATION STORAGE AND PROCESSING AND ELECTROMAGNETIC MEASUREMENTS}

Ronald W. Schafer

Demetrius Paris

Georgia Institute of Technology

Specific topics of research are: multidimensional digital signal processing, signal restoration and detection, morphological systems for multidimensional signal processing, multidimensional processing for sensory arrays, multiprocessor systems and tools for digital signal processing, linear and nonlinear image processing, two-dimensional optical storage and processing, semiconductor quantum wave devices, electromagnetic measurements in the time and frequency domains, and automated radiation measurements for near and far-field transformations.

Reports:

No. $1-11$ in previous editions.

12. Annual Report - Two-Dimensional Signal Processing Optical Information Storage and Processing, and Electromagnetic Measurements, by R.W. Schafer, TR, Jan 91, 79 pp.

13. Studies of Dipole Antennas on Finite Thickness Substrates with Planar Integrated Focusing Elements, by Mark Andrew Gouker, PhD Thesis, 1991, 236 pp.
14. The Use of Surface Impedance Concepts in the Finite-Difference Time-Domain (FDTD) Method, by James G. Maloney, MS, IEEE Trans on Ant and Prop.

15. The Efficient Modeling of Thin Materials Sheets in the Finite-Difference Time-Domain (FDTD) Method, by James G. Maloney, MS, IEEE Trans on Ant and Prop.

16. Measurements of Strip Dipole Antennas on Finite Thickness Substrates at $230 \mathrm{GHz}$, by Mark A. Gouker and Glenn S. Smith, MS, IEEE Microwave and Guided Wave Let.

17. Measured Electrical Constitutive Parameters of Soil as Functions of Frequency and Moisture Content, by Waymond R. Scott, Jr., and Glenn S. Smith, MS, IEEE Trans on Geosci and Remote Sensing.

18. Time Domain Filter Bank Analysis: A New Design Theory, by Kambiz Nayebi et al., MS, IEEE Trans on Acoust, Speech, and Signal Proc.

19. Nonuniform Filter Banks: A Reconstruction and Design Theory, by Kambiz Nayebi et al., MS, IEEE Trans on Acoust, Speech, and Signal Proc.

20. 2-D Zero Phase Rational Filters: Design and BoundaryValued Realization, by T.S. Rao and R.M. Mersereau, MS.

21. Determination of a Coherent Wave Field After Double Passage Through a Diffuser, by William T. Rhodes and Gisele Welch, MS, J Opt Soc Am.

22. Measurement and Calculation of Fields on the Outer Surface of Radomes, by Michael G. Guler et al., MS,

23. Spherical Probing Demonstrated on a Far-Field Range, by R.E. Wilson et al, MS.

24. Range Field Compensation, by D.N. Black et al., MS.

25. Resolution in Spherical Near-Field Microwave Holography, by Michael G. Guler et al., MS

26. Measurement of Edge Coincidence in Image Thresholdings, by Lois Hertz and Ronald W. Schafer, MS, $J$ Visual Commun and Image Representation.

\section{JOINT SERVICES ELECTRONICS PROGRAM}

\section{J.A. Harris, Jr.}

Stanford University

$\mathrm{N}$ - and p-channel MOS thin-film transistors (TFTs) have been fabricated in LPCVD polycrystalline silicon-germanium (poly-SiGe) films using a low-temperature (maximum process temperature $550^{\circ} \mathrm{C}$ ) fabrication process. A poly-SiGe TFT technology allows the use of lower anneal temperatures and shorter anneal times as compared to a poly-Si TFT technology, so that it is attractive for large-area electronics applications.

\section{Reports:}

1. The Entropy of a Randomly Stopped Sequence, by Laura Ekrott and Thomas M. Cover, IEEE Trans on Info Theory 37,1641(1991).

2. Information Theoretic Inequalities, by Amir Dembo et al., IEEE Trans on Info Theory 37,1501(1991). 
3. The Effects of Interfacial Chemistry on the Properties of Schottky Contacts to Gallium Arsenide, by Margaret Leslie Kniffin, PhD Thesis, 1990, 279 pp.

4. JSEP Annual Progress Report No. 1, by James S. Harris, Jr., TR, May 92, 84 pp.

5. Second-Order Susceptibility in Asymmetric Quantum Wells and Its Control by Proton Bombardment, by S.J.B. Yoo et al., Appl Phys Let 58,1724(1991).

6. Successive Refinement of Information, by William H.R. Equitz and Thomas M. Cover.

7. Spectral Weight Changes at the Superconducting Transition of $\mathrm{Bi}_{2} \mathrm{Sr}_{2} \mathrm{CaCu}_{2} \mathrm{O}_{8}+\mathrm{D}$, by D.S. Dessau et al., $J$ Phys Chem Sol 52,1401(1991).

8. A Low Temperature $\left(<550^{\circ} \mathrm{C}\right)$ Silicon-Germanium MOS Thin-Film Transistor Technology for Large-Area Electronics, by Tsu-Jae King and K.C. Saraswat, Proc IEEE, CH $3075,1991, \mathrm{p} 567$.

\section{THE ELECTROMAGNETIC SPECTRUM}

George Flynn

R. Osgood, Jr.

Columbia University

The evolution of interfacial reactions during the deposition of $\mathrm{Pt}$ and $\mathrm{Pd}$ on epitaxial $\mathrm{Si}_{1-x} \mathrm{Ge}_{x}$ alloys was studied using $\mathrm{x}$-ray photoelectron spectroscopy (XPS) for metal coverage up to $10 \AA$. Auger electron depth profiling was performed on a thicker metal overlayer before and after in-vacuo annealing to study the redistribution of composition in the reactions. Researchers found that $\mathrm{Pt}$ and $\mathrm{Pd}$ react mainly with $\mathrm{Si}$ to form silicides at $350^{\circ} \mathrm{C}$, leaving some $\mathrm{Ge}$ to segregate at the surface. These results were correlated with Schottky barrier height measurements. It was found that the Schottky barrier heights of $\mathrm{Pt} / n$ $\mathrm{Si}_{0.8} \mathrm{Ge}_{0.2}$ and $\mathrm{Pd} / n-\mathrm{Si}_{0.8} \mathrm{Ge}_{0.2}$ are about the same, pinned at $0.68 \mathrm{eV}$, which is much smaller than those of $n-\mathrm{Si}$. These barrier heights are quite stable up to $550^{\circ} \mathrm{C}$

\section{Reports:}

No. 1-2 in previous editions.

3. Iodomethane Dissociation on $\mathrm{Cu}(111)$ : Bonding and Chemistry of Adsorbed Methyl Groups, by Jong-Liang Lin and Brian E. Bent, MS, J Vac Sci Tech.

4. Carbon-Carbon Bond Forming Reactions on $\mathrm{Cu}(110) \mathrm{Sur}$ faces, by C.-M. Chiang et al., MS, J Vac Sci Tech.

5. Site-Blocking Effects in Temperature-Programmed Desorption Studies of Surface Decomposition Reactions, by Anumita Paul et al., MS, Surface Sci.

6. Analysis of the Structure of Light-Emitting Porous Silicon by Raman Scattering, by Zhifeng Sui et al., MS

7. Raman Analysis of Light-Emitting Porous Silicon, by Zhifeng Sui et al., Appl Phys Let 60,2086(1992).
8. GaSb-Oxide Removal and Surface Passivation Using an Electron Cyclotron Resonance Hydrogen Source, by Z. Lu et al., MS, J Vac Sci Tech.

9. Temperature and Adsorbate Dependence of the Image-Potential States on $\mathrm{Cu}(100)$, by Z. Wu et al., MS, Phys Rev.

10. Annual Progress Report No. 40. Research Investigation Directed Toward Extending the Useful Range of the Electromagnetic Spectrum, by George W. Flynn and Richard $M$. Osgood, Jr., TR, Dec 91, 85 pp.

11. General Trends in Changing Epilayer Strains Through the Application of Hydrostatic Pressure, by Judah A. Tuchman and Irving P. Herman, Phys Rev B45,11 929(1992).

12. Formation of Stoichiometric SiGe Oxide by Electron Cyclotron Resonance Plasma, by P.W. Li et al., MS.

13. Interfacial Reactions and Schottky Barriers of Pt and Pd on Epitaxial $\mathrm{Si}_{1-\mathrm{x}} \mathrm{Ge}_{\mathbf{x}}$ Alloys, by H.K. Liou et al., MS.

14. Exciton Photoluminescence in Strained and Unstrained ZnSe Under Hydrostatic Pressure, by Judah A. Techman et al., MS.

15. Photoluminescence of $\mathrm{ZnSe} / \mathrm{ZnMnSe}$ Superlattices Under Hydrostatic Pressure, by Judah A. Tuchman et al., MS.

\section{BASIC AND APPLIED RESEARCH IN ELECTRONICS AND OPTICS}

Jonathan Allen

Massachusetts Institute of Technology

InAlAs/InGaAs HFET's fabricated by conventional mesa isolation have a potential parasitic gate-leakage path where the gate metalization overlaps the exposed channel edge at the mesa sidewall. Researchers have unmistakably proven the existence of this path by fabricating special heterojunction diodes with different mesa-sidewall gate-metal overlap lengths. Sidewall leakage is a function of the crystallographic orientation of the sidewall, and increases with channel thickness, sidewall overlap area, and InAs mole fraction in the channel. In HFET's fabricated alongside the diodes, sidewall leakage increased the subthreshold and forward gate leakage currents, and reduced the breakdown voltage. A paper has been prepared which reports on the fabrication of quasi-one dimensional (Q1D) wires on modulation-doped GaAs/AlGaAs using a novel conformable $\mathrm{x}$-ray mask technology which allows one to expose arbitrary sized samples, including samples much smaller than the membrane area, using the laboratory's standard $31 \mathrm{~mm}$-diameter silicon-nitride $\mathrm{x}$-ray mask. After optical alignment, the sample and mask are brought into contact electrically, and then loaded into a specially designed cartridge which allows a vacuum to be pulled between mask and substrate. The vacuum causes the x-ray mask to conform around the sample. A vacuum hold-down is 
necessary to allow easy separation of the sample from the mask with minimal risk to both.

\section{Reports:}

1. Electromagnetic Calculation of Soft X-Ray Diffraction from $0.1 \mathrm{~mm}$-scale Gold Structures, by M.L. Schattenburg et al., MS, J Vac Sci Tech.

2. Femtosecond Time Division Interferometry Technique for Measuring the Tensor Components of $\mathrm{X}^{3}$, by $\mathrm{C}$. de $\mathrm{C}$. Chamon et al., MS, Appl Phys Let.

3. An Anti-Reflection Coating for Use with PMMA at $193 \mathrm{~nm}$, by Anthony Yen et al., J Electrochem Soc 139,616(1992).

4. Self-Focusing-Induced Saturable Loss for Laser Mode Locking, by David Huang et al., Opt Let 17,511(1992).

5. Ab Initio Theory of the Si(111)-7x7 Surface Reconstruction: A Challenge for Massively Parallel Computation, by Karl D. Brommer et al,, Phys Rev Let 68,1355(1992).

6. Proposed Method for Fabricating 50-nm-Period Gratings by Achromatic Holographic Lithography, by Anthony Hen et al., Appl Opt 31,2972(1992).

7. Elimination of Mesa-Sidewall Gate Leakage in InAlAs/InGaAs Heterostructures by Selective Sidewall Recessing, by Sandeep R. Bahl and J.A. del Alamo, IEEE Electron Device Let 13,195(1992).

8. An Achromatic Holographic Configuration for $100 \mathrm{~nm}-\mathrm{Pe}$ riod Lithography, by Anthony Yen et al., MS, Appl Opt.

9. Trapping and Focusing Ground State Atoms with Static Fields, by Wolfgang Ketterle and David E. Pritchard, MS, Appl Phys.

10. Atom Optics Using Microfabricated Structures, by C.R. Ekstrom et al., Appl Phys B54,369(1992).
11. Heterodyne Pump-Probe Technique for Time-Domain Studies of Optical Nonlinearities in Waveguides, by K.L. Hall et al., MS, Opt Let.

12. Classical Amplitude Squeezing for Precision Measurements, by Frank DiFilippo et al., MS, Phys Rev Let.

\section{H. Miscellaneous}

\section{COOPERATIVE RESEARCH IN INTEGRATED CIRCUIT TECHNOLOGY}

Larry W. Sumney

Semiconductor Research Corporation

\section{SC: ARL/EPSD, ARL, NVESD}

The research objective is to foster the U.S. semiconductor industry. The Army Research Office will join with members of the semiconductor industry as well as with government organizations (NIST, NSF, NSA, OSD, AF Wright R \& D Center and DNA) in providing support for the conduct of basic research in semiconductor electronics conducted at leading U.S. universities. Results of this research will be provided to the U.S. Army in the form of written reports as determined by the Semiconductor Research Corporation. 


\section{EUROPEAN RESEARCH PROGRAM}

\section{A. Mathematics and Physics}

\author{
6078 \\ USE AND ANALYSIS OF FINITE \\ ELEMENT METHODS FOR \\ PROBLEMS OF SOLID \\ MECHANICS AND FRACTURE
}

\author{
J.R. Whiteman \\ Brunel University \\ Uxbridge, Middx, UK \\ SC: ARO, ARL/MD, BWL, ARL/WTD.
}

In the context of linear elasticity extensive research continued on adaptive meshing techniques and the exploitation of superconvergence of the recovered gradients of finite element approximations to produce the error estimators which control the adaptivity. Several new recovery techniques were produced and efforts have continued to prove the superconvergence of these. The aim continued to be that of producing single robust schemes for adaptive mesh refinement of problems of linear elastic fracture. In the time dependent context, Whiteman, in collaboration with M.F. Wheeler of Rice University, completed the paper, report (1), which extends the above superconvergence of recovered gradients to discrete time/piece-wise linear Galerkin approximations for linear and nonlinear parabolic problems. This extension to transient problems is important because it opens the way to time dependent problems of solid mechanics. In viscoelasticity the paper (2) on crack growth in the context of Mode I linear viscoelastic fracture was published. At the same time, a complete theoretical error analysis was produced for discrete time/piece-wise linear Galerkin approximations to problems of linear viscoelasticity. These results are significant as they are the first convergence proofs for this approach to quasi-static linear viscoelasticity. A first draft of the long paper on this work has been produced.
Reports:

1. Superconvergence of Recovered Gradients of Discrete Time. Piecewise Linear Galerkin Approximations for Linear and Nonlinear Parabolic Problems, by M.F. Wheeler and J.R. Whiteman, Ms, Numerical Methods for Partial Differential Equations.

2. A Finite Element Model of Crack Growth in a Finite Body in the Context of Mode 1 Linear Viscoelastic Fracture, by M.K. Warby, J.R. Walton and J.R. Whiteman, Comp Meth Appl Mech Eng 97, 375-397, 1992.

3. Numerical Techniques for the Treatment of Quasistatic Solid Viscoelastic Stress Problems, by S. Shaw, M.K. Warby, J.R. Whiteman, C. Dawson and M.F. Wheeler, Ms.

\section{0}

\section{ELECTRONIC AND ATOMIC STRUCTURE OF SEMICONDUCTORS AND HIGH $T_{c}$ SUPERCONDUCTORS}

R. Car

E. Tosatti

Instituti di Fisica dell'Universita di Trieste

Trieste, Italy

\section{SL: ARL/EPSD, ARL, ARL/MD, NVESD, ARL/WTD, ARO}

The research consists of investigations in the electronic and atomic properties of semiconductors and superconductors using computer simulations and $a b$ initio quantum mechanical methods. Four main problems in condensed matter physics central to the research are: (a) microscopic simulation of the amorphous and liquid phase of elemental and compound semiconductors using the Car-Parrinello methods; (b) simulation studies of the diffusion of hydrogen and other defects in crystalline silicon; $(c)$ theory of the electronic and vibrational properties of semiconductors, semiconductor interfaces, and of GaAs based superlattices; and $(d)$ quantum simulations of highly correlated electron systems as models for high- $T_{\mathfrak{c}}$ superconductors. The computer simulations involving Monte Carlo techniques and the "CarParrinello" method will be used to study these topics; results of these calculations will be compared to experimental data and published in the open litera- 
ture. (a) First-principle simulations of hydrogenated amorphous silicon (a-Si:H) have been carried out, and a short review of the main results have been published. Also $n$-dopants in diamond have been studied, in collaboration with an American group. (b) New results on structure and bonding of solid and liquid gallium have been obtained, using CarParrinello techniques similar to those used for Si and GaAs. A first paper is now about to appear. (c) New simulations have been started for semiconductor surfaces, with strong progress mostly for clean $\mathrm{Si}(111) 2 \times 1$, for vacancies on $\mathrm{Si}(111) 2 \times 1$. A study of $\mathrm{Si}$ atom binding and diffusion on $\mathrm{Si}(100)$ has also been conducted in collaboration with an outside group. (d) Vacancy induced self-diffusion in silicon at high temperature has been studied with ab-initio molecular dynamics techniques. These results have been submitted. Research also continued on the vibrational properties of GaAlAs superlattices, focusing mainly on the effect of interface disorder on the Raman spectra.

\section{SYNTHESIS AND APPLICATIONS OF LARGE HETEROMETALLIC ASSEMBLIES}

D.J. Cardin

Trinity College

Dublin, Ireland

SL: NRDEC

SC: ARL/EPSD, ARO, EOARD

The main synthetic thrust in the latest period has been on the preparation of homo- and heterometallic clusters of cobalt (with mercury and cadmium) and ruthenium (with mercury) and of gold clusters. Characterization of the new materials is almost complete and measurements of optical nonlinearities will be reported in the next technical report. Additionally, Au-55 cluster has been donated by Professor G. Schmid (Universität Essen, Germany) and measurements are currently in progress. The incorporation of metal centers into conjugated polymer backbones has been accomplished for a variety of systems, and has been shown to lead to enhanced response. In an attempt to incorporate larger metallic particles, a successful synthesis of a polymer incorporating a bimetallic unit has been achieved. It is worth noting that even the bimetallic palladium cluster investigated exhibits a non-resonant nonlinearity comparable with oligomeric conjugated organic compounds. An apparatus for the preparation of Fullerenes has been built and commissioned. Chromatographic purification of the products has been optimized, and a number of new organometallic compounds have been prepared for further measurements. The compounds reported above have been measured both in the visible and the near infrared regions, with Z-scan and/or degenerate four-wave mixing. Reverse saturable absorption and resonant optical nonlinearity have been studied in detail in C-60 and C-70 and optical limiting effects have been observed and quantified. These results have been submitted for publication in Chemical Physics Letters.

\section{DETERMINISTIC METHODS IN STOCHASTIC CONTROL RESEARCH}

M.H.A. Davis

Imperial College

London, UK

SL: ARDEC

SC: NVESD, ARO, ARL/WTD

The existence of a Lagrange multiplier process was proved such that the cost functional made of the terminal cost of the partially observed optimal control problem, plus an integral Lagrange cost term, is stationary at the value of the optimal adapted control when this cost functional is defined on the class of possibly anticipating controls. A formula was obtained for the multiplier process involving random PDE adjoint to the robust Zakai equation. The computation is based on pathwise calculation of the Frechet derivative for the cost functional. The orthogonality property of the Lagrange multiplier with respect to past adapted integrable processes results for the partially observed maximum principle. If only a particular package of future information is given, which might be corrupted by noise, then the new Lagrange multiplier is equal to the conditional expectation of the old one with respect to the available filtration. It is shown that this Lagrange multiplier process is the unique solution of a second order backward Ito SPDE related to the smoothing problem associated to the given stochastic optimal control problem. The partially anticipative stochastic optimal control problem using an extension-of-filtration approach is considered in which future information is available in the form of a noisy package rather than as the whole future increments filtration. The extension of filtration leads to an extended nonanticipative 
optimal control problem. This is studied from a dynamic programming point of view.

Reports:

1. Random Conservation Laws and Global Solutions of Nonlinear SPDE. Application to the HJBSPDE of Anticipative Control, to appear in Springer Verlag, Lecture Notes in Control, Proceedings of Charlotte SPDE Conference, 1991.

2. Stochastic Partially Observed Optimal Control Via Deterministic Infinite Dimensional Control: On The Value of Information in Partially Observed, by B.H.A. Davis and G. Burstein, submitted to SLAM Joumal on Control and Optimization, 1992.

\section{B. Chemistry and Biological Sciences}

\section{STRUCTURAL AND BIOPHYSICAL CHARACTERIZATION OF A GLUTAMATE RECEPTOR}

P.N.R. Usherwood

University of Nottingham

Nottingham, UK

\section{E.A. Barnard}

University of Cambridge

Cambridge, UK

\section{SL: ERDEC, ARO}

SC: NRDEC

Molecular genetic techniques are being used to obtain a preparation of glutamate receptor (GluR) protein complex sufficient to enable further study of its structure and biophysical properties. The experimental approach taken by Professor Usherwood's group, in collaboration with Professor Barnard's group at Cambridge, is to include purification of GluR from locust muscle; identification and enrichment of the GluR messenger RNA via expression in Xenopus oocytes; cloning the complementary DNA encoding the GluR; incorporation of GluR in lipid bilayers; electrophysiological studies, and biophysical and structural characterization of reconstituted GluR complexes. They have succeeded in isolating and purifying the kainic acid (KA) GluR using the brain of Xenopus where it is exceptionally abundant. All the evidence obtained from the pure receptor indicated that surprisingly it consists of a single protein (containing several subunits) which possesses both the KA and the AMPA (alpha-amino-3hydroxy-5-methyl-isoxazole-propionate) receptor binding sites. The largest subunit (42K) appears to have high homology with a $48 \mathrm{~K}$ polypeptide that was isolated from the amphibian Rana by Wada. Reconstitution into asolectin/cholesterol bilayers has been successful, and channel openings were observed. The peptide sequences obtained for the Xenopus GluR were used to construct DNA primers, which in polymerase chain reaction yielded an 850-base fragment which has now been fully sequenced. This will be used as a probe to screen cDNA libraries from larval locust. The main objective is now to obtain full length cDNAs encoding subunits of the insect non-NMDA receptors of the nervous system. This contract is being extended a fourth and final year to characterize the full length cDNA which has been obtained in recent work. Additional molecular biology and full pharmacological studies will be undertaken. Also additional work with artificial bilayers containing membrane-spanning peptides will be completed. Detailed investigations revealed that a full length $\mathrm{cDNA}$ for a locust glutamate receptor (LGluR1) was not obtained in Professor Barnard's laboratory in Cambridge. Attention was focused, therefore, on two cDNA's that encode glutamate receptor subunits (DGluR1 and DGluR2) which have been isolated recently by the group of Professor Betz at the Max Planck-Institut für Hirnforschung, Frankfurt, Germany. Full length cDNAs encoding these receptors were used to obtain capped RNA transcripts and these were verified as full length transcripts. When the pharmacological properties of DGluR1 and DGluR2 have been fully evaluated an attempt will be made to overexpress them in either bacteria or a eucaryotic cell line. Further work has been undertaken with the TM2 region of LGluR1 emplaced in liquid bilayers. Blocking of spontaneous channels formed by this peptide has been accomplished with a synthetic analog (PhTX-343) of philanthotoxin-433 (PhTX-433).

\section{MOLECULAR IONIZATION AND DISSOCIATIVE IONIZATION AT HYPERTHERMAL SURFACE SCATTERING}

\section{A. Amirav}

Tel Aviv University

Tel Aviv, Israel

\section{SL: ARL/WTD \\ SC: ERDEC}

This research project proposes to study molecular ionization and dissociative ionization induced by a collision with a surface at hyperthermal energies. The 
PI has discovered that stable molecules such as anthracene, aniline, propyliodide, and fluorocarbons undergo collision-induced ionization from surfaces such as single crystal diamond. Molecules with low ionization potentials are ionized to give positive molecular ions. Those containing electronegative groups or atoms such as $\mathrm{Cl}, \mathrm{I}, \mathrm{Br}$, and $\mathrm{CN}$ undergo dissociative ionization to give negative halogen or $\mathrm{NO}_{2}$ ions and positive ions of the rest of the molecule. Recent experiments have shown that rhenium oxide appears to be a universal ionizer for all the organic molecules (and a large portion of inorganics) that have been tested. Ionization yield of close to 10 percent was achieved in a favorable molecule such as piperidine, while the yield in cyclohexanes and octane isomers was $0.001-.0001$ percent. This value is comparable to that of electron impact ionization, but with considerably smaller background. A similar enhancement of the molecular ion was seen with cholesterol. Ionization of clusters of carbon tetrachloride and other organic clusters are planned for future experiments. The main focus of the latest research was on the completion of the new and optimized apparatus, which included a new surface holder and manipulator, optimization of technical rhenium foil as the best practical surface for positive ion HSI, and installation of an electron impact ion source to help calibrate the HSI. HSI of cholesterol has been obtained with good efficiency. The degree of ion fragmentation is fully tunable, and one can obtain HSI-MS dominated by the undissociated molecular ion at low incident kinetic energy. Studies have just begun on isomer effects on the mass spectra of 1-, 2-, and 3-bromopentanes. The research continued as planned, and was focused on fast GC-MS with hyperthermal surface ionization and electron impact mass spectrometry. They have studied the fast GCMS of relatively non-volatile compounds, and a few seconds fast GC-MS was achieved with perylene, coronene, ovalene and phthalocyanine. The last three molecules are usually considered unamendable for conventional GC-MS, yet they are easily separated and detected in a few seconds without peak tailing. The high temperature limitations were studied and found to be the injector temperature. The fast GC-MS was used for the detection of "real world" target compounds such as lead in gasoline (tetraethyl lead), caffeine in instant coffee and codeine in Acamol-Forte drug. Full separation and quantitative results were achieved in just two seconds with hyperthermal surface ionization-single ion monitoring. Researchers found that the decaffeinated coffee is only 94 percent caffeine free and unleaded gasoline contains 5 percent relative lead which is artificially added. Explosives analysis with the fast GC-MS was attempted. Unfortunately, the samples of TNT, RDX and HMX were all impure as shown in the fast GC-MS analysis. TNT gave very characteristic hyperthermal surface ionization mass spectrum, while those of HMX and RDX appeared to be very complex in the fragmentation pattern and exhibited no molecular ion.

\section{MECHANISMS OF LASER INDUCED REACTIONS IN OPAQUE HETEROGENEOUS ENVIRONMENTS}

\section{F. Wilkinson}

Loughborough University of Technology

Leicestershire, UK

\section{SL: ARO}

SC: ERDEC, NRDEC, ARDEC

This research effort will further develop the technique of diffuse reflectance laser flash photolysis that was pioneered by Professor Wilkinson. Studies will include the determination of absorption and emission spectra with decay kinetics of photo-produced transient intermediates at interfaces within zeolite cavities, in polymers, and on dyed fabrics. Elementary reaction rate constants of various excited states and of free radicals will be obtained in these heterogeneous environments. The materials to be studied include organic ketones, aza-aromatics, nitro-compounds, and dyes. Electron transfer from triplet anthracene to methylviologen has been studied in methanol solution prior to studying this same reaction on a silica surface. A germanium based detector for time resolved studies of near infrared luminescence from the singlet delta excited state of oxygen has been successfully assembled and tested. In addition, various microcrystalline samples and dyes adsorbed on cotton fabric have been studied using diffuse reflectance laser flash photolysis, the results of which form part of a recent publication in the Joumal of the Indian Academy of Sciences. The luminescence spectra of $\mathrm{Cu}(\mathrm{I})$ zeolite $4 \mathrm{~A}$ and $\mathrm{Cu}(\mathrm{I})$ zeolite $13 \mathrm{X}$ prepared by various routes have been investigated. These spectra are dependent on the particular zeolite employed, sample preparation method and the water content. Copper zeolites are of current interest as catalysts for the decomposition of nitric oxide, a contributor to air pollution causea by internal combustion engines. A correlation be- 
tween the efficiency of singlet oxygen generation and the rate constant for oxygen quenching of the triplet state has been found for a range of substituted naphthalenes in benzene. Participation of charge transfer interactions is implicated on the basis that the only property which varies significantly with substituent is the oxidation potential of the naphthalene derivative. The investigation of oxygen quenching rates and singlet oxygen generation for a range of substituted naphthalenes in benzene has been extended to cyclohexane and acetonitrile solvents. The effect of varying the solvent is profound for those naphthalenes which have electron donating substituents (e.g. $\mathrm{OCH}_{3}$ ) but is only moderate for those which contain electron withdrawing groups (e.g., $-\mathrm{CN})$. The picosecond absorption measurements of organic molecule- $\mathrm{O}_{2}$ charge transfer complexes have been extended to studies of 1-methoxynaphthalene- $\mathrm{O}_{2}$ complexes which exhibit different behavior to naphthalene and 1-ethylnaphthalene complexes.

\section{CATALYTIC AGENT DEGRADATION ON OXIDE FILMS AND IN MICROHETEROGENEOUS SOLUTION SYSTEMS}

M. Gratzel

Swiss Federal Polytechnic Institute

Lausanne, Switzerland

SL: ERDEC

SC: ARO, ERDEC, NRDEC

This new start at the beginning of FY90 is a continuation of the catalytic photo-oxidation research that was under study during Professor Gratzel's last funding period. He proposes to investigate the destruction of simulants of toxic chemicals on the surfaces of oxide semiconductors, particles, or colloids, in particular oxides of $\mathrm{Ti}, \mathrm{Fe}, \mathrm{Zn}, \mathrm{Ni}$, and $\mathrm{V}$. The principal pathways that have been found are oxidative mineralization and nucleophilic cleavage reactions. In recent experiments, both photocatalytic and dark decompositions of organophosphate compounds diethylbenzylphosphonate and diethylethylphosphonate were investigated in the presence of hydrogen peroxide as oxidant. It was found that ferric sulfate greatly accelerates the complete mineralization of both these phosphonates as well as the substituted aromatic compound nitro-o-xylene. The catalytic and photocatalytic studies were extended to include DREX, a VX simulant, and 4-chlorophenol.
The strong catalytic effect of iron (III) complexes in oxidizing these compounds by $\mathrm{H}_{2} \mathrm{O}_{2}$ and the enhancement by visible light were demonstrated also for these compounds. The pathway is not a Fenton type reaction producing hydroxyl radicals but the oxidation of the organic species proceeds via an intermediate high-valent iron-oxo species. Clay supported-Fe(III) was found to be very active in promoting the oxidation of organic species by $\mathrm{H}_{2} \mathrm{O}_{2}$. The advantage of this heterogeneous system as compared to $\mathrm{Fe}$ (III) homogeneous catalysis is the possibility of reuse of the catalyst. $\mathrm{TiO}_{2}$ films with very large surface areas were found to be another catalytic system for promoting organic compound degradation.

\section{THERMODYNAMICS OF ELECTROLYTE SOLUTIONS FOR USE IN HIGH ENERGY LITHIUM BATTERIES}

\author{
A.F. Danil de Namor \\ University of Surrey \\ Guildford, Surrey, UK \\ SL: ARL/EPSD \\ SC: ARO, ERDEC
}

A cell utilizing a lithium anode requires a non-aqueous solvent. Unfortunately, lithium salts in such solutions have very low electrolytic conductances, due to the low dielectric constants as well as to the tendency to form nonconducting species, such as ion pairs, dimers and as yet unidentified aggregates. To prevent the formation of such nonconducting species in solution, electrolytes are required which have very large ion radii. To decrease the tendency of the lithium ions to associate with the anions, it is also necessary to use lithium salts having anions of very large radius, such as for instance $\mathrm{AsF}_{6}$ - . Solutions to the problems outlined above can be found in the area of macrocyclic chemistry. One of the remarkable properties of ligands such as crown ethers is their capability to solubilize metal salts in nonaqueous solvents. The organic cations formed have higher solubilities than the uncomplexed cations. In addition, the tendency to form nonconducting species, such as ion pairs, will be greatly reduced. The main objective of this project is to obtain thermodynamic data on these complexing reactions which will form a basis for the selection of new electrolytes for use in high energy lithium batteries. The main tool for the determination of the free energy, enthalpy and entropy changes 
will be titration microcalorimetry. Also investigated will be the solution thermodynamics of lithium salts with large anions and of such salts including macrocyclic ligands in non-aqueous solvents. Synthesis and structural characterization of the lithium complexes will be carried out prior to the investigation of the solution properties.

\section{Aeronautics and Mechanics}

\section{RADIAL INFLOW TURBINE STUDY}

R.L. Elder

Cranfield Institute of Technology

Cranfield, UK

\section{SC: TACOM, PROP DIR, ARO}

This research investigation has technical objectives of (a) obtaining an improved basic understanding of the flow processes in high speed radial inflow turbines and $(b)$ obtaining needed experimental data to validate computational codes being developed by the Army and others. The objectives are being achieved by an experimental study of two comparative turbine rotors. The two test rotors are a metal rotor already in use, and a second rotor, also manufactured in metal, but to the design of a ceramic rotor. These rotors are incorporated into an existing experimental rig at Cranfield which is modified to accept the turbine unit. The rig includes conventional instrumentation to define turbine overall performance and laser anemometry to probe the detailed flow within the turbine. The laser anemometer is non-intrusive, has a spatial resolution small enough to measure flows within the passages of interest, and can be used in rotating blade rows (where strobing the data collection electronics with the blade passing frequency will allow the blade to blade variation of the flow velocity to be measured). The major objective of the study is to relate the detailed flow conditions with overall performance parameters obtained from turbine calibration. Such parameters are mass flow and enthalpy, and particular attention is being given to ensuring that the point measurements of the laser anemometer provide "bulk mean" parameters which agree with overall performance values. Laser anemometry measurements have been completed at various positions downstream of the rotor for various values of pressure ratio. Presently, the flow at the turbine inlet (between the guide vanes and the rotor) is being examined.

\section{Materials Science}

\section{THE SOL-GEL-XEROGEL TRANSITION IN SILICA GLASS AND NOVEL ORGANIC DYE/INORGANIC GLASS PHOTOACTIVE MATERIALS}

David Avnir

The Hebrew University of Jerusalem

Jerusalem, Israel

\section{SL: ARL/MD, NVESD}

Photophysical probes will be used to characterize the structural changes during monomer-sol-gel-xerogel transitions and to develop room temperature processes for preparing novel organic dye/- inorganic glass photoactive materials. The phenomenon of room temperature phosphorescence (RTP) of trapped organic molecules, such as Rhodamine 6A/B in silica glass made by sol gel process, the RTP lifetimes and RTP intensities will be determined as a function of gelation time, changing parameters like $\mathrm{pH}$, water/silane ratio, alkoxy groups and the metal atom. Organic photochromic dye/inorganic oxide glass, will be tested for photophysical performance, stability and rate of color changes. The development of photophysical probes for the study of the sol-gel process will continue employing new probes, both of the pyrene family and of other well tested molecules. Small angle $\mathrm{x}$-ray and neutron scatterings will be used for materials characterization, with special emphasis on the application of recent theories of scattering from fractal objects. The role of water in determining the structural properties of the final product as well as the details of the kinetic behavior will be investigated. And, finally, the possibilities of using monolithic blocks or thin films in which organic laser dyes are trapped will be explored as possible substitutes for dye solutions in dye lasers. A new direction in the development of organic dye/inorganic glass photoactive materials is based on the observation that there is a sub-population of the trapped molecules, which, although encapsulated in the matrix, has an accessibility to external reagents through the pore volume. This observation is currently used for the development of sol-gel glasses as photocatalysts. In a preliminary study, photoinduced electron transfer between $\left\{\operatorname{Ir}(\mathrm{bpy})_{2}\left(\mathrm{C}_{3}, \mathrm{~N}^{\prime}\right) \mathrm{bpy}\right\}^{3+}, \mathrm{IR}(\mathrm{III})$ trapped in a porous silica glass generated by the sol-gel process, and 1-4-dimethoxybenzene (DMB) dissolved in a water phase in the pores of the glass, was observed. The kinetics of the redox reactions associated with the Ir(II) ${ }^{+} \mathrm{DMB}^{+}$radical ion pair were studied at dif- 
ferent $\mathrm{pH}$ and ionic strength values. A retardation of 4 orders of magnitude of the back-electron-transfer process with respect to homogeneous solutions was observed at neutral $\mathrm{pH}$ for 25 percent of the generated $\operatorname{Ir}(\mathrm{II})$. The effect is attributed to the immobilization of IR(II) by trapping in the glass matrix and of $\mathrm{DMB}^{+}$by adsorption on the pores' surface. At acidic $\mathrm{pH}$, retardation of the back-reaction leads to catalyzed hydrogen generation from water. One of the major aims of this project has come to completion, namely, the preparation of photoactive sol-gel glasses exhibiting room-temperature phosphorescence. A remarkable manifestation of the special properties of the silica sol-gel cage is the observation that many trapped organic molecules exhibit efficient phosphorescence at room temperature even when exposed to air, and in many instances even without the aid of a heavy atom, and in several cases, even in the wet gel. It is noted that phosphorescence is a delicate process which is usually quenched at temperatures which are not cryogenic and by exposure to oxygen. The generality of the phenomenon was demonstrated with the following molecules: phenanthrene, naphthalene, quinine, 4-biphenyl carboxylic acid, 1-naphthoic acid, eosin-y and pyrene. It was observed that under various gelation conditions, most dyes exhibited not only phosphorescence but also delayed fluorescence. Specific glasses were needed to observe phosphorescence from the various glasses. For example, neutral conditions were sufficient to observe phosphorescence from phenanthrene, naphthalene and quinine, but basic conditions were needed for the two carboxylic acids and for eosin. A heavy atom $\left(\mathrm{Br}^{-}\right)$was needed in order to observe phosphorescence from pyrene, and an $\mathrm{SiO}_{2}$ glass doped with $\mathrm{NaBr}$ was therefore prepared. Very long emission lifetimes were observed. For instance, when biphenylcarboxylic acid was trapped in an $\mathrm{SiO}_{2}$ glass prepared under basic conditions, the lifetime reached the order of several seconds. Lifetimes of the order of milliseconds were obtained even from the wet gels. A detailed mechanistic study of the sol-gel transition was carried out with that carboxylic acid, including an Arrhenius type analysis of the transition between the delayed fluorescence mode and the phosphorescence mode. An obvious potential application of these findings would be an attempt to obtain a phosphorescent laser. A major advancement has been made in the long standing problem of photoinduced charge separation using the sol-gel method, and has recently appeared in Nature 355, 240 (1992). In brief, the problem is the following: A key feature in the intricate mechanism of natural photosynthesis is the initial storage of a substantial fraction of the light energy in the form of a long-lived radical pair. Attempts to mimic this natural process in artificial systems have been one of the major undertakings of modern photochemistry. However, although impressive progress has been made in increasing the efficiency of the forward reaction (1), e.g., between an excited photosensitizer $A^{*}$ and an appropriate electron acceptor, $B$, prevention of the energy-wasting back reaction (2) still constitutes a major challenge:

$$
\begin{aligned}
& \mathrm{A}^{*}+\mathrm{B}-\mathrm{A}^{-}+\mathrm{B}^{-}(1) \\
& \mathrm{A}^{+}+\mathrm{B}^{-}-\mathrm{A}+\mathrm{B}(2)
\end{aligned}
$$

The back-reaction (2), which in homogeneous solutions is usually diffusion-controlled, must be slowed down in order to allow the radical pair formed in reaction (1) to undergo subsequent (catalytic) processes leading to useful energy-rich products. A general approach used to achieve long-lived charge separation is based on the design of a variety of heterogeneous environments for carrying out the reaction. Radical pairs with lifetimes on the order of up to a few minutes have been produced in such systems. The present study has achieved what is believed to be the longest- lived photoinduced charge separation in an artificial photosynthetic system, without the involvement of a secondary ("sacrificial") substrate which reacts with either $\mathrm{A}^{+}$or $\mathrm{B}^{-}$. This result was attained by the trapping of both $A$ and $B$ in a porous solid matrix. Oxidation of $A$ and reduction of $B$ is carried out, indirectly and unidirectionally, by a third mobile charge carrier in the intrapore space. Spatial separation of the immobilized $\mathrm{A}^{+}$and $\mathrm{B}^{-}$pair inhibits the back reaction. The specific photochemical reaction system in which the observation was made in the reduction of sol-gel immobilized N,Ndimethyl-4,4-bipyridinium chloride (methyl viologen, $\mathrm{MV}^{2+}$ ) to $\mathrm{MV}^{+}$by immobilized excited state pyrene $\left(\mathrm{Py}^{*}\right)$, through mediation by the mobile charge carrier, $\mathrm{N}, \mathrm{N}$-tetramethylene-2,2-bipyridinium bromide $\left(\mathrm{TV}^{2+}\right)$. The back electron transfer in this artificial photoredox system is retarded by a factor of seven orders of magnitude with respect to homogeneous solutions. A portion of the generated (immobilized) charge separated pair, $\mathrm{Py}^{+}$and $\mathrm{MV}^{+}$, was observed on a time scale of several hours. The observed retardation factor is larger by at least four orders of magnitude compared to previous data in artificial microenvironments. 


\section{A NEW APPROACH TO TUNABLE} LASER MATERIALS

R. Reisfeld

The Hebrew University of Jerusalem

Jerusalem, Israel

SL: NVESD, ARL/EPSD, ARL

SC: MICOM, ARL/EPSD, ARL, ARL/MD, ARO

The Solid State Tunable Dye Laser has shown good photostability when operated in the range of a few hundreds $\mu \mathrm{j} /$ pulse and with slope efficiency of about 7.5 percent. This is due to a stable perylene dye embedded in a composite glass, which is a sol-gel glass impregnated by a polymethyl methacrylate polymer. Professor Reisfeld's group are currently improving both slope efficiency and optical quality of the laser material. One of the purposes of this contract is the investigation of thin film dye laser, namely waveguide light confined in a thin transparent film doped by a dye. Investigations will follow three directions: 1. Photostable dyes and suitable solid hosts for them. 2. Controlled refractive index of the film for the purpose of matching the thickness and refractive index to obtain a single mode waveguide. 3. Possibility of nonlinear optical effects in the waveguide. 1. Malachite Green introduced into glasses exhibits a characteristic spectral behavior depending on the mode of preparation of the glass and its environment. Neutral mono-cation and di-cation forms can be prepared. Exceptionally high quantum efficiency is obtained with ammonia treatment. An analogy is found in the structure with electron or proton transfer. Very high affinity of this dye to sol-gel glass makes easy the preparation of the doped glasses at various optical densities. This can find application as saturable absorber for femtosecond lasers and also as nonlinear optical waveguide for high laser powers. 2. A red perylene derivative was impregnated into a composite silica gel glass, and characterized as a dye laser material. The absorption spectrum in the range 480 $600 \mathrm{~nm}$, belongs to the $S_{0}-S_{1}$ electron transition, with a structure reflecting the perylene skeletal vibrations of typical energy $1100-1200 \mathrm{~cm}^{-1}$. An additional peak between 400 and $460 \mathrm{~nm}$ belongs to the $S_{0}-S_{2}$ transition. The fluorescence exhibits a mirror image relative to the $S_{0}-S_{1}$ absorption, with Stokes shift of about $40 \mathrm{~nm}$ for the 0-0 transition. Laser tunability was obtained in the range $605-630 \mathrm{~nm}$ using a $532 \mathrm{~nm}$ pump beam (a frequency doubled Nd:YAG laser). This wavelength range is important for medical applications such as photodynamic therapy of some cancer tumors. Maximum laser efficiency was ob- tained at $613 \mathrm{~nm}$. Maximum output was on the order of $0.2 \mathrm{~mJ} / \mathrm{pulse}$ at a repetition rate of $10 \mathrm{~Hz}$. The laser threshold was about $780 \mu \mathrm{J} /$ pulse for excitation with the green $(510 \mathrm{~nm})$ line of a copper vapor laser. The laser output was stable over more than $\sim 500,000$ pulses of excitation of energy density $\sim 40 \mathrm{~mJ} / \mathrm{cm}^{2}$ per pulse.

\section{Reports:}

1. Spectroscopic Behavior of Malachite Green in Sol-Gel Glasses, by V. Chernyak and R. Reisfeld, Chem Phys Let, 181 (1991) 39.

2. New Solid Glass Laser for Photodynamic Therapy, by R. Gvishi and R. Reisfeld, 2nd Intl Conf Laser M2P, Grenoble, July 9-11, 1991. To be published in J Physique (Colloques) 1991.

3. Glasses Including Quantum Dots of Cadmium Sulfide, Silver and Laser Dyes, by $R$. Reisfeld et al, to be published in SPIE Proc 1590 (1991).

4. New Tunable Solid State Glass Lasers in the Visible (Abstract of invited lecture at Seventh Interdisciplinary Laser Science Conference, Monterey, September 1991), by R. Reisfeld, Bull A Phy Soc, 36 (1991) 1964.

\section{HIERARCHICAL STRUCTURE IN ADVANCED POLYMERS: PHASE BEHAVIOR, ORIENTATION, PROPERTIES}

\author{
A. Keller \\ J.A. Odell \\ University of Bristol \\ Bristol, UK
}

\section{SL: ARO, ARL/MD}

This is a new research three year project building upon the recently completed work 5548 reported last year. This project will explore polymeric structure and its relation to properties with special emphasis on the underlying hierarchical principles encompassing phase behavior, including phase segregation in bicomponent systems, with emphasis on molecular flexibility including also natural macromolecules. The specific investigations fall in two categories: 1 . The establishment of phase behavior with relevance to phase morphology structure and resulting properties. This is to embrace: Single component thermotropic systems, bicomponent solvent polymer and polymer-polymer systems capable of liquid-liquid, liquid-liquid crystal and liquid crystal - true crystal phase segregations. The exploration of phase diagrams will involve lyotropic and thermotropic states within the same material and the interception of the phase separation processes by vitrification with the added parameter of pressure where appropriate. 
The exploration of structure and structure property relations on all principal levels of hierarchy will be investigated. These comprise phase structures as arising, e.g., from the bicomponent phase behavior, the texture within the liquid crystalline state and its degree of perfection and crystal morphology. Molecular aspects arising from mesogen spacer ratios will also be included. 2. Orientation - Processing. Chain extension and chain alignment will be followed by special elongational flow methodology. The principal variable is chain flexibility ranging from highly flexible to rigid rod molecules. Special features included will be the relaxation process from the oriented (extended) state which will relate to formation of banded structures, and (in combination with concentration and/or temperature gradients), to the skincore structures in processed liquid crystal forming polymeric materials. The influence of orientation on phase separation and the resulting morphologies on all levels of the structure hierarchy will be explored. The ultimate aim will be the optimization of properties in terms of molecular flexibility, phase behavior, microstructure and orientation/processing parameters, comprising synthetic materials but with lessons being taken from nature. I. Phase Relations. The class in which the liquid crystal phase is metastable, (monotropic) was chosen for in-depth examination as regards the influence of the first formed (but metastable) liquid crystal (LC) phase on the subsequent formation of crystals (C). The two aspects examined were rate of crystallization and morphology. It was found that the overall rate of crystallization was greatly enhanced by the preexisting LC phase in cases where LC formed first. This was found to be due to enhanced nucleation rate rather than growth rate. The enhancement of nucleation rate is at least in qualitative agreement with expectations yet leaving some open ended question on the nature of the crystallization-promoting nuclei. Three distinct morphologies were identified on the level of the optical microscope: $(a)$ Without the pre-existence of LC phase the morphology is the usual spherulites. (b) on transformation from the LC phase at low supercoolings, the characteristic LC texture reorganizes into large scale "spherulite type" textures of two distinct classes: one which is highly birefringent, grows fast, yet nucleates sparsely, and another which is less birefringent, more slowly growing, but which nucleates abundantly, remnants of the original LC texture, (fine mottle) being incorporated into both. (c) on transformation from the LC phase at high supercoolings, the characteristic LC texture (mottle) is preserved without change. The above has many implications on the structure hierarchy and the underlying mechanism of LC-C transformation. II. Large Scale Textures: This effect, discovered in the course of the previous ERO contract, has been pursued. Significant new information was obtained on their origin and structure. Thus, the significance of the orientational effect of elongational flow became apparent, resulting from concentric contraction while in the isotropic phase. This has important implications for processing and handling (even accidental) of melts of intrinsically LC polymers. Further, a fine banded structure arising while passing through the nematic state connects this subject with another major area of LCP studies concerned with the initial relaxation from the oriented state. In this case, the banded structure appearing in combination with the large scale structures offers definitive opportunities for optical analysis of the underlying orientation relations, and of their dependence on flow conditions and sample type (molecular weight). A new successful start with electron microscopy has opened a window into the finer scale aspects of the structure hierarchy underlying these texture features and mechanisms. III. In situ shear-flow study on orientation-disorientation by $\mathrm{x}$-ray diffraction using a synchrotron source. The first experimental series was exceptionally productive, evaluation awaiting data retrieval is currently in progress. The work reported is divided into two parts; one on thermotropic polymers and the other on lyotropic systems. In part one, an optical study was performed on the effects of shear-rate upon the subsequent development of relaxation banding, particularly the band spacing, in thermotropic PHMS 5/7, a mesogenic stilbene-alkyl flexible spacer copolymer. At the highest shear-rates a monodomain texture is observed, free of visual banding. As the shear-rate is progressively reduced, banding is observed, the spacing reduces rapidly, going through a marked minimum before increasing again with further reduced shear-rate. At the lowest shear-rates, the bands subdivide to become narrow again. Speculation upon the molecular origin of these phenomena was given. New results on the morphology of banded textures from TEM and optical microscopy and light scattering studies were discussed, as well as the observation of banded textures which arise from nematic monodomains without applied shear. This is only seen for crystallizable polymers, leading to speculation about the role of free volume in the origin of banding. Concerning the lyotropic PBO/PBZT Poly(Phosphoric Acid) (PPA) system, the report discusses the importance of the PPA solvent to the phase behavior, rheology and structure of 
the materials, and identifies a novel high temperature crystal-solvate phase. TEM/optical studies on relaxation banding in PBO/PBZT identified a transition from sinusoidal banding in the earliest stages of relaxation to a zig-zag structure in the final stages. This is consistent with the idea that the origin of banding lies in the localization of splay within the nematic mesophase.

\section{ADIABATIC SHEAR BANDING AT DIFFERENT IMPACT VELOCITIES}

\author{
J.R. Klepaczko \\ University of Metz \\ Metz, France \\ SL: ARL/MD \\ SC: ARL/WTD
}

This is a new research program funded late in FY90 to perform experimental and numerical studies of adiabatic shear banding in metals at various high strain rates. Material behavior as a function of rate of shearing will be determined. Double-notch specimens, supported by instrumented Hopkinson bar, will be loaded by direct impact and analyzed for materials behavior. Parallel numerical calculations will be made using existing finite-difference codes to introduce different initial conditions of loading expected in the experiments. Various metal alloys and/or composite materials will be tested in consultation with the COR and Army laboratory personnel. A new experimental technique to study adiabatic shear bands at different displacement velocities has been pursued. The technique is in its early stage of development, i.e., the mechanical parts have been machined and the experimental setup has been assembled. The measuring techniques are in preparation. The experimental technique for studying fast shearing events in materials has been made operational and initial tests on low alloy mild steel were conducted to determine the critical shearing parameters as a function of impact velocity. Strain rates on the lower and upper yield limits were determined up to a strain rate on the order of $105 / \mathrm{sec}$. Lower yield stresses of $200 \mathrm{MPa}$ at low strain rates $\mathrm{f} / \mathrm{sec}$ were found to increase sharply to $600-700 \mathrm{MPa}$, at high strain rates $104 / \mathrm{sec}$. Similar trends were found as the upper yield increased from 0.2 to $1.0 \mathrm{GPa}$ shear stress.

\section{E. Environmental Sciences}

\section{PARTICLE DYNAMICS AND \\ GRAVEL STREAM-BED \\ ADJUSTMENTS}

P.A. Carling

Freshwater Biological Association

Windermere Laboratory

Ambleside, Cumbria, UK

This is a new three year research effort, and the objective is to better understand the initial motion processes of particles in coarse mixtures in gradually varied natural flows, the nature of particle motion, and the relationship between local sediment sources and sinks, kinematic waves of particles and rapid changes in bed level. The research program will be carried out in Squaw-Creek in Montana, an ideal study location as it has a snow-melt regime in April and May of each year. Steady changes in stage occur each day in response to diurnal changes in snow pack melt rates which produces daily transport of bedload. The passage of individual particles and kinematic waves of particles will be recorded passing into the experimental reach and exiting from the reach using an existing electromagnetic coil placed across the river. Detailed bed level fluctuations at selected sections will be monitored using in situ profiles and detailed velocity profiles obtained across sections using a boundary-layer rig consisting of six current meters recording simultaneously. This will lead to the establishment of velocity vectors and shear stress distributions across the bed. Bedload samples at critical sections will be obtained using Hollev-Smith samples while individual particle motion will be studied using the radio-tagging and magnetic methods. The results will culminate to develop theoretical bedload transport relationships and models of sedimentary sorting (including the armoring process) which have practical application in river hydraulics. During the Spring of 1990, April to June, field instrumentation commenced. An additional temporary bridge was erected, power supply connected and the field sensors and recording equipment was field tested during a series of moderately high flows. It appears that 1990 was an unusual year with small snow pack and low temperatures. A considerable snow-pack and high air temperatures occurred in 1991, unlike 1990. Long continuous multi-day records of pebble transport were obtained using the detector log. Preliminary observation indicated that transport was in waves approximately every $30 \mathrm{~s}$. 
These records were supplemented by detailed hydraulic data collected during two large hydrographs over 24-hour periods. Data include depth, velocity, and shear stress distributions as well as fluctuations in bed level which can be correlated with the bedload records. The field data for 1991 has been analyzed in a preliminary fashion. This data demonstrates bed level fluctuations during the passage of snow-melt hydrographs when bedload transport occurs. The passage of an unsteady wave of fine bedload over a coarser immobile bed leads to unexpected change in hydraulic roughness as characterized by the DarcyWeisbach roughness coefficient. This is contrary to the usual assumption that total bed roughness falls with rising discharge. This result has implications for modeling flood flows and sediment routing. Four acoustic water level sensors were installed in the field in April 1992 to monitor water level fluctuations at 1 sec intervals during the passage of hydrographs. These proved an improvement over older float operated water level sensors. A vortex-type bedload trap was constructed at the site and tested for sampling efficiency. Unfortunately, runoff this year was slight owing to inadequate snow-pack and consequently no bedload transport occurred. Dr. J. Williams of the Bidstone Observatory of the UK Institute of Oceanographic Sciences completed a series of measurements of turbulent fluctuations in the flow. Preliminary indications are that these are of high quality and represent the first successful measurements of high-resolution fluctuations of current vectors in a mountain stream. In addition, a hydrophone was calibrated to record only the passage of bedload transport, but could not be used in earnest owing to the low flows encountered. Additional measurements of the vertical velocity distribution over rough gravel beds were made using a small dorsally-flattened electromagnetic current meter at one-half to one centimeter intervals in the vertical. This data has yet to be processed, but preliminary plots indicate high-quality definition of the structure and extent of the boundary-layer has been obtained. Additional measurements were also made across the section using impeller flow-meters and electromagnetic meters to determine the nature of secondary-flow circulation patterns. Further improvement and calibration of the magnetic bedload detection system was undertaken by Prof. G. Christaller from Berlin. Prof. Ergenzinger presented a paper concerning results from 1991 at a conference in Bavaria in June 1992.

\section{6}

\section{IMPROVING THE \\ CHARACTERIZATION OF ROCK STRUCTURE GEOMETRY}

\section{J.A. Hudson}

Rock Engineering Consultants

Welwyn Garden City, Herts, UK

In any rock engineering problem, there are a multitude of rock properties that could be measured. Similarly, there are a multitude of proposed rock mechanics mechanisms that can be invoked, and the structure can be designed to defend against many types of potential collapse mechanisms. Furthermore, all these properties and mechanisms influence one another to a greater or lesser extent. One is faced, therefore, with making a decision in an increasingly complex world on how to proceed from the original "top of the flowchart" rock engineering requirement. In order to identify and present the interactions between rock mechanics and rock engineering factors in these conditions, the author has previously proposed the use of rock mechanics interaction matrices. It has recently been realized that this approach is in fact a sub-set of a far wider set of procedural and analysis techniques based on information technology. The purpose of this research is to develop a procedure to enable a client, engineer, contractor or researcher to decide for any project the relevant rock properties and their priority, relevant boundary conditions, rock mechanics mechanisms that should be studied, the interaction between these, and hence the overall type and sequence of site investigation, analysis, design, construction and monitoring that should be conducted for optimal use of the resources available. During 1991, the work was concentrated on analysis of the consequences of different engineering procedures in achieving a given rock engineering objective. This is established by considering the pathways through the interaction matrix - the basic device used to list the primary parameters and all their intentions. The purpose of this research is to optimize engineering design and avoid adverse combinations of parameters; i.e., bad or worse-case scenarios. Task 2, interpretation and development of presentation, was completed and Task 3, computerization of system, is approaching completion. A particularly exciting development is the possibility of neutral network analysis of engineering processes to provide enhanced cognition of the complex rock engineering systems. 


\section{F. Electronics and Computer Sciences}

\section{ENHANCED BACKSCATTERING FROM ROUGH SURFACES}

J.C. Dainty

Imperial College of Science and Technology

London, UK

SL: ARDEC

SC: CECOM

The objective of this project is to conduct unique experiments and data analysis on the scattering of light by rough surfaces. Four primary areas are to be emphasized: 1. Angular scattering by one-dimensional surfaces involving measurements of the average intensity as a function of scattering angle. 2 . Angular scattering by two-dimensional surfaces including measurements of the average intensity as a function of the in-plane scattering angle with regard to polarization. 3. Angular and wavelength correlation of the higher order statistics of the scattered intensity in order to distinguish between single and multiple scattering events. 4. Development of computer codes to simulate scattering from random rough surfaces and from periodic gratings. Experiments are showing significant differences in the angular correlation for single and multiple scattering surfaces. Analytical theory has been developed to deal with the simple scattering case and computer simulations indicate interesting (but not yet understood) differences between the single and multiple scattering case. Multiple scattering investigations from volumes have been initiated in collaboration with Prof. Yu Barabanenker of the Institute of RadioEngineering and Electronics, Moscow. In collaboration with Prof. R. Depine of the University of Buenos Aires, new polarization related efforts have been encountered in gratings with small additional surface roughness.

\section{ION BEAM MIXING IN MULTIPLE QUANTUM WELL STRUCTURES}

B.L. Weiss

University of Surrey

Guildford, Surry, England

SL: ARDEC

SC: ARL/EPSD

The aim of this research is to investigate the use of ion implantation to induce mixing of multiple quan- tum well (MQW) structures and to study its application to the fabrication of optical waveguide devices in III-V semiconductor materials. These experiments are designed to clarify the physical mechanisms involved in the mixing of MOW layers and to determine the optical changes produced by this mixing. Several different III-V semiconductor materials are being studied in this effort along with various implanted ions and annealing techniques. The research also involves development of computer codes to model various optical waveguides including buried, stripe geometry, MQW structures. Waveguide experiments are being carried out at several different infrared wavelengths to establish the optical properties of MQW structures before and after implantation. Comparison with diffusion techniques for achieving MQW layer mixing will be made and practical structures, such as non-square wells, will be analyzed. Ellipsometric measurements are to be performed and changes in the refractive indices are to be correlated with material properties and implantation procedures. This research should yield valuable information relating to the processing of MQW layers for laser fabrication and for optical computing devices required in future Army systems. Over the period of research covered, considerable progress has been made with regard to the implantation induced disordering of $\mathrm{AlGaAs} / \mathrm{GaAs}$ quantum well structures and the modeling of the optical properties (absorption coefficient and refractive index) of quantum well structures. Several papers covering this work have been written and submitted for publication. The project is now nearing its end and the various aspects of this research are being brought to logical conclusions. Basically, this project has developed the understanding of the fabrication and optical properties on non-square quantum well structures for device applications. The realization of devices with improved characteristics remains to be addressed in future projects.

\section{1}

\section{NOVEL OPTOELECTRONIC DEVICES BASED ON COMBINING GaAs AND InP ON Si}

P. Demeester

University of Ghent

Ghent, Belgium

\section{SL: ARDEC, ARO}

This research project addresses some of the basic technological procedures necessary in the fabrication of optoelectronic devices for optical intercon- 
nects and for optical computing. There are four main areas that are included in this research. The first is optimization of the heteroepitaxial growth of GaAs on InP and on Si for the development of high quality optical waveguide and laser structures. The second involves investigation of nonplanar growth techniques for the integration of lasers and optical waveguides in a single-step growth process. The third is based upon advances in the growth techniques and includes efforts to fabricate an optical modulator based on InP multiple quantum wells grown on a $\mathrm{Si}$ substrate. The final topic includes a study of the use of an epi-liftoff technique for the quasi-monolithic integration of III-V devices. This technique is an alternative to heteroepitaxy and may alleviate some the more serious difficulties encountered in such growth. Finally, the results of these investigations are to be combined to develop optical interconnect schemes for use in large volume, high density optoelectronic systems. Such systems are required in massive electronic computers and in optical signal processors for Army target tracking applications. Initial work concentrated on different topics: optimization of the heteroepitaxial growth of InP on GaAs (use of selective growth and shadow masked growth), further development of nonplanar growth for laserwaveguide coupling (and application to broad spectrum LEDS), optimization of $\operatorname{In}(\mathrm{Al}) \mathrm{GaAs} / \mathrm{Al} / \mathrm{GaAs}$ growth on GaAs (and application to lasers and modulators), fabrication of a GaAs/InP OEIC for optical switching. Recent work focused on the following: development and application of nonplanar growth for laser-waveguide coupling and multi-wavelength laser arrays using $\mathrm{GaAs}$ and InP based materials; the use of banding by atomic rearrangement for InP on GaAs and InP on Si devices; and the optimization of epitaxial lift-off technology.

\section{8}

\section{LASER PROPAGATION THROUGH TURBULENCE}

\section{A. Consortini}

University of Florence

Florence, Italy

\section{SL: ARL/BED, ARO}

This research involves the analysis of experimental data concerning the intensity fluctuations of laser radiation propagating through a turbulent atmosphere. The data consist of highly-sampled intensity measurements, taken in collaboration with NOAA, over a very well monitored optical range. Atmospheric conditions varied from weak scintillation to the most saturated scintillation ever observed. The data base can be expected to yield the moments of the intensity, including the scintillation index, as well as the full probability density functions. The evolution of the probability distributions with changing propagation conditions will be investigated along with the effect of the inner scale of turbulence on the laser scintillation index. A deconvolution procedure to remove noise effects from actual intensity fluctuations will be studied. Results of this research will help to verify the applicability of current theoretical models concerning laser propagation through the turbulent boundary layer. From the large data table previously produced in the computer, plots of all quantities were made versus the cycle number for all measurements in order to check the regularity of the data. Scintillation index was analyzed versus wind. Separate files of histograms of intensity scintillation, one for each cycle, and new "filtered" histograms were produced. A deconvolution procedure was applied to data from multiple aperture measurements with good results. Two communications were submitted for a future Scientific Congress. The analysis of the effect of inner scale, structure constant and wind on laser scintillation index has been completed. For the first time, data obtained were compared with theoretical curves and found in good agreement. A computer procedure has been developed to compare experimental probability density graphs and lognormal distributions. Three papers have been presented and two others submitted.

\section{INVESTIGATION OF (HgZn)Te FOR IR DETECTION, EMISSION AND PHOTONICS}

\section{R. Triboulet}

Centre National de la Recherche Scientifique Meudon, France

\section{SL: NVESD, ARO \\ SC: ARL/EPSD, ARL, MICOM, ARO, NVESD}

The objective of this proposal is to investigate the growth and characterization of $\mathrm{HgZnTe}$ semiconductor materials for infrared applications. The research encompasses a full structural, electrical and optical characterization of such materials. The research topics are: 1 . Theoretical modeling of the binding energy, phonon spectra and thermo-statistical properties of $\mathrm{HgZnTe}$. 2. Evaluation of the band structure parameters using high quality crystals. 3 . Determination of the specific structure of the $\mathrm{HgZnTe}$ alloy through electronic and optical mea- 
surements. 4. Investigation of the optical and electrooptical properties of $\mathrm{HgZnTe}$ crystal for photonics applications. The analysis of the specific heat and the linear thermal expansion coefficient data on $\mathrm{Hg}_{1-x} \mathrm{Cd}_{x} \mathrm{Te}$ (MCT) and $\mathrm{Hg}_{1-x} \mathrm{Zn}_{x} \mathrm{Te}$ (MZT) measured between $4 \mathrm{~K}$ and $300 \mathrm{~K}$ indicating a low increase in the covalent part of the bonding with $x$ in MCT and a strong increase of this covalent part in MZT. Specifically, MZT, $x=0.15$, has mechanical properties somewhat better than CdTe (at $80 \mathrm{~K}$ ). Further, an examination of all the processes used to convert the as-grown $p$-type material to $n$-type leads to the conclusion that this conversion is only obtained on MZT with $x=0.15$. Surface passivation experiments have been started on samples with $x=0.18-0.25$. The best results are obtained by covering MZT directly with $\mathrm{SiO}_{2}$, the resulting surface state density is approximately $2 \times 10^{10} \mathrm{~cm}^{-2}$. 


\section{Proposal Numbers}

Note: The Roman numeral refers to the scientific division, and the alphabetic character refers to the division subsection. Proposal numbers are in ascending sequence within the subsections.

\begin{tabular}{|c|c|c|c|}
\hline \multicolumn{2}{|l|}{$\begin{array}{l}\text { Proposal } \\
\text { Number }\end{array}$} & \multicolumn{2}{|l|}{$\begin{array}{l}\text { Proposal } \\
\text { Number }\end{array}$} \\
\hline 5824-ER & IX-C & 26427-MS & Vl-A \\
\hline 5830-ER & IX-F & $26441-\mathrm{CH}$ & . II-E \\
\hline 5884-ER & IX-D & 26442-PH & [-D \\
\hline 5937-ER & $1 \mathrm{X}-\mathrm{B}$ & 26443-EG & V.A \\
\hline 6011-ER & LX-D & 26444-EL & VIII-A \\
\hline 6078-ER & IX-A & 26449-MS & VI-A \\
\hline 6089-ER & LX-B & $26456-\mathrm{EG}$ & V-C \\
\hline 6148-ER & IX-D & $26460-\mathrm{EL}$ & VIHI-E \\
\hline 6170-ER & DX-F & 26485-MS & VI.C \\
\hline 6185-ER & IX-E & 26487-MA & IV-E \\
\hline 6280-ER & IX-A & 26491.GS & VIL-F \\
\hline 6291-ER & IX-F & 26571-MS & VI-C \\
\hline $6294 \mathrm{ER}$ & $I X-B$ & 26579-MS & Vl.C \\
\hline 6296-ER & IX-E & 26582-LS & III-D \\
\hline 6305-ER & IX-B & 26595-EG & V-B \\
\hline 6307-ER & IX-A & 26599-EL & VIIl-C \\
\hline 6366-ER & IX-A & 26601-MS & VI-D \\
\hline 6458-ER & IX-F & 26631-EG & V-B \\
\hline 6563-ER & LX-F & $26636-\mathrm{CH}$ & II-A \\
\hline 6674-ER & IX-B & 26667-MS & VI-A \\
\hline 24381-GS & VII-C & 26673-MS & VI-C \\
\hline 24557-MA & IV-C & $26674-\mathrm{MA}$ & NV-D \\
\hline 25045-EL & VIII-C & 26678-MS & VI-A \\
\hline $25126-\mathrm{LS}$ & HI-C & 26682-PH & I-D \\
\hline 25190-EG & $\mathrm{V}-\mathrm{C}$ & 26686-EL & VIl!-B \\
\hline $25202-\mathrm{MS}$ & VI-C & $26695-\mathrm{PH}$ & I-E \\
\hline 25327-EG & V-D & 26708-EL & VIII-A \\
\hline 25339-EL & VIII-C & 26709-PH & I-B \\
\hline 25345-EG & V-A & 26711-EL & VIll-B \\
\hline 25424-MS & I-B & 26720 -EG & V-C \\
\hline 25461-EG & V-B & 26729-MS & VI-D \\
\hline 25462-EG & V-D & 26735-EL & VIII-E \\
\hline 25467-EG & $V-B$ & $26746-\mathrm{MA}$ & IV-C \\
\hline 25476-LS & III-D & $26748-\mathrm{CH}$ & II-A \\
\hline 25523-CH & II-D & $26750-L S$ & III-B \\
\hline $25546-\mathrm{PH}$ & I-E & 26751-MS & VI-B \\
\hline 25557-EL & VIII-C & 26755-EL & VIII-B \\
\hline 25602-EL & VIII-H & 26761-EL & VIII-A \\
\hline 25623-EG & V-B & 26767-LS & III-F \\
\hline $25630-E G$ & V-D & 26779-MA & IV-E \\
\hline 25663-LS & III-D & 26791-EL & VIII-C \\
\hline $25706-\mathrm{MA}$ & IV-C & 26792-MA & IV-G \\
\hline 25752-LS & III-D & 26797-EG & $\mathrm{V}-\mathrm{C}$ \\
\hline 25833-MS & VI-A & 26802-MA & IV-G \\
\hline 25874-MA & IV-A & $26806-\mathrm{MS}$ & VI-A \\
\hline 26015-PH & I-D & 26811-MA & IV-G \\
\hline 26021-MS & VI-F & 26821-PH & I-B \\
\hline $26031-G S$ & VII-B & $26822-\mathrm{CH}$ & II-C \\
\hline $26061-E G$ & V-D & $26823 \cdot \mathrm{EL}$ & VIII-E \\
\hline $26072-\mathrm{CH}$ & II-E & 26825-MS & Vl-B \\
\hline $26106-\mathrm{CH}$ & II-D & $26839-\mathrm{CH}$ & [I-E \\
\hline $26115-\mathrm{CH}$ & [l-B & $26840-\mathrm{CH}$ & II-E \\
\hline 26123-MS & VI-C & $26863-\mathrm{EG}$ & V-B \\
\hline $26126-\mathrm{CH}$ & []-C & 26883-MS & V-D \\
\hline 26188-EL & VIII-A & 26896-EL & VIII-A \\
\hline 26232-LS & III-D & $26898 \cdot E L$ & VII]-F \\
\hline $26246-\mathrm{MS}$ & VI-F & 26899-PH & I-B \\
\hline $26256-\mathrm{CH}$ & $\mathrm{II}-\mathrm{D}$ & $26902-G S$ & VII-A \\
\hline 26257-PH & I-B & 26908-EG & V-A \\
\hline 26281-GS & VII-B & $26909-\mathrm{MA}$ & IV-A \\
\hline 26284-CH & II-B & $26914-\mathrm{CH}$ & II-B \\
\hline 26392-MS & VI-C & $26930-\mathrm{MA}$ & IV-G \\
\hline 26394-MA & IV-C & 26932-MA & IV-G \\
\hline $26400-\mathrm{MS}$ & VI-A & 26934-EG & V-D \\
\hline 26403-EG & V-C & 26949-EL & VUI-C \\
\hline
\end{tabular}

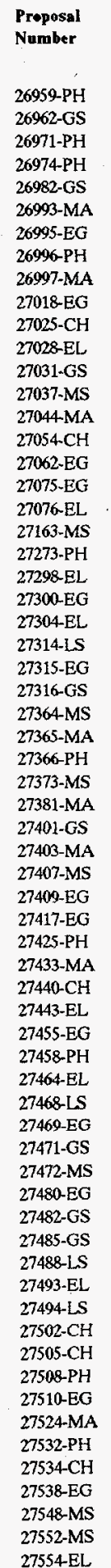

Proposal

Number

I-B

VII-G

I-E

VII-E

IV-C

V-D

IV-C

V-C

[I-D

VIIl-E

VII-D

VI-A

IV-D

II-E

V.B

$V * D$

VIII-E

VI-B

VIII-A

V.D

VIII-C

III-B

$V-A$

VII-B

VI-F

IV-D

I-E

Vl-C

IV-C

VI-B

$\mathrm{V}-\mathrm{A}$

V-C

I-D
IV-A

II-E

VIII-B

V-C

I-D
VIII-E

III-D

Y.C

VII-A

VI-C

$\mathrm{V}-\mathrm{C}$

VD-B

VII-D

III-C

VIII-E

III-A

II-D

I-B

V-D
IV-G

I-B

II-B

V-A

VI-D

VI-A

VIII-H

\begin{tabular}{|c|c|}
\hline 27556-PH & I-E \\
\hline 27557-MA & IV-A \\
\hline 2755\&-EG & V.B \\
\hline 27565-EG & V-C \\
\hline $27567-\mathrm{MA}$ & NV-E \\
\hline $27568-\mathrm{CH}$ & LI-B \\
\hline 27574-MA & IV-C \\
\hline 27578-EL & VIII-B \\
\hline $27580-\mathrm{MA}$ & IV-A \\
\hline 27586-PH & I-E \\
\hline 27591-PH & I-E \\
\hline $27600-\mathrm{CH}$ & [1-A \\
\hline $27603-\mathrm{CH}$ & II-D \\
\hline 27605-MS & VI-D \\
\hline 27620-MA & IV-G \\
\hline 27624-MS & VI-A \\
\hline $27627-\mathrm{EG}$ & $\mathrm{V}-\mathrm{B}$ \\
\hline $27634 \mathrm{LS}$ & III-F \\
\hline 27641-MA & IV-A \\
\hline $27646-\mathrm{PH}$ & I-B \\
\hline $27690 \mathrm{MA}$ & IV-B \\
\hline 27751-EL & VIII-E \\
\hline 27754-EL & VIII-E \\
\hline 27764-MS & VI-D \\
\hline $27766 \mathrm{PH}$ & I-B \\
\hline $27770-\mathrm{CH}$ & [I-C \\
\hline $27775-\mathrm{CH}$ & II-C \\
\hline 27780-PH & I-D \\
\hline 27786-MA & NV-B \\
\hline $27790-\mathrm{MA}$ & IV-C \\
\hline $27808-\mathrm{CH}$ & II-E \\
\hline $27810 \mathrm{MS}$ & VI-F \\
\hline 27817-MA & [V-G \\
\hline 27834-EL & VIII-E \\
\hline $27846-\mathrm{MS}$ & VI-D \\
\hline $27862-\mathrm{MA}$ & IV-C \\
\hline 27863-MS & VI-A \\
\hline $27864-\mathrm{EG}$ & $\mathrm{V}-\mathrm{C}$ \\
\hline 27865-EL & VII-F \\
\hline $27868-\mathrm{MA}$ & IV-C \\
\hline $27869-\mathrm{MA}$ & IV-A \\
\hline $27882-\mathrm{PH}$ & I-E \\
\hline 27883-EL & VIII-B \\
\hline 27886-EL & VIII-E \\
\hline $27887-\mathrm{CH}$ & II.D \\
\hline 27888-PH & I-B \\
\hline $27890-\mathrm{LS}$ & III-C \\
\hline 27892-MA & IV-E \\
\hline 27894-EG & V-B \\
\hline 27899-EL & VIIL-E \\
\hline 27904-EL & VIII-C \\
\hline 27911-GS & VII-D \\
\hline 27916-LS & III-D \\
\hline 27917-EL & VIII-E \\
\hline $27956-\mathrm{LS}$ & III-C \\
\hline 27993-MA & IV-C \\
\hline $27994-\mathrm{EL}$ & VIII-E \\
\hline 27995-GS & VII-F \\
\hline $28002-E G$ & V-B \\
\hline 28004-EL & VIII-B \\
\hline 28005-MS & VI-C \\
\hline $28007-\mathrm{MA}$ & IV-B \\
\hline 28011-LS & [II-C \\
\hline $28013-\mathrm{CH}$ & II-B \\
\hline & \\
\hline
\end{tabular}




\begin{tabular}{|c|c|}
\hline $\begin{array}{l}\text { Proposal } \\
\text { Number }\end{array}$ & \\
\hline $28040-G S$ & VII-B \\
\hline $28043-L S$ & III-C \\
\hline $28052-G S$ & VII-G \\
\hline $28053-\mathrm{CH}$ & II-E \\
\hline $28060-\mathrm{MA}$ & IV-G \\
\hline 28067-MS & VI-C \\
\hline $28030-\mathrm{EL}$ & VIII-D \\
\hline 28071-MA & IV.B \\
\hline 28090 -LS & III-C \\
\hline $28094-G S$ & VII-E \\
\hline 28099-EL & VIII-F \\
\hline 28102-GS & VII-E \\
\hline $28103-\mathrm{CH}$ & II-D \\
\hline $28123-E G$ & V-D \\
\hline $28125-\mathrm{MA}$ & IV.E \\
\hline 28141-MS & VI-D \\
\hline 28143-MA & IV-B \\
\hline $28146-\mathrm{MS}$ & VI-D \\
\hline 28151-EL & VIII-C \\
\hline $28153-\mathrm{CH}$ & II-E \\
\hline 28155-EG & V.D \\
\hline 28159-EG & V-B \\
\hline 28187-EL & VIII-F \\
\hline $28192-\mathrm{MA}$ & IV-B \\
\hline $28215-E G$ & V-B \\
\hline 28219-GS & VII-D \\
\hline 28222-EG & V.A \\
\hline 28249-EG & V-B \\
\hline $28250-E G$ & V-C \\
\hline 28253-EG & V-A \\
\hline 28258-PH & I.E \\
\hline $28271-\mathrm{PH}$ & I-D \\
\hline $28272-\mathrm{MS}$ & VI-E \\
\hline 28283-EG & V-A \\
\hline 28293-EG & V-B \\
\hline 28297-EL & VIII-E \\
\hline 28307-EG & V-A \\
\hline 28309-MA & IV-C \\
\hline 28310-EG & $\mathrm{V}-\mathrm{C}$ \\
\hline 28313-PH & J-E \\
\hline $28314-\mathrm{CH}$ & []-E \\
\hline $28319 \mathrm{CH}$ & Il-C \\
\hline $28320-\mathrm{MA}$ & IV-B \\
\hline $28325-\mathrm{EL}$ & VIII-B \\
\hline $28326-\mathrm{EL}$ & VIII-H \\
\hline $28328-\mathrm{MA}$ & IV-E \\
\hline 28329-EL & VIII-B \\
\hline 28335-EL & VIII-A \\
\hline 28336-PH & I-D \\
\hline 28339-EL & VIII-C \\
\hline 28344 GS & VII-B \\
\hline $28345-\mathrm{EL}$ & VIII-B \\
\hline 28347-EL & VIII-A \\
\hline 28348-PH & I-E \\
\hline 28350-EG & V-D \\
\hline $28351-E L$ & VII-A \\
\hline $28353-\mathrm{CH}$ & II-A \\
\hline 28354-MA & IV-E \\
\hline $28356-\mathrm{PH}$ & I-E \\
\hline 28361-LS & III-C \\
\hline 28362-PH & {$[-D$} \\
\hline 28368-MS & VI-C \\
\hline 28369-MS & VI-A \\
\hline $28371-\mathrm{CH}$ & []-D \\
\hline 28373-CH & I]-E \\
\hline 28377-MA & IV-B \\
\hline 28387-EL & VIII-E \\
\hline 28399-EG & V-D \\
\hline $28402-\mathrm{CH}$ & II-A \\
\hline $28406-\mathrm{EL}$ & VIII-B \\
\hline $28408-\mathrm{MA}$ & IV.G \\
\hline 28416 -PH & I-E \\
\hline $28427-\mathrm{EG}$ & V.C \\
\hline $28428-\mathrm{MA}$ & IV-G \\
\hline 28453-EL & VIIl-H \\
\hline 28457.LS & III-C \\
\hline
\end{tabular}

\begin{tabular}{|c|c|}
\hline $\begin{array}{l}\text { Propesel } \\
\text { Number }\end{array}$ & \\
\hline 28461-EL & VIIl-B \\
\hline 28464-PH & I-D \\
\hline 28467-EL & VIII-E \\
\hline 28468-PH & I-D \\
\hline 28469-CH & II-A \\
\hline $28470-\mathrm{PH}$ & I-D \\
\hline 28472-PH & I-D \\
\hline 28476-MA & IV-G \\
\hline 28479 -LS & III-D \\
\hline $28480-M S$ & VI-B \\
\hline $28483-\mathrm{EL}$ & VII-C \\
\hline $28486-\mathrm{CH}$ & II-A \\
\hline 28489-GS & Vll-G \\
\hline 28493 -EG & V-D \\
\hline $28499-\mathrm{PH}$ & I-B \\
\hline $28502-\mathrm{PH}$ & I-E \\
\hline $28504-G S$ & VII-A \\
\hline 28506-EL & VIL-E \\
\hline 28508-EL & VIIl-B \\
\hline 28509.MS & Vl-A \\
\hline 28511-MA & IV-D \\
\hline $28514 \mathrm{MA}$ & IV-A \\
\hline 28516-EL & VII-C \\
\hline $28525-\mathrm{CH}$ & II-E \\
\hline 28526-PH & I-E \\
\hline $28528 \mathrm{CH}$ & [1]-B \\
\hline 28531-PH & $\mathrm{I}-\mathrm{B}$ \\
\hline 28535-MA & N-B \\
\hline 28548-MA & IV-A \\
\hline $28549-\mathrm{MS}$ & VI-B \\
\hline 28553-MS & VI-D \\
\hline $28555-\mathrm{CH}$ & II-D \\
\hline $28560-M S$ & VI-C \\
\hline 28561-PH & J-B \\
\hline $28565 . \mathrm{CH}$ & II-D \\
\hline $28569-\mathrm{PH}$ & l-B \\
\hline 28575-MS & VI-E \\
\hline 28577-MS & VI-B \\
\hline 28591-PH & I-D \\
\hline $28592-\mathrm{PH}$ & I.E \\
\hline 28594-EL & VIII-A \\
\hline $28599-G S$ & VII-B \\
\hline $28606-\mathrm{MA}$ & IV-A \\
\hline $28608-\mathrm{MS}$ & VI-B \\
\hline 28612-MS & VI-D \\
\hline $28620-\mathrm{MS}$ & VI-F \\
\hline $28628-\mathrm{CH}$ & [I-C \\
\hline $28631-\mathrm{CH}$ & II-E \\
\hline 28639-MS & VI-C \\
\hline $28646-E L$ & VIII-B \\
\hline $28651-E G$ & V-B \\
\hline $28652-\mathrm{PH}$ & I-D \\
\hline $28655-\mathrm{CH}$ & Il-A \\
\hline $28656-\mathrm{CH}$ & II-B \\
\hline 28664-GS & VIJ-F \\
\hline 28669 -LS & II]-C \\
\hline 28674-EL & VIIl-A \\
\hline $28675-\mathrm{CH}$ & [1-E \\
\hline 28679-MA & IV-C \\
\hline $28680-L S$ & III-C \\
\hline 28684-PH & I-D \\
\hline $28686-\mathrm{MA}$ & IV.C \\
\hline 28691-LS & III-A \\
\hline 28699-LS & III-C \\
\hline $28700-\mathrm{CH}$ & [I-D \\
\hline 28701-MA & IV-B \\
\hline $28702-\mathrm{MA}$ & IV-A \\
\hline $28711-\mathrm{CH}$ & II-E \\
\hline $28715-\mathrm{MA}$ & IV.C \\
\hline 28716-MA & IV-B \\
\hline 28717-GS & VII-D \\
\hline 2871\&MA & IV-C \\
\hline $28722-\mathrm{MA}$ & IV-C \\
\hline $28728 \mathrm{MA}$ & IV-A \\
\hline $28729-\mathrm{EL}$ & VII- \\
\hline 28735-MA & IV-B \\
\hline
\end{tabular}

\begin{tabular}{|c|c|}
\hline $\begin{array}{l}\text { Proposal } \\
\text { Number }\end{array}$ & \\
\hline $28740-\mathrm{MA}$ & [V-D \\
\hline 28743-MA & IV-C \\
\hline 28761-MS & VI-B \\
\hline 28766-MA & IV-A \\
\hline $28767-\mathrm{CH}$ & II-C \\
\hline 28T72-GS & VIJ-A \\
\hline 28775 -LS & III-F \\
\hline 28780-MS & VI-B \\
\hline 28784-PH & I-B \\
\hline $28795-\mathrm{CH}$ & II-E \\
\hline 28797-MA & N-A \\
\hline 28799-MA & IV-B \\
\hline 28809-MA & IV-G \\
\hline $28814 \mathrm{CH}$ & [1]-B \\
\hline $28826-\mathrm{MS}$ & Vl-B \\
\hline 28835-MA & IV-D \\
\hline 28852-PH & I-D \\
\hline 28883-MS & VI-C \\
\hline 28884-MS & VI-E \\
\hline $28889-\mathrm{CH}$ & II-D \\
\hline 28895-EL & VII]-B \\
\hline 28898 -EG & V-C \\
\hline $28900-M S$ & VI-C \\
\hline $28905-\mathrm{MA}$ & IV-C \\
\hline 28908-MA & IV-B \\
\hline 28912-EG & V-C \\
\hline 28916-MA & IV-B \\
\hline 28922-EL & Vtil-F \\
\hline $28925-\mathrm{EL}$ & VII-H \\
\hline 28929-LS & IIl-C \\
\hline 28938-MS & VI-C \\
\hline $28945-\mathrm{CH}$ & II-A \\
\hline $28950-\mathrm{MA}$ & IV-E \\
\hline 28954-GS & VI]-P \\
\hline 28955-PH & 1-C \\
\hline 28964 -LS & III-B \\
\hline 28972-LS & III-C \\
\hline 28978-PH & $\mathrm{I}-\mathrm{B}$ \\
\hline 28979-PH & I.D \\
\hline 28982-MA & IV-C \\
\hline $28986-\mathrm{MA}$ & IV-A \\
\hline 28987-MA & IV-A \\
\hline 28994-MA & IV.G \\
\hline $28996-\mathrm{PH}$ & I-A \\
\hline 29015-PH & I-B \\
\hline 29029-EG & $V=D$ \\
\hline $29031-\mathrm{MA}$ & IV-G \\
\hline $29033-\mathrm{CH}$ & IIFD \\
\hline $29036-\mathrm{EL}$ & VIII-B \\
\hline $29037-\mathrm{MS}$ & VI-A \\
\hline $29040-E G$ & V-A \\
\hline 29042-MS & VI-D \\
\hline 29049.EG & V-B \\
\hline $29050-\mathrm{PH}$ & I-E \\
\hline 29053-MA & IV-G \\
\hline 29064-MS & VI-A \\
\hline 29071-MS & VI-A \\
\hline 29097-LS & III-B \\
\hline 29098-MS & VI-C \\
\hline 29113-MA & IV-B \\
\hline 29141-EG & V.C \\
\hline 29167-MA & IV-B \\
\hline $29170-\mathrm{LS}$ & III-F \\
\hline 29184-PH & I-B \\
\hline 29190-MA & IV-G \\
\hline 29194-LS & III-F \\
\hline 29195-LS & III- $\dot{\mathrm{C}}$ \\
\hline 29196-GS & VII-F \\
\hline $29205-G S$ & VII-D \\
\hline 29210-EL & VIII-F \\
\hline 29212-PH & I-D \\
\hline 29218-MS & VI-D \\
\hline 29221-LS & LII-F \\
\hline 29223-EL & Vill-B \\
\hline $29225-\mathrm{CH}$ & Il $-\mathrm{B}$ \\
\hline $29227-\mathrm{CH}$ & II $-\mathrm{C}$ \\
\hline
\end{tabular}

\begin{tabular}{|c|c|}
\hline $\begin{array}{l}\text { Propesal } \\
\text { Number }\end{array}$ & \\
\hline 29282-MS & VI-D \\
\hline 29289-LS & III-C \\
\hline 29297-MA & IV-C \\
\hline $29300-\mathrm{LS}$ & III-D \\
\hline 29302-EG & V-C \\
\hline 29321-PH & I-E \\
\hline 29324-PH & $\mathrm{I}-\mathrm{B}$ \\
\hline 29326-MS & VI-C \\
\hline $29332-L S$ & III-A \\
\hline 29336-EG & V-C \\
\hline 29339-PH & I-E \\
\hline 29341-PH & I-D \\
\hline 29345-LS & III-E \\
\hline 29347-MS & VI-A \\
\hline 29349-MA & IV-G \\
\hline 29351-MA & IV.A \\
\hline $29354-\mathrm{MA}$ & IV-E \\
\hline 29358-MA & IV.C \\
\hline $29360-G S$ & VII-D \\
\hline $29369-G S$ & VII-F \\
\hline 29373-PH & I-B \\
\hline 29375-PH & I-D \\
\hline 29391-PH & I-B \\
\hline $29396-\mathrm{MA}$ & IV-D \\
\hline 29397-MA & IV-D \\
\hline $29404-G S$ & VII-D \\
\hline 29410-MS & VI-C \\
\hline 29412-MA & IV-G \\
\hline 29523-EL & VII-C \\
\hline 29524-MS & VI-C \\
\hline $29530-\mathrm{MA}$ & IV-B \\
\hline 29534-EG & V.C \\
\hline 29537-LS & III-F \\
\hline $29538-\mathrm{CH}$ & [I]C \\
\hline 29540-EL & VIII-K \\
\hline 29541-EL & VIII-B \\
\hline 29543 -EG & V-A \\
\hline $29560-\mathrm{CH}$ & I-A \\
\hline $29572-\mathrm{PH}$ & I-D \\
\hline 29574-MA & IV-B \\
\hline 29576-PH & $I-D$ \\
\hline 29578-PH & I-D \\
\hline 29579-EG & V-E \\
\hline 29594-LS & III-D \\
\hline 29595-PH & I-B \\
\hline $29596, \mathrm{CH}$ & II-D \\
\hline $29606-\mathrm{MS}$ & VI-D \\
\hline 29615-EL & VIII-C \\
\hline 29616-EL & VIII-B \\
\hline $29627-\mathrm{CH}$ & lI-E \\
\hline 29631-EL & VII-B \\
\hline 29633-GS & VII-D \\
\hline 29643-MS & VI-F \\
\hline $29647-\mathrm{MS}$ & VI-F \\
\hline $29653-\mathrm{EL}$ & VII-E \\
\hline 29677-MS & VI-D \\
\hline 29678-MA & IV-C \\
\hline 29692-MA & IV-E \\
\hline $29699 \mathrm{CH}$ & II-B \\
\hline $29708-\mathrm{CH}$ & U-A \\
\hline 29713-MS & VI-D \\
\hline $29725-\mathrm{CH}$ & II-A \\
\hline 29740-MA & IV-C \\
\hline $29750-\mathrm{MS}$ & VI-D \\
\hline 29771-EG & V-C \\
\hline 29808-MA & TV.B \\
\hline 29810-EG & V-C \\
\hline 29850-MA & IV.B \\
\hline 29922-PH & I-D \\
\hline 29923-PH & I-D \\
\hline $30060-\mathrm{MS}$ & VI-C \\
\hline $30061-\mathrm{MA}$ & IV-E \\
\hline 30068-MS & VI-D \\
\hline $30090-\mathrm{EL}$ & VIII-A \\
\hline $30094-\mathrm{CH}$ & II-C \\
\hline 30095-MS & VI-C \\
\hline
\end{tabular}


Propesal

Nember

30102-MS

30108-MS

30113-EG

30124-MS

30141-PH

$30164-\mathrm{CH}$
Propesal

Number

30230-CH

$30289-\mathrm{CH}$

30297-MA

30332-CH

30337-MS

30352-MS
Propesal

Number

30387-GS

30408-PH

30411-MA

30423-PH

30437-EL

$30443-\mathrm{CH}$
Propesal

Number

$30446-\mathrm{CH}$

30488-MS

30500-EL

30549-EL

30595-EL
II-A

VII-F

VIII-F 


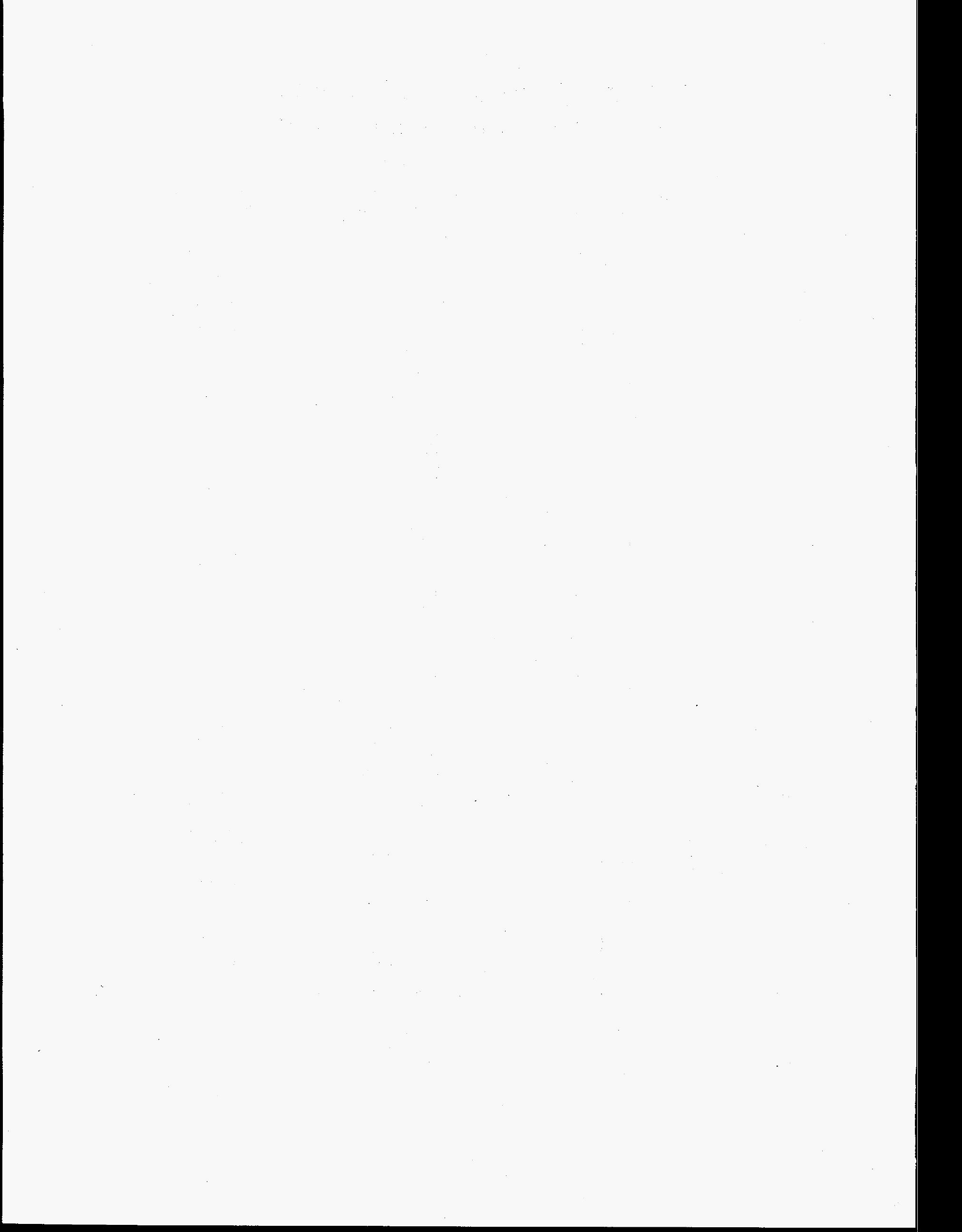




\section{Scientific Liaison and Scientific Cognizance Representatives}

Note: The Roman numeral refers to the scientific division, and the alphabetic character refers to the division subsection. Proposal numbers are in ascending sequence within the subsections.

\begin{tabular}{|c|c|c|}
\hline Advanced Research & V-D-25630 (SL) & II-B-28814 \\
\hline Projects Agency & V-D-26061 & II-B-29225 (SL) \\
\hline I-A-28996 (SL) & V-D-28123 & II-B-29699 \\
\hline I-D-28271 (SL) & & II-C-26126 \\
\hline $\mathrm{J}-\mathrm{E}-27882$ (SL) & & $11-C-26822$ \\
\hline ]-E-28348 (SL) & Benet Weapons & II-C-27770 \\
\hline J-E-28526 (SL) & Laboratories & IJ-C-27775 \\
\hline II-A-29708 (SL) & I-D-29341 (SL) & II-C-27775 (SL) \\
\hline Il-A-30289 (SL) & IV-A-29351 & II-C-28319 \\
\hline II-A-30446 (SL) & IV-B-28535 & II-C-28628 \\
\hline II-E-26072 (SL) & IV-B-29808 (SL) & II-C-28767 \\
\hline VII-A-30437 (SL) & IV-B-30297 (SL) & II-C-29227 \\
\hline VIII-B-29631 & [V-D-29397 (SL) & II-D-26256 \\
\hline \multirow[t]{3}{*}{ VIII-F-28729 (SL) } & V-A-27409 (SL) & II-D-27505 \\
\hline & V-A-28222 (SL) & II-D-27887 \\
\hline & V-A-28253 & II-D-28103 \\
\hline Aeroflightdynamics & V-A-28283 & II-D-28371 \\
\hline Directorate, ATCOM & V-A-28307 (SL) & II-D-28700 \\
\hline Ames Research Center & V-A-29040 & II-D-29033 \\
\hline IV-B-28377 & V-D.27075 (SL) & II-E-26072 \\
\hline IV-D-28835 (SL) & VI-A-26427 (SL) & II-E-26441 \\
\hline V-A-26908 & VI-A-28509 & II-E-26839 \\
\hline V-B-25461 (SL) & VI-A-29037 & II-E-26840 \\
\hline V-B-25467 (SL) & Vl-B-26825 & II-E-28053 \\
\hline V-B-25623 (SL) & VI-B-27163 (SL) & II-E-28153 \\
\hline V-B-26595 (SL) & VI-B-27407 (SL) & II-E-28314 \\
\hline V-B-26631 & VI-C-28639 & II-E-28525 \\
\hline V-B-26631 (SL) & IX-A-6078 & II-E-28631 \\
\hline V-B-27894 & & II-E-28675 \\
\hline V-B-27894 (SL) & & II-E-28675 (SL) \\
\hline V-B-28002 (SL) & Construction Engineering & III-A-28022 (SL) \\
\hline V-B-28159 & Research Laboratory & III-A-29332 (SL) \\
\hline V-B-28159 (SL) & VI-A-26427 & III-B-26750 \\
\hline V-B-28651 & VI-A-29037 & III-C-27890 \\
\hline V-B-28651 (SL) & VI-A-29071 & HII-C-28011 \\
\hline V-B-29049 (SL) & VI-B-27163 & III-C-28043 \\
\hline V-D-25462 (SL) & VI-B-28480 & III-C-28043 (SL) \\
\hline V-D-26061 (SL) & & II]-C-28361 \\
\hline V-D-26934 (SL) & & III-C-28457 \\
\hline$V-D-28123(S L)$ & Corpus Christi Army Depot & III-C-28699 \\
\hline \multirow[t]{3}{*}{ V-D-28493 (SL) } & VI-A-25833 & III-C-29195 (SL) \\
\hline & V1-A-26806 (SL) & III-C-29289 \\
\hline & & III-D-25476 \\
\hline Air Force Astronautics & & III-D-25476 (SL) \\
\hline Laboratory (AFSC) & Edgewood Research, & III-D-25663 \\
\hline \multirow[t]{3}{*}{ II-D-27603 } & Development and & III-D-25663 (SL) \\
\hline & Engineering Center & [II-D-25752 \\
\hline & [-D-29572(SL) & [I]-D-26232 \\
\hline Air Force Engineering and & [1-A-27600 & III-D-27468 \\
\hline Service Center & II-A-28469 & III-D-27916 \\
\hline \multirow[t]{3}{*}{ II-D-28371 (SL) } & Il-A-28486 & III-D-27916 (SL) \\
\hline & II-A-28655 & III-D-28479 \\
\hline & II-A-28945 & III-D-28479 (SL) \\
\hline Air Force Office of & II-A-29560 & III-D-29300 (SL) \\
\hline Scientific Research & II-A-30164 (SL) & [II-F-26767 \\
\hline I-E-28356 (SL) & II-B-26115 & III-F-27634 \\
\hline \multirow[t]{3}{*}{ V-B-26631 } & II-B-26115 (SL) & [11-F-29194 (SL) \\
\hline & II-B-26284 & IV-A-27403 (SL) \\
\hline & II-B-26284 (SL) & IV-A-27433 (SL) \\
\hline Aviation Applied Technology & U]-B-26914 & IV-A-28766 (SL) \\
\hline Directorate, ATCOM & II-B-26914 (SL) & IV-A-28797 (SL) \\
\hline V-A-26908 (SL) & II-B-27568 & V-C-25190 \\
\hline V-B-25461 (SL) & II-B-28013 & V-C-28250(SL) \\
\hline V-B-25467 (SL) & II-B-28656 & VI-A-27624 \\
\hline V-D-25462 (SL) & II-B-28656 (SL) & VI-F-28620 \\
\hline
\end{tabular}

VII-D-27031 (SL)

VII-D-28717 (SL)

VII-D-29205

VII-D-29633 (SL)

VII-E-28102

VII-F-26491

VII-F-27995

VII-F-29196

VIJ-G-26962 (SL)

VIJ-G-28052

VII-G-28052 (SL)

VII-G-28489

VII-G-28489 (SL)

LX-B-5937 (SL)

[X-B-6089

DX-B-6294

IX-B-6305

IX-B-6305 (SL)

IX-B-6674

European Office of

Aerospace R\&D

IX-A-6307

HODA, Office of the

Assistant Secretary of the Army V-B-25461

V-B-25467

V-B-25623

V-D-25462

VIII-H-25602

Naval Research Laboratory

III-B-27314

VII-E-28297

Night Vision and

Flectronic Senso

Directorate

I-B-26899 (SL)

I-B-27532 (SL)

I-B-28784 (SL)

I-B-29391

I-D-27425 (SL)

I-E-28258 (SL)

I-E-28502 (SL)

I-E-28526

[I-A-27502 (SL)

IV-C-27868

IV-C-28715 (SL)

IV.C-28905 (SL)

IV-C-29297 (SL)

IV-C-29678 (SL)

IV-G-29349

VI-A-26427

VI-A-29037

VI-D-26601

VI-D-26883

VI-D-27605 (SL)

VI-D-27846 (SL)

VI-D-28612 (SL) 


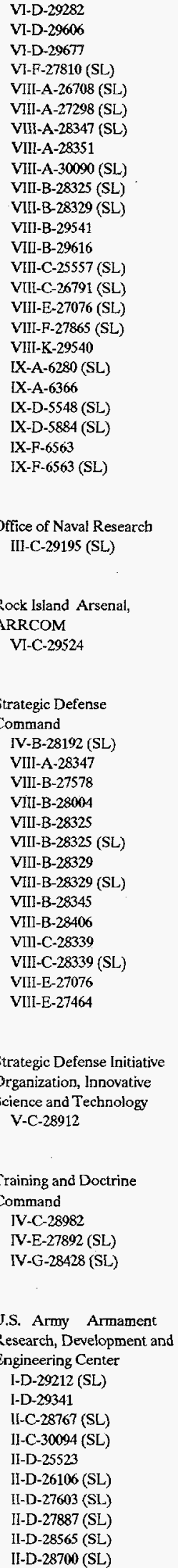

$\begin{array}{ll}\text { II-D-28889 (SL) } & \text { II-A-29560 } \\ \text { II-D-20033 (SL) } & \text { II-C-27775 } \\ \text { II-E-26441 } & \text { Il-D-26106 } \\ \text { II-E-28525 } & \text { Il-E-26441 } \\ \text { II-E-28631 } & \text { II-E-28153 } \\ \text { II-E-28711 (SL) } & \text { II-E-28525 } \\ \text { II-E-28795 } & \text { Il-E-28631 } \\ \text { IV-D-27044 (SL) } & \text { II-E-28711 } \\ \text { IV-D-27365 (SL) } & \text { V-A-27315 (SL) } \\ \text { IV-D-28511 (SL) } & \text { V-C-25190 } \\ \text { IV-D-28740 (SL) } & \text { V-C-27417 } \\ \text { IV-D-28835 (SL) } & \text { V-C-27565 } \\ \text { V-A-25345 } & \text { V-C-28427 } \\ \text { V-A-26443 } & \text { V-C-29141 } \\ \text { V-A-27315 } & \text { VI-A-26427 } \\ \text { V-A-27409 } & \text { VI-A-26449 } \\ \text { V-C-26403 (SL) } & \text { VI-A-26667 } \\ \text { V-C-26720 (SL) } & \text { VI-A-26678 } \\ \text { V-C-27455 } & \text { VI-A-27624 } \\ \text { V-C-29336 (SL) } & \text { VI-A-28369 } \\ \text { V-C-29771 (SL) } & \text { VI-A-29064 } \\ \text { V-D-26995 (SL) } & \text { VI-A-29071 } \\ \text { V-D-27075 } & \text { Vl-B-26751 } \\ \text { V-D-29029 } & \text { Vl-B-28826 } \\ \text { VI-A-25833 (SL) } & \text { VI-C-25202 } \\ \text { VI-A-26400 } & \text { VI-C-26571 } \\ \text { Vl-A-26427 (SL) } & \text { VI-C-27373 } \\ \text { VI-A-26449 } & \text { VI-C-28560 } \\ \text { VI-A-26667 } & \text { VI-C-28883 (SL) } \\ \text { Vl-A-26806 } & \text { Vl-C-29410 } \\ \text { VI-A-27037 } & \text { VI-C-29524 } \\ \text { Vl-A-27552 } & \text { VI-C-30095 } \\ \text { VI-A-27552 (SL) } & \end{array}$

U.S. Army Cold Regions

VI-A-27624

VI-A-27863

VI-A-29037 (SL)

VI-B-26825 (SL)

VI-B-28480

VI-B-28549

VI-B-28761

Vl-B-28780

VI-C-26571

Vl-C-26579

VI-C-27472

VI-C-27472 (SL)

V1-C-28005 (SL)

VI-C-28560

VI-C-28639 (SL)

VI-C-29524

VI-C-30060

VI-C-30095

VI-E-28272 (SL)

VII-D-27911

VIII-A-28674 (SL)

VIII-C-28483 (SL)

VIII-E-27464 (SL)

IX-A-6366 (SL)

[X-B-6294

IX-F-5830(SL)

IX-F-6170 (SL)

IX-F-6291 (SL)

U.S. Army Armament,

Munitions and Chemical

Command

II-D-26106

U.S. Army Aviation and Troop

Command

V-B-25461 (SL)

V-B-25467 (SL)

V-D-25462 (SL)

U.S. Army Betvoir

Research, Development and

Engineering Center

II-A-28655 (SL)
Research and Engineering

Laboratory

III-B-26750

[II-B-27314

VII-A-26902

VII-A-27401 (SL)

VIJ-A-28504 (SL)

VII-B-26031 (SL)

VI]-B-26281 (SL)

VIJ-B-27316

VII-B-27482 (SL)

VII-B-28040

VII-B-28344 (SL)

VII-B-28599 (SL)

VII-C-24381

VII-D-28219

VII-D-30387

VII-E-26982

VIIF-28664

VIJ-F-28954 (SL)

VIJ-F-29196 (SL)

U.S. Army Communications-

Electronics Command

[-B-28978

II-B-27534 (SL)

[I-B-27568 (SL)

IV-E-28328 (SL)

VI-A-29071 (SL)

VI-C.28938 (SL)

VII-D-27031 (SL)

VII-D-27485 (SL)

VIJ-D-27911 (SL)

VII-D-28219 (SL)

VII-D-29205 (SL)

VII-D-29404 (SL)

VII-D-30387 (SL)

VIII-A-28674 (SL)

VIII-C-25045 (SL)

VIII-C-25339 (SL)

VIII-C-26599(SL)

VIII-C-26791 (SL)

VIII-C-26949 (SL)
VIII-C-27304 (SL)

VIII-C-27904 (SL)

VIII-C-28151 (SL)

VIIL-C.28339 (SL)

VIII-C-28483 (SL)

VIII-C-28516 (SL)

VIII-C-29523 (SL)

VIIJ-C-29615 (SL)

VIII-E-27751

VIII-E-27751 (SL)

VIII-E-27834 (SL)

VIII-E-28467 (SL)

VIII-E-28506 (SL)

VIII-E-29653 (SL)

IX-F-5830

\author{
U.S. Army Concepts \\ Analysis Agency \\ [V-B-28143 \\ IV-B-29530 \\ IV-C-26997
}

U.S. Army Dugway Proving Ground

II-B-26284

II-B-26914

II-B-28656

VI!-F-26491

VII-F-27995

VII-F-28664

VII-F-28954

VII-F-29369 (SL)

U.S. Army Engineer

Topographic Laboratories

IV-A-25874

IV-C-26394

VII-A-27471

U.S. Army Infantry School IV-C-28679 (SL)

IV-C-28686 (SL)

U.S. Anny Institute for

Research in Management

Information and Computer

Sciences

IV-E-26487

VIII-E-26823

VIII-E-28467

VIIl-E-28506 (SL)

VIII-E-29653 (SL)

U.S. Army Materiel

Systems Analysis Activity

IV-C-245S7

IV-C-25706

IV.C-26394

IV-C-26746

IV-C-26997

IV-C-27381

IV.C-27574

IV-C-27862

IV-C- 27868

IV-C-27993

IV-C-28679

JV-C-28718

IV.C-29358 (SL)

IV-D-26674

NV-D-27044

IV-D-27365

VIII-E-27834

VIII-E-28297 


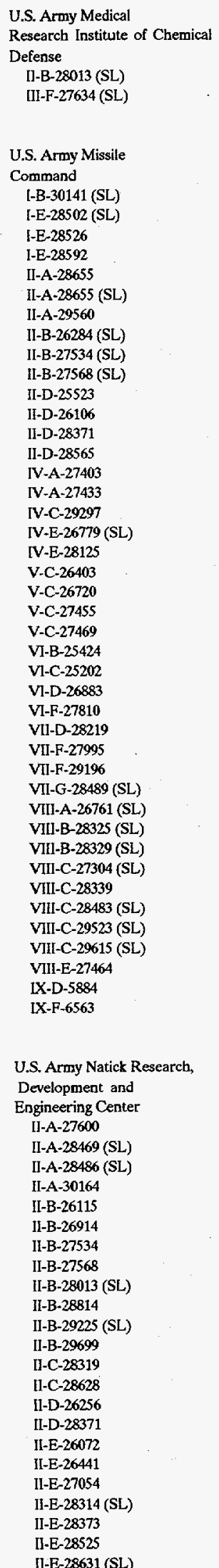

II-E-28675 (SL)

II-E-28711 (SL)

II-E-29627 (SL)

III-A-27494 (SL)

III-A-28691 (SL)

III-B-27314 (SL)

III-C-27488

III-C-27956 (SL)

III-C-28043 (SL)

III-C-28090

III-C-28361

III-C-28457 (SL)

III-C-28680 (SL)

III-C-28699(SL)

III-C-28972 (SL)

II]-C-29289

IIJ-D-25663

III-D-26582

III-D.26582 (SL)

III-D-27468 (SL)

III-D-27916 (SL)

III-E-29345

III-F-26767

III-F-29194 (SL)

VI-A-28369 (SL)

VI-C-26579

VI-C-27373 (SL)

VI-C-28560 (SL)

VI-C-28883 (SL)

VI-C-28900 (SL)

VI-C-30095 (SL)

VI-D-30108

VI.F-28620

[X-A-6307 (SL)

IX-B-5937

IX-B-6294

IX-B-6305

U.S. Army Research

Institute for the Behavioral and

Social Sciences

IV-E-26487 (SL)

IV-E-28125 (SL)

U.S. Army Research

Laboratory

I-A-28996

I-B-28531

I-B-28569

I-B-29391

I-D-26442

I-D-26996

1-D-28336

I-D-28652

I-D-28684

I-D-29212

I-D-29572

I-D-29576

I-D-29578

[-D-29923

I-E-27273

I-E-27586

I-E-28416 (SL)

I-E-28592

VI-D-26729

VI-D-27764

VI-D-28612

VI-D-29042 (SL)

VI-D-29218

VI-D-29282

VI-D-29750

VII-D-28219

VII-D-29360 (SL)

VIII-A-26188 (SL)

VIII-A-26896 (SL)

VIII-A-30090

VIII-B-26686 (SL)
VIII-B-27578

VIII-B-27883

VII]-B-28004 (SL)

VIIJ-B-28646

VIIl-B-28895

VIII-B-29223

VII-B-29616

VII-C-27304 (SL)

VII-E-27886

VIII-E-27917

VIII-F-26898 (SL)

VIII-F-28729 .

VHI-F-29210 (SL)

VIII-K-29540

IX-A-6280 (SL)

IX-D-5884

IX-D-5884 (SL)

IX-F-6563

U.S. Army Research

Laboratory Battlefield

Environments Directorate

I-B-28499

IV-B-28716 (SL)

IV-B-28735 (SL)

IV-B-28916 (SL)

VII-D-28717 (SL)

VII-F-26491

VII-F-26491 (SL)

VII-F-27995 (SL)

VII-F-28664 (SL)

VII-F-29196 (SL)

VII-F-29369 (SL)

VII-G-26962

VII-G-28052 (SL)

VII-G-28489

[X-F-6458 (SL)

U.S. Army Research

Laboratory Electronics \& Power

Sources Directorate

1-A-28996 (SL)

I-B-26821 (SL)

I-B-27532

I-B-28561

l-B-28569

I-B-28569 (SL)

I-B-28784

I-D-27780

I-D-28591 (SL)

I-D-28852 (SL)

I-D-29578

I-D-29923

I-E-28313

I-E-28592

II-A-26636

II-A-26636 (SL)

II-A-26748

II-A-27502

II-C-27770

II-D-26256

II-E-27054

II-E-27054 (SL)

[I-E-27808 (SL)

[II-C-29289 (SL)

IV-A-26909 (SL)

IV-B-28192

IV.B.28916

V-D-28155 (SL)

VI-A-26667 (SL)

VI-B-28780

VI-C-28067

VI-C-28938 (SL)

VI-D-26729

VI-D-27548 (SL)

VI-D-27605

VI-D-27764 (SL)
V-D-28141

VI-D-28146 (SL)

V]-D-28612

VI-D-29218 (SL)

V]-D-29677 (SL)

V1-D.29713 (SL)

VI-D-29750

V]-D-30068 (SL)

VI-F-27810

VIII-A-26444

VIII-A-27298 (SL)

VII-A-28335 (SL)

VII-A-28351 (SL)

VIJ]-A-28594 (SL)

VIII-A-30090 (SL)

VIII-A-30437 (SL)

VIII-B-26686

VIII-B-26711 (SL)

VIII-B-26755 (SL)

VIII-B-27443 (SL)

VIII-B-27578

VIII-B-27883 (SL)

VIII-B-28004

VIII-B-28325 (SL)

VIII-B-28329 (SL)

VIII-B-28345 (SL)

VIII-B-28461

VIII-B-28508

VIII-B-28646

VIIl-B-29223

VIII-B-29541 (SL)

VIII-B-29616

VIII-B-29631

VII-C-26949 (SL)

VIII-C-28339

VIII-C-29523 (SL)

VIII-C-29615 (SL)

VIII-F-27865

VIII-F-28729

VIII-F-28729 (SL)

VII-F-29210 (SL)

VIII-F-30549

VIIl-K-29540

IX-A-6280 (SL)

IX-A-6307

IX-B-6674 (SL)

IX-D-5884

IX-D-5884 (SL)

[X-F-6170

LX-F-6563

U.S. Army Research

Laboratory Human

Research and Engineering

Directorate

IV-G-29349 (SL)

U.S. Army Research

Laboratory Materials

Directorate

I-B-28561

B-29373

I-D-29341 (SL)

I-E-28313

II-A-26636 (SL)

II-A-26748

II-A-27600

II-A-28402

II-A-28469

II-A-29560

II-A-30164

II-B-26115

IJ-B-26914

II-B-28814

II-B-29225

II-C-26126

II-C-28319 (SL) 


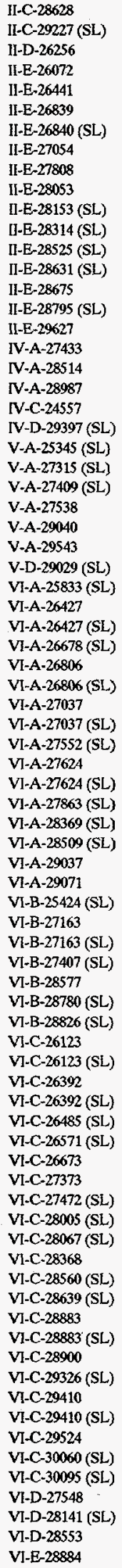

VI-E-28884 (SL)

VI-E-30124

VI-E-30488

VI-F-26021

VI-F-26021 (SL)

Vl-F-27364 (SL)

VI-F-30337

VIII-C-26791

[X-A-6078

IX-A-6280 (SL)

EX-D-5548 (SL)

IX-D-5884

IX-D-6011 (SL)

IX-D-6148 (SL)

U.S. Army Research

Laboratory Vehicle

Propulsion Directorate B-26631

V-B-27627

V-B-28249 (SL)

V-C-26456 (SL)

V-C-27018

V-C-27417 (SL)

V-C-28250

V-C-28898 (SL)

V-C-29141

VI-A-26449

V1-A-27037

V-A-27624

VI-B-26751

VI-C-25202

VI-C-26123

VI-C-26673 (SL)

VI-C-27472

VI-C-28005

VI-C-28067

VI-C-28639

IX-C-5824

U.S. Army Researcb

Laboratory Vehicle

Structures Directorate

V-A-26908

V-A-28222 (SL)

V-A-28307

V-B-25461 (SL)

V-B-25467 (SL)

V-B-26631 (SL)

V-B-28002

V-B-28651

V.D-25462 (SL)

V-D-26061 (SL)

V-D-28493 (SL)

Vl-B-25424

Vl-B-27407

VI-C-26571

VI-C-28900

U.S. Army Research

Laboratory Weapons

Tecbnology Directorate

I-D-27780

I-D-28362

I-D-28652

II-B-27534

II-D-25523

II-D-26106 (SL)

[]-D-27025

[I-D-27603 (SL)

II-D-28103 (SL)

Il-D-28565

II-D-28700 (SL)

II-D-29033
JI-D-29596

II-E-27808 (SL).

IV-A-25874

IV:A-28728

IV-A-28766

IV-A-28986 (SL)

IV-A-28987 (SL)

IV-B-27690 (SL)

IV-B-28320 (SL)

IV-B-28377

IV-B-28535

IV-B-28716

IV-B-28735 (SL)

IV-B-28916

IV-B-28916 (SL)

IV-B-29574

IV-B-29808 (SL)

IV-B-29850 (SL)

IV-C-26394

IV-C-26746

IV-C-26993 (SL)

IV-C-26997

IV-C-28679

IV-C-28686 (SL)

IV-C-28722 (SL)

IV-C-28743

IV-D-29396

V-A-25345

V-A-26443 (SL)

V-A-26908

V-A-27409

V-A-27538

V-A-28283 (SL)

$\mathrm{V}-\mathrm{A}-28307$

$V+A-29040$

V-A-29543 (SL)

$\mathrm{V} \cdot \mathrm{B}-26863(\mathrm{SL})$

V-B-27062

V-B-27558 (SL)

V-B-28249

V-B-28293

V-B-30113

V.C-26403

$\mathrm{V}-\mathrm{C}-26720$

$\mathrm{V}-\mathrm{C}-26797$

V-C-27455 (SL)

V-C-28250

V-C-28310 (SL)

$\mathrm{V}-\mathrm{C}-29336$ (SL)

V-C-29771 (SL)

V-D-28399

VI-B-28577 (SL)

VI-B-28761

VI-C-26392

VI-C-26392 (SL)

VI-C-26485

VI-C-26485 (SL)

VI-C-26673

V.-C-28368

Vl-C-28368 (SL)

VI-C-29326 (SL)

V]-D-28553

VI-E-28272 (SL)

VI-E-28575

VI-E-28575 (SL)

VI-E-28884

VI-E-301.24 (SL)

VI-E-30488

VI-F-26021

VI-F-26021 (SL)

VIl-D-30387 (SL)

VIII-E-27028 (SL)

VIII-E-27493 (SL)

VIII-E-27994

IX-A-6078

LX-A-6280 (SL)

[X-A-6366

IX-B-6089 (SL)

IX-D-6148

\author{
U.S. Army Research Office \\ I-B-26959 (SL) \\ [-B-27646 (SL) \\ I-B-29184 \\ I-E-28592 \\ II-A-26748 (SL) \\ IV-A-28766 \\ IV-B-28799 (SL) \\ V-C-27469 \\ VI-A-26427 \\ VI-F-28620(SL) \\ VIIl-B-26711 (SL) \\ VII-B-26755 (SL) \\ VIII-B-28325 \\ VIII-B-28325 (SL) \\ VIII-B-28329 \\ VIII-B-28329 (SL) \\ VIII-C-28339 (SL) \\ VIII-F-28729 (SL) \\ VIII-F-29210 (SL) \\ [X-A-6078 \\ [X-A-6280 (SL) \\ LX-A-6307 \\ IX-A-6366 \\ IX-B-5937 (SL) \\ IX-B-6294 (SL) \\ IX-B-6305 \\ IX-B-6674 \\ IX-C-5824 \\ IX-D-5884 \\ IX-D-6011 (SL) \\ IX-F-6291 (SL) \\ LX-F-6458 (SL) \\ IX-F-6563 \\ IX-F-6563 (SL)
}

U.S. Atmy Signals Warfare

Center, CECOM

IV-E-26487

IV-E-27892

TV-E-27892 (SL)

IV-G-26802

IV-G-26930

IV-G-27524

[V-G-27817

IV-G-28060

VIII-E-27899 (SL)

U.S. Army Survivability

Management Office

VIII-E-26460

VIII-E-27028

VIII-E-27493

VIII-E-27754

U.S. Army Tank-Automotive

Command

I-E-28356

].E-28416

IV-B-27786 (SL)

IV-B-28007 


V-C-29141 (SL)
V-C-29810
V-D-25630
V-D-26934 (SL)
V-D-26995 (SL)
V-D-27075 (SL)
V-D-29029
VI-A-25833
VI-A-26400
VI-A-26427
VI-A-26667 (SL)
VI-A-27552
VI-A-28369
VI-A-29037
VI-A-29071
VI-B-25424
VI-B-28780 (SL)
VI-B-28826

VI-C-26123
VI-C-26673
VI-C-28005
VI-C-30060
VI-D-26601
VI-E-28884
VI-E-30488
IX-C-5824
J.S. Army Test and Evaluation
Command
VII-E-28102
U.S. Army Toxic and
azardous Materials Agency
II-D-27505
II-D-28371
VII-A-28504

U.S. Army Vulnerability
Assessment Laboratory
VI-D-30108 (SL)
VIII-E-27464
Walter Reed Army Institute of
Researcb
III-C-28090
III-D-25476 (SL)
III-D-28479 (SL)
IV-C-27574
IV-C-28679
IV-C-28686
Waterways Experiment
Station
IV-B-28716
IV-B-28735
IV-B-28916
IV-B-28916 (SL)
IV-B-29808 (SL)
[V-B-30297 (SL)
VII-A-26902 (SL)
VII-A-27401 (SL)
VII-A-28504 (SL)
VII-C-24381 (SL)
VII-D-27485 (SL)
VII-D-29360
VII-E-26982

White Sands Missile Range I-E-28258 (SL)

IV-C-26394

IV-C-26997

VIII-E-26735

VIII-E-27464 


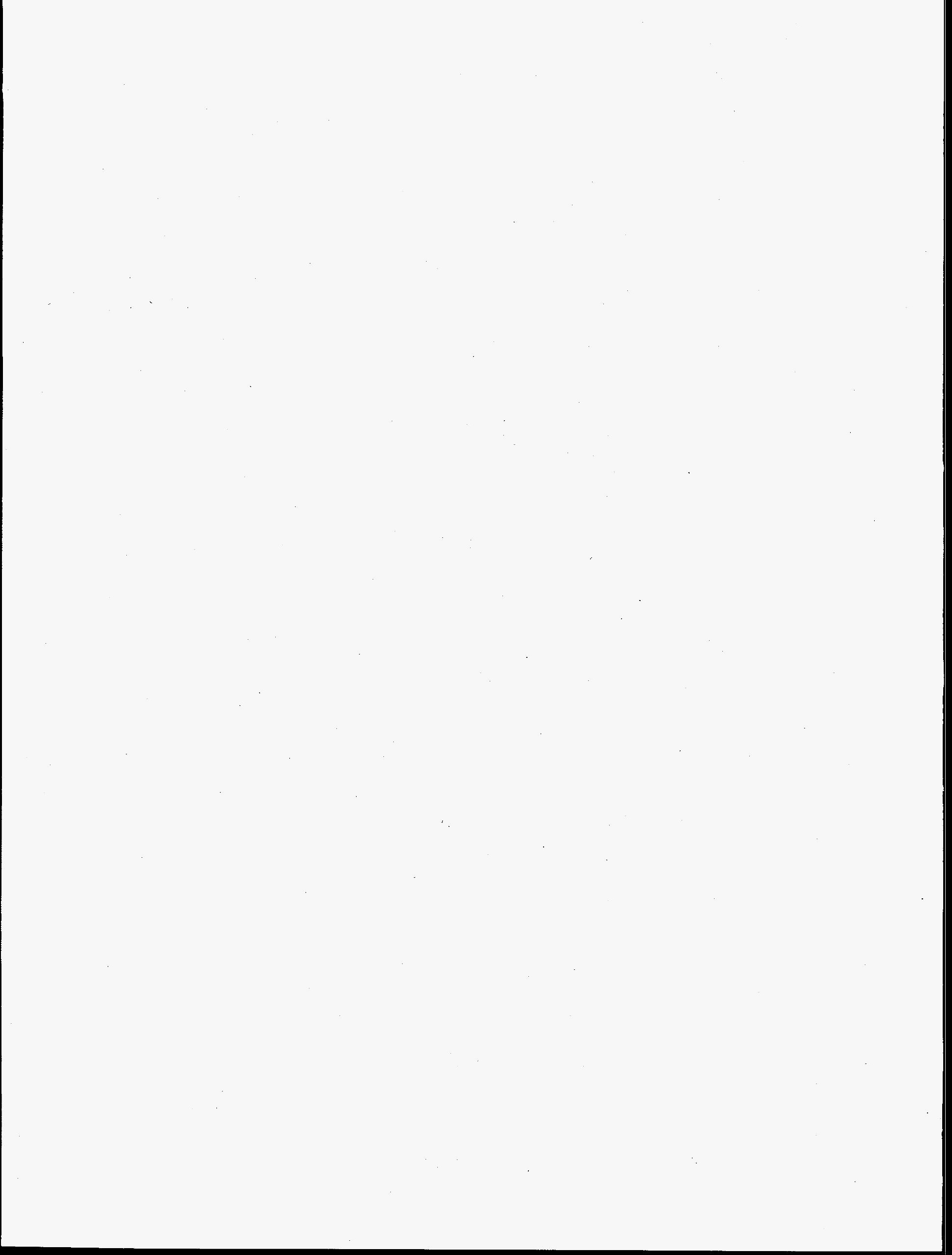




\section{Contractors and Grantees}

Note: The Roman numeral refers to the scientific division, and the alphabetic character refers to the division subsection. Proposal numbers are in ascending sequence within the subsections.

\author{
AT\&T Technology Systems \\ Greensboro, North Carolina \\ I-D-28271 \\ Aerodyne Research, Inc \\ Billerica, Massachusetts \\ []-D-28103 \\ University of Alabama - Huntsville \\ Huntsville, Alabama \\ [1-B-26284 \\ University of Alaska Geophysical Institute \\ Fairbanks, Alaska \\ VII-B-26281

\section{Alfred University \\ Alfred, New York \\ VI-D-27548

Analytic Power Corp.
Bostor, Massacbusetts
II-D-30443
Arizona State University
Tempe, Arizona
V-A-28222
V-B-28002
VI-A-29037
VII-B-26686
VII-B-28461
VIIl-B-28508

University of Arizona
Tucson, Arizona
I-E-26974
I-E-28356
II-E-27440
IV-G-30411
V-B-30113
VI-C-28900

Arnold Engineering and Development

Center

Arnold Air Force Station, Tennessee V-C-28912

Auburn University
Auburn, Alabama
III-F-27634
V-D-26061
BDM Corp.
McLean, Virginia
V-B-28608
Boston College
Chestnut Hill, Massachusetts
I-D-26682
I-D-28652

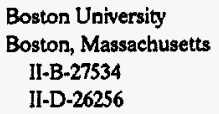

Brookhaven National Laboratory Upton, New York

VI-A-26667

Brooklyn College

Brooklyn, New York

VIIl-B-29036

Brown University

Providence, Rhode Island

III-F-29170

IV-A-27403

IV-B-28071

IV-B-28716

IV-G-26811

IV-G-28428

V-C-28898

VI-E-28575

Brunel University

Uxbridge, Middx, UK

1IX-A-6078

California Institute of Tecbnology
Pasadena, California
I-E-27273
I-E-29321
II-A-28945
IV-A-25874
IV-E-26487
V-C-29534
VI-D-29677
VI-E-28884
VI-E-30352
VII-C-29523

University of California, Berkeley Berkeley, California

IV-C-27993

IV-D-28835

[V-E-27892

VI-A-25833

VII-C-24381

VIIl-C-26949

VIII-E-27886

University of California, Davis

Davis, California

III-D-27916

V-D-27075

\author{
University of California, Irvine \\ Irvine, California \\ V1-C-29410 \\ University of California, Los Angeles \\ Los Angeles, California \\ II-A-29708 \\ III-D-26232 \\ IV-B-28735 \\ IV-B-28916 \\ VIII-A-26896 \\ VIII-C-27904 \\ University of California, San Diego \\ La Jolla, California \\ IV-G-26802 \\ V-C-27455 \\ VI-C-26392 \\ VI-C- 28368 \\ VIII-E-27754 \\ University of California, San Francisco \\ San Francisco, California \\ III-F-29221 \\ University of California, Santa Barbara \\ Santa Barbara, California \\ I-B-28569 \\ I]-B-28656 \\ IV-A-27869 \\ VIII-P-28922 \\ Cambridge, University of \\ Cambridge, UK \\ IX-B-5937

Carnegie-Mellon University
Pittsburgh, Pennsylvania
II-E-28631
IV-A-27557
IV-A-28514
IV-E-29354
IV-G-28994
VI-B-27407
VI-D-28146

Case-Western Reserve University
Cleveland, Ohio
VI-A-27037
VII-D-27911

Central Florida, University of
Orlando, Florida
I-A-28996
VII-A-28594
Centre National de la Recherche
Scientifique
Meudon, France
IX-F-6563 \\ Carnegie-Mellon University \\ Pittsburgh, Pennsylvania \\ IV-A-28514 \\ IV-E-29354 \\ IV-G-28994 \\ VI-B-27407 \\ VI-D-28146
}


University of Cincinnati

Cincinnati, Obio

IV-B-28535

VI-C-26579

VI-C-27373

\section{Clark University \\ Worcester, Massacbusetts \\ II-E-26840 \\ II-E-28795}

\section{Clarkson University \\ Potsdam, New York \\ V-C-26797}

VII-G-26962

\section{Clemson University \\ Clemson, South Carolina \\ VI-C-30095 \\ VIII-C-28151}

Colorado School of Mines Golden, Colorado

III-D-29300

VI-C-30060

Colorado State University

Fort Collins, Colorado

1-D-29375

II-D-28565

IV-B-29530

University of Colorado
Boulder, Colorado
I-B-28561
I-D-30408
I-E-26971
I-E-28592
IV-A-28728
IV-B-28701
IV-E-28950
VII-F-27995
VIII-C-29615

University of Colorado at Colorado Springs Colorado Springs, Colorado I-D-28684

\section{University of Colorado at Denver \\ Denver, Colorado III-C-27488}

University of Colorado at Denver, Heaitb Science

\section{Center}

Denver, Colorado

III-E-29345

\section{Columbia University \\ New York, New York \\ 1-B-29391 \\ I-B-30423 \\ IV.C-28715 \\ V-D-26995 \\ VIII-G-28453}

Connecticut University Health Center

Farmington, Connecticut III-C-27956

University of Connecticut

Storrs, Connecticut

I-D-26442

IV-C-28743
VI-A-27863

VI-C-26571

Cornell University

Itbaca, New York

I-D-26996

II-A-27502

II-D-25523

II-D-28700

III-B-26750

IV-G-26930

JV-G-29031

VI-A-26806

V1-A-27624

VIII-B-26755

Cranfield Institute of Technology

Cranfield, UK

IX-C-5824

DCW Industries, Ine.

La Canada, California

V-B-26863

Dartmouth College

Hanover, New Hampshire

I-B-28531

VI-B-28480

VI-B-27482

VII-B-28599

University of Davton

Dayton, Ohio

VIII-C-25557

\section{University of Delaware \\ Newark, Delaware \\ V]-D-29713 \\ VIII-E-28506}

Drexel University

Philadelphia, Pennsylvania V-C-27417

Duke University

Durham, North Carolina

J-B-27766

l-B-27888

I-D-28468

1-E-27556

IV-B-28799

IV-C.29740

V-D-28493

VI-D-26729

VI-F-30337

VII-A-27401

Duke University Medical Center

Durham, North Carolina

III-F-26767

East Carolina University

Greenville, North Carolina

III-D-25752

Emory University

Atlanta, Georgia

II-A-28469

[]-E-28675

University of Florence

Florence, Italy

[X-F-6458
Florida Atlantic University

Boca Raton, Florida

V-D-28123

Florida State University

Tallabassee, Florida

IV-C-27868

University of Florida

Gainesville, Florida

1-D-28362

II-A-30164

II-B-26914

II-E-26441

VIII-E-26735

Flow Analysis lncorporated Tullahoma, New York

V-B-25623

General Electric Company R\&D Center

Schenectady, New York VIII-F-27865

General Research Corporation

Santa Barbara, California

VI-F-29643

Gent, University of

Gent, Belgium

DX-F-6291

George Mason University

Fairfax, Virginia

IV-C-28309

Georgia Institute of Technology

Atlanta, Georgia

[-E-27366

V-A-29543

V-B-25461

V-D-25327

VI-F-26021

VII-D-27485

VIII-C-28339

VIII-C-28516

VIII-G-27554

Georgia State University

Atlanta, Georgia

I+B-26709

University of Georgia

Athens, Georgia

IV-A-27580

Giner, Inc.

Waltham, Massachusetts

II-A-30289

Hahnemann University

Pbiladelpbia, Pennsyivania

UlI-D-28479

Harvard University

Cambridge, Massachusetts

1-D-27425

IV-C-26993

IV-G-29190 


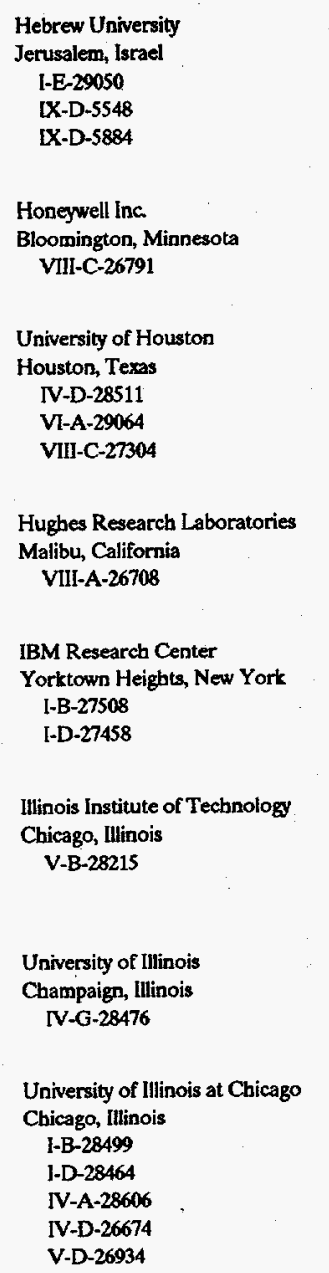

University of Illinois at Urbana-Champaign Urbana, Illinois
II-C-28319
II-D-27025
IV-C-28718
V-B-27558
VII-D-29205
VIII-A-30090
VII-B-26711
VIII-B-28406
VIII-E-27994
VII-F-30595

Imperial College of Science and Tecbnolog London, UK

IX-A-6366

Indiana University - Purdue University at

Indianapolis

lndianapolis, indiana

VI-D-29042

Integrated Systems Inc.

Santa Clara, California

V-D-28350

Iowa State University of Science and

Tecbnology

Ames, lowa

V.B-27062

$$
\begin{aligned}
& \text { University of lowa } \\
& \text { Iowa City, lowa } \\
& \text { J-E-27591 } \\
& \text { III-C-28699 } \\
& \text { V-C-28310 } \\
& \text { VI-B-26031 }
\end{aligned}
$$

Jet Propulsion Laboratory

California Institute of Tecbnology

Pasadena, California

V-C-27018

The Jobns Hopkins University

Baltimore, Marytand

II-C-28767

VI-E-28272

VIII-E-27028

Kansas State University

Manbattan, Kansas II-C-27775

University of Kansas

Lawrence, Kansas

[I]-C-28669

Kent State University

Kent, Obio

IV-B-28908

Lehigh University

Bethlehem, Pennsytvania

[-B-26899

V]-B-28549

VI-D-29282

Lockheed Missiles \& Space Cc., Inc. Palo Alto, California

VI-B-26751

Lockheed Sanders, Inc

Nashua, New Hampshire

I-D-29212

Loughborough University of Technology

Leicestershire, UK

IX-B-6294

Maryland, University of

College Park, Maryland

IV-B-29574

IV-C-29678

IV-E-29692

V-B-25467

VIII-B-28004

Massachusetts Institute of Technology

Cambridge, Massachusetts

[-B-27532

I-B-30141

1-D-29578

II-D-28371

III-C-2S126

III-C-28361

V.A-27538

V-B-27627

VI-A-28369

VI-C-25202

VI-D-28553

VII-A-26902

VIII-B-27883

VII-B-28345

VIII-B-29631

VII-G-28925
MIT Lincoln Laboratory

Lexington, Massachusetts

VI-D-29606

University of Massachusetts, Lowell

Lowell, Massachusetts

III-B-27314

University of Massachusetts

Amherst, Massachusetts

II-B-29699

II-C-26126

II-E-28314

VII-D-29360

VIII-C-26599

VIII-E-26823

VIII-E-27493

McGill University

Montreal, Canada

II-B-28013

MetroLaser

Irvine, California

V-C-29810

University of Metz

Metz, France

LX-D-6148

Michigan State University

East Lansing, Mictigan

III-A-28022

Michigan Technological University

Houghton, Michigan

V-A-27409

VI-A-29347

University of Michigan

Ann Arbor, Michigan

I-D-29922

I-D-29923

I-E-28339

IV-D-27044

V-D-27510

VI-A-28509

VIJ-D-30387

VIII-B-28325

VIII-C-28483

VIII-E-27834

Minnesota Supercomputer Center, Inc. Minneapolis, Minnesota

$$
\text { IV-D-29396 }
$$

University of Minneapolis at Minnesota Minneapolis, Minnesota

II]-D-29594

N-A-28986

[V-A-28987

IV-B-29850

IV-D-27365

IV-G-28408

VI-D-29218

VIII-A-26761

VIII-B-27578

VIII-B-29223

VII-B-29616

VIII-E-27076 
University of Minnesota, St. Paul

St. Paul, Minnesota

IV.A-28797

University of Mississippi, Medical Center

Jackson, Mississippi

III-C-28972

University of Missouri, at Columbia

Columbia, Missouri

IJ-A-28486

VI-A-26400

University of Missouri, at Rolla

Rolla, Missouri

V-A-26443

V-A-28283

Modell Development Corp.

Framingham, Massacbusetts

II-D-30230

Montana State University

Bozeman, Montana

VII-B-28040

National Acadeny of Sciences

Wasbington, District of Columbia IV-D-29397

NASA Ames Research Center Moffett Field, California

v-C-27469

National Center for Atmospheric

Research

Boulder, Colorado

VIl-F-28954

National Institute of Standards and

Technology

Gaithersburg, Maryland

II-C-30094

II-D-29596

VI-C-26123

National Oceanic and Atmospheric

Administration

Boulder, Colorado

VII-E-28094

VII-F-28664

Naval Postgraduate School

Monterey, California

IV-E-28328

V-B-27894

University of Nebraska

Lincoln, Nebraska

I-D-27780

VII-D-28219

University of New Hampshire

Durham, New Hampshire

VI-A-26449

University of Medicine \& Dentistry

New Jersey

Piscataway, New Jersey

III-C-29289
New Mexico State University

Las Cruces, New Mexico

VII-G-28052

University of New Mexico

Albuquerque, New Mexico

II-C-28628

New York Institute of Technology

Old Westbury, New York

VIII-A-28674

New York State College of Ceramics at Alfred

University

Alfred, New York

VI-C-29098

New York University Courant lnstitute of

Mathematics

New York, New York

IV-A-29351

University of New York City

New York, New York

]-D-29572

University of New York State at Albany

Albany, New York

II-E-26072

VII-D-28717

University of New York State at Buffalo Buffalo, New York

III-D-26582

III-F-28775

University of New York State at Stony Brook

Stony Brook, New York

II-E-28153

IV-A-27433

IV-B-29808

Nielsen Engineering \& Research

Incorporated

Mountain View, California

V-B-29049

North Carolina Agricultural and Technical State

University

Greensboro, North Carolina

V-E-29579

North Carolina State University

Raleigh, Nortb Carolina

[-D-28336

I-E-27586

I-E-28313

II-E-28373

UII-B-29697

IV-A-28702

IV-B-27786

[V-B-28192

IV-E-27567

VI-B-26825

VI-C-26673

VI-D-26601

VI-F-27364

VI.F-27810

VIII-D-28070

VIII-F-28099

VII-F-28187

VIII-F-30549
University of North Carolina at Chapel Hill

Chapel Hill, North Carolin

II-C-29538

IV-C-29297

V.-D-27846

VI-F-26246

VII-A-28504

University of North Carolina at Chariotte Charlotte, Nortb Carolina

!-E-28416

VIII-B-27443

Northeastern University

Boston, Massachusetts

IV.C-27790

IV-C-29358

IV-D-28740

Nortbwestern University

Evanston, Illinois

II-E-27054

IV-B-28320

V-C-28905

VI-B-27163

University of Nottingham

Nottingham, UK

IX-B-5937

Optoelectronics Industry Development

Association

Palo Alto, Califomia

VIII-A-30437

Obio State University

Columbus, Ohio

[-B-29324

I]-A-29560

V-A-29040

V-B-26595

$\mathrm{V} \cdot \mathrm{B}-28249$

VI-C-29524

VI-D-28141

VI-D-30108

VIII-E-28467

VIII-E-29653

Oklahoma State Universit

Stillwater, Oklahoma

[-D-28472

II-D-2610

II-E-26839

Old Dominion University

Norfolk, Virginia

l-B-2682

IV-G-29053

Oregon Graduate lnstitute of Science and

Technology

Beaverton, Oregon

VII-E-28102

Oregon State University

Corvaltis, Oregon

1-D-28852

VI1-F-26491 


\section{University of Oregon \\ Eugene, Oregon \\ I-B-29015}

[-E-25546

\section{The Pennsytvania State University \\ University Park, Pennsylvania \\ I-B-28784 \\ I-E-28502 \\ II-C-27770 \\ II-E-28711 \\ IV-G-26792 \\ V-C-28427 \\ V-C-29336 \\ VI-C-29326 \\ VI-D-30668 \\ VII-A-27471 \\ VII-E-26982 \\ VILF-29196 \\ VIII-A-26444}

University of Pennsylvania

Philadelphia, Pennsylvania

IV-C-28982

IV-E-26779

IV-E-28125

IV-E-30061

\section{University of Pittsburgh \\ Pittsburgh, Pennsylvania \\ II-C-29227 \\ III-C-28011 \\ HI. - - -28043}

Polytechnic Institute of New York,

Farmingdale

Farmingdale, New York

Ii-A-26636

Princeton Combustion Research Laboratory, Inc.

Monmouth Junction, New Jersey V-C-26403

Princeton University

Princeton, New Jersey

I-D-26015

II-D-28555

II-D-28889

[V-A-26909

IV-A-28766

IV-C-26997

V-C-27565

V-C-29141

VII-A-28772

VIII-A.28335

Purdue University

Lafayette, Indiana

II-B-28528

III-C-28929

$\mathrm{V} \cdot \mathrm{A}-28307$

V.D-27300

V-D-29029

VII-B-27316

VIII-E-27464

Rensselaer Polytechnic Institute

Troy, New York

[-D-28591

IV-B-29167

V-D-25462

V1-B-28577

VI-D-27605

VIII-B-28329

\author{
University of Rhode Island \\ Kingston, Rhode Istand \\ III-D-25476 \\ IV-G-27620
}

Rice University

Houston, Texa

I-B-29373

II-A-30446

IV-C-28686

IV-G-26932

University of Rochester

Rocbester, New York

I-B-29184

I-D-28470

Rack Engineering Consultants

Welwyn Garden City, Herts, UK IX-E-6296

Rockwell International Corporation Canoga Park, California

$$
\text { II-A-28655 }
$$

Rutgers, The State University of New

Jersey

New Brunswick, New Jersey

II-B-29225

III-A-27494

IV-C-27862

V-B-28651

Rutgers, The State University of New

Jersey

Piscataway, New Jersey

II-B-27568

V-D-28155

VI-B-25424

SCS Telecom, Inc.

Port Washington, New York VIII-E-27751

SRI International

Menlo Park, California

I-B-26959

I-C-28955

V-C-27864

VI-E-30488

Statistical Signal Processing Inc.

Yountville, California

VIII-E-27917

Sandia National Laboratories

Livermore, California

II-D-27887

Science Research Laboratory, Inc. Somerville, Massacbusetts

II-D-30332

Scientific Research Associates, lnc

Glastonbury, Connecticut

VII]-B-28646

The Scripps Research Institute

La Jolla, California

III-C-29195
Semiconductor Research Corporation

Research Triangle Park, North Carolina VIII-K-29540

Signal Processing Technology, Ltd.

Palo Alto, California

VIII-E-27899

University of South Carolina

Columbia, South Carolin

[II-D-25663

University of Soutb Florida

Tampa, Florida

VI-D-27764

University of Southern California Los Angeles, California

[-B-26257

I-B-29595

V-B-28826

VIII-A-27298

VIII-F-30500

Southern Methodist University

Dallas, Texas

VIII-C-25339

Soutbwest Research Institute

San Antonio, Texas

VI-F-29647

Stanford University

Stanford, California

I-B-28978

I-D-29576

I-E-26695

I-E-28348

II-A-28353

IV-A.28548

IV-B-28143

[V-B-28377

IV-C-26394

IV-E-28354

IV-G-27817

IV.G-28060

IV.G-28809

IV-G-29412

V-A-28253

V-C-27480

VI-B-28761

VI-D-26883

VIII-E-28297

VIII-E-28387

VIII-F-28729

VIII-F-29210

VIII-G-25602

VIII-G-28326

Stevens Institute of Technology

Hoboken, New Jersey

V-C. 25706

VI-C-28938

Surface Optics Corp.

San Diego, California VII-D-27031

University of Surrey

Guildford, Surrey, UK

[X-B-6674

DX-F-6170 
Swiss Federal Polytechnic Institute Lausanne, Switzerland IX-B-6305

TTTAN Systems, Inc., ARAP Group

Princeton, New Jersey VII-F-29369

TRW, Jnc.

Redondo Beach, California I-D-28979

Tel Aviv University

Tel Aviv, tsrael [X-B-6089

University of Tennessee, Knoxville

Knoxwille, Tennessee

IV-B-29113

V1-A-26678

Texas A \& M University

College Station, Texas

III-D-27468

IV-C-27574

IV-G-27524

Texas Christian University

Fort Worth, Texas

II-E-29627

Texas Tech University

Lubbock, Texas

[V-C-26746

University of Texas at Arlington

Arlington, Texas

II-E-27808

University of Texas at Austin

Austin, Texas

I-E-28258

II-A-26748

II-C-26822

II-E-28053

Il-E-28525

[V-B-30297

V-A.27315

V-B-28293

VIII-A-28351

VIII-B-28895

VIII-C-25045

University of Texas at Dallas Richardson, Texas

$\mathrm{V}-\mathrm{C}-28250$

University of Trieste

Trieste, ltaly

LX-A-6280

Trinity College

Dublin, Ireland

IX-A-6307
Tufts University

Medford, Massachusetts

I-E-27882

I-E-28526

V-A-25345

University of Tulsa

Tulsa, Okjahoma

III-F-29537

Uniformed Services of the Health Sciences Bethesda, Maryland

III-C-27890

United Technologies Research Center

East Hartford, Connecticut

V-B-26631

V.C-26456

University of Utab

Salt Lake City, Utab

II-D-27603

III-A-29332

VI-D-28612

Virginia Polytechnic Institute and State University Blacksburg Virginia

V-A-26908

V-D-25630

VI-C-28560

VI-C-28883

VII-D-29633

Vanderbilt University

Nastiville, Tennessee

II-A-28402

V.C-26720

V1-A-29071

Virginia Commonwealth University

Richmond, Virginia

I-D-29341

University of Virginia

Charlottesville, Virginia

$$
\begin{aligned}
& \text { II-A-29725 } \\
& \text { III-P-29194 } \\
& \text { V-D-28399 } \\
& \text { VI-C-28067 } \\
& \text { VI-C-28639 } \\
& \text { VIII-A-28347 }
\end{aligned}
$$

Washington State University

Pullman, Washington

V-B-28159

VI-E-30124

Washington University

St. Louis, Missouri

IV-G-29349

University of Washington

Seattle, Wasbington

V-C-29771
VII-B-28344

VII-D-29404

Wayne State University

Detroit, Michigan

II-B-26115

V-C-25190

V-C-29302

VI-B-28780

VII-B-29541

Wesleyan University

Middletown, Connecticut III-C-28090

Western Michigan University Kalamazoo, Michigan II-D-27505

Windermere Laboratory Ambleside, Cumbria, UK IX-E-6185

University of Wisconsin - Madison Madison, Wisconsin
I-B-27646
II-A-27600
II-D-29033
IV-A-27641
IV-B-27690
IV.C-27381
IV-C-28679
IV-C-28722
VI-A-27552
VI-C-27472
VI-C-28005
V.D. 29750
VIII-E-26460

University of Wisconsin - Mihwaukee Milwaukee, Wisconsin

$$
\text { VI-A-26427 }
$$

Worcester Polytechnic Institute Worcester, Massacbusetts

$$
\text { VIII-A-26188 }
$$

University of Wyoming

Laramie, Wyoming

II-B-28814

III-C- 28457

Yale University

New Haven, Connecticut

III-B-28964

III-C- 28680

IV-B-28007

VII-G-28489

Zernow Technical Services, Inc. San Dimas, California

V1.C-26485 


\section{Principal Investigators}

Note: The Roman numeral refers to the scientific division, and the alphabetic character refers to the division subsection. Proposal numbers are in ascending sequence within the subsections.

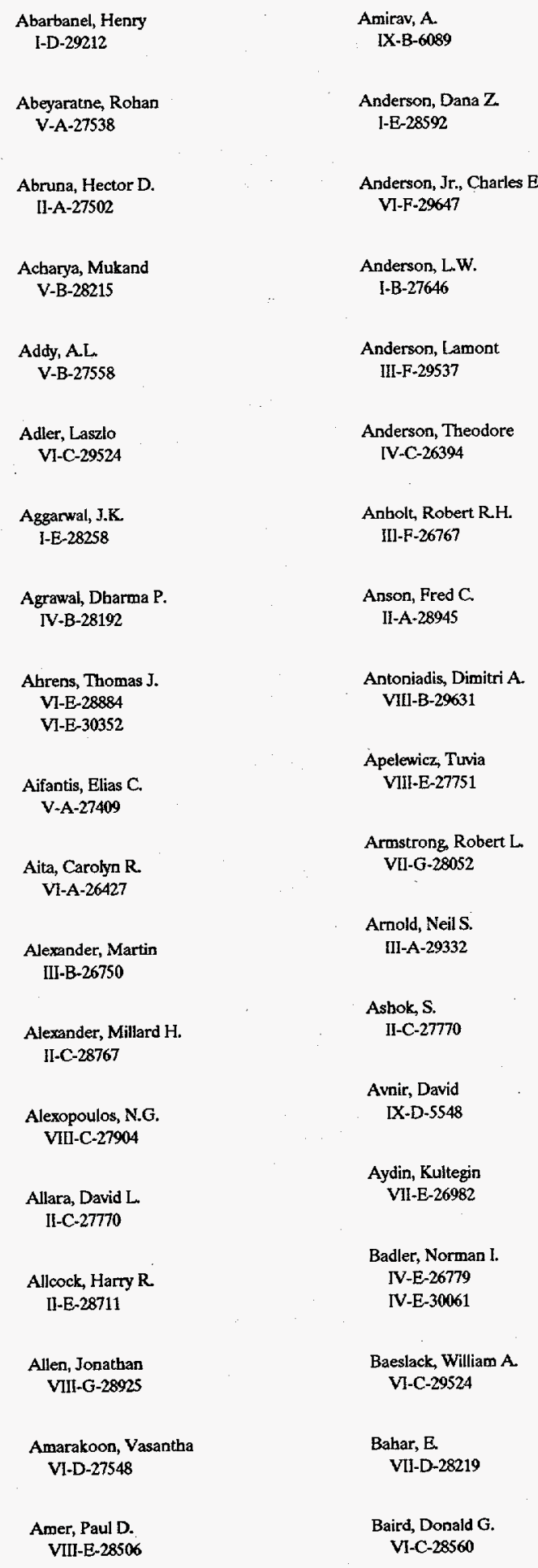

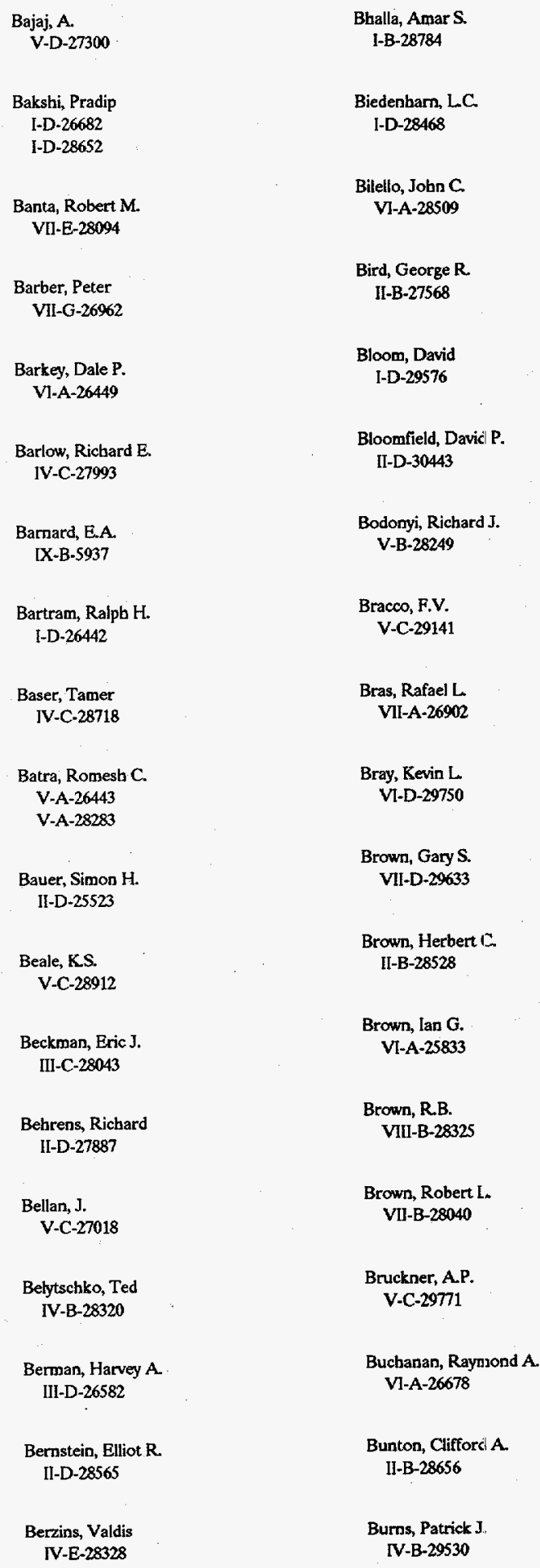




\section{$X$ indexes}

\begin{tabular}{|c|c|}
\hline $\begin{array}{l}\text { Butler, Chalmers M. } \\
\text { VIII-C-28151 }\end{array}$ & $\begin{array}{c}\text { Chew, Weng Cho } \\
\text { VII-D-29205 }\end{array}$ \\
\hline $\begin{array}{l}\text { Butler, Jerome K } \\
\text { VIII-C-25339 }\end{array}$ & $\begin{array}{l}\text { Chiang, Yet-Ming } \\
\text { V1-C-25202 }\end{array}$ \\
\hline $\begin{array}{c}\text { Butler, P. Barry } \\
\text { V-C-28310 }\end{array}$ & $\begin{array}{l}\text { Chin, Jik } \\
\text { II-B-28013 }\end{array}$ \\
\hline $\begin{array}{l}\text { Byer, Robert L. } \\
\text { L-E-28348 }\end{array}$ & $\begin{array}{c}\text { Chlamtac, Imrich } \\
\text { VIII-E-26823 }\end{array}$ \\
\hline $\begin{array}{l}\text { Caflisch, Russell } \\
\text { IV-B-28916 }\end{array}$ & $\begin{array}{c}\text { Cbopra, Inderjit } \\
\text { V-B-25467 }\end{array}$ \\
\hline $\begin{array}{l}\text { Catvert, Paul } \\
\text { VI-C-28900 }\end{array}$ & $\begin{array}{c}\text { Chou, Stephen Y. } \\
\text { VII]-B-27578 } \\
\text { VIII-B-29223 }\end{array}$ \\
\hline $\begin{array}{c}\text { Camiey, R.E. } \\
\text { I-D-28684 }\end{array}$ & Chou, Y.T. \\
\hline $\begin{array}{l}\text { Campbell, Stephen L } \\
\text { IV-B-27786 }\end{array}$ & V]-B-28549 \\
\hline $\begin{array}{l}\text { Car, R } \\
\qquad \text { XX-A-6280 }\end{array}$ & $\begin{array}{c}\text { Christe, Karl O. } \\
\text { Il-A-28655 }\end{array}$ \\
\hline $\begin{array}{l}\text { Cardin, D.J. } \\
\text { IX-A-6307 }\end{array}$ & $\begin{array}{c}\text { Chu, Benjamin } \\
\text { [1-E-28153 }\end{array}$ \\
\hline $\begin{array}{r}\text { Carling, P.A } \\
\text { DX-E-6185 }\end{array}$ & $\begin{array}{c}\text { Chui, Charles K } \\
\text { IV-G-27524 }\end{array}$ \\
\hline $\begin{array}{c}\text { Case, Steven T. } \\
\text { III-C-28972 }\end{array}$ & $\begin{array}{l}\text { Chylek, Petr } \\
\text { VII-D-28717 }\end{array}$ \\
\hline $\begin{array}{l}\text { Casey, H. Craig } \\
\text { I-E-27556 }\end{array}$ & $\begin{array}{c}\text { Ciftan, Mikael } \\
\text { ]-D-28468 }\end{array}$ \\
\hline $\begin{array}{l}\text { Cebe, Pegby } \\
\text { VI-A-28369 }\end{array}$ & $\begin{array}{l}\text { Clark, Douglas S. } \\
\text { III-C-28699 }\end{array}$ \\
\hline $\begin{array}{l}\text { Cernansky, Nicholas P. } \\
\text { V-C-27417 }\end{array}$ & $\begin{array}{l}\text { Clark, Kenneth D. } \\
\text { IV-B-27786 }\end{array}$ \\
\hline $\begin{array}{c}\text { Chaganty, N.R. } \\
\text { IV-G-29053 }\end{array}$ & $\begin{array}{c}\text { Clark, Noel A. } \\
\text { [-E-26971 }\end{array}$ \\
\hline $\begin{array}{l}\text { Chan, Tony F.C. } \\
\text { IV-B-28735 }\end{array}$ & $\begin{array}{l}\text { Clifton, R.J. } \\
\text { VI-E-28575 }\end{array}$ \\
\hline $\begin{array}{l}\text { Cbandrasekhara, M.S. } \\
\text { V-B-27894 }\end{array}$ & $\begin{array}{l}\text { Cohen, Donald S. } \\
\text { IV-A-25874 }\end{array}$ \\
\hline $\begin{array}{c}\text { Chang Fu-Kuo } \\
\text { V-A-28253 }\end{array}$ & $\begin{array}{l}\text { Coldren, Larry } \\
\text { VIII-F-28922 }\end{array}$ \\
\hline $\begin{array}{l}\text { Chang Leory } \\
\text { I-D-27458 }\end{array}$ & $\begin{array}{l}\text { Cole, Barry } \\
\text { VIIl-C-26791 }\end{array}$ \\
\hline $\begin{array}{l}\text { Chang, Richard K. } \\
\text { VIl-G.28489 }\end{array}$ & $\begin{array}{l}\text { Collin, R.E. } \\
\text { VII-D-27911 }\end{array}$ \\
\hline $\begin{array}{c}\text { Chapman, D.R. } \\
\text { V-C-27480 }\end{array}$ & $\begin{array}{l}\text { Condrate, Robert } \\
\text { Vl-D-27548 }\end{array}$ \\
\hline $\begin{array}{l}\text { Chern, Rey T. } \\
\text { II-E-28373 }\end{array}$ & $\begin{array}{r}\text { Conlisk, A.T. } \\
\text { V-B-26595 }\end{array}$ \\
\hline
\end{tabular}

\begin{tabular}{|c|c|}
\hline $\begin{array}{c}\text { Conover, W.J. } \\
\text { IV-C-26746 }\end{array}$ & $\begin{array}{l}\text { DeBarber, Peter A. } \\
\text { V-C-29810 }\end{array}$ \\
\hline $\begin{array}{r}\text { Conrad, Hans } \\
\text { VI-B-26825 }\end{array}$ & $\begin{array}{l}\text { Deiwert, George S. } \\
\text { V-C-27469 }\end{array}$ \\
\hline $\begin{array}{c}\text { Conrad, John R. } \\
\text { VI-A-27552 }\end{array}$ & $\begin{array}{c}\text { De Boor, Carl } \\
\text { IV-B-27690 }\end{array}$ \\
\hline $\begin{array}{l}\text { Consortini, A. } \\
\text { IX-F-6458 }\end{array}$ & $\begin{array}{l}\text { De Namor, A.F. Danil } \\
\text { [X-B-6674 }\end{array}$ \\
\hline $\begin{array}{l}\text { Cooper, David M. } \\
\text { V.C-27469 } \\
\end{array}$ & $\begin{array}{l}\text { Delucia, Frank C. } \\
\text { I-B-29324 }\end{array}$ \\
\hline $\begin{array}{l}\text { Cooper, Loon N. } \\
\text { JII-F-29170 }\end{array}$ & $\begin{array}{l}\text { Demain, Amold L. } \\
\text { III-C-25126 } \\
\text { III-C-28361 }\end{array}$ \\
\hline $\begin{array}{l}\text { Courtney, Thomas H } \\
\text { Vl-C-28639 }\end{array}$ & $\begin{array}{c}\text { Demeester, P. } \\
\text { DX-F-6291 }\end{array}$ \\
\hline $\begin{array}{l}\text { Crandall, Micbael G. } \\
\text { IV-A-27869 }\end{array}$ & $\begin{array}{l}\text { Dempster, Arthur P. } \\
\text { IV-C-26993 }\end{array}$ \\
\hline $\begin{array}{l}\text { Crim, F. Fleming } \\
\text { I]-D-29033 }\end{array}$ & $\begin{array}{c}\text { Dennis, Jobn E. } \\
\text { IV-G-26932 }\end{array}$ \\
\hline $\begin{array}{l}\text { Cronin-Golomb, Mar } \\
\text { I-E-27882 } \\
\text { I-E-28526 }\end{array}$ & $\begin{array}{l}\text { Deutsch, Stuart J. } \\
\text { IV-C-29358 }\end{array}$ \\
\hline $\begin{array}{r}\text { Crosley, D.R. } \\
\text { V-C-27864 }\end{array}$ & $\begin{array}{l}\text { Dlott, Dana D. } \\
\text { II-D-27025 }\end{array}$ \\
\hline $\begin{array}{l}\text { Cross, L Eric } \\
\text { [-B-28784 }\end{array}$ & $\begin{array}{c}\text { Dobbins, RA. } \\
\text { V-C-28898 }\end{array}$ \\
\hline $\begin{array}{l}\text { Crowson, Andrew } \\
\text { VI-F-30337 }\end{array}$ & $\begin{array}{c}\text { Dodd, Ricbard A. } \\
\text { VI-A-27552 }\end{array}$ \\
\hline $\begin{array}{l}\text { Curran, Donald R. } \\
\text { VI-E-30488 }\end{array}$ & $\begin{array}{l}\text { Dolling, David S. } \\
\text { V-B-28293 }\end{array}$ \\
\hline $\begin{array}{l}\text { Cusanovich, Michael } \\
\text { I-E-28356 }\end{array}$ & $\begin{array}{c}\text { Dongarra, Jack } \\
\text { IV-B-29113 }\end{array}$ \\
\hline $\begin{array}{l}\text { Cushman, Jobn H. } \\
\text { VII-B-27316 }\end{array}$ & $\begin{array}{l}\text { Dordick, Jonathan S. } \\
\text { III-C-28699 }\end{array}$ \\
\hline $\begin{array}{l}\text { Dafermos, C.M. } \\
\text { IV-A-27403 }\end{array}$ & $\begin{array}{l}\text { Douglas, Kenneth } \\
\text { I-D-30408 }\end{array}$ \\
\hline $\begin{array}{l}\text { Dagdigian, Paul J. } \\
\text { II-C-28767 }\end{array}$ & $\begin{array}{l}\text { Dow, Jobn D. } \\
\text { VIII-B-28508 }\end{array}$ \\
\hline $\begin{array}{l}\text { Dainty, J.C. } \\
\text { IX-F-5830 }\end{array}$ & $\begin{array}{l}\text { Dowell, Eart H. } \\
\text { V-D-28493 }\end{array}$ \\
\hline $\begin{array}{l}\text { Dapkus, P.D. } \\
\text { VIII-F-26898 } \\
\text { VIII-F-30500 }\end{array}$ & $\begin{array}{l}\text { Drago, Russell S. } \\
\text { II-B-26914 }\end{array}$ \\
\hline $\begin{array}{l}\text { Davies, P. } \\
\text { V-D-27300 }\end{array}$ & $\begin{array}{l}\text { Duerksen, Gary L. } \\
\text { 1-D-28271 }\end{array}$ \\
\hline $\begin{array}{l}\text { avis, M.H.A. } \\
\text { IX-A-6366 }\end{array}$ & $\begin{array}{l}\text { Duffy, Christopher J. } \\
\text { VII-A-27471 }\end{array}$ \\
\hline
\end{tabular}




\begin{tabular}{|c|c|}
\hline $\begin{array}{l}\text { Dupuis, Russell D. } \\
\text { VIII-A-28351 }\end{array}$ & $\begin{array}{l}\text { Fox, Alvin } \\
\text { III-D-25663 }\end{array}$ \\
\hline $\begin{array}{l}\text { Dutton, J. Craig } \\
\text { V-B-27558 }\end{array}$ & $\begin{array}{l}\text { Franciosi, A. } \\
\text { VIII-A-26761 }\end{array}$ \\
\hline $\begin{array}{l}\text { Dutton, Robert W. } \\
\text { VIIl-E-28297 } \\
\text { VIII-F-29210 }\end{array}$ & $\begin{array}{l}\text { Freeman, Benny } \\
\text { II-E-28373 }\end{array}$ \\
\hline $\begin{array}{l}\text { Eastman, Lester F. } \\
\text { VIII-B-26755 }\end{array}$ & $\begin{array}{l}\text { Freidlin, Mark } \\
\text { IV-C-29678 }\end{array}$ \\
\hline $\begin{array}{l}\text { Eckman, Richard M. } \\
\text { VIl-F-28664 }\end{array}$ & $\begin{array}{l}\text { Frencb, Donald A } \\
\text { IV-B-28535 }\end{array}$ \\
\hline $\begin{array}{l}\text { Eisenstat, Stanley C. } \\
\text { IV-B-28007 }\end{array}$ & $\begin{array}{l}\text { Freudenstein, F. } \\
\text { V-D-26995 }\end{array}$ \\
\hline $\begin{array}{l}\text { Ekerdt, John G. } \\
\text { II-A-26748 }\end{array}$ & $\begin{array}{l}\text { Frey, Jeffrey } \\
\text { ViII-B-28004 }\end{array}$ \\
\hline $\begin{array}{l}\text { El-Fakahany, Esam E. } \\
\text { III-D-29594. }\end{array}$ & $\begin{array}{l}\text { Frieden, B. Roy } \\
\text { IV-G-30411 }\end{array}$ \\
\hline $\begin{array}{l}\text { Elder, R.L. } \\
\text { IX-C-5824 }\end{array}$ & $\begin{array}{l}\text { Friedlander, Benjamin } \\
\text { VIII-E-27899 }\end{array}$ \\
\hline $\begin{array}{l}\text { Elman, Howard C. } \\
\text { IV-B-29574 }\end{array}$ & $\begin{array}{l}\text { Frisch, Harry L } \\
\text { II-E-26072 }\end{array}$ \\
\hline $\begin{array}{l}\text { Farbat, Nabil H. } \\
\text { IV-E-28125 }\end{array}$ & $\begin{array}{r}\text { Fuller, Gerald } \\
\text { VI-D-26883 }\end{array}$ \\
\hline $\begin{array}{l}\text { Fasel, Hermann } \\
\text { V-B-30113 }\end{array}$ & $\begin{array}{c}\text { Gaonkar, G.H. } \\
\text { V-D-28123 }\end{array}$ \\
\hline Fauchet, Philippe & $\begin{array}{l}\text { Gardner, Carl L. } \\
\text { IV-B-28799 }\end{array}$ \\
\hline $\begin{array}{l}\text { I-B-29184 } \\
\text { I-D-28470 }\end{array}$ & $\begin{array}{l}\text { Gardner, William A. } \\
\text { VIII-E-27917 }\end{array}$ \\
\hline $\begin{array}{l}\text { Favro, Lawrence D. } \\
\text { VI-B-28780 }\end{array}$ & $\begin{array}{l}\text { Garrison, Jr., Warren M. } \\
\text { V1-B-27407 }\end{array}$ \\
\hline $\begin{array}{l}\text { Feldman, Michael R. } \\
\text { l-E-28416 }\end{array}$ & $\begin{array}{l}\text { Gerbardt, Pbilipp } \\
\text { III-A-28022 }\end{array}$ \\
\hline $\begin{array}{l}\text { Ferry, David } \\
\text { VIIl-B-28461 }\end{array}$ & $\begin{array}{l}\text { German, Randall M. } \\
\text { VI-C-29326 }\end{array}$ \\
\hline $\begin{array}{l}\text { Firsbein, William } \\
\text { III-C-28090 }\end{array}$ & $\begin{array}{l}\text { Ghosh, Somnath } \\
\text { V-A-29040 }\end{array}$ \\
\hline $\begin{array}{l}\text { Flaherty, Joseph E } \\
\text { IV-B-29167 }\end{array}$ & $\begin{array}{l}\text { Ghosh, Sumit } \\
\text { IV-G-28428 }\end{array}$ \\
\hline $\begin{array}{l}\text { Flynn, George } \\
\text { V[I]-H-28453 }\end{array}$ & $\begin{array}{l}\text { Gibbons, James F. } \\
\text { VIII-F-28729 }\end{array}$ \\
\hline Fonstad, Clifton G. & \\
\hline $\begin{array}{l}\text { I-D-29578 } \\
\text { VIII-B-27883 }\end{array}$ & $\begin{array}{l}\text { Gibson, Walter } \\
\text { Il-E-26072 }\end{array}$ \\
\hline $\begin{array}{l}\text { Ford, Warren T. } \\
\text { IJ-E-26839 }\end{array}$ & $\begin{array}{l}\text { Gidas, Basilis } \\
\text { IV-G-26811 }\end{array}$ \\
\hline $\begin{array}{c}\text { Fosdick, Roger } \\
\text { IV-A-28986 }\end{array}$ & $\begin{array}{l}\text { Gillespie, David } \\
\text { III-D-28479 }\end{array}$ \\
\hline
\end{tabular}

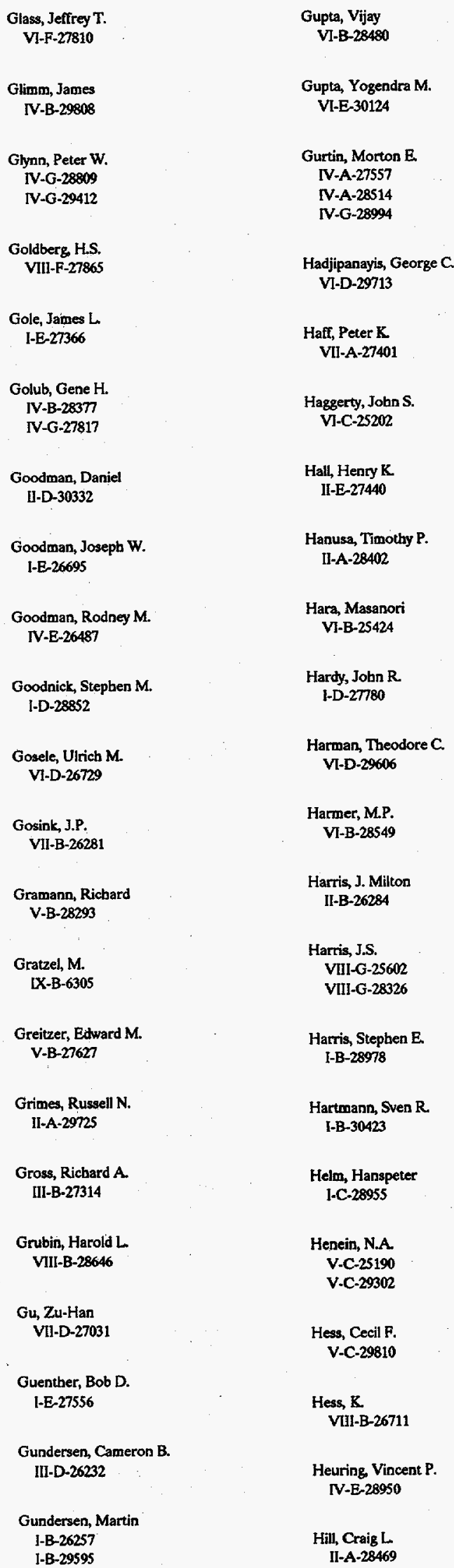




\begin{tabular}{|c|c|}
\hline $\begin{array}{l}\text { Hill, Steven C. } \\
\text { VII-G-26962 }\end{array}$ & $\begin{array}{c}\text { Jackson, David R. } \\
\text { VII-C-27304 }\end{array}$ \\
\hline $\begin{array}{l}\text { Hilvert, Donald } \\
\text { III-C-29195 }\end{array}$ & $\begin{array}{r}\text { Jacob, Jonah } \\
\text { II-D-30332 }\end{array}$ \\
\hline $\begin{array}{l}\text { Hindman, Richard G. } \\
\text { V-B-27062 }\end{array}$ & $\begin{array}{c}\text { Jacox, Marilyn E. } \\
\text { II-C-30094 }\end{array}$ \\
\hline $\begin{array}{l}\text { Hislop, David W. } \\
\text { NV-E-27567 }\end{array}$ & $\begin{array}{l}\text { Jaeger, David A. } \\
\text { II-B-28814 }\end{array}$ \\
\hline $\begin{array}{l}\text { Ho, Yu-Chi } \\
\text { IV-G-29190 }\end{array}$ & $\begin{array}{l}\text { James, Richard } \\
\text { IV-A-28987 }\end{array}$ \\
\hline $\begin{array}{l}\text { Hodges, D.H. } \\
\text { V-D-25327 }\end{array}$ & $\begin{array}{l}\text { Jeffries, Jay B. } \\
\text { I-B-26959 }\end{array}$ \\
\hline $\begin{array}{l}\text { Holmes, J. Fred } \\
\text { VII-E-28102 }\end{array}$ & $\begin{array}{l}\text { Jena, } P . \\
\text { I-D-29341 }\end{array}$ \\
\hline $\begin{array}{l}\text { Holonyak, N. } \\
\text { VIII-A-30090 }\end{array}$ & $\begin{array}{c}\text { Jones, Alan A. } \\
\text { II-E-26840 }\end{array}$ \\
\hline $\begin{array}{l}\text { Horgan, C.O. } \\
\text { V-D-28399 }\end{array}$ & $\begin{array}{l}\text { Jones, II, Guilford } \\
\text { II-B-27534 }\end{array}$ \\
\hline $\begin{array}{l}\text { Houser, Thomas } \\
\text { I1-D-27505 }\end{array}$ & $\begin{array}{l}\text { Jones, Pbillip L. } \\
\text { VI-F-30337 }\end{array}$ \\
\hline $\begin{array}{l}\text { Houston, Paul L. } \\
\text { II-D-28700 }\end{array}$ & $\begin{array}{c}\text { Jones, Richard A. } \\
\text { [I-A-26748 }\end{array}$ \\
\hline $\begin{array}{l}\text { Hu, Qing } \\
\text { I-D-29578 }\end{array}$ & $\begin{array}{l}\text { Joseph, Daniel D. } \\
\text { IV-A-28797 }\end{array}$ \\
\hline $\begin{array}{l}\text { Hudson, J.A. } \\
\text { IX-E-6296 }\end{array}$ & $\begin{array}{l}\text { Josbi, Aravind K. } \\
\text { IV-E-26779 }\end{array}$ \\
\hline $\begin{array}{l}\text { Huennekens, John P. } \\
\text { I-B-26899 }\end{array}$ & $\begin{array}{l}\text { Kabamaba, Pierre T. } \\
\text { V-D.27510 }\end{array}$ \\
\hline $\begin{array}{l}\text { Hughes, Brian L } \\
\text { VIII-E-27028 }\end{array}$ & $\begin{array}{c}\text { Kacbanov, Mark } \\
\text { V-A-25345 }\end{array}$ \\
\hline $\begin{array}{l}\text { Hurley, Francis X. } \\
\text { V-E-29579 }\end{array}$ & $\begin{array}{l}\text { Kailath, Thomas } \\
\text { IV-G-28060 }\end{array}$ \\
\hline $\begin{array}{l}\text { afrate, Gerald J. } \\
\text { VIII-F-30549 }\end{array}$ & $\begin{array}{l}\text { Kak, Avi C. } \\
\text { VIII-E-27464 }\end{array}$ \\
\hline $\begin{array}{l}\text { glebart, Donald L } \\
\text { IV-G-28809 }\end{array}$ & $\begin{array}{l}\text { Kallianpur, Gopinath } \\
\text { IV-C-29297 }\end{array}$ \\
\hline IV-G-29412 & $\begin{array}{c}\text { Kaner, Ricbard B. } \\
\text { II-A-29708 }\end{array}$ \\
\hline $\begin{array}{l}\text { nglefield, Paul T. } \\
\text { II-E-26840 } \\
\text { II-E-28795 }\end{array}$ & $\begin{array}{r}\text { Kapania, RK } \\
\text { V-A-26908 }\end{array}$ \\
\hline $\begin{array}{l}\text { coh, Tatsuo } \\
\text { VIII-C-25045 }\end{array}$ & $\begin{array}{l}\text { Karalamangala, A.S. } \\
\text { IV-G-28476 }\end{array}$ \\
\hline $\begin{array}{l}\text { er, K.R. } \\
\text { VI-F-27364 }\end{array}$ & $\begin{array}{l}\text { Karatzas, Ioannis } \\
\text { IV-C-28715 }\end{array}$ \\
\hline $\begin{array}{l}\text { ccodine, Ralph J. } \\
\text { VI-D-29282 }\end{array}$ & $\begin{array}{l}\text { Kassoy, David R } \\
\text { IV-A-28728 }\end{array}$ \\
\hline
\end{tabular}

\begin{tabular}{|c|c|}
\hline $\begin{array}{l}\text { Katehi, Linda P.B. } \\
\text { VIII-C-28483 }\end{array}$ & $\begin{array}{c}\text { Kosut, Robert } L \\
\text { V-D-28350 }\end{array}$ \\
\hline $\begin{array}{c}\text { Katritzky, Alan R. } \\
\text { II-A-30164 }\end{array}$ & $\begin{array}{l}\text { Krajcinovic, D. } \\
\text { V-A-28222 }\end{array}$ \\
\hline $\begin{array}{l}\text { Keller, A. } \\
\text { IX-D-6011 }\end{array}$ & $\begin{array}{l}\text { Kramer, Edward J. } \\
\text { VI-A-27624 }\end{array}$ \\
\hline $\begin{array}{l}\text { Kelly, Jeffery W. } \\
\text { III-D-27468 }\end{array}$ & $\begin{array}{l}\text { Krishnaswamy, J. } \\
\text { VI-D-26601 }\end{array}$ \\
\hline $\begin{array}{l}\text { Kempa, K. } \\
\text { I-D-26682 } \\
\text { I-D-28652 }\end{array}$ & $\begin{array}{l}\text { Kruse, Paul } \\
\text { VIII-C-26791 }\end{array}$ \\
\hline $\begin{array}{l}\text { Khargonekar, Pramod P. } \\
\text { IV-D-27044 }\end{array}$ & $\begin{array}{l}\text { Kuech, T.F. } \\
\text { VI-D-29750 }\end{array}$ \\
\hline $\begin{array}{l}\text { Kim, K-W. } \\
\text { VIII-F-28187 }\end{array}$ & $\begin{array}{l}\text { Kumar, P.R. } \\
\text { IV.C-28718 }\end{array}$ \\
\hline Kinderlebrer, David & $\begin{array}{c}\text { Kumar, Vipin } \\
\text { IV-G-28408 }\end{array}$ \\
\hline IV-G-28994 & $\begin{array}{c}\text { Kuo, Kenneth K } \\
\text { V-C-29336 }\end{array}$ \\
\hline $\begin{array}{l}\text { Kingon, Angus I. } \\
\text { VI-C-26673 }\end{array}$ & \\
\hline & $\begin{array}{l}\text { Kustin, Kenneth } \\
\text { [II-A-28691 }\end{array}$ \\
\hline $\begin{array}{l}\text { Klabunde, Kenneth J. } \\
\text { II-C-27775 }\end{array}$ & \\
\hline & $\begin{array}{l}\text { LaConti, Anthony B. } \\
\text { II-A-30289 }\end{array}$ \\
\hline $\begin{array}{l}\text { Klepaczko, J.R. } \\
\text { IX-D-6148 }\end{array}$ & \\
\hline & $\begin{array}{l}\text { Lagowski, Jacek } \\
\text { VI-D-27764 }\end{array}$ \\
\hline Knight, Doyle & \\
\hline V-B-28651 & $\begin{array}{l}\text { Lai, M.-C. } \\
\text { V.C-29302 }\end{array}$ \\
\hline Koberstein, Jeffirey T. & \\
\hline V1-A-27863 & $\begin{array}{l}\text { Lakdawala, V.K. } \\
\text { I-B-26821 }\end{array}$ \\
\hline Koc, C.K & \\
\hline IV-D-28511 & $\begin{array}{l}\text { Langdon, Terence G. } \\
\text { VI-B-28826 }\end{array}$ \\
\hline Koch, Stephan & \\
\hline I-E-26974 & $\begin{array}{l}\text { Langer, Stanley H. } \\
\text { II-A-27600 }\end{array}$ \\
\hline $\begin{array}{l}\text { Kohn, Robert V. } \\
\text { IV-A-29351 }\end{array}$ & $\begin{array}{l}\text { Lansman, Jeffry B. } \\
\text { [II-F-29221 }\end{array}$ \\
\hline $\begin{array}{l}\text { Soksini, Jozef L. } \\
\text { III-A-27494 }\end{array}$ & $\begin{array}{l}\text { Lavernia, EJJ. } \\
\text { VI-C-29410 }\end{array}$ \\
\hline $\begin{array}{l}\text { Colawa, Elabieta } \\
\text { Vl-D-29677 }\end{array}$ & $\begin{array}{l}\text { Law, Chung K } \\
\text { V-C-27565 }\end{array}$ \\
\hline $\begin{array}{l}\text { Colb, Charles E. } \\
\text { II-D.28103 }\end{array}$ & $\begin{array}{l}\text { Law, K.H. } \\
\quad \text { IV-B-28377 }\end{array}$ \\
\hline $\begin{array}{l}\text { Oolbas, Robert M. } \\
\text { VIII-D-28070 }\end{array}$ & $\begin{array}{l}\text { Lawler, J.E. } \\
\text { I-B-27646 }\end{array}$ \\
\hline $\begin{array}{l}\text { orpel, Adrian } \\
\text { I-E-27591 }\end{array}$ & $\begin{array}{l}\text { Lax, Melvin } \\
\text { I-D-29572 }\end{array}$ \\
\hline $\begin{array}{l}\text { osek, Jobn A. } \\
\text { II-A-30289 }\end{array}$ & $\begin{array}{l}\text { Leburton, Jean } \\
\text { VIII-B-28406 }\end{array}$ \\
\hline
\end{tabular}




\begin{tabular}{|c|c|}
\hline $\begin{array}{l}\text { Lee, M. Howard } \\
\text { IV-A-27580 }\end{array}$ & $\begin{array}{l}\text { MacCormack, Robert W. } \\
\text { V-C-27480 }\end{array}$ \\
\hline $\begin{array}{l}\text { Lee, Peter C.Y. } \\
\text { [V-A-26909 }\end{array}$ & $\begin{array}{l}\text { MacKnight, William J. } \\
\text { II-E-28314 }\end{array}$ \\
\hline $\begin{array}{l}\text { Leibowitz, Michael J. } \\
\text { III-C-29289 }\end{array}$ & $\begin{array}{l}\text { Madbukar, Anupam } \\
\text { VIII-A-27298 }\end{array}$ \\
\hline $\begin{array}{l}\text { Leone, Stephen R. } \\
\text { I-B-28561 }\end{array}$ & $\begin{array}{l}\text { Mabrt, Larry } \\
\text { VII-F-26491 }\end{array}$ \\
\hline $\begin{array}{l}\text { Lewis, Aaron } \\
\text { I-E-29050 }\end{array}$ & $\begin{array}{l}\text { Majda, Andrew J. } \\
\text { IV-A-28766 }\end{array}$ \\
\hline $\begin{array}{l}\text { Lewis, Randolph V. } \\
\text { III-C-28457 }\end{array}$ & $\begin{array}{l}\text { Manson, Steven T. } \\
\text { I-B-26709 }\end{array}$ \\
\hline $\begin{array}{l}\text { leberman, Edward M. } \\
\text { III-D-25752 }\end{array}$ & $\begin{array}{l}\text { Maracas, George N. } \\
\text { VIII-B-26686 }\end{array}$ \\
\hline $\begin{array}{l}\text { Ljechti, Kenneth M. } \\
\text { V-A-27315 }\end{array}$ & $\begin{array}{l}\text { Margrave, Jobn L. } \\
\text { Il-A-30446 }\end{array}$ \\
\hline $\begin{array}{l}\text { Lin, S.P. } \\
\text { V-C-26797 }\end{array}$ & $\begin{array}{c}\text { Mark, James E. } \\
\text { VI-C-27373 }\end{array}$ \\
\hline $\begin{array}{r}\text { Liu, Ming-Tsan } \\
\text { VIII-E-28467 }\end{array}$ & $\begin{array}{l}\text { Marquis, Robert E. } \\
\text { III-A-28022 }\end{array}$ \\
\hline VIII-E-29653 & $\begin{array}{l}\text { Martin, Sandra L } \\
\text { III-E-29345 }\end{array}$ \\
\hline $\begin{array}{l}\text { Liu, Tai-Ping } \\
\text { [V-A-28548 }\end{array}$ & $\begin{array}{l}\text { Matyjaszewski, Krzysztof } \\
\text { II-E-28631 }\end{array}$ \\
\hline $\begin{array}{l}\text { Loewy, Robert G. } \\
\text { V-D-25462 }\end{array}$ & $\begin{array}{l}\text { Mayer, James W. } \\
\text { VI-A-26806 }\end{array}$ \\
\hline $\begin{array}{l}\text { Logan, Kathryn V. } \\
\text { V1-F-26021 }\end{array}$ & $\begin{array}{l}\text { McCauley, James W. } \\
\text { VI-C-29098 }\end{array}$ \\
\hline $\begin{array}{l}\text { Lob, Wei-Yin } \\
\text { [V-C-28679 }\end{array}$ & $\begin{array}{l}\text { McCormick, Rodney I. } \\
\text { I-B-29324 }\end{array}$ \\
\hline $\begin{array}{l}\text { Long, G.G. } \\
\text { VI-C-26123 }\end{array}$ & $\begin{array}{l}\text { McDonald, John F. } \\
\text { VIII-B-28329 }\end{array}$ \\
\hline $\begin{array}{l}\text { Lorber, Peter F. } \\
\text { V-B-26631 }\end{array}$ & $\begin{array}{l}\text { McDowell, David L. } \\
\text { V-A-29543 }\end{array}$ \\
\hline $\begin{array}{l}\text { Lorents, Donald C. } \\
\text { I-C-28955 }\end{array}$ & $\begin{array}{l}\text { McElwee-White, Lisa } \\
\text { [1-A-28353 }\end{array}$ \\
\hline $\begin{array}{l}\text { Lovas, Francis J. } \\
\text { II-D-29596 }\end{array}$ & $\begin{array}{l}\text { McGervey, John D. } \\
\text { VI-A-27037 }\end{array}$ \\
\hline $\begin{array}{l}\text { Lu, Toh-Ming } \\
\text { VI-D-27605 }\end{array}$ & $\begin{array}{l}\text { McGrath, James E. } \\
\text { VI-C-28883 }\end{array}$ \\
\hline $\begin{array}{l}\text { Louk, Franklin T. } \\
\text { IV-G-26930 }\end{array}$ & $\begin{array}{l}\text { Mclntosh, Kobert E. } \\
\text { VII-D-29360 }\end{array}$ \\
\hline $\begin{array}{l}\text { Luo, Ren C. } \\
\text { IV-E-27567 }\end{array}$ & $\begin{array}{l}\text { McKoy, Vincent } \\
\text { V-C-29534 }\end{array}$ \\
\hline $\begin{array}{l}\text { Luskin, Mitchell } \\
\text { IV-A-28987 }\end{array}$ & $\begin{array}{l}\text { MeManus, Samuel P. } \\
\text { II-B-26284 }\end{array}$ \\
\hline
\end{tabular}

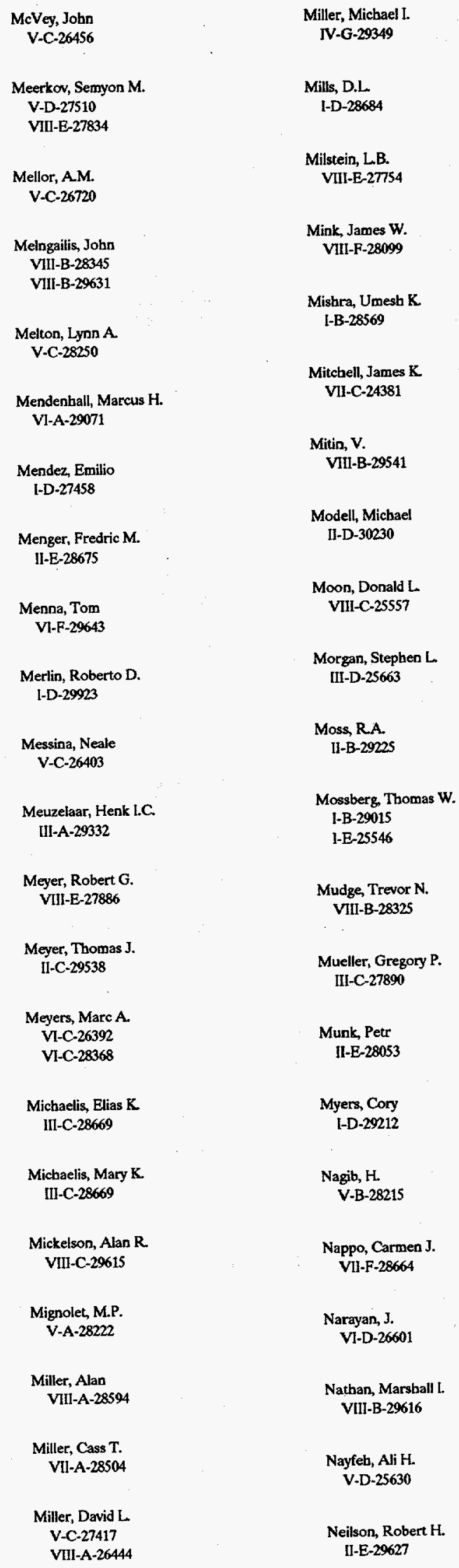




\begin{tabular}{|c|c|}
\hline $\begin{array}{l}\text { Nelson, Donald F. } \\
\text { VIII-A-26188 }\end{array}$ & $\begin{array}{l}\text { Paris, Demetrius } \\
\text { VIII-G-27554 }\end{array}$ \\
\hline $\begin{array}{l}\text { Nelson, Ron } \\
\text { VII-A-30437 }\end{array}$ & $\begin{array}{l}\text { Parks, Leo } \\
\text { [I]-B-29097 }\end{array}$ \\
\hline $\begin{array}{l}\text { Nelson, Wilfred H. } \\
\text { III-D-25476 }\end{array}$ & $\begin{array}{c}\text { Parrish, Phillip } \\
\text { VI-B-28608 }\end{array}$ \\
\hline $\begin{array}{l}\text { Nerode, Anil } \\
\text { [V-G-29031 }\end{array}$ & $\begin{array}{l}\text { Parzen, Emanuel } \\
\text { IV-C-27574 }\end{array}$ \\
\hline $\begin{array}{l}\text { Nicolet, Marc-A } \\
\text { V-D-29677 }\end{array}$ & $\begin{array}{c}\text { Patton, Carl E. } \\
\text { I-D-29375 }\end{array}$ \\
\hline $\begin{array}{l}\text { Nieh, T.G. } \\
\text { VI-B-26751 }\end{array}$ & $\begin{array}{l}\text { Paul, Donald R. } \\
\text { II-E-28525 }\end{array}$ \\
\hline $\begin{array}{l}\text { Nixon, Wilfred A. } \\
\text { VI]-B-26031 }\end{array}$ & $\begin{array}{l}\text { Paydar, Nasser H. } \\
\text { VI-D-29042 }\end{array}$ \\
\hline $\begin{array}{l}\text { O'Donnell, Kevin } \\
\text { Vll-D-27485 }\end{array}$ & $\begin{array}{l}\text { Pederson, Donald O. } \\
\text { VIIL-E-27886 }\end{array}$ \\
\hline $\begin{array}{l}\text { O'Handley, R.C. } \\
\text { VI-D-28553 }\end{array}$ & $\begin{array}{l}\text { Peng, Song-Tsuen } \\
\text { VIII-A-28674 }\end{array}$ \\
\hline $\begin{array}{l}\text { Odell, J.A. } \\
\text { IX-D-6011 }\end{array}$ & $\begin{array}{l}\text { Perepezko, John H. } \\
\text { VI-C-27472 }\end{array}$ \\
\hline $\begin{array}{l}\text { Oden, J. Tinsley } \\
\text { IV-B-30297 }\end{array}$ & VI-C-28005 \\
\hline $\begin{array}{l}\text { Ogilby, Peter R. } \\
\text { II-C-28628 }\end{array}$ & $\begin{array}{l}\text { Petrenko, Victor F. } \\
\text { VIJ-B-28599 }\end{array}$ \\
\hline $\begin{array}{l}\text { Olson, David L. } \\
\text { VI-C-30060 }\end{array}$ & $\begin{array}{l}\text { Petrucci, Sergio } \\
\text { II-A-26636 }\end{array}$ \\
\hline $\begin{array}{l}\text { Olson, Gregory B. } \\
\text { VI-B-27163 }\end{array}$ & $\begin{array}{c}\text { Petuskey, W.T. } \\
\text { VI-A-29037 }\end{array}$ \\
\hline $\begin{array}{c}\text { Oncley, Steven P. } \\
\text { VII-F-28954 }\end{array}$ & $\begin{array}{r}\text { Petzold, Linda } \\
\text { IV-B-29850 }\end{array}$ \\
\hline $\begin{array}{l}\text { Ornston, L. Nicholas } \\
\text { III-B-28964 }\end{array}$ & $\begin{array}{l}\text { Peyghambarian, Nasse } \\
\text { [-E-26974 }\end{array}$ \\
\hline $\begin{array}{l}\text { Osgood, Jr., R.M. } \\
\text { I-B-29391 }\end{array}$ & $\begin{array}{l}\text { Pinsky, Mark A } \\
\text { IV-C-28905 }\end{array}$ \\
\hline $\begin{array}{l}\text { Osgood, Jr., R } \\
\text { VIII-G-28453 }\end{array}$ & $\begin{array}{r}\text { Platzer, M.F. } \\
\text { V-B-27894 }\end{array}$ \\
\hline $\begin{array}{l}\text { Osterkamp, T.E. } \\
\text { VII-B-26281 }\end{array}$ & $\begin{array}{l}\text { Plotkin, Serge A. } \\
\text { IV-B-28143 }\end{array}$ \\
\hline $\begin{array}{l}\text { Packard, Andrew } \\
\text { IV-D-28835 }\end{array}$ & $\begin{array}{c}\text { Poetz, Walter } \\
\text { I-D-28464 }\end{array}$ \\
\hline $\begin{array}{l}\text { Packard, William E. } \\
\text { VIII-B-28508 }\end{array}$ & $\begin{array}{l}\text { Pollak, Fred H. } \\
\text { VIII-B-29036 }\end{array}$ \\
\hline $\begin{array}{l}\text { Paesler, Michael A } \\
\text { l-E-28313 }\end{array}$ & $\begin{array}{l}\text { Poon, S. Joseph } \\
\text { VI-C-2806? }\end{array}$ \\
\hline $\begin{array}{l}\text { Parbi, Keshab K. } \\
\text { VIII-E-27076 }\end{array}$ & $\begin{array}{l}\text { Popovic, Zoya B. } \\
\text { VII-C-29615 }\end{array}$ \\
\hline
\end{tabular}

\begin{tabular}{|c|c|}
\hline $\begin{array}{l}\text { Post, Madison J. } \\
\text { VII-E-28094 }\end{array}$ & $\begin{array}{c}\text { Rehfield, L.W. } \\
\text { V-D-25327 }\end{array}$ \\
\hline $\begin{array}{l}\text { Powell, Richard C. } \\
\text { I-D-28472 }\end{array}$ & $\begin{array}{l}\text { Reisenthel, Patrick H. } \\
\text { V-B-29049 }\end{array}$ \\
\hline $\begin{array}{l}\text { Prater, John T. } \\
\text { V]-F-27810 }\end{array}$ & $\begin{array}{l}\text { Reisfeld, R. } \\
\text { IX-D-5884 }\end{array}$ \\
\hline $\begin{array}{l}\text { Pritchard, David E. } \\
\text { I-B-30141 }\end{array}$ & $\begin{array}{l}\text { Reynolds, John R. } \\
\text { II-E-27808 }\end{array}$ \\
\hline $\begin{array}{l}\text { Pursley, Michael B. } \\
\text { VIIJ-E-27994 }\end{array}$ & $\begin{array}{l}\text { Rhodes, Charles } \\
\text { I-B-28499 }\end{array}$ \\
\hline $\begin{array}{l}\text { Quin, Louis D. } \\
\text { II-B-29699 } \\
\text { II-C-26126 }\end{array}$ & $\begin{array}{l}\text { Robbins, Herbert } \\
\text { IV-C-27862 }\end{array}$ \\
\hline $\begin{array}{l}\text { Rabinowitz, Paul H. } \\
\text { IV-A-27641 }\end{array}$ & $\begin{array}{l}\text { Robinson, Stephen M. } \\
\text { IV-C-27381 }\end{array}$ \\
\hline $\begin{array}{l}\text { Rabitz, Herschel } \\
\text { IJ-D-28555 } \\
\text { II-D-28889 }\end{array}$ & $\begin{array}{l}\text { Rodin, Gregory J. } \\
\text { V-A-27315 }\end{array}$ \\
\hline $\begin{array}{c}\text { Ramaprian, B.R. } \\
\text { V-B-28159 }\end{array}$ & $\begin{array}{l}\text { Roe, RJ. } \\
\text { VI-C-26579 }\end{array}$ \\
\hline $\begin{array}{l}\text { Ramesh, KT. } \\
\text { Vl-E-28272 }\end{array}$ & $\begin{array}{l}\text { Ron, Amos } \\
\text { IV-B-27690 }\end{array}$ \\
\hline $\begin{array}{l}\text { Rao, Bbaskar D. } \\
\text { IV-G-26802 }\end{array}$ & $\begin{array}{r}\text { Roth, J. Reece } \\
\text { Vl-A-26678 }\end{array}$ \\
\hline $\begin{array}{l}\text { Rao, C.R } \\
\text { IV-G-26792 }\end{array}$ & $\begin{array}{c}\text { Roth, Steven F. } \\
\text { IV-E-29354 }\end{array}$ \\
\hline $\begin{array}{l}\text { Ratner, Mark A. } \\
\text { II-E-27054 }\end{array}$ & $\begin{array}{r}\text { Ruden, P. Paul } \\
\text { VIIJ-B-29616 }\end{array}$ \\
\hline $\begin{array}{l}\text { Ravani, Babram } \\
\text { V-D-27075 }\end{array}$ & $\begin{array}{c}\text { Russell, Alan J, } \\
\text { III-C-28011 } \\
\text { III-C-28043 }\end{array}$ \\
\hline $\begin{array}{l}\text { Ravishanker, Nalini } \\
\text { IV-C-28743 }\end{array}$ & $\begin{array}{l}\text { Rutledge, David } \\
\text { VIII-C-29523 }\end{array}$ \\
\hline $\begin{array}{l}\text { Raymer, Michael G. } \\
\text { 1-E-25546 }\end{array}$ & $\begin{array}{c}\text { Sabin, Jobn R. } \\
\text { [-D-28362 }\end{array}$ \\
\hline $\begin{array}{l}\text { Raymond, Charles F. } \\
\text { VII-B-28344 }\end{array}$ & $\begin{array}{l}\text { Sachs, Frederick } \\
\text { III-F-28775 }\end{array}$ \\
\hline $\begin{array}{l}\text { Rebeiz, Gabriel M. } \\
\text { VIII-C-28483 }\end{array}$ & $\begin{array}{l}\text { Sadwick, L.P. } \\
\text { VI-D-28612 }\end{array}$ \\
\hline $\begin{array}{l}\text { Reddy, J.N. } \\
\text { V-A-26908 }\end{array}$ & $\begin{array}{c}\text { Salama, Kamel } \\
\text { VI-A-29064 }\end{array}$ \\
\hline $\begin{array}{l}\text { Redner, Sidney } \\
\text { II-D-26256 }\end{array}$ & $\begin{array}{l}\text { Salo, Timotby J. } \\
\text { IV-D-29396 }\end{array}$ \\
\hline $\begin{array}{l}\text { Reeber, Robert R. } \\
\text { VI-F-26246 }\end{array}$ & $\begin{array}{c}\text { Santavicca, D.A. } \\
\text { V-C-28427 }\end{array}$ \\
\hline $\begin{array}{l}\text { Reeves, Roger J. } \\
\text { I-D-28472 }\end{array}$ & $\begin{array}{l}\text { Sarwate, D.V. } \\
\text { VIII-E-27994 }\end{array}$ \\
\hline
\end{tabular}




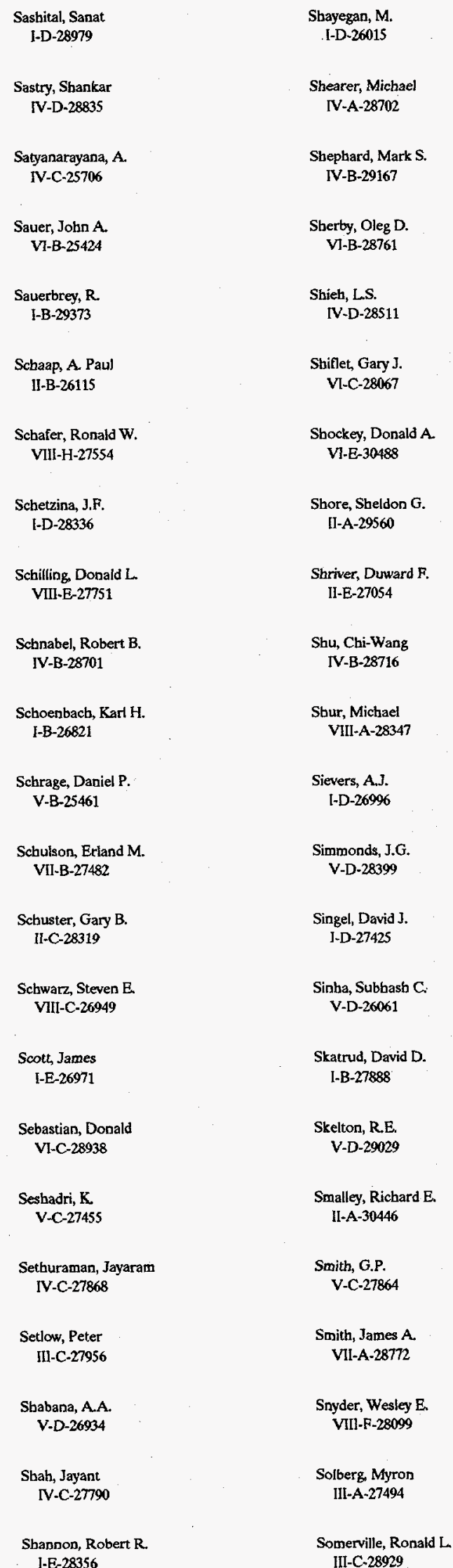

\begin{tabular}{|c|c|}
\hline $\begin{array}{l}\text { Sorokin, Peter P. } \\
\text { I-B-27508 }\end{array}$ & $\begin{array}{l}\text { Sung, CS.P. } \\
\text { Vl-C-26571 }\end{array}$ \\
\hline $\begin{array}{l}\text { Spicer, Eleanor K. } \\
\text { III-C-28680 }\end{array}$ & $\begin{array}{l}\text { Swanson, M.L. } \\
\text { V]-D-27846 }\end{array}$ \\
\hline $\begin{array}{l}\text { Srivastav, Ram P. } \\
\text { IV-A-27433 }\end{array}$ & $\begin{array}{l}\text { Sykes, R.l. } \\
\text { VII-F.29369 }\end{array}$ \\
\hline $\begin{array}{l}\text { Stalder, Kenneth R. } \\
\text { I-B-26959 }\end{array}$ & $\begin{array}{l}\text { Syvanen, Michael } \\
\text { III-D-27916 }\end{array}$ \\
\hline $\begin{array}{l}\text { Stangle, Gregory C. } \\
\text { Vl-C-29098 }\end{array}$ & $\begin{array}{l}\text { Tadmor, Gilead } \\
\text { IV-D-28740 }\end{array}$ \\
\hline $\begin{array}{l}\text { Steel, Duncan G. } \\
\text { I-D-29922 }\end{array}$ & $\begin{array}{l}\text { Tan, Teh Y. } \\
\text { V-D-26729 }\end{array}$ \\
\hline $\begin{array}{l}\text { Steele, J. Michael } \\
\text { JV-C-28982 }\end{array}$ & $\begin{array}{l}\text { Tannenbaum, Allen } \\
\text { IV-D-27365 }\end{array}$ \\
\hline $\begin{array}{c}\text { Stegeman, G. } \\
\text { I-A-28996 }\end{array}$ & $\begin{array}{l}\text { Tantaratana, Sawasd } \\
\text { VIII-E- } 27493\end{array}$ \\
\hline $\begin{array}{l}\text { Steinhoff, John } \\
\text { V-B-25623 }\end{array}$ & $\begin{array}{l}\text { Taylor, Fred J. } \\
\text { VIII-E-26735 }\end{array}$ \\
\hline $\begin{array}{l}\text { Stephan, Karl D. } \\
\text { VIII-C-26599 }\end{array}$ & $\begin{array}{l}\text { Tester, Jefferson W. } \\
\text { II-D-28371 }\end{array}$ \\
\hline $\begin{array}{l}\text { Stewart, John M. } \\
\text { III-C-27488 }\end{array}$ & $\begin{array}{c}\text { Thies, Mark C. } \\
\text { VI-C-30095 }\end{array}$ \\
\hline $\begin{array}{l}\text { Stillman, G.E. } \\
\text { VIII-F-30595 }\end{array}$ & $\begin{array}{l}\text { Thomas, John E. } \\
\text { I-B-27766 }\end{array}$ \\
\hline $\begin{array}{l}\text { Stonebraker, Micbael } \\
\text { IV-E-27892 }\end{array}$ & $\begin{array}{l}\text { Thomas, Robert L. } \\
\text { V1-B-28780 }\end{array}$ \\
\hline $\begin{array}{l}\text { Streetman, Ben G. } \\
\text { VIII-B-28895 }\end{array}$ & $\begin{array}{l}\text { Thompson, Carl V. } \\
\text { VI-D-28553 }\end{array}$ \\
\hline $\begin{array}{l}\text { Stringfellow, G.B. } \\
\text { VI-D-28612 }\end{array}$ & $\begin{array}{l}\text { Thompson, Donald L. } \\
\text { Ll-D-26106 }\end{array}$ \\
\hline $\begin{array}{l}\text { Stroscio, M.A. } \\
\text { VIII-F-28187 }\end{array}$ & $\begin{array}{l}\text { Thompson, James R. } \\
\text { IV-C-28686 }\end{array}$ \\
\hline $\begin{array}{l}\text { Stroud, David G. } \\
\text { V]-D-30108 }\end{array}$ & $\begin{array}{l}\text { Thompson, Richard C. } \\
\text { II-A-28486 }\end{array}$ \\
\hline $\begin{array}{l}\text { Subrabmanian, V.S. } \\
\text { IV-E-29692 }\end{array}$ & $\begin{array}{r}\text { Thorsos, Eric I. } \\
\text { VII-D-29404 }\end{array}$ \\
\hline $\begin{array}{l}\text { Suenram, Richard D. } \\
\text { II-D-29596 }\end{array}$ & $\begin{array}{l}\text { Thynell, Stefan T. } \\
\text { V-C-29336 }\end{array}$ \\
\hline $\begin{array}{l}\text { Sugama, Tosbifumi } \\
\text { VI-A-26667 }\end{array}$ & $\begin{array}{l}\text { Tiersten, Harry F. } \\
\text { I-D-28591 }\end{array}$ \\
\hline $\begin{array}{l}\text { Summers, Christopber J. } \\
\text { VIII-C-28339 }\end{array}$ & $\begin{array}{l}\text { Ting T.C.T. } \\
\text { N-A-28606 }\end{array}$ \\
\hline $\begin{array}{c}\text { Sumney, Larry W. } \\
\text { VIII-K-29540 }\end{array}$ & $\begin{array}{l}\text { Tirrell, Matthew } \\
\text { IV-A-28797 }\end{array}$ \\
\hline $\begin{array}{l}\text { Sun, C.T. } \\
\text { V-A-28307 }\end{array}$ & $\begin{array}{l}\text { Tomozawa, Minoru } \\
\text { VJ-B-28577 }\end{array}$ \\
\hline
\end{tabular}




\begin{tabular}{|c|c|}
\hline $\begin{array}{l}\text { Tosatti, E. } \\
\text { IX-A-6280 }\end{array}$ & $\begin{array}{c}\text { Virgin, Lawrence N. } \\
\text { IV-C-29740 }\end{array}$ \\
\hline $\begin{array}{l}\text { Tove, Shirley R } \\
\text { III-B-29097 }\end{array}$ & $\begin{array}{r}\text { Vitter, Jeffrey } \\
\text { IV-B-28071 }\end{array}$ \\
\hline $\begin{array}{r}\text { Triboulet, R. } \\
\text { IX-F-6563 }\end{array}$ & $\begin{array}{l}\text { Vodyanoy, Vitali } \\
\text { III-F-27634 }\end{array}$ \\
\hline $\begin{array}{l}\text { Tricamo, Stephen } \\
\text { Vl-C-28938 }\end{array}$ & $\begin{array}{c}\text { Voorhees, Kent J. } \\
\text { [II-D-29300 }\end{array}$ \\
\hline $\begin{array}{l}\text { Tsong, I.S.T. } \\
\text { VI-A-29037 }\end{array}$ & $\begin{array}{l}\text { Vranos, A } \\
\text { V-C-26456 }\end{array}$ \\
\hline $\begin{array}{l}\text { Tsu, Rapbael } \\
\text { VIII-B-27443 }\end{array}$ & $\begin{array}{c}\text { Waber, James T. } \\
\text { V1-A-29347 }\end{array}$ \\
\hline $\begin{array}{l}\text { Tsui, D.C } \\
\text { I-D-26015 } \\
\text { VII-A-28335 }\end{array}$ & $\begin{array}{l}\text { Wagener, Ken B. } \\
\text { II-E-26441 }\end{array}$ \\
\hline $\begin{array}{l}\text { Tufts, Donald W. } \\
\text { IV-G-27620 }\end{array}$ & $\begin{array}{c}\text { Wager, John F. } \\
\text { 1-D-28852 }\end{array}$ \\
\hline $\begin{array}{l}\text { Tukey, J.W. } \\
\text { [V-C-26997 }\end{array}$ & $\begin{array}{r}\text { Wallace, W.E } \\
\text { V1-D-28146 }\end{array}$ \\
\hline $\begin{array}{r}\text { Uchino, Kenji } \\
\text { VI-D-30068 }\end{array}$ & $\begin{array}{l}\text { Walsh, John } E \\
\text { 1-B-28531 }\end{array}$ \\
\hline $\begin{array}{l}\text { Ulaby, Fawwaz T. } \\
\text { VII-D-30387 }\end{array}$ & $\begin{array}{l}\text { Wang, } K . L . \\
\text { VIII-A-2689 }\end{array}$ \\
\hline $\begin{array}{l}\text { Ullman, Frank G. } \\
\text { I-D-27780 }\end{array}$ & $\begin{array}{l}\text { Wang Paul } \\
\text { IV-B-28908 }\end{array}$ \\
\hline $\begin{array}{l}\text { Ullman, Jeffrey D. } \\
\text { IV-E-28354 }\end{array}$ & $\begin{array}{l}\text { Weaver, James C. } \\
\text { IIl-C-28361 }\end{array}$ \\
\hline $\begin{array}{l}\text { Usberwood, P.N.R } \\
\text { IX-B-5937 }\end{array}$ & $\begin{array}{c}\text { Weaver, John H. } \\
\text { V-D-29218 }\end{array}$ \\
\hline $\begin{array}{c}\text { Van Veen, Barry } \\
\text { VIII-E-26460 }\end{array}$ & $\begin{array}{l}\text { Webber, Stephen E. } \\
\text { II-E-28053 }\end{array}$ \\
\hline $\begin{array}{l}\text { VanderLugh, Anthony } \\
\text { 1.E-27586 }\end{array}$ & $\begin{array}{l}\text { Wegman, Edward J. } \\
\text { N.-C-28309 }\end{array}$ \\
\hline
\end{tabular}

Weidman, Scott $T$. IV-D-29397

Weil, Jeffrey C.

VII-F-27995

Weiss, B.L.

IX-F-6170

Weller, Robert A.

VI-A-29071

Wells, Valana L. V-B-28002

West, Lawrence C.

I-D-2827]

White, Heniy W.

VI-A-26400

White, John M

II-C-26822

Whiteman, J.R

IX-A-6078

Wigen, Philip E.

V]-D-28141

Wight, Charles A

Il-D-27603

Wilcox, David C.

V-B-26863

Wild, James R.

III-D-27468

Wilkinson, F.

IX-B-6294

Williams, Writiam 0.

[V-A-27557

Wilson, Robert G VIII-A-26708
Wiltse, James

VII-C-28339

VIII-C-28516

Wisian-Neilson, Patty Il-E-29627

Wong, Ngai Chuen

I-B-27532

Wong Shan S.

III-B-27314

Wooley, Bruce A. VIII-E-28387

Wyngaard, John C VII-F-29196

Yalisove, Steven $\mathbf{M}$.

VI-A-28509

Yang, Jie

III-F-29194

Yariv, Amnon

I-E-27273

[-E-29321

Yates, Jobn T.

II-C-29227

Yau, Stephen

[V-D-26674@

Yong, Yook-Kong

V-D-28155

Young, Donald R

VI-D-29282

Yu, Bin

IV-C-28722

Yu, Francis T.S.

l-E-28502

Zernow, Louis

Vl-C-26485 


\section{Subject Index}

Note: The Roman numeral refers to the scientific division, and the alphabetic character refers to the division subsection. Proposal numbers are in ascending sequence within the subsections.

ATM Networks IV-D-29396 Ablation II-D-27025

Abrasion Resistance VI-C-29098 Absorption Coefficients VII-D-28717 Absorption Spectra 1-B-27508, IX-B-6294 Acceleration I-D-28591, V-C-26720, V-D-26934

Acetamides II-A-26636

Acetylcholine III-D-25752

Acetylcholinesterase III-D-26582

Acetylenes V-C-28898, VI-C-28883

Acid Neutralization II-D-30230

Acids VIII-B-27883

Acinetobacter calcoaceticus III-B-28964

Acoustic Phonon Scattering VIII-B-28406

Acoustic Processing I-E-28592

Acoustic Signals IV-B-28916

Acoustic Waves [V-F-28994

Acquisition Schemes VIII-E-27493

Acrylates II-A-28945, III-C-28043, Vl-C-28900

Acrylonitriles [1-E-27808

Actuator Materials VI-D-30068

Actuators IV-D-28835, V-B-25461

Adaptive Control IV_C-28718,

IV-D-28835, V-D 28350

Adhesion V-A-27315, VI-A-26667,

VI-A-27552, VI-A-27624, VI-C-26579, VI-C-29098

Adbesives V-A-27315, V-A-27409,

VI-A-26667

Adiabatic Shear Bands V-A-28283, IX-D-6148

Adsorption IJ-C-27775, II-C-29227, DX-A-6307

Aerodynamics V-B-25461, V-B-25467 V-B-25623, V-B-26631, V-B-28002, V-D-25462, V-D-28123, V-D-28493

Aeroelasticity V-B-25461, V-D-25327, V-D-28123, V-D-28493

Aeromechanical Stability V-B-25467

Aerosols III-A-29332, Vll-D-28717, VIJ-G-28052

Aging VI-A-27037, VI-B-26825

Air Purification II-C-27775

Air Separation Il-E-28373

Airborne Contaminants II-A-30164

Aircraft V-B-26631

Airfojls JV-F-26932, V-B-25467

V-B-25623, V-B-26631, V-B-27894,

$V-B-28002$ V-B-28159, V-B-28249,

V-B-28651, V-B-29049

Airframes V-B-26595

Albedo VII-D-28717

Aldehydes [1-E-27440

Algebra IV-B-27786, IV-B-28007,

N-B-29113, IV-B-29850, IV-D-26674

Algorithms 1-E-28258, I-E-28416,

IV-B-27786, IV-B-28071, IV-B-28143, IV-B-28192, IV-B-28320, IV-B-28701, IV-B-28735, IV-B-29113, IV-B-29530, IV-B-29574, IV-B-29808, IV-B-29850, IV-C-25706, IV-C-26993, IV-C-26997 [V-C-27381, IV-C-28309, IV_C-28718, IV-C-28982, IV-D-27044, [V-D-28511 IV-E-27567, IV-E-27892, IV-E-28328, IV-E-29354, IV-E-29692, IV-F-26802, IV-F-26811, IV-F-26930, IV-F-26932,
IV-F-27524, IV-F-27817, IV-F-28060, IV-F-28408, IV-F-28476, IV-F-28809, IV-F-29031, IV-F-29190, IV-F-29412, IV-F-30411, V-B-28651,

VIII-A-26188, VII-B-26711

VIILE-26460, VIII-E-27464,

VIII-E-27899, VIII-E-28506

Aliphatic Polyamides II-E-28525

Alkali Diatomic Molecules L-B-26899

Alkali Halides I-D-26996

Alkali Metal Sulfates 11-D-27780

Alkaline Earth Compounds I-D-26442, II-A-28402

Alkaline Earth Molecules 1-B-26899

Alkane Thiols Il-C-27770

Alkenes II-E-26839

Alkyl Carbonates 11-A-26636

Alkyl Metaphosphates II-C-26126

Alloying VI-C-28639, VI-C-29326

Alloys

Deformation V1-E-28272, VI-E-28575

Epitaxial Growth VI-D-29606

Fatigue VI-A-25833

Impact Tests VI-F-29647, IX-D-6148

Joining VI-C-29524

Magnetic Properties VI-D-29713

Mechanical Properties V-C-28005 VI-C-28067, VI-E-28272

Microstructure VI-C-27472

VI-C-28005, V1-C-28067

VI-C-29326

Phase Transformations IV-A-27557

Processing VI-B-26825

Stress Corrosion Cracking Vl-A-25833

Aluminum

Corrosion Protection VI-A-26400

VI-A-26667, VI-A-26678

Self Diffusion Vl-C-29410

Welding Flux Additive Vl-C-30060

Aluminum Alloy Matrix Composites

VI-C-29524

Aluminum Alloys VI-B-26825,

VI-C-27472, V1-C-28005, VI-C-28067,

V]-C-29524, VI-C-30060, VI-D-29713

Aluminum Arsenides VIIl-A-26444,

VIII-A-26761, VIII-B-26755,

VII]-B-27883, VIII-B-28461,

VIII-G-25602

Aluminum Borides VI-F-26246

Aluminum Chalcogenides II-A-26748

Aluminum Gallium Arsenide Heterostructures VIII-B-28461

Aluminum Gallium Arsenide Microprocessors VIII-B-28329

Aluminum Gallium Arsenide Quantum Wells VIII-B-28895, VIII-F-28922

Aluminum Gallium Arsenides I-A-28996,

I-B-28569, I-D-26015, I-D-27458,

I-D-28470, VI-D-29750, VIII-A-28594, IX-F-6170

Aluminum Glasses VI-C-28067

Aluminum lons I-B-26709, [-D-28472

Aluminum Matrix Composites VI-A-26400

VI-A-29064, VI-C-29410

Aluminum Nickel Powder Mixtures

VI-F-27364

Aluminum Nitrides I1-A-29560,

VI-C-26673
Aluminum Oxide Vl-B-2675

VI-B-28480, VI-B-28577,

VI-C-26392, VI-C-28005, VI-C-29524,

VI-F-26021, VII-A-30090

Aluminum Oxide Matrix Composites VI-B-28826

Aluminum Oxide Waveguides

VIII-C -25339

Aluminum Phosphides II-A-26748

Amides II-A-26748, II-E-27440

Amines II-A-26748, II-B-26914

Aminoglycoside Antibiotics III-F-29221

Ammonia II-C-28767

Ammunition V-C-29336

Amorphous Silicon IX-A-6280

Amphibians LX-B-5937

Amplifiers I-E-28356, VIII-C-29523,

VIII-F-30595

Amplitude Modulators I-D-28271

Analog Digital Conversion VIIL-E-28387

Aniline IX-B-6089

Anions II-A-29560

Anisotropy IV-A-28606, IV-F-29031,

V-A-27409, V-A-29543

Annealing IV-F-26811, VI-A-26427,

$$
\text { IX-F-6170 }
$$

Antenna Feed Networks VIII-C-27304

Antennas VIII-C-25045, VIII-C-27304

VIII-C-27904, VIII-C-28151,

VIII-C-28483, VIII-C-28516,

VIII-G-27554

Antbracene IX-B-6089, IX-B-6294

Anthropometry [V-E-30061

Antibiotics [I]-F-29221

Antibody Catalysis III-C-291.95

Antimonides ]-B-26257, V1-D-29606

VIII-A-26708, VIII-B-28508

Aperture Antennas VIJ-C-27904

Appalactian Region VIJ-A-28772

Applied Mathematics IV-B-28535

Approximation IV-B-27690, IV-F-27620

Arc Welding Flux V1-C-30060

Argon V-C-28898, VI-A-26427, VI-A-29071

Arithmetic Coding IV-B-28071

Arithmetic Fourier Transform IV-F-27620

Armor Vl-B-28608, V]-C-26485,

VI-F-26021, VI-F-29647

Aromatic Compounds III-B-26750,

IX-B-6294

Array Processing VIII-E-27899

Arsenic VIII-B-29616

Arsenides

Crystal Defects VI-D-27764

Crystal Growtb !-D-26015,

VIII-A-28351, VIII-F-27865

Diffusion Mechanisms VI-D-26729

Electrical Properties I-B-26821 VIII-A-26444

Electron Emission I-B-28569

Electronic Properties IX-A-6280

Epitaxial Growth VI-D-29750,

VIII-B-26686, IX-F-6291

Epitaxiai Layers VIII-A-26761

Etching VIII-B-27883

Gate Leakage VIII-G-28925

Heterostructures 1-A-28996, I-D-29922,

VII-G-28925 
Ion Bombardment VIIl-A-26708 Ion Implantation IX-F-6170

Magnetotransport Properties I-D-27458

Optical Properties I-D-28470,

VIII-A-26188, VIII-B-26755

Processing VIII-F-27865

Properties II-A-26748

Quantum Well Structures VII-G-25602

Scanning Tunneling Microscopy

VII-B-28508

Schottky Contacts VIII-G-28326

Simulation VIII-B-26711

Surface Properties []-C-27770

VIIl-B-28508

Switching Properties l-B-26821

Transport Properties VII-A-28594,

VII-G-25602

Vibrational Properties LX-A-6280

Articulated Figures IV-E-26779

Artificial Intelligence IV-E-26779

Asymptotic Properties IV-A-27580,

$$
\text { IV-F-26792 }
$$

Asymptotic Series IV-C-29678

Asynchronous Data Transfer IV-D-29396

Atmospheric Boundary Layer VII-F-29196

VI-F-29369

Atmospheric Gravity Waves VIl-F-28664

Atmospberic Surface Layer VII-F-28954

Atmospheric Turbulence IV-F-30411,

$$
\text { IX-F-6458 }
$$

Atomic Collisions [-B-26709

Atomic Force Microscope III-F-29194

Atomic Hydrogen I-B-26709, 1-B-27646,

$$
\text { I-D-28362 }
$$

Atomic Interferometty 1-B-30141

Atomic Layer Epitaxy VIIl-F-26898

VIII-F-30500

Atomic Ordering I-D-28362

Atomic Position Measurement I-B-27766

Atomic Structure [X-A-6280

Atomization V-C-26797

Austenite IV-A-28987

Autocorrelation Function IV-A-27580

Autoignition V-C-25190

Automatic Target Acquisition V-D-27075

Automatic Target Recognition

VIII-E-27464

Axisymmetric Wakes V-B-30113

Axons III-D-25752

Azaacomatic Compounds IX-B-6294

Azaethylenes II-E-27440

Azides II-A-26748, II-A-28655,

II-E-28631

Azobenzenes II-A-28353

Bacillus megaterium III-A-28022,

III-C-27956

Bacillus subtilis III-C-27956, III-C-28090

Bacillus III-D-25476

Backlighted Thyratrons I-B-26257

Backscattering VII-D-27031, VII-D-29633, VII-E-28102, IX-F-5830

Bacteria III-C-28361, III-C-28972,

III-D-25476, III-D-27468,

III-D-29300, III-F-29537, IX-B-5937

Bacterial Polyesters III-B-27314

Bacterial Spores III-A-28022, III-C-27956, III-D-25476

Ball Bearings V-D-27075

Ball Screw Mechanisms V-D-27075

Ballisties V-A-28307, VI-F-26021

Bandpass Filters VIII-C-25045

Barium I-B-26709

Barium Fluorides [-D-26442

Barium lons II-A-28402

Barium Magnesium Germanates I-D-27425

Barium Sodium Niobates I-E-26971

Barium Titanates I-E-28526, VI-D-27548

Batteries IX-B-6674
Battlefield Simulation IV-B-29530 Beam Formers VII-E-26460

Beams (Structural Forms) V-B-25467,

V-D-25327, V-D-25630

Bearingless Rotors V-B-25467

Bearings V-D-27075

Belief Functions IV-C-26993

Benzaldehydes II-D-27505

Benzenes II-A-28353, II-C-27775,

LX-B-6294

Benzoquinone Il-A-27600

Beryllium VIII-A-26444

Bifurcation V-D-27300

Bilayer Strips V-D-28155

Biliproteins III-F-29537

Binary Matrices [V-C-27868

Biocatalytic Reactions III-C-28043

Biochemistry III-B-29097, III-D-27468, UIL-E-29345

Biodegradation III-B-26750, III-B-27314, III-B-28964

Biological Agent Detection II-B-26115

Biological Membranes VI-C-28900

Biological Tissue I-E-29339

Biomarkers IIIL-D-29300

Biomechanics IV-E-30061

Biophysical Properties IX-B-5937

Biopolymers III-C-28972

Biotechnology VI-C-28900

Biphenyls III-B-26750

Birefringence I-D-27780

Bismuth Antimonides VI-D-29606

Bismuth lodides I-E-26974

Bistable States I-D-28468

Blade Tip Aerodynamics V-B-25461

Blade Tip Vortices V-B-26595

Blade Vortex Interactions V-B-25461

Blood Cells III-F-29194

Body Temperature III-E-29345

Bond Fission Reactions I-D-26106

Bonding Mechanisms VI-A-29037

Boost Pbase V-C-27469

Bootstrap Methods IV-C-28679

Borane Anions II-A-29560

Boranes II-D-25523

Borides 1-B-26257, II-A-29560

VI-C-26392, V-C-28368, VI-D-29713,

VI-E-30488, VI-F-26246

Boron Il-A-29560, VI-D-29750,

VIII-F-28729

Boron Alloys V1-C-27472, VI-D-29713

Boron Nitrides II-A-29560 VI-E-28884

Boundary Conditions IV-A-26909,

VII-C-28483, VIII-F-28187

Boundary Layers V-B-26595, V-B-26863,

V-B-27558, V-B-28293, VII-E-28094,

VII-F-27995, VII-F-28664,

VII-F-29196, VII-F-29369

Boundary Value Problems IV-B-27786,

IV-B-29574

Box Beams V-B-25467

Box Splines IV-B-27690

Brain III-C-27890

Broadband Networks VIII-E-28467

Broadband Photodetectors 1-B-29184

Bromine IX-B-6089

Brownian Diffusion VII-B-27316

Brownian Motion IV-C-28905

Bubble Oscillations IV-B-28916

Buckling V-D-25630

Butadienes II-E-27440

Butyl Rubber II-E-26840

Butyl Sulfides Il-B-26914

Butyrylcholinesterase III-D-26582

Cables V-D-25630

Cadmium IX-A-6307

Cadmium Manganese Fluorides I-D 28472

Cadmium Manganese Tellurides I-D-27458
Cadmium Sulfides IX-D-5884

Cadmium Tellurides I-D-27458,

VIII-A-28347

Caffeine IX-B-6089

Calcium [I-C-28767, III-D-25752,

$$
\text { VI-D-28141 }
$$

Cakcium Fluorides I-D-26442

Calcium lons II-A-28402, VI-A-25833

Calcium Oxides II-C-27775

Calcium Salts VI-B-25424

Cantilever Beams V-D-25630

Capacitance VIII-B-28646

Carbide Reinforced Composites

VI-C-25202, VI-C-29410

Carbides VI-B-27163, VI-B-28761,

VI-B-28826, VI-C-26392, VI-C-26673

VI-C-28368, VI-D-29713, VI-E-28884

VIII-B-28508

Carbobydrates III-A-27494

Carbon IL-C-26822, VIII-G-28453

Carbon Dioxide II-D-28371,

II-E-26840,III-C-28043, V-C-28898

Carbon Fibers VI-C-30095

Carbon Monoxide 11-C-26822,

IJ-C-29227, II-D-28371, V-C-27417, $\mathrm{V}-\mathrm{C}-2745$

Carbon Tetrachloride [X-B-6089

Carbonates II-A-26636, II-D-30230

Carbophosphazenes II-E-28711

Carboxylic Acids LX-D-5548

Cascade Superfluorescence I-B-30423

Catalysis II-A-27600, II-A-28469,

Il-D-26256, II-E-29627, III-C-27488,

III-C-28699, III-C-29195, III-D-27468, DX-B-6305

Catalysts II-B-26914, II-B-28013,

II-C-29227, II-E-26839, II-E-28631,

[X-B-6294, IX-B-6305

Catalytic Properties II-E-28675

Caucby Singular Integral Equations

$$
\text { [V-A-27433 }
$$

Cavitation Vl-B-26825

Cells (Biology) III-C-27890, III-C-28361,

III-C-28972, III-D-25752, III-F-29194

Central Nervous System III-C-27890

Centrifugal Compressors V-B-27627

Ceramic Armor VI-B-28608, VI-F-26021

Ceramic Ferroelectrics I-E-26971

Ceramic Films I-E-26971

Ceramic Matrix Composites VI-B-26751,

VI-B-28480, VI-B-28608, VI-B-28826

VI-C-25202

Ceramic Rotors DX-C-5824

Ceramics

Bonding VI-A-29037

Combustion Synthesis VI-C-26392. VI-C-28368

Compacting VI-C-26392, VI-C-28368

Cracking VI-B-28549, VI-E-30488

Crystal Chemistry VI-F-26246

Deformation V]-E-30124

Densification V1-C-26392

Electrical Properties VI-D-30068

Extrusion VI-B-26751

Flaw Testing VI-B-28549

Fracture Toughness V-A-25345,

VI-B-28577, VI-E-30488

Grain Boundary Engineering VI-D-27548

Impact Tests VI-E-30124, VI-E-30488

Interfaces VI-B-28480

Ion Beam Modification VI-A-26806

Mecbanical Properties VI-A-26806, Vl-E -30352

Microstructure VI-A-26806,

VI-C-26123, VI-C-26399 VI-D-27548

Phase Transformations VI-E-30352

Processing VI-C-26123, V-C-26392 
Shock Loading V1-E-30124, V1-E-30352 Sintering VI-B-28577, V]-C-26123

Superplastic Properties VI-B-2675

Cerenkov Lasers I-B-28531

Cerium Alloys VI-C-28067

Cerium lons [-E-2697]

Cesium I-B-28978, 1-D-28362

Cesium Antimonides 1-B-26257

Cesium lon Bombardment VII-A-26708

Cesium Lithium Sulfates 1-D-27780

Cesium Zinc lodides [-D-27780

Chalcogenides II-A-26748

Change Analysis IV-C-27574

Channels (Waterways) VII-A-26902

Chaos I-D-29212, I-D-29375, I-E-28526,

V-B-25461, V-D-27300

Charge Carriers IV-B-28799

Charge Separation IX-D-5548

Charge Transfer IX-B-6294

Charge Transport 1-D-28464, [-E-27054,

VIII-A-28594, VID-B-28406,

VIII-B-28646, VIII-B-29203

Chemical Agents IJ-B-26115, II-B-28814, II-C-27775

Chemical Beam Epitaxy VI-D-28612

Chemical Bonds II-D-27603,

VII-G-28453

Chemical Heat Generators III-A-28691

Chemical Kinetics V-C-2745S

Chemical Lasers I-E-2736

Chemical Markers III-A-28691, II]-D-25663

Cbemical Reactions II-A-27600,

II-A-28353, IJ-B-26284, II-B-28656,

II-C-28767, II-C-30094, II-D-26106,

II-D-27505, II-D-28700, II-D-30230,

V-C-27455, V-C-27469, VIII-G-28453, IX-B-6674

Chernical Vapor Deposition 1-B-27646

Chemically Enhanced Oxidation VI-D-29282

Chemiluminescence 1L-B-26115

Chemisorption l-D-29341

Chiral Compounds II-B-28528

Chioride induced Corrosion VI-A-26678

Chlorides I-D-27780, ll-A-26748,

II-D-28371, II-D-30230, III-D-25752,

UI-F-29194, V1-A-26449, VIII-B-28508

Chlorine I-B-28561, 1-D-28336, IX-B-6089

Chlorine Compounds a-C-26822

Chlorobenzene II-C-27775

Chloroform II-A-26636

Cholesterol IX-B-6089

Choline Transporters III-D-26232

Chromatographic Sticicon Dioxide

$$
\text { II-A-27600 }
$$

Chromium I-D-27425, VI-A-27552, VI-B-28480, VI-D-26729 Chromium Alloys VI-A-25833, VI-C-28005, VI-C-28639

Cbromium Complexes I-D-26442

Chromium lons I-D-28472, VI-A-25833 VI-A-26678

Chromium Silicides VI-D-2067

Chromophores III-F-29537

Chromosome Structure III-C-27956

Chymobelizymes III-C-27488

Cinnamaldebyde IL-E-27440

Circuit Design VIII-E-27886

Clinical Trial IV-C-2699?

Cloning III-C-25126, III-C-28090,

[II-C-28669, III-D-26232, III-D-27916

Clostridium perfringens III-C-27956

Clostridiun thermocellum IJI-C-25126

Clotbing IV-E-30061

Clouds (Meteorolow) VI1-D-28717,

VII-F-29369
Clustering III-B-28964, NV-B-28071, IV-C-26997

Clitter VII-D-29360, VII-D-30387

Coatings I-D-29576, V-A-27409,

V-A-28253, VI-A-26400, VI-A-26667,

VI-A-26678, V-A-27552,

V]-A-28509, VI-B-28480, VI-D-26601 VI-D-30108

Cobalt Alloys VI-B-27163, VI-C-28067,

VI-C-29326, VI-D-28146, VI-D-29713

Cobalt Clusters DX-A-6307

Cobalt Complexes II-A-28945, II-E-28675

Cobalt Containing Polymers 11-E-28373

Codeine IX-B-6089

Coding ]-E-28416, [V-B-28071

IV-B-28908, IV-C-28722

VIII-E-27994

Cognition IV-E-28125

Coherent Electron Beams I-E-27556

Coberent Interference Suppression VIII-E-26460

Cold Starting V-C-25190

Collision Cross Sections V-C-29534

Colloidal Catalysts II-E-26839

Colloids II-B-28656, IX-B-6305

Combat Simulation IV-B-29530,

$$
\text { IV-C-29358 }
$$

Combinatorial Optimization N-B-28143

Combustion Il-C-30094, 11-D-28103,

IV-A-28548, IV-A-28728, V-C-25190,

V-C-26456, V-C-27018, V-C-27565,

V-C-29141, V-C-29302, V-C-29810

Combustion Synthesis VI-C-26392,

VI-C-28368, VI-F-27810

Command, Control, Communications Systems IV-F-28428, IV-F-29190

Communication Networks VIIl-E-26823,

VIII-E-27834, VIII-E-28467

Communication Protocols IV-D-29396,

VIII-E-28467, VIII-E-28506, VIII-E-29653

Communication Systems IV-F-27620, IV-F-29053, VII-E-27028, VIII-E-27899, VII-E-27994

Communications IV-B-28071

Compacting VI-C-26392

Comparators VIIL-E-28387

Complexing Reactions [X-B-6674

Composite Beams V-B-25467, V-D-25327,

$$
\text { V-D-25630 }
$$

Composite Fuselage Frames V-D-25462

Composite Materials

Corrosion Protection V1-A-26400

Creep Properties VI-B-28826

Cure Monitoring VI-C-26571

Deformation V-A-29040, VI-B-26751

Discontinuities V-B-25467

Elastic Properties IV-A-28606,

$$
\text { V-B-2675! }
$$

Fabrication I-D-30408, IV-A-28797

Impact Tests V-A-28253, V-A-28307

VI-B 28608 , IX-D-6148

Interfaces VI-B-28480

Manufacturing VI-C-28938

Mechanical Properties IV-A-28797,

IV-A-29351, VI-C-25202,

VI-C-28560, V1-C-29410

V1-F-26246

Microstructure IV-A-29351

Nondestructive Testing VI-A-29064

Optical Properties VI-D-30108

Positron Annihilation Studies VI-F-30337

Process Defects V-A-28222

Processing VI-C-25202, VI-C-28560, VI-C-29410

Properties II-A-30446, VI-C-28938

Superplastic Properties VI-B-26751

Composite Plates V-D-25462, V-D-25630,
V-D-28155

Composite Rocket Propellants V-C-26720

Composite Shells V-D-25630

Composite Structures V-B-25467,

V-D-28399

Composite Tubes V-D-25462

Compressibility V-C-2679?

Compressive Loading V-C-26403

Compressive Stresses IV-C-27993

Compressive Tests VII-B-27482

Compressors V-B-27627

Computation IV-C-27381, IV-F-29031

Computer Aided Design V-D-26995

Computer Architecture VIIL-B-28325

VIII-B-28329

Computer Generated Holograms I-E-28416

Computer Graphics IV-B-28071,

IV-E-30061

Computer Networks IV_D-29396

VIII-E-27834, VIII-E-28467

Computer Programming IV-E-26779

Computer Programs IV-C-28686

IV-E-28328, IV-E-28950, V-B-27894

VIII-B-28325, VIII-F-29210

Computer Vision IV-F-26811

Computer Work Stations VIII-B-28329

Computerized Animation IV-E-26779

Computerized Simulation IV-B-29530,

[V-C-28722, IV-C-29358, IV-E-27567

IV-E-28328, IV-E-30061, JV-F-28800,

V-D-27075, VIl-A-27401,

VII-B-27316, VIII-F-29210,

LX-A-6280, IX-F-6170

Computers IV-B-28192

Condensed Matter Physics IX-A-6280

Conducting Polymers 1-B-29373,

VI-A-26400, VI-A-26449

Conservation Laws IV-A-28548,

$$
\text { IV-B-28799 }
$$

Contaminants 11-A-30164

Continuum Mechanics IV-A-28514,

IV-F-28994, V-A-27538

Control IV_C-27862

Control Algotithms IV-B-27786

Control Charts IV-C-29358

Control Laws [V-C-28718

Control Problems IV-B-28535

Control Systems IV-D-28511, IV-D-28740,

IV-F-26930, V-B-25461, VIII-F-27865 Control Theory IV-D-27044, IV-D-27365,

V-8-25467, V-D-27510, IX-A-6366

Controller Design IV-D-28835,

V-D-26061, V-D-29029

Controller Synthesis IV-D-27044

Convection IV-A-28797, IV-B-29574,

V-B-25623

Convective Boundary Layers VII-F-27995

Convective Systems VII-E-26982

Convergence IV-F-26932, IV-F-29031

Conversion Coatings VI-A-26400

Cooking III-A-27494

Copolymers II-E-27808, II-E-28053

Copper

Diffusivity VI-D-29677

Electrodeposition II-A-27502

Mechanical Properties VI-B-2682

Surface Properties VIII-G-28453

Thermodynamic Properties VI-D-29677

Copper Alloys VI-C-28067, VI-C-28639,

VI-C-29524, VI-D-28553, VI-D-29713

Copper Chlorides VID-B-28508

Copper Compounds V]-D-29677

Copper Films VI-D-28553

Copper Ions II-E-28675

Copper Substrates VI-D-26601

Copper Surfaces I-B-29391

Copper Vapor Laser IX-D-5884

Copper Zeolites [X-B-6294

Commeal III-A-27494 
Coronenes IX-B-6089

Corrosion VI-A-27552

Corrosion Inhibition VI-A-26449,

V1-A-26678

Corrosion Protection VI-A-26400,

V1-A-26667, V1-A-27552

Corrosion Resistance VI-A-25833,

VI-C-29098

Cortisol IV-C-26993

Coulomb Friction V-D-26995

Coumarin Dyes I1-B-27534

Crack Propagation VI-B-28780,

VI-E-30488, VII-B-26031

VII-B-27482, DX-A-6078

Cracking (Fracturing) V-A-25345,

V-D-25462, VI-A-25833, VI-A-26806,

VI-B-28549, VI-D-26601, VI-E-30488, VI-B-27482

Crasbworthiness V-B-25467, V-D-25462

Creep (Mechanics) V-A-27315,

VI-B-28826, VI-C-26673,

VI-C-29410, VI-D-29042, VII-B-27482

Critical States V-A-28222

Crystal Chemistry VI-F-26246

Crystal Defects IV-A-28987, V-A-28222,

VI-A-29037, Vl-D-26729,

VI-D-27764, VI-D-27846,

VI-D-29750, LX-A-6280

Crystal Growth VI-B-28577, V-C-28900

VI-F-26246, VI-F-27810,

VIII-A-28351

VIII-B-28895, VIII-B-29036,

VIII-F-27865, VIII-F-30595

Crystal Structure II-A-28655, III-B-28964

Crystallization VI-A-28369, VI-C-27472,

IX-D-6011

Crystallography VI-D-28146

Cure Monitoring V]-C-26571

Curing V-A-28222

Curvature IV-C-28743

Cyanamide II-E-27440

Cyanobacteria III-F-29537

Cyclic Feature Anatysis VIII-E-27917

Cyclohexanes IX-B-6089

Cylinders V-A-28283

Dampers V-B-25467

Damping V-D-28123

Data Analysis IV-C-26997, IV-C-27574,

IV-C-27993, IV-C-28686

Data Fusion I-E-28258

Data Networks VIII-E-27834

Data Processing IV-B-28071,

VII-E-26460, VIII-E-28387,

VIII-F-28099

Data Structures IV-B-28071, ]V-F-28408

Data Transfer IV-D-29396

Database Languages IV-E-28354

Database Management Systems IV-E-27892

Databases IV-E-26779, IV-E-29692

IV-F-29031

Decision Processes IV-F-28428

Decision Theory IV-E-29354

Decoder Architectures VIII-E 27076

Decomposition II-D-25523, II-D-27887,

Il-D-28700, II-D-29033, []-D-29596

Decontamination Il-A-28469, II-B-26284,

II-B-26914, II-B-28656, II-E-26839,

III-D-27916

Deductive Databases IV-E-29692

Defect Engineering V]-D-27764

Deformation IV-A-27557, IV-A-28702

IV-A-28987, V-A-26443, V-A-26908,

V-A-27315, V-A-28222, V-A-28283,

V-A-28307, V-A-29040, V-A-29543,

V-C-26403,V_C-26720, V-D-25327,

V-D-26934, V-D-28399, VI-A-26667,

VI-A-27037, VI-B-26751, VI-B-26825,

VI-C-28560, V]-D_26601, V]-E-28272
V1-E-28884, VI-E-30124, VII-B-27482 VIL-B-28040

Delamination V-A-28307

Densification VI-F-26021

Densities I-B-27646, IV_C-28722

Deoxyribonucleic Acids III-C-27956

III-C-28090, II]-C-28669, III-D-27916

III-F-29194, LX-B-5937

Descartes Ring Scattering VU-G-28052 VII-G-28489

Design Automation V-D-26995

Detection II-A-30164

Detonation Hazards V-C-28310

Detonation Waves IV-A-28728, IV-A-28766

Detonations V-C-28310

Deuterium VI-A-29347

Diakoptic Theory VIII-C-28151

Diamond VI-C-26673, V-E-28884,

$$
\mathrm{IX}-\mathrm{A}-6280, \mathrm{IX}-\mathrm{B}-6089
$$

Diamond Films 1-B-26959, I-B-27646,

VI-A-26400, VI-D-26601, VI-F-27810

Dielectric Coatings ]-D-29576

Dielectric Interfaces VII-C-27304

Dielectric Materials II-A-26636,

VI-B-28480, VIL-D-29633

Dielectric Properties II-A-26636,

II-E-27808, IV-A-29351, VL-C-25202

Dielectric Resonators IV-A-26909

Dielectric Waveguides VII-C-25339,

VIIl-C-28483

Dienes II-E-27440

Diesel Engines V-C-25190, V-C-27565,

V-C-29141, V-C-29302

Diesel Fueis V-C-29141, V-C-29302

Differential Equations IV-A-25874

IV-A-27403, IV-A-27869, IV-A-28702,

IV-B-27786, IV-B-28535, IV-B-29167,

IV-B-29850, IV-C-29297; IV_C-29678

IV-D-26674, IV-F-28994

Differential Geometrical Methods IV-C-28743

Diffusers V $-B-27627$

Diffusion I-A-28996, II-E-26072

II-E-26840, III-A-27494, IV-A-25874,

IV-A-28797, IV-B-29574, IV-C-27790

IV-C-27868, IV-F-28994, IV-F-29349

V-C-27469, VI-C-28005, VI-C-29410,

Vl-D-26729, Vl-D-29282

VIl-A-27401,

VII-B-27316, VIII-B-29541,

VIII-F-28729, IX-A-6280

Diffusion Flames V-C-27455, V_C-28898 V-C-29302

Diffusivity VI-D-29677

Digital Communication Systems IV-F-29053

Digital Elevation Maps VIJ-A-26902

Digital Radio 1-E-27586

Digital Signal Processing IV-F-27817, VIII-G-27554

Diodes VIII-A-28347, VIIL-C-26599,

VIII-F-28729, VIII-G-25602

Dioxetanes II-B-26115

Dipole Antennas VII-G-27554

Direction Finding IV-F-29349,

VIII-E-27899

Dirichlet Problem IV-F-29031

Dislocations (Crystal Defects) V-A-27409,

VI-A-29037, VI-B-22163, VI-D-28553

Dispersion VI-F-27995

Display Systems VIII-C-2555?

Dissociative Ionization LX-B-6089

Distributed Decision Processes IV-F-28428

Distributed Memory Systems IV-B-28007

Distributed Systems IV-D-27365

Distribution Planning IV-E-29354

Domain Decomposition IV-B-28735

Doped Glasses IX-D-5844
Doping 1-B-26821, I-C-28955,

I-D-27425, VIII-A-28351

Dosimetry II-E-26072

Drag V-D-28123

Drive Shafts V-D-25462

Drops (Liquids) V-C-27018, V-C-28250

V-C-28427, VII-D-28717,

VII-G-26962,

VI]-G-28052, V]L-G-28489

Ductile Brittle Transition VII-B-27482

Durability V-A-27315

Dye Lasers IX-D-5548, IX-D-5884

Dyed Fabrics 1X-B-6294

Dyes IL-B-27534, II-B-27568, II-D-27025,

VI-D-30108, IX-B-6294

Dynamic Loading V-A-28283, V-C-26403

VI-C-26392, VI-E-28575

Dynamic Response V-A-26908

Dynamic Simulation V-D-27075

Dynamic Stall V-B-25461, V-B-25467,

V-B-26631, V-B-27894, V-B-28159, V-B-29049, V-D-28123

Dynamic Systems IV-A-27403

IV-C-28905, IV-F-29190, V-B-25461

V-D-25630, V-D-26061, V-D-26934

Dynamics IV-C-29740

Dysprosium VI-D-28146

Earth Atmosphere VII-F-29196

Eddies V-C-27018

Edge Effects V-D-28399

Eigenvalues IV-B-28377, IV-C-27868 V-D-28399

Elastic Properties IV-A-28514,

IV-A-28606, IV-A-29351, V-A-25345,

V-A-27409, V-A-27538, V-A-29543,

V-C-26403, V-D-25327, V-D-28399,

VI-B-26825, VI-D-29042, IX-A-6078

Elastic Tubes V-D-28399

Elastodynamics [V-B-30297

Elastomeric Dampers V-B-25467

Elastomers Il-E-26840, II-E-28711,

$$
\text { VI-C-27373 }
$$

Elastoplastic Materials IV-A-28702

Electric Circuits VIII-E-2829?, VII-F-30595.

Electric Contacts VI-D-29677

Electric Fields ]-D-29923, 1-E-26971

VI-A-29037, VI-B-26825, VI-D-30068, VIII-C-28483

Electric Switches I-B-26257

Electric Waves VIII-A-28674

Electrical Properties I-B-26821, I-B-29373, 1-D-28362, I-D-28852, II-C-27770,

VI-D-27548, VI-D-29282, VI-D-29606,

V]-D-29750, VI-B-28599,

VIII-A-26444,

VII-B-26686, [X-F-6563

Electrical Resistivity Vul-A-26708

Electroactive Materials II-A-29725

Electrochemistry I-D_30408,

II-A-28945, II-A-30289, II-A-30446,

II-C-29538, II-E-27054, II-E-28711

VI-A-26449, VI-A-26678

Electrodeposition I-D-30408, II-A-27502

Electrodes II-A-28945, II-A-30289,

V1-A-25833, V]-A-29347

Electroluminescent Devices I-D-28852

Electroluminescent Sources I-E-29050

Electrolytes II-A-28945, III-D-26583

IX-B-6674

Electromagnetic Fields VI-D-28219

Electromagnetic Measurements VII-G-27554

Electromagnetic Pulses I-B-28978

Electromagnetic Radiation VII-D-28717

Electromagnetic Scattering VI-D_27485

VII-D-27911, VII-D-28219,

VII-D-29205, 
VIII-A-28674, VIII-C-27904 Electromagnetic Spectrum VII-G-28453 Electromagnetic Waves I-B-28499, IV-A-26909, VHI-A-28674

Electromagnetically Induced Transparency 1-B-28978

Electromechanical Properties V1-C-29098 Electron Beam Lithography I-B-29595,

VIII-B-27578, VIII-B-29223

Electron Beam Radiolysis II-D-30332

Electron Beams 1-B-26257, 1-B-26821, I-B-28531, L-E-27556

Electron Collisions V-C-29534,

VIII-B-28004

Electron Emission I-B-28569, VI-A-27552 Electron Impact V-C-29534

Electron Paramagnetic Resonance 1-D-27425

Electron Phonon Interactions VIII-B-26755 Electron Processes VII-F-30549

Electron Sxattering 1-D-28470,

VII-A-28347, VIII-B-26711,

VIII-B-29541, VIII-F-28187 Electron Systems I-D-26015

Electron Transfer II-C-29538, LX-B-6294, IX-D-5548

Electron Transport VIIL-G-25602

Electron Tunneling ]-D-29572, [-D-29578,

$$
\text { VIII-G-25602 }
$$

Electronic Ceramics VI-D-27548

Electronic Devices $[-\mathrm{B}-28569$

VIII-B-29223, VIII-B-29631

VIII-E-28297

Electronic Materials VIII-A-27298

Electronic Networks IV-F-29412

Electronic Properties I-D-28684,

VIII-B-29036

Electronic Structure $1-D-29341$,

[X-A-6280

Electronic Transport I-D-26015

Electronic Warfare IV-F-27620

Electro-optic Devices I-D-28979

Electro-optic Displays VIII-C-25557

Electro-optical Materials VI-D-27605

Electro-optical Signal Processors 1-E-27273

Electro-optics VI-D-26883, VIII-C-29615

Electrophysiology IX-B-5937

Electroporation III-C-28361

Electrostatic Control HI-D-26582

Elevation Maps VII-A-26902

Elliptic Control Problems IV-B-28535

Elliptic Partial Differential Equations

IV-A-27641

Elliptical Waveguides VIII-C-28516

Elpasolites I-D-26442

Emission Spectra IX-B-6294

Emulsions II-B-26914, Il-E-28675

Energetic Materials II-A-28655,

[1-D-26106, II-D-27025, II-D-27887,

II-D-28565, II-D-28700, V-C-26720, V-C -28310

Energy V-C-27864

Energy Levels 1-C-28955

Energy Transfer I-D-28472, I-E-27366 II-D-26106, V-C-27864

Energy Transport Models IV-B-28716 Engineering Design V-D-28350

Engines V-C-29302

Enthalpy $[X-B-6674$

Entropy IV-A-28548, VIII-G-28326, IX-B-6674

Enzymes II-B-28013, III-B-27314,

III-B-28964, III-C-27488, III-C-28011,

III-C-28699, III-D-27468, II]-D-27916 Epitaxial Films V1-D-28553

Epitaxial Growth 1-D-27458, V1-D-26601,

VI-D-28612, VI-D-29606, VI-D-29750,

VIII-A-26444, VIIl-A-26761,

VIII-A-27298, VIII-A-28351,
VIII-B-26686,

VIII-B-28895, VII-B-29036,

VIII-B-29616, VIII-F-26898

VIII-F-28729, VIII-F-30500,

VIII-G-28453, DX-F-6291

Epoxidation II-E-26839

Epoxides IL-C-26126

Epoxy Resins II-E-28795

Equations of Motion [V-A-28797

Erbium VIII-A-26708

Erbium Alloys VI-C-27472

Error Identification JV-C-28718

Escherichia coli III-C-25126, III-C-28090, III-C-28929

Esterification III-C-28043

Esters II-B-28013, III-B-27314,

Estimating IV_C-27862, IV-F-26792

$$
\text { IV-F-27620 }
$$

Estimation Algebra [V-D-26674

Etchants VIII-B-2788

Etching I-B-28561, VIII-B-27578,

VIII-B-27883, VIII-F-28922

Etbanes Il-A-29560, BI-C-28043

Ethers II-B-26914

Ethylenes II-E-27440, III-C-28043, V-C-28898

Evaporation V-C-27018

Evolution Problem IV-B-28535

Excimer Emission I-B-268999

Excitation I-B-26709, 1-B-26959,

I-B-27508, I-B-28499, I-B-29184,

I-B-30423, I-C-28955, I-D-26442

I-D-28684, I-E-26974, II-B-27568,

[]-C-28628, II-C-28767, II-C-29227,

II-C-29538, II-D-26106, II-D-28565,

II-D-29033, III-D-25752, V-C-27864,

V-C-29534, V-D-25630, V-D-27300,

VI-C-26571,VII-G-28489, IX-B-6294, DX-D-5884

Excitons 1-D-29922, L-E-26974,

VIII-G-28453

Exhaust Plumes V-C-27469, V-C-28912

Exoenzymes III-B-27314

Exothermic Mixtures VI-F-27364

Expert Systems IV-E-26487, V-D-26995

Explosives V-C-28310

Exponential Distributions IV-C-27993

Exponential Signals IV-F-26792

Extinction Coefficients VII-D-28717

Extrusion III-A-27494, VI-B-26751,

VI-C-28560

Fabrication VI-C-29098

Fabrics [X-B-6294

Factorization IV-B-28377

Failure IV-C-27868, IV_C-27993, VIII-B-28004

Far Infrared Lasers I-D-29578

Far Infrared Radiation I-D-26996

Fatigue (Mechanics) V1-A-25833

VI-B-25424, VI-B-26825, VI-D-29042 VII-B-26031

Fault Tolerance IV-F-27817, IV-F-28060

Feed Networks VIII-C-27304

Feedback I-D-29572, IV-D-27044 V-D-28350, VII-E-27834

Fermion Boson Systems I-D-28464

Ferroelectric Crystals [-E-26695, 1-E-26971

Ferroelectric Memories I-E-26971

Ferroelectric Thin Films 1-D-28979

Ferroelectric Transitions [-D-27780

Ferromagnetism ]-D-28468, IV-F-28994

Fiber Optic Receivers VII-E-28387

Fiber Reinforced Composites

Impact Tests V-A-28253

Field Effect Transistors VIII-A-30090

VIII-B-28004, VIIL-B-28461,
VIII-B-29036, VIII-B-29616

File Systems IV_D-29396

Film Growth VII-B-2834

Filtering [-E-27586, IV-C-28718,

IV-C-29297, IV-D-26674, V-B-27894

VIII-G-27554

Filters I-E-27273, V-D-28155,

VIII-C-25045, VIII-E-2707,

Finite Difference Time Domain

VIII-G-27554

Finite Element Technique IV-B-28320,1 V-B-28377, IV-B-28535, IV-B-28716, V-B-28908, IV-B-30297, V-A-29940, V-D-26995, VI-D-29042, VIII-F-29210 IX-A-6078

Fish Protein III-D-26232

Flame Propagation IV-A-28728, V-C-26456

Flame Spreading V-C-29336

Flame Structure V-C-27455

Flames V-C-28898, V-C-29302

Flash Photolysis IX-B-6294

Flavor III-A-27494

Flaw Testing VI-B-28549

Flexible Robotics V-D-26934

Flexible Systems V-D-28350

Flight Control Systems V-B-25461

Flight Dynamics V-B-25467

Flood Forecasting VII-A-26902

Floods VII-A-28772

Flow Fields V-B-25623, V-B-27558 V-B-27894, V-C-27480, V-C-29534, $\mathrm{V}-\mathrm{C}-29771$

Flow Kinetics V_C-27864

Flow Problems IV-B-28143

Flow Properties V-B-27627, IX-E-6185

Flow Separation IV-B-28916, V-B-26595, V-B-27558, V-B-28293, V-B-28651

Flow Transitions V-B-26863

Fluid Dynamics IV-A-28797, IV-B-28916, V-B-27062, V-B-27558, V-B-27627, V-B-29049, V-C-29771, V-E-29579

Fluid Flow V-C-27864, VII-A-27471, Vll-A-28504

Fluid Mechanics IV-A-28766

Fluids IV-A-27557, IV-B-29808

Fluorescence I-B-26959, 1-B-30423,

[1-E-28053, [X-D-5548

Fluorides

Electrical Properties I-D-28362

Energy Transfer Processes [-D-28472

Optical Properties I-D-26442

Properties II-A-28655

Solvent Properties III-C-28043

Fluorinated Ferroelectrics L-E-26971

Fluorinators II-A-28655

Fluorine I-A-28996, II-B-28528, VI-D-29282

Fluoroacetopbenones V1-C-28883

Fluoroacrylonitriles II-E-27808

Fluorobenzenes [1-E-26072

Fluorocarbons IX-B-6089

Fluoroform III-C-28043

Flux Measurements VIl-F-27995

Foams II-E-26072, VI-C_27373

Foliage VIJ-D-28219

Food III-A-27494

Food Processing Ill-A-27494

Forsterite l-D-27425, I-D-28472

Forward Flight V-B-25467, V-B-25623,

V-B-28002, V-D-28123, V-D-28493

Fourier Analysis IV-F-27620

Fourier Transform IV-F-27620,

VIILE-27994

Fracture Mecbanics V-A-28307

Fracture Properties IV-A-27557,

V-A-25345, V-C-29336, VI-A-27624,

VI-B-25424, V1-B-28549, VI-C-28639

VI-E-30488, IX-A-6078 
Fracture Toughness V_C-26403,

VI-B-25424, VI-B-27163, VI-B-27407,

VI-B-28480, VI-B-28577, VI-B-28780,

VI-C-28560, VI-E-30488, VII-B-26031

Free Boundaty Problem IV-F-28994

Free Electron Lasers 1-B-28531

Free Energy lX-B-6674

Free Piston II-D-30443

Free Radicals II-C-28767

Freezing III-F-29194, VII-B-27316

Frequency Conversion I-D-28472, I-E-28348

Frequency Division I-B-27532

Freshwater Ice VII-B-26031

Fresnel Zone Plates VIII-C-28516

Fretting Wear V1-A-27552

Friction V-D-26995, V]-A-26806,

VIJ-B-27482

Front Propagation IV-A-27403

Frost Heaving VIl-B-27316

Fuel Additives V-C-28898

Fuel Blends V-C-27417

Fuel Cells II-A-30289, II-D-30443

Fuel Injection V-C-29302

Fuel Injectors V-C-29141

Fuel Properties V-C-27565

Fuel Sprays V-C-25190, V-C-26797,

V-C-28250, V-C-28427, V-C-29302

Fullerenes J-C-28955, II-A-28655,

II-A-29708, Il-A-30446, IX-A-6307

Fullerite VI-A-26400

Fungi 11]-B-29097

Furans II-E-27440, II-E-28153

Fuselage Frames V-D-25462

Gadolinium Alloys VI-C-28067

Gadolinium Garnets I-D-27425

Gain Dynamics I-D-28470

Galerkin Methods IV-F-29031, IX-A-6078

Gallium IX-A-6280

Gallium Aluminum Arsenides

$$
\text { I-D-27458, DX-A }-6280
$$

Gallium Antimonides VHI-A-26708

Gallium Arsenide Devices VIILE-28387

Gallium Arsenide Heterostructures

$$
\text { VIIl-B-28461 }
$$

Gallium Arsenide Microprocessor

VIII-B-28325, VIII-B-28329

Gallium Arsenide Phosphides 1-A-28996

Gallium Arsenide Quantum Wells

VIIl-B-28895

Gallium Arsenide Surfaces VIII-F-26898

Gallium Arsenide Transistors

VIII-A-28335

Gallium Arsenides

Crystal Defects VI-D-27764

Crystal Growth VII]-F-27865

Diffusion Mechanisms VI-D-26729

Electrical Properties I-B-26821 VII-A-26444

Electron Emission I-B-28569

Electronic Properties LX-A-6280

Epitaxial Growth VI-D-29750,

VII-B-26686, IX-F-6291

Epitaxial Layers VIII-A-26761

Heterostructures I-A-28996, J-D-29922

Ion Bombardment VIII-A-26708

Ion Implantation IX-F-6i70

Optical Properties I-D-28470

VIII-B-26755

Photoluminescence I-D-27458

Processing VII]-F-27865

Properties Il-A-26748

Quantum Effects I-D-27458

Scanning Tunneling Microscopy VIII-B-28508

Schottry Contacts VIII-G-28326

Simulation VIII-B-26711

Superlattices VIII-A-26444
Surface Properties [1-C-27770

Switching Properties l-B-2682

Transport Properties VIII-A-28594 VIII-G-25602

Gallium Chalcogenides II-A-26748

Gallium Chlorides II-A-26748

Gallium Nitrides II-A-26748

Gallium Phosphides II-A-26748

Garnets I-D-27425, I-D-28472

I-D-29375, VI-D-28141

Gas Dynamics IV-A-28548, IV-A-28728, V-C-29771

Gas Permeability II-E-28711

Gas Phase Densities I-B-27646

Gas Turbines V-C-26456

Gases II-E-26840

Gauss Machine VIII-E-26735

Gelatinization III-A-27494

Gelation V1-C-27373

Genes III-B-28964, III-C-25126,

[I]-C-27890, III-C-28090, III-C-28929,

III-C-28972, UL-E-29345

Genetic Search Algorithms IV-E-29354

Genetics IIL-D-27468

Geology VIL-A-27401

Geometric Methods V-C-28743,

IV-D-26674, V-D-26995

Germanides VIII-A-26896, VIII-F-28729, VIII-G-25602

Germanium V1-D-29282

Germanium Silicides V1-D-29282

Glass Ceramics VI-B-28577

Glasses

Doping IX-D-5884

Fracture Properties VI-A-27624

Interfaces [1-C-29538, V]-A-27624

Mechanical Properties VI-C-28067

Microstructure VI-C-28067

Penetration II-E-26\&40

Structural Cbanges IX-D-5548

Glassy Polymers IV-A-25874

Glial Cells III-D-25752

Glutamate Receptors III-C-28669 IX-B-5937

Glutamates Ill-D-25752

Glutathione Transferases IIl-D-27916

Glycoproteins III-F-26767

Gold VI-B-28480, VII-D-27031,

VIII-B-28345, VIII-G-28925,

[X-A -6307

Grain Boundary Engineering VI-D-27548

Grain Growth Vl-C-29326

Granular Transport Vll-A-27401

Graph Algorithms IV-B-28143

Graph Simulation IV-C-27868

Graph Theoretical Madels IV-C-28718

Graphics IV-B-28071, IV-E-30061

Graphite 1-8-26257, I-D-28362

VI-A-26400, V1-E-28884

Graphitization l-B-29373

Grating Couplers [-D-28652

Gratings I-E-27882, Vll-D-27031,

VIIl-A-28674, VII]-C-25339,

VIIL-C-27304,

VIII-G-28925

Gravel Stream Beds IX-E-6185

Gravity Waves VII-F-28664

Grid Amplifiers V11I-C-29523

Grid Oscillators VIII-C-29615

Growtb Models IV-F-29031

Guided Waves VIIl-C-25045

Gun Propellants V-C-26403, V-C-29336

Hafnium lons VI-A-25833

Halide Elpasolites I-D-26442

Halides [-D-26996

Hall Domain I-D-26682

Hall Effect 1-D-26015, 1-D-27458

Halogen Oxidizers [l-A-28655
Hamiltonian Systems IV-A-27641

Hardening VI-B-26825

Harmonic Oscillators IV_C-28905

Harmonic Overtones V-D-28155

Hazardous Chemicals Destruction

Hazards V_C-28310

Heart Rate III-E-2934

Heat Conduction IV-C-28905

Heat Generation III-A-28691

Heat Pulses II-D-27025

Heat Resistance III-A-28022, VI-C-29098

Heat Transfer II-D-28371, V-A-29040,

V-B-28249, V_C-29336, VI-A-27552,

VI-B-28344

Heavy Alloys VI-C-29326

Helicopter Rotor Blades [V-C-29740.

$$
\text { V-B-25623 }
$$

Helicopter Rotors V-B-26631, V-D-28123

Helicopters V-B-25467, V-B-25623,

V-B-26595, V-B-26631, V-D-26061

Helium II-C-28767

Helizymes III-C-27488

Hessenberg Systems IV-B-27786

Heterojunctions I-D-26015, VIII-A-26444

VIIl-A-26761, VIII-F-30500

Heterometallic Clusters IX-A-6307

Heterostructures I-A-28996, I-D-27458,

I-D-28464, J-D-29922, I-E-28356,

VIII-A-30090, VIII-B-27883,

VIII-B-28461, VIII-B-29036,

VII-B-29616, VII-F-28729,

VIIL-F-30595, VIIL-G-28925

Heuristic Algorithms IV-B-28192

Hexadecane V-C-28250

Hibernation III-E-29345

High Energy Lasers VII-G-28489

High Frequency VIII-E-27886

High Performance Computing IV-B-28071

High Power Devices I-B-2625?

High Power Lasers I-E-28348

High Power Switching 1-B-26821

High Pressure V-C-27417, VI-E-28272

High Temperature VI-A-27863,

VI-B-28826, VI-C-28005

High Temperature Coatings VI-A-28509

High Temperature Synthesis VI-C-29098

Hingeless Rotor Blades V-D-28123,

$$
V-D-28493
$$

Hole Burning [-D-28470

Hoimium I-D-28472, VI-D-28146

Holograms l-E-27273, l-E-28416,

$$
\text { [-E-28592 }
$$

Holograpbic Interferometry V-C-29810

Holograpby I-E-27273, I-E-28526,

1-E-29339, VIII-G-27554,

VIII-G-28925

Homeostasis III-D-25752

Hormones [V-C-26993

Hom Antennas VIII-C-28483

Hot Electron Physics [-D-28852

Hot Pressing VI-F-26121

Houseflies III-D-27916

Hover V-B-25467, V-B-28002, V-D-28123

Human Anatomy, Physiology IV-E-26779, IV-E-30061

Humidity II-B-26284, V-A-27315

Hybrid Systems [V-F-29031

Hydration III-C-28043

Hydraulics IX-E-6185

Hydrides II-B-28528

Hydrocarbons II-A-30289, III-B-26750,

V-C-27417, V-C-27455, V-C-27565

Hydrodynamics IV-A-28797, [V-B-28799,

VIII-E-28297

Hydrofurans II-E-28153

Hydrogen I-B-26709, I-B-27646,

I-D-28362, I-D-29341, II-C-26822

V-C-27455, V-C-29302, VI-A-29071 
VI-D-27846, VIII-A-26708, [X-A-6280 Hydrogen Cyanide V-C-27455

Hydrogenation VII-A-26896

Hydrology VII-A-27471, V]1-A-28772, VII-E-26982

Hydrolysis II-B-28013, II-B-28656,

II-E-26839, III-C-27488, III-D-27468 III-D-29594

Hydrophobic Substances II-E-28053

Hydroquinone Oxidation II-A-27600

Hydroxides VI-A-26449

Hydroxyl I-B-26959, []-B-28656, II-B-29699

Hydroxylation II-B-26914

Hyperbolic Conservation Laws IV-A-28548

Hyperbolic Equations IV-A-27403, JV-B-30297

Hyperbolic Partial Differential Equations IV-A-28702

Hypersonic Flow V-B-26863, V-C-27469, V-C-27480, V-C-29534

Hyperthermal Surface Scattering [X-B-6089

Ice VII-B-26031, VII-B-27482,

VII-B-28599

Identification IV_C-28718

Ignition IV-A-28728, V-C-25190

V-C-26720, V-C-27018, V-C-27417,

V-C-27565, V-C-28310, V-C-29302, V-C-29336

Image Analysis IV-F-26792, IV-F-26811

lmage Compression IV-B-28071

Image Degradation IV-F-30411

Image Formation IV-F-28476

Image Intensity IV-C-27790

Image Processing I-B-28784, I-E-27591

IV-B-28071, IV-C-28309, IV-F-26811,

VV-F-27620, V1-B-28780, V1I-E-26982,

VIII-C-25557, VIII-E-26735,

VII-E-27076, VIII-G-27554

lmage Restoration IV-F-30411

$$
\text { VIII-C-25557 }
$$

Image Segmentation IV-C-27790

lmaging I-E-29339, II-D-27025,

VI-B-28780

Imidization VI-C-26571

Imines [I-E-27440

Impact lgnition V-C-26720

Impact lonization VIII-B-26711

Impact Tests V-A-26908, V-A-28253

V-A-28307, VI-E-28272; VI-E-28575

VI-E-30124, VI-E-30488, VI-F-27364,

VI-F-29647, [X-D-6148

Impactor Shape Effects V-A -28253

Impedance VII-G-27554

Impurities I-A-28996, I-D-26996,

VI-D-27846, VI-D-29750,

VIII-A-28347, VIII-F-28729,

VIII-G-25602, IX-A-6280

Indium VIII-B-26686

Indium Alloys IV-A-28987

Indium Aluminum Arsenides Vlil-G-2892

Indium AJuminum Gallium Arsenides $\mathrm{X}-\mathrm{F}-6291$

Indium Antimonides VIll-A-26708, VIII-B-28508

Indium Arsenide Gallium Arsenide Devices VIII-D-28070

Indium Arsenides VIII-A-26444

VIII-B-26711, VIII-B-27883,

VIII-B-28508

Indium Compounds VIII-B-27883

Indium Gallium Arsenide Pbosphides VIII-A-26188, VII-A-28351

Indium Gallium Arsenide Quantum Wells VIIIF-28922

Indium Gallium Arsenides VI-D-29750,

VIII-A-26188, VIII-A-28351

VIII-B-26686, VIII-B-27883,
VIII-B-28461, VIII-G-28925 Indium Manganese Arsenides 1-D-27458

Indium Phosphides I-D-28470,

VI-D-27764, V]-D-29750,

VIII-A-26188,

VIIl-A-28351, VIII-B-26686,

VIII-B-26711, VIII-B-28508,

$$
\text { IX-F-6291 }
$$

Indium Phosphorus Dimers II-A-26748

Indium Vacancy Complexes VI-D-27846

Information Exchange IV-F-29412

information Storage VIII-G-27554

Information Theoretic Inequalities

$$
\text { VIII-G-28326 }
$$

Infrared Absorption VIII-A-26896

Infrared Bandgap Superlattices

VLII-A-26188

infrared Detection IX-F-6563

Infrared Detectors VIII-A-26896,

VIII-A-28335

Infrared Lasers I-D-28271

Infrared Radiation 1-B-27888, I-B-28784, II-A-26636

Infrared Sensors [-E-28356

Infrared Spectral Hole Burning I-D-26996

Infrared Spectroscopy VI-A-26400

Insects [X-B-5937

Insulators ]-D-28684

Integral Equations IV-A-27433

Integrated Circuits I-B-29373,

VIII-B-28345, VIII-C-25045,

VII]-E-27886, VIII-F-26898,

VIIl-F-30500, VIII-F-30595,

VIII-H-29540

Integrated Optoelectronic Transmitters

VIII-A-28351

Intelligent Control VIII-F-27865

Intelligent Polymers VI-C-28900

Interconnects [-A-28996

lnterface Bonding V-A-28253

Interface Phonons VIII-B-28461,

$$
\text { VIII-F-28187 }
$$

Interfaces I-D-28852, II-B-28656

II-C-27770, II-C-29538, IV-A-28987,

IV-B-29808, IV-E-27892, IV-F-28994,

VI-A-26667, VI-A-27624, VI-B-28480

VI-D-29282, VIII-C-27304,

VIII-G-28326,

VII]-G-28453, [X-A-6280, IX-B-6294

Interference I-E-27273, VIII-E-26460

VIII-E-27028

Interferometry [-B-30141, I-E-28526,

I-E-29339, V-B-27894, V-C-29810,

VIII-G-28925

Intermetallics Vl-C-2800

Internal Reflections I-E-2697

Internal Resonance V-D-25630

Interpolation IV-B-27690, IV-D-27365

lodides $I-D-27780, I-E-26974$

lodine IX-B-6089

Jodomethane VIII-G-2845

Ion Beam Etching VIII-F-28922

Ion Beam Fabrication Tecbnology

VIII-B-29631

Ion Beams VI-A-27552, V!-A-29071

VIII-A-26708, VIII-B-28345, IX-F-6170

Ion Binding II-B-28656

Ion Bombardment VIII-A-26708

Ion Channels III-F-28775, III-F-2922

Ion Clusters VI-D-29218

Ion Implantation VI-A-25833, V1-A-26678

VI-A-26806, VI-A-27552, VIII-A-26708,

$$
\text { [X-F-6170 }
$$

Ion Sources IJ-A-28402, VI-A-25833

Ion Transport Il-E-27054

Ion Traps III-A-29332

[onic Homeostasis II]-D-25752

Ionic Molecular Solids I-D-27780
Lonization I-B-26709, J-B-28499,

VIII-B-26711, IX-B-6089

Jonomers VI-B-25424

Ions II-A-27600, VI-D-28141, [X-B-6674

Iron VI-D-26601

Iron Alloys VI-A-25833, VI-B-27163,

VI-C-27472, VI-C-28067, VI-C-28639,

VI-C-29326, VI-D-28146, VI-D-29713

VI-E-28575

ron Carbides VI-B-28761

Iron lons III-C-29195, VI-D-28141

Iron Oxides II-C-27775, VI-C-30060, DX-B-6305

Iron alloys VI-C-30060

Isomers III-F-27634

Isotope Exchange II-C-27775

Iteration IV-B-28735, IV-C-26394,$$
\text { IV-F-27620 }
$$

Iterative Encoding Methods I-E-28416

Joining VI-C-29524

Joint Transform Correlators I-E-28502

Josephson Coupling Energies II-E-26072

Kalman Filter IV-C-26993

Ketones II-B-28528, IX-B-6294

Xinematic Models IV_E_-26779

Kinematic Waves IX-E_6185

Kinetic Energy VII-F-29196

Kinetic Energy Penetrators VI-C-29326

Knowledge Bases ]-E-28258, IV-E-26779

Krypton VI-A-26427

Lactones [1-E-2644

Laminar Flames V-C-27455

Laminar Flow V-B-26863, V-C-28427

Laminates II-E-28675, V-A-26908,

V-A-28253, V-A-28307, V-D-25630

V-D-28155, VI-A-2850

Landmarks IV-E-27567, IV-F-26792

Lanthanum Borides I-B-26257, II-A-29560

Laser Ablation II-D-27025

Laser Beams V-C-28912, VII-G-28052

Laser Dyes [I-B-27534, []-B-27568

IX-D - 5548

aser Flash Photolysis IX-B-6294

Laser Induced Reactions l-B-26959,

IX-B-6294

Laser Interactions VI-G-28489

Laser Irradiation I-B-29373

Laser Materials !-D-26442, I-D-28472,$$
\text { LX-D-5884 }
$$

Laser Propagation IX-F-6458

Laser Pyrolysis IL-D-27603, III-A-29332

Laser Radar 1-E-28258, VIII-F-28099

Laser Radiation I-B-29391, LX-F-6458

Laser Spallation VI-B-28480

Lasers I-A-28996, I-B-28499, ]-B-28978

I-B-29015, L-D-29572, I-D-29578,

I-E-27366, I-E-28348, I-E-28356,

I-E-29050, II-D-28889, VIII-A-28351,

VIII-A-30090, VIII-F-28922,

VIII-F-30595, VIII-G-28925,

IX-D-5884, IX-F-6291

Latexes 11-E-26839

Lattice Dynamics I-D-26442

Lattice Filters VIII-E-27076

Lattice Vibrations 1-E-26974

Lead Alloys VI-D-29042

Lead Detection [X-B-6089

Lead Electrodes V-A-25833

Lead Ions I-D-26442

Lead Titanates I-E-26971, VIII-C-26791

Learning IV-B-28071, IV-C-28722

Least Squares Algorithm IV-D-27044

Lift IV-D-27365, V-D-28123

Lifting Surfaces V-B-25467

Ligands III-C-27890, III-F-28775

Light (Visible Radiation) Il-D-2702S 
Light Emitting Porous Silicon VIII-G-28453

Light Modulators I-D-29576

Light Scattering l-D-29572, VII-D-27031 IX-F-5830

Light Sources [-E-29050

Light Transmission VII-D-28717

Limit Theorems IV-F-29053

Linear Programming IV-F-26932

Liouville Equations VIII-B-28646

Lipid Membranes VI-C-28900

Lipids III-A-27494

Liposomes II-B-2922

Liquid Crystal Polymers VI-C-28560 VI-F-30337

Liquid Crystal Television I-E-28502

Liquid Crystals I-E-26695, I-E-26971, II-C-28319, IV-F-28994, IX-D-601

Liquid Jets V-C-26797, V-C-27018

Lithium I-D-28362, VIII-A-26708

Lithium Alloys VI-C-29524

Lithium Batteries IX-B-6674

Lithium Fluorides I-D-28362

Lithium Ions IX-B-6674

Lithium Niobates VI-D-27548,

VII-A-26708

Lithium Oxides VI-B-28577

Lithium Salts IX-B-6674

Lithium Tantalates VIII-A-26708

Lithography 1-B-29595, VIII-B-27578

VII-B-29223, VIII-G-28925

Liver III-E-29345

Local Area Networks TV-D-29396

Locust Muscle IX-B-5937

Logic Devices VIII-A-28594

Logical Database Languages IV-E-28354

Lubricants II-A-30446

Lubrication V1-A-27863

Luminance VIII-C-25557, VII-F-28099

Luminescence IX-B-6294

Lysozymes III-A-27494

Macbine Vision I-E-28258

IV-E-26779, VIl-E-27464

Magnesium VI-C-30060

Magnesium Alloys VI-C-29524, VI-C-30060

Magnesium Oxide I1-C-27775,

VI-A-26806, V1-C-26123

Magnetic Anisotropy VI-D-28553

Magnetic Fields I-D-29578, II-C-28628

Magnetic Hardening V1-D-29713

Magnetic Materials VI-D-28146

Magnetic Multilayers I-D-286\&

Magnetic Phase Transformations 1-D_-28684

Magnetic Properties l-D-27425,

I-D-27458, I-D-28468, 1-D-28684,

I-D-29341, I-D-29375, IV-A-27580,

VI-D-28141, VI-D-28146, VI-D-29713

Magnetic Resonance Imaging VII-F-28099

Magnetic Semiconductors I-D-27458,

VI-D-28141

Magnetic Thin Films VI-D-28553

Magnetic Waves VIII-A-28674

Magnetization I-D-27458

Magnetooptics 1-D-29922

Magnetoresistance VII]-B-28461

Magnetostriction IV-A-28987, IV-F-28994

Magnetotransport Properties I-D-27458

Magnetotunneling Spectroscopy I-D-29578

Magnets VI-D-29713

Malachite Green IX-D-5884

Mammalian Cells [1I-C-27890,

III-D-25752

Mammalian Liver III-E-29345

Man Machine Interactions IV-E-2935

Manganese Alloys VI-C-29326

Manganese lons III-C-29195

Manufacturing IV-F-28994, VI-C-28938
Many Body Systems l-D-28464,

IV-A-27580

Maps, Mapping IV-C-28722, IV-F-26811, VIL-A-26902

Maritime Snow Pack VII-B-28344

Markov Chains IV-C-27868

Martensite IV-A-28987

Martingale Representation IV_C-28715

Mass Spectrometry I-B-26959

III-A-29332, III-D-29300,

VII]-A-26708

Mass Transport IV-F-28994, V-C-29336,

II-A-28504, IX-E-6185

Materials Processing VI-C-2800S,

VIII-F-27865

Materials Science [V-A-27557,

IV-A-28514

Materials Science Reports IV-D-29397

Mathematical Models I-D-29212,

I-E-28356, J-E-29339, V-A-25874,

IV-A-27580, IV-A-28548, IV-B-28716

IV-B-28799, IV-B-29808, IV-C-26993

IV-C-27574, IV-C-27868, IV-C-28718,

IV-C-28743, IV-C-29358, IV-D 27044

IV-E-26779, IV-F-29031, IV-F-29190,

V-A-27409, V-A-27538, V-A-28307,

V-B-25467, V-B-26595, V-B-26863,

V-C-27455, V-C-27469, V-D 25630

VI-D-28141, VI-D-29042, VI-E-28575

VII-A-26902, VII-A-27471

VII-A-28504

VII-A-28772, VIl-E-26982

VII-F-27995,

VIII-A-28594, VIII-B-28406,

VII-C-29523

Matrices (Mathematics) IV-B-29113, IV-C-27868

Matrix Approximation IV-F-27620

Mechanical Alloying VI-C-28639

Mechanical Design V-D-26995

Mechanical Properties II-E-28314

IV-A-28986, IV-A-29351, V-A-25345, V-A-26908, V-A-27538, V-C-26403,

V-C-26720, V-D-25462, V1-A-26806,

VI-A-28509, VI-B-25424, VI-B-26751,

V1-B-26825, V1-B-28480, VI-C-25202,

VI-C-27373, VI-C-28005, VI-C-28067,

VI-C-28560, V]-C-29098, V]-C-29326

VI-C-29410, VI-C-30060, VI-D-29042

VI-E-28272, VI-E-30352, VI-F-26246,

VII-B-28344, IX-F-6563

Mechanical Systems IV-B-29850

V-D-26061,

V-D-26934, V-D-26995, V-D-27075, V-D-27300

Mechanics IV-A-28548, IV-A-28986

Melting VI-B-28577, VII-B-28344, DX-E-6185

Membrane Mimetic Chemistry II-B-29225

Membranes UI-E-26072, III-C-28090, III-F-29170, VI-C-28900

Memories (Storage Devices) I-E-2697

Memory I-E-25546

Memory Systems I-E-29050, [V-E-27892

Mercury V11-D-27031, IX-A-6307

Mercury Cadmium Tellurides VI-D-27846,

Vill-A-26708, VIII-A-28347

Mercury Zinc Tellurides IX-F-6563

Mesb Generation V-D-26995

Mesogens II-E-28314

Mesophase Pitch VI-C-30095

Message Routing VIII-E-2783

Metabolic Rate III-E-29345

Metal Activation II-B-28013

Metal Borides II-A-29560

Metal Films VI-A-29037, VIII-B-2834

Metal Ion Complexes II-A-28486

Metal Ion Implantation V1-A-25833

Metal lons II-A-27600
Metal Laminates VI-A-28509

Metal Matrix Composites VI-A-26400,

VI-A-29064, VI-C-29410, VI-F-26246

Metal Organic Compounds VI-D-29218

Metal Oxides II-C-27775, [1-C-29538,

V]-A-26427

Metal Surfaces I-B-29391

Metallacarborane Complexes II-A-2972

Metallizing VII-B-28461

Metallocenes II-A-28402

Metalloenzymes [1-B-28013

Metalloid Oxides [-E-2736

Metals V-A-29543, VI-B-26825,

VI-B-28480, VI-C-28639, IX-D-6148 Metastable State I-E-27366, [X-D-6011

Meteorology VII-E-26982

Methacrylates III-C-28043, VI-C-28900

Methane 11-A-30289, II-B-26914,

II-C-30094, V-C-28898, V-C-29302

Methanococcus jannaschii III-C-28011

Methanol II-A-30289

Metbides II-A-29560

Methyl I-B-27646, VIII-G-28453

Methyl Acetamides II-A-26636

Metbyl Carbonates 11-A-26636

Methyl Gallium Azides 11-A-26748

Methyl Viologens IX-B-6294

Micelles [I-B-28656, II-E-28053, II-E-28675

Microbial Degradation IIJ-B-27314

Microbial Mineralization III-B-26750

Microbial Spores III-A-2802

Microcomputer Systems VIII-B-28325

Microcracking V-D-25462, VI-D-26601

Microelectronics 1-E-27556, IV-B-28716,

VIII-B-28325, VIII-B-28345,

VIII-B-29631

Microemulsions II-B-26914, II-E-28675

Microorganisms III-D-25476, III-D-25663

Microscopy I-E-27591, I-E-28313;

III-F-29194, VI-A-29037

Microstrip Antennas VIII-C-27904,

Vil-C-28151

Microstructure IV-A-28986, IV-A-28987

V-A-29351, IV-F-28994, V-A-26443,

V-A-28283, VI-A-26806, VI-A-27552,

VI-A-28509, VI-C-26123, VI-C-26392

VI-C-27472, VI-C-28005, VI-C-29098,

V]-C-29326, VI-C-29410, VI-C-29524

VI-D-27548, VI-D-28146, VI-D-29713,

VI-E-30124, VI-F-27810, VI-B-28040 VIIL-B-28345

Microwave Bandpass Filters VIII-C-25045

Microwave Digital Radios [-E-27586

Microwave Holography VIII-G-27554

Microwave Oscillations VIII-E-27886

Microwaves I-D-29375, I-E-27556

Military Networks IV-F-28428

Millimeter Wave Antennas VII-C-27304, VIII-C-28483

Millimeter Wave Circuits VIII-C-26949

Millimeter Wave Devices VIII-C-25045,

Vili-C-25339

Millimeter Wave Power Combining

VIIl-C-28339

Millimeter Waves I-B-29324

i-D-29375, VIL-D-29360, VII-D-30387, VIII-C-29523

Mine Detection IV-F-26811

Mineralization III-B-26750, IX-B-6305

Minerals VI-F-26246

Mixing V-C-28427

Mixtures V_C-27417, VI-F-27364,

LX-E-6185

Mobile Robots I-E-28258

Mode Locking VIIl-G-28925

Model Validation IV-D_27044

Modeling IV-C-28309, [V-E-30061

IV-F-27620, V-D-26995, VI-D-29042, 
VI-F-29647, VII-A-27471, VIII-A-28594, VIII-B-28004

Modulation I-E-27556, I-E-27586

Modulators I-A-28996, I-D-29576, VIII-A-28594, VIII-B-29616, VIII-F-30595, [X-F-6291

Moisture III-A-27494, VII-E-28094, VII]-G-27554

Molecular Beam Epitaxy I-D-27458, I-D-28336, VI-D-29606,

VIII-A-26761, VIII-A-27298,

VII-B-26686, VIII-B-28895,

VIII-B-29036, VIII-B-29616

Molecular Beams LI-D-28700

Molecular Clustering I-D-26442

Molecular Collisions I-B-27888,

II-C-28767, II-D-28700, V-C-29534

Molecular Decomposition []-D-29033

Molecular Dissociation 1-B-27508, II-D-26256

Molecular Dynamics II-D-26106, II-D-27025, JI-D-28555, II-D-28889, [V-A-28797, VI-A-27037 Molecular Engineering VI-A-27863 Molecular Films Il-C-27770 Molecular Free Radicals II-C-28767 Molecular Graphics II-E-28711 Molecular Ionization LX-B-6089 Molecular Mechanics II-B-27568 Molecular Motion II-D-28555 Molecular Prohes IIl-F-29221 Molecular Properties III-A-27494 Molecular Relaxation VII-B-27316 Molecular Structure II-D-27887, II-E-28314, []-E-28631, II-E-28711 Molecular Systems I-B-29324

Molecular Transport III-C-2836]

Molecular Weight II-E-28373

Molybdate lons II-E-26839

Molybdenum II-A-28486, II-C-29227,

VI-A-27552, VI-A-28509, VI-C-29410

Molybdenum Alloys VI-C-28005,

VI-C-29326

Molybdenum Ions VI-A-26678

Molybdenum Silicides VI-C-29410 V!-D-29677

Momentum Management Controller IV-D-28511

Monte Carlo Simulation VIII-B-26711

Motion Planning IV-E-27567 Mountain Terrain VII-A-27471 Multibody Systems V-D-26934 Multicommodity Flow IV-B-28143 Multidegree of Freedom Systems V-D-27300

Multihop Radio Networks VIIL-E-26823

Multimedia Communications VIII-E-29653

Multiphase Systerns VII-A-28504

Multiple Quantum Well Structures VIII-A-28594, [X-F-6170

Multiple Scattering VII-D-29205

Multiple Target Tracking IV-F-26792

Multiprocessor Systems IV-F-28060, VIII-G-27554

Multiprocessors IV-B-28192

Multivariable Systems IV-D-28511

Multivariate Analysis IV-C-26394,

$$
\text { IV-F-26792 }
$$

Multivariate Data IV_C-28309

Multivariate Probability Distributions

IV_C 27993

Multivariate Spline Approximation

IV-B-27690

Muscarinic Receptors II]-D-29594

Muscle Fibers III-F-29221

Mustard II-A-28469, II-B-26284,

II-B-26914

Nanofabrication VIII-B-29631
Nanostructures 1-D-30408, VIII-B-27578 Naphthalenes II-D-27025, II-E-28053,

V-C-28250, IX-B-6294, IX-D-5548 Naphthoic Acids IX-D-5548

Natural Language Processing IV-E-26779 Natural Surfaces VII-D-29360

Nematic Liquid Crystals II-C-28319

Neodymium VIII-A-26708

Neodymium Alloys VI-D-29713

Neodymium Lasers l-E-28348

Neon V1-A-26427, VI-A-29071

Nerve Agents II-E-28675

Nerve Cells III-C-27890

Nervous Systems III-D-25752, IX-B_5937

Network Arcbitectures VIIl-E-28467

Network Management IV-E-26487

Network Reliability IV_C-2S706

Networks I-E-26695, IV-B-28071,

IV-F-28428, VIII-E-27834,

VIII-E-27994, VIII-E-28467

Neural Computation VIII-G-25602

Neural Networks IV-E-28125, IV-F-27524

Neural Source Location IV-F-26802

Neurodynamic Systems IV-E-28125

Neuronal Ligands IH-C-27890

Nickel VI-C-26392, VI-D-28553

Nickel Alloys VI-A-25833, V1-B-27163, VI-C-27472, VI-C-28067, VI-C-28639,

VI-C-29326, VI-D-28553, VI-E 28575

Nickel Aluminum Powder Mixtures V]-F-27364

Nickel Ions III-C-29195, VI-A-25833

Nickel Oxides IX-B-6305

Niobates [-D-28979, ]-E-26971,

I-E-27273, V]-D-27548, VIII-A-26708

Niobium J-D-29341, V]-A-26427,

$$
\text { VI-B-28480 }
$$

Niobium Alloys VI-C-28005, VI-C-28639

Niobium Ions VI-A-25833

Niobium Oxides VI-A-26427

Niobium Silicides VI-D-20677

Nitramines [J-C-30094, II-D-27601

II-D-27887, II-D-28103, II-D-29033, IL-D-29596

Nitrates I-E-29321, II-D-29033 III-F-29194

Nitrene Complexes II-A-28353

Nitric Oxide V-C-27455, V-C-27864 [X-B-6294

Nitrides II-A-28353, 11-A-29560

VI-A-26427, VI-A-27552, VI-C-26673,

VI-D-29677, V1-D-29713, VI-E-28884

Nitriding VI-D-2928

Nitrites II-D 29033

Nitro Compounds II-D-29033, IX-B-6294

Nitrogen I-B-29373, V-C-27469,

$$
\mathrm{V}-\mathrm{C}-28898
$$

Nitrogen Compounds II C-26822, II-D-27505

Nitrogen Dioxide V-C-27455

Nitrogen Fluocides II-A-28655

Nitrogen lons V]-A-26678

Nitromethane II-C-30094

Nitrostilbenes VI-D-27605

Nitrous Oxide ]-B-28490

Noble Gases l-B-26899, V]-A-26806

Noise IV-F-27524, VIII-B-29541

Noise (Sound) IV-C-28905

Nondestructive Testing VI-A-29064, VI-C-28938

Nonlinear Analysis IV-A-27641, IV-F-28994

Nonlinear Beam Theory V-B-25461

Nonlinear Continuum Mechanics

$$
\text { IV-A-28514 }
$$

Nonlinear Controller Designs IV-D-28835

Nonlinear Differential Equations IV-A-27869

Nonlinear Diffusion IV_C-27790
Nonlinear Dynamics IV-C-29740, V-D-28493

Nonlinear Equations IV-A-27869

Nonlinear Filtering IV-D-26674

Nonlinear Flexible Systems V-D-28350

Nonlinear Magnetooptics I-D-29922

Nonlinear Mathematics IV-A-27557

Nonlinear Mechanical Systems V-D-27300

Nonlinear Numerical Methods IV-B-28916

Nonlinear Optical Frequency Conversion

$$
1-E-28348
$$

Nonlinear Optical Interactions VIL-G-28489

Nonlinear Optical Materials I-D-28472 I-E-29321, VI-D-27605

Nonlinear Optical Properties VI-D-30108 IX-A-6307

Nonlinear Optical Scattering VIl-G_26962

Nonlinear Optical Waveguides I-A-28996

Nonlinear Optics 1-B-28978, I-D-29922, I-E-28526

Nonlinear Optimization IV-B-28701

Nonlinear Partial Differential Equations IV-A-27403, IV-B-28535

Nonlinear Problems IV-A-25874

Nonlinear Systems IV-D-27365, V-D-26061, VIII-E-27917

Nonlinear Time Series IV-C-27868,

$$
\text { IV-F-26792 }
$$

Nonlinear Vibration IV-C-29740

Nonlinear Waves [V-A-28548, IV-B-29808

Nucleic Acids II]-D-28479

Nucleophilic Cleavage [X-B-6305

Nucleophilic Decontamination Agents II-B-26284

Numerical Analysis IV-A-25874, -

IV-B-28535, IV-B-28716, IV-B-28916, IV-B-29574, V-D-28155

Numerical Approximation IV-B-28535

Numerical Methods IV-A-25874,

IV-A-27433, IV-B-27786, IV-B-29113

IV-B-29850

Nylon II-E-28525

Object Oriented Image Segmentation IV-C-27790

Object Recognition IV-F-26811

Object Tracking IV-F-29349

Octanes IX-B-6089

Odorant Recognition III-P-26767, III-F-27634

Olefins III-C-29195

Olfactomedin IIl-F-26767

Olfactory Reception [I]-F-26767, III-F-27634

Oligomer Films VI-A-27863

Oligopeptides III-D-29300

Operating Systems VIII-B-28325

Optical Bistability [-E-26971

Optical Coefficients [-E-28348

Optical Dephasing I-E-26974

Optical Fields I-B-27766

Optical Filters l-E-27273

Optical Frequency Conversion [-E-28348

Optical Frequency Division I-B-27532

Optical Gratings I-E-27882, VII-D-27031

Optical Images IV-F-30411

Optical Interactions VII-G-2848

Optical Interconnects I-E-26695,

VIII-E-28387, IX-F-6291

Optical Interferometry I-E-27273

Optical Materials 1-D-28472, I-E-29321,

VI-D-26883

Optical Memory VIIl-B-28895

Optical Memory Systems 1-E-29050

Optical Microscopy I-E-27591

Optical Modulators VII-A-28594,

IX-F-6291 
Optical Parametric Oscillators 1-B-27532, I-D-28472

Optical Phonons VIII-B-29541, VII]-F-28187

Optical Pbotons I-E-26974

Optical Processing 1-E-29339 Optical Properties I-B-28978, l-B-29184,

I-D-26442, I-D-27425, 1-D-27458

I-D-28470, I-D-29572, I-E-26971

I-E-28356, VI-D-26883, VI-D-29750,

V]-D-30108, VII-D-28717,

VIII-A-26188, VIII-A-27298,

VIII-B-26686,

VIII-B-26755, IX-A-6307, LX-D-5884, IX-F-6170, IX-F-6563

Optical Pumping [-B-27766

Optical Remote Sensing VIl-E-28102

Optical Scattering VII-G-26962

Optical Signal Processing I-E-27586

Optical Sources I-E-29050

Optical Storage VIII-G-27554

Optical Switching 1-A-28996,

VII-A-28594

Optical Temporal Waveforms [-E-25546

Optical Vibrations VIII-B-26755

Optical Waveguides I-A-28996,

IX-F-6170, 1X-F-6291

Optimal Coding IV-C-28722

Optimal Control IV-D-27044,

IV-D-28511, [X-A-6366

Optimizing IV-B-28143, IV-B-28701

IV-C-28686, IV-F-26932, IV-F-29190

Optoelectronics I-B-29184, I-D-28271,

VII-A-27298, VIII-A-28351,

VIII-A-30090, VIII-A-30437,

VIII-B-29223, VIII-E-28297,

VIII-F-26898, VIII-F-30500,

VIII-F-30595, LX-F-6291

Organic Acids VIII-B-27883

Organic Aerosols [11-A-29332

Organic Clusters [X-B-6089

Organic Compounds II-D-27505,

II-D-29033, [l-D-30230, III-B-26750

VI-D-29218

Organic Laser Dyes [X-D-5548

Organic Molecule Complexes II-C-28628

Organic Molecules DX-B-6089, IX-D-5548

Organic Optical Materials VI-D-27605

Organic Solvents II]-C-28699

Organometallic Compounds II-A-28402,

Organometallic Polyphospbazenes II-E-28711

Organophosphates III-D-27468

$$
\text { IX-B-6305 }
$$

Organophosphorus Compound II-C-27775, III-D-27468

Oscillating Airfoils V-B-25623

V-B-26631, V-B-27894, V-B-28159

Oscillations I-E-28526, IV-B-28916, VIII-B-28461

Oscillators I-B-27532, I-B-28531, I-B-28978, I-D-28362, I-D-28472

IV-C-28905, VIII-C-25045,

VIII-C-26599, VIII-C-26949,

VIILC-20615, VIL-E-27886

Osmium [I-C-29538

Osmium Antimonides [-B-2625]

Osmium Oxide Fluorides II-A-28655

Ovalenes LX-B-6089

Oxidation II-A-27600, 11-A-28469

II-A-28655, II-A-30289, I]-B-26914,

[1-B-28656, II-C-26126, II-C-29227,

[1-D-25523, [I-D-28371, II-D-30230,

[I-E-26839, III-C-29195, V-C-27417

V-C-27455, V1-D-27548, VI-D-29282, IX-B-6305

Oxidation Resistance VI-A-25833

Oxidative Mineralization lX-B-6305
Oxide Films VI-A-26427, IX-B-6305

Oxide Particles II-C-27775

Oxide Removal VIII-G-28453

Oxide Semiconductors [X-B-6305

Oxides

Adsorption II-C-29227

Ballistic Properties VI-F-26021

Decomposition [X-B-6294

Diffusion II-E-26840

Dynamic Loading V-C-26392

Fracture Toughness VI-B-28577

Interfaces II-C-29538

Ionization [-B-28499

Joining VI-C-29524

Laminations V-D-28155

Microstructure V-A-26806

Nanocrystalline Powders Vl-C-26673

Optoelectronic Properties VII-A-30090

Oxidation Il-D-28371

Properties II-A-28655

Shock Tube Studies. V-C-27864

Sintering VI-C-26123

Surface Decontamination IX-B-6305

Transport Properties VIII-B-27443

Oxidizers [1-A-28655

Oxygen I-B-29373, 11-C-26822,

II-C-28628, V-C-27455, V-C-27469,

VT-D-29750, IX-B-6294

Oxygen Binding II-E-28373

Oxygen Compounds II-D-27505

Oxygen lon Bombardment VIII-A-26708

Oxygen Ions VI-D_28141

Oxynitride Filus VI-A-26427

Packet Radio Networks VIH-E-26823,

VII-E-27028, VIIL-E-27994

Palladium VI-A-29347, VIII-G-28453

Palladium Clusters [X-A-6307

Parabolic Equations IV-A-27403

Parabolic Problems IX-A_6078

Parallel Algorithms IV-B-29530

IV-B-29574, IV-B-29808, JV-F-28408

Parallel Processing IV-B-29167,

IV-E-26779, IV-F-26932, IV-F-28060 VIII-B-28329

Parallel Processors IV-B-28007,

IV-B-28377, IV-B-28735, IV-F-28408

Parallel Programming IV-B-28908

Parallelism Detection IV-B-28192

Partial Differential Equations IV-A-27403,

IV-A-27641, IV-A-28702, IV-B-28535,

IV-B-29167, IV-B-30297, [V-C-29678,

N-D-26674, IV-F-26932, IV-F-28994

Particle Collisions 1-B-26709

Particle Dynamics LX-E-6185

Passivation I-D-29576

Passive Films VI-A-26400, VI-A-26678

Pathogen Detection III-D-28479

Pattern Recognition I-E-28503 IV-E-28125

Pebble Transport [X-E-6185

Pendulums V-D-27300

Penetrants II-E-26840, IV-A-25874

Penetration IV-B-28320

Penetration Mechanics V-A-26443,

$$
\text { V-A-28307 }
$$

Penetrators V1-C-29326

Penicillium funiculosum III-B-27314

Peptides III-C-28680, III-D-29300

Periodic Systems IV-D-27365, V-D-26061

Permafrost VII-B-26281

Permanent Magnet Materials V]-D-28146

Permanent Magnets VI-D-29713

Permittivity II-A-26636, II-E-27808

Perovskites I-D-26442

Peroxo Metal Ion Complexes II-A-28486

Peroxomolybdate lons II-E-26839

Peroxomolybdenum Complexes II-A-28486

Personal Computer Market IV-C-28686
Perturbation Theory VII-D-29404 Perylenes II-B-27568, XX-B-6089,

IX-D-5884

Pesticides III-D-27468

Petroleum Pitch VI-C-30095

Phase Boundaries IV-F-28994

Phase Conjugate Intensity I-E-28526

Phase Diagrams II-E-28314

Phase Modulation VIIl-E-27994

Phase Transformations 1-D-27780

[-D-28468, I-D-28684, ]-E-27273, I

IV-A-27403, IV-A-27557

IV-A-28514, IV-A-28987, IV-B-28535,

V-A-27538, V-A-28283, V]-C-29524,

VI-E-30352

Phased Array Lasers VIIl-F-30595

Phased Arrays VIII-C-2659

Phenantbrenes III-B-26750, DX-D-5548

Ptenobarbital III-D-27916

Pbonon Scattering VIII-B-26686,

VIII-B-28406

Phonons I-D-28852, VIII-B-28461,

VIII-B-29541, VIII-F-28187

Phosphatases 1I-B-26115

Pbosphate Esters II-B-28013

Phospbates I-B-27532, II-B-28656,

II-B-29699, II-C-26126, III-D-25752,

DI-D-27468, VI-A-26400

Phosphazenes II-E-28631, 11-E-28711, Il-E-29627

Pbospbides I-D-28470, VI-D-27764,

VI-D-29750, VII-A-26188,

VII-A-28351, VIII-B-26686,

VII-B-26711, VIII-B-28508,

LX-F-6291

Phosphine [I-C-29227

Pbospbine Azides [1-E-28631

Phosphoesterase II-B-28013

Phosphonates $\mathrm{XX}-\mathrm{B}-6305$

Phosphoramidates II-C-26126

Phosphoranimines II-E-28631

Phosphorescence IX-D-5548

Phasphorolvtic Chemistry II-B-29225

Ptosphorus II-C-29227, II-E-28631, VIII-B-29616

Phosphorus Chemistry II-C-26126

Phosphorus Compounds il-C-26822 II-E-28675

Phosphoryl [I-B-29699

Pbosphorylation II-B-29699

Photoactive Materials LX-D-5548

Photuchemistry II-A-28469, II-B-27534, II-C-28319, II-C-29538

Photodegradation II-B-27534

Photodetectors I-B-29184, VIII-B-28895

Pbotodiodes VIII-F-30595

Photodissociation l-B-2750

Photoelasticity V-A-27409

Photoemission I-B-29391, VII-A-26896

Photoexcitation I-C-28955, I-D-28470, II-C-29227

Photoionization I-B-26709

Photoluminescence I-D-27458, J-D-28336

I-D-29923, VIII-A-26708,

VIIl-A-26896,

VIII-B-26686, VIII-B-28895, VIII-G-28453

Photolysis II-C-26126, [1-C-28767, [X-B-6294

Photon Scanning Tunneling Microscope I-E-28313

Photonics IX-F-6563

Photons I-E-26974

Phot-oxidation IX-B-6305

Pbotopbysics [I-B-27534

Photoreflectance VIII-B-29036

Photorefractive Materials 1-D-28472,

L-E-27273, [-E-27882, I-E-29321

Photorefractive Nonlinear Optics 
[-E-28526

Photorefractive Systems I-E-28592

Pbotostability LX-D-5884

Photostrictive Actuators VI-D-30068

Phthalocyanine [X-B-6089

Phthalocyanine Dyes VI-D-30108

Physical Mapping IV-C-28722

Pbysical Properties VI-D-26601,

VII-B-28599

Piezoelectric Ceramics V-B-25461,

VI-D-30068

Piezoelectric Composites IV-A-29351

$V-D-28155$, Vi-B-28608

Piezoelectric Effect V-D-25630,

V-D-2815S, VIII-B-29616

Pinball Algorithm IV-B-28320

Pipelining VII-E-27076

Piperidine [X-B-6089

Piston Rings V-C-29302

Pitch VI-C-30095

Pitching Airfoils V-B-28651, V-B-29049

Pitting Corrosion VI-A-26678

Planar Networks IV_C-25706

Planetary Boundary Layer VII-F-27995, VI1-F-28664

Plasma Devices I-B-29595

Plasma Instabilities I-D-28652

Plasma lon implantation V1-A-26678

Plasma Sprayed Tungsten VI-C-29410

Plasmas (Pbysics) I-B-28499, 1-D-26682,

I-D-28652, IV-A-27580

Plasmid Stabilization III-C-25126

Plastic Deformation V-A-27409,

V-C-26403, VI-D-26601, VI-E-28272,

VI-E-28884, VI-E-30124

Plastic Flow V-A-27409

Plasticity V-A-27409

Plasticizers VI-B-25424

Plates V-D-25462, V-D-25630,

V-D-27300, V-D-28155, V]-E-28575,

VI-E-30124

Platinum VII-G-28453

Platinum Electrodes Il-A-27502

Platinum lons V-A-25833

Point Defects VI-D-26729, VI-D-27846

Poisson Problem IV-B-28735

Polarization l-D-26682, I-E-26974

VIII-B-29616, VIII-E-27899

Pollution IV-C-29297

Polyacrylates II-A-28945

Polyacrylonitriles V]-C-27373

Polyalkyl/arylphosphazenes II-E-29627

Polyamides II-E-28525, V-A-28253

Polyanilines VI-A-26400

Polyarylene Ethers V1-C-28883

Polybenzoxazoles VI-C-28883

Polybutadienes VI-C-26579

Polybutylene Terephthalate VI-A-28369

Potycarbonates [I-E-27054, VI-A-27037

V1-B-28780

Polycarboxylic Acids VII-B-27883

Polyelectrolytes Il-A-28945, II-E-27054

Polyesters III-B-27314

Polyetheretherketone VI-A-28369

Polyimides I-B-29373, VI-C-28883

Polyisobutylene II-E-26840

Polymer Additives II-E-28153

Polymer Blends [I-E-27808, II-E-28525

IV-A-28797, VI-A-28369, VI-B-28780,

VI-C-26579, VI-C-28560

Polymer Coatings II-A-30164

Polymer Films II-B-26284, IJ-E-27808,

V]-A-26449, V]-A-27863,

VI-D-26883, VI-D-2760

Polymer Gels VI-C-27373

Polymer Glasses V-A-27037

Polymer Laminates II-E-28675

Polymer Latexes II-E-26839

Polymer Matrix Composites IV-A-28797,
V-A-28253, V]-A-26667, VI-C-28560, VI-C-28938

Polymer Mixtures II-E-28153

Polymer Reduction VI-A-26449

Polymer Synthesis V]_C-28900, [X-A-6307

Polymeric Coatings Vl-A-26667

Polymeric Proteins III-C-28972

Polymerization II-E-26441, II-E-27440

II-E-28373, II-E-28631, JI-E-29627,

V-A-28222, VI-C-28900, VII-A-28504

Polymers

Adbesion V1-A-27624

Aging VI-A-27037

Biocatalytic Processing III-C-28043

Catalytic Properties Il-E-28675

Chemical Modification II-E-29627

Cracking VI-B-28780

Crystallization VI-A-28369, IX-D-601

Curing V-A-28222

Deformation V1-A-27037

Dielectric Properties II-E-27808

Effects on Laser Dyes II-B-27534

Electrical Properties I-B-29373,

$$
\text { VI-A-26449 }
$$

Extrusion VI-C_28560

Fracture Toughness VI-B-25424,

VI-B-28780, VI-C-28560

Hierarchical Structure IX-D-6011

Interfaces VI-A-27624, LX-B-6294

Manufacturing VI-C-28938

Mechanical Properties II-E-28314

Molecular Dynamics VI-A-27037

Molecular Properties VI-A-27863

Molecular Structure II-A-26748,

I]-E-28314, II-E-28631, II-E-28711

Molecular Weight II-E-28373

Penetration II-E-26072, II-E-26840, IV-A-25874

Permeability II-E-28711

Properties VI-C-28938

Surface Chemistry II-E-28711

Synthesis II-E-28675, II-E-28711

Tensile Properties VI-A-27037,

$$
\text { VI-C-28560 }
$$

Thermodynamic Properties II-D-27025,

I]-E-27808, [1-E-28153, 1]-E-2831

Polymetbylthiophene Films VI-A-26449

Polyoxometalates [1-A-28469

Polypeptides IX-B-5937

Polyphenyletheretherketone VI-A-26667

Polyphosphazenes II-E-27054, II-E-28631, II-E-28711

Polypropylenes VI-C-28560, VI-F-30337

Polypyridyl Complexes II-C-29538

Polysiloxanes II-E-27054

Polyspectra VIII-E-27917

Polystyrenes II-E-26072, II-E-26840,

II-E-28053, [1-E-28153, [1-E-28675,

VI-A-27624, VI-B-25424, VI-B-28780,

VI-C-25202, V]-C-26579, Vl-C-27373

Polyurethanes II-E-28314, V]-C-27373

Polyzirconosiloxane films VI-A-26667

Positron Annibilation VI-F-30337

Potassium I-D-28362

Potassium lons III-D-25752

Potassium Magnesium Fluorides I-D-26442

Potassium Nitrates I-D-27780

Potassium Tantalum Niobates I-D-28979, I-E-27273

Potassium Tantalum Nitrates I-E-29321

Potassium Titanyl Phosphates 1-B-27532

Potassium Vapor I-B-26899

Potassium Zinc Cblorides I-D-27780

Powder Metallurgy VI-C-29410

Power Combining VIII-C-26599,

VIII-C-28339

Power Plants II-D-30443

Power Spectra VIII-E-27994

Praseodymium 1-C-28955
Precipitation (Meteorology) VII-E-26982

Pressure Recovery II-D-30443

Pressure Regulation III-C-28011

Printed Circuits VII-C-27904

Probability IV-C-26993, IV-C-27574,

IV-C-27868, IV-C-27993, IV-C-28715

IV-C-28722, IV-C-28982

Programming Languages IV-B-28192

Projectiles l-D-28362, V-C-29771

Propane 1I-A-30289, V-C-27417

Propellants II-C-30094, II-D-27603,

II-D-28103, II-D-29596, V-C-26403,

V-C-26720, V-C-28310, V-C-28912

$\mathrm{V}-\mathrm{C}-2933$

Propene V-C-27417

Propyliodide [X-B-6089

Protective Coatings VI-A-26667

Protein Interactions III-C-28929

Proteins III-A-27494, III-C-27956,

[I]-C-28457, III-C-28669, III-C-28680

III-C-28972, III-D-26232, III-D-26582

IIl-F-29194, III-F-29537, [X-B-5937

Protocol Engineering VIII-E-28467,

VIII-E-29653

Protocype System Description Language IV-E-28950

Prototyping IV-D-28835

Pseudomonas putida III-B-28964

Pulsed Lasers II-D-28889, VIII-D-28070

Pulsed Power Plasma Devices I-B-29595

Pyrenes [X-D-5548

Pyrolysis II-A-26748, II-C-27775,

III-D-27603, II-D-27887, II-D-29596,

III-A-29332, III-D-25663, III-D-29300

Pyrometallurgy V]-C-30060

Pyro-optic Techniques [-B-28784

Quadratic State Regulators IV-D-28511 Quantum Confinement VIII-B-27443

Quantum Devices VIII-A-26896

Quantum Dots I-D-26682, IX-D-5884

Quantum Effects I-D-27458, VIII-B-28004

Quantum Electronics VilI-G-28326 •

Quantum Hydrodynamies [V-B-28799

Quantum Mechanics VIII-B-28406,

LX-A-6280

Quantum Optics I-D-28468, 1-E-25546

Quantum Scattering II-C-28767

Quantum Statistical Microdynamics 1-D-28468

Quantum Transport VII-B-28461 VIII-F́-30549

Quantum Wave Devices VII-B-27578, VIII-G-27554

Quantum Well Amplitude Modufators I-D-28271

Quantum Well Avalanche Photodiodes VII-F-30595

Quantum Well Base Transistors VIII-B-27883

Quantum Well Devices VIII-D-28070

Quantum Well Lasers VIIL-A-30090

Quantum Well Structures VII-A-26188

VIII-A-27298, VIII-A-28594,

VII-B-28646, VIII-G-25602, IX-F-6170

Quantum Wells 1-A-28996, I-D-26015,

[-D-27458, ]-D-28336, I-D-29572,

I-D-29578, I-D-29923, I-E-26974,

I-E-28356, VIII-A-26708,

VIII-A-26896, VII-B-26686,

VIII-B-26755, VIII-B-27443,

VIII-B-28461, VIII-B-28895,

VIII-F-28187, VIII-F-28922

VIII-G-28326

Quantum Wire Lasers VIII-F-28922

Quantum Wires Vill-B-28406,

VIII-B-29541, VII-F-28187

Quartz Resonators V-D-28155 
Quasi-Optical Power Combining VIIJ-C-28339

Quasi-Optical Signals VII-C-29523 Quinines [X-D-5548

Radar I-D-29212, IV-F-27524, IV-F-27620, VII-A-26902, VII-A-28772, VII-D-30387, VII-E-26982, VII-F-28099

Radar Cross Sections VI-D-28219

Radar Targets IV-E-28125

Radial Inflow Turbines IX-C-5824

Radiation Detection VIII-C-2679

Radiation Interactions I-B-26709

Radiation Signatures V-C-27469,

V-C-27480, V-C-28912

Radiation Sources I-D-26682

Radical Beams [-B-27646, VIII-F-28922

Radio Networks VIII-E-26823

Radio Systems VIII-E-27994

Radiolysis [l-D-30332

Radium [-B-26709

Radomes VIII-G-27554

Radon I-B-26709

Rainfall VII-A-26902, VII-A-28772,

VII-B-28344, VII-E-26982

Ram Accelerator Projectiles V-C-29771

Raman Seattering I-D-27780, I-E -25546 VIII-A-26188

Raman Spectroscopy I-E-26971,

III-D-25476, IX-A-6280

Rana IX-B-5937

Random Processes IV-C-29678

Rank Transformation Tests IV-C-26746

Rare Earth Intermetallic Alloys V1-D-29713

Rare Earth Metals I-D-29341

Rare Earths VI-D-28146

Ration Heaters III-A-28691

Reaction Intermediates IL-C-30094

Reaction Kinetics II-D-26256, II-D-27505

Reaction Processing VIII-F-28729

Reaction Rates II-B-28656, V-C-27469

Reactive Armor VI-C-26485

Reactive Polymers II-E-28373

Real Time l-E-28502, l-E-28592,

IV-B-29850, IV-C-27574, IV-F-26930,

[V-F-27524, [V-F-27817, V]-A-28369

Reasoning IV-E-29692

Receptors III-F-29170

Recognition IV-E-27567

Recursive Partitioning Methods IV-C-28679

Red Water Disposa! II-D-30332

Redox Catatysis []-A-28469

Redox Chemistry II-A-28486

Reduced Instruction Set Computers

VII-B-28325, VIII-B-28329

Refractivity II-A-26636, VII-F-29196,

VIII-A-26708, [X-D-5884, LX-F-6170

Refractory Materials VI-A-28509,

VI-C-29098, VI-C-29410

Regression Models IV_C-28679, [V-C-28722

Reliability IV_C-25706, IV-C-27868,

[V-F-27817, V]-D-27548,

VIII-B-28004

Remote File Systems IV-D-29396

Remote Sensing VII-E_28102

Repair Models IV-C-27868

Reproduction [V-E-29354

Residual Stresses VI-A-28509

Resins VI-C-28883

Resonance I-B-27766, I-D-27425, I-D-29375, V-D-25462, V-D-25630, V-D-27300

Resonant Tunneling 1-D-29923

Resonant Tunneling Diodes VIII-C-26599, VIII-G-25602
Resonators 1-D-28591, IV-A-26909, V-D-28155

Respiratory Rate III-E-29345

Retina III-F-29170

Rhenium Foils IX-B-6089

Rhenium Oxides DX-B-6089

Rheology III-A-27494, IV-A-28797

Rheometers II-E-28153

Rhodamine Dyes II-B-27534, IX-D-5548

Ribonucleic Acids III-C-29289,

$$
\text { III-D-28479 }
$$

River Basins VII-A-26902

River Hydraulics IX-E-6185

Robotics I-E-28258, IV-C-29740

IV-E-26779, IV-E-27567, IV-F-26811

V-D-26934, V-D-26995, V-D-27075

Rock Properties IX-E-6296

Rocket Exhaust V-C-27469, V-C-28912

Rocket Launch V-C-27469

Rocket Propellants V-C-26720

Rods V-D-26061

Rotary Wings V-B-25623, V-B-26631,

V-B-28002, V-D-25462, V-D-26061

V-D-28123, V-D-28493

Rotational Energy V-C-27864

Rotational Relaxation l-B-27888

Rotor Blades IV-C-29740, V-B-25461,

V-B-25623, V-B-26595, V-D-25327,

V-D-26061

Rotor Noise V-B-28002

Rotor Shafts V-D-25462

Rotor Tips V-B-25467

Rotor Wake V-B-25467, V-B-25623

Rotorcraft V-B-25461, V-B-25467,

V-D-25462, V-D-28493

Rotors V-D-28123, 1X-C-5824

Rough Surfaces VIL-D-27031,

V]I-D-27485, VII-D-27911,

VII-D-28219, VII-D-29404

VII-D-29633,

IX-F-5830

Rough Terrain VI-D-28219

Routing VIII-E-26823, VIII-E-27834

Rubber [I-E-26840

Rubidium I-D-28362

Rubidium Zinc Chlorides I-D-27780

Ruthenium II-C -29538

Rutbenium Clusters IX-A-6307

Ruthenium Complexes 11-B-26914

Rydberg States II-D-28565

Saccharomyces cerevisiae III-B-29097, IIL-C-29280

Saline Ice VII-B-27482

Salt Crystals III-F-29194

Salt Movement VII-B-2628]

Sands VII-C-24381

Sapphire Cerenkov Lasers 1-B-28531

Scanning Tunneling Microscopy VI-A-29037, VIIl-B-28508

Scattering VII-D-28219, VII-D-28717

VII-D-29205, VII-D-29404,

VII-D-29633, VII-G-26962

VII-G-28052, VII-G-28489,

VIII-B-26711, VIII-B-26755,

VIII-B-29541, VIII-C-27304,

VIII-C-27904

Scenario Analysis IV-C-27381

Scheduling IV-B-28071; IV-B-28192

IV-E-29354, IV-F-26802, IV-F-28060

Schottky Barriers II-C-27770,

VIII-B-26686

Schottky Contacts VII - G-28326

Schottky Diodes VIII-C-29523

Schrödinger Equations IV -A-27641

Schwann Cells III-D-25752

Scintillation IX-F-6458

Search Algoritbms IV-F-28408

Sediments III-B-26750, VI-A-27401,
IX-E-6185

Selenides I-D-27458, II-A-26748,

VIII-A-26761, VIII-G-28453

Self Ignition IV-A-28728

Self Tuning Control TV-D-28511

Semiconductor Devices IV-B-28716,

IV-B-28799, VIIL-B-28461,

VIII-F-29210

Semiconductor Dielectric Waveguides

VIII-C-2S339

Semiconductor Electronics VIII-H-29540

Semiconcuctor Film Lasers [-B-28531

Semiconductor Heterojunctions

VIII-A-26761, VIII-F-30500

Semiconductor Heterostructures VIII-F-30595

Semiconductor Lasers 1-D-28271,

I-D-28336, l-D-29572, I-E-28356

Semiconductor Materials IX-F-6170

Semiconductor Nanostructures

VIII-F-28187

Semiconductor Superlattices VIU-B-26686

Semiconductor Surfaces I-B-29391

Semiconductor Switches I-B-26257,

I-B-26821

Semiconđuctors

Alloys I-D-28464

Atomic Structure 1X-A-6280

Chemical Vapor Deposition [I-A-26748

Contacts VI-D-29677

Deposition 1-B-2856

Electrical Properties II-C-27770,

VIII-A-27298

Electron Emission 1-B-28569

Electronic Properties VIII-B-29036

Electronic Structure I-D-28464,

IX-A-6280

Etching I-B-28561

Heterostructures I-D-28464, I-D-29922, I-E-28356

Interfaces II-C-27770, [X-A-6280

Ionization VIII-B-26711

Magnetotransport Properties I-D-27458

Optical Properties I-D-28470

Optoelectronic Properties VIII-A-27298, VIII-A-30090

Piezoelectric Properties VIII-B-29616

Plasma Stabilities I-D-26682

Reliability VIII-B-28004

Scanning Tunneling Microscopy VIII-B-28508

Superlattices [-D-29923

Surface Decontamination IX-B-6305

Surface Properties II-C-27770,

VIII-B-28508, IX-A-6280

Transport Properties VIII-A-28347,

VIII-A-28594, VIII-B-26711,

VIII-B-28461, VIII-B-28646

Sensor Data Fusion I-E-28258

Sensors I-E-28356, Il-A-30164,

V-B-25461, VIII-G-25602

Sensory Processing N-E-27567

Sequential Acquisition Schemes

VII-E-27493

Shape Representation IV-F-26811

Shape Theory IV-F-26792

Shear Bands IV-A-28702, V-A-27409,

V-A-28283, IX-D-6148

Shear Deformation V-A-27315

Shear Stresses IV_C-27993

Shells (Structural Forms) V-A-28253,

$$
\text { V-D-25630 }
$$

Shock (Mechanics) II-D-27025

Shock Consolidation VI-E-28884

Shock Loading V-C-28310, V-C-29336

Vl-E-30124, VI-E-30352

Shock Tube Studies V-B-27062

V-C-29336

Shock Waves IV-B-28916, V-A-28307, 
V-B-27558, V-B-28293, V-C-27469, V-C-28310, VI-B-28608, VI-F-27364 Signal Anatysis IV-F-27524

Signal Computation VIII-E-26460

Signal Detection I-D-29212, IV-F-26792 VIII-E-26460

Signal Direction Finding VIII-E-27899

Signal Interception VIII-E-27754

Signal Processing [-D-28684, I-D-29212

I-D-29375, I-E-27586, III-F-29170,

IV-F-26792, IV-F-26802, JV-F-26930

V-F-27524, V-F-27620, IV-F-27817,

IV-F-28060, N-F-28476, VII-E-28102

VIII-E-26735, VIII-E-27076,

VIII-E-27886, VIII-G-275S4

Signal Processors I-E-27273

Silanes II-E-26441

Silica Glass IX-D-5548

Silicides VI-D-29282, VI-D-29677

Silicon

Crystal Defects VI-D-27764

Defects IX-A-6280

Electric Field Effects VI-A-29037

Electron Emission I-B-2856

Epitaxial Layers VIII-A-26761

Etching 1-B-28561

Ion Bombardment VIII-A-26708

Laminations V-D_28155

Light Emission VIII-G-2845

Nanocrystalline Powders VI-C-26673

Nitriding Vl-D-29282

Oridation VI-D-29282

Surface Atoms VI-A-29037

Surface Properties I-D-29576

Transport Properties VIII-B-26011 VIIJ-B-27443

Silicon Alloys V-C-28005

Silicon Carbides VI-A-29064, VI-B-28826,

VI-C-25202, VI-C-26673, VI-C-29410

V-E-28884, VIII-B-28508

Silicon Devices VID-B-28004, VIII-E-28387

Siliçon Dioxide II-A-27600, VI-B-28577, VIII-B-27443

Silicon Germanides VIII-A-26896, VIII-F-28729

Silicon Germanium Alloys VII-G-28453

Silicon Germanium Films VIII-G-28326

Silicon Ions VI-A-25833

Silicon Nitride Matrix Composites

VI-C-25202

Silicon Nitrides V-C-26673, VI-E-28884

Silicon Oxides [-A-28996

Silicon Substrates VI-A-28509,

VI-D-26601, IX-F-6291

Silk III-C-28457

Siloxanes II-E-2644

Sitver IX-D-5884

Silver Alloys VI-C-29524

Simulation VIILE-28297

Simulation Networks IV-F-29412

Single Crystal Films VI-D-28553

Single Crystals VIII-F-27865

Singular Value Decomposition

IV-F-26930, IV-F-27817

Sintering VI-B-28577, VI-C-26123,

VI-C-29326

Slurries II-D-30230

Swart Materials VI-B-28608, Vl-C-28900

Stmart Structures V-B-25461

Smell III-F-2676?

Smoothing VI-D-29633

Snow VII-B-28040

Snow Pack VII-B-28344, 1X-E-6185

Sodium I-D-28362

Sodium Barbital III-D-27916

Sodium Cbloride II-D-28371, III-F-29194

Sodium Chloride Solutions VI-A-26678

Sodium Nitrate UI-F-29194
Sodium Sals V-B-25424

Sodium Sulfate []-D-28371

Software Engineering IV-E-28950

Software Maintenance IV-E-28328

Soil Moisture VII-A-27471

Soil Properties III-B-26750, VIII-G-27554

Solder Joints V]-D-29042

Solid Mechanics IV-A-28086, [X-A-6078

Solid Polyelectrolytes II-E-27054

Solid Propellants II-C-30094, II-D-27603,

1]-D-28103, II-D-29596, V-C-28912

Solid State Devices I-E-27556

Solid State Laser Materials 1-D-28472

Solid State Lasers I-B-28531, I-D-27425,

]-E-28348, LX-D-5884

Solid State Plasmas I-D-26682

Solid State Reactions VI-C-26571

Solidification V-A-29040, VI-C-27472,

$$
\text { VI-C-29410 }
$$

Solitons l-D-29375, l-E-28356

Solubilities II-D-28371

Solute Transport VIl-A-28504

Solutions IX-B-6305, IX-B-6674

Solvent Effects II-B-27534

Soot Formation V-C-28898, V_C-29302

Sorting Algoritbms IV-B-28071

Sound (Noise) 1-E-28592

Space Stations IV-D-28511

Spalling VI-B-28480

Sparse Matrix Algorithms IV-F-28408

Sparse Matrix Factorization IV-B-28377

Specific Heat V-A-27538

Spectral Hole Burning I-E-28356

Spectroscopy 1-B-27508, I-B-27646

I-C-28955, 1-D-26442, I-D-29578,

II-C-30094, III-A-29332, III-D-29300,

V1-A-26400, VI-A-29037, VI-C-29524 IX-D-5884

Speecb Recognition IV-F-26811

Spheres VII-D-28717, VII-D-29205

Spider Silk III-C-2845?

Spider Venom III-F-28775

Spinning Viscous Fluids IV-A-28797

Spline Approximation IV-B-27690

Spore Protease 1II-C-27956

Spores III-A-28022, [II-C-27956,

III-D-25476

Sporulation HI-C-27956

Spread Spectrum VII-E-2799

Sputtering I-D-28979, V]-A-26427,

VI-A-27552, VIII-C-26791

Squid III-D-25752

Stability IV-A-28514, IV-C-28905,

IV-D-27044, V-B-25467, V-D-25327,

V-D-25462, V-D-26061, V-D-28123, VIL-F-28664

Stacking Faults VI-D-29282

Stall V-B-25461, V-B-25467, V-B-26631,

V-B-27894, V-B-28159, V-B-29049,

V-D-28123, V-D-28493

Starcb III-A-27494

Stark Ladders I-D-27458

State Regulators N-D-28511

Statistical Data Analysis IV_C-27574

Statistical Estimation IV-C-27862

Statistics IV-C-27868, IV-C-28722

IV_C-28982, VII-D-30387

Steel Matrix Composites V1-A-26400

Steels V-A-27409, V-A-28283,

VI-A-25833, VI-A-26667,

VI-A-27552, VI-B-26825, VI-B-27163

VI-B-27407, VI-C-27472

Sterols III-B-29097

Stochastic Comparison Algorithm

$$
\text { IV-F-29190 }
$$

Stochastic Control IV-C-28715, IX-A-6366

Stochastic Differential Equations

IV-C-29297

Stochastic Harmonic Oscillators
IV-C -28905

Stochastic Learning IV-C-28722

Stochastic Numerical Metbods IV-B-28916

Stochastic Processes IV-C-26394,

IV-C-28686, IV-C-29297, N-F-26792

IV-F-26811, IV-F-28994, IV-F-29031,

VII-F-27995

Stocbastic Systems IV_C-28686

Stochastic Waves IV-B-29808, IV-C-28905

Storm Rainfall VII-A-28772

Strain (Mechanics) II-B-28013,

V-A-29543, VI-D-29042, VI-D-29282,

VI-E-30352, VIIL-G-28453

Strain Energy V-D-28399

Strain Fields V-A-28222

Strain Induced Polarization Effects

VIII-B-29616

Strain Rate V-A-26443, VI-C-29410,

VI-F-29647, [X-D-6148

Stream Beds DX-E-6185

Stress Analysis V-A-25345

Stress Concentration IV-A-27433

Stress Corrosion Cracking VI-A-25833

Stress Fields V-A-28222 VI-B-27163

Stress Sensitivity IV-A-26909

Stress Waves [V-B-30297

Stresses IV-A-27557, IV-A-28514,

IV-A-29351, IV-C-27993, V-A-26908,

V-A-29543, V-C-26720, V-A-28509,

VI-B-28480, VI-E-30488,

VII-C-24381,

IX-A 6078

Stripline Array Antennas VIII-C-28151

Strob Formalism IV-A-28606

Strontium Barium Niobates I-E-26971

Strontium Fluorides I-D-26442

Strontium lons II-A-28402

Structural Design V-D-29029

Structured Programming IV-B-29530

Styrenes II-E-28525

Submillimeter Waves [-B-27888,

I-B-29324, VIII-C-28483,

VIII-C-29523

Subsoils III-B-26750

Substrate Preparation VIII-F-28922

Sugar III-A-27494

Sulfates I-D-27780, II-D-28371

Sulfides I-D-28336, L-D-28852

II-A-26748, IJ-B-26914, III-C-29195, IX-D-5884

Sulfur II-C-26822

Sulfur Compounds II-C-26822, II-D-27505

Sulfur Hexafluoride [I]-C-28043

Super Hydrides II-B-28528

Supercapacitors II-A-26636

Supercomputers VII-D-28219

VIII-B-28325

Superconducting Films I-D-26996,

VI-A-29037

Superconductive Optoelectronis

I-B-29184

Superconductivity II-A-28655

Supercanductors II-A-29708, II-E-26072

VIII-G-28326, IX-A-6280

Supercritical Fluid Extraction VI-C-30095

Supercritical Fluids III-C-28043

Supercritical Water II-D-27505,

II-D-28371, [1-D-30230

Superfluorescence I-B-30423

Superlattices I-D-26015, 1-D-27458,

I-D-28684, I-D-29375, I-D-29923,

VI-D-26729, VI-D-29606,

VIII-A-26188, VII-A-26444,

VIII-A-26761, VIII-A-26896,

VIII-A-28335, VIII-B-26686,

VIII-B-26755, VIII-D-28070,

VIII-F-28922, VIII-F-30595,

VIII-G-28453, IX-A-6280

Superplastic Ceramics VI-B-28761 
Supersonic Flow V-B-27558, V-B-30113 Superstructures VIII-A-26896

Supraoperonic Clustering III-B-2896-

Surface A coustic Wave Devices II-A-3016 Surface Chemistry II-C-26822, II-C-29227 [I-E-28711

Surface Impedance VII]_G-27554

Surface Measurements VII-D-29360

Surface Modifications II-C-26126

VI-A-25833

Surface Properties I-B-29391, I-D-29576,

II-C-27770, II-E-28795, VI-A-26400,

VIII-B-28345, VIII-B-28508,

VIII-F-26898, VIII-G-28453,

[X-A-6280,

IX-B-6305

Surface Scattering IX-B-6089

Surfactants Il-A-30164, II-B-28814, II]-B-26750

Suspensions IV-A-28797

Swept Sine Excitation V-D-27300

Switching I-E-26971

Switching Networks I-E-26695, VIII-E-27751

Synaptic Plasticity III-F-29170

Syntax VIII-E-28467

Synthesis (Chemistry) II-E-27440, II-E-28314, II-E-28711

Synthetic Apertures IV-F-27620

System Design IV-D-28740

System Failure IV-C-27868

TCP/IP Networks IV-D-29396

Tactical Switched Network Systems VIII-E-27751

Tantalates VIII-A-26708

Tantalum J-B-26959

Tantalum lons VI-A-25833

Tantalum Silicides VI-D-29677

Target Acquisition V-D-27075

Target Classification IV-F-26811

Target Recognition IV-E-28125, VII-E-27464

Target Tracking IV-F-29349

Techpology Transfer [V-E-28950

Tellurides

Ion Bombardment V1U-A-26708 Monte Carlo Simulation VIII-A-28347 Properties II-A-26748 Surface Properties VIII-B-28508 Transitions I-D_27458 Transport Properties VIII-A-28347 Vacancy Complexes VI-D-27846

Temperature III-E-29345, V-C-28912, VII-F-29196

Tensile Strength VI-A-27037, VI-C-28560

Tensile Stresses [V_C-27993

Tensile Tests VI-B-25424

Terbium VI-D-28146

Terbium Ions I-D-26996

Terrain VII-A-27471, VIL-D-28219, VII-D-30387

Tetanus Toxins III-C-27890

Tetraethyl Lead IX-B-6089

Tetrahydrofurans II-E-28153

Text to Speech Conversion IV-F-26811

Textures III-A-27494, IV-E-26487

Thallium Alloys [V-A-28987

Thermal Adaptation IEl-A-28022

Thermal Conductivity V-C-27469

Thermal Conversion Efficiency I-B-28784

Thermal Decomposition II-D-27603

II-D-27887, II-D-29596, III-D-29300, VIII-F-26898

Thermal Equilibria VI-D-26729

Thermal Excitation II-C-29227

Thermal Expansion V-A-27538

Thermal Fatigue VI-D-29042

Thermal Focusing [-E-2697]
Thermal Fragmentation Il-C-26126

Thermal Imaging Systems VIII-C-25557

Thermal Imidization VI-C-26571

Thermal Lenses I-E-26971

Thermal Oxidation Il-A-28469

Thermal Properties I-D-28684, II-D-27025, II-E-26072, II-E-27808

Thermal Shock Resistance Vl $\_$C-29098

Thermal Stability V]-C-28005

Thermal Wave Imaging V1-B-28780

Thermals VII-F-26491

Thermistors V1-D-27548

Thermochemical Modeling V_C-27469

Thermochemical Transport Models $\mathrm{V}-\mathrm{C}-28310$

Thermodynamic Properties II-E-28153, I1-E-28314, III-F-27634, NV-A-27580, IV-A-28514, TV_F-28994, VI-D-29677, IX-B-6674

Thermoelastic Phase Transformations V-A-27538

Thermoelectric Properties VI-D-29606

Thermomechanical Properties VI-C-29098

Thermomechanics IV-F-28994, V-A-27538

Thermoplastic Composites Vl-C-28560, VI-F-30337

Thermoplastics V-A-28253, VI-A-28369, V1-C-28560, VI-C-28883

Thermosetting Resins V-A-28222 VI-C-28883

Thermotropic Liquid Crystal Polymers VI-F-30337

Thin Film Devices [-D-28852

Thin Films 1-B-26959, 1-B-27646, I-B-29184, 1-D-26996, [-D-28336, I-D-28362, I-D-28470, I-D-28684, I-D-28979, I-D-29375, I-E-26971, I-E-28526, II-B-26284, II-B-26914, II-C-27770, [I-D-27603, II-E-26072, II-E-28675, V-D-28155, VI-A-26400, V]-A-26427, V]-A-27552, VI-A-27863, VI-A-28509, VI-A-29037, VI-D-26601 VI-D-26883, VI-D-27605, VI-D-28553, VI-D-29218, VI-D-29606, VI-F-27810

VII-B-27316, VII-D-27031,

VIII-A-27298, VIII-A-28351

VIII-B-28345, VIII-C-26791,

VIII-G-25602, VIII-G-28326,

VIII-G-28453, IX-D-5548, IX-D 5884

Thioethers II-A-28469, II-B-26914

Thiols II-C-27770

Thulium 1-D-28472

Thyratrons I-B-26257

Time Delay Systems I-E-28592

Time Dependent Problems IV-B-3029?

Time Evolution IV-A-27580

Time Series IV_C-27868

Time Series Analysis I-D-29212 IV-C-26394, IV-C-26993, IV-C-28743 IV-F-26792, VIII-E-27917

Time Series Models [V-C-28743

Time Varying System Design [V-D-28740

Tin Alloys VI-D-29042

Titanates 1-E-26971, I-E-28526,

VI-D-27548, VIILC-C-26791

Titanium VI-A-26449, VI-B-26825

Titanium Alloys VI-A-26806, VI-C-27472,

VI-C-28005, V1-D-28146

Titanium Aluminide VI-A-25833

Titanium Borides VI-C-26392 VI-C-28368, VI-E-30488

Titanium Carbides VI-C-26392 VI-C-26673, VI-C-28368

Titanium Ions VI-A-25833

Titanium Nitrides VI-C-26673

Titanium Oxides VI-C-26673, IX-B-6305

Toluenes II-E-26840, II-E-28675,

VI-C-30095

Tomography IV-F-26811, IV-F-28476,
VII-E-26982

Topographic Maps IV-F-26811

Topography VII-A-27471

Torsion V-A-27409

Tosylates II-B-26284

Total Internal Reflection l-E-26971

Toughening V-A-28253, VI-B-27163

Toxic Chemicals IX-B-6305

Toxins and Antitoxins III-C-27890, III-D-27916, In-F-28775

Tradeoff Modeling IV_C-27381

Transistors IV-B-28790, VII-A-26444,

VII-A-28335, VII-A-30090,

VIII-B-27883, VIII-B-28004,

VIII-B-28461, VIII-B-29036,

VIIL-B-29616, VIII-G-25602, VIII-G-28326

Transition Metal Alloys VI-D-29713

Tránsition Metal Nitride Coating VI-A-27552

Transition Metals I-D-29341

Translational Energy V-C-27864

Transmission Lines VIII-C-25045, VIII-C-26949, VIII-C-28151, VIII-C-29523

Transonic Flow V-B-27062

Transparency l-B-28978

Transport Mechanics VII-A-2740

Transport Properties I-D-27458,

I-D-28464, I-D-28852, I-D-29572,

II-E-26840, II-E-27054, IV-B-28716,

V-A-28222, V-C-27469, VIII-A-28347,

VIII-A-28594, VIII-B-26711,

VIII-B-27443, VIII-B-28406,

VIII-B-28646, VIII-B-29223,

VII-B-29541, VIII-E-28297,

VII-F-30549, VIII-G-25602

Tribology II-A-30446, VI-A-27863

Trimethylgallium VI-D-28612, VIII-F-26898

Trinitrotoluene II-D-30332

Tubes V-D-25462, V-D-28399

Tunable Laser Materials IX-D-5884

Tunable Lasers I-D-26442, I-D-27425

Tungsten Alloys VI-C-28639, VI-C-29326,

VI-E-28272, VI-E-28575, VI-F-29647

Tungsten [ons VI-A-25833, V1-A-26678

Tungsten Nitrene Complexes II-A-28353

Tungsten Nitrides II-A-28353

Tungsten Silicides VI-D-29677

Tunnel Diodes IV-B-28799

Turbines IX-C 5824

Turbulence IV-A-28766, IV-A-29351,

IV-B-28716, IV-F-30411, V-B-26863,

V-B-28159, V-B-28293, V-B-30113,

V-C-28427, VII-F-28954, IX-E-6185, [X-F-6458

Turbulent Boundary Layers V-B-26863, V-B-28293

Turbulent Diffusion Flames V-C-29302

Turbulent Eddies V-C-27018

Turbulent Flow V-C-28427

Turbulent Kinetic Energy VII-F-29196

Turbulent Microfronts VIL-F-2649]

Turbulent Mixing V-B-26863

Two Photon Excitation I-B-30423

Two Photon Lasers I-B-29015

Ultrasonic Spectroscopy VI-C-29524

Ultraviolet Laser Radiation I-B-29391

Ultraviolet Radiation 1-B-28499

Ultraviolet Radiation Resistance III-C-27956

Ultraviolet Signatures V-C-29534

Universal Cading IV-C-28722

Vacuum Microelectronic Devices I-B-28569 
Validation Schemes IV_C-29358

Vanadium Oxides VI-A-26427, IX-B-6305

Vanadium Silicides VI-D-29677

Vapor Phase Epitaxy VI-D-29750

Vaporization V-C-28427

Variational Analysis IV-C-26997

Vector Quantization IV-B-28071

Vegetation VII-D-28219

Velocimeters V-B-27558

Venom III-F-28775

Venusian Topography IV-F-26811

Vesicles II-B-28814, II-E-28675

Vibration IV-C-29740, V-A-26908, V-B-25461, V-B-25467, V-D-27300,

$$
\text { V-D-27510, V-D-28155 }
$$

Vibrational Cooling II-D-2702

Vibrational Energy V-C-27864

Vibrational Relaxation 1-B-27888

Vibrational Spectra II-A-28655

Video Transmission Techniques VIII-E-27751

Vinyl Pyridines I]-E-28153

Vinyinaphthalenes II-E-28053

Viscoelastic Materials IV-F-28994

Viscoelastic Properties IV-B-28535,

$$
\text { [X-A-6078 }
$$

Viscosity lI-E-28153, IV-A-27869,

$$
\text { IV-A-28797, V-C-27469 }
$$

Viscous Aerodynamics V-B-25461

Viscous Fluids IV-A-28797

Vision IV-F-26811

Visual Cortex III-F-29170

Visualization Methods IV-C-28309

Voltage VIII-B-28646

Vortex Loops V-B-26595

Vortices IV-B-28916, IV-F-28994,

V-B-25461, V-B-25623, V-B-28159,

V-B-28215, V-B-29049, V-C-26456,

V-C-27018

Voter Systems IV-F-29031

Wakes V-B-25467, V-B-25623,
V-B-28159, V-B-30113, V-D-28123

Waste Treatment II-D-28371

Water []-D-27505, I]-D-28371,

II-D-30230, III-A-27494,

VII-A-27401, VIL-A-27471

Water Activated Heat Generation

III-A-28691

Water Clouds VII-D-28717

Water Movement VII-B-26281

Water Penetration VII-B-28344

Wave Equations IV-B-28535, IV-C-28905

Wave Initiation IV-A-28728

Wave Propagation [-B-28499, IV-A-26909 IV-A-28728, IV-F-27620, IV-F-28994,

V-A-26908, V-D-26934, VI-E-30352,

VII-D-29205, VIII-C-28516

Wave Scattering VIIIC-27304

Waveforms I-E-25546, I-E-28592,

$$
\text { IV-F-27620 }
$$

Waveguides [-A-28996, ]-E-26695, 1-E-27273, VIII-A-26708,

VIII-A-28594, VIII-A-28674,

VII-C-25045 VIII-C-25339,

VIII-C-26599, VII-C-28151

VIII-C-28483, VIII-C-28516,

VIII-G-28925, IX-D-5884, IX-F-6170,

IX-F-6291, IX-F-6563

Wavelets IV-F-27524

Wear VI-A-27552

Wear Resistance VI-C-29098

Weather Fronts VIl-F-26491

Weather Radar VI-A-26902

Welding VI-C-28639, VI-C-29524

Welding Flux VI-C-30060

Weldments VI-C-29524, VI-C-30060

Whisker Reinforced Composites VI-B-28826

Whiskers (Single Crystals) VI-E-28884

Wind (Meteorology) VI-A-27401,

VII-E-28094, VII-E-28102,

VII-F-26491

Wind Tunnel Tests V-B-27558,
V-B-28215, V-B-28293

Wing - Body Juncture Flow V-B-28249

Wing Tip Vortices V-B-28159

X Rays I-B-28978

$X$-Ray Lasers 1-B-28499

$X$-Ray Scattering VI-A-28369

$X-R a y$ Sources I-B-28499

Xenon VI-A-26806

Xenopus III-D-26232, IX-B-5937

Xylene VI-C-25202

Yeasts III-B-29097, III-C-28361,

$$
\text { III-C-29289 }
$$

Yttrium Alloys VI-C-27472

Yttrium Aluminum Garnets I-D-28472, [-E-28348

Yttrium Barium Copper Oxide Films

I-B-29184

Yttrium lons VI-A-25833

Yttrium Iron Garnets I-D-29375,

VI-D-28141

Zeolites IX-B-6294

Zinc VI-D-26729

Zinc Alloys VI-D-29713

Zinc Cadmium Sulfides 1-D-28336

Zinc Diffusion I-A-28996

Zinc Oxides V-D-28155, DX-B-6305

Zinc Phosphates VI-A-26400

Zinc Selenides I-D-27458, VIII-A-26761

VIII-G-28453

Zinc Sulfides 1-D-28336, I-D-28852

Zinc Tellurides VIII-B-28508, IX-F-6563

Zirconium Alloys VI-C-29524

Zirconium Oxides VI-A-26806,

VI-B-26751, VI-B-28577

Zone Plate Antennas VIII-C-28516 